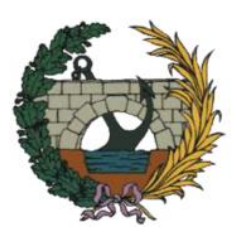

UNIVERSIDAD POLITÉCNICA DE MADRID

E.T.S. INGENIEROS DE CAMINOS, CANALES Y PUERTOS

\title{
MODELOS DE PREDICCIÓN DE ESFUERZOS HIDRODINÁMICOS Y SOCAVACIÓN APLICADOS \\ A INGENIERÍA OFFSHORE
}

TESIS DOCTORAL

\author{
Autor: Adrián Escobar Pastor \\ Ingeniero de Caminos, Canales y Puertos
}

Año 2017 

DEPARTAMENTO DE HIDRÁULICA, ENERGÍA Y MEDIO AMBIENTE

E.T.S. INGENIEROS DE CAMINOS, CANALES Y PUERTOS

\section{MODELOS DE PREDICCIÓN DE ESFUERZOS HIDRODINÁMICOS Y SOCAVACIÓN APLICADOS A INGENIERÍA OFFSHORE}

Autor: $\quad$ Adrián Escobar Pastor Ingeniero de Caminos, Canales y Puertos

Directores: José Santos López Gutiérrez

Dr. Ingeniero de Caminos, Canales y Puertos

María Dolores Esteban Pérez

Dr. Ingeniero de Caminos, Canales y Puertos

Año 2017 

TESIS DOCTORAL

\title{
MODELOS DE PREDICCIÓN DE ESFUERZOS HIDRODINÁMICOS Y SOCAVACIÓN APLICADOS A INGENIERÍA OFFSHORE
}

\author{
Autor: $\quad$ Adrián Escobar Pastor \\ Ingeniero de Caminos, Canales y Puertos \\ Directores: José Santos López Gutiérrez \\ Dr. Ingeniero de Caminos, Canales y Puertos \\ María Dolores Esteban Pérez \\ Dr. Ingeniero de Caminos, Canales y Puertos
}

\section{TRIBUNAL CALIFICADOR}

Presidente:

Secretario:

Vocal:

Vocal:

Vocal:

Acuerdan otorgarle la calificación de

Madrid, de de 2017 

A mis padres, Ana y Javier A mi hermano, Jorge A mi abuelo, Manuel A Laura 



\section{Agradecimientos}

La realización de la presente Tesis Doctoral ha supuesto una gran aventura en la que, al mirar atrás, veo a toda la gente que me ha acompañado a lo largo de este viaje y solo puedo sentir el más profundo agradecimiento y cariño hacia todos ellos por el apoyo y la ayuda que generosamente me han prestado. Como en todas las aventuras, ha habido muchos momentos buenos de euforia y gratificación y, también, momentos malos de duda, incertidumbre o frustración. Estos últimos han sido los más importantes dado que me han brindado la oportunidad de madurar personalmente y aprender a superar las adversidades, sean cuales sean, mediante esfuerzo, tenacidad y dedicación.

Esta aventura no podría haber llegado a buen puerto si no hubiera contado con el inestimable apoyo de mis tutores José Santos López y María Dolores Esteban y, el apoyo de Vicente Negro, a los que dedico mi más profundo agradecimiento por la gran confianza que han depositado en mí y, la paciencia y dedicación que me han regalado. Ellos me han dado luz cuando las márgenes del camino se perdían en la niebla, me han transmitido calma cuando parecía que me iban a fallar las fuerzas y me han guiado con generosidad y honestidad aportándome su empuje, su fuerza y su aliento, para que pudiera llegar a la cima de la colina. Únicamente pido el haber sido capaz de hacerles sentir orgullosos y espero haber estado a la altura de sus expectativas durante la realización de este trabajo.

Gracias a mis padres Ana y Javier, a mi abuelo Manuel y a mi hermano Jorge por haberme apoyado de manera incondicional durante todo este tiempo. Para mí sois un ejemplo, y vuestro amor y entrega me ha permitido ir dando todos y cada uno de los pasos que he seguido en mi vida y que me han llevado a convertirme en la persona que soy hoy. Gracias a Laura, por su amor, su apoyo y su paciencia. Durante estos años has creído en mí y me has enseñado una lección de actitud positiva ante la vida y los problemas, haciendo posible el que haya llegado al final de este gran reto.

Por último, gracias a todos mis amigos, familiares y compañeros, que han sabido prestarme su apoyo y atención cuando lo he necesitado, ayudándome a relativizar en los tiempos más difíciles y celebrando conmigo los mejores momentos. 



\section{ÍNDICE GENERAL}

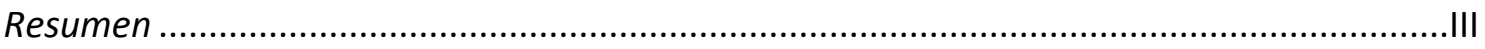

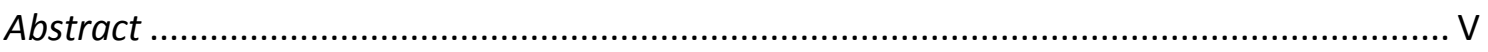

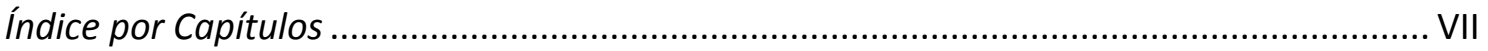

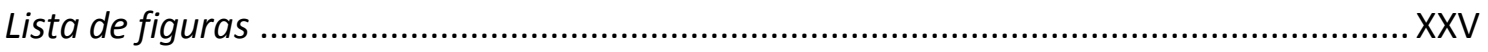

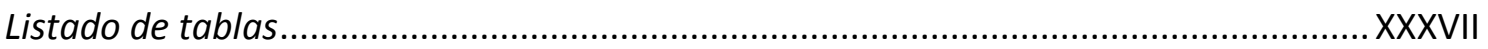

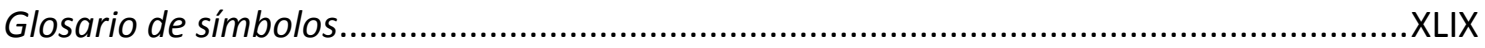


Modelos de predicción de esfuerzos hidrodinámicos y socavación aplicados a ingeniería offshore 


\section{Resumen}

Los parques eólicos offshore permiten generar una mayor cantidad de energía que sus equivalentes onshore. Sin embargo, el diseño, construcción, mantenimiento y operación de estos parques offshore, lleva asociado un importante incremento de costes debido a la dificultad de posicionar una estructura de este tipo en medio del mar en vez de en tierra firme. La tendencia actual que está sufriendo este campo es la de posicionar estructuras cada vez más grandes, a profundidades mayores y a una mayor distancia de la costa. Por lo tanto, es fundamental el poder establecer un modelo de cálculo preciso que permita estimar las acciones generadas por el oleaje sobre las estructuras y sus respectivas cimentaciones. De esta forma, la energía eólica offshore podrá continuar creciendo y se podrán instalar nuevos parques, cada vez, en zonas más profundas y alejadas de la costa, donde la producción energética será también mayor.

La acción del oleaje presenta el inconveniente de que su correcta determinación está plagada de incertidumbres que obligan al ingeniero diseñador a considerar escenarios de diseño sobredimensionados, en la mayor parte de los casos, como solución paliativa de las posibles imprecisiones del cálculo. Además, incluso aunque la caracterización de las cargas se realice de forma precisa, es necesario considerar todos los efectos de interacción entre el oleaje y la estructura, así como los problemas que surgirán sobre su cimentación. Efectos que tendrán como límite intrínseco su dificultad de cálculo y su importante coste computacional.

La presente Tesis Doctoral se centra en resolver todos estos problemas, en relación con el correcto cálculo de estructuras offshore, arrojando luz sobre las distintas incertidumbres que condicionan el problema y aportando un nuevo método avanzado de cálculo, que será comparado con el análisis tradicional, permitiendo dimensionar de forma más precisa todos los elementos de la estructura que va a ser el soporte del aerogenerador.

Este método de cálculo avanzado ha sido desarrollado como una herramienta de software compacta que ha permitido integrar todos los elementos intervinientes en el diseño de estructuras offshore frente a cargas hidrodinámicas en un único modelo numérico de gran 
eficiencia y potencia, estableciendo así una solución general, válida para el dominio físico al completo.

En la elaboración de esta herramienta se ha realizado una profunda revisión del estado del conocimiento, que ha permitido extractar las principales teorías actuantes para caracterizar cada uno de los fenómenos que intervienen en el proceso y, ha permitido detectar las deficiencias y debilidades que presentan cada una de ellas, definiendo así sus rangos válidos de aplicación. Por otro lado, ha sido posible el realizar la introducción de variables, nunca antes incluidas de forma explícita en este tipo de cálculos, como la temperatura y la salinidad del agua. Además, se ha desarrollado una nueva formulación de cálculo para incluir los efectos dinámicos vibratorios en el cálculo del comportamiento estructural para los primeros 25 modos de vibración. Esta nueva formulación y su implantación mediante algoritmos de alta eficiencia han permitido realizar la propuesta de un método de cálculo que permita incorporar en el análisis los efectos de acoplamiento entre el oleaje y la estructura, sin dilatar el tiempo de cálculo de una forma excesiva y sin perder potencia o precisión en los resultados obtenidos. Por último, se ha propuesto una modificación de una de las teorías más utilizadas y contrastadas para la evaluación de la máxima profundidad de socavación en equilibrio y se ha demostrado que dicha aportación ha reducido el error existente entre los valores teóricos de cálculo, y los valores procedentes de las mediciones reales realizadas en campo.

Además, la utilización de esta herramienta ha permitido posteriormente, la realización de una profunda reflexión comparativa, para detectar los principales errores y las limitaciones que presenta el método de cálculo más simplificado y tradicional, con respecto a este nuevo método de cálculo avanzado, así como la afección que dichas limitaciones presentan sobre las estructuras construidas hoy en día, con mayores diámetros y a mayores profundidades. 


\begin{abstract}
Offshore wind farms can generate more energy than their onshore counterparts. However, the design, construction, maintenance and operation of these offshore wind farms has associated a significant increase in costs due to the difficulty of building such a structure, in the middle of the sea instead of on land. The current trend that this field is undergoing it is to position ever larger structures, at greater depths and further away from the shore. Therefore, it is fundamental to be able to establish an accurate calculation model to estimate the actions generated by the sea waves on the structures and their foundations. In this way, the field of offshore wind energy will be able to keep growing, and new facilities may be installed, in deeper areas away from the coast, where energy production will also increase.
\end{abstract}

Wave action has the disadvantage that its correct determination is plagued by uncertainties that oblige the designer engineer to consider oversized design scenarios in most cases as a palliative solution to the possible imprecision of the calculation. In addition, even if the characterization of the loads is performed accurately, it is necessary to consider all the effects of interaction between the sea waves and the structure, as well as the problems that will arise on its foundations. Effects that will have as intrinsic limit their difficulty of calculation and their important computational cost.

The present Doctoral Thesis focuses on solving all these problems, in relation to the correct calculation of offshore structures, shedding light on the different uncertainties that condition the problem and providing a new advanced method of calculation, which will be compared with the traditional analysis, allowing to accurately design all the elements of the structure that will be the support of the wind turbine.

This advanced calculation method has been developed as a compact software tool that has allowed to integrate all the elements involved in the design of offshore structures against hydrodynamic loads in a single numerical model of high efficiency and power, thus establishing a general, valid solution for the entire physical domain. 
In the development of this tool, a thorough review of the state of knowledge has been carried out, which has allowed the main theories to be extracted to characterize each one of the phenomena that take part in the process, and has allowed to detect the deficiencies and weaknesses of each of them, defining their valid ranges of application. On the other hand, it has been possible to perform the introduction of variables, never explicitly included in such calculations, such as temperature and water salinity. In addition, a new calculation formulation has been developed to include dynamic vibrational effects in the calculation of structural behavior for the first 25 vibration modes. This new formulation and its implementation by means of high efficiency algorithms have made possible the proposal of a calculation method that allows incorporating in the analysis the effects of coupling between the sea waves and the structure, without increasing the calculation time very much and without losing power or precision in the results obtained. Finally, it has been proposed a modification of one of the most used and contrasted theories for the evaluation of the maximum depth of scouring in equilibrium and it has been demonstrated that this contribution has reduced the existing error between the theoretical values of calculation, and the values from actual field measurements.

In addition, the use of this tool has subsequently allowed a deep comparative analysis to detect the main errors and limitations of the most simplified and traditional method of calculation with respect to this new advanced calculation method, as well as the influence that these limitations present on the structures built today, with larger diameters and built at greater depths. 


\section{Índice por Capítulos}





\section{Capítulo 1}

\section{INTRODUCCIÓN, OBJETIVOS Y ESTRUCTURA DEL DOCUMENTO}

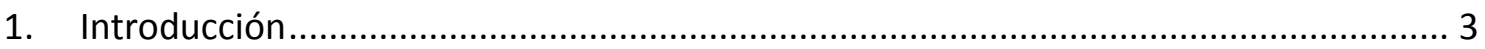

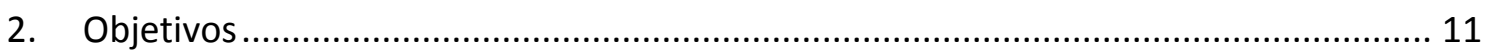

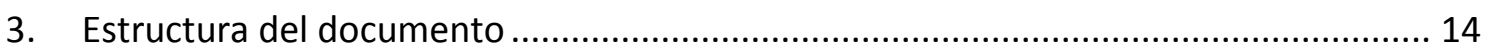





\section{Capítulo 2}

\section{ESTADO DEL CONOCIMIENTO}

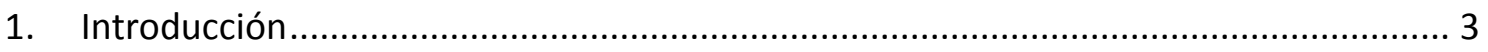

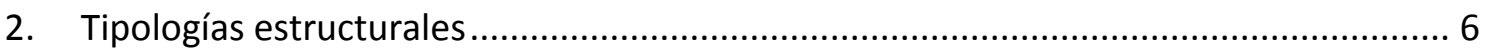

2.1. Estructuras apoyadas en el lecho marino ......................................................... 7

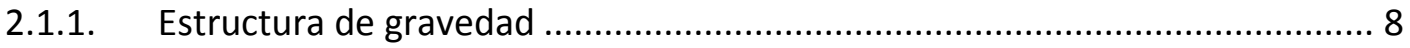

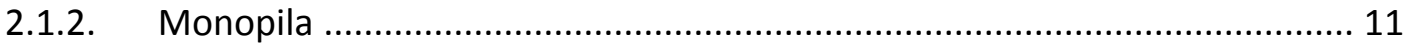

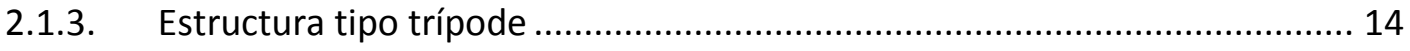

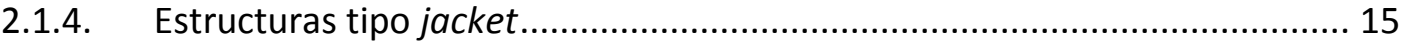

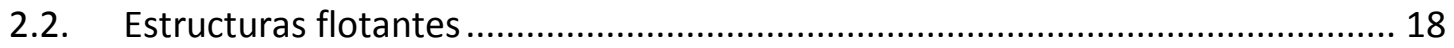

2.2.1. Lastre estabilizado boya-larguero ............................................................ 20

2.2.2. Plataforma con línea de fondeo estabilizada con tirantes (TLP) .................. 22

2.2.3. Plataforma con flotabilidad estabilizada .................................................. 24

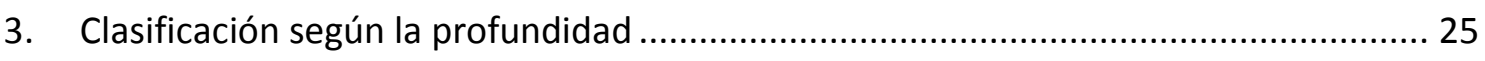

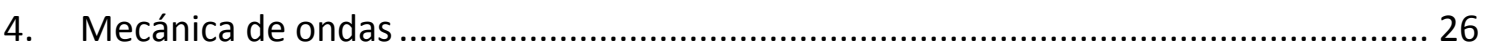

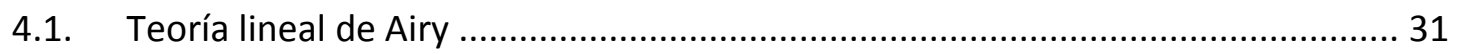

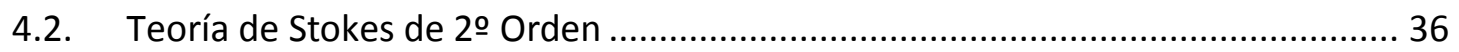

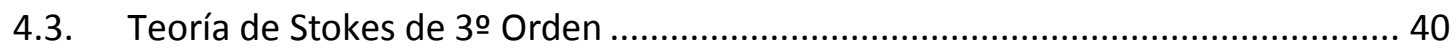

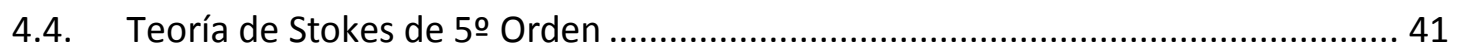

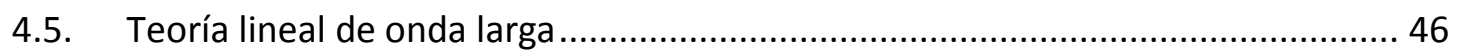

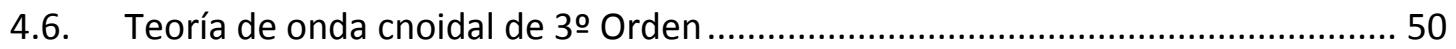

4.7. Teoría de onda cnoidal de 5o Orden (onda hiperbólica) .................................... 53

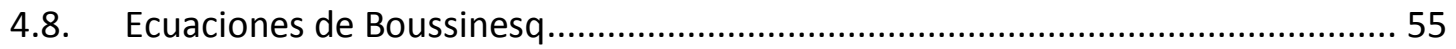

4.9. Función de corriente o Stream Function .......................................................... 58

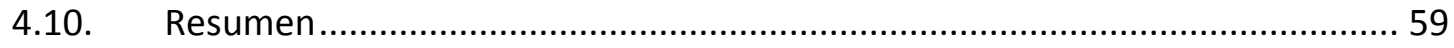




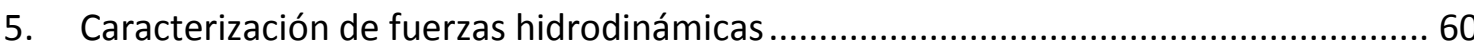

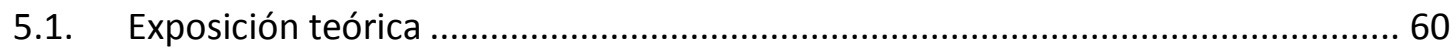

5.2. Acción de un fluido sobre un cuerpo sumergido ..............................................69

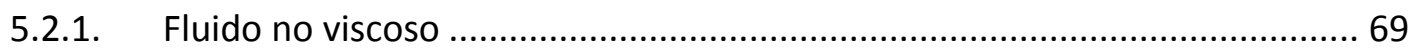

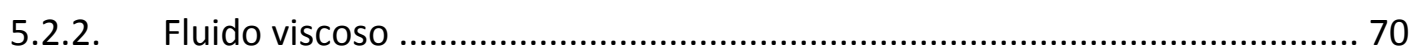

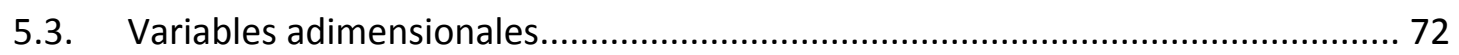

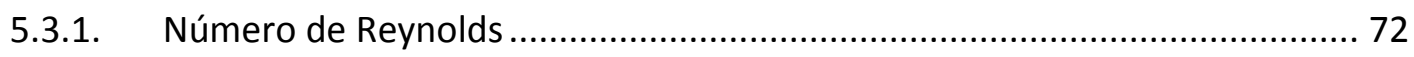

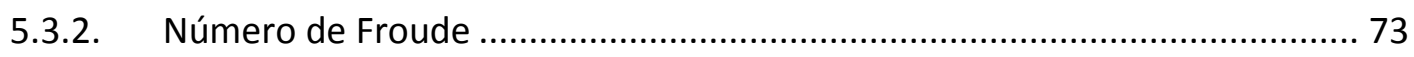

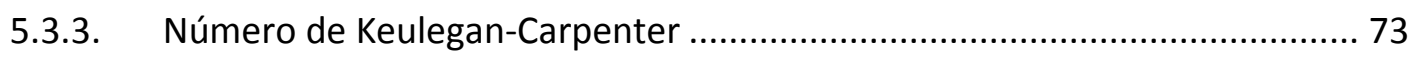

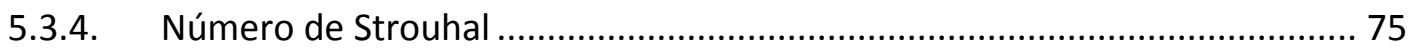

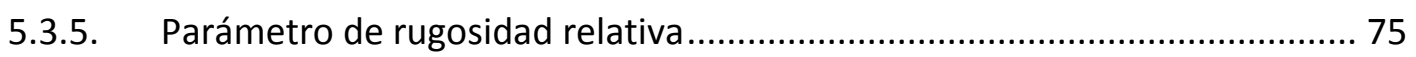

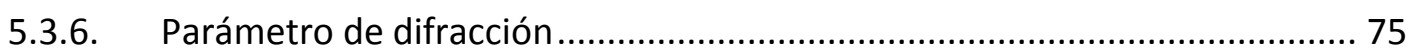

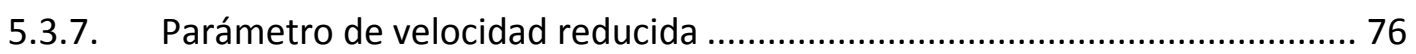

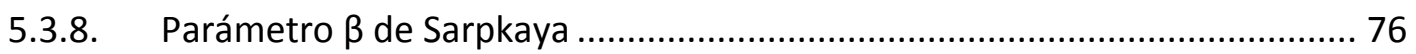

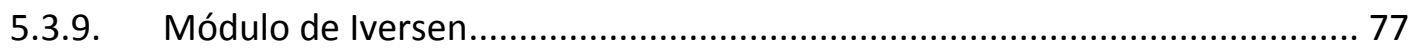

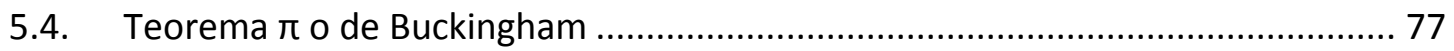

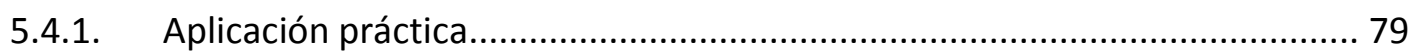

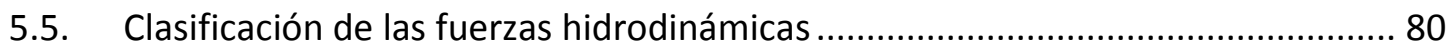

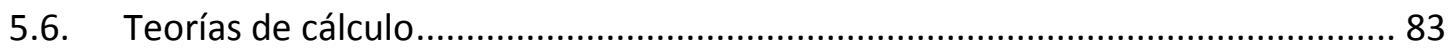

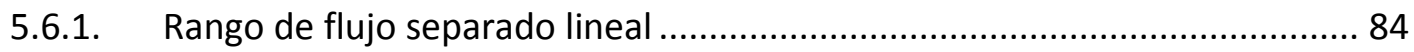

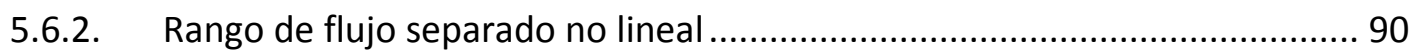

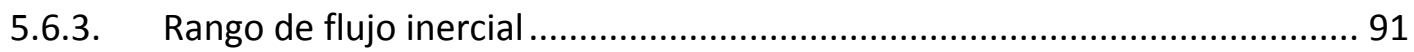

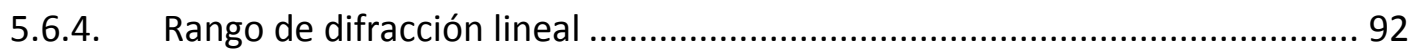

5.6.5. Rango de difracción no lineal ................................................................. 97

6. Descripción de las vibraciones sobre la estructura ................................................. 102

6.1. Vibraciones sobre una viga empotrada .......................................................... 102 
7. Fenómeno de socavación

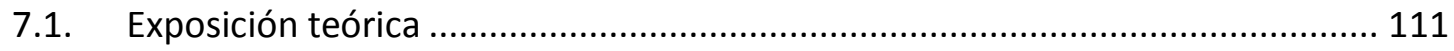

7.2. Socavación bajo condiciones de oleaje ............................................................. 116

7.3. Caracterización del fenómeno de la socavación a partir del campo de aceleraciones . 



\section{Capítulo 3}

\section{METODOLOGÍA DE LA INVESTIGACIÓN}

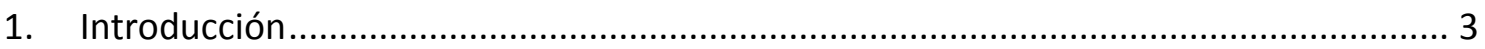

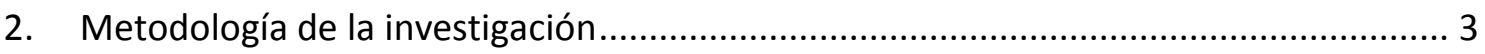

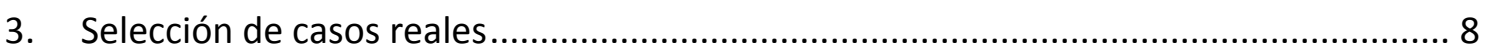

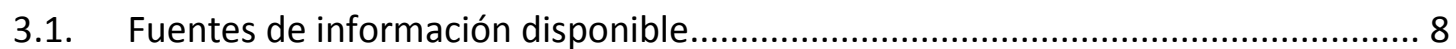

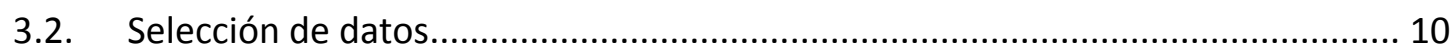

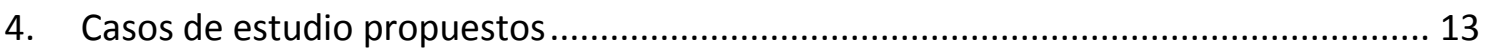

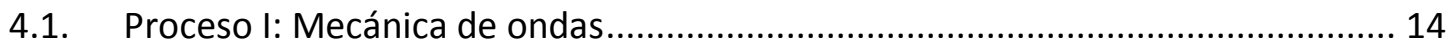

4.2. Proceso II: Cálculo de las fuerzas hidrodinámicas ........................................... 17

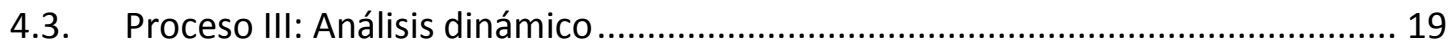

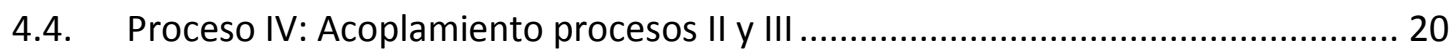

4.5. Proceso V: Comportamiento de la cimentación. ................................................. 21 



\section{Capítulo 4}

\section{DESARROLLO DE LA HERRAMIENTA}

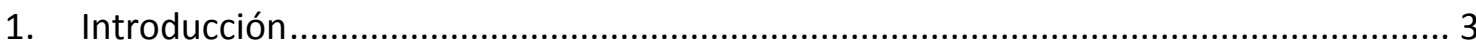

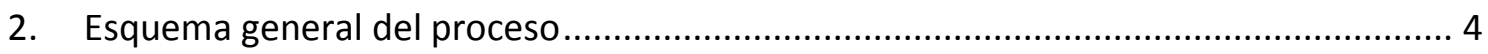

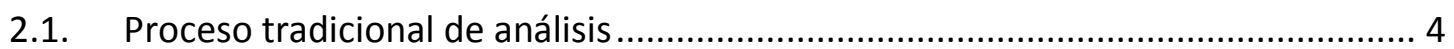

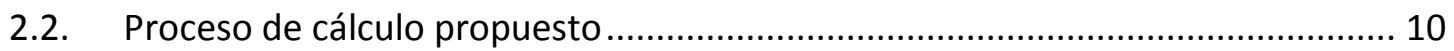

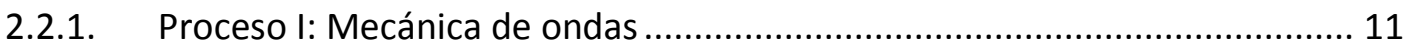

2.2.2. Proceso II: Cálculo de las fuerzas hidrodinámicas ........................................ 16

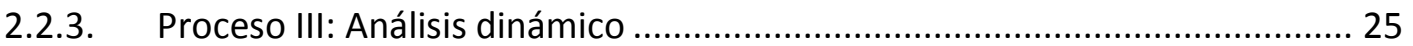

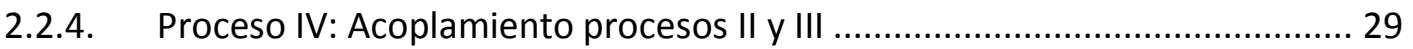

2.2.5. Proceso V: Comportamiento de la cimentación.......................................... 31

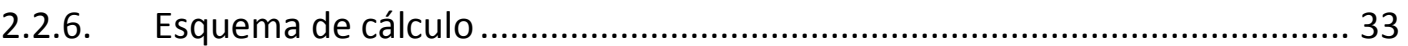





\section{$\underline{\text { Capítulo } 5}$}

\section{APLICACIÓN DE LA HERRAMIENTA A LOS CASOS DE ESTUDIO}

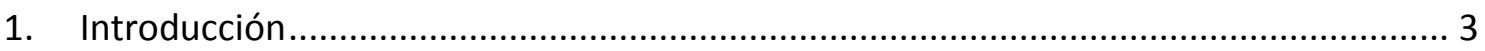

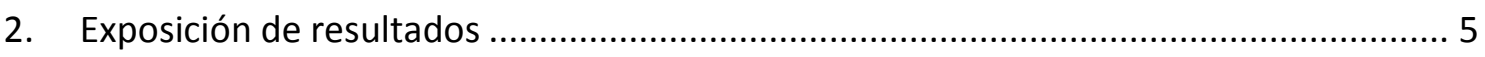

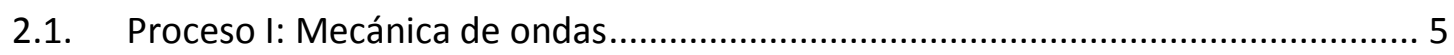

2.2. Proceso II: Cálculo de las fuerzas hidrodinámicas ............................................. 9

2.2.1. Comparación entre método tradicional y método avanzado ....................... 10

2.2.2. Análisis de influencia de la temperatura y la salinidad ............................. 17

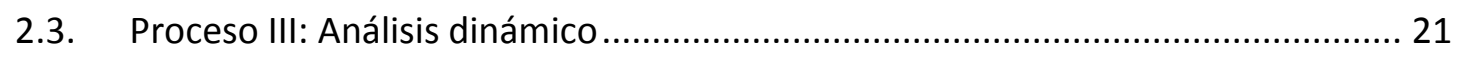

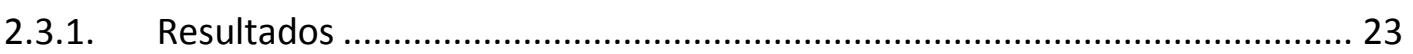

2.3.2. Comparación entre el método tradicional y el avanzado ............................. 23

2.3.3. Análisis de influencia de la temperatura y la salinidad ................................. 26

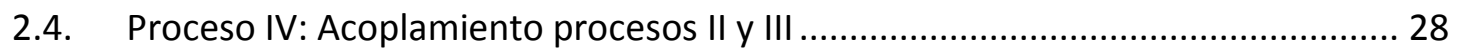

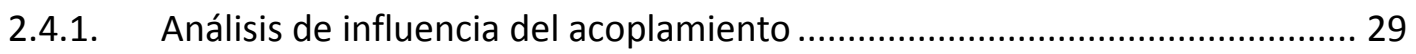

2.5. Proceso V: Comportamiento de la cimentación. .................................................. 31

2.5.1. Análisis de influencia del campo de aceleraciones sobre la socavación ........ 33

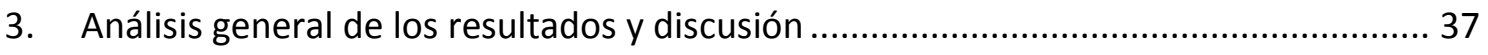

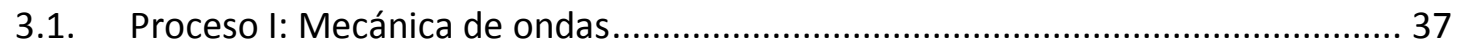

3.2. Proceso II: Cálculo de fuerzas hidrodinámicas .................................................... 40

3.3. Proceso III: Análisis dinámico de la estructura ..................................................... 46

3.4. Proceso IV: Acoplamiento de procesos II y III ....................................................... 51

3.5. Proceso V: Comportamiento de la cimentación ................................................ 53 



\section{Capítulo 6}

\section{CONCLUSIONES}

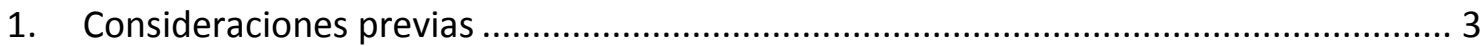

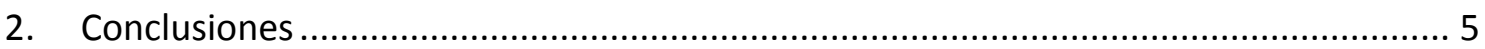

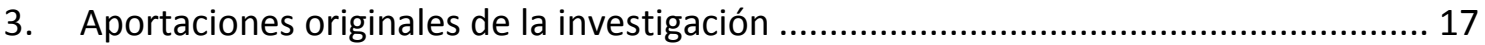

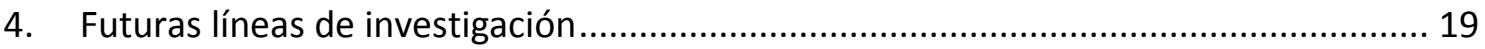





\section{$\underline{\text { Capítulo } 7}$}

\section{REFERENCIAS BIBLIOGRÁFICAS}

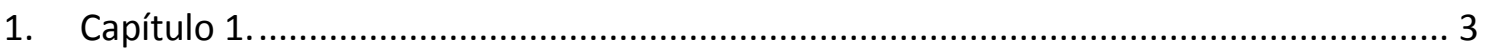

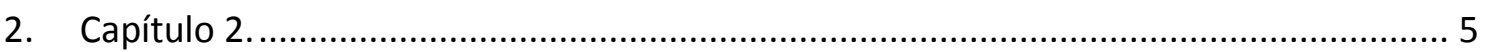

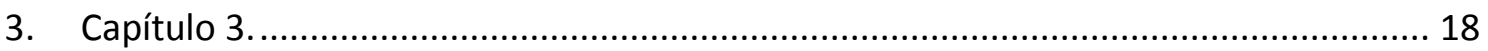

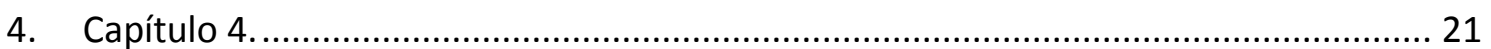

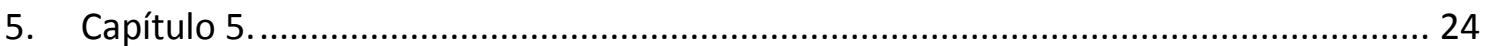





\section{Lista de figuras}





\section{Capítulo 1}

\section{ÍNDICE DE FIGURAS}

Figura 1. Evolución de la evolución de las distintas capacidades energéticas instaladas en

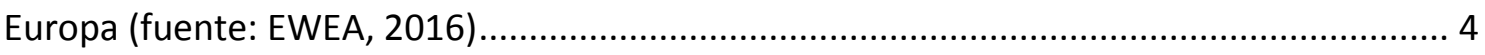

Figura 2. Distribución de la energía eólica offshore en Europa (fuente: EWEA, 2016) ..... 5

Figura 3. Comparativa entre distancia a la costa y profundidad, de los parque eólicos offshore europeos (fuente: EWEA, 2016) 7 



\section{$\underline{\text { Capítulo } 2}$}

\section{ÍNDICE DE FIGURAS}

Figura 1. Clasificación estructural de las estructuras offshore(fuente: elaboración propia)

Figura 2. Tipologías estructurales aplicadas a los aerogeneradores offshore (fuente: www.nrel.gov/) 7

Figura 3. Tipos de cimentación (fuente: www.offshorewindenergy.org) 8

Figura 4. Esquema básico de una cimentación de gravedad (fuente: Carrol et al., 2010) 9

Figura 5. Cimentación monopila (fuente: www.4coffshore.com) 12

Figura 6. Colocación de estructuras de tipo trípode en el parque eólico de Alpha Ventus (fuente: www.areva.com)

Figura 7. Cimentación tipo jacket en el parque de Thornton Bank, Bélgica (fuente: www.foundocean.com)

Figura 8. Pieza de transición en una estructura jacket, parque de Thornton Bank, Bélgica (fuente: www.offshorewind.biz)

Figura 9. Esquema de una cimentación tipo jacket (La figura de la izquierda es de fuente: DNV, 2013; la de la derecha: Esteban, 2009 )

Figura 10. Tipologías de estructuras flotantes (fuente: Butterfield et al., 2005)...... 19

Figura 11. Ejemplo de tipología flotante con lastre estabilizado (fuente: Quallen et al., 2016)

Figura 12. Imagen de la Hywind en Noruega (fuente: www.statoil.com/)

Figura 13. Tipología estabilización mediante línea de amarre (fuente: Thiagarajan et al., 2014)

Figura 14. Modelo TLP Wind de Iberdrola (fuente: Amate, 2014) ................................ 24

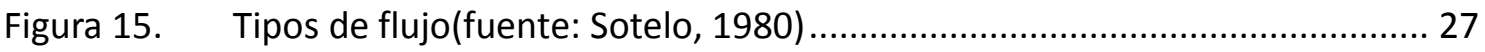

Figura 16. Clasificación de las distintas teorías de mecánica de ondas (fuente: Le Méhauté, 1976).... 30 
Figura 17. Esquema de la teoría lineal de ondas (fuente: elaboración propia).

Figura 18. Trayectorias de una partícula de agua. (fuente: Shore Protection Manual Vol.

$1,1984)$ 35

Figura 19. Perfil del oleaje (fuente: Brorsen, 2007) 37

Figura 20. Representación de onda cnoidal y onda solitaria (fuente: Hereman, 2011) 48

Figura 21. Clasificación de las teorías de mecánica de ondas y rango de aplicación (fuente: elaboración propia) 59

Figura 22. Verificación de la teoría de Lighthill (fuente: Sarpkaya, 2001) 65

Figura 23. Coeficiente $C_{D}$ en relación con Reynolds y K (fuente: Sarpkaya, 1981) .........66

Figura 24. Coeficiente $C_{M}$ en relación con Reynolds y K (fuente: Sarpkaya, 1981)........66

Figura 25. Coeficiente $C_{D}$ en relación con Reynolds y rugosidad (fuente: Sarpkaya, 1981)

Figura 26. Coeficiente $C_{D}$ en relación con Reynolds y rugosidad (fuente: Sarpkaya, 1981)

Figura 27. Líneas de flujo de corriente que varían su trayectoria a causa de un obstáculo (fuente: Vannucci, 2006)

Figura 28. Perfil de velocidades a partir de la superficie del obstáculo (fuente: Vannucci, 2006).

Figura 29. Clasificación de fuerzas hidrodinámicas (fuente: elaboración propia) 83

Figura 30. Obtención del coeficiente $C_{D}$, de acuerdo a los estudios realizados por distintos autores (fuente: Shore Protection Manual, 1975)

Figura 31. Esquema de viga empotrada(fuente: Sonalla, 1989) 102

Figura 32. Ábaco de Shields, 1936 (fuente: Matutano, 2013) 113

Figura 33. Curva de Soulsby (1997) como adaptación de la curva de Shields (1936). (Fuente: De Vos, 2008)

Figura 34. Representación de tiempo característico. (fuente: Sumer y Fredsoe, 2002) .... 


\section{Capítulo 3}

\section{ÍNDICE DE FIGURAS}

Figura 1. Metodología de la investigación (fuente: elaboración propia)

Figura 2. Localización de los parques eólicos offshore estudiados (fuente: elaboración propia)

Figura 3. Ejemplo del flujo de velocidades calculado por el software (fuente: elaboración propia)

Figura 4. Ejemplo del flujo de aceleraciones calculado por el software (fuente: elaboración propia) 16

Figura 5. Distribución de fuerzas y localización de puntos pésimos (fuente: elaboración propia) 18

Figura 6. Movimiento de la estructura según el método tradicional con análisis estático y según el método avanzado con análisis dinámico (fuente: elaboración propia) 20 



\section{Capítulo 4}

\section{ÍNDICE DE FIGURAS}

Figura 1. Los cuatro pilares que definen el problema estudiado (fuente: elaboración propia)

Figura 2. Movimiento de la partículas según la Teoría de Airy (fuente: Shore Protection Manual Vol. 1 p. 2-17)

Figura 3. Perfiles de oleaje según distintas teorías de cálculo (fuente: elaboración propia) 5

Figura 4. Esfuerzos hidrodinámicos producidos sobre la pila (fuente: elaboración propia)

Figura 5. Clasificación de teorías de mecánica de ondas aplicadas a oleaje (fuente: Le Méhauté, 1976) 6

Figura 6. Estructura sin influencia sobre el flujo incidente (fuente: Bengt, 2011) ............ 7

Figura 7. Estructura con influencia sobre el oleaje incidente (fuente: Han et al., 2015) .. 7

Figura 8. Análisis estático de viga empotrada (fuente: www.codecogs.com) .................. 8

Figura 9. Modos de vibración de una viga empotrada(fuente: elaboración propia)......... 8

Figura 10. Esquema tradicional de cálculo para pilas (fuente: elaboración propia)....... 9

Figura 11. Procesos actuantes en el cálculo (fuente: elaboración propia) ..................... 10

Figura 12. Representación de los contornos del problema (fuente: elaboración propia)..

Figura 13. Proceso de cálculo (fuente: elaboración propia) ........................................ 29

Figura 14. Proceso de cálculo general propuesto (fuente: elaboración propia)............. 33 



\section{$\underline{\text { Capítulo } 5}$}

\section{ÍNDICE DE FIGURAS}

Figura 1. Velocidades para $\mathrm{H}=7,7 \mathrm{~m}, \mathrm{~h}=24,0 \mathrm{~m}$ y $\mathrm{T}=9,7 \mathrm{~s}$ según Stokes de 3o Orden .......... 5

Figura 2. Velocidades para $\mathrm{H}=7,7 \mathrm{~m}, \mathrm{~h}=24,0 \mathrm{~m}$ y $\mathrm{T}=9,7 \mathrm{~s}$ según teoría lineal .................... 6

Figura 3. Aceleraciones para $\mathrm{H}=7,7 \mathrm{~m}, \mathrm{~h}=24,0 \mathrm{~m}$ y $\mathrm{T}=9$, $7 \mathrm{~s}$ según Stokes de 3O Orden ....... 6

Figura 4. Aceleraciones para $\mathrm{H}=7,7 \mathrm{~m}, \mathrm{~h}=24,0 \mathrm{~m}$ y $\mathrm{T}=9$, $7 \mathrm{~s}$ según teoría lineal .................. 6

Figura 5. Momento flector máximo según método avanzado para $H=7,7 m, h=24,0 m$,

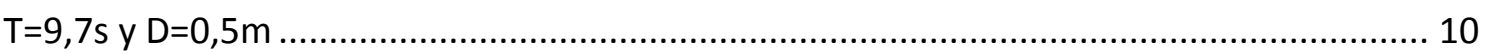

Figura 6. Momento flector máximo según método tradicional para $H=7,7 m, h=24,0 m$,

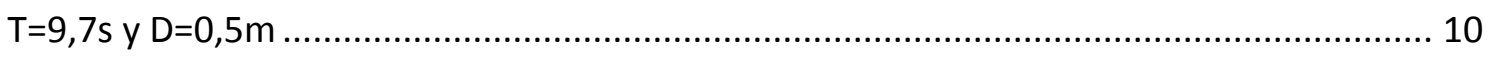

Figura 7. Fuerza en superficie, mostrada a lo largo del tiempo.................................... 20

Figura 8. Esquema del método de cálculo según el proceso III .................................... 22

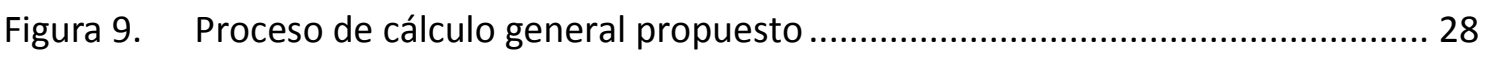

Figura 10. Comparación de velocidades entre teorías de ondas ................................... 37

Figura 11. Comparación de aceleraciones entre teorías de ondas .............................. 39

Figura 12. Comparación de fuerza total entre método avanzado y tradicional, en relación al diámetro

Figura 13. Comparación de fuerzas de arrastre entre método avanzado y tradicional, en relación al diámetro

Figura 14. Comparación de fuerzas de inercia entre método avanzado y tradicional, en relación al diámetro

Figura 15. Variación de fuerza total para variaciones de temperatura y salinidad, en relación al diámetro

Figura 16. Variación de los esfuerzos en el lecho marino, en relación al diámetro ...... 47

Figura 17. Variación de los desplazamientos en superficie, en relación al diámetro .... 49 
Modelos de predicción de esfuerzos hidrodinámicos y socavación aplicados a ingeniería offshore

Figura 18. Variación de los esfuerzos en la cimentación debido a la temperatura y la salinidad, en relación al diámetro 50

Figura 19. Variación de los movimientos de la estructura debido a la temperatura y la salinidad, en relación al diámetro 50

Figura 20. Variación de los esfuerzos en la cimentación debido a la consideración del acoplamiento, en relación al diámetro

Figura 21. Variación de la socavación entre el método avanzado y el método tradicional ambos con KC tradicional

Figura 22. Variación de la socavación entre el método avanzado utilizando KC tradicional y KC modificado 55

Figura 23. Comparación de la socavación máxima en equilibrio 56

Figura 24. Error generado en cada uno de las dos formulaciones de cálculo. 57 


\section{Listado de tablas}





\section{Capítulo 1}

\section{INDICE DE TABLAS}

Tabla 1. Porcentaje de capacidad offshore instalada en Europa (fuente: EWEA, 2016) .. 5 



\section{Capítulo 2}

\section{INDICE DE TABLAS}

Tabla 1. Profundidades máximas que se pueden alcanzar con cada una de las tipologías (fuente: elaboración propia) 25

Tabla 2. Teoría de Airy (fuente: Shore Protection Manual Vol. 1, 1984) 36

Tabla 3. Formulación de Stokes de 2o Orden (fuente: Chakrabarti, 2005) 40

Tabla 4. Números adimensionales que rigen el comportamiento del problema físico (fuente: elaboración propia) 80

Tabla 5. Clasificación de fuerzas hidrodinámicas (fuente: elaboración propia)..... 83

Tabla 6. Determinación del coeficiente $\mathrm{C}_{\mathrm{M}}$, de acuerdo a distintos autores (fuente: Shore Protection Manual, 1975) 88

Tabla 7. Primeros cinco modos de vibración de una viga (fuente: elaboración propia) ..... 



\section{Capítulo 3}

\section{INDICE DE TABLAS}

Tabla 1. Parques eólicos instalados en Europa, estudiados en la presente Tesis (fuente: Harris et al., 2010, Carroll et al., 2010, Whitehouse et al., 2011, y Matutano et al., 2015) .. 9 Tabla 2. Características de las cimentaciones (fuente: Harris et al., 2010, Carroll et al., 2010, Whitehouse et al., 2011, y Matutano et al., 2015) 10

Tabla 3. Variables principales de clima marítimo para período de retorno de 50 años (fuente: Harris et al., 2010, Carroll et al., 2010, Whitehouse et al., 2011, y Matutano et al., 2015)

Tabla 4. Parques eólicos offshore analizados (fuente: Harris et al., 2010, Carroll et al., 2010, Whitehouse et al., 2011, y Matutano et al., 2015)

Tabla 5. Número de casos a estudiar (fuente: elaboración propia) 13

Tabla 6. Variables principales de caracterización del oleaje (fuente: elaboración propia a partir de los estudios referenciados en el párrafo anterior) 



\section{Capítulo 4}

\section{INDICE DE TABLAS}

Tabla 1. Influencia de la salinidad y la temperatura sobre la densidad y la viscosidad del agua (fuente: elaboración propia a partir de Chakravarti, 2005) 15

Tabla 2. Clasificación de fuerzas hidrodinámicas (fuente: elaboración propia) 18

Tabla 3. Constantes vibratorias de una viga empotrada en un extremo (fuente: elaboración propia) 26 



\section{$\underline{\text { Capítulo } 5}$}

\section{INDICE DE TABLAS}

Tabla 1. Error de los resultados de velocidades entre la teoría lineal y las no lineales ... 7

Tabla 2. Error relativo de los resultados de velocidades entre la teoría lineal y las no

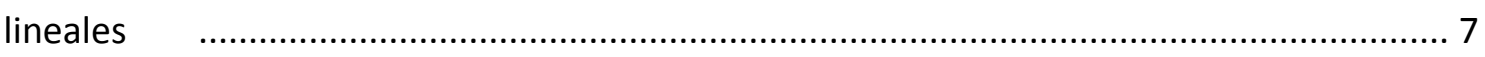

Tabla 3. Error de los resultados de aceleraciones entre la teoría lineal y las no lineales ...

Tabla 4. Error relativo de los resultados de aceleraciones entre la teoría lineal y las no

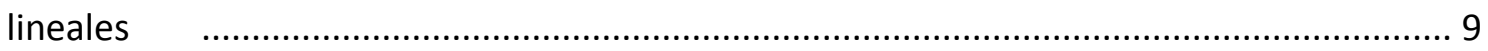

Tabla 5. Comparación entre método avanzado y tradicional para $\mathrm{T}^{\mathrm{a}}=1^{\circ} \mathrm{C}$ y $\mathrm{S}=0,0 \% \ldots 12$

Tabla 6. Comparación entre método avanzado y tradicional para $\mathrm{T}^{\mathrm{a}}=22^{\circ} \mathrm{C}$ y $\mathrm{S}=35,0 \% .13$

Tabla 7. Comparación a nivel relativo entre método avanzado y tradicional para $T^{a}=1^{\circ} \mathrm{C}$

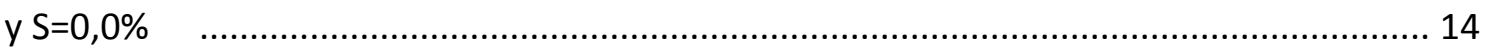

Tabla 8. Comparación a nivel relativo entre método avanzado y tradicional para $\mathrm{T} \underline{\mathrm{a}}=22^{\circ} \mathrm{C}$ y $\mathrm{S}=35,0 \%$ 15

Tabla 9. Comparación entre método avanzado y tradicional para $\mathrm{T}^{\mathrm{a}}=1^{\circ} \mathrm{C}$ y $\mathrm{S}=0,0 \% \ldots 16$

Tabla 10. Comparación entre método avanzado y tradicional para $\mathrm{T}^{\mathrm{a}}=22^{\circ} \mathrm{C}$ y $\mathrm{S}=35,0 \%$. 16

Tabla 11. Variación debida al cambio de temperatura y salinidad según el método avanzado

Tabla 12. Variación relativa debida al cambio de temperatura y salinidad según el método avanzado

Tabla 13. Variación debida al cambio de temperatura y salinidad según el método avanzado

Tabla 14. Rigideces utilizadas en los casos de cálculo

Tabla 15. Comparación entre método avanzado y tradicional para Temperatura de $1^{\circ} \mathrm{C}$ y Salinidad del 0,0\%. 
Tabla 16. Comparación entre método avanzado y tradicional para Temperatura de $22^{\circ} \mathrm{C}$ y Salinidad del $35 \%$

Tabla 17. Comparación entre método avanzado y tradicional para Temperatura de $1^{\circ} \mathrm{C}$ y Salinidad del 0,0\%

Tabla 18. Comparación entre método avanzado con acoplamiento y sin acoplamiento para Temperatura de $1^{\circ} \mathrm{C}$ y Salinidad del $0,0 \%$

Tabla 19. Comparación entre método avanzado con acoplamiento y sin acoplamiento para Temperatura de $22^{\circ} \mathrm{C}$ y Salinidad del $35 \%$

Tabla 20. Variables para el cálculo de la socavación

Tabla 21. Comparación entre el método avanzado con KC según fórmula tradicional y el método tradicional

Tabla 22. Comparación entre método avanzado de socavación y el método avanzado con KC según fórmula tradicional 35 
Glosario de símbolos 



\section{Glosario de símbolos}

$A\left[\mathrm{~m}^{2}\right]$

$\mathrm{a}[\mathrm{m}]$

$\mathrm{a}^{\prime}[\mathrm{m}]$

a。[adim.]

$\mathrm{a}_{\mathrm{x}}\left[\mathrm{m} / \mathrm{s}^{2}\right]$

$a_{r}\left[m / s^{2}\right]$

$a_{z}\left[\mathrm{~m} / \mathrm{s}^{2}\right]$

$\mathrm{C}_{\mathrm{A}}$ [adim.]

$C_{D}$ [adim.]

$\mathrm{C}_{\mathrm{M}}$ [adim.]

$\mathrm{c}[\mathrm{m} / \mathrm{s}]$

$\mathrm{c}^{(1)}[\mathrm{m} / \mathrm{s}]$

$c^{(2)}[\mathrm{m} / \mathrm{s}]$

$\mathrm{c}^{(3)}[\mathrm{m} / \mathrm{s}]$

cn

$\mathrm{D}[\mathrm{m}]$

$D^{\prime}$

$D_{S}[\mathrm{~m}]$

$D_{*}$ [adim.

$\mathrm{dn}$

$\mathrm{E}\left[\mathrm{N} / \mathrm{m}^{2}\right]$

EI $\left[N \cdot m^{2}\right]$

e [adim.]
Área de la sección de la estructura.

Mitad de la altura de ola, $\mathrm{H}=2 \mathrm{a}$.

Mitad del diámetro de la estructura, $\mathrm{D}=2 \mathrm{a}$ '.

Parámetro adimensional de escala aplicado a la amplitud de onda.

Componente horizontal de la aceleración de una partícula de agua.

Aceleración relativa entre un fluido y el objeto inmerso en él.

Componente vertical de la aceleración de una partícula de agua.

Coeficiente de masa añadida.

Coeficiente de arrastre.

Coeficiente de inercia.

Celeridad de la onda.

Componente de primer orden de la celeridad de la onda.

Componente de segundo orden de la celeridad de la onda.

Componente de tercer orden de la celeridad de la onda.

Función coseno elíptico de Jacobi

Diámetro de la estructura.

Tensor de estiramiento.

Diámetro medio de las partículas de sedimento.

Diámetro adimensional de las partículas de sedimento.

Función delta amplitud elíptica de Jacobi

Módulo de elasticidad de Young.

Módulo de rigidez a flexión de la estructura.

Número adimensional de la rugosidad relativa. 
$\mathrm{F}[\mathrm{N}]$

$\mathrm{F}_{\mathrm{D}}[\mathrm{N}]$

$\mathrm{F}_{\text {dif }}$ [adim.]

$\mathrm{F}_{\mathrm{R}}[$ adim.]

$\mathrm{f}_{\mathrm{c}}$ [adim.]

$f_{s}[1 / s]$

$f_{w}$ [adim.]

$\mathrm{g}\left[\mathrm{m} / \mathrm{s}^{2}\right]$

$\mathrm{H}[\mathrm{m}]$

$\mathrm{H}_{\mathrm{s}}[\mathrm{m}]$

$\mathrm{h}[\mathrm{m}]$

$\mathrm{h}_{\mathrm{o}}$ [adim.]

$\mathrm{h}_{\mathrm{R}}[\mathrm{m}]$

$I_{v}$ [adim.]

$J^{\prime}{ }_{1}$

KC [adim.]

K

$\mathrm{K}_{\mathrm{r}}[\mathrm{m}]$

$\mathrm{k}[\mathrm{rad} / \mathrm{m}]$

$\mathrm{k}_{\mathrm{o}}$ [adim.]

$\mathrm{L}[\mathrm{m}]$

I [m]

m [adim.]

m' [adim.]
Fuerza horizontal ejercida por el oleaje.

Fuerza de arrastre ejercida por el oleaje.

Número adimensional de la difracción.

Número de Froude.

Coeficiente de fricción adimensional inducido por una corriente continua.

Frecuencia de resonancia de la estructura.

Coeficiente de fricción adimensional inducido por el oleaje.

Aceleración de la gravedad.

Altura de ola.

Altura de ola significante en régimen extremal.

Calado.

Parámetro adimensional de escala aplicado a la profundidad.

Profundidad hidráulica.

Módulo de Iversen.

Función de Bessel de primer tipo.

Número de Keulegan-Carpenter.

Integral elíptica completa de primer orden.

Rugosidad relativa.

Número de onda.

Parámetro adimensional de escala aplicado al número de onda.

Longitud de onda.

Longitud de la estructura.

Parámetro de las funciones elípticas de Jacobi.

Masa adicional movilizada o masa hidrodinámica 


\begin{tabular}{|c|c|}
\hline $\mathrm{m}_{\text {est }}[\mathrm{kg} / \mathrm{m}]$ & Masa de la estructura por metro lineal. \\
\hline $\mathrm{p}\left[\mathrm{N} / \mathrm{m}^{2}\right]$ & Presión en el fluido. \\
\hline $\mathrm{p}_{\mathrm{atm}}\left[\mathrm{N} / \mathrm{m}^{2}\right]$ & Presión atmosférica. \\
\hline$Q[\mathrm{~m}]$ & Constante de Bernoulli. \\
\hline $\mathrm{R}[\mathrm{N}]$ & $\begin{array}{l}\text { Respuesta estructural de la estructura offshore sometida a la acción } \\
\text { del oleaje. }\end{array}$ \\
\hline Re [adim.] & Número de Reynolds. \\
\hline $\mathrm{S}[\%]$ & Salinidad del agua de mar. \\
\hline $\mathrm{S}_{\mathrm{e}}[\mathrm{m}]$ & Socavación en equilibrio. \\
\hline $\mathrm{S}_{\max }[\mathrm{m}]$ & Socavación máxima en equilibrio. \\
\hline $\mathrm{S}_{\mathrm{t}}[\mathrm{m}]$ & Socavación a lo largo del tiempo. \\
\hline$s^{\prime}[\operatorname{adim}]$. & Densidad relativa de las partículas del lecho marino. \\
\hline sn & Función seno elíptico de Jacobi \\
\hline $\mathrm{T}_{\mathrm{a}}\left[{ }^{\circ} \mathrm{C}\right]$ & Temperatura del agua. \\
\hline $\mathrm{T}_{\mathrm{car}}[\mathrm{s}]$ & Tiempo característico. \\
\hline $\mathrm{T}[\mathrm{s}]$ & Periodo del oleaje. \\
\hline $\mathrm{T}_{\mathrm{p}}[\mathrm{s}]$ & Período de pico en régimen extremal. \\
\hline $\mathrm{t}[\mathrm{s}]$ & Tiempo. \\
\hline $\mathrm{U}_{\mathrm{c}}[\mathrm{m} / \mathrm{s}]$ & Velocidad media del flujo bajo condiciones de corriente continua. \\
\hline$U_{f l}[\mathrm{~m} / \mathrm{s}]$ & Velocidad característica del fluido. \\
\hline $\mathrm{U}_{\mathrm{m}}[\mathrm{m} / \mathrm{s}]$ & Velocidad media del flujo bajo condiciones de oleaje. \\
\hline $\mathrm{U}_{\mathrm{s}}$ [adim.] & Parámetro de Ursell. \\
\hline $\mathrm{u}[\mathrm{m} / \mathrm{s}]$ & Componente horizontal de la velocidad de una partícula de agua. \\
\hline $\mathrm{u}_{\mathrm{r}}[\mathrm{m} / \mathrm{s}]$ & Velocidad relativa entre un fluido y un objeto inmerso en él. \\
\hline$V_{m}\left[m^{3}\right]$ & Volumen del cilindro. \\
\hline
\end{tabular}




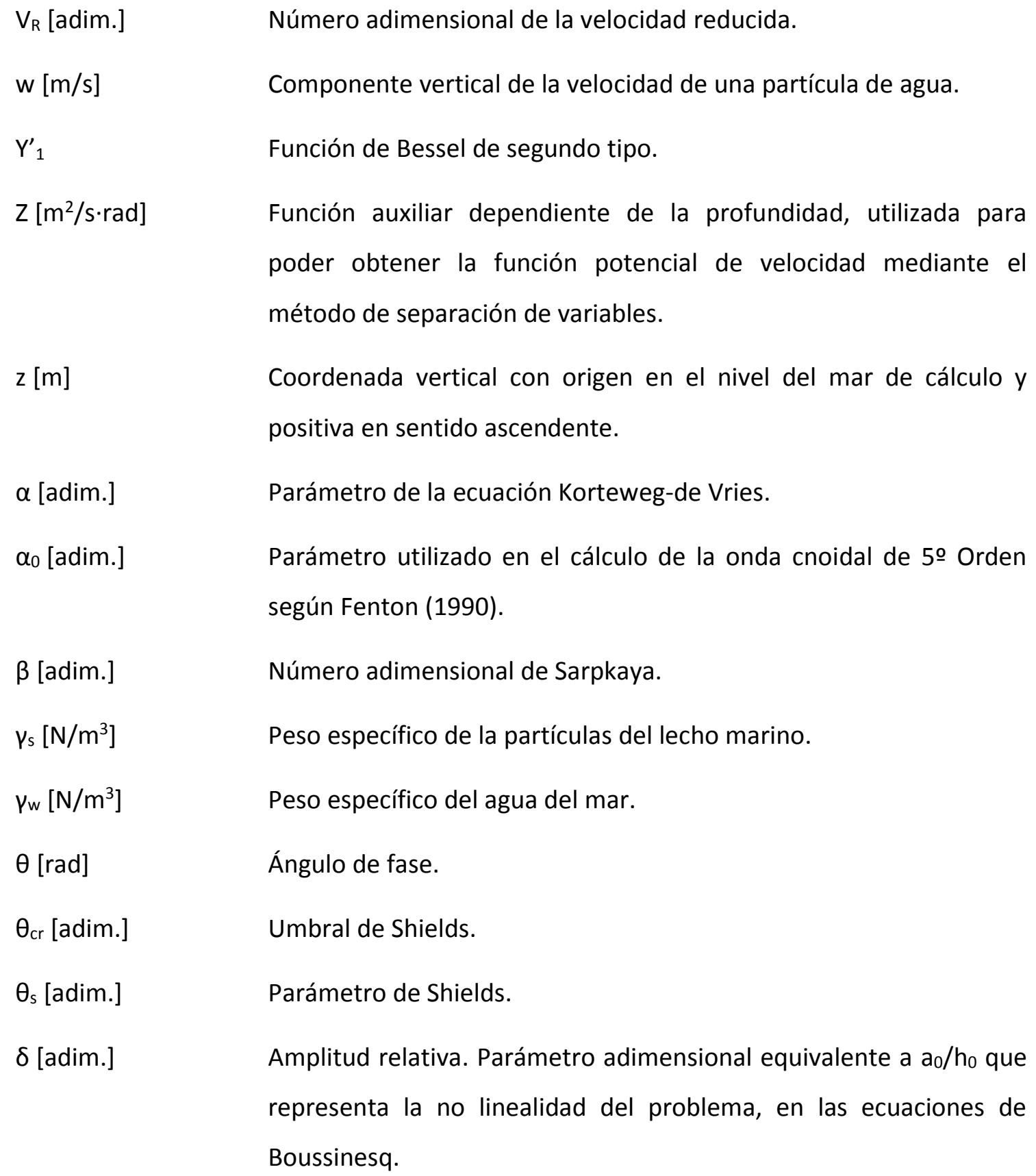

$\eta[\mathrm{m}] \quad$ Desplazamiento vertical del agua en la superficie libre. 
$\kappa$ [adim.]

$\lambda[1 / \mathrm{m}]$

$\lambda_{2}$ [adim.]

$\mu\left[N / s \cdot m^{2}\right]$

$\mu_{\mathrm{r}}[\operatorname{adim}$.

$\mu_{2}$ [adim.]

$v\left[\mathrm{~m}^{2} / \mathrm{s}\right]$

$\rho\left[\mathrm{kg} / \mathrm{m}^{3}\right]$

$\rho_{\text {est }}\left[\mathrm{kg} / \mathrm{m}^{3}\right]$

$\rho_{\text {est }}\left[\mathrm{kg} / \mathrm{m}^{3}\right]$

$\tau[\mathrm{N} / \mathrm{m}]$

$\tau_{c}\left[\mathrm{~N} / \mathrm{m}^{2}\right]$

$\tau_{m}\left[\mathrm{~N} / \mathrm{m}^{2}\right]$

$\tau_{\max }\left[\mathrm{N} / \mathrm{m}^{2}\right]$

$\tau_{w}\left[\mathrm{~N} / \mathrm{m}^{2}\right]$

$\emptyset[0]$

$\Phi\left[\mathrm{m}^{2} / \mathrm{s} \cdot \mathrm{rad}\right]$

$\phi\left[\mathrm{m}^{2} / \mathrm{s} \cdot \mathrm{rad}\right]$

$\phi_{1}\left[\mathrm{~m}^{2} / \mathrm{s} \cdot \mathrm{rad}\right]$
Módulo de las integrales y de las funciones elípticas en la solución de onda cnoidal de 3 ㅇ orden desarrollada por Isobe (1988).

Constante utilizada para calcular los Stokes de 5o Orden según la formulación de Skjelbreia et al. (1960).

Parámetro adimensional igual a $\left(\left(1-k^{\wedge} 2\right)\right) / k^{\wedge} 2$ utilizado en la solución de onda cnoidal de 3oo orden desarrollada por Isobe (1988).

Viscosidad dinámica del agua de mar.

Profundidad relativa. Parámetro adimensional que representa la dispersión de la frecuencia en las ecuaciones de Boussinesq.

Parámetro adimensional igual a $\mathrm{E} /\left(\mathrm{K}^{\wedge} 2 \mathrm{~K}\right)$ utilizado en la solución de onda cnoidal de 3 o orden desarrollada por Isobe (1988).

Viscosidad cinemática del agua de mar.

Densidad del agua.

Densidad del elemento estructural.

Densidad de la estructura.

Tensión superficial del fluido.

Esfuerzo cortante en el lecho debido a una corriente continua.

Esfuerzo cortante medio en el lecho marino.

Esfuerzo cortante máximo en el lecho marino.

Esfuerzo cortante en el lecho debido al oleaje.

Ángulo entre la dirección de propagación del oleaje y la dirección de la corriente.

Función auxiliar dependiente del espacio tiempo, utilizada para poder obtener la función potencial de velocidad mediante el método de separación de variables.

Función potencial de velocidad.

Función potencial de velocidad del oleaje incidente. 
Modelos de predicción de esfuerzos hidrodinámicos y socavación aplicados a ingeniería offshore

$\phi_{\mathrm{D}}\left[\mathrm{m}^{2} / \mathrm{s} \cdot \mathrm{rad}\right] \quad$ Función potencial de velocidad del oleaje difractado.

$\phi_{\mathrm{R}}\left[\mathrm{m}^{2} / \mathrm{s} \cdot \mathrm{rad}\right] \quad$ Función potencial de velocidad del flujo de radiación.

$\psi\left[\mathrm{m}^{2} / \mathrm{s} \cdot \mathrm{rad}\right] \quad$ Función de corriente.

$\omega[\mathrm{rad} / \mathrm{s}] \quad$ Velocidad angular. 


\section{Capítulos}



Capítulo 1

INTRODUCCIÓN, OBJETIVOS Y ESTRUCTURA DEL DOCUMENTO 



\section{Capítulo 1}

\section{INTRODUCCIÓN, OBJETIVOS Y ESTRUCTURA DEL DOCUMENTO}

\section{Introducción}

El viento ha sido una de las principales fuentes de energía utilizadas por el hombre desde la antigüedad. Desde un punto de vista histórico, la utilización de la energía eólica siempre ha ido de la mano de la innovación y ha generado grandes cambios evolutivos en las civilizaciones que supieron utilizarla en su favor, para conseguir: mejoras en la producción agrícola, mejoras alimenticias, como medio de transporte para expandir los límites conocidos por una determinada población, etc. Este es el caso del uso de la energía eólica destinada al riego, la molienda o en la navegación a vela (Fernández Díez, 1993).

La primera máquina considerada como la precursora de los actuales aerogeneradores fue diseñada a finales del siglo XIX. No obstante, el aprovechamiento de la energía eólica en forma de energía eléctrica no empezó a cobrar fuerza hasta el año 1973, coincidiendo con la crisis del petróleo (Espejo Martín, 2004).

La necesidad de asegurar un modelo energético bajo en carbón en los países más industrializados, la ha convertido en una gran garantía de abastecimiento seguro y limpio, contribuyendo además a la independencia energética de los países y al desarrollo socioeconómico de las comunidades. Por ello, en 1997, tras la firma del Protocolo de Kioto, una serie de países se comprometieron a llevar a cabo determinadas medidas encaminadas a reducir las emisiones de gases de efecto invernadero, entre las cuales destacaba la utilización de energías renovables (Naciones Unidas, 1998). Esto supuso un gran impulso para la energía eólica que es, dentro de las renovables, la que más crecimiento ha experimentado desde entonces.

No obstante, el desarrollo principal de la energía eólica se ha llevado a cabo, fundamentalmente, mediante la implantación de aerogeneradores en tierra (en adelante, 
onshore), siendo muy pocos los megavatios (MW) instalados en el mar (en adelante, offshore) dentro de los límites de control económico de los países. De hecho, a principios del año 2009, de los 120.791 MW eólicos instalados en todo el mundo, sólo unos 1.500 MW correspondían a parques eólicos offshore (www.thewindpower.net, Pág. Web de Wind Power). Esto encuentra su principal justificación en que las instalaciones eólicas offshore están sujetas a costes notablemente superiores y requieren una tecnología más avanzada (Dong-Sheng, 2008).

Posteriormente, de acuerdo a la información estadística presentada por la organización European Wind Energy Association (EWEA) a comienzos de 2013 existían 5.538 MW de eólica marina instalados a nivel mundial, de los cuales el $90 \%$ se encuentran situados en países de la Unión Europea. Como también se recoge en dicha publicación, en Europa había una capacidad instalada de $4.995 \mathrm{MW}$ a lo largo de 55 parques eólicos offshore repartidos en 10 países europeos.

Actualmente, de acuerdo con las últimas estadísticas presentadas por la EWEA para el año 2016, la energía eólica ha superado al carbón, convirtiéndose en la segunda forma predominante de generación de energía en Europa, con una capacidad total instalada de $153,7 \mathrm{GW}$, tal y como muestra la figura 1.

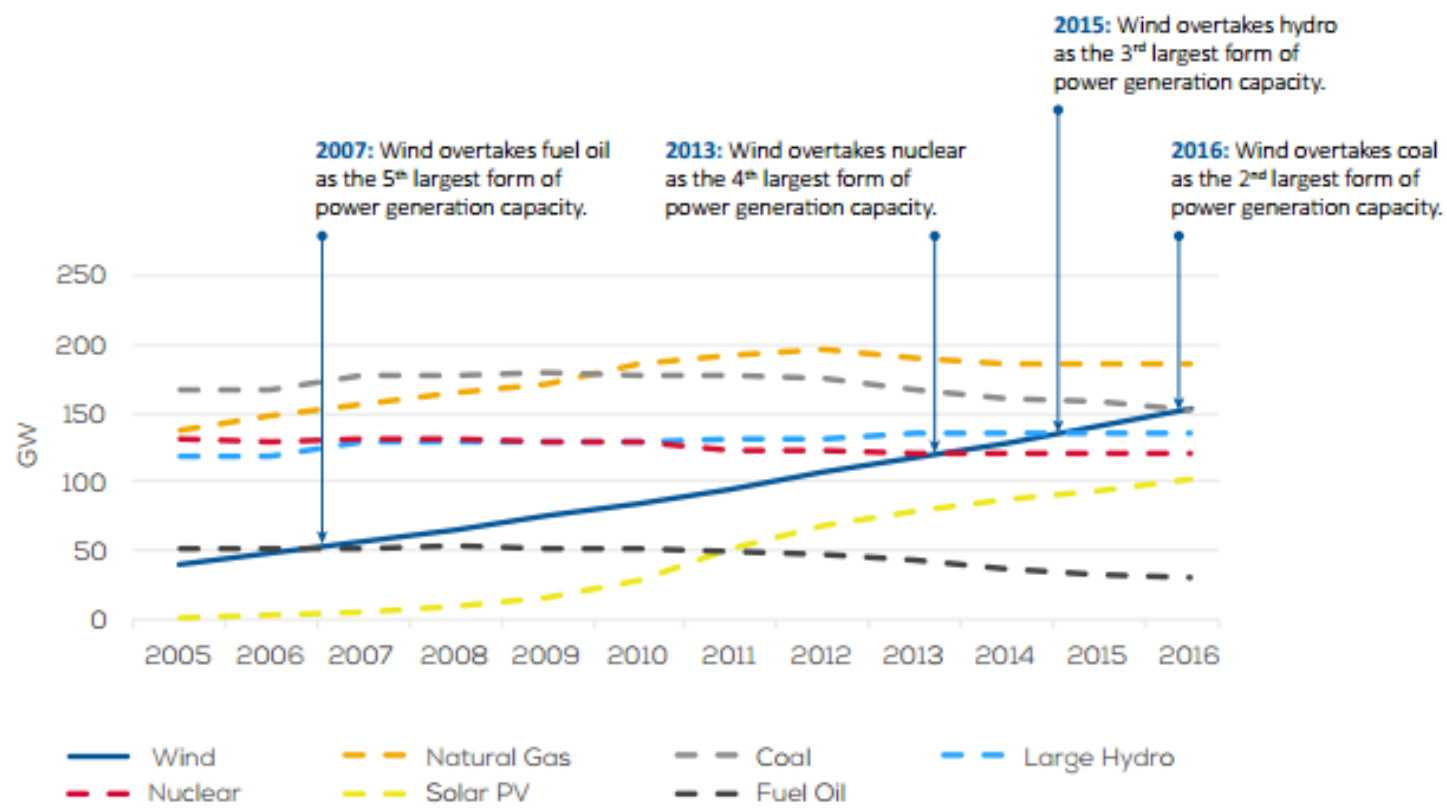

Figura 1. Evolución de la evolución de las distintas capacidades energéticas instaladas en Europa (fuente: EWEA, 2016) 
Dentro de esta potencia instalada, la cantidad correspondiente a generación offshore asciende a la cantidad de $12.631 \mathrm{MW}$, repartida principalmente en un 73\% en el Mar del Norte, un $15 \%$ en el Mar de Irlanda y 12\% en el Mar Báltico, tal y como muestra la figura 2.

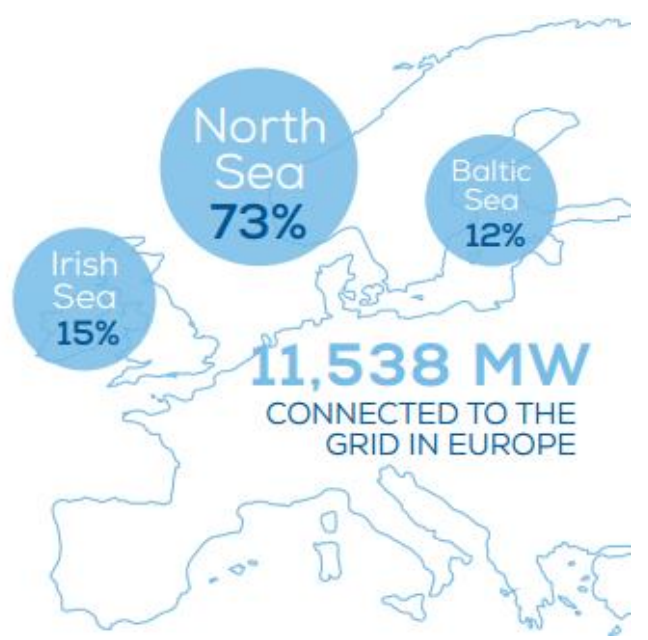

Figura 2. Distribución de la energía eólica offshore en Europa (fuente: EWEA, 2016)

De acuerdo con estos datos, tal y como se muestra en la tabla 1, el $40,82 \%$ de la capacidad offshore instalada pertenece a Reino Unido, seguida de cerca por Alemania con un 32,52\%. Posteriormente se encuentran Dinamarca con un $10,1 \%$ y Holanda con un $8,8 \%$. Por último, Bélgica con un 5,6\%, Suecia con un 1,6\%, Finlandia con una $0,3 \%$, Irlanda con un $0,2 \%$, España con un $0,04 \%$ y Noruega con un $0,02 \%$.

\begin{tabular}{|c|c|}
\hline País & \% Capacidad offshore instalada en Europa \\
\hline Reino Unido & $40,82 \%$ \\
\hline Alemania & $32,52 \%$ \\
\hline Dinamarca & $10,10 \%$ \\
\hline Holanda & $8,80 \%$ \\
\hline Bélgica & $5,60 \%$ \\
\hline Suecia & $1,60 \%$ \\
\hline Finlandia & $0,30 \%$ \\
\hline Irlanda & $0,20 \%$ \\
\hline España & $0,04 \%$ \\
\hline Noruega & $0,02 \%$ \\
\hline
\end{tabular}

Tabla 1. Porcentaje de capacidad offshore instalada en Europa (fuente: EWEA, 2016) 
En definitiva, a la cabeza del actual desarrollo se encuentran países como: Reino Unido, Alemania, Dinamarca, Holanda y Bélgica; seguidos de lejos por un numeroso grupo de países. España puede considerarse encuadrada dentro de este último grupo, manifestándose, en el año 2007, una posición optimista por parte de las empresas promotoras, tras la publicación del Real Decreto 1028/2007, de 20 de julio, por el que se establece el procedimiento administrativo para la tramitación de las solicitudes de autorización de instalaciones de generación eléctrica en el mar territorial (BOE, 2007). No obstante, a día de hoy las políticas en España encaminadas en esta dirección se encuentran totalmente congeladas, y no hay abierto ningún concurso específico.

Las cimentaciones de tipo monopila son las estructuras más habituales en la instalación de aerogeneradores marinos. Según los datos del 2016, presentados por la EWEA, el 80,8\% de todas las unidades instaladas actualmente presentan una cimentación de tipo monopila (monopile), seguido por un $7,5 \%$ de estructuras de gravedad, un 6,6\% de estructuras tipo jacket, un 3,2\% de estructuras tipo trípode y un 1,9\% de estructuras tripila.

Por otro lado, los parques eólicos marinos se han movido más lejos de la costa y a aguas más profundas. A finales de 2015 , la profundidad media del agua de los parques eólicos conectados a la red era de $27,1 \mathrm{~m}$ y la distancia media a la costa era de $43,3 \mathrm{~km}$. Esto es principalmente el resultado de un mayor despliegue en Alemania durante 2015, donde los emplazamientos de parques eólicos offshore tenían un promedio de 52,6 $\mathrm{km}$ de distancia a la costa.

En comparación, los proyectos del Reino Unido tenían una distancia promedio de 9,4 km de distancia a la costa y los proyectos holandeses una distancia media de $31,4 \mathrm{~km}$ de distancia a la costa.

En 2016, la profundidad media ha alcanzado un valor de $29,2 \mathrm{~m}$, siendo la distancia media a la costa de 43,5 km, lo cual supone un ligero incremento de ambos valores, con respecto a los obtenidos en 2015. En la figura 3, se muestran las estadísticas de distancia a la costa y profundidad, con respecto a cada uno de los países. 


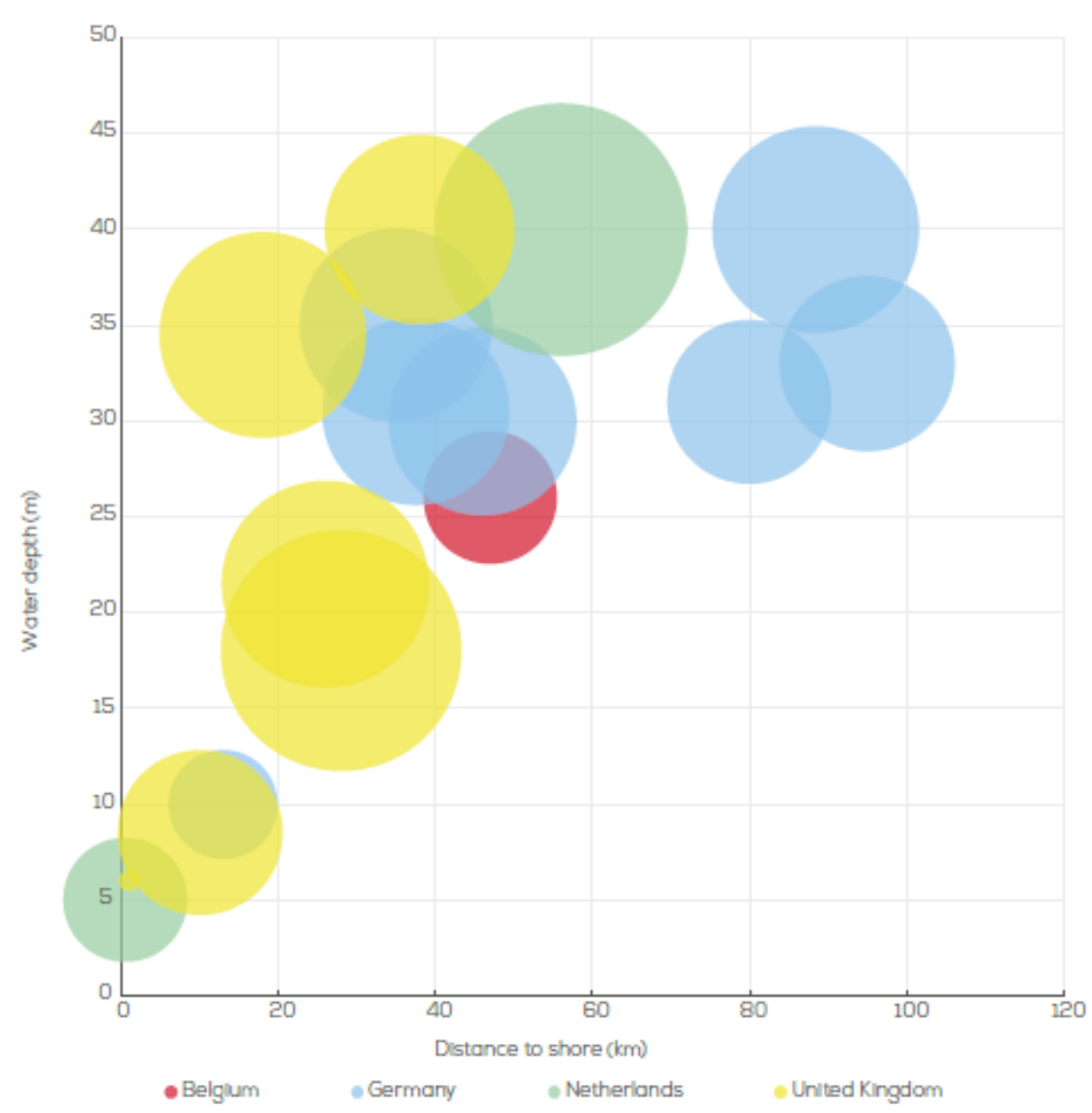

Figura 3. Comparativa entre distancia a la costa y profundidad, de los parque eólicos offshore europeos (fuente: EWEA, 2016)

No obstante, el mercado de la energía eólica offshore no solo está experimentando un importante crecimiento en Europa. A nivel global, puede observarse que el impulso se está produciendo de forma simultánea en otros muchos mercados, como por ejemplo el mercado asiático.

Dentro del mercado asiático, China está aumentando de forma exponencial la capacidad instalada de eólica offshore, teniendo a finales de 2015 una capacidad acumulada de 1.015 MW. Por otro lado, Japón poseía a finales de 2015 una capacidad de eólica offshore de 52,6 MW e India inició los trabajos destinados a desarrollar la implantación de este tipo de energía, mediante la publicación oficial de sus medidas políticas con respecto al desarrollo de la energía offshore (Global Wind Energy Council, GWEC, 2015).

En definitiva, se puede afirmar que a lo largo de los últimos años, la implantación de la energía eólica a nivel mundial ha experimentado un crecimiento exponencial, aparejado al hecho de que el fuerte crecimiento de la demanda energética mundial está creando 
dificultades a la hora de cumplir los objetivos relacionados con la reducción de emisiones de $\mathrm{CO}_{2}$ y gases de efecto invernadero. Por ello, es esencial mantener este crecimiento en el número de MW eólicos instalados, y conseguir que la implantación de este tipo de energía pueda también extenderse a países menos desarrollados tecnológicamente.

Con este objetivo en mente, es necesario que se desarrollen varias acciones de manera simultánea, tales como: establecer un marco regulatorio favorable en las zonas en las que aún no existe para estimular la inversión en este tipo de energía; mejorar las instalaciones de la red de transporte de energía eléctrica de manera que se aumente la capacidad de transporte de esta; perfeccionar la tecnología de los aerogeneradores para conseguir un mejor aprovechamiento del recurso eólico, de manera que zonas en las que actualmente estas instalaciones no son rentables, con los incentivos económicos marcados, lo puedan ser en un futuro; realizar una mayor inversión económica en I+D+i para lograr aprender de las distintas experiencias; repotenciar las instalaciones en funcionamiento, o lo que es lo mismo, sustituir las máquinas instaladas en parques eólicos ya existentes por otras de mayor potencia; y, por último, impulsar la implantación de parques eólicos offshore (Esteban, 2009).

Además, no se puede ignorar el hecho de que los parques eólicos offshore permiten generar una mayor cantidad de energía que sus equivalentes onshore. Sin embargo, el diseño, construcción, mantenimiento y operación de estos parques offshore, lleva asociado un importante incremento de costes debido a la dificultad de posicionar una estructura de este tipo, en medio del mar en vez de en tierra firme. Por lo tanto, es fundamental el poder establecer un modelo de cálculo preciso que permita estimar las acciones generadas por el oleaje, sobre las estructuras y sus respectivas cimentaciones. De esta forma, la energía eólica offshore podrá continuar creciendo, y se podrán instalar nuevos parques, cada vez, en zonas más profundas y alejadas de la costa, donde la producción energética será también mayor.

Con respecto a las acciones que debe resistir la estructura, es importante destacar que las estructuras offshore sufrirán cargas importantes y mucho mayores que sus homólogas onshore, debido fundamentalmente a las acciones generadas por el oleaje. La acción del 
oleaje presentará además el inconveniente añadido de que su correcta determinación está plagada de incertidumbres (Negro et al., 2014). Estas incertidumbres se deben principalmente a dos aspectos. En primer lugar, a la dificultad intrínseca en la realización del propio cálculo para estimar las fuerzas hidrodinámicas, debido a la complejidad del fenómeno, y por lo tanto, a la complejidad de la formulación asociada a la resolución del mismo. En segundo lugar, a la escasez de datos fiables, necesarios para caracterizar correctamente la mecánica de ondas que modelizará el oleaje, de una forma totalmente precisa.

Debido a estos motivos, la fuerte dispersión de resultados en lo referente a la correcta estimación de las cargas generadas por el oleaje, obliga a considerar escenarios de diseño sobredimensionados en la mayor parte de los casos, como solución paliativa de las posibles imprecisiones del cálculo.

Por otro lado, es necesario tener en cuenta en el diseño, que incluso aunque la caracterización de las cargas se realice de forma precisa, se deberán considerar todos los efectos de interacción entre el oleaje y la estructura. Efectos que, nuevamente, tendrán como límite intrínseco su dificultad de cálculo y su importante coste computacional. Estos efectos son muy importantes dado que estas cargas hidrodinámicas pueden generar un acoplamiento dinámico con la propia estructura, que podrá derivar en efectos de amplificación dinámica o de disminución de la vida útil de la estructura a causa de la fatiga.

Además, a parte de los esfuerzos generados sobre la estructura, también será necesario tener en cuenta los problemas que surgirán en la cimentación, dado que el lecho marino tendrá generalmente una menor capacidad portante que los terrenos en tierra firme. Esto obliga a plantear soluciones que implicarán una mayor dificultad, tanto de diseño como de construcción, apareciendo efectos importantes que deberán ser estudiados en detalle, como por ejemplo: la socavación; lo cual complicará aún más el diseño, y obligará al diseñador a predecir el posible efecto de este fenómeno sobre la vida útil de la estructura, así como el diseño de las oportunas medidas de protección para evitar su aparición o reducir su efecto. 
La presente Tesis Doctoral se centra en resolver todos estos problemas, en relación con el correcto cálculo de estructuras offshore, arrojando luz sobre las distintas incertidumbres que condicionan el problema y aportando un nuevo método avanzado de cálculo, que será comparado con el análisis tradicional, y que permitirá dimensionar de forma más precisa todos los elementos de la estructura, que va a ser el soporte del aerogenerador.

Este método de cálculo avanzado será desarrollado como una herramienta compacta que permitirá, de una forma sencilla y eficiente, calcular perfectamente el campo de velocidades y aceleraciones del agua de mar, sometida a un oleaje con cualquier tipo de características, en lo referente a: altura de ola, profundidad y periodo; teniendo como única limitación que no se trate de una ola rota. Este cálculo tendrá en cuenta todos los efectos no lineales, la difracción, y otras variables que hasta el momento nunca habían sido añadidas de forma explícita en este tipo de cálculo, como son, la temperatura y la salinidad del agua. Además, tendrá en cuenta todos los posibles efectos de interacción existentes entre este oleaje y la estructura, permitiendo simular efectos de amplificación dinámica o resonancia, para los primeros 25 modos de vibración de la estructura.

Debido a que la tipología estructural utilizada de forma mayoritaria en parques eólicos offshore es la estructura de tipo monopila, todos los casos a analizar estarán compuestos por una estructura con forma cilíndrica en posición vertical.

Por último, este método también incluirá una mejora en los modelos de predicción de la socavación, al desarrollar una nueva expresión que va a tener en cuenta los efectos del campo de aceleraciones, de una forma explícita.

En conclusión, el método planteado permitirá aumentar la precisión en el diseño estructural de parques eólicos offshore, haciendo que se reduzcan las limitaciones que sufre este campo, debido a las diversas incertidumbres que afectan al diseño, y permitiendo también que se puedan ajustar más los costes asociados a la construcción de estas estructuras. 


\section{Objetivos}

El objetivo principal de esta investigación es la creación de un modelo numérico de cálculo avanzado, que permita tener en cuenta todas las variables que definen el diseño estructural de una estructura offshore con cimentación monopila, que se encuentra sometida únicamente a la acción del oleaje. Por lo tanto, todos los casos de análisis seleccionados estarán formados por un fuste cilíndrico vertical y una cimentación monopila.

Se ha elegido la cimentación monopila debido a que actualmente es la más utilizada en los parques eólicos offshore instalados en Europa, perteneciendo a esta tipología el 80,8\% de todas las instalaciones construidas actualmente.

Este modelo avanzado de cálculo permitirá mejorar la precisión de los resultados que aportan los modelos actuales. Para ello, la investigación se centra en el estudio del campo de fuerzas hidrodinámicas que se generan con el impacto de una ola sobre una estructura cilíndrica vertical, en la afección que dichas fuerzas generan sobre la estructura, y en segunda instancia, en la afección generada sobre la cimentación.

Para ello, se han desarrollado cuatro líneas de estudio para analizar de forma separada los principales aspectos físicos que definen el problema:

1. La teoría de ondas.

2. La caracterización de fuerzas hidrodinámicas.

3. La teoría de vibración aplicada a una estructura.

4. La teoría de socavación.

A continuación, se procede a realizar la exposición de los objetivos específicos que se engloban dentro de cada uno de los cuatro procesos de cálculo.

En primer lugar, se ha realizado un compendio de todas las teorías englobadas dentro de la mecánica de ondas, aplicadas al oleaje y se ha expuesto cuál es su rango de aplicación. De esta forma se podrá realizar una simulación precisa de los movimientos que sufrirá cada partícula del fluido, que esté sometido a un oleaje regular de unas determinadas 
condiciones, para cada una de las teorías englobadas dentro de la mecánica de ondas. Los objetivos particulares dentro de este primer proceso de cálculo, son los siguientes:

- Comparación de la variación del campo de velocidades y aceleraciones entre la teoría lineal y el resto de teorías para los casos de estudio seleccionados.

- Descomposición espectral del campo de velocidades y aceleraciones el oleaje con el objeto de identificar las principales frecuencias que lo componen.

- Comparación de la amortiguación que sufre el campo de velocidades y de aceleraciones con la profundidad, entre los resultados aportados por la teoría lineal y los resultados obtenidos tras aplicar las teorías de mecánica de ondas que corresponden a cada uno de los casos de cálculo.

En segundo lugar, se han recopilado y ordenado todas las teorías hidrodinámicas que permiten estimar las presiones generadas por el oleaje sobre una estructura, a partir de los movimientos, velocidades y aceleraciones conocidas de las partículas de dicho fluido, incluyendo el cálculo de efectos no lineales y de difracción. Este segundo grupo de teorías también ha sido clasificado por rangos de aplicación, permitiendo la solución del problema para cualquier oleaje y para cualquier diámetro de la estructura cilíndrica vertical.

Los objetivos particulares dentro de este segundo proceso de cálculo, son los siguientes:

- Comprobación de las fuerzas hidrodinámicas generadas por el método avanzado que tiene en cuenta cualquier oleaje y cualquier tamaño de la estructura, con respecto a las generadas por el método tradicional, que utiliza la fórmula de Morison et al. y la teoría lineal de ondas.

- Análisis de la influencia de la temperatura y la salinidad sobre la distribución y magnitud de las cargas.

- Análisis de la influencia de los efectos no lineales sobre la distribución y magnitud de las cargas hidrodinámicas.

- Análisis de los puntos que reciben las mayores cargas para cada teoría de mecánica de ondas.

- Amortiguación de las distintas fuerzas con la profundidad.

- Variación del número de Reynolds.

- Variación del número de Keulegan-Carpenter. 
- Variación del parámetro de Sarpkaya.

En tercer lugar, se ha desarrollado un algoritmo que permite aplicar las fuerzas hidrodinámicas halladas sobre una estructura tipo ménsula y analizar su comportamiento en los primeros 25 modos de vibración de una forma eficiente. Este proceso puede acoplarse con el primero permitiendo obtener los resultados teniendo en cuenta que la estructura se mueve, mientras el oleaje impacta contra ella.

Los objetivos específicos de este tercer proceso de cálculo son los siguientes:

- Análisis de los esfuerzos generados en la cimentación entre los casos de cálculo tradicional y aquellos resueltos mediante el modelo optimizado.

- Análisis de movimientos que sufre la estructura comparando los generados por el modelo estático con los del modelo dinámico.

- Determinar la influencia que tiene el acoplamiento de la estructura con el oleaje sobre los esfuerzos que sufre la estructura.

- Determinar la influencia que tiene el acoplamiento de la estructura con el oleaje sobre los desplazamientos que sufre la estructura.

- Determinar la influencia del acoplamiento frente a la aparición de posibles efectos de resonancia a ciertas frecuencias.

- Determinar cómo varía esta influencia dependiendo del diámetro y de la teoría hidrodinámica aplicada.

- Determinar cómo varía este acoplamiento con respecto a la teoría de ondas utilizada para caracterizar el oleaje.

Por último, se analizará la predicción de posibles fenómenos de socavación en la estructura a partir de los valores calculados del campo de velocidades en la cimentación de la estructura, teniendo en cuenta los efectos de difracción y efectos no lineales.

Los objetivos específicos de este último proceso de cálculo son los siguientes:

- Influencia de la teoría de ondas a aplicar sobre la socavación.

- Influencia del diámetro sobre la socavación.

- Influencia del campo de aceleraciones. 


\section{Estructura del documento}

La investigación llevada a cabo en la presente Tesis Doctoral, que se ha desarrollado de acuerdo a la metodología de trabajo expuesta anteriormente, se organiza en el presente documento de acuerdo a la siguiente estructura:

\section{Capítulo 1. Introducción, objetivos y estructura del documento}

En este capítulo se encuentran recogidas: una introducción sobre la investigación, la motivación del estudio, el planteamiento de los objetivos y la estructura final del documento.

\section{Capítulo 2. Estado del conocimiento}

En este capítulo se expone y analiza el estado del arte en cuanto a las distintas tipologías de estructuras offshore desarrollando su clasificación estructural. Además, se analizan las formulaciones empleadas para la predicción de los esfuerzos hidrodinámicos sobre estructuras offshore, teniendo en cuenta las cuatro líneas principales del estudio: mecánica de ondas, caracterización hidrodinámica, fenómeno vibratorio sobre la estructura y el proceso de socavación de la cimentación.

\section{Capítulo 3. Metodología de la investigación}

En este capítulo se realiza una descripción detallada de la metodología seguida durante el proceso de investigación, definiendo claramente cada uno de los pasos o etapas desarrolladas y el motivo de las mismas. Además, se recogen las principales fuentes de datos, se justifica la información empleada y se describen en detalle las hipótesis seleccionadas en cada uno de los casos de cálculo.

\section{Capítulo 4. Desarrollo de la herramienta}

En este capítulo se describe el desarrollo completo del modelo numérico empleado durante la investigación. Además, se describe la formulación obtenida para el desarrollo del método avanzado de cálculo de esfuerzos hidrodinámicos sobre estructuras offshore. 
En lo referente a la formulación desarrollada por la presente Tesis Doctoral, se explicará en detalle el procedimiento de resolución utilizado en el modelo y se presentarán los principales avances propuestos en relación a:

- La modelización del fluido sometido a la acción del oleaje.

- La resolución de los distintos estados dinámicos de la estructura mediante el uso de la teoría vibratoria.

- El proceso de acoplamiento entre oleaje y estructura.

- El desarrollo de la nueva formulación desarrollada para estudiar el fenómeno de la socavación.

\section{Capítulo 5. Aplicación de la herramienta a los casos de estudio}

En este capítulo se lleva a cabo una discusión y análisis sobre los resultados para cada uno de los cinco procesos que componen el método de cálculo:

- Proceso I: Mecánica de ondas.

- Proceso II: Caracterización hidrodinámica.

- Proceso III: Análisis dinámico.

- Proceso IV: Acoplamiento del oleaje con la estructura.

- Proceso V: Socavación.

En cada uno de ellos, se analizan en detalle las diferencias entre los resultados calculados mediante el método avanzado desarrollado en la presente Tesis Doctoral y los calculados mediante el método tradicional.

Como puede observarse, se ha pasado de cuatro procesos de cálculo a cinco. Esto se debe a que a efectos de cálculo, la tercera etapa de análisis será dividida en dos procesos de cálculo diferentes. Por un lado, se desarrollará como tercer proceso de cálculo, el proceso que englobaría el análisis dinámico de la estructura, teniendo en cuenta los fenómenos vibratorios de la estructura. Por otro lado, se desarrollará como cuarto proceso de cálculo, el proceso que analiza los efectos de acoplamiento entre la estructura y el oleaje. 


\section{Capítulo 6. Conclusiones y nuevas líneas de investigación}

En el Capítulo 6 se recogen las principales conclusiones alcanzadas tras la investigación y se proponen las nuevas líneas de investigación, que se abren tras la finalización de esta Tesis Doctoral.

\section{Capítulo 7. Referencias bibliográficas}

En este capítulo se recoge el conjunto de referencias bibliográficas consultadas durante el desarrollo de la presente Tesis Doctoral.

\section{Apéndices}

Además se incluyen dos apéndices a la presente Tesis Doctoral con el objeto de reducir el cuerpo principal del documento.

\section{Apéndice 1. Resultados de los casos de estudio}

En este apéndice se ha incorporado toda la información relevante en referencia a los resultados obtenidos durante las distintas simulaciones realizadas en el proceso de investigación.

\section{Apéndice 2. Indicios de calidad}

En este apéndice se adjuntan las principales publicaciones que se han elaborado a partir de los resultados obtenidos en la presente Tesis Doctoral, incluyendo además una breve descripción de la revista o medio en la que se ha producido dicha publicación. 


\section{Capítulo 2}

\section{ESTADO DEL CONOCIMIENTO}





\section{Capítulo 2}

\section{ESTADO DEL CONOCMIENTO}

\section{Introducción}

El término estructura offshore hace referencia a una estructura que se construye en aguas marinas, encontrándose por lo tanto sometida entre otros fenómenos: a la acción del oleaje, a la acción de corrientes, a la acción del hielo, etc.; y, en definitiva, a unas condiciones meteoceánicas adversas. Estas acciones deben ser tenidas en cuenta de forma adecuada en el cálculo y diseño de este tipo de estructuras, ya que la acción meteoceánicas es una de las principales causas de fallo en las estructuras offshore.

Las estructuras offshore pueden ser apoyadas sobre el lecho marino o ser flotantes. La función principal para la que fueron concebidas inicialmente es la exploración y producción, de gas y petróleo. No obstante, a lo largo de los años han ido surgiendo otras funciones diferentes como: soportes de aerogeneradores, aprovechamiento de la energía del mar, bases de edificios, aeropuertos, etc.

Aunque para todas estas funciones se pueden aplicar los mismos principios de diseño y construcción, es necesario darse cuenta de que el proyecto debe ser financieramente viable, lo que implica que las consideraciones constructivas de mantenimiento y de diseño vendrán condicionadas por los rendimientos potenciales que sea capaz de generar la estructura objeto de proyecto. Esto se traduce en que, para determinados lugares y profundidades, no se podrá construir la estructura en cuestión, o se deberá optar por una tipología estructural específica, para garantizar la existencia de una rentabilidad mínima en la instalación. Por lo tanto, existirá una restricción por usos de las distintas zonas según la profundidad, reservando las áreas más profundas, a las actividades más rentables. 
A lo largo de este capítulo se va a exponer en detalle el estado del conocimiento actual, en relación con el cálculo y el diseño de estructuras offshore, en lo referente a la resistencia que presentan estas estructuras frente a las fuerzas hidrodinámicas generadas por el oleaje. Para ello, se van a abordar todos y cada uno de los distintos puntos que intervienen en el problema, y que serán desarrollados a lo largo de los distintos capítulos que componen la presente Tesis Doctoral.

Por lo tanto, se va a proceder a dividir el estado del conocimiento en:

- Tipologías estructurales.

- Clasificación según profundidad.

- Mecánica de ondas.

- Caracterización de fuerzas hidrodinámicas.

- Fenómeno de socavación.

En primer lugar, se realizará la exposición de los distintos tipos de estructuras offshore que existen hoy en día en el campo de la generación de energía eléctrica y se explicarán las particularidades que definen a una determinada tipología, como la más idónea, en relación con las condiciones físicas del lugar en que se va a implantar la estructura.

Posteriormente, se describirá la principal influencia que supone la profundidad en el uso de cada una de las distintas tipologías estructurales.

Después, se desarrollarán las principales teorías dentro de la mecánica de ondas, que se aplican actualmente, para caracterizar y modelizar el oleaje, definiendo las principales hipótesis que se deben asumir en el cálculo y el rango de aplicación en el que cada teoría es válida. Es necesario destacar en este punto, que la correcta caracterización del oleaje jugará un papel muy importante en la precisión de los resultados finales, obtenidos de los procesos de cálculo, desarrollados durante la presente Tesis Doctoral.

A continuación, se expondrá el estado del arte en lo referente a las teorías de caracterización hidrodinámica. El objeto principal que persiguen estas teorías es obtener las fuerzas hidrodinámicas que ejerce el oleaje sobre una estructura. En este apartado se 
mostrarán las distintas formulaciones de aplicación, así como la influencia de los distintos números adimensionales sobre la definición del problema.

Posteriormente, se realizará la exposición teórica de la metodología de cálculo utilizada para el análisis dinámico de la estructura y su estado del arte.

Por último, se hará un breve desarrollo de las distintas teorías que estudian el fenómeno de la socavación bajo condiciones de oleaje y corriente continua. 


\section{Tipologías estructurales}

La estructura de soporte del aerogenerador es uno de los puntos más críticos de toda explotación destinada al aprovechamiento de la energía eólica, y especialmente si esta es offshore. Si la estructura de soporte no es diseñada adecuadamente, el aerogenerador será incapaz de resistir las cargas y los esfuerzos impuestos por su entorno, debidos principalmente: al oleaje, al viento y a las corrientes. Las estructuras offshore se pueden clasificar en dos grandes grupos: las apoyadas en el lecho marino y las flotantes; tal y como se muestra en las figuras 1 y 2 .

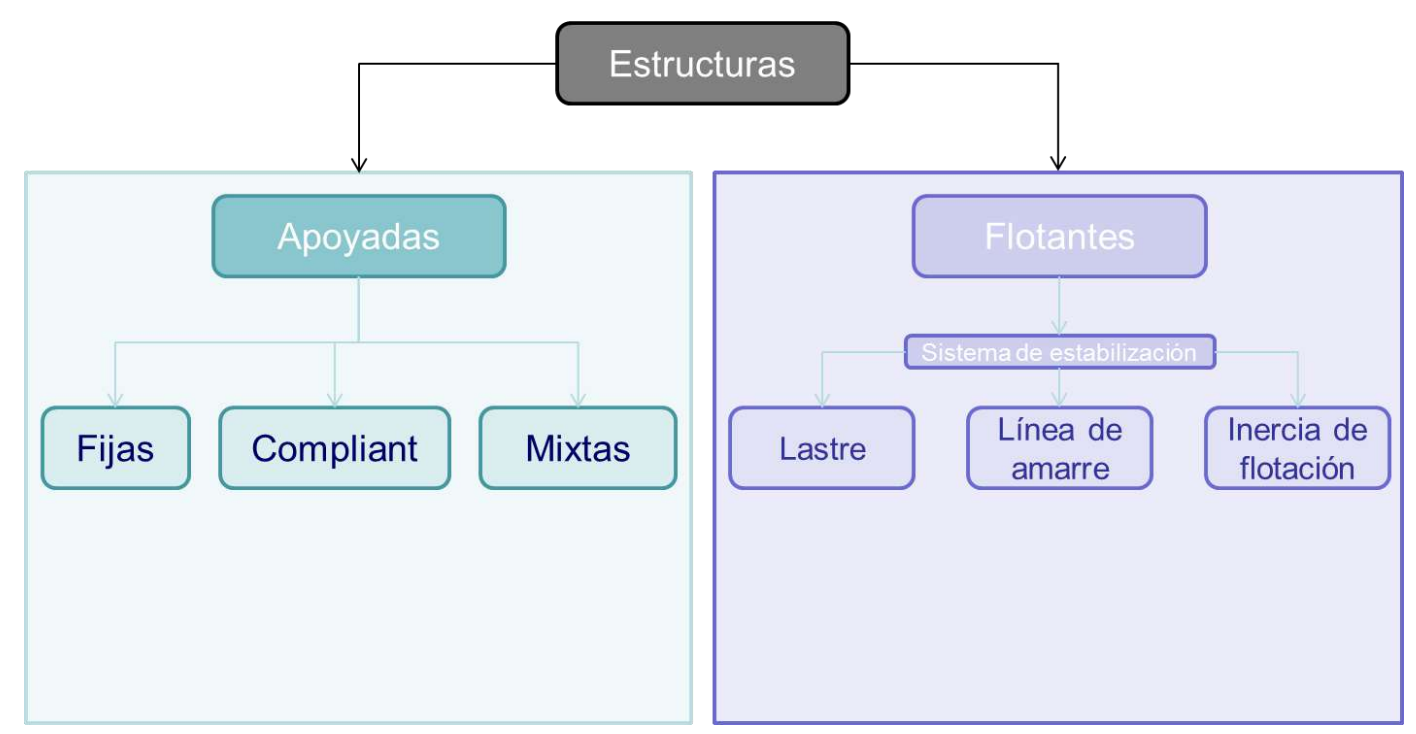

Figura 1. Clasificación estructural de las estructuras offshore (fuente: elaboración propia) 


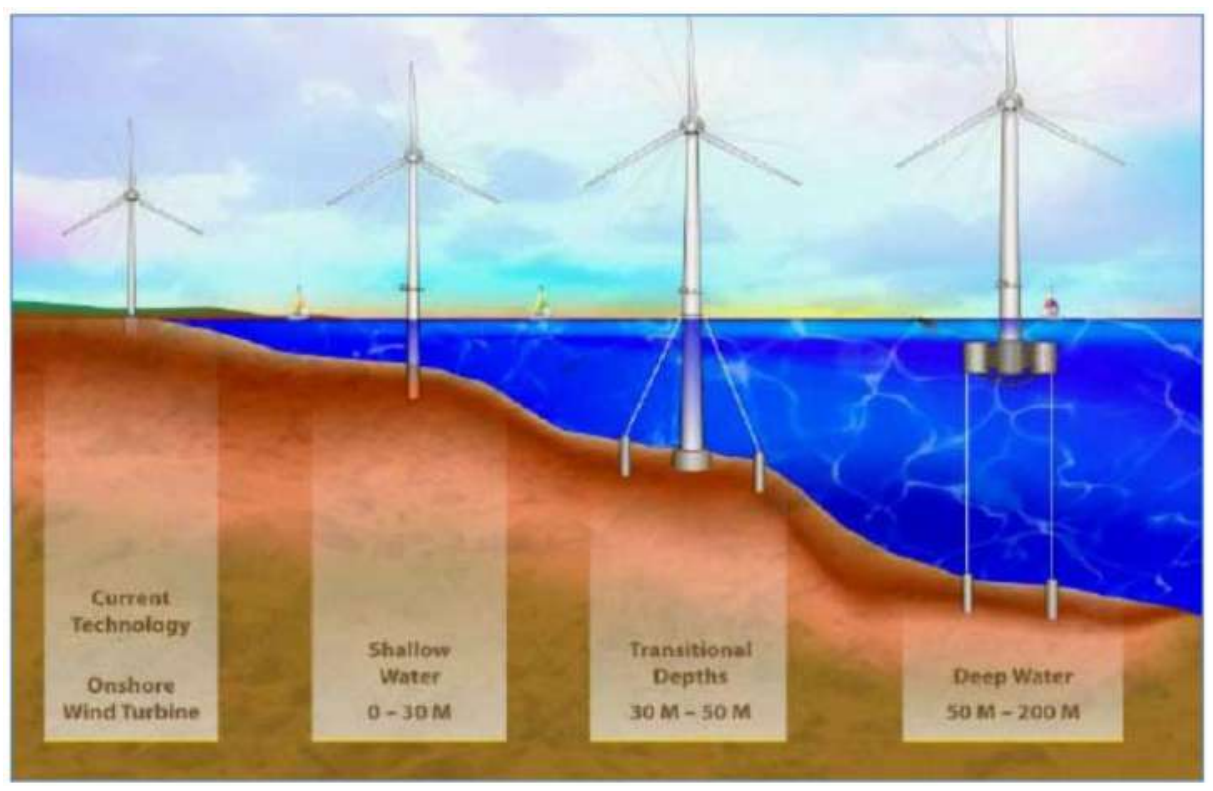

Figura 2. Tipologías estructurales aplicadas a los aerogeneradores offshore (fuente: www.nrel.gov/)

\subsection{Estructuras apoyadas en el lecho marino}

En el caso de aguas someras o aguas de transición, se utilizan sistemas donde la torre descansa o va pilotada sobre el lecho marino. Las estructuras apoyadas en el lecho marino están normalmente formadas por perfiles tubulares de acero, que se sueldan para actuar como un entramado que soporta el peso de la total de la estructura, así como las fuerzas debidas al oleaje, las corrientes marinas, el viento, etc. No obstante, las estructuras apoyadas en el lecho marino también pueden ser construidas en hormigón. Las estructuras tipo jacket o tipo monopila, por el contrario, son construidas generalmente con perfiles tubulares de acero.

Según su comportamiento estructural se diferencian principalmente tres tipos (Chakrabarti, 2005):

- Fijas: pertenecen a esta tipología cuando la frecuencia natural más baja de la estructura se encuentra por encima de la frecuencia más alta de excitación de la ola significante. Se comportan como un cuerpo rígido y deben resistir todas las fuerzas dinámicas del ambiente.

- Flexible: se consideran de este tipo cuando la frecuencia natural más baja está por debajo de la frecuencia más alta de excitación de la ola significante. Las cargas actuantes provocan que este tipo de estructuras sufran pequeños movimientos, 
reduciendo así la magnitud de las cargas dinámicas que sufriría la estructura, si esta fuera rígida. Este hecho permite que este tipo de estructuras sea más económico para aguas más profundas que las estructuras fijas.

- Mixta: Existe una estructura intermedia que en ambientes suaves se comporta como fija y cuando se excede de un determinado límite se comporta como dócil o flexible.

La figura 3 muestra los sistemas de fijación más destacados para aerogeneradores offshore (Byrne y Houlsby, 2003), en profundidades de hasta 50 metros (EWEA, 2013):

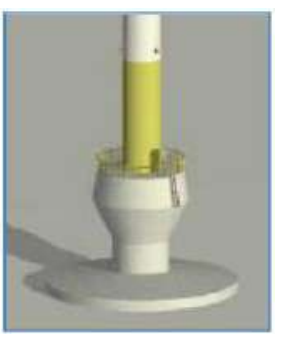

Cimentación de gravedad

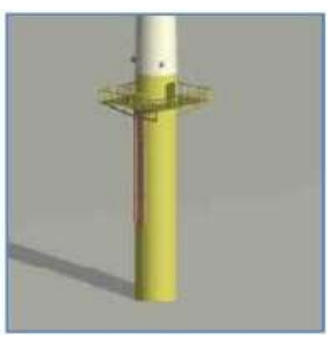

Cimentación monopila

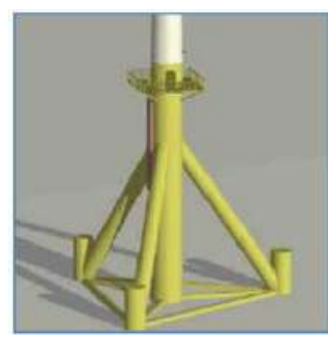

Cimentación trípode

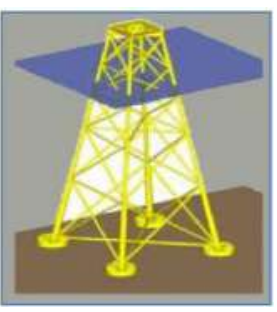

Cimentación jacket

Figura 3. Tipos de cimentación (fuente: www.offshorewindenergy.org)

\subsubsection{Estructura de gravedad}

El primer parque eólico offshore del mundo que se puso en funcionamiento fue el parque de Vindeby y se implantó sobre cimentaciones de gravedad. Once estructuras de hormigón enormes, con un peso promedio de 908 toneladas, se colocaron en las aguas cercanas a la costa de Lolland, en Dinamarca en el año 1991 (Karimirad, 2014).

Una cimentación por gravedad, tal y como su nombre indica, requiere de un gran peso propio para poder desempeñar su función principal, es decir, servir de cimiento a una determinada estructura. Por ello, normalmente, consiste en un bloque de hormigón de grandes dimensiones, al cual va unido el fuste o estructura que soporta el aerogenerador (Dean, 2010). Este bloque normalmente se realiza en hormigón, y al principio está vacío en su interior, por lo que flota. Esto hace que pueda ser remolcado hasta el punto de instalación y allí sea llenado con arena, hormigón y grava, para poder así asentarlo en la base del fondo marino (Clauss, 1992). No obstante, no siempre se aplica este método de transporte dado que, si el tamaño de los elementos que conformarán la estructura de gravedad lo permite, 
en muchas ocasiones el transporte se realiza directamente mediante la utilización de grandes barcazas.

Debido al gran peso de conjunto, este bloque es capaz de soportar los esfuerzos impuestos por el oleaje y el viento, que actúan sobre la estructura, sin requerir de la realización de ningún tipo de perforación en el suelo. No obstante, se tendrá que preparar el fondo marino para que acepte la estructura, $y$, en definitiva, para que la base quede bien asentada y no se mueva.

Las cimentaciones de gravedad suelen ser construidas de hormigón armado y/o pretensado, como se muestra en la figura 4.

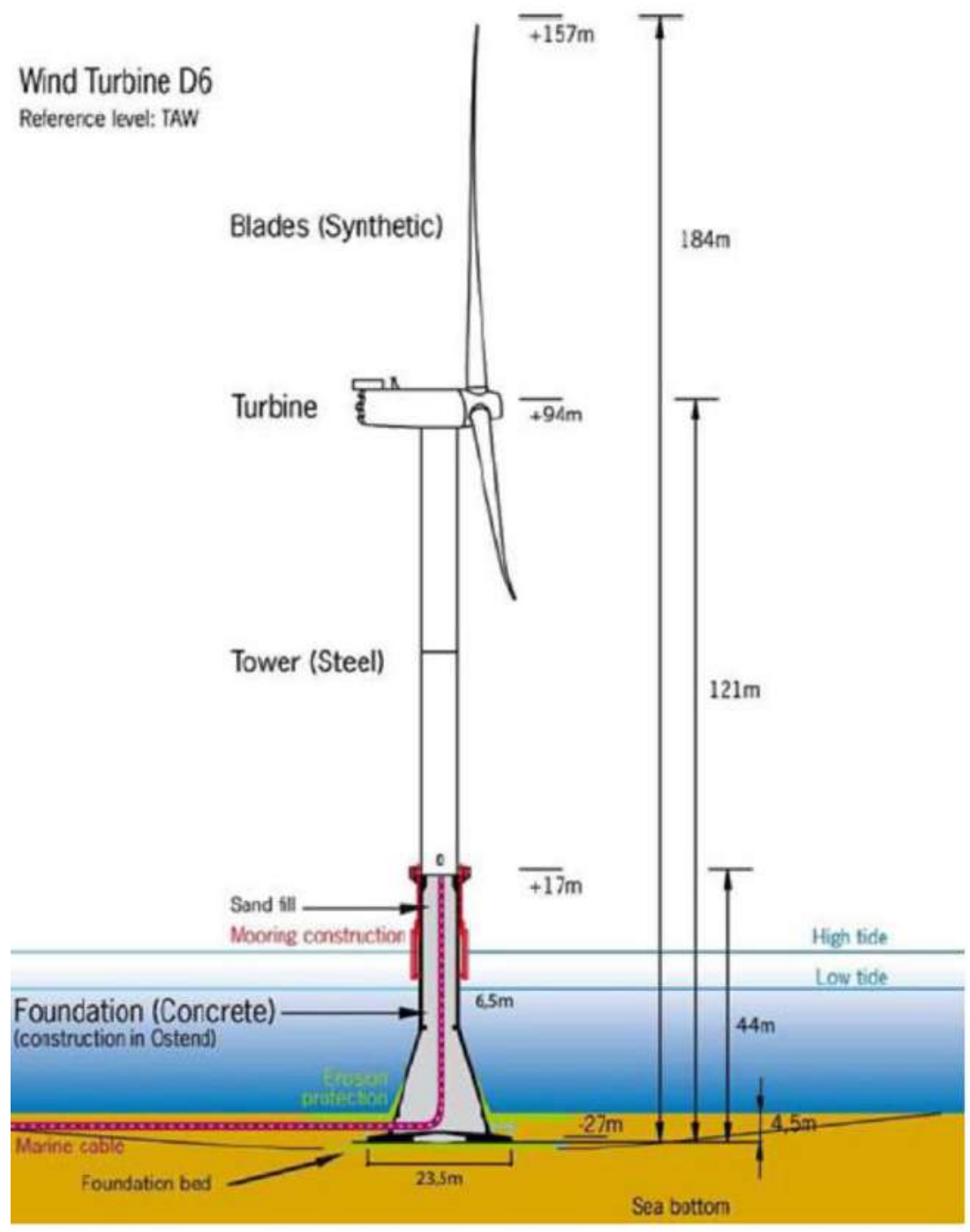

Figura 4. Esquema básico de una cimentación de gravedad (fuente: Carrol et al., 2010) 
En estos últimos años se ha conseguido reducir bastante el coste de ejecución de estas cimentaciones, debido principalmente a avances y modificaciones, realizados para facilitar su proceso de construcción. De hecho, uno de los avances ha consistido en experimentar con bases de acero, ya que son estructuras más ligeras que permiten un transporte más sencillo, y además tienen unos costes de fabricación menores. No obstante, el coste de estas cimentaciones sigue siendo elevado.

A continuación, se muestran las principales características de las cimentaciones de gravedad:

- Se recomienda el uso de este tipo de cimentación cuando las acciones ambientales no son especialmente agresivas y las cargas debidas al peso propio son dominantes. Además, esta tipología es la más competitiva si los medios capaces de instalar otra cimentación no están disponibles o existe un alto coste de movilización de los mismos al emplazamiento donde se pretende construir la instalación.

- La cimentación por gravedad tiene generalmente forma de cono, teniendo la base inferior de dicho cono entre 30 y 40 metros de diámetro, aunque se pueden encontrar otras formas (Mathis, 2007). No obstante, dichas dimensiones dependen de multitud de factores, que deben ser correctamente tenidos en cuenta durante la fase de diseño.

- Existen diferentes criterios en relación con la profundidad a la cual se puede considerar que esta tipología de cimentación es válida. Algunos autores afirman que estas cimentaciones son adecuadas para estructuras situadas en emplazamientos cuyos calados varíen entre 0 y 10 metros (Ashuri y Zaaijer, 2007), mientras otros amplían este rango hasta los 25 metros (DNV, 2013). La realidad es que hoy en día se puede plantear este tipo de cimentación en profundidades de hasta 50 metros (Costa, 2015).

- Para poder hacer uso de este tipo de cimentación, se debe garantizar que el lecho sobre el que se va a apoyar la estructura posee una determinada capacidad portante. Este hecho generalmente obliga a realizar una eliminación de las capas superficiales de lecho marino, para poder garantizar que la cimentación se apoye sobre una superficie preparada, y que posea unas prestaciones mínimas. 
- Por otro lado, en este tipo de cimentación es necesario tener en cuenta el posible efecto generado por el fenómeno de la socavación que pueda sufrir el pie de la estructura, haciendo indispensable en muchos casos el estudiar y definir los elementos de protección pertinentes, que evite o reduzca los efectos de la socavación.

\subsubsection{Monopila}

Esta tipología posee el diseño más sencillo de todos. Su estructura consiste generalmente en un pilote de acero hueco hincado de forma directa al lecho marino, que se conecta al aerogenerador mediante una pieza de transición. En ocasiones, si el lecho marino presenta una resistencia importante, son necesarias labores de perforación para poder realizar la instalación de la monopila.

Esta sencillez aporta una gran versatilidad y flexibilidad en lo referente al método de fabricación, no necesitando ningún método de carácter específico, que sea exclusivo de este tipo de estructuras. En la figura 5 se puede observar un ejemplo de esta tipología. Además, el impacto ambiental generado debido a la ocupación del lecho marino es mucho menor en comparación con la tipología de gravedad, y se encuentra más localizado. No obstante, como contrapartida presentan problemas importantes de generación de ruido durante el proceso de hincado.

Hoy en día, esta tipología es la estructura predominante en la mayor parte de los aerogeneradores offshore instalados (De Vries, 2011). 


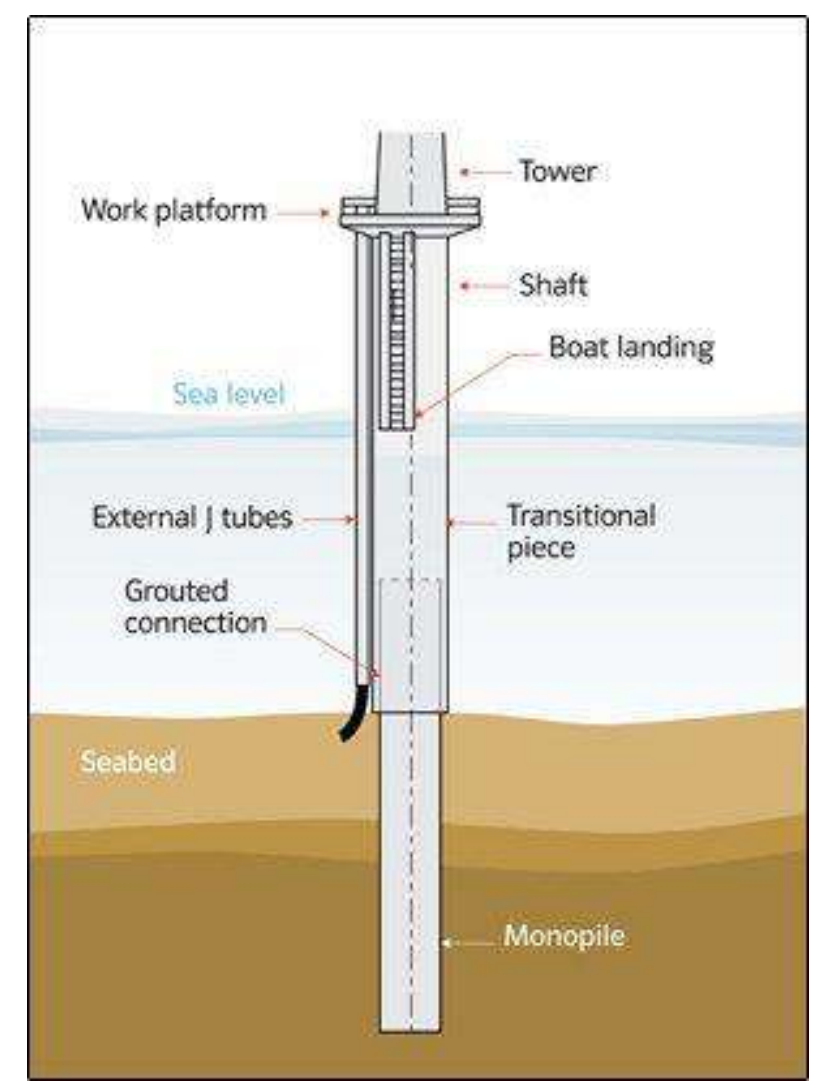

Figura 5. Cimentación monopila (fuente: www.4coffshore.com)

Cabe destacar, que, para garantizar la estabilidad y la firmeza suficiente en el soporte, existen diferentes técnicas, cuyo rango de aplicación dependerá de las características presentes en el lecho marino, y de la profundidad a la que se pretende ejecutar la instalación.

La estructura deberá poseer una sección mínima que será calculada para resistir los esfuerzos axiles, de flexión, de cortante y de fatiga; de acuerdo con las cargas recibidas del medio marino y de las características del aerogenerador. Dicha sección aumentará de forma significativa al aumentar la profundidad de instalación, haciendo que para calados profundos (>30 m), este sistema comienza a dejar de ser válido. No obstante, dentro de esta tipología estructural ha aparecido en los últimos años el nuevo término de cimentación monopila XXL, para referirse a los últimos desarrollos de parques offshore con cimentación monopila en los que se están alcanzando profundidades que superan los 30 metros de profundidad. Algunos ejemplos pertenecientes a esta tipología serían el parque Baltic II, el parque Gode Wind Il y el parque Veja Mate, en Alemania. 
Algunas de las principales características de las cimentaciones de este tipo son las siguientes:

- Se recomienda la utilización de esta tipología en aquellas zonas donde el lecho marino presenta un cierto espesor de terreno blando en las capas superficiales, antes de llegar a la capa de material competente. Por otro lado, el diseño de estructuras offshore, obliga a tener en cuenta los posibles fenómenos de socavación que se puedan producir. Esta tipología estructural presenta menores profundidades de socavación que las existentes en la tipología de cimentación por gravedad, tal y como afirman diferentes autores (Rudolph et al., 2007).

- Para aerogeneradores de tamaño pequeño y mediano, esta es la estructura más competitiva, pese a que existen ya ejemplos de cimentación monopila para grandes turbinas. Durante el proceso de construcción, quizá el punto más problemático es el de tener acceso a las embarcaciones adecuadas para realizar el transporte de los distintos componentes y su montaje, dado que la posterior instalación es sencilla y el proceso de fabricación se realiza en serie.

- La composición más común que se encuentra en esta tipología de cimentación, es que se encuentren formadas por estructuras metálicas tubulares con espesores de entre 70 y 100 milímetros, y con diámetros de hasta 8 metros, aunque cada vez más se está empezando a introducir la utilización de estructuras de hormigón y la fabricación de pilas de mayor diámetro. Tal y como se afirmaba anteriormente, las dimensiones de la estructura dependen de una gran cantidad de condicionantes de diseño, por lo que existen multitud de variedades. Por otro lado, dependiendo de las condiciones del lecho marino y de las cargas actuantes, se deberá ajustar la penetración de las monopilas, en el mismo.

- Con respecto a su rango de aplicación, existen autores indican que dichas estructuras son adecuadas para profundidades entre 0 y 25 metros (DNV, 2013), mientras que otros indican que dicho rango puede extenderse hasta los 30 metros de profundidad (Ashuri y Zaaijer, 2007). No obstante, su uso es desaconsejable en profundidades mayores debido a que pueden aparecer problemas de vibración y deflexión, debido a la flexibilidad de la estructura (DNV, 2013; Krolis et al., 2007). 


\subsubsection{Estructura tipo trípode}

La estructura principal está compuesta por un trípode localizado bajo el agua, que soporta el fuste del aerogenerador principal. Esta tipología está formada por tres pilotes pequeños que se anclan en el suelo marino, impidiendo así el movimiento de la estructura.

Este sistema presenta como ventaja principal, que con carácter general no se requiere ningún tipo de tratamiento especial en el lecho marino. La única operación necesaria es el llenado del cilindro central de soporte, con lechada. Además, la utilización de tres puntos de apoyo en vez de uno único, como en el caso de cimentación tipo monopila, hace que la estructura mejore su rigidez y su estabilidad contra el vuelco, permitiendo así su instalación a profundidades mayores. El rango de aplicación de esta tipología varía entre 20 y 50 metros.

Sin embargo, en esta tipología aparece el problema de que los buques de servicio y mantenimiento pueden chocar con alguno de los pilotes de la estructura, cuando esta se encuentra a profundidades pequeñas. Además, en comparación con las estructuras tipo jacket, el trípode sufrirá mayores cargas hidrodinámicas, debido al gran diámetro de los tubos de acero que lo conforman. La articulación principal en la columna central supone una importante dificultad de diseño, dado que será un elemento altamente sensible a la fatiga y muy complejo de diseñar. En la figura 6, se observan dos fotografías de la puesta en obra de los elementos que conforman la cimentación de estas estructuras.
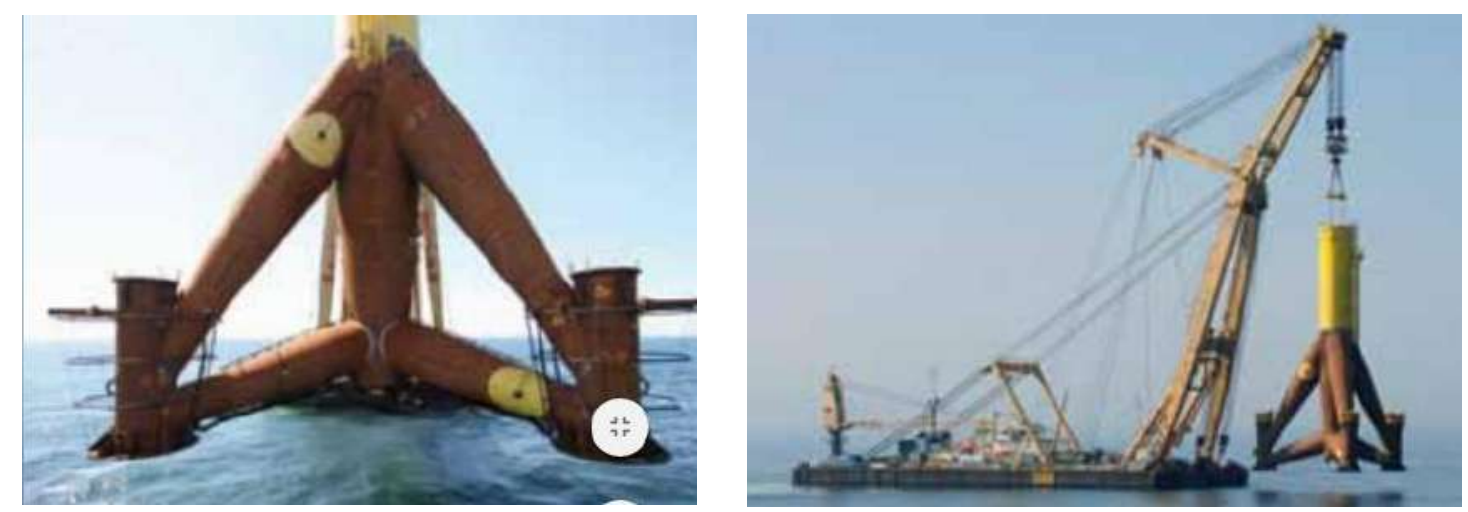

Figura 6. Colocación de estructuras de tipo trípode en el parque eólico de Alpha Ventus (fuente: www.areva.com) 
Hasta ahora, sólo en Alemania, en el parque eólico Alpha Ventus, se utilizan trípodes para apoyar a seis de sus turbinas de viento, Areva Multibrid m5000.

En los próximos años, se tiene previsto instalar trípodes en Borkum West II Riffgrund, Alemania (43 Uds.), Costa de Alabastro, Francia (21 Uds.) y Galveston, EE.UU. Pero, aun así, dicha tipología sigue siendo sólo una pequeña minoría en relación con el resto de diseños.

\subsubsection{Estructuras tipo jacket}

Hasta el año 2007, otros sistemas de soporte como las estructuras de gravedad o las estructuras monopila, sólo habían sido capaces de alcanzar profundidades en el entorno de los 20 metros.

Sin embargo, el proyecto Beatriz cambió radicalmente este hecho, permitiendo a la ingeniería de parques eólicos offshore dar un salto desde los 20 hasta los 45 metros de profundidad, y postulando a la estructura jacket como tipología adecuada para alcanzar mayores profundidades. El proyecto Beatriz pertenece a un consorcio entre la empresa petrolera británica Talisman Energy y la eléctrica escocesa Scottish Southern Energy (SSE), compañías que colaboran con otras empresas europeas.

El concepto de diseño de las estructuras tipo jacket procede de la industria del petróleo y del gas, donde se utilizan para poder apoyar equipos de perforación, a profundidades superiores a los 100 metros. La estructura jacket está formada por cuatro patas principales, que se conectan entre sí mediante multitud de arriostramientos a modo de celosía. Este sistema es similar al de una torre estándar de alta tensión.

De forma similar a la mostrada en la tipología de trípode, la base de la estructura se fija al lecho marino mediante cuatro pequeños pilotes. Todos los elementos que componen la estructura son tubulares, a diferencia de sus homólogos onshore que se hacen generalmente a partir de perfiles angulares. La figura 7 muestra un ejemplo de esta tipología en el parque eólico offshore de Thornton Bank en Bélgica. 


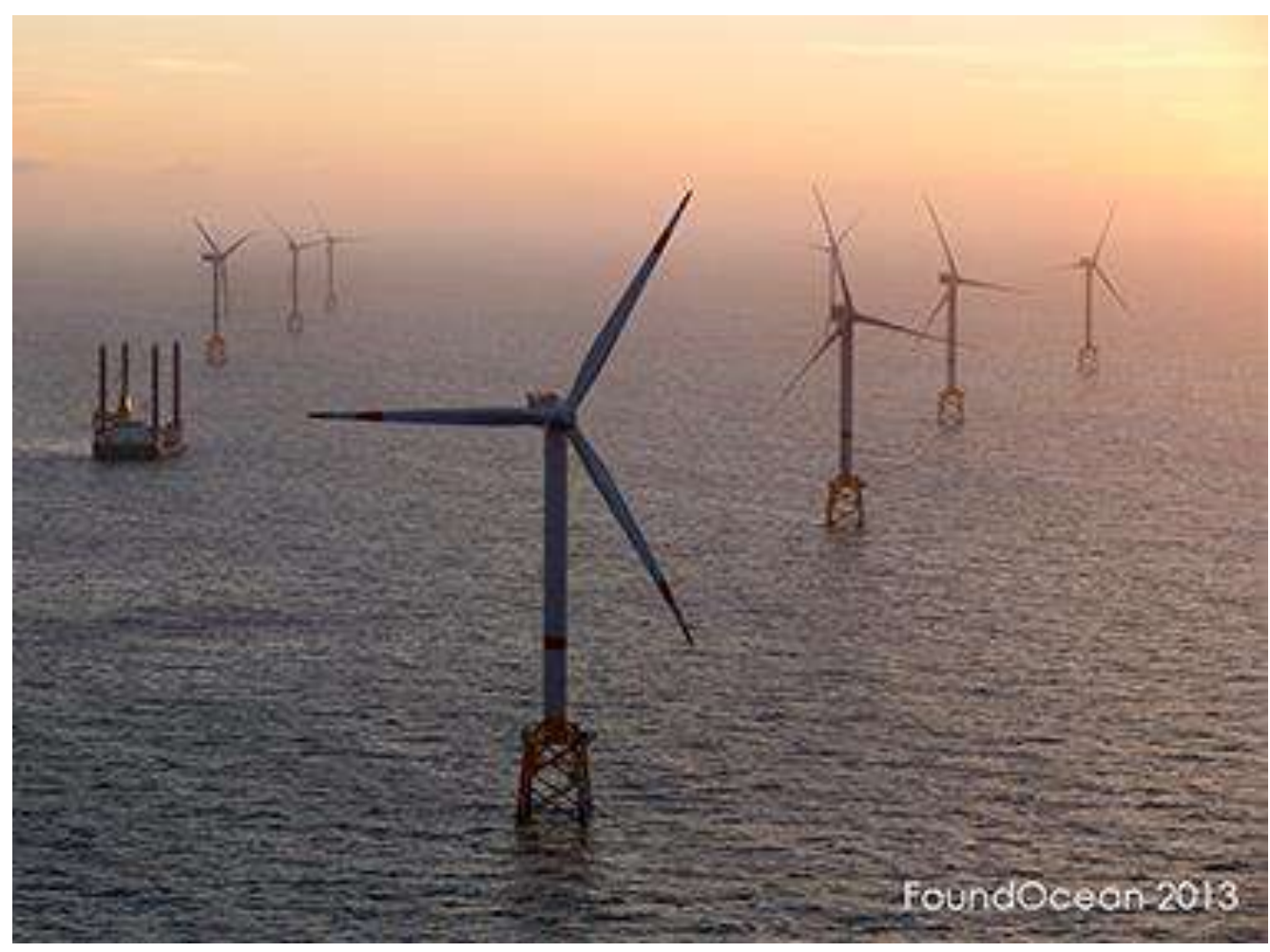

Figura 7. Cimentación tipo jacket en el parque de Thornton Bank, Bélgica (fuente: www.foundocean.com)

Los distintos elementos que componen la estructura se conectan entre ellos a través de las articulaciones tubulares. Estas articulaciones constituyen uno de los aspectos más críticos durante la fase de construcción. En la actualidad las juntas tubulares están soldadas, y debido al gran número de articulaciones, es necesaria la realización de una gran cantidad de soldadura de tipo manual, haciendo indispensable la utilización de un gran número soldadores para terminar la estructura en un tiempo razonable. Además, estas articulaciones son los puntos más débiles de la estructura, en términos de fatiga.

Al igual que en los casos de la tipología trípode, en las estructuras tipo jacket en necesario el uso de una pieza de transición para apoyar la torre del aerogenerador, que también incluye la plataforma de trabajo justo debajo de dicha torre. A continuación, en la figura 8 se observa un ejemplo de esta pieza de transición: 
Modelos de predicción de esfuerzos hidrodinámicos y socavación aplicados a ingeniería offshore

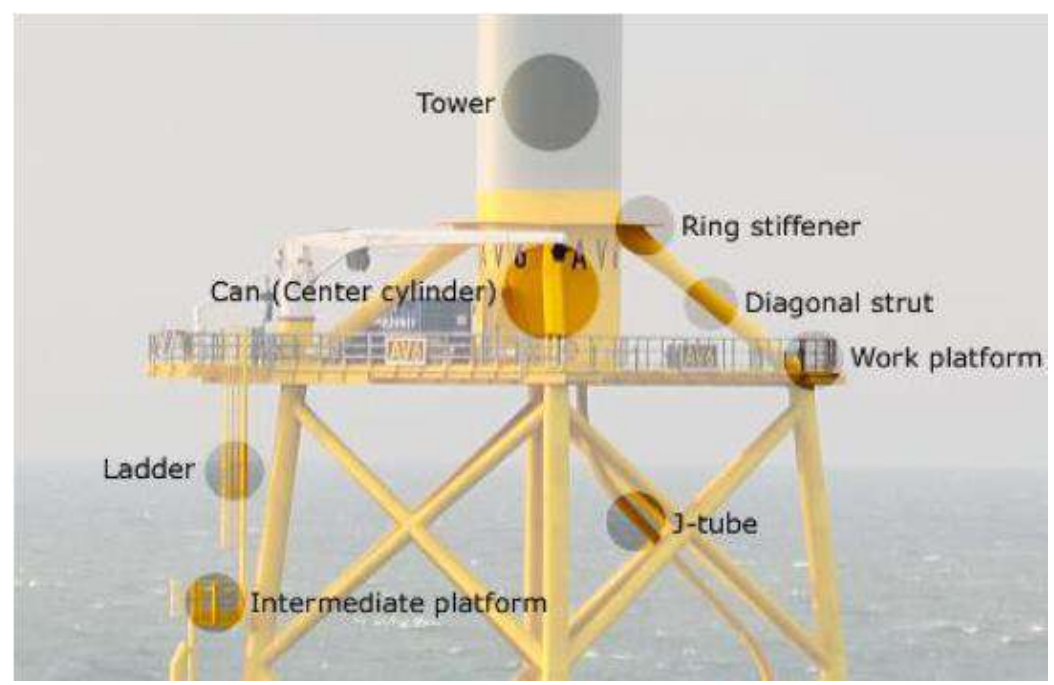

Figura 8. Pieza de transición en una estructura jacket, parque de Thornton Bank, Bélgica (fuente: www.offshorewind.biz)

La estructura tipo jacket a menudo se ancla al lecho marino mediante pilotes. No obstante, también se puede encontrar el uso de cimentaciones de gravedad o de anclajes de tracción. En la figura 9, se observan esquemas de estructuras tipo jacket:
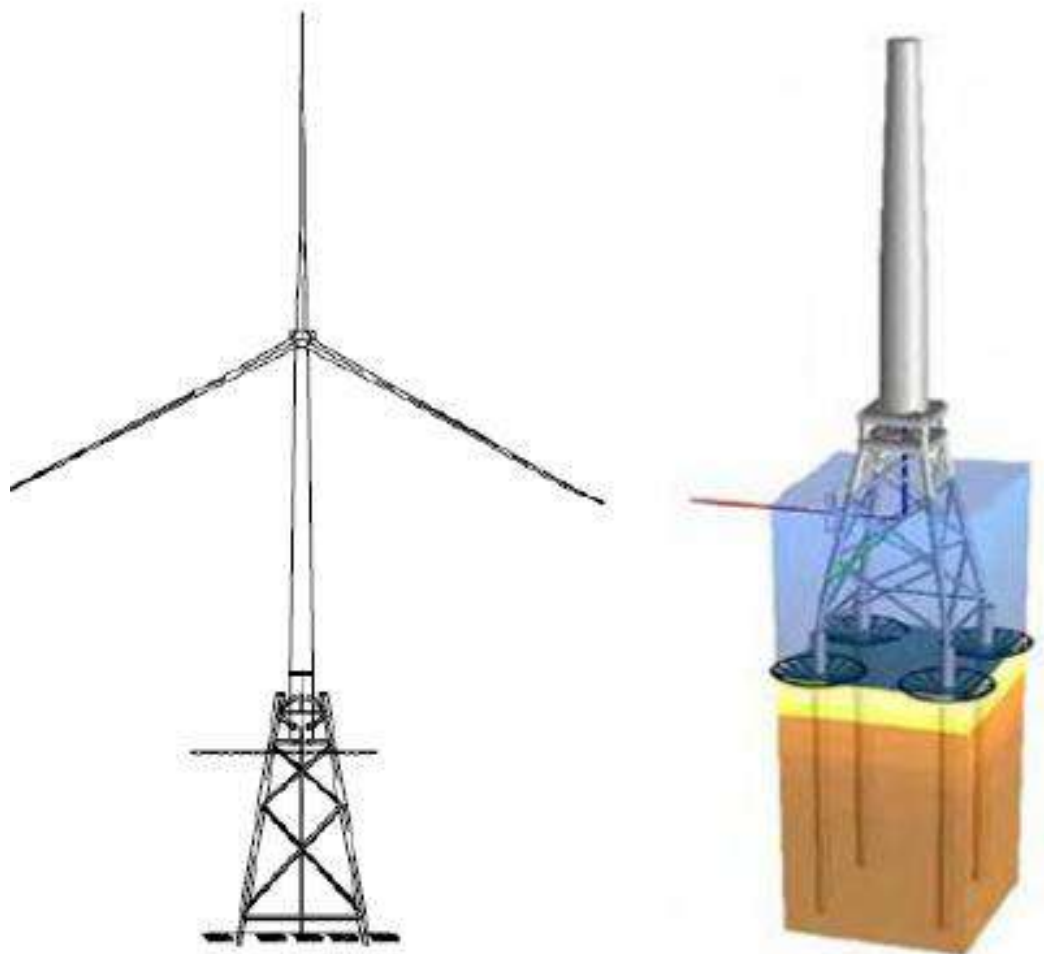

Figura 9. Esquema de una cimentación tipo jacket (La figura de la izquierda es de fuente: DNV, 2013; la de la derecha: Esteban, 2009)

Las principales características que presentan las cimentaciones tipo trípode y jacket son: 
- Están compuestas por elementos cilíndricos metálicos, de reducido diámetro en comparación con la tipología de monopila. Estos elementos poseen diámetros de entre 1 y 2 metros, generalmente (Seidel, 2007).

- El rango de aplicación de estas estructuras, viene definido por algunas referencias para profundidades entre 25 y 50 metros (DNV, 2013), mientras otras amplían este rango de aplicación, para todas aquellas profundidades que sean mayores de 20 metros (Ashuri y Zaaijer, 2007). Como puede observarse, son profundidades superiores a las recomendadas para monopilas y para cimentaciones de gravedad. La viabilidad financiera del proyecto es la que establece el límite de aplicación en los 50 metros de profundidad en el momento actual, dado que para profundidades mayores solo será rentable la instalación si se encuentra dedicada a la extracción de gas o petróleo.

- Estas estructuras pueden encontrarse pilotadas o sobre bases de gravedad de hormigón, dependiendo de la capacidad portante y de las características del lecho marino. Este hecho supone una importante ventaja, permitiendo que esta tipología se pueda adaptar a cualquier tipo de lecho marino.

\subsection{Estructuras flotantes}

En la actualidad, para profundidades superiores a los 40-50 metros, es necesario recurrir a estructuras de tipo flotante, dado que las estructuras fijas presentan una instalación muy cara y dejan de ser viables económicamente. A día de hoy, estos sistemas flotantes se encuentran en fase de desarrollo, pudiéndose diferenciar principalmente tres tipologías.

- Ballast Stabilized Spar-Buoy: que será denominado a lo largo del texto como tipologías de lastre estabilizado boya-larguero.

- Mooring Line Stabilized with Tension Leg Platform (TLP): que será denominado a lo largo del texto como plataformas con línea de fondeo estabilizada con tirantes, o plataformas con patas en tensión.

- Buoyancy Stabilized: que será denominado a lo largo del texto como plataformas con flotabilidad estabilizada.

En la figura 10 se observa un esquema de cada una de las tres tipologías: 


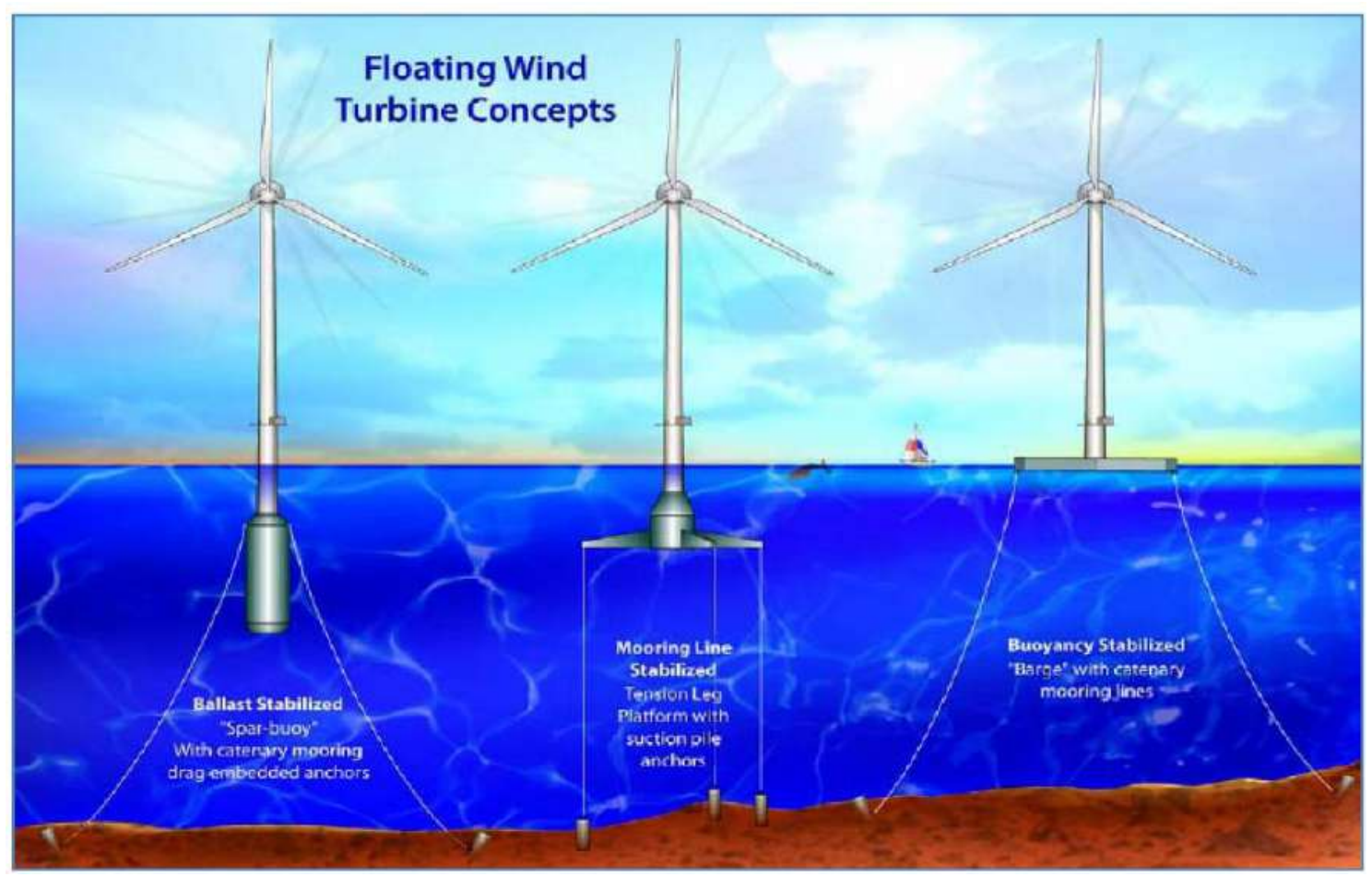

Figura 10. Tipologías de estructuras flotantes (fuente: Butterfield et al., 2005)

El proyecto Windfloat desarrollado mediante la colaboración entre la empresa norteamericana Principle Power y EDP, frente a la costa de Aguçadoura en Portugal, supuso el primer indicio en Europa de este tipo de estructuras. Aunque posteriormente, el proyecto Deepwind en Dinamarca, permitió al país nórdico, establecer su liderazgo en el desarrollo de estructuras flotantes.

No obstante, existen multitud de ejemplos en Europa. En Noruega el proyecto HiPRWind, fue utilizado como banco de pruebas para el desarrollo de aerogeneradores offshore flotantes, contando con la participación de la empresa española Acciona. Además, también se realizó en Noruega el proyecto Hywind, que supuso el desarrollo de la primera turbina eólica a nivel mundial, con una estructura de soporte flotante.

Por otro lado, otras empresas españolas, como por ejemplo Iberdrola, han optado también por apostar en este nuevo concepto de diseño, invirtiendo grandes cantidades de tiempo y recursos para investigar la implantación de estas nuevas tecnologías. En el caso concreto de Iberdrola, la empresa ha utilizado su implantación en Reino Unido, para iniciar su proyecto 
TLPWind, en colaboración con la Universidad de Strathclyde y con ORE Catapult, con el objeto de desarrollar estructuras de la tipología de plataformas con patas en tensión.

Por otra parte, existen además varios modelos a escala, que se encuentra flotando ya en el mar: el Blue $\mathrm{H}$, cerca de Italia, y el Sway, en las aguas de Noruega.

Las estructuras flotantes, como su nombre indica, son aquellas cuyo sostenimiento o apoyo proviene directamente del agua, en vez de estar soportadas por el lecho marino. No obstante, estas estructuras necesitan ser ancladas al lecho mediante cables de amarre, largueros o tirantes.

La concepción de esta tipología nació en la industria petrolera y de gas, pero la industria eólica está desarrollando nuevas modificaciones e híbridos, que le permitan obtener la máxima eficiencia y adaptar esta tecnología a sus características particulares.

\subsubsection{Lastre estabilizado boya-larguero}

Esta tipología está formada por un gran casco cilíndrico que se sitúa en la parte inferior, que está hecho de acero o de hormigón. Además, posee unos cables que anclan la estructura al lecho marino, reduciendo así la posible amplitud de movimiento que pueda sufrir la instalación. De las tres tipologías flotantes, esta es la que permite una mayor profundidad de instalación. En la figura 11 se muestra una infografía que representa dicha tipología: 


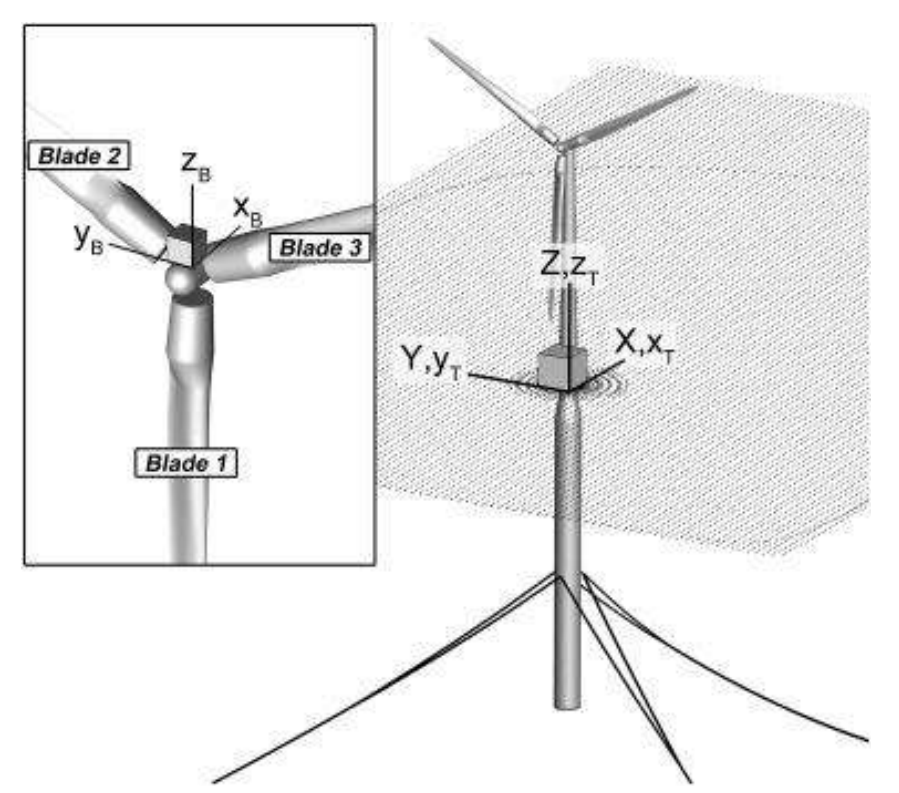

Figura 11. Ejemplo de tipología flotante con lastre estabilizado (fuente: Quallen et al., 2016)

La función principal del casco cilíndrico es la de proporcionar estabilidad, por lo tanto, las secciones inferiores de este son, por ejemplo: tanques de almacenamiento de aceite, depósitos de arena, etc.; para actuar de lastre. Las principales ventajas de este sistema son:

- El movimiento inducido por el oleaje es mínimo.

- Presenta unos costes estructurales reducidos.

- Durante el proceso de transporte y montaje, se puede aprovechar que la estructura es flotante.

No obstante, pese a todas estas ventajas, esta tipología presenta una relativa amplitud de movimientos debidos a que los cables de anclaje no se encuentran sometidos a una gran tensión, lo que puede dificultar las labores de operación y mantenimiento. Este hecho es una importante desventaja que debe ser analizada durante la fase de diseño, cuando se prevea el uso de este tipo de estructuras.

En la figura 12 se muestra el Hywind diseñado por la empresa noruega Statoil, que pertenece a esta tipología. El cilindro de acero alcanza los 100 metros de profundidad, tiene un diámetro de 8,3 metros (6 metros en la línea de agua), y está construido a partir de 1.500 toneladas de acero. Teniendo en cuenta el peso del lastre y de la turbina, el peso total es de 5.300 toneladas. La turbina del prototipo Hywind es una Siemens de 2,3 MW. 
El flotador se ancla al fondo marino mediante líneas de amarre, y presenta una ligera inclinación debida al efecto el agua y del viento sobre la estructura. Este hecho supone uno de los principales inconvenientes, ya que los aerogeneradores están diseñados para ser apoyados sobre una base estable, que garantice un ángulo que no diverja más de $0,5^{\circ}$ de inclinación respecto de la vertical.
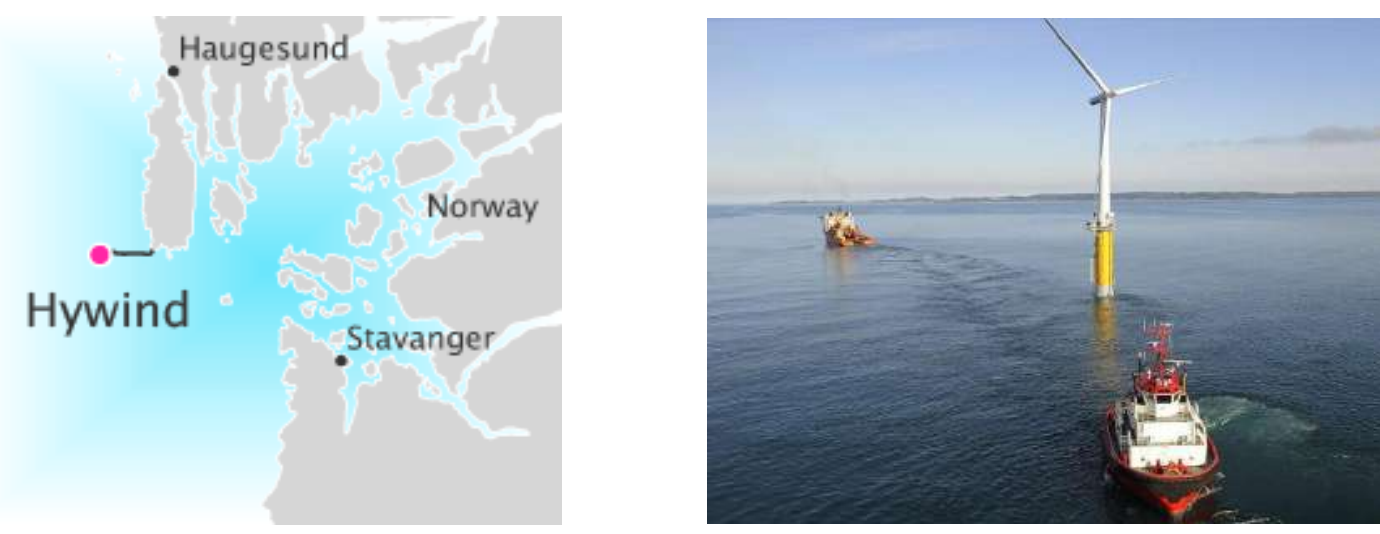

Figura 12. Imagen de la Hywind en Noruega (fuente: www.statoil.com/)

El peso es el parámetro de diseño fundamental para esta tipología. Cuanto mayor sea el lastre, menores serán los movimientos que se produzcan en la estructura. Para Hywind, la inclinación llega a alcanzar hasta $3^{\circ}$ fuera de la vertical, y el balanceo de la estructura tiene un periodo de entre 20 y 30 segundos. Además, la ventaja de esta tipología, en comparación con otras estructuras flotantes, es la pequeña sección transversal en superficie. De esta forma, la estructura recibe menores cargas hidrodinámicas del oleaje.

\subsubsection{Plataforma con línea de fondeo estabilizada con tirantes (TLP)}

Esta tipología está compuesta por una plataforma vertical flotante anclada mediante cuatro cables macizos de alta resistencia al fondo marino. Los cables se mantienen siempre con una alta tensión, permitiendo que la base permanezca con una flotación constante.

Cualesquiera que sean las condiciones de viento y de oleaje, gracias a la tensión de los cables de anclaje, se consigue estabilizar la flotabilidad y la posición vertical de la turbina. El parámetro fundamental de diseño de esta tipología es la tensión que presentarán los cables 
de anclaje, dado que cuanto mayor sea menor será la libertad de movimientos que tendrá la estructura. En la figura 13, se muestra un ejemplo de la tipología TLP:

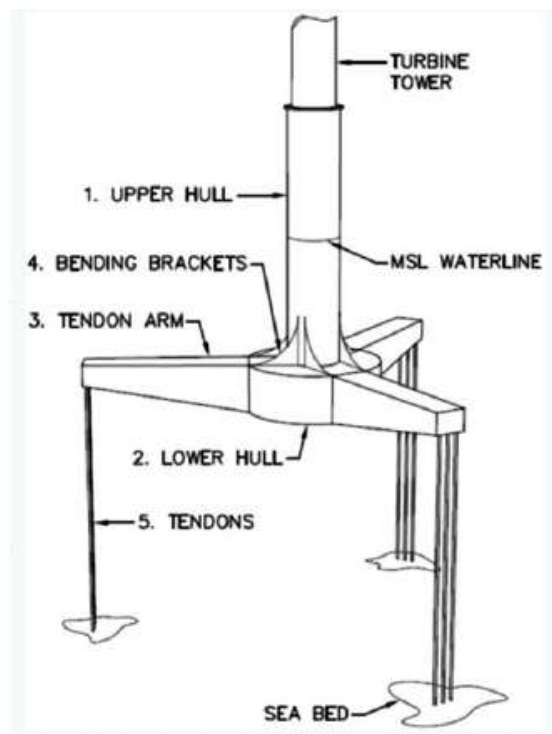

Figura 13. Tipología estabilización mediante línea de amarre (fuente: Thiagarajan et al., 2014)

La mayor desventaja de este tipo de estructura será el garantizar la correcta fijación de los cables de anclaje, siendo necesaria la utilización de complejos sistemas que no son aptos para todo tipo de lechos. Existen ejemplos de esta tipología utilizados para grandes profundidades en la industria de extracción de petróleo desde la década de los 80 . La primera plataforma de extracción petrolífera que utilizó esta tipología se instaló en el yacimiento de Hutton en el verano de 1984. Actualmente, la empresa holandesa Blue $\mathrm{H}$ ha construido un prototipo de escala 3/4 frente a las costas de Italia. Además, Iberdrola mediante el proyecto TLPWind pretende implantar y promover la instalación de aerogeneradores de esta tipología estructural a lo largo de las aguas que rodean Reino Unido, dado que hay gran multitud de zonas en las que la implantación de parques eólicos offshore no es viable debido a que se encuentran a grandes profundidades. En la figura 14 se muestra un ejemplo esquemático del modelo de TLPWind de Iberdrola. 


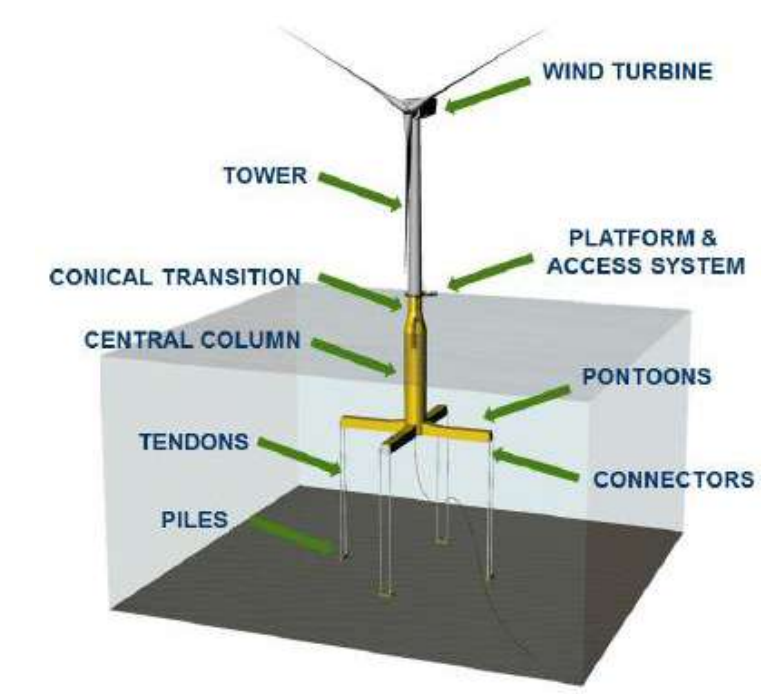

Figura 14. Modelo TLP Wind de Iberdrola (fuente: Amate, 2014)

De las tres tipologías, esta es la que garantiza una menor amplitud de movimiento en la estructura, aunque, por otro lado, también es la que presenta una instalación más cara.

\subsubsection{Plataforma con flotabilidad estabilizada}

Este sistema consiste en una plataforma flotante semisumergible, cuya flotabilidad está estabilizada. Su diseño ha tenido como objetivo principal el proporcionar una estructura flotante más rentable y de fácil instalación. El principio de diseño es el de proveer una plataforma muy ligera que presente una importante área de flotación que permita garantizar la estabilidad de la estructura en condiciones de carga. Dispone de unos cables anclados en el lecho marino, cuya misión principal es evitar el vuelco de la estructura, en condiciones extremas, así como su desplazamiento lejos de la localización de diseño. No obstante, esta tipología no será válida para zonas con un oleaje extremo, ya que al ser un sistema cuya estabilidad depende exclusivamente del área de flotación, presenta una importante fragilidad frente a condiciones medioambientales muy adversas. Las principales ventajas de esta tipología son sus reducidos costes de instalación y transporte, siendo posible que sea arrastrada desde el puerto por cualquier remolcador, lo que reduce bastante los costes de desplazamiento. 


\section{Clasificación según la profundidad}

En función de la profundidad en la que se encuentre el aerogenerador habrá que recurrir a la tipología adecuada que permita que el proyecto sea viable. Conforme la profundidad aumenta se debe recurrir a estructuras cada vez menos rígidas, que sean capaces de absorber mejor la energía de impacto del oleaje, utilizando finalmente, las estructuras flotantes para las mayores profundidades.

De acuerdo con este criterio, se ha elaborado la clasificación expuesta en la tabla 1 de acuerdo con los datos obtenidos de los parques eólicos, que actualmente se encuentran en operación.

\begin{tabular}{|c|c|}
\hline Profundidad máxima & Tipología \\
\hline$<30$ metros & Monopila \\
\hline$<50$ metros & Monopila XXL \\
\hline$<50$ metros & Estructura de gravedad \\
\hline $20-50$ metros & Jacket \\
\hline$>50$ metros & Otros tipos y flotantes \\
\hline
\end{tabular}

En ingeniería offshore aplicada a la generación de energía eléctrica a partir del aprovechamiento de la energía eólica, las estructuras dejan de ser rentables en profundidades superiores a los 50 metros. A partir de esta profundidad, la elección de una de las tres tipologías flotantes frente al uso de estructuras tipo jacket fijas o flexibles, atiende a otros condicionantes como la capacidad portante del lecho marino, los condicionantes de la operación y el mantenimiento que exigen pocas amplitudes de movimiento de la estructura, etc. 


\section{Mecánica de ondas}

En este apartado se va a proceder a realizar una exposición detallada sobre la caracterización física del oleaje y los factores que influyen sobre sus magnitudes fundamentales, mostrando cada una de las principales teorías desarrolladas por diferentes autores, que se han establecido a lo largo de la historia, para definir este fenómeno en cada uno de los diferentes casos que se presentan en la naturaleza.

El proceso de propagación de oleaje, a través de un fluido, es no lineal y se ve influenciado por un gran número de variables y efectos, cuya interacción es complicada de estudiar desde un punto de vista teórico. Por este motivo, tradicionalmente, ha sido necesario establecer una serie de hipótesis de partida que simplifican el problema para poder plantear una solución analítica del mismo. Las consideraciones básicas que se suelen adoptar son:

1. Se supone que el agua se comporta como un fluido incompresible $(\Delta \rho=0)$.

2. No se tiene en cuenta la tensión superficial.

3. Las principales fuerzas a tener en cuenta son las fuerzas gravitatorias y las fuerzas derivadas del gradiente de presión. Por lo tanto, se supone que el fluido es no viscoso $(v=0)$ y que se pueden despreciar las tensiones tangenciales.

4. Se reduce el análisis del problema a una componente horizontal $u$ y otra vertical $w$, aceptando que el modelo físico es bidimensional, pese a que en la realidad el movimiento siempre será tridimensional.

5. Se acepta que el fondo es fijo e impermeable.

6. Se supondrá que el oleaje es regular y periódico, y que la única fuerza exterior que tiene efecto sobre el fluido es la gravedad terrestre.

7. El efecto de Coriolis y las pérdidas de energía por rotura de la ola son despreciables. 
8. También se asume que se trata de un movimiento irrotacional $\nabla \times u=0$, lo que permite definir el problema en forma de potencial de velocidades $\nabla \phi=u$. Esta asunción permite plantear el comportamiento del fluido mediante un flujo laminar, es decir, el movimiento de las partículas del fluido se produce siguiendo trayectorias bastante regulares, separadas y perfectamente definidas dando la impresión de que se tratara de láminas o capas más o menos paralelas entre sí, las cuales se deslizan suavemente unas sobre otras sin que exista mezcla macroscópica o intercambio transversal entre ellas. Esto implica que no se producirán pérdidas locales de energía debidas a la formación de turbulencias, tal y como se muestra en la figura 15.

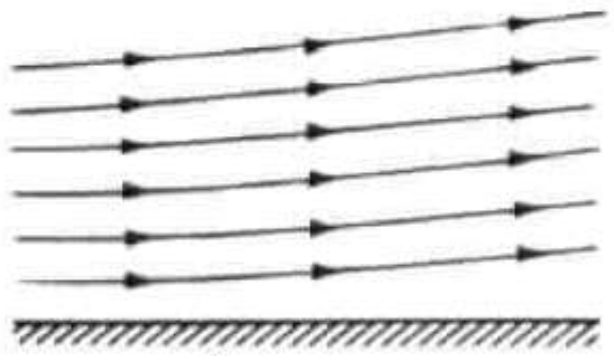

Flujo laminar

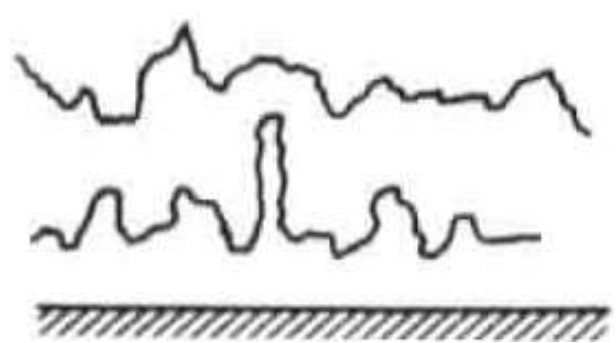

Flujo turbulento

Figura 15. Tipos de flujo (fuente: Sotelo, 1980)

Las ecuaciones que definen el proceso de propagación del oleaje en un fluido que cumple las condiciones anteriormente descritas, son:

Ecuación de Laplace. Esta ecuación se cumple en los flujos incompresibles e irrotacionales y considera que no hay variación en el tiempo, ni de volumen ni de masa (incompresibilidad y conservación de la masa).

$$
\frac{\partial^{2} \phi}{\partial x^{2}}+\frac{\partial^{2} \phi}{\partial z^{2}}=0
$$

Primera condición de contorno en la superficie libre. Esta condición de contorno establece que las partículas de agua en la superficie libre $\eta(x, t)$ permanecen en esta:

$$
\frac{\partial \phi}{\partial z}=\frac{\partial \eta}{\partial t}+\frac{\partial \phi}{\partial x} \cdot \frac{\partial \eta}{\partial x} \quad w=\frac{\partial \eta}{\partial t}+u \cdot \frac{\partial \eta}{\partial x}
$$


donde $\eta(x, t)$ es la función que define el desplazamiento del agua en la superficie libre con referencia al nivel medio del agua $(z=0)$ y $w$ es la velocidad vertical.

Segunda condición de contorno en la superficie libre (ecuación de Bernoulli).

$$
\frac{\partial \vec{\phi}}{\partial t}+\frac{p_{a t m}}{\rho}+\frac{1}{2}(\nabla \phi)^{2}+g \eta=0
$$

Condición de contorno por fondo. Esta condición de contorno establece que la velocidad vertical es nula en las partículas del fondo. La función que define el fondo marino es $z=-h(x)$.

$$
w=-u \cdot \frac{\partial h}{\partial x}=0
$$

Condición de contorno lateral. La condición de oleaje periódico y regular establece la periodicidad en una longitud de onda $L$ y periodo $T$.

$$
\begin{aligned}
& \phi(x, t)=\phi(x+L, t) \\
& \phi(x, t)=\phi(x, t+T)
\end{aligned}
$$

A continuación, se muestra un breve resumen de las teorías físicas propuestas por distintos autores para determinar la propagación del oleaje y los efectos que durante ella se producen. Cada teoría resuelve el problema en un rango determinado de valores de la longitud de onda en comparación con la profundidad y la altura de ola.

Teoría de ondas de Airy o Stokes de primer orden. Dicha teoría presenta una dispersión de frecuencias completa, por tanto, es válida para profundidades y longitudes de onda arbitrarias, pero se trata de una teoría lineal sin dispersión en la amplitud, quedando limitado su uso a amplitudes pequeñas de onda, es decir, a alturas de ola pequeñas en comparación con la profundidad.

Teoría de Stokes de órdenes superiores a la unidad. La teoría de Stokes se compone de una suma en serie de términos que aumentan en orden y que permiten describir ondas 
débilmente no lineales y dispersivas. Esta formulación es especialmente acertada en aguas más profundas para longitudes de onda pequeñas, con respecto a la profundidad de agua.

Teoría de onda larga. Para ondas largas la aproximación de Boussinesq o la aplicación de la ecuación de Korteweg-de Vries son más utilizadas. Esto es debido a que en aguas poco profundas las series de Stokes necesitan muchos términos antes de converger hacia la solución, debido a las esbeltas crestas y a los valles planos y largos, característicos de las ondas no lineales. Los modelos de Korteweg-de Vries o de Boussinesq dan buenas aproximaciones para estas ondas no lineales largas.

Ecuación de Korteweg-de Vries. Esta formulación describe la propagación frontal de ondas en régimen débilmente no lineal y dispersivo para longitudes de onda $L>7 h$, siendo $L$ la longitud de onda del oleaje estudiado y h la profundidad media. La ecuación de Kortewegde Vries es la que da origen a toda la teoría cnoidal.

Ecuaciones de Boussinesq. En su forma clásica, estas ecuaciones tienen el mismo rango de validez que la ecuación de Korteweg-de Vries, pero permiten estudiar la propagación del oleaje en direcciones arbitrarias y no solo hacia delante. El problema fundamental de esas ecuaciones es que, normalmente, son más difíciles de resolver que las ecuaciones de Korteweg-de Vries, y en multitud de aplicaciones el oleaje reflejado es pequeño y puede ser eliminado de las ecuaciones.

Ecuaciones de aguas someras. Estas ecuaciones son también de carácter no lineal y presentan dispersión en su amplitud, pero no en su frecuencia. Estas ecuaciones son válidas para longitudes de onda $L>20 \mathrm{~h}$.

Se muestra a continuación, en la figura 16, la clasificación de Le Méhauté (1976) para definir el rango de aplicación de cada una de las distintas teorías. 


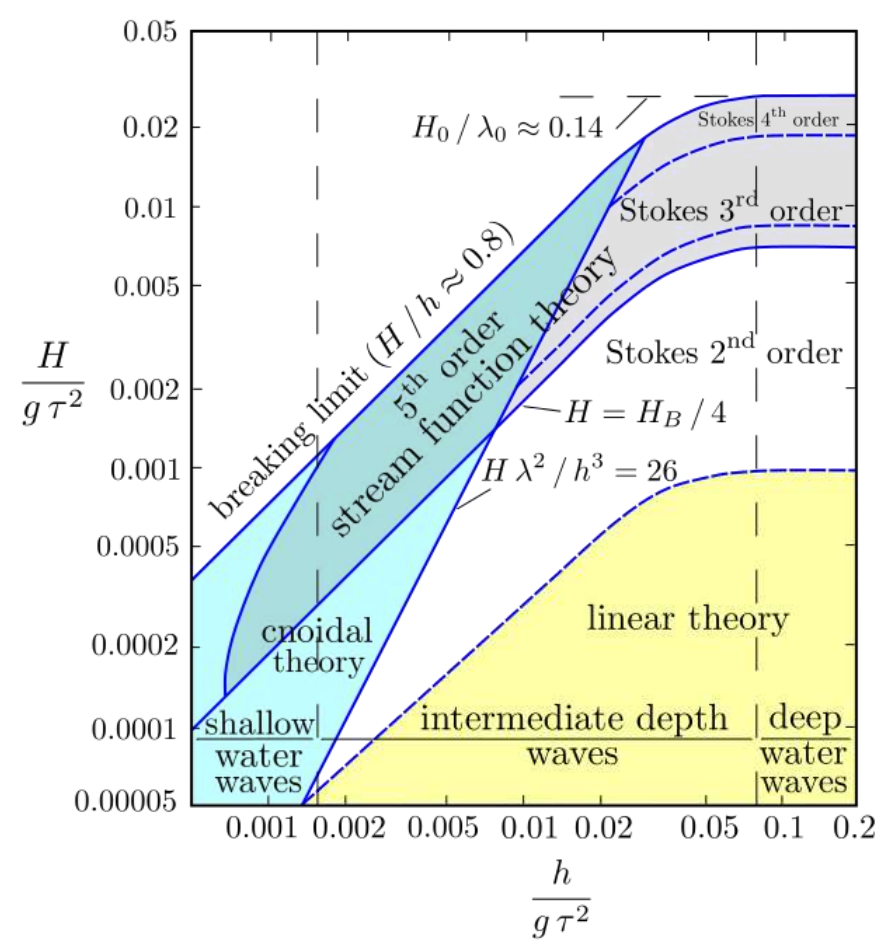

Figura 16. Clasificación de las distintas teorías de mecánica de ondas (fuente: Le Méhauté, 1976)

La teoría de onda de Stokes y la teoría de onda cnoidal pertenecen a la categoría de soluciones de perturbación, en las cuales cada solución es expresada como serie de potencias en términos de un cierto parámetro adimensional. La teoría de Stokes será válida para aguas relativamente profundas y la teoría cnoidal para aguas relativamente someras. Existen otras muchas soluciones desarrolladas también en forma de perturbación, no obstante, estas dos formulaciones han demostrado ser las que mejor se adaptan a la realidad, siendo su error con respecto a la condición de contorno no lineal que parametriza la superficie libre, el más pequeño de todas las teorías desarrolladas.

La formulación desarrollada se postula como fuente principal del estudio, dado que es la base a partir de la cual se realizan todos los modelos que predicen las cargas hidrodinámicas que una estructura sufrirá al estar sometida a un oleaje de unas determinadas características y a una determinada profundidad. Por lo tanto, se van a exponer las distintas teorías que estudian el oleaje según los diferentes dominios, que, fundamentalmente, se diferenciarán por la profundidad en la que se encuentre el punto de estudio y la longitud de onda del oleaje, tal y como se mostraba en la figura 16 con la clasificación de las distintas teorías según Le Méhauté. 


\subsection{Teoría lineal de Airy}

El fenómeno del oleaje está definido por un conjunto de ecuaciones, que forman un sistema no lineal de ecuaciones diferenciales. Para hacer frente a este hecho, la teoría lineal de Airy realiza una simplificación matemática y física del problema, permitiendo modelizar el fenómeno de una forma compacta y sencilla (Horikawa, 1988).

En la figura 17 se muestra el esquema general y el sistema de coordenadas utilizado en el cálculo realizado según la teoría lineal.

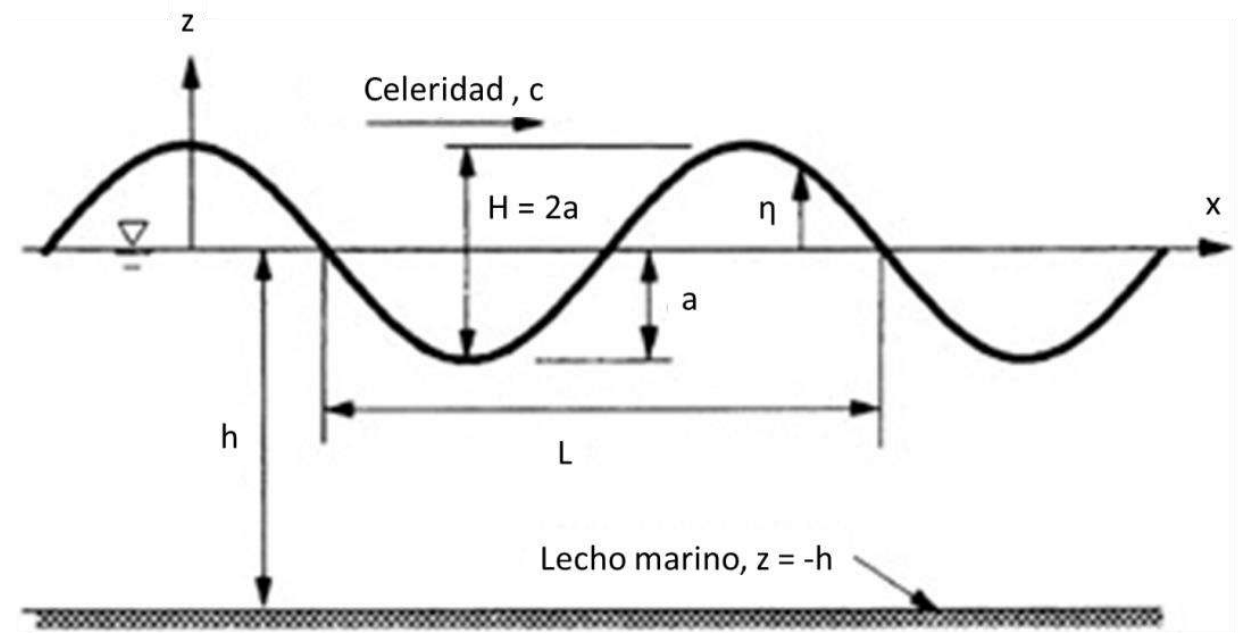

Figura 17. Esquema de la teoría lineal de ondas (fuente: elaboración propia)

Para amplitudes de ola pequeñas en relación con la longitud de onda, las condiciones de contorno en la superficie libre quedan reducidas a (Sarpkaya et al., 1981):

$$
\begin{array}{ll}
\frac{\partial \phi}{\partial z}-\frac{\partial \eta}{\partial t}=0 & \text { en } \quad z=0 \\
\frac{\partial \vec{\phi}}{\partial t}+g \eta=0 & \text { en } z=0
\end{array}
$$

La cuales se pueden combinar para dar:

$$
\frac{\partial^{2} \phi}{\partial t^{2}}+g \frac{\partial \phi}{\partial z}=0 \quad \text { en } \quad z=0
$$




$$
\eta=-\frac{1}{g}\left(\frac{\partial \phi}{\partial t}\right)_{z=0} \quad \text { en } \quad z=0
$$

La solución del problema puede ser obtenida por medio de la técnica de separación de variables donde $\phi$ será definida de la siguiente forma:

$$
\phi=Z(z) \Phi(x-c t)
$$

Sustituyendo esta expresión de $\phi$ dentro de la ecuación de Laplace, se obtienen dos ecuaciones diferenciales ordinarias:

$$
\begin{aligned}
& \frac{\partial^{2} Z}{\partial z^{2}}-k^{2} Z=0 \\
& \frac{\partial^{2} \Phi}{\partial x^{2}}+k^{2} \Phi=0
\end{aligned}
$$

donde el signo de la constante $\mathrm{k}^{2}$ ha sido elegido para proporcionar una solución periódica en $(x-c t)$ de tipo hiperbólico. Las soluciones generales de $Z$ y $\Phi$ son:

$$
\begin{gathered}
Z=A_{1} \cdot \cosh (k z)+A_{2} \cdot \operatorname{senh}(k z) \\
\Phi=A_{3} \cdot \cos (k(x-c t))+A_{4} \cdot \operatorname{sen}(k(x-c t))
\end{gathered}
$$

donde $A_{1}, A_{2}, A_{3}$ y $A_{4}$ son constantes que deberán ser determinadas a partir de las condiciones de contorno.

La variable tiempo, $t$, se define para que su instante inicial de valor $t=0$ coincida con el paso de la cresta de la ola por el punto $x=0$. Teniendo en cuenta la segunda condición de contorno:

$$
\eta=-\frac{1}{\mathrm{~g}}\left(\frac{\partial \phi}{\partial t}\right)_{z=0}
$$


se puede observar que $A_{3}=0$. La condición de contorno en fondo implicará que $A_{2}=A_{1} \cdot \tanh (k h)$. Por lo tanto, sustituyendo dentro de la definición que se ha establecido de la función de velocidad potencial, las expresiones obtenidas para Z y $\Phi$, se obtiene:

$$
\phi=A \cdot \frac{\cosh (k(z+h))}{\cosh (k h)} \operatorname{sen}(k(x-c t))
$$

donde la nueva constante es $A=A_{1} A_{4}$. La constante $A$ puede ser determinada por medio de la segunda condición de contorno que aporta una expresión que relaciona $\eta$ con la altura de ola $\mathrm{H}$, obteniendo que $\mathrm{A}=\mathrm{gH} / 2 \mathrm{kc}$. La expresión anterior cumple la condición de contorno lateral al mostrar que la función de velocidad potencial es periódica en la dirección del eje $x$ con una longitud de onda $L=2 \pi / k$ y es periódica en el tiempo con un periodo $T=2 \pi / k c$.

El número de onda viene representado por $k$, la frecuencia angular de la onda es $\omega=k c$ y la celeridad de la onda es $c=L / T=\omega / k$. De acuerdo con estas definiciones se puede reescribir la primera condición de contorno para obtener la relación de dispersión que define c o $\omega$ en términos de $\mathrm{k}$ :

$$
\omega^{2}=g k \tanh (k h)
$$

o bien,

$$
c^{2}=\frac{g}{k} \tanh (k h)
$$

Esta expresión describe como aumenta la velocidad de la onda con la longitud de onda. En vista de las expresiones anteriores, finalmente, se puede escribir la solución de la velocidad potencial como:

$$
\phi=\frac{H}{2} \cdot \frac{g}{\omega} \cdot \frac{\cosh (k(h+z))}{\cosh (h+z)} \cdot \operatorname{sen}(k x-\omega t)
$$

o bien como:

$$
\phi=\frac{\pi \mathrm{H}}{k T} \cdot \frac{\cosh (k(h+z))}{\cosh (h+z)} \cdot \operatorname{sen}(k x-\omega t)
$$


El desplazamiento de la superficie libre del agua se obtiene tras aplicar la segunda condición de contorno en la superficie libre:

$$
\eta=\left.\frac{1}{g} \cdot \frac{\partial \phi}{\partial t}\right|_{z=0}=\frac{H}{2} \cdot \operatorname{sen}(k x-\omega t)
$$

Es importante destacar que la aplicación de la teoría de Airy permite predecir varias características importantes del comportamiento del oleaje como, por ejemplo, el movimiento de las partículas del agua a distintas profundidades, a pesar de las simplificaciones establecidas, siempre y cuando se cumpla la condición de que la amplitud sea significativamente pequeña en comparación con la longitud de onda.

El movimiento seguido por las partículas dependerá de la relación existente entre la profundidad y la longitud de onda, estableciéndose tres zonas bien diferenciadas:

- En aguas profundas las partículas del fluido describen órbitas circulares, cuyo diámetro decrece exponencialmente con la profundidad.

- En aguas intermedias las órbitas de las partículas son elípticas y los ejes mayor y menor disminuyen exponencialmente con la profundidad, siendo el eje mayor paralelo a las superficies de nivel.

- En aguas poco profundas las órbitas son como las de aguas intermedias, pero el eje mayor es independiente de la profundidad.

En la figura 18 se muestra la órbita elíptica de las partículas de agua para el caso de aguas poco profundas y la órbita circular para el caso de profundidades indefinidas. 

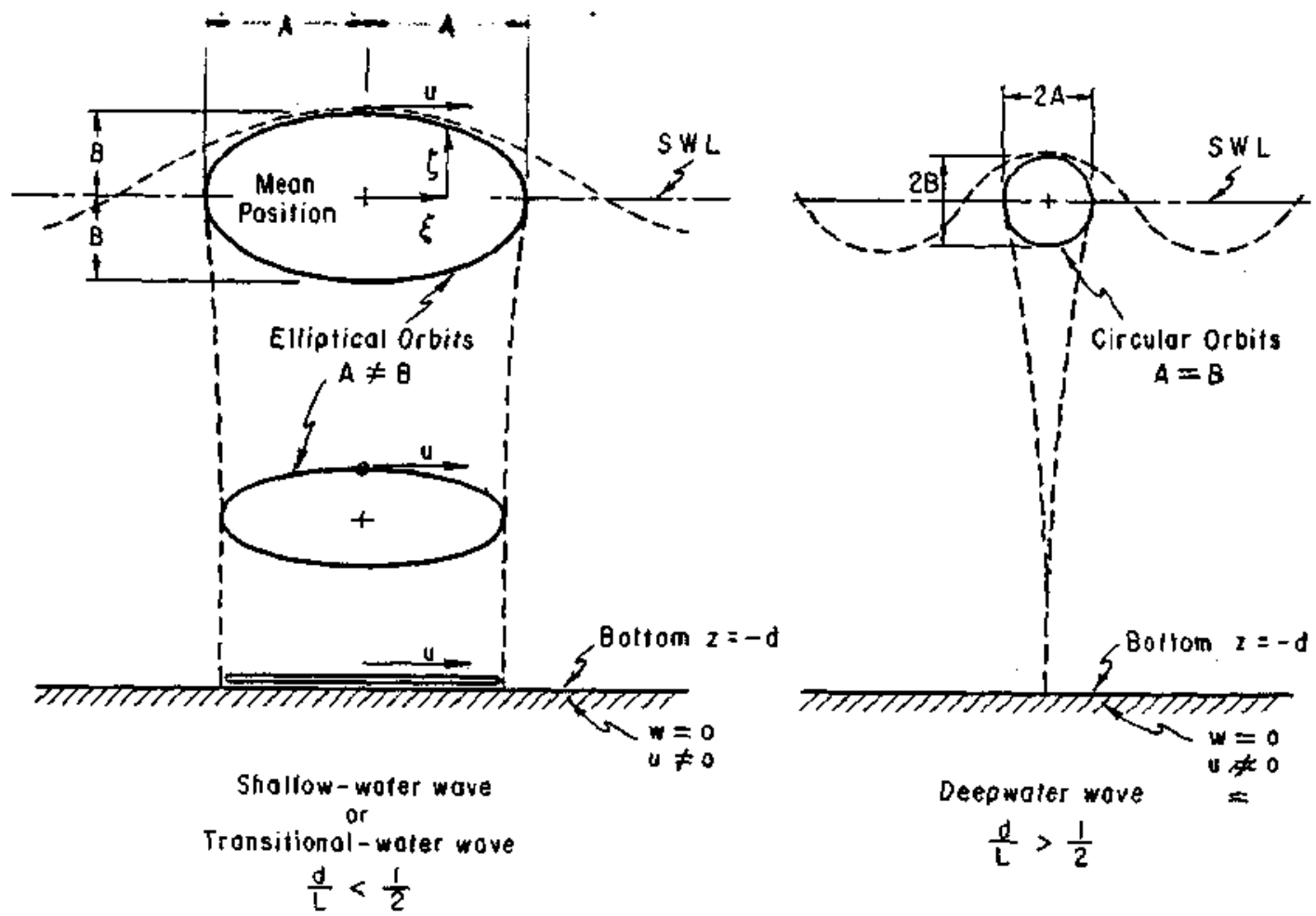

Figura 18. Trayectorias de una partícula de agua (fuente: Shore Protection Manual Vol. 1, 1984)

A continuación, se muestran en la tabla 2 todas las fórmulas derivadas de la resolución de la teoría lineal, que permiten hallar entre otras cosas, los desplazamientos, las velocidades y las aceleraciones, de cada una de las partículas presentes en el agua. 


\begin{tabular}{|c|c|c|c|}
\hline Relative Depth & $\begin{array}{c}\text { Shallow IVater } \\
\qquad \frac{d}{L}<\frac{1}{25}\end{array}$ & $\begin{array}{l}\text { Transitional Water } \\
\qquad \frac{1}{25}<\frac{d}{L}<\frac{1}{2}\end{array}$ & $\begin{array}{c}\text { Deep Water } \\
\frac{d}{L}<\frac{1}{2}\end{array}$ \\
\hline 1. Wave peofile & Sans As $>$ & $\eta=\frac{H}{2} \cos \left[\frac{2 \pi x}{L}-\frac{2 \pi t}{T}\right]=\frac{H}{2} \cos 0$ & $<$ SameAs \\
\hline 2. Wave celcrity & $C=\frac{L}{T}$ & $C=\frac{L}{T}=\frac{g T}{2 \pi} \tanh \left(\frac{2 \pi d}{L}\right)$ & $C=C_{0}=\frac{L}{T}=\frac{g T}{2 \pi}$ \\
\hline 3. Wavelraghth & $L=T \sqrt{8}$ & $L=\frac{g T^{2}}{2 \pi} \tanh \left(\frac{2 \pi d}{L}\right)$ & $L=L_{0}=\frac{g T^{2}}{2 \pi}=C_{0} T$ \\
\hline 4. Group velocity & $C_{x}=C$ & $C_{x}=n C=\frac{1}{2}\left[1+\frac{4 \pi d H L}{\sinh (4 \pi d L)}\right] C$ & $C_{z}=\frac{1}{2} C=\frac{\mathrm{g} T}{4 \pi}$ \\
\hline $\begin{array}{l}\text { 5. Waserparticle } \\
\text { velocky } \\
\text { (a) Hotizonal } \\
\text { (b) Verical }\end{array}$ & $\begin{array}{c}u=\frac{H}{2} \sqrt{\frac{g}{d}} \cos \theta \\
w=\frac{H \pi}{T}\left(1+\frac{z}{d}\right) \sin \theta\end{array}$ & $\begin{array}{l}u=\frac{I I}{2} \frac{g T}{L} \frac{\cosh [2 \pi(z+d) / L]}{\cosh (2 \pi d / L)} \cos 0 \\
w=\frac{H}{2} \frac{g T}{L} \frac{\sinh [2 \pi(z+d) / L]}{\cosh (2 \pi d / L)} \sin 0\end{array}$ & $\begin{array}{l}u=\frac{\pi H}{T} e^{\left(\frac{2 x}{L}\right)} \cos 0 \\
w=\frac{\pi H}{T} e^{\left(\frac{2 x}{L}\right)} \sin 0\end{array}$ \\
\hline $\begin{array}{l}\text { 6. Waser particle } \\
\text { acoelentiogs } \\
\text { (a) Horizonal } \\
\text { (b) Vertical }\end{array}$ & $\begin{array}{c}a_{x}=\frac{H \pi}{T} \sqrt{\frac{g}{d}} \sin \theta \\
a_{z}=-2 H\left(\frac{\pi}{T}\right)^{2}\left(1+\frac{z}{d}\right) \cos \theta\end{array}$ & $\begin{array}{l}a_{x}=\frac{g \pi H}{L} \frac{\cosh [2 \pi(z+d) / L]}{\cosh (2 \pi d L)} \sin 0 \\
a_{z}=-\frac{g \pi / I}{L} \frac{\sinh [2 \pi(z+d) / L]}{\cosh (2 \pi d L)} \cos 0\end{array}$ & $\begin{array}{l}a_{x}=2 H\left(\frac{\pi}{T}\right)^{2} e^{\left(\frac{2 x}{L}\right)} \sin \theta \\
a_{z}=-2 H\left(\frac{\pi}{T}\right)^{2} e^{\left(\frac{2 x}{L}\right)} \cos \theta\end{array}$ \\
\hline $\begin{array}{l}\text { 7. Weser particle } \\
\text { displacemens } \\
\text { (a) Hotizonal } \\
\text { (b) Vertical }\end{array}$ & $\begin{array}{l}\xi=-\frac{H T}{4 \pi} \sqrt{\frac{g}{d}} \sin \theta \\
\xi=\frac{H}{2}\left(1+\frac{\Xi}{d}\right) \cos \theta\end{array}$ & $\begin{array}{c}\xi=-\frac{H}{2} \frac{\cosh [2 \pi(z+d) / L]}{\sinh (2 \pi d / L)} \sin \theta \\
\zeta=\frac{H}{2} \frac{\sinh [2 \pi(z+d) / L]}{\sinh (2 \pi d L)} \cos \theta\end{array}$ & $\begin{array}{l}\xi=-\frac{H}{2} e^{\left(\frac{2 \pi}{L}\right)} \sin 0 \\
\zeta=\frac{H}{2} e^{\left(\frac{2 x}{L}\right)} \cos \theta\end{array}$ \\
\hline $\begin{array}{l}\text { 8. Sabsurface } \\
\text { pessufe }\end{array}$ & $p=\rho g(\eta-z)$ & $p=\rho g \eta \frac{\cosh [2 \pi(z+d V L]}{\cosh (2 \pi d / L)}-\rho g z$ & $p=\rho g \eta e^{\left(\frac{x_{0}}{L}\right)}-\rho g z$ \\
\hline
\end{tabular}

Tabla 2. Teoría de Airy (fuente: Shore Protection Manual Vol. 1, 1984)

En la tabla anterior, el parámetro $d$ representa la profundidad (en inglés depth), que es representada por la letra $h$ a lo largo de todo el documento.

\subsection{Teoría de Stokes de 2o Orden}

En la práctica, en muchas ocasiones el oleaje presenta una esbeltez o peralte, $\mathrm{H} / \mathrm{L}$, tan grande que los cálculos realizados mediante la teoría lineal de ondas presentan resultados que se alejan notablemente de la realidad. 
Como se vio en el punto anterior, la solución del problema de primer orden está basada en una solución exacta de la ecuación de Laplace, considerando una aproximación lineal de las condiciones de contorno. No obstante, las medidas muestran que la solución que presenta la teoría lineal de primer orden puede dirigir a resultados inaceptables, como se muestra en la figura 19.

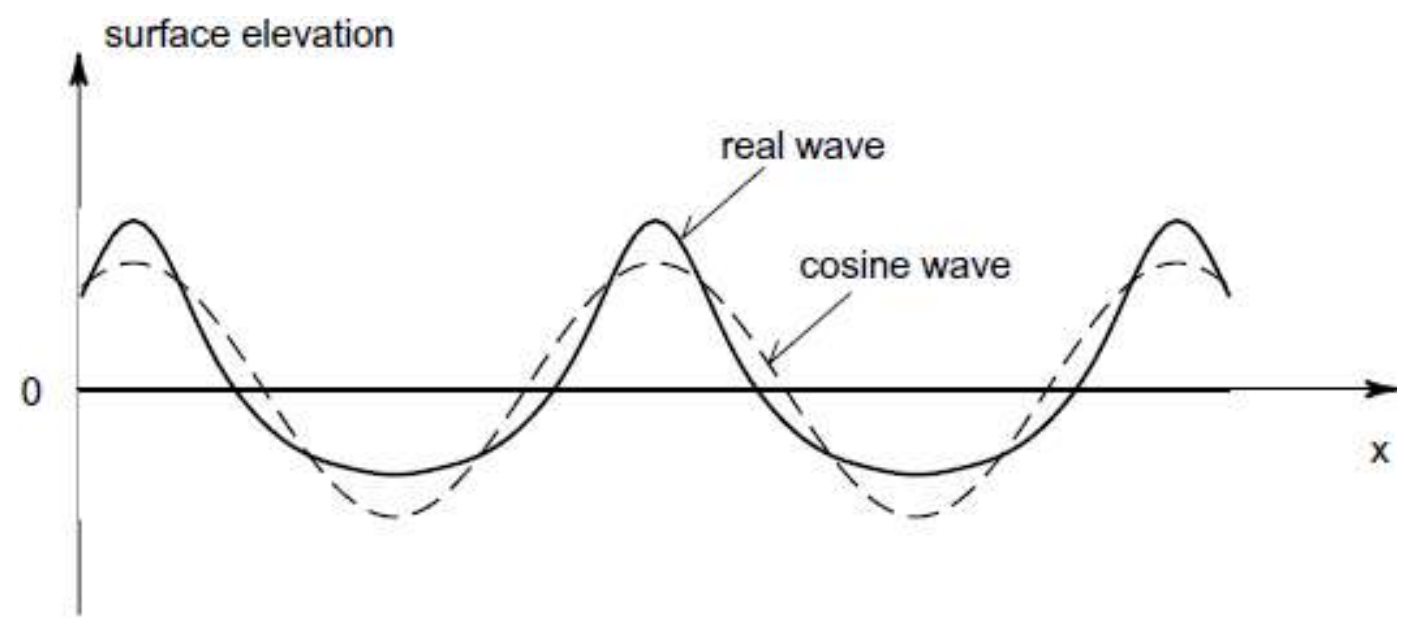

Figura 19. Perfil del oleaje (fuente: Brorsen, 2007)

Con la intención de resolver el problema de flujo no lineal, se aplica un método de perturbación (Stokes, 1847). Este método asume que todas las variables pueden ser expresadas como series:

$$
\varphi=\varphi^{(1)}+\varphi^{(2)}+\cdots+\varphi^{(i)}
$$

donde,

$$
o\left(\varphi^{(i+1)}\right)=o\left(\varphi^{(i)} \cdot \frac{H}{L}\right)=o\left(\varphi^{(i-1)} \cdot\left(\frac{H}{L}\right)^{2}\right)=o\left(\varphi^{(1)} \cdot\left(\frac{H}{L}\right)^{i}\right)
$$

siendo o( ) el orden de magnitud de la expresión entre paréntesis. Las expresiones que determinan la elevación de la superficie y la presión dinámica son:

$$
\begin{gathered}
\eta=\eta^{(1)}+\eta^{(2)}+\cdots+\eta^{(i)} \\
p^{+}=p^{+(1)}+p^{+(2)}+\cdots+p^{+(i)}
\end{gathered}
$$

Según la teoría de Stokes de orden "i", es necesario sustituir el desarrollo del flujo potencial (ф) y de la sobreelevación de la superficie en la ecuación diferencial parcial y en las 
condiciones de contorno. Después se descartan todos aquellos términos que tengan un factor $(H / L)^{n}$ (siendo $\left.n \geq i\right)$ en su orden de magnitud.

De acuerdo con todo esto la teoría de Stokes de segundo orden se enunciaría de la siguiente forma:

$$
\begin{gathered}
\varphi=\varphi^{(1)}+\varphi^{(2)} \\
\eta=\eta^{(1)}+\eta^{(2)}
\end{gathered}
$$

donde $\varphi^{(1)}$ y $\eta^{(1)}$ son conocidos a partir de la teoría lineal de ondas de primer orden y dado que $\varphi^{(1)}$ satisface la ecuación de Laplace:

$$
\frac{\partial^{2} \varphi^{(1)}}{\partial x^{2}}+\frac{\partial^{2} \varphi^{(1)}}{\partial z^{2}}=0
$$

Al sustituir en la ecuación de Laplace la nueva expresión de la velocidad potencial, se deberá cumplir:

$$
\frac{\partial^{2} \varphi^{(2)}}{\partial x^{2}}+\frac{\partial^{2} \varphi^{(2)}}{\partial z^{2}}=0
$$

Con la intención de encontrar $\varphi^{(2)}$, es necesario resolver la ecuación diferencial con sus correspondientes condiciones de contorno. Igual que en la teoría de primer orden, el problema con la posición de superficie libre es resuelto mediante expansión del problema en series de Taylor a partir de $z=0$, de las condiciones de contorno dinámicas y cinemáticas, respectivamente. Aunque, en esta ocasión se descartarán los términos con un orden de magnitud $(H / L)^{2}$ veces inferior, con respecto a los términos dominantes.

La expresión en serie de Taylor de la condición de contorno cinemática puede expresarse como:

$$
\left(\frac{\partial \varphi}{\partial z}-\frac{\partial \eta}{\partial t}-\frac{\partial \varphi}{\partial x} \frac{\partial \eta}{\partial x}\right)_{z=0}+\eta \frac{\partial}{\partial z}\left(\frac{\partial \varphi}{\partial z}-\frac{\partial \eta}{\partial t}-\frac{\partial \varphi}{\partial x} \frac{\partial \eta}{\partial x}\right)_{z=0}+\cdots=0
$$


existiendo una expresión similar para la condición de contorno dinámica. Operando la nueva formulación en la condición de contorno cinemática y en la dinámica, y eliminando los términos cuyo orden de magnitud sea $(H / L)^{2}$ inferior, es posible eliminar $\eta^{(2)}$ de ambas condiciones de contorno, y combinarlas en la siguiente ecuación (Svendsen, 1985).

$$
\frac{\partial \varphi^{(2)}}{\partial z}+\frac{1}{g} \frac{\partial^{2} \varphi^{(2)}}{\partial t^{2}}=-\frac{3 \omega}{4 k}(k H)^{2} \frac{\operatorname{sen}(2(\omega t-k x))}{\operatorname{senh}(2 k h)}
$$

Tras encontrar el valor de $\varphi^{(2)}$, los componentes de segundo orden de la velocidad se calculan a partir de derivar $\varphi^{(2)}$.

$$
\begin{aligned}
u^{(2)} & =\frac{\partial \varphi^{(2)}}{\partial x} \\
w^{(2)} & =\frac{\partial \varphi^{(2)}}{\partial z}
\end{aligned}
$$

Finalmente, $\mathrm{c}^{(2)}, \mathrm{\eta}^{(2)}$ y $\mathrm{p}^{(2)}$ se derivan del mismo modo que en la teoría de primer orden y se comprueba que $\mathrm{c}^{(2)}=0$ dando:

$$
c=c^{(1)}+c^{(2)}=c^{(1)}=\sqrt{\frac{g}{k} \cdot \tanh k h}
$$

La velocidad de propagación no varía de la calculada según la teoría lineal de primer orden, como se observa en la tabla 3: 
Modelos de predicción de esfuerzos hidrodinámicos y socavación aplicados a ingeniería offshore

\begin{tabular}{|c|c|c|}
\hline Quantity & First-Order Component & Second-Order Component \\
\hline Dispersion relationship & $c^{2}=\frac{g}{k} \tanh k d$ & $c^{2}=\frac{g}{k} \tanh k d$ \\
\hline Wave profile & $\eta=\frac{H}{2} \cos (k x-\omega t)$ & $\eta=\frac{\pi H^{2}}{8 L} \frac{\cosh k d}{\sinh ^{3} k d}[2+\cosh 2 k d] \cos 2(k x-\omega t)$ \\
\hline Horizontal velocity & $u=\frac{\pi H}{T} \frac{\cosh k s}{\sinh k d} \cos (k x-\omega t)$ & $u=\frac{3}{4 c}\left(\frac{\pi H}{T}\right)^{2} \frac{\cosh 2 k s}{\sinh ^{4} k d} \cos 2(k x-\omega t)$ \\
\hline Vertical velocity & $v=\frac{\pi H}{T} \frac{\sinh k s}{\sinh k d} \sin (k x-\omega t)$ & $v=\frac{3}{4 c}\left(\frac{\pi H}{T}\right)^{2} \frac{\sinh 2 k s}{\sinh ^{4} k d} \sin 2(k x-\omega t)$ \\
\hline Horizontal acceleration & $\dot{u}=\frac{2 \pi^{2} H}{T^{2}} \frac{\cosh k s}{\sinh k d} \sin (k x-\omega t)$ & $\dot{u}=\frac{3 \pi}{2 L}\left(\frac{\pi H}{T}\right)^{2} \frac{\cosh 2 k s}{\sinh ^{4} k d} \sin 2(k x-\omega t)$ \\
\hline Vertical acceleration & $\dot{v}=-\frac{2 \pi^{2} H}{T^{2}} \frac{\sinh k s}{\sinh k d} \cos (k x-\omega t)$ & $\dot{v}=-\frac{3 \pi}{4 L}\left(\frac{\pi H}{T}\right)^{2} \frac{\sinh 2 k s}{\sinh ^{4} k d} \cos 2(k x-\omega t)$ \\
\hline Dynamic pressure & $p=\rho g \frac{H}{2} \frac{\cosh k y}{\cosh k d} \cos [k(x-c t)]$ & $\begin{aligned} p= & \frac{3}{4} \rho g \frac{\pi H^{2}}{L} \frac{1}{\sinh 2 k d}\left[\frac{\cosh 2 k s}{\sinh ^{2} k d}-\frac{1}{3}\right] \cos 2(k x-\omega t) \\
& -\frac{1}{4} \rho g \frac{\pi H^{2}}{L} \frac{1}{\sinh k d}[\cosh 2 k s-1]\end{aligned}$ \\
\hline
\end{tabular}

Tabla 3. Formulación de Stokes de 2o Orden (fuente: Chakrabarti, 2005)

En la tabla anterior, el parámetro $d$ representa a la profundidad (en inglés depth), que es representada por la letra $h$ a lo largo de todo el documento.

\subsection{Teoría de Stokes de 3o Orden}

El resultado más importante de la teoría de tercer orden es que la velocidad de propagación $c^{(3)} \neq 0$, dando:

$$
c=c^{(1)}+c^{(2)}+c^{(3)}=c^{(1)}+c^{(3)}=\sqrt{\frac{g}{k} \cdot \tanh k h}+c^{(3)}
$$

La expresión general para $c^{(3)}$ viene dada por Svendsen \& Jonsson (1980). Para el caso de aguas profundas, la expresión es:

$$
c=\sqrt{\frac{g}{k}} \cdot \sqrt{1+\left(\frac{k H}{2}\right)^{2}}=c^{(1)} \cdot \sqrt{1+\left(\frac{k H}{2}\right)^{2}}
$$

Como puede observarse, en la solución que aporta la teoría de tercer orden la celeridad de onda depende de $\mathrm{H}$. 


\subsection{Teoría de Stokes de 5o Orden}

La extensión de los cálculos aumenta de forma importante cada vez que un término extra es incluido en el desarrollo en serie de cada variable. A pesar de ello, la solución de la teoría de 5o orden fue publicada en 1960 por Skjelbreia et al.

La longitud de onda $\mathrm{L}$ y el coeficiente $\lambda$ se hallan mediante el cálculo iterativo de las siguientes dos expresiones.

$$
\begin{aligned}
& L=\frac{g T^{2}}{2 \pi} \tanh (k h) \cdot\left(1+\lambda^{2} C_{1}+\lambda^{4} C_{2}\right) \\
& \pi H=L\left(\lambda+\lambda^{3} B_{33}+\lambda^{5}\left(B_{35}+B_{55}\right)\right)
\end{aligned}
$$

Los coeficientes $B_{l m}=B_{l m}(k h)$ y $C_{n}=C_{n}(k h)$ dependen de $k h$, donde $k=2 \pi / L$. La iteración es iniciada en $\lambda=0$, dado que este valor corresponde $a=L^{(1)}$, es decir, a la longitud de onda calculada según la teoría lineal. En la práctica, los valores de $L$ y $\lambda$ se obtienen mediante solución iterativa de las ecuaciones anteriores reescritas de la siguiente forma:

$$
\begin{gathered}
F(k, \lambda)=\omega^{2}-g k \cdot \tanh (k h) \cdot\left(1+\lambda^{2} C_{1}+\lambda^{4} C_{2}\right)=0 \\
f(\lambda, k)=\frac{k H}{2}-\left(\lambda+\lambda^{3} B_{33}+\lambda^{5}\left(B_{35}+B_{55}\right)\right)=0
\end{gathered}
$$

El proceso iterativo deberá mantenerse hasta obtener, por ejemplo:

$$
\left|k_{i}-k_{i-1}\right|<0,0001 k_{i}
$$

Tras calcular $L$ y $\lambda$ la velocidad potencial $\phi$ es calculada a partir de la expresión:

$$
\varphi=-\frac{c}{k} \sum_{j=1}^{5} D_{j} \cdot \cosh j k(z+h) \cdot \operatorname{sen} j \theta
$$

donde:
$C \quad=\quad \mathrm{L} / \mathrm{T}$
$\theta=\omega t-k x$ 

$D_{1}=\lambda A_{11}+\lambda^{3} A_{13}+\lambda^{5} A_{15}$
$D_{2}=\lambda^{2} A_{22}+\lambda^{4} A_{24}$
$D_{3}=\lambda^{3} A_{33}+\lambda^{5} A_{35}$
$\mathrm{D}_{4} \quad=\quad \lambda^{4} \mathrm{~A}_{44}$
$D_{5} \quad=\quad \lambda^{5} A_{55}$

siendo, $A_{l m}=A_{l m}(k h)$ funciones conocidas de $k h$.

Los componentes de la velocidad se hallan a partir de derivar la función de flujo potencial, dando:

$$
\begin{gathered}
u=\frac{\partial \varphi}{\partial x}=\frac{\partial \varphi}{\partial \theta} \cdot \frac{\partial \theta}{\partial x}=\frac{\partial \varphi}{\partial \theta} \cdot(-k) \\
u=c \sum_{j=1}^{5} j \cdot D_{j} \cdot \cosh j k(z+h) \cdot \cos j \theta \\
w=\frac{\partial \varphi}{\partial z} \\
w=-c \sum_{j=1}^{5} j \cdot D_{j} \cdot \operatorname{senh} j k(z+h) \cdot \operatorname{sen} j \theta
\end{gathered}
$$

Las aceleraciones se hallan a partir de:

$$
\frac{d u}{d t}=\frac{\partial u}{\partial t}+u \frac{\partial u}{\partial x}+w \frac{\partial u}{\partial z}
$$

donde:

$$
\begin{gathered}
\frac{\partial u}{\partial t}=\frac{\partial u}{\partial \theta} \cdot \frac{\partial \theta}{\partial t}=\frac{\partial u}{\partial \theta} \cdot \omega=-c \omega \sum_{j=1}^{5} j^{2} \cdot D_{j} \cosh j k(z+h) \operatorname{sen} j \theta \\
\frac{\partial u}{\partial x}=c k \sum_{j=1}^{5} j^{2} \cdot D_{j} \cosh j k(z+h) \operatorname{sen} j \theta \\
\frac{\partial u}{\partial z}=c k \sum_{j=1}^{5} j^{2} \cdot D_{j} \operatorname{senh} j k(z+h) \cos j \theta
\end{gathered}
$$


Dado $\omega=c k$, puede observarse que:

$$
\frac{\partial u}{\partial t}=-c \frac{\partial u}{\partial x}
$$

Finalmente, $\eta$ se calcula como:

$$
\eta=\frac{1}{k} \sum_{j=1}^{5} E_{j} \cos j \theta
$$

donde:
$E_{1}=\lambda$
$E_{2}=\lambda^{2} B_{22}+\lambda^{4} B_{24}$
$E_{3}=\lambda^{3} B_{33}+\lambda^{5} B_{35}$
$\mathrm{E}_{4} \quad=\quad \lambda^{4} \mathrm{~B}_{44}$
$\mathrm{E}_{5} \quad=\quad \lambda^{5} \mathrm{~B}_{55}$

siendo cada $B_{l m}=B_{l m}(k h)$ la misma función aplicada bajo el cálculo de L.

La solución analítica completa es también presentada por Nishimura et al. (1977) y Isobe et al. (1978):

$$
\begin{aligned}
& \eta=\frac{1}{k}\left[\sum_{n=1}^{5} A_{n} \cos n(k x-\omega t)\right] \\
& u=c_{0}\left[-B_{0}+\sum_{n=1}^{5} B_{n} \cosh n k(h+z) \cos n(k x-\omega t)\right] \\
& w=c_{0}\left[\sum_{n=1}^{5} B_{n} \operatorname{senh} n k(h+z) \operatorname{sen} n(k x-\omega t)\right]
\end{aligned}
$$

donde:

$$
A_{1}=\varepsilon_{1}+\varepsilon_{1}^{3} \frac{3\left(-9 c t^{6}+3 c t^{4}-3 c t^{2}+1\right)}{64}
$$




$$
\begin{aligned}
& 188325 c t^{16}+203310 c t^{14} \pm 764046 c t^{12}-340410 c t^{10}+53932 c t^{8} \\
& +\varepsilon_{1}^{5} \frac{-6070 c t^{6}+14622 c t^{4}+2370 c t^{2}-225}{12288\left(5 c t^{2}+1\right)\left(5 c t^{2}+3\right)} \\
& A_{2}=\varepsilon_{1}^{2} \frac{c t\left(3 c t^{2}-1\right)}{4}+\varepsilon_{1}^{4} \frac{c t\left(-324 c t^{8}+792 c t^{4}-352 c t^{2}+12\right)}{384} \\
& A_{3}=\varepsilon_{1}^{3} \frac{3\left(9 c t^{6}-3 c t^{4}+3 c t^{2}-1\right)}{64} \\
& +\varepsilon_{1}^{5} \frac{-14580 c t^{14}-7776 c t^{12}+46980 c t^{10}-5328 c t^{8}+900 c t^{6}-1008 c t^{4}-180 c t^{2}}{4096\left(5 c t^{2}+1\right)} \\
& A_{4}=\varepsilon_{1}^{4} \frac{c t\left(405 c t^{10}+81 c t^{8}+522 c t^{6}-262 c t^{4}+c t^{2}+21\right.}{384\left(5 c t^{2}+1\right)} \\
& A_{5}=\varepsilon_{1}^{5} \frac{5\left(\begin{array}{c}
6075 c t^{16}+8910 c t^{14}+25866 c t^{12}-498 c t^{10}-3896 c t^{8} \\
+2618 c t^{6}-570 c t^{4}-150 c t^{2}+45
\end{array}\right)}{12288\left(5 c t^{2}+1\right)\left(5 c t^{2}+3\right)} \\
& B_{0}=\varepsilon_{1}^{2} \frac{c t}{2 k h}+\varepsilon_{1}^{4} \frac{c t\left(-9 c t^{6}-3 c t^{4}-7 c t^{2}+3\right)}{64 k h} \\
& B_{1}=\frac{1}{\operatorname{senh} k h}\left[\varepsilon_{1}+\varepsilon_{1}^{3} \frac{-27 c t^{6}-3 c t^{4}-41 c t^{2}+39}{64}\right. \\
& 62775 c t^{16}+89370 c t^{14}-199602 c t^{12}-91438 c t^{10} \\
& +\varepsilon_{1}^{5} \frac{-131460 c t^{8}-75986 c t^{6}+30114 c t^{4}+12262 c t^{2}+861}{4096\left(5 c t^{2}+1\right)\left(5 c t^{2}+3\right)} \\
& B_{2}=\frac{2}{\operatorname{senh} 2 k h}\left[\varepsilon_{1}^{2} \frac{3 c t\left(c t^{2}-1\right)}{4}+\varepsilon_{1}^{4} \frac{c t\left(-162 c t^{8}+81 c t^{6}+261 c t^{4}+19 c t^{2}-103\right.}{192}\right] \\
& B_{3}=\frac{3}{\operatorname{senh} 3 k h}\left[\varepsilon_{1}^{3} \frac{27 c t^{6}-57 c t^{4}+17 c t^{2}+13}{64}\right. \\
& \left.+\varepsilon_{1}^{5} \frac{-3645 c t^{14}+4131 c t^{12}+10935 c t^{10}-7569 c t^{8}}{1024\left(5 c t^{2}+1\right)}\right] \\
& B_{4}=\frac{4}{\operatorname{senh} 4 k h}\left[\varepsilon_{1}^{4} \frac{c t\left(405 c t^{10}-1269 c t^{8}+342 c t^{6}+1466 c t^{4}-747 c t^{2}-197\right)}{384\left(5 c t^{2}+1\right)}\right]
\end{aligned}
$$

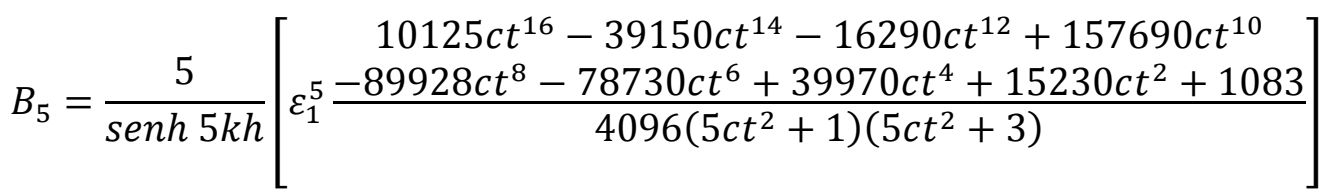


siendo:

$$
\begin{gathered}
\varepsilon_{1}=\frac{k H}{2} \\
c t=\operatorname{coth} k h \\
c_{0}=\sqrt{\left(\frac{g}{k}\right) \tanh k h}
\end{gathered}
$$

El parámetro $c_{0}$ debe ser calculado usando el valor de $\mathrm{k}$ de 5 o orden. Conocido $h, T$ y $H$, el valor de k es calculado mediante la siguiente relación de dispersión:

$$
\begin{aligned}
\omega^{2}=g k \tanh & k h[1 \\
+ & \varepsilon^{2}\left\{\frac{9 c t^{4}-10 c t^{2}+9}{16}\right. \\
& \left.-\frac{c t}{2 k h}\right\}+\varepsilon^{4}\left\{\frac{-405 c t^{10}-117 c t^{8}+2454 c t^{6}-2194 c t^{4}+351 c t^{2}+39}{1024}\right. \\
& \left.\left.-\frac{c t\left(-9 c t^{6}-3 c t^{4}-7 c t^{2}+3\right)}{64}\right\}\right]^{2}
\end{aligned}
$$

Obteniendo las aceleraciones al igual que en el caso de Skjelbreia et al. (1960), mediante las siguientes expresiones:

$$
\begin{gathered}
a_{x}=\frac{d u}{d t}=\frac{\partial u}{\partial t}+u \frac{\partial u}{\partial x}+w \frac{\partial u}{\partial z} \\
a_{z}=\frac{d w}{d t}=\frac{\partial w}{\partial t}+u \frac{\partial w}{\partial x}+w \frac{\partial w}{\partial z}
\end{gathered}
$$

Fenton (1985), desarrolla su propia solución de para la teoría de Stokes de 5o orden, siendo dicha teoría corregida en 1987, debido a una errata en la formulación. 


\subsection{Teoría lineal de onda larga}

Esta teoría se encarga de estudiar oleajes que se producen en zonas de escasa profundidad relativa y amplitud relativa, es decir, que tienen una longitud de onda grande en comparación con la profundidad y con la altura del oleaje. Este dominio de estudio se caracteriza por ignorar los posibles efectos de disipación, dado que $\mu_{\mathrm{r}} \ll<1$, y considera una solución prácticamente lineal del problema, $\delta<<1$.

La ecuación Korteweg-de Vries, denominada de aquí en adelante como KdV, fue originalmente derivada para describir oleajes someros de larga longitud de onda y pequeña amplitud. En la derivación, $\mathrm{KdV}$ asume que todo el movimiento es uniforme en el eje $\mathrm{Y}$, a lo largo de la cresta de las olas. En este caso, la superficie de elevación de la ola se propaga según la dirección del eje $\mathrm{X}$ y es una función de la posición horizontal y del tiempo, $Z=$ $\eta(X, T)$. La ecuación $\mathrm{KdV}$ se expresa como:

$$
\frac{\partial \eta}{\partial T}+\sqrt{g h} \frac{\partial \eta}{\partial X}+\frac{3}{2} \frac{\sqrt{g h}}{h} \eta \frac{\partial \eta}{\partial X}+\frac{1}{2} h^{2} \sqrt{g h}\left(\frac{1}{3}-\frac{\tau}{\rho g h^{2}}\right) \frac{\partial^{3} \eta}{\partial X^{3}}=0
$$

donde $\mathrm{h}$ es la profundidad uniforme del agua, $\mathrm{g}$ es la gravedad, $\rho$ es la densidad y $\tau$ representa la tensión superficial. De hecho, el parámetro $\tau /\left(\rho g h^{2}\right)$ también se conoce como el número de Bond y permite medir la fuerza relativa entre las tensiones superficiales y las fuerzas gravitatorias.

De la expresión anterior se observa que la celeridad asociada a la onda larga es $c=\sqrt{g h}$. De hecho, se trata de la máxima velocidad de propagación alcanzable por olas de gravedad de pequeña amplitud. Sin embargo, la velocidad de propagación de olas solitarias de pequeña amplitud es ligeramente superior. De acuerdo con la fórmula empírica de Russell's, la celeridad es igual a $c=\sqrt{g(h+k)}$, siendo k la altura de la onda solitaria.

La ecuación KdV puede ser expresada en variables adimensionales de la siguiente forma:

$$
u_{t}+\alpha u u_{x}+u_{x x x}=0
$$

donde el parámetro $\alpha$ usa valores como $\alpha= \pm 1$ o $\alpha= \pm 6$. 
El término $u_{t}$ describe la evolución temporal de la propagación de la onda en una dirección. Por ello, se denomina a la expresión anterior como ecuación evolutiva. El término no lineal $\alpha u u_{x}$ tiene en cuenta la esbeltez de la ola y el término de dispersión $u_{x x x}$ describe la propagación de la onda.

El término no lineal de esbeltez puede ser equilibrado con el efecto de la dispersión. Por tanto, el resultado de que se contrarresten los dos efectos que proporcionan la no linealidad al problema es una ola estable y solitaria que se propaga de forma similar a como lo haría una partícula. En definitiva, una onda solitaria tendrá una amplitud finita y se propagará a una velocidad constante sin sufrir cambio alguno en su forma durante distancias relativamente largas.

La formulación compacta de la solución de una onda solitaria, según la teoría lineal para ondas larga, viene dada por:

$$
u(x, t)=\frac{\omega-4 k^{3}}{\alpha k}+\frac{12 k^{2}}{\alpha} \operatorname{sech}^{2}(k x-\omega t+\delta)=\frac{\omega-8 k^{3}}{\alpha k}-\frac{12 k^{2}}{\alpha} \tanh ^{2}(k x-\omega t+\delta)
$$

No obstante, como demostró Korteweg and de Vries (1895), también existe otra función de carácter periódico que resuelve las expresiones anteriores. Esta solución periódica se ha denominado solución de onda cnoidal por estar formada por funciones elípticas de Jacobi. La formulación compacta de la onda cnoidal, según la teoría lineal para ondas largas, viene dada por:

$$
u(x, t)=\frac{\omega-4 k^{3}(2 m-1)}{\alpha k}+\frac{12 k^{2} m}{\alpha} c n^{2}(k x-\omega t+\delta ; m)
$$

donde $\mathrm{cn}$ es la función coseno elíptico de Jacobi con módulo m, siendo m:

$$
0<\mathrm{m}<1
$$

A continuación, se muestra en la figura 20 el perfil de elevación de una onda solitaria frente al de una onda cnoidal. 


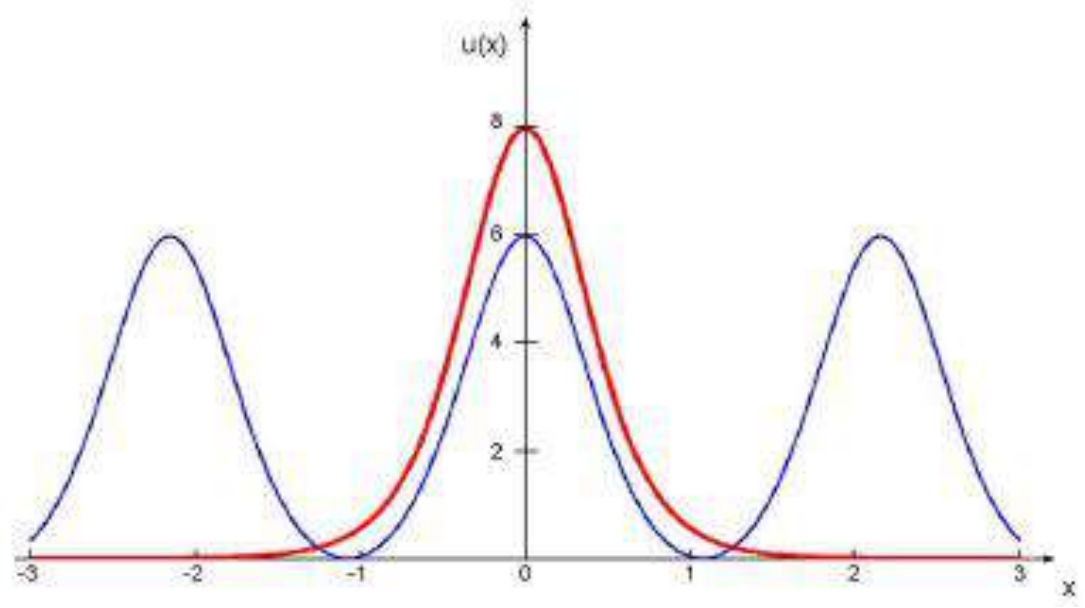

Figura 20. Representación de onda cnoidal y onda solitaria (fuente: Hereman, 2011)

A continuación, se muestran las ecuaciones y los parámetros para la formulación de la teoría de onda cnoidal de segundo orden (Laitone, 1960):

Trough depth, to

$$
\frac{h}{d}=1-h_{1}-\epsilon^{2} h_{2}+0\left|\varepsilon^{3}\right|
$$

Surface elevation, $\eta$

$$
\left.\frac{\eta}{d}=e\left(\operatorname{cn}^{2} q-h_{1}\right)-e^{2}\left[\frac{3}{4} \operatorname{con}^{2} q\left(1-\operatorname{cn}^{2} q\right)+h_{2}\right]+0 \mid \varepsilon^{3}\right)
$$

Wave celerity, $c$

$$
\begin{aligned}
& \frac{c}{\sqrt{g d}}=1+e c_{1}+c^{2} c_{2}+0\left|c^{3}\right| \\
& \frac{c^{3}}{\sqrt{g d}}=1+\varepsilon c_{1}+\epsilon^{2} c_{3}^{4}+0\left[\epsilon^{3}\right]
\end{aligned}
$$

Wave length, $L$

$$
\frac{\mathbb{L}}{\mathbb{d}}=\frac{4 k \mathbb{K}}{\sqrt{3 \epsilon}}\left\{\mathbb{1}-\varepsilon Q_{\mathbb{Q}}+0\left\|\epsilon^{2}\right\|\right\}
$$

W/ave period, $T$

$$
\begin{aligned}
& \frac{d}{g T^{2}}=\frac{3 \varepsilon}{16 \kappa^{2} K^{2}}\left\{\left(\frac{1+\epsilon c_{1}+\epsilon^{2} c_{2}}{1-\epsilon Q_{1}}\right)^{3}+0\left|\varepsilon^{3}\right|\right\} \\
& \frac{d}{g T^{2}}=\frac{3 \epsilon}{16 x^{2} K^{2}}\left\{\left(\frac{1+\epsilon c_{1}+\varepsilon^{2} c_{2}^{3}}{1-e^{2}}\right)^{2}+0\left[\epsilon^{3}\right]\right\}
\end{aligned}
$$


Horizontal particle velocity, $t:$

Vertical particle velocity。 w

Horizonal pasticle acceleration, $\partial u / \partial t$

Vertical particle acceleration, $\partial w / \partial t$

Pressure, $\mathrm{p}$ $\frac{1}{g} \frac{\partial w}{\partial t}=\frac{3 \varepsilon^{2}}{2 k^{2}}\left(\frac{s}{d}\right)\left(k^{\prime 2}+2\left(2 k^{3}-1\right) \mathrm{cn}^{2} q-3 k^{2} \mathrm{~cm}^{4} q\right)+0\left[e^{3}\right]$

Average energy

density, $\mathbb{E}$

Energy đlux, P

$$
\frac{p}{a g d}=\frac{7}{d}+1-\frac{s}{d}-e^{2}\left(\frac{3}{4 x^{3}}\right)\left[\left(\frac{s}{d}\right)^{2}-1\right]
$$

$$
\left[x^{2}+2\left(2 x^{2}-1\right) c n^{2} q-3 x^{2} c n^{4} q\right]+0\left[c^{3}\right]
$$

$$
\begin{aligned}
& \frac{u}{\sqrt{g d}}=\epsilon\left(\mathrm{cm}^{2} q-b_{1}\right)+e^{2}\left\{\left(f_{1}+f_{2} \operatorname{cn}^{2} q_{2}-\cos ^{4} q\right)\right. \\
& -\frac{3}{4 k^{2}}\left(\frac{3}{d}\right)^{2}\left|x^{2}+2\left(2 x^{2}-1\right) c n^{2} q-3 x^{2} c n^{4} d\right| \\
& +0\left|e^{3}\right| \\
& \frac{q^{*}}{\sqrt{g d}}=\epsilon\left(\mathrm{cn}^{2} q-h_{q}\right)+\epsilon^{2}\left\{\left(f_{q}^{*}+\delta_{2} \mathrm{cn}^{2} q-\mathrm{cn}^{6} q\right)\right. \\
& \left.-\frac{3}{4 k^{2}}\left(\frac{s}{d}\right)^{3}\left[x^{2}+2\left(2 x^{2}-1\right) \operatorname{cn}^{2} q-3 x^{2} \operatorname{cn}^{2} q\right]\right\} \\
& +0\left|e^{3}\right|
\end{aligned}
$$$$
\frac{w}{\sqrt{g d}}=\frac{e \sqrt{3 \varepsilon}}{n}\left(\frac{s}{d}\right) \operatorname{con} q d n q \operatorname{sn} q\left\{1+\varepsilon\left[f_{3}-2 \mathrm{cn}^{2} q\right.\right.
$$$$
\left.-\left(\frac{s}{d}\right)\left\{\left(\frac{2 k^{3}-1}{2 k^{2}}-\frac{3}{2} \operatorname{cm}^{2} q\right)\right]+0\left|\varepsilon^{2}\right|\right\}
$$

$$
\begin{aligned}
& \frac{1}{g} \frac{\partial u}{\partial t}=\frac{\epsilon \sqrt{3 \epsilon}}{\omega} \cos q d n q \sin g d t+\epsilon\left[r_{4}-2 \cos ^{2} q\right. \\
& \left.\left.-\left(\frac{s}{d}\right)^{2}\left[\frac{3\left(2 x^{2}-1\right)}{2 x^{2}}+\frac{9}{2} \mathrm{cn}^{2} q\right)\right]+0\left|\varepsilon^{2}\right|\right\}
\end{aligned}
$$

$$
\frac{E}{\rho g d^{2}}=\frac{1}{2} e^{2}\left\{e_{\varepsilon}+z e_{2}+0\left[\epsilon^{2}\right]\right\}
$$

$$
\frac{p}{\rho g d^{2} \sqrt{g d}}=e^{2}\left\{p_{1}+\epsilon p_{2}+0\left[e^{2} \mid\right\}\right.
$$

where

$$
\begin{aligned}
& \mathrm{q}=\frac{K \theta}{\pi}=\frac{K}{\pi}(\mathrm{kx}-\omega t) \\
& \mathrm{h}_{1}=\left\{\gamma-\kappa^{\prime 2}\right\} / x^{2} \\
& h_{2}=\left\{\gamma\left(k^{3}-2\right)+2 \kappa^{\prime 2}\right\} / 4 \kappa^{3}
\end{aligned}
$$




$$
\begin{aligned}
& c_{1}=\left\{2-x^{2}-3 \gamma\right\} / 2 k^{2} \\
& c_{2}=\left\{-5 \gamma\left(15 y+19 k^{2}-38\right)-18 k^{4}-88 k^{3}\right\} / 120 k^{8} \\
& c_{2}^{4}=\left\{5 \gamma\left(3 \gamma-k^{8}+2\right)-6 k^{3}-16 k^{2}\right\} / 40 k^{4} \\
& Q_{3}=\left\{12 \gamma+5 k^{2}-10\right\} / 8 \kappa^{2} \\
& f_{1}=\left\{-\gamma\left(6 \gamma+11 k^{2}-16\right)+k^{\prime 2}\left(9 k^{2}-10\right)\right\} / 12 k^{4} \\
& \Upsilon_{1}^{1}=\left\{\gamma\left(2 \gamma-\kappa^{2}\right)+\kappa^{\prime 2}\left(3 \kappa^{2}-2\right)\right\} / 4 \kappa^{8} \\
& \mathbb{I}_{2}=\left\{2 \gamma+7 x^{2}-6\right\} / 4 x^{8} \\
& f_{3}=\left\{16 \gamma+19 k^{2}-22\right\} / 8 k^{2} \\
& f_{4}=\left\{4 \gamma+15 \kappa^{2}-14\right\} / 8 \kappa^{2} \\
& \left.e_{1}=2\left\{-9\left(3 \gamma+2 k^{2}-4\right)-x^{2}\right\}\right] / 3 \kappa^{4} \\
& e_{2}=\left\{\gamma\left(15 \gamma^{2}-2 k^{4}-17 k^{\prime 2}\right)-k^{\prime 2}\left(k^{2}-2\right)\right\} / 15 k^{6} \\
& p_{1}=\left\{-\gamma\left(3 \gamma+2 k^{2}-4\right)-k^{\prime 2}\right\} / 3 x^{4} \\
& D_{2}=\left\{\gamma\left(75 \gamma^{2}+60 \gamma\left(k^{2}-2\right)+8 x^{8}+53 \kappa^{\circ}\right)+4 k^{\prime 2}\left(k^{2}-2\right)\right\} / 30 \kappa^{6}
\end{aligned}
$$

\subsection{Teoría de onda cnoidal de 3 o Orden}

Como se ha explicado en el punto anterior, Korteweg y de Vries (1895) derivaron y resolvieron una ecuación para oleaje en aguas someras, llamada la ecuación KdV, teniendo en cuenta los efectos de dispersión (profundidad finita) y no linealidad (amplitud finita). Esta es la teoría de onda cnoidal de primer orden. Laitone $(1960 ; 1965)$ calculó una solución de segundo orden y desarrolló la segunda definición para la celeridad de la onda. Por otro lado, Chappelear (1962), Yasuda (1978), Isobe et al. (1978), Tsuchiya et al. (1985) y Isobe (1988); calcularon soluciones de tercer orden, a partir de desarrollos físicos diferentes. A continuación, se muestra la solución de tercer orden de la onda cnoidal (Isobe, 1988):

$$
\begin{aligned}
& \eta=h \sum_{n=0}^{3} A_{n} c n^{2 n}\left\{2 K\left(\frac{x}{L}-\frac{t}{T}\right)\right\} \\
& u=\sqrt{g h} \sum_{n=0}^{3} \sum_{m=0}^{2} B_{n m}\left(\frac{h+z}{h}\right)^{2 m} c n^{2 n}\left\{2 K\left(\frac{x}{L}-\frac{t}{T}\right)\right\} \\
& w=\sqrt{g h} \cdot \operatorname{sn}\left\{2 K\left(\frac{x}{L}-\frac{t}{T}\right)\right\} \cdot d n\left\{2 K\left(\frac{x}{L}-\frac{t}{T}\right)\right\} \\
& \cdot \frac{4 K h}{L} \sum_{n=1}^{3} \sum_{m=0}^{2} \frac{n}{2 m+1} B_{n m}\left(\frac{h+z}{h}\right)^{2 m+1} c n^{2 n-1}\left\{2 K\left(\frac{x}{L}-\frac{t}{T}\right)\right\}
\end{aligned}
$$




$$
p=\rho g(\eta-z)+\rho g h \sum_{n=0}^{3} \sum_{m=0}^{2} P_{n m}\left(\frac{h+z}{h}\right)^{2 m} c n^{2 n}\left\{2 K\left(\frac{x}{L}-\frac{t}{T}\right)\right\}
$$

donde:

$$
\begin{aligned}
& A_{0}=\varepsilon_{2}\left(\lambda_{2}-\mu_{2}\right)+\frac{\varepsilon_{2}^{2}\left(-2 \lambda_{2}+\mu_{2}-2 \lambda_{2}^{2}+2 \lambda_{2} \mu_{2}\right)}{4} \\
& +\frac{\varepsilon_{2}^{3}\left(133 \lambda_{2}-16 \mu_{2}+399 \lambda_{2}^{2}-466 \lambda_{2} \mu_{2}+100 \mu_{2}^{2}+266 \lambda_{2}^{3}-466 \lambda_{2}^{2} \mu_{2}+200 \lambda_{2} \mu_{2}^{2}\right)}{400} \\
& A_{1}=\varepsilon_{2}+\varepsilon_{2}^{2}\left(-\frac{3}{4}\right)+\varepsilon_{2}^{3}\left(\frac{50-\lambda_{2}-60 \mu_{2}}{80}\right) \\
& A_{2}=\varepsilon_{2}^{2}\left(\frac{3}{4}\right)+\varepsilon_{2}^{3}\left(\frac{-151+\lambda_{2}+60 \mu_{2}}{80}\right) \\
& A_{3}=\varepsilon_{2}^{3}\left(\frac{101}{80}\right) \\
& B_{00}=\varepsilon_{2}\left(\lambda_{2}-\mu_{2}\right)+\frac{\varepsilon_{2}^{2}\left(\lambda_{2}-\mu_{2}-2 \lambda_{2}^{2}+2 \mu_{2}^{2}\right)}{4} \\
& +\frac{\varepsilon_{2}^{3}\left(-71 \lambda_{2}+47 \mu_{2}-23 \lambda_{2}^{2}+97 \lambda_{2} \mu_{2}-50 \mu_{2}^{2}+153 \lambda_{2}^{3}-153 \lambda_{2}^{2} \mu_{2}-25 \lambda_{2} \mu_{2}^{2}+25 \mu_{2}^{3}\right)}{200} \\
& B_{01}=\varepsilon_{2}^{2}\left(-\frac{3 \lambda_{2}}{4}\right)+\varepsilon_{2}^{3}\left(\frac{6 \lambda_{2}+24 \lambda_{2}^{2}-21 \lambda_{2} \mu_{2}}{8}\right) \\
& B_{02}=\varepsilon_{2}^{3}\left(\frac{3 \lambda_{2}-3 \lambda_{2}^{2}}{16}\right) \\
& B_{10}=\varepsilon_{2}+\frac{\varepsilon_{2}^{2}\left(1-6 \lambda_{2}+2 \mu_{2}\right)}{4}+\frac{\varepsilon_{2}^{3}\left(-19-27 \lambda_{2}+10 \mu_{2}+101 \lambda_{2}^{2}-100 \lambda_{2} \mu_{2}+15 \mu_{2}^{2}\right)}{40} \\
& B_{11}=\frac{\varepsilon_{2}^{2}\left(-3+3 \lambda_{2}\right)}{2}+\frac{\varepsilon_{2}^{3}\left(6+36 \lambda_{2}-21 \mu_{2}-24 \lambda_{2}^{2}+21 \lambda_{2} \mu_{2}\right)}{4} \\
& B_{12}=\frac{\varepsilon_{2}^{3}\left(6-39 \lambda_{2}+6 \lambda_{2}^{2}\right)}{16} \\
& B_{20}=\varepsilon_{2}^{2}(-1)+\frac{\varepsilon_{2}^{3}\left(-2+32 \lambda_{2}-15 \mu_{2}\right)}{10} \\
& B_{21}=\varepsilon_{2}^{2}\left(\frac{9}{4}\right)+\frac{\varepsilon_{2}^{3}\left(30-120 \lambda_{2}+63 \mu_{2}\right)}{8} \\
& B_{22}=\frac{\varepsilon_{2}^{3}\left(-45+45 \lambda_{2}\right)}{16} \\
& B_{30}=\varepsilon_{2}^{3}\left(\frac{6}{5}\right)
\end{aligned}
$$




$$
\begin{aligned}
& B_{31}=\varepsilon_{2}^{3}\left(-\frac{15}{2}\right) \\
& B_{32}=\varepsilon_{2}^{3}\left(\frac{45}{16}\right) \\
& P_{00}=\varepsilon_{2}^{2}\left(\frac{3 \lambda_{2}}{4}\right)+\frac{\varepsilon_{2}^{3}\left(-9 \lambda_{2}-21 \lambda_{2}^{2}+12 \lambda_{2} \mu_{2}\right)}{16} \\
& P_{01}=\varepsilon_{2}^{2}\left(-\frac{3 \lambda_{2}}{4}\right)+\frac{\varepsilon_{2}^{3}\left(3 \lambda_{2}+24 \lambda_{2}^{2}-18 \lambda_{2} \mu_{2}\right)}{8} \\
& P_{02}=\frac{\varepsilon_{2}^{3}\left(3 \lambda_{2}-3 \lambda_{2}^{2}\right)}{16} \\
& P_{10}=\varepsilon_{2}^{2}\left(-\frac{3+3 \lambda_{2}}{2}\right)+\frac{\varepsilon_{2}^{3}\left(-18-33 \lambda_{2}+24 \mu_{2}+42 \lambda_{2}^{2}-24 \lambda_{2} \mu_{2}\right)}{16} \\
& P_{11}=\varepsilon_{2}^{2}\left(-\frac{3+3 \lambda_{2}}{2}\right)+\frac{\varepsilon_{2}^{3}\left(3+36 \lambda_{2}-18 \mu_{2}-24 \lambda_{2}^{2}+18 \lambda_{2} \mu_{2}\right)}{4} \\
& P_{12}=\frac{\varepsilon_{2}^{3}\left(6-39 \lambda_{2}+6 \lambda_{2}^{2}\right)}{16} \\
& P_{20}=\varepsilon_{2}^{2}\left(-\frac{9}{4}\right)+\frac{\varepsilon_{2}^{3}\left(15+75 \lambda_{2}-36 \mu_{2}\right)}{16} \\
& P_{21}=\varepsilon_{2}^{2}\left(\frac{9}{4}\right)+\frac{\varepsilon_{2}^{3}\left(39-120 \lambda_{2}+54 \mu_{2}\right)}{8} \\
& P_{22}=\frac{\varepsilon_{2}^{3}\left(-45+45 \lambda_{2}\right)}{16} \\
& P_{30}=\varepsilon_{2}^{3}\left(\frac{15}{16}\right) \\
& P_{31}=\varepsilon_{2}^{3}\left(-\frac{33}{4}\right) \\
& P_{32}=\varepsilon_{2}^{3}\left(\frac{45}{16}\right)
\end{aligned}
$$

siendo:

$$
\begin{gathered}
\varepsilon_{2}=\frac{H}{h} \\
\lambda_{2}=\frac{\left(1-\kappa^{2}\right)}{\kappa^{2}} \\
\mu_{2}=\frac{E}{\kappa^{2} K}
\end{gathered}
$$


siendo $\mathrm{cn}$, sn y dn funciones elípticas de Jacobi; $\mathrm{K}$ y E son las integrales elípticas completas de primer y segundo orden, respectivamente; у $\kappa$ es el módulo de las integrales y de las funciones elípticas.

Una vez conocidos $h, T, y H$, el módulo $k$ puede calcularse a partir de la siguiente expresión, que relaciona el parámetro de Ursell para aguas someras con $\kappa$.

$$
U_{s}=\frac{\left[\frac{16 \kappa^{2} K^{2}}{3}\right]}{1+\varepsilon_{2} \frac{-1-2 \lambda_{2}}{4}+\varepsilon_{2}^{2} \frac{\left(8+33 \lambda_{2}-10 \mu_{2}+33 \lambda_{2}^{2}-20 \lambda_{2} \mu_{2}\right)}{40}}
$$

siendo el parámetro de Ursell para aguas someras:

$$
U_{s}=\frac{g H T^{2}}{h^{2}}
$$

Una vez hallado $k$, la longitud de onda L puede calcularse según la expresión:

$L$

$$
\begin{aligned}
& =T \sqrt{g h}\left[1+\varepsilon_{2}\left(\frac{1+2 \lambda_{2}-3 \mu_{2}}{2}\right)+\varepsilon_{2}^{2} \frac{\left(-6-16 \lambda_{2}+5 \mu_{2}-16 \lambda_{2}^{2}+10 \lambda_{2} \mu_{2}+15 \mu_{2}^{2}\right)}{40}\right. \\
& \left.+\frac{\varepsilon_{2}^{3}\left(150+1079 \lambda_{2}-203 \mu_{2}-2337 \lambda_{2}^{2}-2653 \lambda_{2} \mu_{2}+350 \mu_{2}^{2}+1558 \lambda_{2}^{3}-2653 \lambda_{2}^{2} \mu_{2}+700 \lambda_{2} \mu_{2}^{2}+175 \mu_{2}^{3}\right)}{2800}\right]
\end{aligned}
$$

Si $\kappa \rightarrow 1$, entonces $U_{S} \rightarrow \infty, h / L \rightarrow 0, c n \rightarrow \operatorname{sech}$, y la solución coincide con la solución de onda solitaria. Si por el contrario $\kappa \rightarrow 0$, entonces $c n \rightarrow \cos$, y la solución coincide con la solución de ola de pequeña amplitud en aguas someras.

\subsection{Teoría de onda cnoidal de 5o Orden (onda hiperbólica)}

Iwagaki (1968) propone un método aproximado para calcular la onda cnoidal de 5o orden. En primer lugar, sugiere una simplificación, que viene justificada en que para olas de poca amplitud y esbeltas, los valores del parámetro $k$ rondan la unidad. Este hecho sugiere la simplificación de que en cualquier parte que aparezca dicho parámetro, este se sustituya por la unidad, $k=1$, lo cual permite reducir notablemente la formulación a llevar a cabo. 
Esta teoría podrá ser aplicada para ondas largas donde $k \geq 0,96$. Iwagaki observó que en muchas aplicaciones de la teoría cnoidal $k$ puede ser mantenido como 1 sin pérdida apreciable en la precisión del modelo. Él presentó resultados para el segundo orden y denominó a las ondas resultantes "ondas hiperbólicas", dado que las funciones elípticas de Jacobi se aproximan a funciones hiperbólicas para este límite. En Fenton (1990) se muestran también los resultados teóricos para 5o orden.

$$
\begin{aligned}
& \eta=h\left(1+\varepsilon c n^{2}+\varepsilon^{2}\left(-\frac{3}{4} c n^{2}+\frac{3}{4} c n^{4}\right)+\varepsilon^{3}\left(\frac{5}{8} c n^{2}-\frac{151}{80} c n^{4}+\frac{101}{80} c n^{6}\right)\right. \\
& +\varepsilon^{4}\left(-\frac{8209}{6000} c n^{2}+\frac{11641}{3000} c n^{4}-\frac{112393}{24000} c n^{6}+\frac{17367}{8000} c n^{8}\right) \\
& +\varepsilon^{5}\left(\frac{364671}{196000} c n^{2}-\frac{2920931}{392000} c n^{4}+\frac{2001361}{156800} c n^{6}-\frac{17906339}{1568000} c n^{8}\right. \\
& \left.\left.+\frac{1331817}{313600} c n^{10}\right)\right) \\
& \alpha_{0}=\sqrt{\frac{3 \varepsilon}{4 m}}\left(1-\frac{5 \varepsilon}{8}+\frac{71 \varepsilon^{2}}{128}-\frac{100627 \varepsilon^{3}}{179200}+\frac{16259737 \varepsilon^{4}}{28672000}\right) \\
& \delta_{0}=\frac{4 \alpha_{0}^{2}}{3}
\end{aligned}
$$

Velocidad horizontal del fluido:

$$
\begin{aligned}
& \frac{\bar{U}}{\sqrt{g h}}=-1+\delta_{0}\left(-\frac{1}{2}+c n^{2}\right) \\
& +\delta_{0}^{2}\left(-\frac{19}{40}+\frac{3}{2} c n^{2}-c n^{4}+\left(\frac{Y}{h}\right)^{2}\left(-\frac{3}{2} c n^{2}+\frac{9}{4} c n^{4}\right)\right) \\
& +\delta_{0}^{3}\left(\begin{array}{c}
\left.-\frac{55}{112}+\frac{71}{40} c n^{2}-\frac{27}{10} c n^{4}+\frac{6}{5} c n^{6}+\left(\frac{Y}{h}\right)^{2}\left(-\frac{9}{4} c n^{2}+\frac{75}{8} c n^{4}-\frac{15}{2} c n^{6}\right)\right) \\
+\left(\frac{Y}{h}\right)^{4}\left(\frac{3}{8} c n^{2}-\frac{45}{16} c n^{4}+\frac{45}{16} c n^{6}\right)
\end{array}\right)
\end{aligned}
$$


$+\delta_{0}^{4}\left(\begin{array}{c}-\frac{11813}{22400}+\frac{53327}{42000} c n^{2}-\frac{13109}{3000} c n^{4}+\frac{1763}{375} c n^{6}-\frac{197}{125} c n^{8} \\ +\left(\frac{Y}{h}\right)^{2}\left(-\frac{213}{80} c n^{2}+\frac{3231}{160} c n^{4}-\frac{729}{20} c n^{6}+\frac{189}{10} c n^{8}\right) \\ +\left(\frac{Y}{h}\right)^{4}\left(\frac{9}{16} c n^{2}-\frac{327}{32} c n^{4}+\frac{915}{32} c n^{6}-\frac{315}{16} c n^{8}\right) \\ +\left(\frac{Y}{h}\right)^{6}\left(-\frac{3}{80} c n^{2}+\frac{189}{160} c n^{4}-\frac{63}{16} c n^{6}+\frac{189}{64} c n^{8}\right)\end{array}\right)$

$+\delta_{0}^{5}\left(\begin{array}{c}-\frac{57159}{98560}-\frac{144821}{156800} c n^{2}-\frac{1131733}{294000} c n^{4}+\frac{757991}{73500} c n^{6}-\frac{298481}{36750} c n^{8}+\frac{13438}{6125} c n^{10} \\ +\left(\frac{Y}{h}\right)^{2}\left(-\frac{53327}{28000} c n^{2}+\frac{1628189}{56000} c n^{4}-\frac{192481}{2000} c n^{6}+\frac{11187}{100} c n^{8}-\frac{5319}{125} c n^{10}\right.\end{array}\right)$

Velocidad vertical del fluido:

$$
\frac{V}{\sqrt{g h}}=2 \alpha_{0} \cdot \operatorname{cn}() \cdot \operatorname{sn}() \cdot d n() \cdot \sum_{i=1}^{5} \delta_{0}^{i} \sum_{j=0}^{i-1}\left(\frac{Y}{h}\right)^{2 j+1} \sum_{k=1}^{i} c n^{2(k-1)}() \frac{k}{2 j+1} \Phi_{i j k}
$$

Los coeficientes $\Phi_{i j k}$ son los mismos que aparecen en la expresión para la velocidad horizontal.

\subsection{Ecuaciones de Boussinesq}

La teoría clásica de Boussinesq ofrece un abanico de ecuaciones evolutivas para el estudio del oleaje en el límite de la no linealidad débil y dispersión débil. Esto implica un estudio del problema partiendo de la base de que el ratio entre la altura de ola y profundidad es $\delta<<1$, y que el ratio entre la profundidad y la longitud de onda es $\mu_{r}<<1$ (Kirby, 2003).

El uso de estos modelos resultó complicado, debido a que requerían grandes cantidades de tiempo y una gran potencia de cálculo. Por ello, muy pocos cálculos específicos de 
propagación de oleaje y ningún estudio de procesos en zonas superficiales fueron realizados utilizando modelos basados en la teoría de Boussinesq.

Casi todos los tipos de modelos basados en la teoría de Boussinesq derivan de los supuestos de flujo incompresible y no viscoso. En primer lugar, se debe elegir una escala apropiada con respecto al régimen donde la longitud de onda excede a la profundidad.

$$
\begin{gathered}
(x, y)=\left(k_{0} x^{\prime}, k_{0} y^{\prime}\right) \\
z=\frac{z^{\prime}}{h_{0}} \\
t=\sqrt{g h_{0} k_{0}{ }^{2}} \cdot t^{\prime} \\
\eta=\frac{\eta^{\prime}}{a_{0}} \\
\phi=\left(\frac{a_{0} \sqrt{g h_{0}}}{k_{0} h_{0}}\right)^{-1} \cdot \phi^{\prime}
\end{gathered}
$$

donde los primeros términos denotan las variables dimensionales de espacio y tiempo, $h_{\circ}$ es una escala aplicada a la profundidad, $a_{o}$ es una escala aplicada a la amplitud de onda y $k_{\circ}$ es una escala inversa de la longitud de onda horizontal, siendo las variables dependientes el desplazamiento superficial $\eta$ y la función de velocidad potencial $\phi$. Como se vio en los apartados anteriores las componentes de la velocidad vendrán dadas por:

$$
u=(u, v)=\left(\frac{\partial \phi}{\partial x}, \frac{\partial \phi}{\partial y}\right)
$$

El problema resultante, tras haber sido escalado, se caracteriza por dos ratios adimensionales:

$$
\mu^{\prime}=k_{0} h_{0} \quad, \quad \delta^{\prime}=\frac{a_{0}}{h_{0}}
$$


El parámetro $\mu_{\mathrm{r}}$ caracteriza la dispersión de la frecuencia y el límite $\mu_{r} \rightarrow 0$ representa el límite de no dispersión. La designación, débilmente disperso, se refiere a un régimen con $\mu_{\mathrm{r}}<<1$. El parámetro $\delta$ caracteriza la no linealidad y el límite $\delta \rightarrow 0$ representa el límite de linealidad. Por consiguiente, la denominación, débilmente disperso, se refiere a un régimen con $\delta<<1$. El set completo de ecuaciones escaladas es dado por:

$$
\begin{array}{ll}
\nabla^{2} \phi+\frac{1}{\mu^{2}} \phi_{z z}=0, & -\mathrm{h} \leq \mathrm{z} \leq \delta \eta \\
\nabla h \cdot \nabla \phi+\frac{1}{\mu^{2}} \phi_{z}=0, & \mathrm{z}<-\mathrm{h} \\
\eta+\phi_{t}+\frac{\delta}{2}\left(|\nabla \phi|^{2}+\frac{1}{\mu^{2}}\left(\phi_{z}\right)^{2}\right)=0, & \mathrm{z}=\delta \eta \\
\eta_{t}+\delta \nabla \eta \cdot \nabla \phi-\frac{1}{\mu^{2}} \phi_{z}=0, & \mathrm{z}=\delta \eta
\end{array}
$$

Después de resolver las ecuaciones anteriores se tiene que:

$$
\phi(x, y, z, t)=\sum_{n=0}^{\infty}(-1)^{n} \mu^{\prime 2 n}\left(\frac{z^{2 n}}{(2 n) !} \nabla^{2 n} \phi^{(0)}+\frac{z^{2 n+1}}{(2 n+1) !} \nabla^{2 n} \phi^{(1)}\right)
$$

donde $\phi^{(0)}$ y $\phi^{(1)}$ representan $\phi$ y $\phi_{z}$ evaluados en $z=0$, que serán desconocidos antes de aplicar las condiciones de contorno.

Partiendo de la suposición de que $\mathrm{H}<<\mathrm{h}$ y $\mathrm{h}<<\mathrm{L}$ el problema sigue una formulación débilmente dispersiva y prácticamente lineal. De acuerdo con las ecuaciones anteriores la relación de dispersión que define la propagación del oleaje viene dada por:

$$
\omega^{2}=g k \cdot \tanh k h
$$

o de forma equivalente,

$$
c^{2}=g h \frac{\tanh k h}{k h}
$$


donde $\omega$ es la frecuencia angular, $k$ es el número de onda y c es la celeridad de la onda.

\subsection{Función de corriente o Stream Function}

Dean (1965) introdujo un innovador método numérico para la predicción bidimensional de las características de una ola, basándose en la representación del flujo mediante su correspondiente función de corriente. Dicha teoría fue desarrollada para examinar numéricamente oleajes completamente no lineales.

Dado que el flujo es bilineal, una función de corriente $\psi$ existe, y la condición de irrotacionalidad implica que este debe satisfacer la ecuación de Laplace dentro del fluido:

$$
\frac{\partial^{2} \psi}{\partial x^{2}}+\frac{\partial^{2} \psi}{\partial z^{2}}=0
$$

Además, este está sujeto a las condiciones de contorno del lecho marino y de la superficie libre:

$$
\begin{array}{cc}
\frac{\partial \psi}{\partial x}=0 & \text { en } \mathrm{z}=-\mathrm{h} \\
w=u \frac{\partial \eta}{\partial x} & \text { en } \mathrm{z}=\boldsymbol{\eta} \\
\frac{1}{2 g}\left(u^{2}+w^{2}\right)+\eta=Q & \text { en } \mathrm{z}=\eta
\end{array}
$$

donde $Q$ es la constante de Bernoulli. De acuerdo con las expresiones anteriores, la función de corriente tendrá la siguiente forma:

$$
\psi(x, z)=c z+\sum_{n=1}^{N} X_{n} \operatorname{senh}(n k(z+h)) \cos (n k x)
$$

Dicha función es dependiente de kx y satisface la ecuación de Laplace, la condición de contorno en fondo y la condición de contorno en superficie. 
Para hallar el valor de $k$ y de $X_{n}$, Dean propone un método iterativo cuyo enfoque pretende reducir a cero el error producido en el ajuste con la condición de contorno dinámica en la superficie libre. Este error puede expresar de la forma siguiente:

$$
E=\frac{1}{N} \sum_{i=1}^{N}\left(Q_{i}-\bar{Q}\right)^{2}
$$

\subsection{Resumen}

En definitiva, todas las teorías de ondas que permiten caracterizar el oleaje, y que han sido explicadas en los puntos anteriores, se pueden clasificar tal y como muestra la figura 21 :

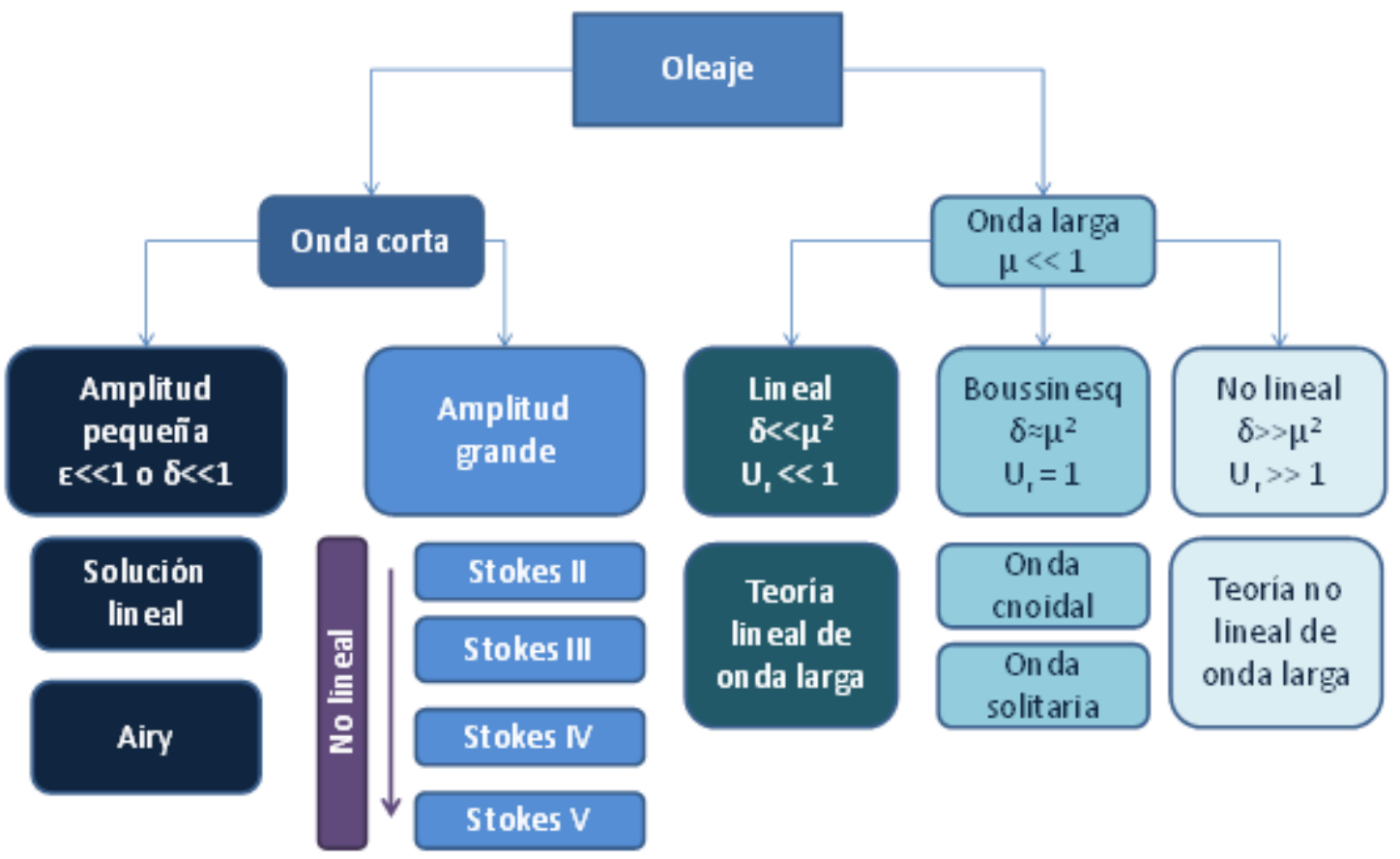

Figura 21. Clasificación de las teorías de mecánica de ondas y rango de aplicación (fuente: elaboración propia)

siendo:

$\varepsilon=H / L$, peralte.

$\delta=\mathrm{H} / \mathrm{h}$, amplitud relativa.

$\mu_{r}=h / L$, profundidad relativa.

$\mathrm{U}_{\mathrm{r}}=\delta / \mu_{\mathrm{r}}^{2}$, parámetro de Ursell. 


\section{Caracterización de fuerzas hidrodinámicas}

En este apartado se va a proceder a realizar una exposición de los principales modelos existentes que permiten realizar el cálculo de las fuerzas hidrodinámicas a partir del campo de velocidades y aceleraciones del oleaje.

\subsection{Exposición teórica}

Dependiendo del tipo y tamaño de los miembros de una estructura en alta mar, en comparación con la longitud de onda, se emplean diferentes métodos de cálculo en la predicción de las fuerzas hidrodinámicas y las respuestas resultantes (Sarpkaya y Isaacson, 1981; Hooft ,1982; Faltinsen, 1990a; Chakrabarti, 1990).

Los métodos existentes para hallar las fuerzas hidrodinámicas en estructuras offshore se basan principalmente en una de las siguientes técnicas, bien la utilización de la fórmula de Morison et al.; o las técnicas que usan el potencial de velocidades para fuentes y sumideros en flujo bidimensional o tridimensional. Entre estos métodos, el método tridimensional se utiliza, generalmente, para estructuras que comprenden grandes elementos estructurales. Por otro lado, si una estructura consta de elementos pequeños se requerirá la utilización de la ecuación de Morison et al. o la utilización del método bidimensional de distribución fuente-sumidero.

Morison et al. (1950) sugirieron que se pueden superponer los dos regímenes de flujo que generan las fuerzas de inercia y las fuerzas de arrastre, para obtener la carga total variable en el tiempo por unidad de longitud de un cilindro, que está parado en un campo de flujo plano con velocidad de flujo libre arbitraria.

La fuerza del oleaje sobre pilas circulares, teniendo en cuenta el potencial de difracción, fue calculada por primera vez por MacCamy y Fuchs (1954), que propusieron la existencia de una solución matemática exacta, presentada por el oleaje según la teoría lineal de ondas.

Chakrabarti (1972) obtuvo las fuerzas hidrodinámicas provocadas por oleajes no lineales sobre un cilindro vertical, incluyendo el uso de la teoría de difracción mediante una 
formulación compacta, y, posteriormente, comparó la formulación propuesta con resultados experimentales. Chakrabarti et al. (1975) propone una formulación general para la ecuación de fuerza del oleaje aplicable a un cilindro sumergido o semisumergido orientado de forma aleatoria. Posteriormente, también son propuestas las expresiones para las presiones y las fuerzas de oleaje sobre varios cilindros verticales con tamaños y separaciones distintas, hallando las fuerzas hidrodinámicas debidas a la interacción de las distintas ondas lineales propagándose con direcciones arbitrarias.

Goda y Yoshimura (1972) presentaron una solución analítica para la difracción del oleaje sobre un cilindro elíptico mediante la resolución de la ecuación de Helmholtz utilizando el método de separación de variables.

La aplicación del método de elementos finitos fue utilizada por primera vez para la resolución de problemas de difracción por Bettess y Zienkiewicz (1977). La modelización de un dominio no acotado mediante infinitas condiciones de contorno de radiación fue uno de los grandes desafíos en los análisis de elementos finitos. Para resolver dicho problema, se comenzó a utilizar un modelo híbrido como, por ejemplo, el desarrollado por Houston (1981), el de Tsay y Liu (1983), o el de Tsay et al. (1989). El método híbrido combina las ventajas de los análisis mediante elementos finitos en dominios acotados con las ventajas aportadas por los métodos con condiciones de contorno de radiación. Este método híbrido pasó a llamarse método de elementos de contorno (BEM).

Lighthill (1979) hace un análisis metodológico describiendo los fenómenos físicos básicos junto con los métodos de aplicación utilizados para la estimación de las fuerzas hidrodinámicas. Su principal contribución al cálculo de la fuerza de oleaje mediante el uso de la ecuación de Morison et al. reside en la proposición de una corrección de segundo orden en la misma. Las fuerzas turbulentas inducidas (flujo separado) en los cilindros sometidos a oleaje y algunos métodos para la predicción de tales fuerzas son discutidos por Graham (1979).

La carga hidrodinámica sufrida por cuerpos de varios componentes se calcula mediante el método de elementos finitos descrito por Eatock Taylor y Ziestman (1982). Lighthill (1986) 
desarrolló ideas muy importantes que validan una separación de cargas hidrodinámicas en las fuerzas de flujo turbulento y de flujo potencial, aclarando el importante papel de los componentes de segundo orden de las fuerzas de flujo potencial.

Chaplin (1988) hizo mediciones experimentales que revelan una importante contribución no lineal a la fuerza de oleaje para números de Reynolds altos en un cilindro horizontal sumergido con su eje paralelo a las crestas de las olas. Se midieron las fuerzas de las olas que actúan sobre un cilindro que emerge en un campo de olas regulares y las compararon con las de los cálculos basados en la formulación de Morison et al. (Koterayama y Nakamura, 1988).

Cook y Simiu (1989) también trabajaron en las fuerzas hidrodinámicas sobre cilindros verticales, aplicando la corrección de Lighthill, con el fin de incluir los efectos no lineales en el cálculo.

Rainey (1989) presenta una variación de una ecuación para la carga potencial de flujo aplicada a una estructura de tipo reticular en movimiento parcialmente sumergido sometida a la acción del oleaje.

Paulling (1970) propuso un método aproximado de predecir las fuerzas hidrodinámicas y el movimiento producido en plataformas formadas por estructuras tubulares localizadas mar adentro.

Hooft (1971) hizo una contribución significativa al proponer un método aproximado de cálculo de las fuerzas hidrodinámicas y de las fuerzas derivadas de la propia excitación de la onda aplicadas a elementos semisumergidos.

Hogben (1976) discute los métodos de estimación de cargas hidrodinámicas sobre estructuras, utilizados en diversos proyectos de investigación financiados por el Departamento de Energía del Reino Unido y llevados a cabo por los Laboratorios Nacionales de Física. Una investigación de las fuerzas de inercia y las fuerzas de arrastre debidas al oleaje en buques fue presentada por Brown y Patel (1985). 
El Comité de Ingeniería Oceánica del 170 International Towing Tank Conference, conocido por las siglas ITTC, llevó a cabo una comparación entre los métodos para calcular el movimiento de un semisumergible. Concluyendo con un resumen con los resultados finales del proyecto, en el que se utilizaron 34 programas informáticos de 28 organizaciones diferentes, que fue posteriormente descrito por Takagi et al. (1985).

Ostergaard y Schellin (1987) presentan los cálculos hidrodinámicos de aplicación a una variedad de estructuras offshore, discutiendo la validez y exactitud de los resultados previstos, en comparación con las soluciones de formulaciones compactas o de mediciones en modelos de ensayo.

Faltinsen (1990b) hace un análisis detallado sobre los movimientos lineales y no lineales inducidos por el oleaje y las cargas de segundo orden, así como los problemas no lineales y las cargas viscosas. En este estudio se concluyó que la fórmula de Morison et al. y los métodos basados en la teoría del potencial se asemejan favorablemente entre ellos y con las mediciones experimentales realizadas en campo o laboratorio.

El Doctor Dr. Turgut Sarpkaya es uno de los autores más prolíficos de los últimos 50 años en lo referente al estudio de mecánica de ondas y caracterización de las fuerzas hidrodinámicas producidas por el oleaje.

De entre sus estudios más importantes cabe destacar, en primer lugar, su demostración de que el rango de soluciones englobadas dentro del rango de flujo separado, en el cual se encuentra la formulación de Morison et al. y la Lighthill, no cumplen en la realidad una de las hipótesis fundamentales sobre las que se establecen, la separación del flujo. Sarpkaya (2001) afirma que la fuerza de arrastre y la fuerza de inercia no pueden operar de forma independiente y que, por lo tanto, no es posible dividir la medición de las fuerzas dependientes del tiempo en la suma de una fuerza de inercia no viscosa y una fuerza viscosa de arrastre. Sus resultados muestran que la generación, convección y difusión de turbulencia afectan a ambos componentes de la fuerza, porque el flujo inestable no es ni una yuxtaposición de estados de flujo estables ni una yuxtaposición de estados de flujo 
inestables impulsivamente iniciados. En definitiva, demuestra que la descomposición de Lighthilll es solo válida para $\mathrm{KC} \rightarrow 0, \beta \rightarrow \infty$. No obstante, Sarpkaya no discute la validez de la fuerza total dada por la expresión de Lighthill:

$$
F=-\sigma \rho \frac{\partial}{\partial t} \int x \wedge \omega d^{3} x+\rho V_{b} \frac{\partial U}{\partial t}
$$

Discute la expresión que Lighthill obtiene tras rescribir la ecuación de Morison et al. reducida para un flujo con velocidad $\mathrm{U}(\mathrm{t})=-\mathrm{U}_{\mathrm{m}} \cos \omega \mathrm{t}$, y las hipótesis basadas en el teorema de energía mínima de Kelvin que asumen que el movimiento de un fluido infinito puede ser expresado como la suma lineal de un flujo potencial que satisface las condiciones de contorno, y un movimiento turbulento residual que cumple las condiciones de contorno que deben ser cero en la interfase y en el infinito. La fórmula reducida de Lighthill resultante de utilizar el teorema de energía mínima viene dada por la siguiente expresión:

$$
C_{F}=-C_{d}|\cos \omega t| \cos \omega t+C_{m}^{*} \frac{\pi^{2}}{K C} \operatorname{sen} \omega t
$$

donde $\mathrm{C}_{\mathrm{m}}^{*}$ es el valor ideal de coeficiente de inercia, que para el caso de un cilindro es igual a 2.

Dicha expresión, al ser comparada con ensayos de campo, midiendo las fuerzas producidas por un flujo incidente sobre un cilindro, con $\mathrm{KC} \approx 15, \mathrm{Re} \approx 40.000$, muestra diferencias significativas como muestra la figura 22. 


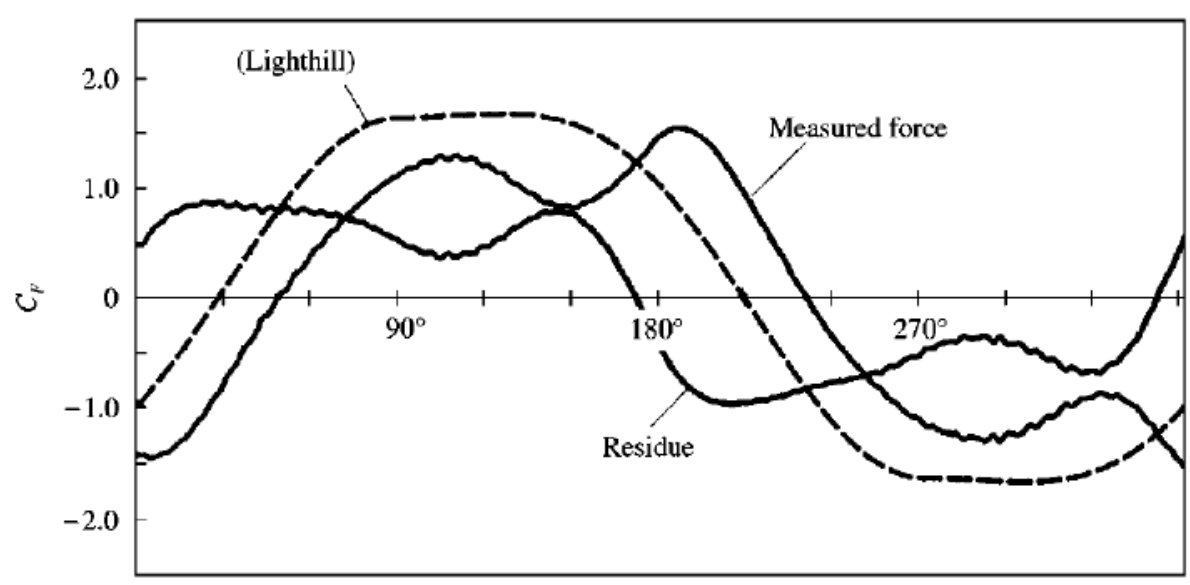

Figura 22. Verificación de la teoría de Lighthill (fuente: Sarpkaya, 2001)

Para valores muy reducidos de KC la fórmula teórica de Lighthill coincidirá con la fuerza medida, sin embargo, para valores más elevados ocurrirá lo mismo que se muestra en la figura anterior. Sarpkaya realiza una reducción de primer orden del residuo mediante un análisis de Fourier, corrigiendo la expresión anterior.

$$
C_{F}=-C_{d}|\cos \omega t| \cos \omega t+C_{m}^{*} \frac{\pi^{2}}{K C} \operatorname{sen} \omega t-\frac{\left(C_{m}^{*}-C_{m}\right) \pi^{2}}{K C} \operatorname{sen} \omega t
$$

En definitiva, Sarpkaya modifica el modelo de Morison et al. para aumentar su precisión en el régimen dominado por las fuerzas de inercia y arrastre (rango de flujo separado) mediante el uso de un tercer término, expresado en términos de los dos coeficientes existentes, y un nuevo parámetro basado en:

$$
\left(\pi^{2} / K C\right) \cdot\left(C_{m}^{*}-C_{m}\right)
$$

Además, es el autor que más predominio dará en sus estudios al parámetro $\beta$, explicado en detalle en el apartado 5.3, y que será el precursor de su universalización en 1976 para la resolución de problemas de flujo separado inestable y completamente no lineal. No obstante, pese a que muchos autores lo han denominado como el parámetro de Sarpkaya, el parámetro $\beta$ fue definido por primera vez en 1851 por Stokes, en su análisis lineal de un flujo viscoso no separado para bajas oscilaciones sobre un tornillo cilíndrico y una esfera.

Sarpkaya también realizó estudios para hallar los coeficientes $C_{D}$ y $C_{M}$ para una gran cantidad de casos diferentes, incluyendo la determinación de las fuerzas producidas sobre dos 
cilindros para distintas distancias de separación entre ellos y distintas orientaciones del flujo incidente, como se observa en las figuras 23 y 24.

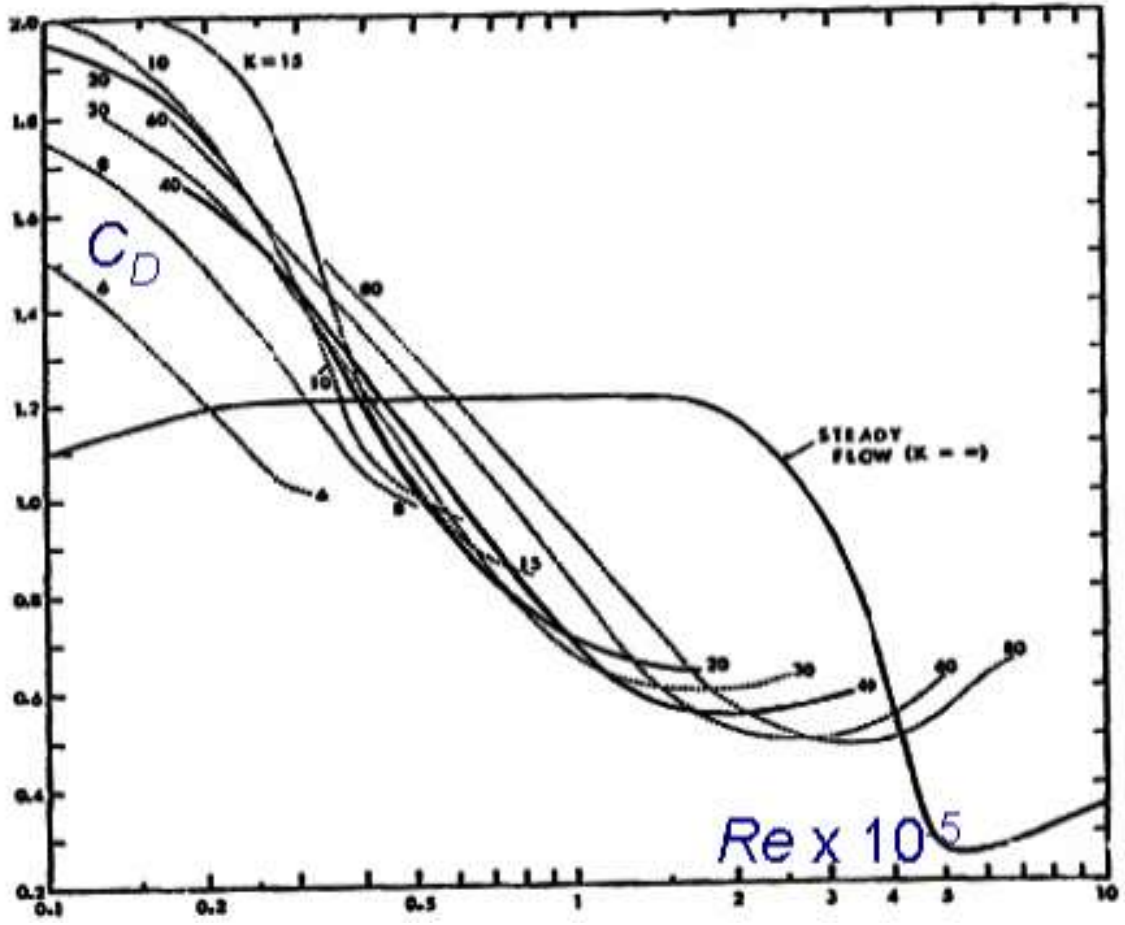

Figura 23. Coeficiente $C_{D}$ en relación con Reynolds y K (fuente: Sarpkaya, 1981)

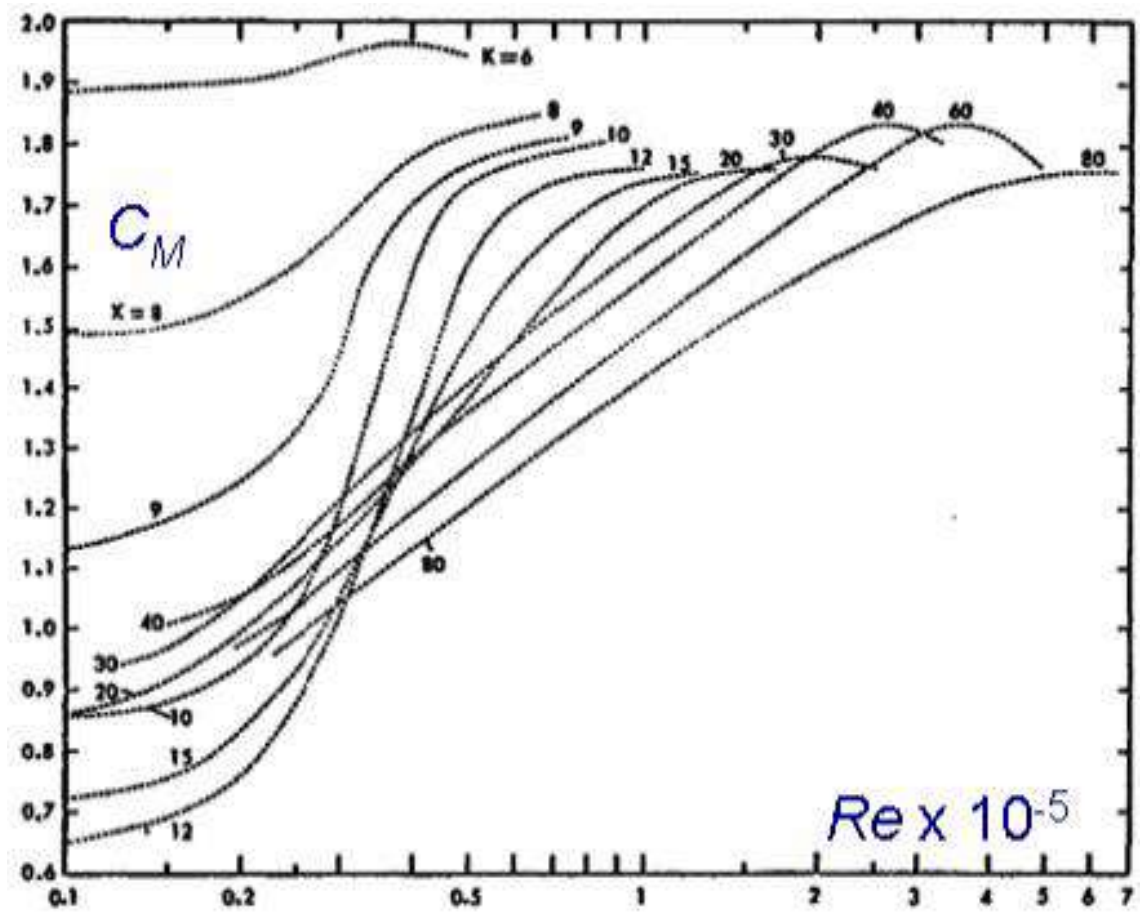

Figura 24. Coeficiente $C_{M}$ en relación con Reynolds y K (fuente: Sarpkaya, 1981) 
Estos diagramas experimentales fueron realizados por Sarpkaya en 1976, para hallar los coeficientes $C_{D}$ y $C_{M}$ en función de distintos valores del número de Reynolds, y para valores constantes del número de Keulegan-Carpenter (KC). También realizó los mismos diagramas para un $\mathrm{KC}=50$ y valores constantes de la rugosidad relativa del cilindro, como se muestra en las figuras 25 y 26.

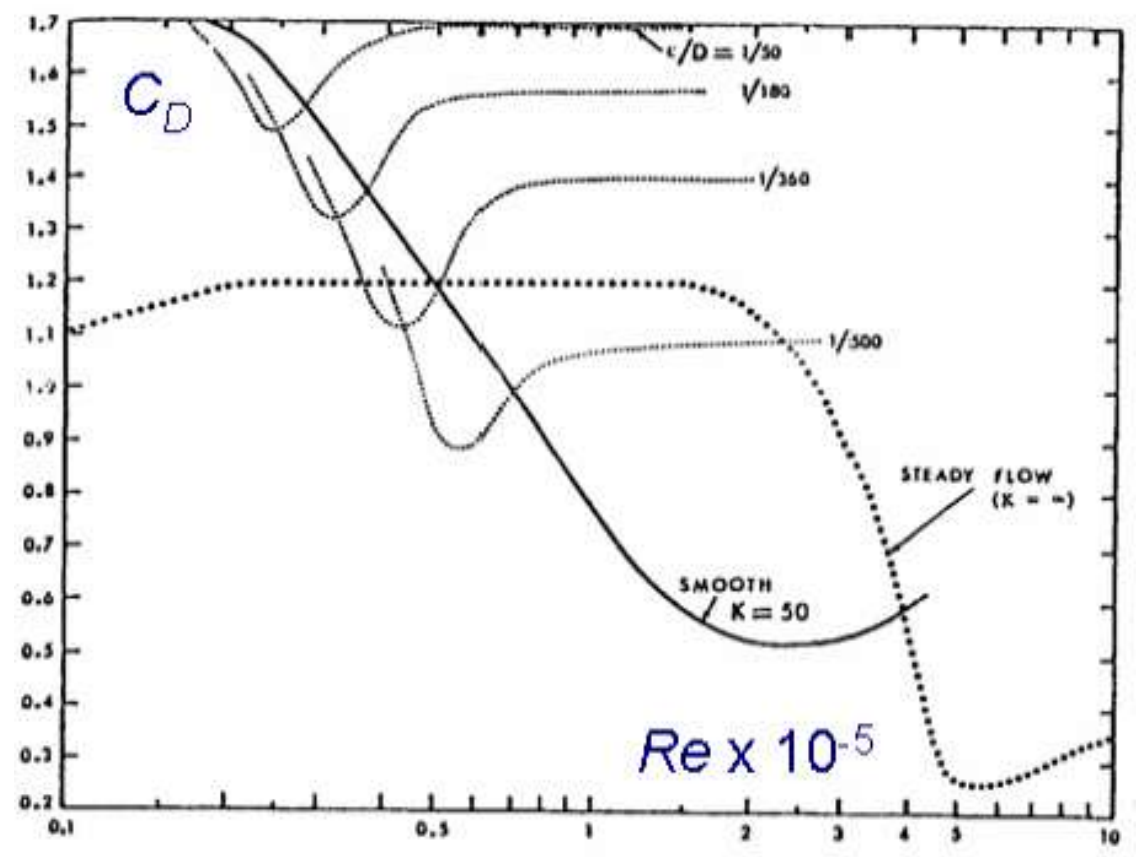

Figura 25. Coeficiente $C_{D}$ en relación con Reynolds y rugosidad (fuente: Sarpkaya, 1981)

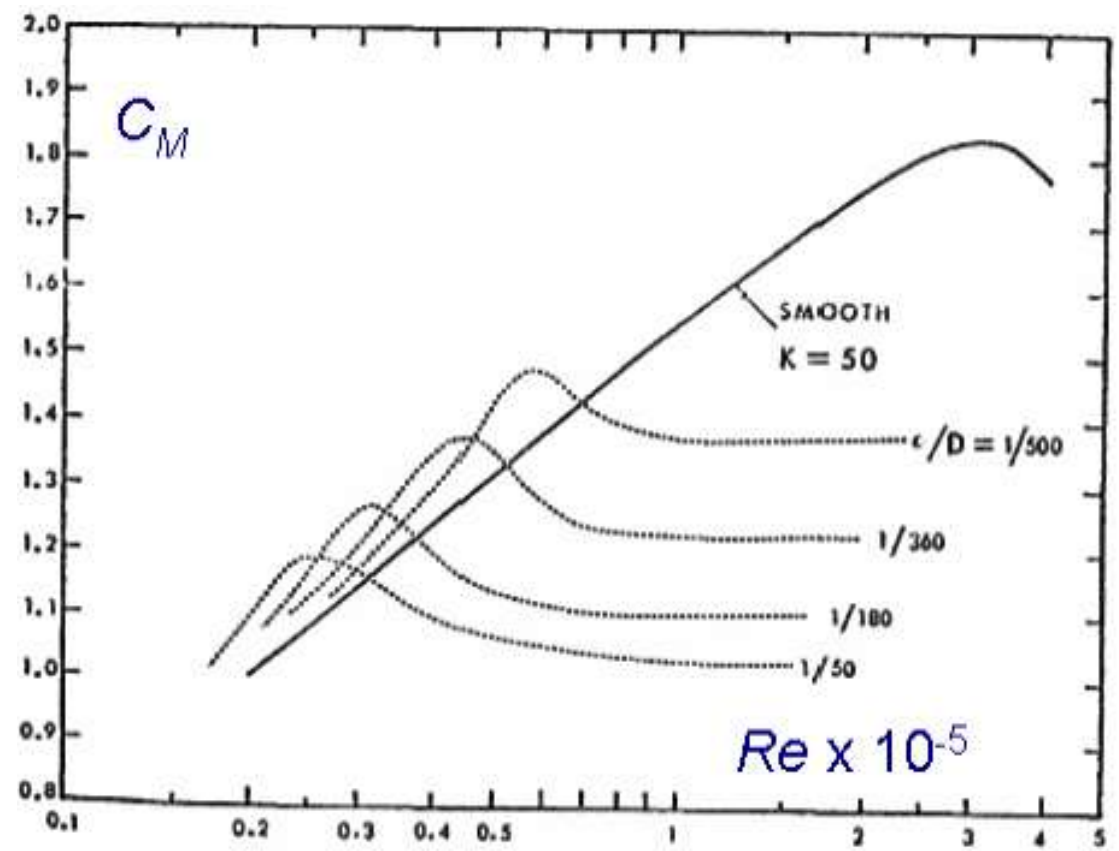

Figura 26. Coeficiente $C_{D}$ en relación con Reynolds y rugosidad (fuente: Sarpkaya, 1981) 
Tras el aumento del poder computacional por medio del desarrollo de la ingeniería de hardware y software, métodos nuevos y más sofisticados han sido introducidos en los últimos años, para calcular el problema de interacción entre el oleaje y la estructura.

Koutandos (2009) presenta un estudio numérico en la interacción de la onda con barreras verticales rígidas y calcula las velocidades y la energía cinética de turbulencia en las proximidades de la estructura. Zhu (2013) investiga la interacción del oleaje con una fila de pilotes utilizando la teoría de onda completa, basándose en los estudios de Isaacson et al. (1998). Zheng et al. (2015) presentan un modelo analítico para predecir la difracción de la onda tridimensional de un cilindro flotante situado en frente de una pared vertical a una profundidad de agua finita.

Feng et al. (2015) proporcionan una solución para el problema de interacción no lineal estructura-oleaje en dos dimensiones por un método integral no singular en combinación con un método de Euler-Lagrange mixto. Dongfeng et al. (2015) desarrollan un modelo a escala 1/10 con agua dulce para estudiar la influencia de la degradación de la cimentación sobre la respuesta dinámica de una plataforma offshore tipo jacket, incluyendo el diseño de modelos de elementos finitos para el análisis del comportamiento dinámico de la plataforma jacket.

A lo largo de este apartado se va a realizar una descripción de las principales fuerzas que se producen sobre un cuerpo cuando este se encuentra en la trayectoria de un fluido en movimiento y se mostrará cuál es su caracterización física.

Además, también se definirán los principales números que juegan un papel importante en el proceso y que permiten conocer cuáles de las fuerzas serán predominantes y si existirán efectos de segundo orden que deban ser estudiados.

A partir de todo ello se dividirá el dominio físico de estudio en los seis subdominios siguientes: dominio de flujo separado lineal, dominio de flujo separado no lineal, dominio inercial lineal, dominio inercial no lineal, dominio de difracción lineal y dominio de difracción no lineal. En cada uno de estos dominios de cálculo se realizará una descripción de las 
principales características que lo definen y se expondrán una o varias teorías que resuelven los casos que se encuentra dentro de dicho dominio.

\subsection{Acción de un fluido sobre un cuerpo sumergido}

\subsubsection{Fluido no viscoso}

En el caso de un cuerpo inmerso en un fluido infinitamente no viscoso, la única fuerza, aparte de la fuerza de Arquímedes, actuante sobre el cuerpo sumergido es la fuerza de Froude-Krylov, debida a la presión transitoria que se ejerce sobre él. En la figura 27, puede observarse el efecto de un fluido que ve interrumpido su movimiento a causa de un obstáculo de sección circular.

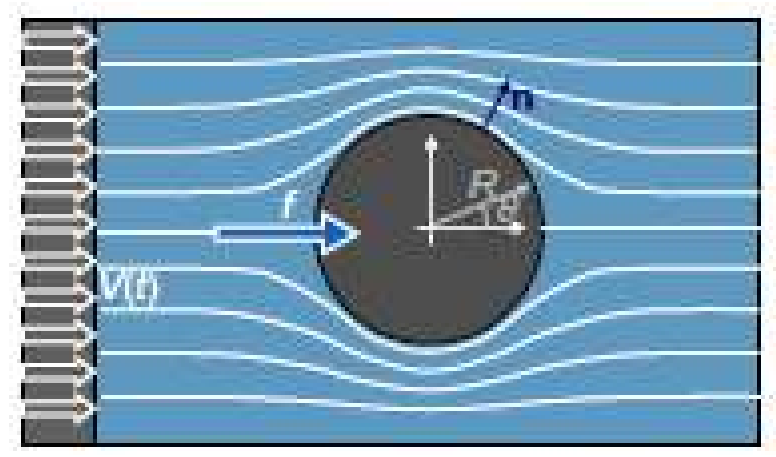

Figura 27. Líneas de flujo de corriente que varían su trayectoria a causa de un obstáculo (fuente: Vannucci, 2006)

Según la teoría de Froude-Krylov la fuerza producida por el fluido sobre el cuerpo sumergido es:

$$
f=-\int_{\partial \Omega} p \boldsymbol{n} d s=\rho \int_{\partial \Omega} \frac{\partial \varphi}{\partial t} \boldsymbol{n} d s
$$

Para el caso de un cilindro:

$$
f=2 \pi \rho a_{x} R^{2}
$$

siendo $a_{x}$ la aceleración del fluido en un flujo uniformemente acelerado. Por lo tanto, la velocidad de flujo inalterado será $V(t)=a_{x} t$. También se desprende como segunda conclusión de la expresión anterior que la fuerza sobre el cilindro es nula si $\mathrm{a}_{\mathrm{x}}=0$. 
La fuerza de Froude-Krylov puede ser dividida en dos partes, una dependiente del volumen y otra dependiente de la forma que, reescribiendo la formula anterior queda de la forma siguiente:

$$
f=C_{M} \rho \Omega \alpha e_{\alpha}
$$

$\Omega$ es el volumen del cuerpo y $C_{M}$ es el coeficiente de inercia:

$$
C_{M}=1+C_{A}
$$

$C_{A}$ se denomina como el coeficiente de masa añadida del cuerpo, y viene determinado por:

$$
C_{A}=\frac{\mu_{c a}}{\rho \Omega}
$$

siendo $\mu_{c a}$ un parámetro que se determina empíricamente. Para el caso de un cilindro, normalmente, se toma $C_{A}=1$ y $C_{M}=2$, no obstante, el valor de estos coeficientes depende de otros factores como se verá en los puntos siguientes.

Sin embargo, cuando ese mismo cilindro se mueve con una aceleración $\alpha$ en dirección $e_{\alpha}$, a través de un fluido en reposo, la fuerza de resistencia al avance que produce dicho fluido sobre el cilindro es:

$$
f=\mu \alpha e_{\alpha}=C_{A} \rho \Omega \alpha e_{\alpha}
$$

Es decir, la resistencia al avance producida para el caso de un cilindro que se mueve con una aceleración $\alpha$, es la mitad que la fuerza que se produce sobre un cilindro en reposo, cuando el fluido que lo rodea se mueve con una aceleración $\mathrm{ax}_{\mathrm{x}}$.

\subsubsection{Fluido viscoso}

En el caso de cuerpos con forma afilada, la viscosidad presente en el fluido da lugar a una fuerza de arrastre viscosa. La fuerza de arrastre viene definida por la siguiente fórmula:

$$
f_{v}=2 \mu \int_{\partial \Omega} D^{\prime} \boldsymbol{n} d s
$$


siendo $D^{\prime}$, el tensor de estiramiento, que en otras palabras es la parte simétrica del gradiente de velocidad.

$$
D^{\prime}=\frac{\nabla u+\nabla u^{T}}{2}
$$

Para el caso de un obstáculo cuya sección es fina y afilada, si la capa límite del fluido se mantiene muy cerca de la superficie del cuerpo, se puede decir que la única fuerza actuante es esta fuerza viscosa. En la figura 28, se observa el perfil de velocidades a partir de la superficie de un obstáculo situado en medio del flujo de corriente de agua.

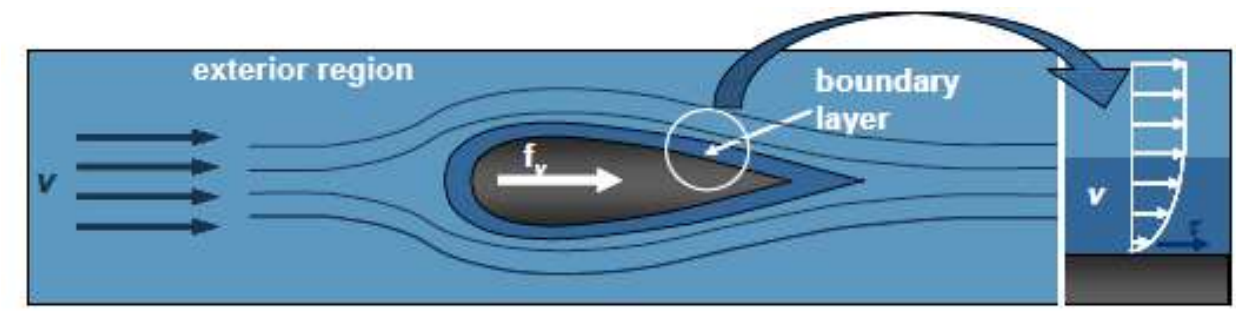

Figura 28. Perfil de velocidades a partir de la superficie del obstáculo (fuente: Vannucci, 2006)

Para el caso de cuerpos que presenten formas romas, la estela de separación no permite obtener la solución analítica del problema hidrodinámico, y la fuerza actuante sobre el cuerpo es producida solo indirectamente por la viscosidad. En realidad, para este caso, la denominada fuerza de arrastre, es debida al desequilibrio presente en el campo de presiones alrededor del cuerpo y se puede expresar mediante la forma semi-empírica:

$$
f_{d}=\frac{1}{2} C_{D} \rho A u^{2}
$$

siendo:

- A: el área de la sección del cuerpo.

- $\mathrm{u}$ : la velocidad del flujo inalterado.

- $C_{D}$ : el coeficiente de arrastre, que depende de la forma del cuerpo, del número de Reynolds, de la rugosidad de la superficie; y que se determina de forma empírica.

Para el caso de un cilindro de diámetro $D$, la fuerza de arrastre producida sobre él por unidad de longitud es:

$$
f_{d}=\frac{1}{2} C_{D} \rho D u^{2}
$$


Muchos autores han hallado una gran cantidad de valores de $C_{M}$ y $C_{D}$, para multitud de casos con distintas condiciones. Más adelante se verán algunas de las fórmulas más aceptadas en el campo de la ingeniería offshore.

\subsection{Variables adimensionales}

Para poder caracterizar de una forma precisa las condiciones físicas del caso de estudio, es necesario definir en primer lugar las variables adimensionales presentes en el problema.

Una correcta definición de las mismas aportará a priori una gran cantidad de información importante como, por ejemplo, cuáles son las fuerzas predominantes en el modelo, qué formulación e hipótesis se pueden tomar para simplificar el problema y cuáles no, etc. En definitiva, marcan el camino a seguir para determinar la solución analítica y permiten entender las clasificaciones aportadas por algunos autores, que se exponen posteriormente en este apartado.

\subsubsection{Número de Reynolds}

El número de Reynolds es quizá el número adimensional más importante en el estudio de dinámica de fluidos, dado que muestra el ratio existente entre las fuerzas de inercia y las fuerzas viscosas presentes en un medio de estudio sometido a unas determinadas condiciones físicas. Su cálculo viene dado por la siguiente fórmula:

$$
R e=\frac{\rho u_{r} D}{\mu}=\frac{\rho u_{r}}{v}
$$

donde:

- $\rho$ : Densidad de fluido.

- $u_{r}$ : Velocidad relativa entre el fluido y el objeto inmerso en él.

- D: Diámetro del objeto.

- $\mu$ : Viscosidad dinámica del fluido.

- $\quad v$ : Viscosidad cinemática del fluido.

El número de Reynolds es utilizado para determinar si un fluido está actuando en régimen laminar o régimen turbulento. Si Re es superior a 4.000, las fuerzas de inercia dominan sobre las fuerzas de viscosidad y el flujo es turbulento. Si Re es menor a 2.300, las fuerzas viscosas 
dominan y el flujo está en régimen laminar. Es llamado número de Reynolds en honor a su descubridor, el físico e ingeniero británico Osborne Reynolds (1842 - 1912), de la Universidad de Manchester.

Para valores altos en el número de Reynolds, el flujo tiene un comportamiento muy turbulento siendo la variabilidad de la velocidad muy grande, y, por consiguiente, aumentando notablemente los esfuerzos cortantes que se producen en la masa del fluido. Esto genera un aumento en la disipación energética que se produce en el fluido fruto de su propio movimiento.

\subsubsection{Número de Froude}

El número de Froude es otra variable adimensional de gran importancia a la hora de estudiar la mecánica de fluidos y es usado para indicar la influencia de las fuerzas gravitatorias frente a las fuerzas de inercia de un fluido en movimiento. Viene dado por la siguiente expresión:

$$
F_{r}=\frac{u}{\sqrt{g h_{R}}}
$$

donde:

- u: Velocidad del fluido.

- $h_{R}$ : Profundidad hidráulica.

- g: Aceleración de la gravedad.

El número de Froude se asocia, en primer lugar, con los efectos sobre la superficie libre, mientras que las fuerzas del oleaje se ejercen sobre un elemento cilíndrico que se extiende una longitud apreciable bajo la superficie libre del agua, lo cual implica que no se genera ninguna perturbación en dicha superficie. Por ello, el número de Froude no es tan relevante a la hora de estudiar las fuerzas hidrodinámicas que genera el oleaje.

\subsubsection{Número de Keulegan-Carpenter}

El cálculo de las fuerzas hidrodinámicas sobre un cuerpo sumergido puede ser llevado a cabo mediante una base teórica dependiente de tres factores principales, que son: la altura relativa o esbeltez $(\mathrm{H} / \mathrm{L})$, la profundidad relativa (D/L) y el número de Keulegan-Carpenter (KC). Este último se define mediante la siguiente fórmula: 


$$
K C=\frac{\mathrm{u}_{\mathrm{r}} \cdot T}{D}=\frac{g H k T}{2 \omega D}=2 \pi \frac{g H k}{2 \omega^{2} D}=\pi \frac{H}{D \tanh k h}=\frac{\pi(H / L)}{(D / L) \tanh k h}=\frac{\pi H}{D} \cdot \frac{1}{\tanh k h}
$$

donde:

- $\mathrm{u}_{\mathrm{r}}$ : Velocidad relativa entre el fluido y el objeto inmerso en él.

- T: Periodo del flujo oscilante.

- D: Diámetro del objeto.

El número de Keulegan-Carpenter es, en definitiva, una cantidad adimensional que describe la importancia relativa de las fuerzas de arrastre más las fuerzas de inercia producidas por un fluido con movimiento oscilatorio sobre los objetos inmersos en él. El número de Keulegan-Carpenter lleva el nombre de Garbis H. Keulegan (1890-1989) y Lloyd H. Carpenter.

Además, puede relacionarse directamente con las ecuaciones de Navier-Stokes mediante la observación de las escalas características de los términos de aceleración:

- Aceleración convectiva: $(u \cdot \nabla) u \sim V^{2} / L$.

- Aceleración local: $\partial u / \partial t \sim V / T$.

Dividiendo estas dos escalas de aceleraciones se obtiene el número de Keulegan-Carpenter. Como aplicación práctica para entender la importancia de este parámetro basta con observar la ecuación de Morison et al. y realizar el ratio entre las fuerzas de inercia y las fuerzas de arrastre.

$$
\frac{F_{D \text { max }}}{F_{I \max }}=\frac{\frac{1}{2} C_{D} \rho D u_{\text {max }}^{2}}{\frac{\pi D^{2}}{4} C_{M} \rho \omega u_{\max }}=\frac{2}{\pi} \frac{C_{D}}{C_{M}} \frac{u_{\max }}{\omega D}=\frac{K C}{\pi^{2}} \frac{C_{D}}{C_{M}}
$$

Dado que los coeficientes $C_{D}$ y $C_{M}$ se encuentran normalmente en un rango entre 0,6 y 2,0, se puede observar que el número de Keulegan-Carpenter será un buen indicador para determinar las fuerzas predominantes en un determinado caso de estudio. Por lo tanto, para valores pequeños de KC, las fuerzas de inercia predominarán sobre las de arrastre, ocurriendo lo contrario para valores grandes de KC. En otras palabras, para valores pequeños de $\mathrm{KC}$ se podrán despreciar las fuerzas de arrastre. 


\subsubsection{Número de Strouhal}

Un parámetro muy similar a este, y que también es utilizado por gran cantidad de autores, es el número de Strouhal, que presenta una reciprocidad matemática con el número de Keulegan-Carpenter. No obstante, la diferencia entre ambos radica en que el número de Strouhal da la frecuencia de turbulencia resultante de la colocación de un objeto en un flujo constante, por lo que, describe el flujo de inestabilidad como resultado de una inestabilidad del flujo aguas abajo del objeto. Mientras que el número Keulegan-Carpenter está relacionado con la frecuencia de oscilación de un flujo inestable, en el que se coloca el objeto.

\subsubsection{Parámetro de rugosidad relativa}

La rugosidad de la superficie de la estructura influye sobre las fuerzas hidrodinámicas que se generan, sobre todo cuando se trata de una estructura pequeña. La rugosidad de una estructura se verá, principalmente, incrementada por el crecimiento marino en la parte sumergida de la estructura. En particular, los valores de los coeficientes hidrodinámicos de inercia y arrastre diferirán, significativamente, dependiendo de la rugosidad superficial de la estructura. La rugosidad relativa es determinada por el tamaño medio de las partículas de una superficie mediante el parámetro $\mathrm{K}$ normalizado para el diámetro equivalente de la sección del miembro estructural. La rugosidad relativa se determina mediante la siguiente fórmula:

$$
e=\frac{K_{r}}{D}
$$

donde:

- K: Parámetro de rugosidad superficial.

- D: Diámetro del objeto.

\subsubsection{Parámetro de difracción}

El parámetro de difracción indica si los efectos de dispersión del oleaje a partir de la superficie de la estructura son lo suficientemente importantes para ser considerados en el cálculo. Para grandes estructuras, el oleaje en los alrededores de la estructura presenta gran cantidad de olas difractadas causando un significante efecto en los esfuerzos generados por el oleaje sobre la estructura. El parámetro de difracción viene dado por la siguiente expresión: 


$$
F^{\prime}{ }_{\text {dif }}=\frac{\pi D}{L}
$$

donde:

- D: Diámetro del objeto.

- L: Longitud de onda del oleaje incidente.

\subsubsection{Parámetro de velocidad reducida}

En un flujo estable, un miembro estructural flexible estará sujeto a una vibración longitudinal. El parámetro de velocidad reducida determina si la respuesta vibratoria de la estructura sometida al flujo estable es lo suficientemente significativa y debe ser considerada en los cálculos. Este parámetro viene dado por la fórmula:

$$
V_{R}=\frac{u_{r}}{f_{s} D}
$$

donde:

- $u_{r}$ : Velocidad relativa entre el fluido y el objeto inmerso en él.

- $\mathrm{f}_{\mathrm{s}}$ : Frecuencia de vibración de la estructura.

- D: Diámetro del objeto.

\subsubsection{Parámetro $\beta$ de Sarpkaya}

Sarpkaya e Isaacson (1981) llevaron a cabo numerosos experimentos utilizando un tubo en U para generar un flujo oscilatorio. Como forma más conveniente de mostrar los resultados de sus experimentos ellos descubrieron el parámetro $\beta$, también denominado parámetro de Sarpkaya:

$$
\beta=\frac{D^{2}}{v \cdot T}
$$

donde:

- D: Diámetro del objeto.

- T: Periodo del flujo oscilante.

- $\quad v$ : Viscosidad cinemática del fluido.

No obstante, el parámetro de Sarpkaya no revela ninguna información nueva de cara al estudio, dado que operando un poco se demuestra que: 


$$
\beta=\frac{D^{2}}{v \cdot T}=\frac{\frac{\mathrm{D} \cdot \mathrm{V}}{\mathrm{V}}}{\frac{\mathrm{V} \cdot T}{D}}=\frac{R e}{K C}
$$

Es decir, el parámetro de Sarpkaya es únicamente el ratio entre el número de Reynolds y el número de Keulegan-Carpenter.

\subsubsection{Módulo de Iversen}

Otro de los parámetros utilizados por algunos autores es el módulo de Iversen. Fue un parámetro desarrollado por Iversen y Balent (1951) antes de que Keulegan y Carpenter realizaran su trabajo.

$$
I_{v}=\frac{a_{r} \cdot D}{u_{r}^{2}}
$$

donde:

- $a_{r}$ : Aceleración relativa entre el fluido y el objeto inmerso en él.

- $u_{r}$ : Velocidad relativa entre el fluido y el objeto inmerso en él.

- D: Diámetro del objeto.

Este parámetro tampoco aporta información nueva y no será utilizado en el estudio, dado que conociendo que en una onda sinusoidal $a_{r}=\frac{2 \pi}{T} v_{r}$, se demuestra que:

$$
I_{v}=\frac{2 \pi}{K C}
$$

En la práctica el parámetro de Keulegan-Carpenter es más utilizado y se ha extendido más a nivel internacional.

\subsection{Teorema $\pi$ o de Buckingham}

El concepto de análisis dimensional surge de la aplicación a la física de los conceptos de semejanza y proporción. Aplicado por Galileo y Newton entre otros, fue Buckingham quien dedujo el conocido teorema $\Pi$ en 1914. Sus ideas básicas son las siguientes:

- El concepto de magnitud física es intuitivo pero puede racionalizarse.

- Dos entes físicos son comparables cuando existe una relación operacional y universal de la razón. 
- Una magnitud está formada por dos partes, una numérica que mide su valor y otra que representa el término de comparación.

- El enlace físico entre magnitudes da lugar a ecuaciones.

- Cualquier magnitud puede expresarse mediante la ecuación de dimensiones.

- En mecánica clásica el modelo se obtiene, o bien, mediante el espacio, la masa y el tiempo; o bien, mediante el espacio, la fuerza y el tiempo.

- Cada magnitud derivada depende de " $m$ " magnitudes fundamentales y se dice que está en dependencia tipo monomio.

Sobre estas premisas se definen las variables correspondientes a un sistema físico, que tendrán unas unidades concretas.

El teorema de Buckingham establece que, dada una relación física expresable mediante una ecuación en la que están involucradas " $\mathrm{n}$ " magnitudes físicas o variables y si dichas variables se expresan en términos de " $\mathrm{k}$ " cantidades físicas dimensionalmente independientes, entonces la ecuación puede expresarse de forma equivalente como una ecuación con una serie de " $n-k$ " números adimensionales construidos con las variables originales.

Consideremos un fenómeno físico en el que intervienen un número " $n$ " de variables $q_{1}, q_{2}$ ..., $q_{n}$ expresadas en términos de unidades físicas independientes $L_{1}, L_{2} \ldots, L_{k}$ donde $n>k$. $L a$ ecuación de dimensiones de cada variable $q_{i}$ puede expresarse en términos de las dimensiones básicas como sigue:

$$
\left[q_{i}\right]=L_{1}^{a_{1 i}} \cdot L_{1}^{a_{2 i}} \cdot \ldots \cdot L_{1}^{a_{j i}}
$$

donde los $a_{\mathrm{ji}}($ siendo $\mathrm{i}=1,2 \ldots \mathrm{n} \mathrm{y} \mathrm{j}=1,2 \ldots, \mathrm{k}$ ) son los exponentes de la magnitud en cada dimensión básica.

Con estos exponentes se crea la matriz " $\mathrm{A}$ " de dimensiones " $\mathrm{n} \times \mathrm{k}$ " y de rango " $\mathrm{r}$ " igual al número de dimensiones fundamentales " $k$ ". De esta forma se tiene una base algebraica.

$$
A=\left[\begin{array}{ccc}
a_{11} & \cdots & a_{k 1} \\
\vdots & \ddots & \vdots \\
a_{1 n} & \cdots & a_{k n}
\end{array}\right]
$$


El teorema $\Pi$ afirma que:

- Con las variables $q_{1}, q_{2} \ldots, q_{n}$ se pueden formar " $n-k$ " parámetros adimensionales independientes $\pi_{1}, \pi_{2} \ldots, \pi_{n-k}$.

- El funcional $f\left(q_{1}, q_{2}, \ldots, q_{n}\right)=0$ se puede reescribir de forma equivalente como $F\left(\pi_{1}, \pi_{2} \ldots, \pi_{n-k}\right)=0$.

Cada parámetro adimensional $\pi \mathrm{m}$ relaciona las distintas variables a través de ecuaciones del tipo:

$$
\pi_{m}=q_{1}^{a 1} \cdot q_{2}^{a 2} \ldots q_{n}^{a n}
$$

donde an son números enteros.

De esta forma el teorema ofrece un método de construcción de parámetros adimensionales, incluso cuando la forma de la ecuación es desconocida.

\subsubsection{Aplicación práctica}

Con la intención de entender el efecto del medio sobre la estructura y las fuerzas resultantes que esta experimenta, se han mostrado ciertos parámetros adimensionales que juegan un papel importante y se han descartado en los puntos anteriores algunos de ellos por no ofrecer información adicional o por no presentar una relevancia significativa en el proceso físico objeto de la presente Tesis Doctoral.

La importancia relativa que presentan estos parámetros adimensionales puede ser examinada mediante un simple análisis. Un análisis dimensional puede ser realizado para obtener la dependencia de las distintas fuerzas actuantes, con respecto a los parámetros adimensionales. La fuerza sobre una estructura sujeta a la acción del oleaje depende de un conjunto de variables independientes, presentadas en la siguiente expresión:

$$
f=F\left(t, T, D, K, f_{s}, L, u_{r}, \rho, v\right)
$$


En conclusión, los parámetros adimensionales necesarios y determinantes para estudiar las fuerzas hidrostáticas que genera el oleaje sobre una estructura son:

$$
\frac{f}{\rho V^{2} D}=F\left(\frac{t}{T}, \frac{u_{r} \cdot T}{D}, \frac{u_{r} \cdot D}{v}, \frac{\pi D}{L}, \frac{K}{D}, \frac{u_{r}}{f_{s} D}\right)
$$

En la tabla 4, se muestran los principales parámetros adimensionales que influyen en el fenómeno estudiado:

\begin{tabular}{|l|c|}
\hline Número de Reynolds & $R e=\frac{\rho u_{r} D}{\mu}$ \\
\hline Número de Keulegan-Carpenter & $K C=\frac{u_{r} \cdot T}{D}$ \\
\hline Rugosidad relativa superficial & $e=\frac{K}{D}$ \\
\hline Parámetro de difracción & $F^{\prime}{ }^{\prime}$ if $=\frac{\pi D}{L}$ \\
\hline Velocidad reducida & $V_{R}=\frac{u_{r}}{f_{s} D}$ \\
\hline
\end{tabular}

Tabla 4. Números adimensionales que rigen el comportamiento del problema físico (fuente: elaboración propia)

\subsection{Clasificación de las fuerzas hidrodinámicas}

Como ha podido verse a lo largo del apartado anterior, los diferentes autores coinciden en que las fuerzas hidrodinámicas que se producen sobre un cuerpo sometido a la acción de un fluido en movimiento, si este fluido presenta una determinada aceleración, son de dos tipos:

- Fuerzas de arrastre (también llamadas, de tipo viscoso).

- Fuerzas de inercia.

Existen tres conjuntos principales de teorías que se dedican al estudio de dichas fuerzas y que prevén las solicitaciones totales que producirá el oleaje sobre la estructura.

- Rango de separación (Separation range).

- Rango inercial (Inertial range). 
- Rango de difracción (Difraction range).

La elección de la teoría apropiada dependerá fundamentalmente del dominio físico en el que se encuentre el problema. Además, en las tres regiones existirá una cuarta región interna a ellas, en la que los efectos no lineales no podrán ser despreciados por ser significativamente importantes en los resultados obtenidos. Se entienden por efectos no lineales a aquellos derivados de las interacciones existentes entre las fuerzas de inercia y las fuerzas de arrastre. Para poder conocer en qué dominio se encuentra el problema en estudio y qué teoría física es de aplicación, se deberán observar dos valores: el número de Keulegan-Carpenter y el ratio $\mathrm{D} / \mathrm{L}$, diámetro del elemento estructural entre longitud de onda del oleaje incidente.

Las teorías dentro del rango de separación estudian las fuerzas hidrodinámicas de carácter inercial y de arrastre, de forma separada, sumando posteriormente sus efectos. Este dominio es válido para estructuras con un diámetro reducido en relación a la longitud de onda del oleaje. El ratio límite entre el diámetro y la longitud de onda que se deberá cumplir para que estas teorías sean de aplicación es:

$$
\frac{D}{L} \leq 0,2
$$

El desarrollo de la primera formulación precisa para resolver la solicitación hidrodinámica en estructuras que cumplían estas condiciones fue realizado por Morison, Johnson, Schaaf y O’Brien (1950).

Dentro de este dominio, Lighthill (1979) desarrolló una corrección a la ecuación de Morison et al. para incorporar los efectos no lineales que aparecen a causa de la interacción entre las fuerzas de inercia y las de arrastre.

Las teorías dentro del rango inercial ignoran las fuerzas de arrastre debido a que tienen una baja o nula influencia en comparación con las fuerzas de inercia. Por lo tanto, dentro de este rango se estudia la fuerza de Froude-Krylov como única causante de las solicitaciones de la estructura. 
Pertenecen a este dominio aquellos problemas que tienen un bajo número de KeuleganCarpenter y a la vez cumplen el ratio explicado en el párrafo anterior para rango de separación:

$$
\frac{D}{L} \leq 0,2 \quad K C \leq 4
$$

En último lugar, las teorías englobadas dentro del dominio de difracción se dedican a estudiar elementos estructurales de gran tamaño, entendiendo por gran tamaño, a aquellos que cumplan con el siguiente ratio:

$$
\frac{D}{L}>0,2
$$

Dentro de las formulaciones que pertenecen a este grupo, se encuentran las teorías propuestas por Mc Camy y Fuchs, Garrett, y Ogilvie, entre otros autores.

Dentro de las teorías de difracción, también existe un conjunto que engloba a aquellas en las que los efectos no lineales no pueden ser despreciados.

A continuación, se muestra en la tabla 5 la formulación a utilizar según el ratio $D / L$ y según el número de Keulegan-Carpenter. Además, se indica el rango en el que deben ser considerados los efectos lineales. 
Modelos de predicción de esfuerzos hidrodinámicos y socavación aplicados a ingeniería offshore

\begin{tabular}{|c|c|c|c|c|}
\hline & $\begin{array}{l}K C<4 \\
H / L<0,5 \cdot H / L_{\max }\end{array}$ & $\begin{array}{l}K C<4 \\
H / L>0,5 \cdot H / L_{\max }\end{array}$ & $\begin{array}{l}K C>4 \\
H / L<0,5 \cdot H / L_{\max }\end{array}$ & $\begin{array}{l}K C>4 \\
H / L>0,5 \cdot H / L_{\max }\end{array}$ \\
\hline$D / L<0,2$ & $\begin{array}{l}\text { Rango Inercial } \\
\text { Teoría lineal de } \\
\text { oleaje } \\
\text { Fuerza: Froude- } \\
\text { Krylov }\end{array}$ & $\begin{array}{l}\text { Rango Inercial } \\
\text { Teoría no lineal de } \\
\text { oleaje } \\
\text { Fuerza: no lineal de } \\
\text { Froude-Krylov }\end{array}$ & $\begin{array}{l}\text { Rango Separación } \\
\text { Teoría lineal de oleaje } \\
\text { Fuerza: inercial más } \\
\text { arrastre }\end{array}$ & $\begin{array}{l}\text { Rango Separación } \\
\text { Teoría no lineal de } \\
\text { oleaje } \\
\text { Fuerza: inercial más } \\
\text { arrastre más términos } \\
\text { cuadráticos }\end{array}$ \\
\hline$D / L>0,2$ & $\begin{array}{l}\text { Rango Difracción } \\
\text { Teoría lineal de } \\
\text { oleaje } \\
\text { Fuerza: Teoría lineal } \\
\text { de difracción }\end{array}$ & $\begin{array}{l}\text { Rango Difracción } \\
\text { Teoría no lineal de } \\
\text { oleaje } \\
\text { Fuerza: Teoría no } \\
\text { lineal de difracción }\end{array}$ & - & - \\
\hline
\end{tabular}

También se muestra la información anterior en forma de gráfico en la figura 29:

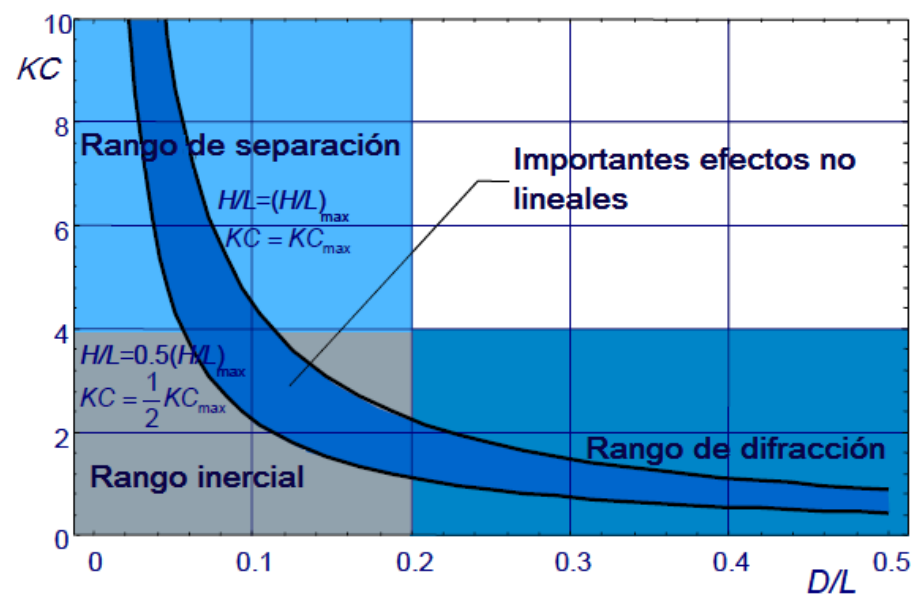

Figura 29. Clasificación de fuerzas hidrodinámicas (fuente: elaboración propia)

\subsection{Teorías de cálculo}

En este punto se procede a realizar un breve repaso de cada uno de los autores relevantes y del dominio en el que es de aplicación su formulación. Por ello se va a seguir el siguiente esquema:

- Rango de separación o de flujo separado:

- Sin efectos no lineales: Morison.

- Con efectos no lineales: Lighthill.

- Rango de flujo inercial:

- Sin efectos no lineales: Froude-Krylov. 
- Con efectos no lineales: Lighthill modificado.

- Rango de difracción:

- Sin efectos no lineales: McCamy \& Fuchs, Garrett y Ogilvie.

- Con efectos no lineales: Adaptación no lineal de McCamy \& Fuchs.

\subsubsection{Rango de flujo separado lineal}

\subsubsection{Morison et al.}

Morison et al. (1950) sugirieron que se pueden superponer los dos regímenes de flujo que generan las fuerzas de inercia y las fuerzas de arrastre para obtener la carga total variable en el tiempo por unidad de longitud de un cilindro, que está parado en un campo de flujo plano con velocidad de flujo libre arbitraria.

En el dominio de aguas profundas, para estudiar el régimen de oleaje que afecta a un cuerpo cilíndrico fijo, se debe hacer una descomposición de las distintas fuerzas actuantes. Para ello las fuerzas que ejerce el oleaje sobre el mismo se pueden clasificar en:

a.- $\quad$ Fuerzas de arrastre (también llamadas, de tipo viscoso).

a.1 "Form drag" $\bar{F}_{p}$ asociada a las tensiones normales.

a.2 "Friction drag" $\bar{F}_{f}$ asociada a la fricción superficial.

b.- $\quad$ Fuerzas de inercia.

Dichas fuerzas, según la teoría ondulatoria, proceden del potencial de velocidades $\Phi$ :

$$
\Phi=\Phi_{\mathrm{I}}+\Phi_{\mathrm{D}}+\Phi_{\mathrm{R}}
$$

- $\Phi_{\mathrm{I}} \quad$ Oleaje incidente.

- $\Phi_{\mathrm{D}} \quad$ Difracción del oleaje.

- $\Phi_{\mathrm{R}} \quad$ Radiación del oleaje.

siendo el potencial del oleaje incidente, tal y como su nombre indica, el generado por el propio oleaje que choca contra la estructura. Por otro lado, el potencial de difracción es aquél que se genera sobre la superficie del propio cuerpo sumergido cuando este tiene unas 
dimensiones lo suficientemente grandes, en comparación con la longitud de onda del oleaje incidente, que generan una influencia y modifican el campo de onda incidente. Por último, el potencial de radiación que se genera incluso en ausencia de una onda incidente, cuando los cuerpos sumergidos se encuentran en movimiento, generando ondas y por ende fuerzas de onda.

Cuando el diámetro de la estructura, D, es mucho más pequeño que la longitud de la onda, $L$, es decir, $D<<L$, el oleaje incidente no se modifica significativamente con la presencia del cuerpo. Por lo tanto, se puede despreciar la componente de difracción y de radiación, $\Phi_{D}$ V $\Phi_{\mathrm{R}}$. La fuerza que actúa sobre un cilindro, debida a un flujo continuo de corriente es:

$$
F_{D}=\frac{1}{2} \cdot \rho \cdot C_{D} \cdot D \cdot u \cdot|u|
$$

donde $F_{D}$ es la fuerza por unidad de longitud del cilindro, $\rho$ la densidad del fluido y $C_{D}$ es el coeficiente de arrastre. Se escribe la velocidad al cuadrado de la forma $u \cdot|u|$ para asegurar que la dirección de la fuerza de arrastre va en la misma dirección de la velocidad del flujo.

Sin embargo, el oleaje es una corriente oscilatoria y, en este caso, habrá dos términos adicionales que contribuyen a la totalidad de la fuerza.

$$
F=\frac{1}{2} \cdot \rho \cdot C_{D} \cdot D \cdot u \cdot|u|+m^{\prime} \cdot a_{x}+\rho \cdot V_{m} \cdot a_{x}
$$

donde $m^{\prime} \cdot a_{x}$ se llama la fuerza de masa adicional movilizada, en inglés hydrodynamic-mass force, donde $\mathrm{m}^{\prime}$ es la masa adicional movilizada o masa hidrodinámica, que en inglés se denomina hydrodynamic mass. Por otro lado, se denomina a $\rho \cdot V_{m} \cdot a_{x}$ como la fuerza Froude-Krilov, siendo $V_{m}$ el volumen del cilindro. Si tomamos una unidad de longitud del cilindro, como rango de cálculo, dicha variable queda reducida a A, que es el área de la sección transversal.

La masa adicional movilizada se define como la masa del fluido alrededor del cuerpo que es acelerada con el movimiento del cuerpo debido a la acción de la presión. 
Cuando el cuerpo se mantiene inmóvil y el agua se mueve con aceleración $\dot{U}$, existen dos efectos. Primero, el agua que rodea el cuerpo sufrirá una aceleración como ya se ha descrito anteriormente, $y$, por lo tanto, la fuerza debida a la masa hidrodinámica estará presente. EI segundo efecto será que el movimiento acelerado del fluido en la región externa del flujo generará un gradiente de presiones de acuerdo a:

$$
\frac{\partial p}{\partial x}=-\rho \cdot \frac{d u}{d t}
$$

Donde $u$ es la velocidad del fluido alejado del cilindro. Este gradiente de presiones producirá una fuerza adicional en el cilindro, que denominamos fuerza de Froude -Krylov.

Esta fuerza puede ser calculada por medio de la siguiente integración:

$$
F_{P}=-\int_{S} p \cdot d S
$$

siendo S la superficie del cuerpo. Por el teorema de Ostrogadski - Gauss tenemos:

$$
F_{P}=-\int_{V_{m}} \frac{\partial p}{\partial x} \cdot d V_{m}
$$

Si tenemos en cuenta que el gradiente de presiones es constante:

$$
F_{p}=\rho \cdot V_{m} \cdot a_{x} \quad, \text { con } a_{x}=\frac{\partial u}{\partial t}
$$

Para un cilindro de área transversal $A$, la fuerza por unidad de longitud, $F_{p}$, es:

$$
F_{p}=\rho \cdot A \cdot a_{x}
$$

La fuerza $\mathrm{F}$ total viene dada por las expresiones anteriores, teniendo en cuenta la fuerza de masa hidrodinámica y la fuerza de Froude-Krylov: 


$$
F=\frac{1}{2} \cdot \rho \cdot C_{D} \cdot D \cdot u \cdot|u|+\rho \cdot C_{m} \cdot A \cdot a_{x}+\rho \cdot A \cdot a_{x}
$$

donde:

Fuerza de arrastre.

Fuerza de masa adicional movilizada.

Fuerza de Froude-Krylov.

$$
\frac{1}{2} \cdot \rho \cdot C_{D} \cdot D \cdot u \cdot|u|
$$

$$
\rho \cdot C_{m} \cdot A \cdot a_{x}
$$

$$
\rho \cdot A \cdot a_{x}
$$

No obstante, se puede escribir de nuevo la ecuación de la siguiente forma:

$$
F=\frac{1}{2} \cdot \rho \cdot C_{D} \cdot D \cdot u \cdot|u|+\rho \cdot\left(C_{m}+1\right) \cdot A \cdot a_{x}
$$

Sabiendo que $C_{M}=C_{m}+1$, se obtiene la clásica expresión de Morison, Johnson, Schaaf y O’Brien (1950):

$$
F=\frac{1}{2} \cdot \rho \cdot C_{D} \cdot D \cdot u \cdot|u|+\rho \cdot C_{M} \cdot A \cdot a_{x}=\rho \cdot \frac{D}{2} \cdot C_{D} \cdot u \cdot|u|+\rho \cdot \frac{\pi \cdot D^{2}}{4} \cdot C_{M} \cdot \frac{\partial u}{\partial t}
$$

donde:

- $u$ : Velocidad relativa horizontal entre fluido y estructura.

- $\frac{\partial u}{\partial t}$ : Aceleración relativa horizontal entre fluido y estructura.

- $\rho:$ Densidad del agua.

- D: Diámetro del cilindro vertical.

- A: Área de la sección del cilindro vertical.

- $\mathrm{C}_{\mathrm{M}}$ : Coeficiente de inercia debido a la masa hidrodinámica.

- $C_{D}$ : Coeficiente de arrastre.

\subsubsection{Determinación de los coeficientes $C_{M}$ y $C_{D}$}

A pesar de la aparente sencillez de la ecuación de Morison et al., su aplicación conlleva una serie de dificultades que conviene tener en cuenta. En primer lugar, la determinación de los coeficientes $C_{D}$ y $C_{M}$. 
Ambos son de carácter empírico y no es objeto de la presente Tesis Doctoral profundizar en su obtención. No obstante, sí es importante destacar que se han realizado multitud de estudios por parte de muchos autores para realizar su obtención. En la tabla 6, se resumen algunos de los más importantes:

\begin{tabular}{|c|c|c|c|}
\hline Investigador & $\mathbf{R}_{\mathrm{e}}$ aproximado & $C_{M}$ & Tipo de experimento y teoría usada \\
\hline $\begin{array}{l}\text { Keulegan y Carpenter } \\
\text { (1956) }\end{array}$ & $<3 \cdot 10^{4}$ & $1,5-2,5$ & Laboratorio, flujo oscilatorio (A/D 6) \\
\hline Bretschneider (1957) & $\begin{array}{l}1,6 \cdot 10^{5} \text { a } 2,3 \cdot 10^{5} \\
3,8 \cdot 10^{5} \text { a } 6 \cdot 10^{5}\end{array}$ & $\begin{array}{l}2,26-2,02 \\
1,74-1,23\end{array}$ & $\begin{array}{l}\text { Experimentos de Campo } \\
\text { Teoría lineal }\end{array}$ \\
\hline Wilson (1965) & $>5 \cdot 10^{5}$ & 1,53 & Experimentos de campo, análisis espectral \\
\hline Skjelbreia (1960) & $>5 \cdot 10^{5}$ & $1,02 \pm 0,53$ & $\begin{array}{l}\text { Experimentos de campos, Teoría de } \\
\text { Stokes de } 5 \text { o orden }\end{array}$ \\
\hline $\begin{array}{l}\text { Dean y Aagaard } \\
(1970)\end{array}$ & $2 \cdot 10^{5}$ a $2 \cdot 10^{6}$ & $1,2-1,7$ & $\begin{array}{l}\text { Experimentos de campo, Teoría de } \\
\text { función de flujo }\end{array}$ \\
\hline Evans (1970) & $>5 \cdot 10^{5}$ & $1,76 \pm 1,05$ & $\begin{array}{l}\text { Experimentos de campo, Teoría de Stokes } \\
\text { de 5o orden y teoría numérica de ondas }\end{array}$ \\
\hline Wheeler (1970) & $>5 \cdot 10^{5}$ & 1,5 & $\begin{array}{l}\text { Experimentos de campo, Análisis de } \\
\text { espectro modificado. }\end{array}$ \\
\hline
\end{tabular}

Tabla 6. Determinación del coeficiente $C_{M}$, de acuerdo a distintos autores (fuente: Shore Protection Manual, 1975)

A partir de los distintos estudios mostrados en la tabla anterior, el criterio que propone el Shore Protection Manual para el establecimiento del coeficiente $C_{M}$ es:

$$
\begin{array}{ll}
C_{M}=2,0 & \text { cuando } \mathrm{R}_{\mathrm{e}}<2,5 \cdot 10^{5} \\
C_{M}=2,5-\frac{R_{e}}{5 \cdot 10^{5}} & \text { cuando } 2,5 \cdot 10^{5}<\mathrm{R}_{\mathrm{e}}<5 \cdot 10^{5} \\
C_{M}=1,5 & \text { cuando } \mathrm{R}_{\mathrm{e}}>5 \cdot 10^{5}
\end{array}
$$

Para hallar el coeficiente $C_{D}$, uno de los criterios a seguir es el criterio seguido por el Shore Protection Manual, a partir de los estudios de Achenbach (1968).

$$
C_{D}=1,2 \quad \text { cuando } \mathrm{R}_{\mathrm{e}}<1 \cdot 10^{5}
$$




$$
\begin{array}{ll}
C_{D}=1,2-\frac{1,2-0,7}{3 \cdot 10^{5}} \cdot\left(R_{e}-10^{5}\right) & \text { cuando } 1 \cdot 10^{5}<\mathrm{R}_{\mathrm{e}}<4 \cdot 10^{5} \\
C_{D}=0,7 & \text { cuando } \mathrm{R}_{\mathrm{e}}>4 \cdot 10^{5}
\end{array}
$$

Como puede observarse en la figura 30 procedente del Shore Protection Manual de 1975, una gran cantidad de autores han realizado estudios para determinar el coeficiente $C_{D}$, y los resultados aportados por Achenbach se postulan como una envolvente de todos los resultados.

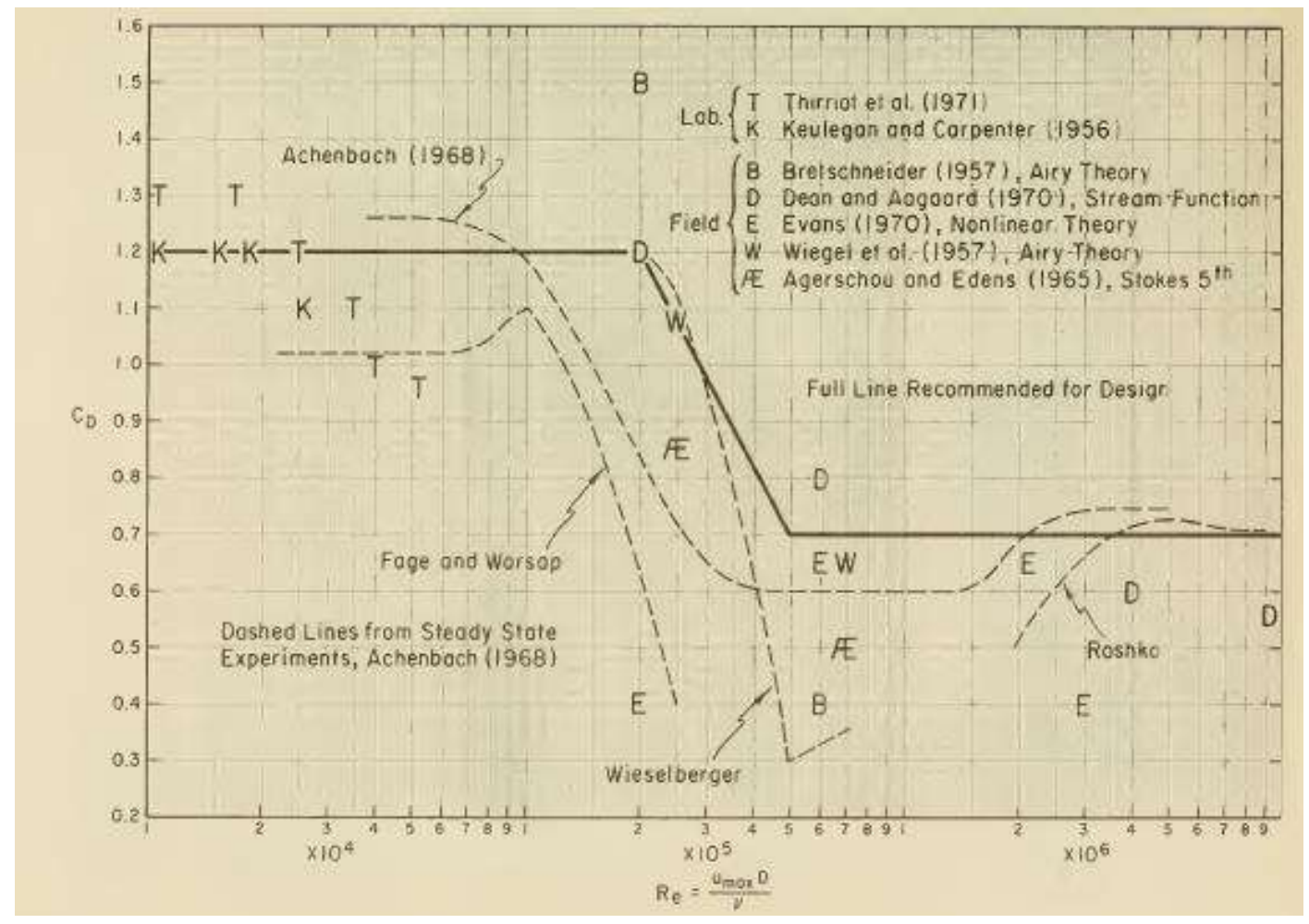

Figura 30. Obtención del coeficiente $C_{D}$, de acuerdo a los estudios realizados por distintos autores (fuente: Shore Protection Manual,

1975)

No obstante, ambos coeficientes también dependerán de la rugosidad relativa y la forma de la sección del elemento estructural y del número de Keulegan-Carpenter. Para realizar análisis más precisos, será necesario hallarlos de forma empírica mediante la creación de modelos de laboratorio reducidos u observación en ensayos de campo. 


\subsubsection{Rango de flujo separado no lineal}

\subsubsection{Lighthill}

Lighthill (1979) hace un análisis metodológico describiendo los fenómenos físicos básicos junto con los métodos de aplicación utilizados para la estimación de las fuerzas hidrodinámicas. Su principal contribución al cálculo de la fuerza de oleaje mediante el uso de la ecuación de Morison et al. reside en la proposición de una corrección de segundo orden en la misma. Las fuerzas turbulentas inducidas (flujo separado) en los cilindros sometidos a oleaje y algunos métodos para la predicción de tales fuerzas son discutidos también por Graham (1979).

Lighthill propone una fórmula que se compone de cinco términos, de los cuales dos coinciden con la formulación propuesta por Morison et al., siendo los tres restantes, términos que tienen en cuenta los efectos de segundo orden. Estos efectos cuadráticos o de segundo orden se convierten en más significativos conforme aumenta la esbeltez de la ola:

$$
\mathrm{F}=\mathrm{F}_{1}+\mathrm{F}_{2}+\mathrm{F}_{3}+\mathrm{F}_{4}+\mathrm{F}_{5}
$$

$F_{1}$ : Fuerza de Froude-Krylov.

$$
F_{1}=-\rho \int_{\partial \Omega} \frac{\partial \varphi_{l}}{\partial t} \boldsymbol{n} d s=\frac{\pi D^{2}}{4} \rho g \frac{H}{2} C_{M} \cos \omega t
$$

$F_{2}$ : Fuerza de arrastre.

$$
F_{2}=\frac{1}{4} C_{D} \rho g\left(\frac{H}{2}\right)^{2} D \operatorname{sen} \omega t|\operatorname{sen} \omega t|
$$

$F_{3}$ : Término cuadrático de la velocidad potencial en Stokes de segundo orden, según descomposición potencial.

$$
F_{3}=-\rho \int_{z=d} w\left(\nabla \varphi_{l}\right)^{2} d s=\frac{\pi}{2} \rho g\left(\frac{H}{2}\right)^{2}\left(\frac{D}{2}\right)^{4} k^{3}\left(16 \ln \frac{0,56}{k D}-3\right) \operatorname{sen}(2 \omega t)
$$


F4: Presión cinemática debía al potencial lineal.

$$
F_{4}=\frac{1}{2} \rho \int_{S}\left(\nabla \varphi_{l}\right)^{2} \cos \theta d s=\frac{\pi}{4} \rho g\left(\frac{H}{2}\right)^{2}\left(\frac{D}{2}\right)^{2} k \operatorname{sen}(2 \omega t)
$$

F5: Término presente sólo si el cilindro cruza la superficie libre.

$$
F_{5}=\frac{\rho}{2 g} \oint_{C}\left(\frac{\partial \varphi_{l}}{\partial t}\right)^{2} \cos \theta d l=4 \cdot F_{4}
$$

Lighthill (1986) desarrolló ideas muy importantes que validan una separación de cargas hidrodinámicas en las fuerzas de flujo turbulento y de flujo potencial, aclarando el importante papel de los componentes de segundo orden de las fuerzas de flujo potencial.

\subsubsection{Rango de flujo inercial}

Si el problema a resolver se encuentra en el rango de flujo inercial, son aplicables las fórmulas mostradas en el apartado anterior para el rango de flujo separado, pero eliminando los términos que determinan las fuerzas viscosas debido a que en este dominio son prácticamente despreciables en comparación con las fuerzas de inercia.

\subsubsection{Rango inercial lineal}

En el rango inercial lineal la única fuerza actuante sobre el cilindro será la fuerza de FroudeKrylov, es decir, la fuerza de inercia que figuraba como término en la fórmula de Morison et al.

$$
F=\rho \cdot C_{M} \cdot A \cdot a_{x}=\rho \cdot \frac{\pi \cdot D^{2}}{4} \cdot C_{M} \cdot a_{x}
$$

\subsubsection{Rango inercial no lineal}

En el caso de oleaje escarpado o esbelto, se deberá incluir la fuerza de inercia no lineal dada por la corrección de Lighthill exceptuando el término de las fuerzas de arrastre.

$$
\mathrm{F}=\mathrm{F}_{1}+\mathrm{F}_{3}+\mathrm{F}_{4}+\mathrm{F}_{5}
$$




$$
\begin{gathered}
F=\frac{\pi D^{2}}{4} \rho g \frac{H}{2} C_{M} \cos \omega t \\
+\frac{\pi}{2} \rho g\left(\frac{H}{2}\right)^{2}\left(\frac{D}{2}\right)^{4} k^{3}\left(16 \log \frac{0,56}{k D}-3\right) \operatorname{sen}(2 \omega t)+ \\
+5 \cdot\left(\frac{\pi}{4} \rho g\left(\frac{H}{2}\right)^{2}\left(\frac{D}{2}\right)^{2} k \operatorname{sen}(2 \omega t)\right)
\end{gathered}
$$

\subsubsection{Rango de difracción lineal}

\subsubsection{McCamy y Fuchs}

La solución analítica al problema de difracción lineal fue tratada inicialmente por Havelock (1940) para el rango de profundidades indefinidas. Posteriormente Omer y Hall (1949) estudiaron el problema para el caso de aguas someras y, finalmente, McCamy y Fuchs (1954) obtuvieron la solución analítica para una profundidad general.

McCamy y Fuchs propusieron la existencia de una solución matemática exacta presentada por el oleaje según la teoría lineal de ondas.

$$
\mathrm{F}_{\mathrm{MCF}}=\frac{4 \cdot \rho \cdot \mathrm{g} \cdot \mathrm{H} / 2}{\mathrm{k}^{2}} \cdot \frac{\tanh (\mathrm{kd})}{\sqrt{\left(\mathrm{J}_{1}^{\prime 2}(\mathrm{~kb})+\mathrm{Y}_{1}^{\prime 2}(\mathrm{~kb})\right.}} \cdot \cos (\omega \mathrm{t}-\theta)
$$

siendo el ángulo de fase $\theta$ :

$$
\theta=-\arctan \frac{\mathrm{Y}_{1}^{\prime}(k b)}{\mathrm{J}_{1}^{\prime}(\mathrm{kb})}
$$

Si B es la flotabilidad de un cilindro que tiene el mismo radio que el actual, y es tan alto como la altura del oleaje incidente $\mathrm{H} / 2$ :

$$
B=\pi \rho g \frac{H}{2}\left(\frac{D}{2}\right)^{2}
$$

Entonces se puede expresar la forma adimensional de $F_{M C F}$ de la siguiente forma:

$$
\bar{F}_{M C F}=\frac{F_{M C F}}{B}=\frac{4}{\pi \mu^{2}} \frac{\tanh \lambda}{\sqrt{J_{1}^{\prime 2}(\mu)+Y_{1}^{\prime 2}(\mu)}} \cos (\omega t-\delta)
$$


siendo $\mathrm{J}_{1}{ }_{1}$ e $\mathrm{Y}_{1}{ }_{1}$ respectivamente las funciones de Bessel de primer y segundo tipo, ambas de primer orden, $\lambda=k d$ y $\mu=k D / 2$.

Para $\mu<1$, la expresión anterior puede aproximarse a la siguiente formula, que resulta de utilizar las expresiones asintóticas de las funciones de Bessel:

$$
F_{M C F}=\frac{8}{\sqrt{16+\pi^{2} \mu^{4}}} \tanh \lambda \cos \left(\omega t-\delta_{x}\right)
$$

El momento de vuelco debido a la fuerza del oleaje sobre el centro de la base del cilindro sobre el lecho marino es:

$$
M_{M C F}=\frac{2 \rho g H}{k^{3}} \frac{1+k d \operatorname{senh} k d-\cosh k d}{\cosh k d} \frac{\cos (\omega t-\delta)}{\sqrt{J_{1}^{\prime 2}(k b)+Y_{1}^{\prime 2}(k b)}}
$$

Su expresión adimensional viene dada por:

$$
\bar{M}_{M C F}=\frac{M_{M C F}}{B \cdot \frac{D}{2}}=\frac{4}{\pi \mu^{3}} \frac{1+\lambda \operatorname{senh} \lambda-\cosh \lambda}{\cosh \lambda} \frac{1}{\sqrt{J_{1}^{\prime 2}+Y_{1}^{\prime 2}}} \cos (\omega t-\delta)
$$

Para $\mu<1$, la expresión puede aproximarse nuevamente mediante el uso de las expresiones asintóticas de las funciones de Bessel:

$$
\bar{M}_{M C F}=\frac{1+\lambda \operatorname{senh} \lambda-\cosh \lambda}{\cosh \lambda} \frac{8}{\mu \sqrt{16+\pi^{2} \mu^{4}}} \cos (\omega t-\delta)
$$

Los resultados de la teoría de difracción lineal sobre un cilindro vertical, expresados en su forma adimensional, se adjuntan a continuación: 
Flujo potencial

$$
\frac{\phi}{g H / \omega}=-\frac{1}{2} \frac{\cosh (k s)}{\cosh (k d)}\left\{\sum _ { m = 0 } ^ { \infty } i \beta _ { m } \left[J_{m}(k r)\right.\right.
$$

$$
\left.\left.-\frac{J_{m}^{\prime}\left(k a^{\prime}\right)}{H_{m}^{(1)^{\prime}}\left(k a^{\prime}\right)} H_{m}^{(1)}(k r)\right] \cos (m \theta)\right\} e^{-i \omega t}
$$

Superficie Libre

$$
\left(\frac{\eta}{H}\right)_{r=a \prime}=\left\{\sum_{m=0}^{\infty} \frac{i \beta_{m} \cos (m \theta)}{\pi k a^{\prime} H_{m}^{(1)^{\prime}}\left(k a^{\prime}\right)}\right\} e^{-i \omega t}
$$

Presión

$$
\left(\frac{p}{\rho g H}\right)_{r=a^{\prime}}=-\frac{z}{H}+\frac{\cosh (k s)}{\cosh (k d)} \sum_{m=0}^{\infty} \frac{i \beta_{m} \cos (m \theta)}{\pi k a^{\prime} H_{m}^{(1)^{\prime}}\left(k a^{\prime}\right)} e^{-i \omega t}
$$

Variación de fuerza con

$$
\frac{\partial F / \partial s}{\rho g H a^{\prime}}=2 \cdot \frac{A\left(k a^{\prime}\right)}{k a^{\prime}} \frac{\cosh (k s)}{\cosh (k d)} \cos (\omega t-\theta)
$$

la profundidad

$$
\frac{F}{\rho g H a^{\prime} d}=2 \cdot \frac{A\left(k a^{\prime}\right)}{k a^{\prime}} \frac{\tanh (k d)}{k d} \cos (\omega t-\theta)
$$

Momento

$$
\frac{M}{\rho g H a^{\prime} d^{2}}=2 \cdot \frac{A\left(k a^{\prime}\right)}{k a^{\prime}}\left[\frac{k d \operatorname{senh}(k d)+1-\cosh (k d)}{k d^{2} \cosh (k d)}\right] \cos (\omega t
$$

Fuerza de Froude- $\frac{F_{k}}{\rho g H a^{\prime} d}=-\pi J_{1}\left(k a^{\prime}\right) \frac{\tanh (k d)}{k d} \operatorname{sen}(\omega t-\theta)$
Krylov

siendo:

$$
\begin{aligned}
& s=z+d \\
& \beta_{0}=1, \beta_{m}=2 i^{m} \text { Para } m>1 \\
& A(k a)=\left[J_{1}^{\prime 2}(k a)+Y_{1}^{\prime 2}(k a)\right]^{-\frac{1}{2}} \\
& \theta=-\tan ^{-1} \frac{Y_{1}^{\prime}(k a)}{J_{1}^{\prime}(k a)} \\
& a^{\prime}=D / 2
\end{aligned}
$$

A partir de las expresiones anteriores es posible despejar la expresión que define la fuerza por unidad de longitud del cilindro:

$$
F_{z}=\frac{\pi}{8} \rho g H k D^{2} \frac{\cosh (k(z+d))}{\cosh (k d)} \frac{4 A(k a)}{\pi(k a)^{2}} \cos (\omega t-\theta)
$$




\subsubsection{Garrett}

La solución de Garret (1971), a diferencia de la solución propuesta por McCamy y Fuchs, está adaptada para un cilindro flotante cuya base se encuentra separada del fondo marino una distancia $\mathrm{h}^{\prime}$.

La solución de Garret es analítica, pero no presenta una forma compacta, es decir, es expresada como una serie de funciones especiales cuyos coeficientes son la solución de un sistema lineal.

Vannucci (1993) da una fórmula simplificada por hallar la acción sobre el cilindro flotante, aplicando simplificaciones a la formulación inicialmente propuesta por Garrett.

La expresión para determinar fuerza horizontal generada por el oleaje sobre el cilindro, expresada en forma adimensional es:

$$
\frac{F_{x}}{B}=\frac{4 \chi_{x}}{\pi \mu^{2}} \frac{\operatorname{senh} \lambda-\operatorname{senh} v}{\cosh \lambda} \frac{1}{\sqrt{J_{1}^{\prime 2}+Y_{1}^{\prime 2}}} \cos \left(\omega t-\delta_{x}\right)
$$

donde:

$$
\begin{aligned}
v & =k \cdot h^{\prime} \\
\lambda & =k \cdot h \\
\mu & =k \cdot \frac{D}{2}
\end{aligned}
$$

Pudiendo aproximar las funciones de Bessel a su forma asintótica cuando $\mu<1$, pudiendo rescribirse la fórmula anterior como:

$$
\frac{F_{x}}{B}=\frac{8 \chi_{x}}{\sqrt{16+\pi^{2} \mu^{4}}} \frac{\operatorname{senh} \lambda-\operatorname{senh} v}{\cosh \lambda} \cos \left(\omega t-\delta_{x}\right)
$$

El parámetro $\chi_{x}$ es un coeficiente de reducción que tiene en cuenta el efecto sobre la base del cilindro y va en función de $\lambda, \mu$ y $v$.

La expresión de la fuerza vertical en forma adimensional es: 


$$
\frac{Z}{B}=\frac{4 \chi_{z}}{\pi \mu \nu} \frac{\xi(\mu, v)}{\sqrt{J_{0}^{\prime 2}+{Y_{0}^{\prime}}^{2}}} \frac{\operatorname{senh} v}{\cosh \lambda} \cos \left(\omega t-\delta_{z}\right)
$$

donde:

$$
\xi(\mu, v)=\frac{1}{2}+\frac{2 v}{\pi \mu} \sum_{n=1}^{\infty} \frac{1}{n\left(1+\frac{n^{2} \pi^{2}}{v^{2}}\right)} \frac{I_{l}\left(\frac{n \pi \mu}{v}\right)}{I_{0}\left(\frac{n \pi \mu}{v}\right)}
$$

Para $\mu<1$, la expresión anterior puede aproximarse a:

$$
\frac{Z}{B}=\frac{4 \chi_{z}}{\sqrt{16+\pi^{2} \mu^{4}}} \frac{\operatorname{senh} v}{\cosh \lambda} \frac{v+e^{-v}}{v}
$$

El momento de vuelco para las fuerzas horizontales y verticales es:

$$
\begin{gathered}
\frac{M_{1}}{b B}=-\frac{2 \mu \chi_{x}}{\sqrt{16+\pi^{2} \mu^{4}}} \frac{v+e^{-v}}{v} \frac{\operatorname{senh} v}{\cosh v} \\
\frac{M_{2}}{b B}=\frac{8 \chi_{x}}{\mu \sqrt{16+\pi^{2} \mu^{4}}} \frac{\lambda \operatorname{senh} \lambda-\cosh \lambda-v \operatorname{senh} v+\cosh v}{\cosh \lambda}
\end{gathered}
$$

\subsubsection{Ogilvie}

La solución de Ogilvie (1963) es normalmente de aplicación al caso de emisarios o tuberías en el mar. La solución se aplica a un cilindro horizontal y paralelo al lecho marino.

La solución presenta una gran complejidad, no obstante, el propio autor propuso una importante simplificación para la determinación de la fuerza por unidad de longitud para el caso en que el radio o la distancia de inmersión del cilindro, o ambos, sea pequeño en comparación con la longitud de onda. Este suele ser el caso más habitual, a la hora de resolver problemas de ingeniería offshore.

La expresión simplificada de la fuerza por unidad de longitud es: 


$$
f=2 \pi \rho g k \frac{H}{2}\left(\frac{D}{2}\right)^{2} e^{-k\left(p-\frac{D}{2}\right)}
$$

donde $p$ es la distancia desde la superficie del agua hasta el centro de la sección del cilindro.

\subsubsection{Rango de difracción no lineal}

La teoría de difracción no lineal generalmente halla la fuerza ejercida por el oleaje como superposición de otras tres fuerzas:

- Una fuerza de primer orden, oscilando a una frecuencia $\omega$ (Fuerza de Froude-Krylov).

- Una fuerza dependiente del tiempo de segundo orden, oscilando a una frecuencia $2 \omega$.

- Una fuerza constante de segundo orden, "drift force" o fuerza de deriva.

La técnica usada en la teoría de difracción para tener en cuenta los efectos de las fuerzas no lineales, es justo la misma que se usa para la teoría de ondas de Stokes, es decir, se descomponen la velocidad potencial y el perfil de la onda en dos términos: un término lineal y otro cuadrático.

$$
\begin{gathered}
\varphi=\varepsilon_{1} \varphi_{1}+\varepsilon_{1}^{2} \varphi_{2}=\varepsilon_{1}\left(\varphi_{1 I}+\varphi_{1 D}\right)+\varepsilon_{1}^{2}\left(\varphi_{2 I}+\varphi_{2 D}\right) \\
\eta=\varepsilon_{1} \eta_{1}+\varepsilon_{1}^{2} \eta_{2}=\varepsilon_{1}\left(\eta_{1 I}+\eta_{1 D}\right)+\varepsilon_{1}^{2}\left(\eta_{2 I}+\eta_{2 D}\right)
\end{gathered}
$$

siendo el subíndice I utilizado para el potencial incidente y el subíndice D para el potencial difractado. Tanto el potencial lineal como el cuadrático deben ser armónicos y satisfacer las condiciones de contorno en el lecho marino, en la superficie del elemento estructural y en la superficie libre.

$$
\begin{gathered}
\Delta \varphi_{i I}=\Delta \varphi_{i D}=0 \\
\frac{\partial \varphi_{i I}}{\partial z}=\frac{\partial \varphi_{i D}}{\partial z}=0 \quad \operatorname{Para} z=0 ; i=1,2
\end{gathered}
$$




$$
\begin{gathered}
\frac{\partial \varphi_{i I}}{\partial n}=-\frac{\partial \varphi_{i D}}{\partial n} \quad \text { En } \partial \Omega \\
\frac{\partial^{2} \varphi_{1 j}}{\partial t^{2}}+g \frac{\partial \varphi_{1 j}}{\partial z}=0 \text { Para } z=d ; j=I o D \\
\frac{\partial^{2} \varphi_{2 j}}{\partial t^{2}}+g \frac{\partial \varphi_{2 j}}{\partial z}=-\frac{\partial\left(\nabla \varphi_{1 j}\right)^{2}}{\partial t}-\eta_{1 j} \frac{\partial}{\partial z}\left(\frac{\partial^{2} \varphi_{1 j}}{\partial t^{2}}+g \frac{\partial \varphi_{1 j}}{\partial z}\right) \text { Para } z=d ; j=I o D
\end{gathered}
$$

Además, el potencial disperso debe satisfacer una condición de radiación, la condición de Sommerfeld para $\varphi_{1 I}$ y su equivalente para $\varphi_{2 I}$.

Los componentes del perfil de la ola se calculan de la forma habitual.

$$
\begin{gathered}
\eta_{1 j}=-\frac{1}{g} \frac{\partial \varphi_{1 j}}{\partial t} \\
\eta_{2 j}=-\frac{1}{g}\left[\frac{1}{2}\left(\nabla \varphi_{1 j}\right)^{2}+\eta_{1 j} \frac{\partial^{2} \varphi_{1 j}}{\partial t \partial z}+\frac{\partial \varphi_{2 j}}{\partial t}\right]
\end{gathered}
$$

para $j=I$ o $D$.

Para realizar el cálculo de la fuerza, también se descompone esta, en una parte lineal y otra cuadrática:

$$
F=\varepsilon_{1} F_{1}+\varepsilon_{1}^{2} F_{2}
$$

siendo el parámetro de perturbación en la teoría de Stokes:

$$
\varepsilon_{1}=k \cdot \frac{H}{2}=\frac{\pi H}{L}
$$

Se observa que la parte lineal corresponde con la fuerza de Froude-Krylov, y la parte cuadrática fue expresada por Molin (1979). 


$$
\begin{gathered}
\varepsilon_{1} F_{1}=\rho \int_{s} \frac{\partial \varphi_{1}}{\partial t} n d s \\
\varepsilon_{1}^{2} F_{2}=-\frac{\rho}{2 g} \oint_{C}\left(\frac{\partial \varphi_{1}}{\partial t}\right)^{2} n d l+\frac{\rho}{2} \int_{\partial \Omega}\left(\nabla \varphi_{1}\right)^{2} n d s+\rho \int_{\partial \Omega} \frac{\partial \varphi_{1}}{\partial t} n d s
\end{gathered}
$$

Si se expresan los potenciales en su forma compleja se tiene:

$$
\begin{gathered}
\varphi_{1}=\operatorname{Re}\left[\phi_{1}(x, y, z) e^{-i \omega t}\right] \\
\varphi_{2}=\operatorname{Re}\left[\phi_{2}(x, y, z) e^{-2 i \omega t}\right]
\end{gathered}
$$

A partir de su forma compleja se pueden obtener las fuerzas de segundo orden, $F_{2}$, utilizando las fórmulas anteriores.

$$
F_{2}=F_{2 c}+\operatorname{Re}\left(F_{2 t} e^{-2 i \omega t}\right)
$$

En la expresión anterior, la fuerza constante es la fuerza de deriva o drift force, que es debida, exclusivamente, a la condición de segundo orden en la superficie libre y al término cuadrático de la presión cinemática debida al potencial de primer orden, y que puede ser expresada como:

$$
F_{2 c}=-\frac{\omega^{2} \rho}{4 g} \int_{C} \phi_{1} \overline{\phi_{1}} n d l+\frac{\rho}{4} \int_{\partial \Omega} \nabla \phi_{1} \cdot \nabla \overline{\phi_{1}} n d s
$$

donde la barra superior indica la conjugada.

El valor de la fuerza dependiente del tiempo de segundo orden, oscilando a una frecuencia $2 \omega$, viene dado por:

$$
F_{2 t}=-\frac{\omega^{2} \rho}{4 g} \int_{C} \phi_{1}^{2} n d l-\frac{\rho}{4} \int_{\partial \Omega}\left(\nabla \phi_{1}\right)^{2} n d s+2 i \omega \rho \int_{\partial \Omega} \phi_{2} n d s
$$


Para estructuras de tipo flexible, las fuerzas de segundo orden son muy importantes, de hecho, la fuerza de deriva genera los mayores desplazamientos en la estructura y la fuerza oscilatoria de segundo orden es la que puede causar los mayores problemas de fatiga.

La solución analítica de las expresiones anteriores puede ser obtenida para el caso de un cilindro vertical apoyado en el fondo marino, adaptando la solución de McCamy y Fuchs, se puede expresar la fuerza de deriva como:

$$
F_{2 c}=\frac{2 \rho g\left(\frac{H}{2}\right)^{2} \frac{D}{2}}{\pi \mu^{2}}\left(1+\frac{2 \lambda}{\operatorname{senh} 2 \lambda}\right) \sum_{m=0}^{\infty} \Psi_{m}(\mu)\left[\frac{m(m+1)}{\mu^{2}}-1\right] e_{1}
$$

donde:

$$
\Psi_{m}(\mu)=\frac{J_{m}^{\prime} Y_{m+1}^{\prime}-J_{m+1}^{\prime} Y_{m}^{\prime}}{\left({J_{m}^{\prime}}^{2}+Y_{m}^{\prime 2}\right)\left(J_{m+1}^{\prime}{ }^{2}+Y_{m+1}^{\prime}{ }^{2}\right)}
$$

La evaluación de la fuerza oscilatoria de segundo orden, dependiente del tiempo, es mucho más complicada. Si se divide dicha fuerza en dos términos, el primero dependiente de $\varphi_{1} \mathrm{y}$ el segundo dependiente de $\varphi_{2}$, es posible expresar el primero de forma analítica:

$$
\begin{gathered}
F_{2 t}=F_{2 t 1}+F_{2 t 2} \\
F_{2 t 1}=-\frac{2 \rho g\left(\frac{H}{2}\right)^{2}\left(\frac{D}{2}\right)}{\pi \mu^{2}} \sqrt{A^{2}+B^{2}} \cos (2 \omega t-\delta)
\end{gathered}
$$

donde:

- $\quad A=\sum_{m=0}^{\infty}(-1)^{m} \frac{A_{m}}{C_{m}} f_{m}$.

- $\quad B=\sum_{m=0}^{\infty}(-1)^{m} \frac{B_{m}}{C_{m}} f_{m}$.

- $\delta=\arctan \frac{B}{A}$.

- $f_{m}=\frac{3 \operatorname{senh} 2 \lambda-2 \lambda}{\operatorname{senh} 2 \lambda}+\frac{m(m+1)}{\mu^{2}} \frac{2 \lambda+\operatorname{senh} 2 \lambda}{\operatorname{senh} 2 \lambda}$.

- $A_{m}=J_{m}^{\prime} Y_{m+1}^{\prime}+Y_{m}^{\prime} J_{m+1}^{\prime}$.

- $B_{m}=J_{m}^{\prime} J_{m+1}^{\prime}-Y_{m}^{\prime} Y_{m+1}^{\prime}$.

- $C_{m}=\left(J_{m}^{\prime 2}+Y_{m}^{\prime 2}\right)\left(J_{m+1}^{\prime}{ }^{2}+Y_{m+1}^{\prime}{ }^{2}\right)$. 
El cálculo del segundo componente de $F_{2 T}$ es mucho más complejo de realizar pudiendo ser obtenido mediante la técnica propuesta por Haskind (1957) y basada sobre un flujo de irradiación dual. Desafortunadamente, incluso para los casos más sencillos, no se puede obtener una solución analítica.

No obstante, puede comprobarse fácilmente que dicho término corresponde con el término $F_{3}$ de la corrección de Lighthill, y, por lo tanto, como ocurría con el término $F_{3}$, el segundo término de $\mathrm{F}_{2 \mathrm{~T}}$ es prácticamente despreciable en comparación con el resto de fuerzas de segundo orden. 


\section{Descripción de las vibraciones sobre la estructura}

En este apartado se procederá a hacer una descripción detallada de la teoría matemática que permite caracterizar la respuesta estructural frente a las cargas hidrodinámicas.

\subsection{Vibraciones sobre una viga empotrada}

Una viga es un elemento estructural que tiene una dimensión, la longitud, mucho mayor que sus otras dos dimensiones, ancho y canto. Puede tratarse de un elemento recto o curvo. No obstante, a lo largo de este apartado se estudiará el caso de elemento recto. Las características vibratorias de las vigas juegan un importante papel en el diseño de estructuras que puedan estar sujetas a cargas dinámicas y ser susceptibles de sufrir efectos vibratorios.

Cómo de grande es la máxima tensión producida sobre la viga empotrada, debida a la flexión dinámica, en comparación con la máxima tensión debida a la flexión estática es una importante cuestión a resolver. Además, también es interesante tratar de responder a las siguientes preguntas:

- ¿La vibración mantiene un movimiento periódico después de que desaparezca la carga inicial?

- ¿Cuánto tarda la viga empotrada en volver a su forma original de deflexión estática, después de que desaparezca la carga inicial?

- ¿Cómo influyen las frecuencias más altas sobre el movimiento de la viga cuando esta es desplazada de su punto de equilibrio?

La viga empotrada mostrada en la figura 31 tiene una longitud $L$ y una sección constante a lo largo de todo su eje. Está hecha de material homogéneo y la sección tiene dos ejes de simetría.

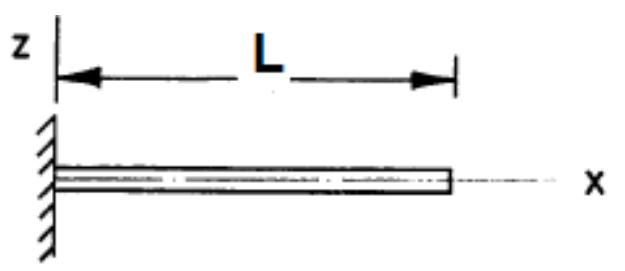

Figura 31. Esquema de viga empotrada (fuente: Sonalla, 1989) 
La viga está sujeta a varias cargas iniciales. Se estudia el movimiento vibratorio consecutivo después de desaparecer estas cargas iniciales. Se asume que todos los movimientos vibratorios tienen estrictamente lugar en la dirección $z$ con una componente de desplazamiento $w=w(x, t)$.

Las hipótesis usuales dentro de la resistencia de materiales serán utilizadas aquí para los movimientos transversales de la viga. Esto hace que el problema sea unidimensional, tanto matemáticamente como físicamente. Además, el movimiento de la viga está completamente determinado por el movimiento de su eje central, de tal forma que w debe ser definido en realidad como el desplazamiento del eje central de la viga respecto de su punto de equilibrio en posición recta.

Además, la teoría clásica de la viga de Euler - Bernoulli, que asume que el plano de la sección transversal normal al eje de la viga, se mantiene plano y normal durante la deformación, también se tomará como hipótesis de cálculo.

Por simplicidad, se supondrá que la viga resistirá vibraciones de una amplitud pequeña. Además, se supondrá que está compuesta por un material homogéneo y elástico, de sección constante. La ecuación diferencial del movimiento debido a vibraciones libres es:

$$
E I \frac{\partial^{4} w}{\partial x^{4}}+\rho A \frac{\partial^{2} w}{\partial t^{2}}=0
$$

donde "A" es el área de la sección transversal, " $\rho$ " es la masa por unidad de volumen y "El" es la rigidez a flexión, siendo "E" el módulo de Young del material e "I" el momento de inercia de la sección transversal con respecto a la fibra neutra.

Usando el procedimiento de separación de variables para resolver la ecuación anterior, la solución puede escribirse como:

$$
w(x, t)=X(x) \cdot T(t)
$$


y, por lo tanto,

$$
E I \cdot X^{I V} T+\rho A \cdot X T^{I I}=0
$$

Si se agrupan las constantes en un único término y se divide por $X \cdot T$, se obtiene la siguiente expresión:

$$
\frac{X^{I V}}{X}=-\left(\frac{\rho A}{E I}\right) \frac{T^{I I}}{T}
$$

Dado que ambas variables han sido separadas y deben ser iguales a una constante, que denominaremos $\alpha^{4}$, el problema puede reescribirse nuevamente como:

$$
\begin{aligned}
& X^{I V}-\alpha^{4} X=0 \\
& T^{I I}+\omega^{2} T=0
\end{aligned}
$$

donde:

$$
\begin{gathered}
\omega^{2}=\frac{E I \alpha^{4}}{\rho A} \\
\alpha=\frac{1}{l} \cdot \sqrt{\frac{2 \pi}{T} \cdot l^{2} \cdot \sqrt{\frac{\rho A}{E I}}}
\end{gathered}
$$

Para resolver la primera ecuación, se puede realizar una factorización de tal forma que se tendrán que resolver dos ecuaciones diferenciales de segundo orden, en vez de resolver una, de orden cuarto. Si $\psi=d / d x$, entonces:

$$
\begin{gathered}
\left(\psi^{4}-\alpha^{4}\right) X=0 \\
\left(\psi^{2}+\alpha^{2}\right)\left(\psi^{2}-\alpha^{2}\right) X=0
\end{gathered}
$$

$Y$ se tiene entonces:

$$
\begin{aligned}
& \left(\psi^{2}+\alpha^{2}\right) X=0 \\
& \left(\psi^{2}-\alpha^{2}\right) X=0
\end{aligned}
$$


Asumiendo que $\alpha^{2}$ es positivo, existe una solución en términos de funciones trigonométricas para la primera ecuación y en términos de funciones exponenciales que serán expresados mediante su forma hiperbólica, para la segunda ecuación. Por lo tanto, la solución será del tipo:

$$
X(x)=C_{1} \operatorname{sen} \alpha x+C_{2} \cos \alpha x+C_{3} \operatorname{senh} \alpha x+C_{4} \cosh \alpha x
$$

donde $C_{1}, C_{2}, C_{3}$ y $C_{4}$ son las constantes de integración. Las ecuaciones anteriores muestran que, si $\alpha^{2}$ es negativo, la solución es la misma, y que si $\alpha^{2}=0$, la solución será la trivial. Por otro lado, la solución del segundo sistema es la siguiente:

$$
T(t)=A \sin \omega t+B \cos \omega t
$$

donde A y B, son las constantes adicionales de integración. Por lo tanto, la solución general del problema será:

$$
w(x, t)=X(x) \cdot(A \operatorname{sen} \omega t+B \cos \omega t)
$$

Hay ocho constantes combinadas de integración. Todas ellas son determinadas a partir de las condiciones de contorno y de las condiciones iniciales del problema.

Las condiciones físicas que existen en los dos extremos de la viga deben ser expresadas matemáticamente como condiciones de contorno. Cuando estas condiciones son aplicadas a la ecuación diferencial se obtiene la solución al problema. Las condiciones de contorno a tener en cuenta son las siguientes:

- En el extremo empotrado, se supondrá a modo de simplificación que el giro y el movimiento serán nulos:

$$
w=0 \quad, \quad \frac{\partial w}{\partial x}=0
$$

- En el extremo libre, el momento y el cortante son nulos:

$$
\frac{\partial^{2} w}{\partial x^{2}}=0 \quad, \quad \frac{\partial^{3} w}{\partial x^{3}}=0
$$


De la primera condición de contorno se obtiene que:

$$
\begin{aligned}
& X(0)=0 \\
& X^{\prime}(0)=0
\end{aligned}
$$

Por lo tanto, sustituyendo en $\mathrm{X}$ se obtiene que:

$$
\begin{aligned}
& X(0)=C_{2}+C_{4}=0 \quad \rightarrow \quad C_{4}=-C_{2} \\
& X^{\prime}(0)=C_{1}+C_{3}=0 \quad \rightarrow \quad C_{3}=-C_{1}
\end{aligned}
$$

De la segunda condición de contorno se obtiene que:

$$
\begin{aligned}
& X^{\prime \prime}(l)=0 \\
& X^{\prime \prime \prime}(l)=0
\end{aligned}
$$

Sustituyendo se obtiene que:

$$
\begin{aligned}
& X^{\prime \prime}(l)=-C_{1} \operatorname{sen} \alpha l-C_{2} \cos \alpha l+C_{3} \operatorname{senh} \alpha l+C_{4} \cosh \alpha l=0 \\
& X^{\prime \prime \prime}(l)=-C_{1} \cos \alpha l+C_{2} \operatorname{sen} \alpha l+C_{3} \cosh \alpha l+C_{4} \operatorname{senh} \alpha l=0
\end{aligned}
$$

Si se sustituyen las constantes de acuerdo a la primera condición de contorno se obtiene que:

$$
\begin{gathered}
C_{3}(\operatorname{sen} \alpha l+\operatorname{senh} \alpha l)+C_{4}(\cos \alpha l+\cosh \alpha l)=0 \\
C_{3}(\cos \alpha l+\cosh \alpha l)+C_{4}(-\operatorname{sen} \alpha l+\operatorname{senh} \alpha l)=0
\end{gathered}
$$

Reordenando y dividiendo ambas ecuaciones por $C_{4}$, se obtiene que el ratio $C_{3} / C_{4}$ es:

$$
\frac{C_{3}}{C_{4}}=\frac{\cos \alpha l+\cosh \alpha l}{\operatorname{sen} \alpha l+\operatorname{senh} \alpha l}=\frac{\operatorname{sen} \alpha l-\operatorname{senh} \alpha l}{\cos \alpha l+\cosh \alpha l}
$$

Para obtener la autofunción, $C_{4}$, puede ser factorizado para obtener: 


$$
X(x)=C_{4}[(\cosh \alpha x-\cos \alpha x)-\gamma(\operatorname{senh} \alpha x-\operatorname{sen} \alpha x)]
$$

donde $\gamma=-C_{3} / C_{4}$ es el autovector y $C_{4}$ se determina a partir de las condiciones iniciales. Omitiendo el coeficiente $\mathrm{C}_{4}$, la ecuación anterior puede escribirse como:

$$
X_{m}(x)=\left(\cosh \alpha_{m} x-\cos \alpha_{m} x\right)-\gamma_{m}\left(\operatorname{senh} \alpha_{m} x-\operatorname{sen} \alpha_{m} x\right)
$$

donde los subíndices $\mathrm{m}$ han sido añadidos para identificar los $\mathrm{m}$ modos de forma. Por lo tanto, finalmente, se puede expresar la función solución que satisface las condiciones de contorno y resuelve la ecuación diferencial como:

$$
w(x, t)=\sum_{m=1}^{\infty} X_{m}(x) \cdot\left(A_{m} \operatorname{sen} \omega_{m} t+B_{m} \cos \omega_{m} t\right)
$$

donde $\omega_{m}$ es la frecuencia natural de cada uno de los modos de vibración con una amplitud definida por $A_{m}$ y $B_{m}$. Además, el movimiento queda representado como una superposición de todos los modos de vibración libre, vibrando cada uno con su propia frecuencia.

Las frecuencias de vibración libre y los modos vibratorios para la viga son determinados a partir de la siguiente expresión:

$$
\left[\begin{array}{cc}
\operatorname{sen} \alpha l+\operatorname{senh} \alpha l & \cos \alpha l+\cosh \alpha l \\
\cos \alpha l+\cosh \alpha l & -\operatorname{sen} \alpha l+\operatorname{senh} \alpha l
\end{array}\right]\left[\begin{array}{l}
C_{3} \\
C_{4}
\end{array}\right]=\left[\begin{array}{l}
0 \\
0
\end{array}\right]
$$

Para una solución no trivial $\left(C_{3} \neq 0\right.$ y $\left.C_{4} \neq 0\right)$, manteniendo el determinante de la matriz de coeficientes igual a cero, expandiéndolo, y utilizando algunas identidades, resulta la ecuación de la frecuencia característica:

$$
\cos \alpha_{m} l \cosh \alpha_{m} l+1=0
$$

Al resolver esta expresión se obtienen los autovalores del sistema. Posteriormente, al utilizar la ecuación que relaciona $C_{3} / C_{4}$ con $\alpha l$ se obtienen los autovectores $\gamma_{m}$. En la tabla 7 
se muestran los autovalores y autovectores correspondientes a los primeros cinco modos de vibración.

\begin{tabular}{|c|c|c|}
\hline Modos & Autovalores $\left(\boldsymbol{\alpha}_{\boldsymbol{m}} \boldsymbol{l}\right)$ & Autovectores $\left(\boldsymbol{v}_{\mathrm{m}}\right)$ \\
\hline 1 & 1,87510410 & 0,7340955 \\
\hline 2 & 4,69409113 & 1,01846644 \\
\hline 3 & 7,85475743 & 0,99922450 \\
\hline 4 & 10,99554074 & 1,00003355 \\
\hline 5 & 14,13716839 & 0,99999855 \\
\hline \multicolumn{2}{|c|}{ Tabla 7. Primeros cinco modos de vibración de una viga (fuente: elaboración propia) } \\
\hline
\end{tabular}

Posteriormente, utilizando la función que relaciona los autovalores con las frecuencias, se pueden obtener cada una de las frecuencias propias de vibración:

$$
(\alpha l)^{2}=\omega l^{2} \sqrt{\frac{\rho A}{E I}}
$$

Las amplitudes $A_{m}$ y $B_{m}$ se deben hallar mediante las condiciones iniciales. Supongamos que la viga sufre un desplazamiento inicial y una velocidad inicial que vienen determinadas por $f(x)$ y $g(x)$, respectivamente, de forma que:

$$
\begin{gathered}
w(x, 0)=f(x) \\
\frac{\partial w}{\partial t}(x, 0)=g(x)
\end{gathered}
$$

Se observa que:

$$
\begin{gathered}
f(x)=\sum_{m=1}^{\infty} B_{m} X_{m} \\
g(x)=\sum_{m=1}^{\infty} A_{m} \omega_{m} X_{m}
\end{gathered}
$$


Multiplicando ambos lados por $X_{n}$, donde $X_{n}$ representa la enésima autofunción, $e$ integramos sobre la longitud de la viga, se obtiene que:

$$
\int_{0}^{l} f(x) X_{n} d x=\int_{0}^{l} \sum_{m=1}^{\infty} B_{m} X_{m} X_{n} d x
$$

Intercambiando el orden entre el sumatorio y la integral en el lado derecho de la ecuación anterior, y considerando la ortogonalidad de $X_{n}$ y $X_{m}$, se puede observar que todos los términos de la suma infinita en el lado derecho desaparecen, excepto $m=n$.

siendo la solución:

$$
B_{m}=\frac{\int_{0}^{l} f(x) X_{m}(x) d x}{\int_{0}^{l} X_{m}^{2}(x) d x}
$$

Siguiendo el mismo proceso para $A_{m}$, se obtiene que:

$$
A_{m}=\frac{1}{\omega_{m}} \frac{\int_{0}^{l} g(x) X_{m}(x) d x}{\int_{0}^{l} X_{m}^{2}(x) d x}
$$

Los coeficientes $A_{m}$ y $B_{m}$ podrán ser obtenidos mediante el uso de las fórmulas anteriores para cada uno de los modos de vibración dado que la viga generará una respuesta a los desplazamientos y/o velocidades inicialmente impuestos.

Este planteamiento teórico del análisis dinámico de una viga empotrada fue descrito por primera vez por Sonalla (1989). No obstante, en los últimos años, estudios como el realizado por Repetto et al. (2012) han realizado una mejora del planteamiento teórico inicial, aportando ensayos de laboratorio para verificar la caracterización de este fenómeno.

Sedighi et al. (2012) también desarrollan una aportación importante en este campo al realizar un desarrollo matemático que permite realizar una aproximación no lineal de las vibraciones que sufre una viga empotrada. Djidrov et al. (2014) establecen un método para enfocar el cálculo de vibraciones de la viga empotrada hacia la detección de posibles daños que se generen en la misma. Por último, Khalkar et al. (2017) desarrolla un método de 
Modelos de predicción de esfuerzos hidrodinámicos y socavación aplicados a ingeniería offshore

análisis de vibraciones en vigas empotradas para poder caracterizar la aparición de fisuración oblicua.

Capítulo 2. Pág. 110 


\section{Fenómeno de socavación}

Por último, se va a realizar una exposición del fenómeno de la socavación, observando las aportaciones que han realizado diversos autores al campo de la ingeniería marítima, en lo referente a este proceso.

\subsection{Exposición teórica}

La socavación es un fenómeno físico que se encuentra directamente relacionado con el movimiento de los sedimentos que componen el lecho marino. La presencia de cualquier obstáculo apoyado en el fondo marino, provoca cambios en las condiciones del flujo del agua, en el entorno próximo a dicho obstáculo. Todas estas modificaciones en el flujo producen un incremento en la capacidad de transporte de sedimentos, provocando así el proceso de la socavación. En este sentido, es inmediato observar, que el fenómeno de socavación que se produce en el mar, es mucho más complejo que el desarrollado en el medio fluvial, dado que en el medio marítimo el oleaje es la acción predominante, y en los ríos, el fenómeno se produce bajo condiciones de corriente continua.

Debido a la fricción que ejerce la acción de las corrientes y el oleaje sobre el lecho marino, se produce el arrastre de partículas de sedimento, y por lo tanto la socavación del mismo (De Vos, 2008). Esta fricción se define como el esfuerzo cortante que se produce en el lecho marino.

Por un lado, existirá un esfuerzo cortante debido al oleaje y por otro un esfuerzo cortante debido a las corrientes. La suma de ambos define los esfuerzos cortantes que se producen en el fondo marino, debido a la acción del oleaje y las corrientes respectivamente.

Ha sido estudiado en detalle el cálculo del esfuerzo cortante en el fondo bajo la acción combinada del oleaje y las corrientes, llegándose a proponer diferentes modelos o teorías como las desarrolladas por Fredsoe y Deigaard (1992) o Soulsby (1997).

Soulsby (1997) Ilevó a cabo el cálculo de los esfuerzos cortantes medio $\left(\tau_{m}\right)$ y máximo $\left(\tau_{\max }\right)$ en el lecho marino. Las formulaciones desarrolladas fueron las siguientes: 


$$
\begin{gathered}
\tau_{m}\left(N / m^{2}\right)=\tau_{c}\left(1+1,2\left(\frac{\tau_{w}}{\tau_{c}+\tau_{w}}\right)^{3,2}\right) \\
\tau_{\max }\left(N / m^{2}\right)=\left(\left(\tau_{m}+\tau_{w} \cos \emptyset\right)^{2}+\left(\tau_{w} \operatorname{sen} \emptyset\right)^{2}\right)^{1 / 2}
\end{gathered}
$$

donde,

- $\tau_{c}\left(N / m^{2}\right)=$ Esfuerzo cortante en el lecho debido a una corriente continua.

$$
\tau_{c}=\frac{1}{2} \rho f_{c} U_{c}^{2}
$$

- $\rho\left(k g / \mathrm{m}^{3}\right)=$ Densidad del agua.

- $\mathrm{f}_{\mathrm{c}}=$ Coeficiente de fricción adimensional inducido por una corriente continua.

- $\mathrm{U}_{\mathrm{c}}(\mathrm{m} / \mathrm{s})=$ Velocidad media del flujo bajo condiciones de corriente continua.

- $\emptyset(\stackrel{o}{)}$ = Ángulo entre la dirección de propagación del oleaje y la dirección de la corriente.

- $\tau_{w}\left(N / m^{2}\right)=$ Esfuerzo cortante en el lecho debido al oleaje.

$$
\tau_{w}=\frac{1}{2} \rho_{w} f_{w} U_{m}^{2}
$$

- $\mathrm{f}_{\mathrm{w}}=$ Coeficiente de fricción adimensional inducido por el oleaje.

- $\mathrm{U}_{\mathrm{m}}(\mathrm{m} / \mathrm{s})=$ Velocidad media del flujo bajo condiciones de oleaje.

Como se recoge en Soulsby (1997), dicho autor propone que el cálculo del esfuerzo cortante medio en el lecho es necesario para calcular la difusión de los sedimentos, mientras que el cálculo del esfuerzo cortante máximo permite conocer el inicio del transporte de sedimentos.

Uno de los factores adimensionales claves a definir dentro del estudio del fenómeno del transporte de sedimentos es el parámetro de Shields, $\theta$. Este parámetro definido a través del esfuerzo cortante en el lecho, así como de las características del sedimento, permite establecer una relación entre las fuerzas de arrastre de la partícula y las fuerzas estabilizadoras o peso que mantienen a la partícula sólida en su lugar. 
De este modo, Shields (1936), a través de la definición de un valor crítico propuso una sencilla metodología para la determinación del inicio del transporte de sedimentos, estableciendo que este fenómeno tiene lugar cuando se supera dicho valor umbral $\left(\theta_{\mathrm{s}}>\theta_{\mathrm{cr}}\right)$ :

$$
\theta_{s}=\frac{\tau_{b}}{\left(\gamma_{s}-\gamma_{w}\right) D_{s}}
$$

donde $\gamma_{s}$ y $\gamma_{w}$, son los pesos específicos de las partículas y del agua respectivamente, y $D_{s}$, es el diámetro medio de las partículas.

Otra de las grandes aportaciones de la citada investigación fue el desarrollo del ábaco de Shields. Dicho gráfico permitió, y permite a día de hoy, calcular el inicio del movimiento de las partículas en el fondo a través del número de Reynolds, parámetro adimensional que evalúa la turbulencia y se define como:

$$
R e=\frac{U_{f l} D_{s}}{v}
$$

donde $U_{f l}$ es la velocidad característica del fluido, $D_{s}$ es el diámetro medio de las partículas, y $v$ es la viscosidad cinemática del fluido.

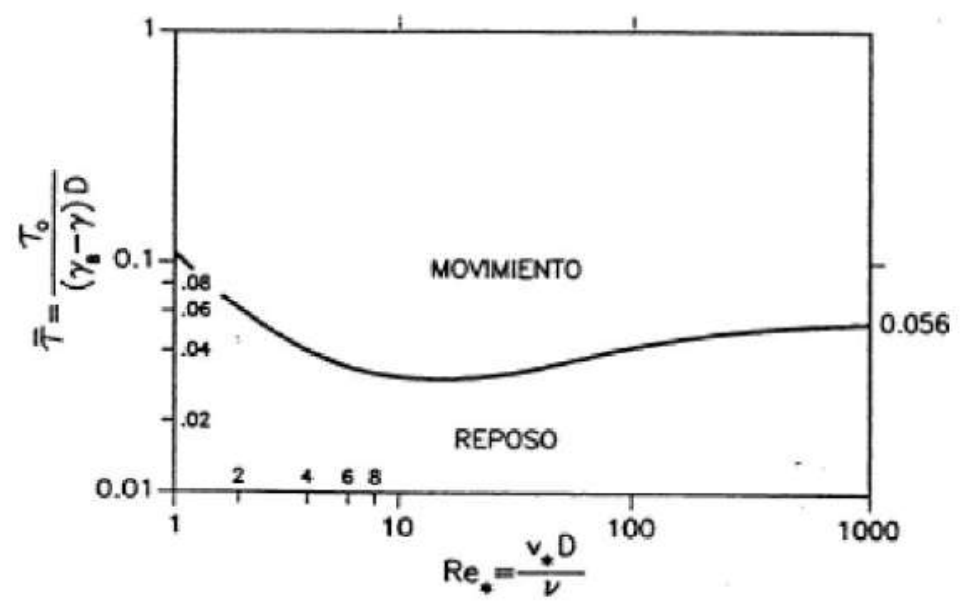

Figura 32. Ábaco de Shields, 1936 (fuente: Matutano, 2013)

Como se puede observar en la figura 32, para números de Reynolds elevados, el parámetro crítico de Shields tiende a tomar un valor constante de 0,056. 
En 1975, autores como Grant, y Komar y Miller, aseguraron que los resultados de sus investigaciones acerca del transporte de sedimentos bajo flujos no constantes (oleaje) no diferían en gran medida de los presentados por Shields en su Tesis, basados en entornos de corriente continua (De Vos, 2008).

Por su parte, Soulsby (1997), tras analizar el fenómeno del transporte de sedimentos en un entorno marino elaboró una propuesta similar a la curva original de Shields, desarrollando la figura 33 en la que el parámetro de Shields aparece en función del diámetro adimensional de las partículas.

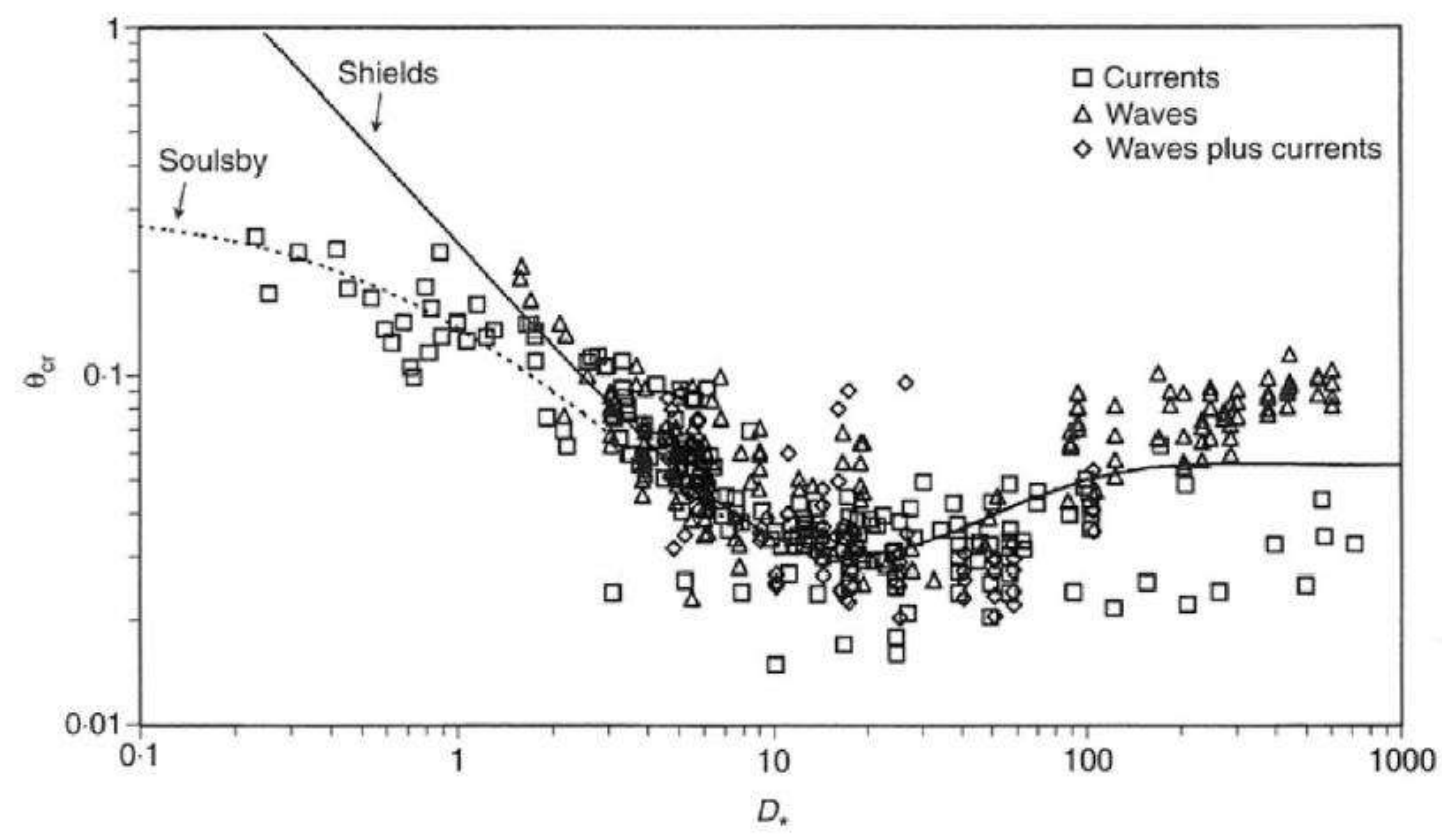

Figura 33. Curva de Soulsby (1997) como adaptación de la curva de Shields (1936). (Fuente: De Vos, 2008)

Soulsby y Whitehouse (1998) propusieron una expresión mediante la cual se podía llevar a cabo el cálculo del diámetro de las partículas en función del parámetro de Shields, aportación que en la actualidad permite calcular de manera rápida el tamaño crítico de las partículas en el inicio del movimiento. La fórmula anteriormente descrita es la siguiente:

$$
\theta_{c r}=\frac{0,3}{1+1,2 D_{*}}+0,055 \cdot\left[1-e^{0,020 \cdot D_{*}}\right]
$$

siendo:

- $D_{*}$ : Diámetro adimensional de las partículas. 


$$
D_{*}=\left[\frac{g\left(s^{\prime}-1\right)}{v^{2}}\right]^{\frac{1}{3}} \cdot D_{s}
$$

- $D_{s}$ : Diámetro medio de la partícula de sedimento.

- g: aceleración de la gravedad.

- $s^{\prime}=\frac{\rho_{s}}{\rho_{w}}$ : Densidad relativa del árido.

- $\quad v$ : Viscosidad cinemática del fluido.

- $\theta_{c r}$ : Parámetro crítico de Shields correspondiente al inicio del movimiento.

Los factores que condicionan el proceso de socavación no están completamente definidos debido a las grandes lagunas existentes en cuanto al conocimiento de la socavación inducida por la acción del oleaje, y a la complejidad inherente del problema en cuanto a las interacciones flujo-estructura.

Aunque de acuerdo a diferentes publicaciones (Sumer y Fredsoe, 2002; Whitehouse, 1998) el desarrollo de este fenómeno se encuentra influenciado en gran medida por factores como la composición del terreno, la geometría de la estructura situada sobre el lecho marino, el clima marítimo o la profundidad.

El fenómeno de la socavación es un proceso que se desarrolla a lo largo del tiempo desde el instante en el que la pila se hinca en el fondo marino.

De acuerdo a Whitehouse (1998), el desarrollo de la socavación a lo largo del tiempo puede describirse según una ley exponencial $\mathrm{S}_{\mathrm{t}}(\mathrm{t})$ en función de la profundidad de socavación en equilibrio $\left(\mathrm{S}_{\mathrm{e}}[\mathrm{m}]\right)$ y del tiempo característico $\left(\mathrm{T}_{\mathrm{car}}[\mathrm{s}]\right)$ para las condiciones hidráulicas consideradas. El tiempo característico para el desarrollo de la socavación puede definirse como el tiempo necesario que debe transcurrir para que se produzca el desarrollo del $63 \%$ de la profundidad de socavación en equilibrio.

$$
S_{t}(t)=S_{e}\left[1-e^{-\frac{t}{T_{c a r}}}\right]
$$

De acuerdo a Sumer y Fredsoe (2002), el tiempo característico también podría calcularse a través de la intersección entre la recta que define la profundidad de socavación en equilibrio 
$\left(\mathrm{S}_{\mathrm{e}}[\mathrm{m}]\right)$ y la recta tangente a la curva que representa la profundidad de socavación $(\mathrm{S}[\mathrm{m}])$ frente al tiempo $(\mathrm{t}[\mathrm{s}])$ trazada desde el origen $(\mathrm{t}=0)$, tal y como muestra la figura 34.

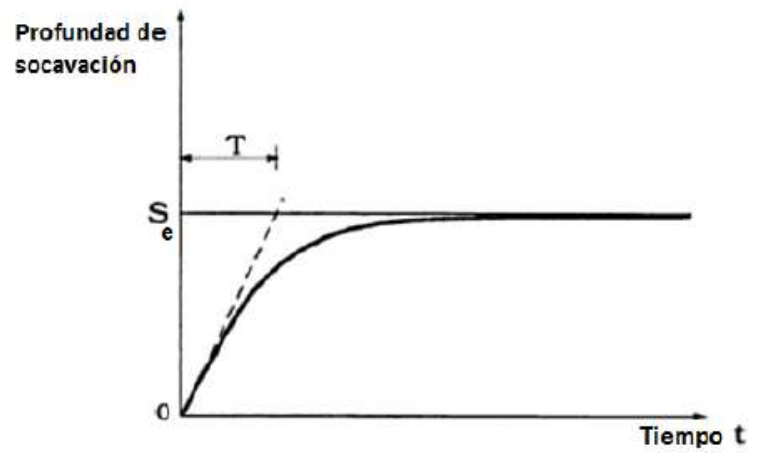

Figura 34. Representación de tiempo característico. (fuente: Sumer y Fredsoe, 2002)

\subsection{Socavación bajo condiciones de oleaje}

Numerosas investigaciones se han realizado para conocer en mayor profundidad el fenómeno de la socavación alrededor de pilas verticales, debido a la acción del oleaje.

En el año 1992, Sumer et al. publicaron los primeros datos experimentales sobre la profundidad máxima de socavación en equilibrio alrededor de una pila expuesta simplemente a oleaje. Su investigación derivó en el desarrollo de la siguiente fórmula adimensional:

$$
\frac{S_{\max }}{D}=1,3\left(1-e^{-0,03(K C-6)}\right) \quad \text { para } K C \geq 6
$$

donde $\mathrm{KC}$ es el número de Keulegan-Carpenter y $\mathrm{D}[\mathrm{m}]$ es el diámetro de la pila. Para oleaje regular, de acuerdo a Sumer et al. (1992), el número KC resulta ser:

$$
K C=\frac{\left(U_{w} \cdot T\right)}{D}
$$

donde $T[\mathrm{~s}]$ es el periodo de oleaje y $\bigcup_{\mathrm{w}}[\mathrm{m} / \mathrm{s}]$ es la velocidad orbital de las partículas en el fondo. 
De acuerdo a estos mismos autores, en la mayoría de las localizaciones donde se encuentran ubicados los parques eólicos marinos, el parámetro KC adopta valores menores a 10-15.

Zanke (1982), también ofrece una formulación similar para caracterizar dicho fenómeno:

$$
\frac{S_{\max }}{D}=K C-6
$$

En el año 2002, Sumer et al. proponen una modificación de su fórmula de 1992 para incluir el efecto de la velocidad de corriente en el lecho marino. De esta forma se puede predecir la profundidad máxima de socavación en equilibro, teniendo en cuenta de forma conjunta condiciones de oleaje y corriente.

$$
\mathrm{S}_{\max }=1,3 \cdot \mathrm{D} \cdot\left(1-e^{-\mathrm{A}(K C-\mathrm{B})}\right)
$$

donde:

$$
\begin{gathered}
A=0,03+\frac{3}{4} U_{c w}^{2,6} \\
B=6 \mathrm{e}^{-4,7 \mathrm{U}_{\mathrm{cw}}} \\
U_{c w}=\frac{U_{c}}{U_{c}+U_{m}}
\end{gathered}
$$

siendo $U_{c}$, la velocidad de la corriente y $U_{m}$ la velocidad del agua, debida al oleaje.

En 2006, Rudolph y Bos desarrollaron una nueva formulación para la predicción de la socavación alrededor de monopilas situadas sobre un lecho marino no cohesivo (arenas) bajo condiciones de oleaje y corriente.

$$
\mathrm{S}_{\max }=1,3 \cdot \mathrm{D} \cdot\left(1-e^{-\mathrm{A}_{1}\left(K C-\mathrm{B}_{1}\right)} \cdot\left(1-U_{c w}\right)^{C_{1}}\right) \quad \text { para } K C \geq B_{1}
$$

donde:

$$
A_{1}=0,03+\frac{3}{2} U_{c w}^{4}
$$




$$
\begin{gathered}
B_{1}=6 \mathrm{e}^{-5 \mathrm{U}_{\mathrm{cw}}} \\
C_{1}=0,1
\end{gathered}
$$

En 2008, Raaijmakers y Rudolph, presentaron una nueva fórmula de predicción de la socavación:

$$
S_{\text {max }}=1,5 \cdot D \cdot \tanh \left(\frac{h}{D}\right) \cdot K_{w} \cdot K_{h}
$$

donde:

$$
\begin{gathered}
K_{h}=\left(\frac{h_{p}}{h}\right)^{0,67} \\
K_{w}=1-e_{2}^{-A} \\
A_{2}=0,012 K C+0,57 K C^{1,77} U_{c w}^{3,67}
\end{gathered}
$$

donde $K_{h}$ es un factor corrector de forma, $K_{h}$ un factor de corrección de tamaño de sedimento y $h_{p}$ la altura de la pila.

No obstante, como puede observarse en la formulación anterior, la socavación va a depender fuertemente del campo de velocidades del fluido, pero no se va a ver influenciada por el campo de aceleraciones del fluido. Por ello, a lo largo de los siguientes capítulos, se va a explorar la influencia existente que ejerce el campo de aceleraciones sobre dicha formulación, teniendo además en cuenta otros parámetros importantes como el efecto de la temperatura y la salinidad.

\subsection{Caracterización del fenómeno de la socavación a partir del campo de aceleraciones}

Puede observarse que la formulación anterior que caracteriza el fenómeno de la socavación, no incluye la aceleración del fluido como variable explícita. Tras haber realizado una revisión crítica, a lo largo de este capítulo, sobre las distintas teorías que definen la caracterización de las fueras hidrodinámicas que produce el oleaje sobre una estructura, es inmediato 
observar que la aceleración tendrá una gran importancia sobre el fenómeno de socavación, para todos aquellos casos en los que dominen las fuerzas de inercia.

De acuerdo a la teoría de Morison et al. (1950) desarrollada en el punto 3.4 .1 la fuerza producida por el oleaje sobre un cilindro viene dada por la expresión:

$$
F=\frac{1}{2} \cdot \rho \cdot C_{D} \cdot D \cdot u \cdot|u|+\rho \cdot C_{M} \cdot A \cdot a_{x}=\rho \cdot \frac{D}{2} \cdot C_{D} \cdot u \cdot|u|+\rho \cdot \frac{\pi \cdot D^{2}}{4} \cdot C_{M} \cdot \frac{\partial u}{\partial t}
$$

Lo cual permite afirmar que las fuerzas en el fondo podrán ser de carácter viscoso o inercial, siendo las primeras dominadas por el campo de velocidades y las segundas por el campo de aceleraciones.

A modo de ejemplo, si se toma la teoría lineal de ondas en aguas profundas, aplicada sobre un cilindro vertical de sección circular, se puede deducir mediante la teoría de Morison et al. la relación existente entre la fuerza máxima producida en el fondo marino con la teoría de socavación, de la forma siguiente:

La determinación de la fuerza máxima, requerirá la obtención de la fuerza máxima generada por las fuerzas de arrastre y la fuerza máxima generada por las fuerzas inerciales. Para ello, es necesario conocer los valores de aceleración y velocidad máxima

$$
a_{\max }=\frac{g \cdot \pi \cdot H}{L} \cdot \frac{\cosh \frac{2 \pi(h+z)}{L}}{\cosh \frac{2 \pi h}{L}} \cdot \operatorname{sen} \theta
$$

Para el valor máximo, el $\operatorname{sen} \theta$ se hace igual a 1 , y se calcula la función hiperbólica en el lecho, es decir, para $z=-h$, resultando:

$$
a_{\text {max }}=\frac{g \cdot \pi \cdot H}{L} \cdot \frac{1}{\cosh \frac{2 \pi h}{L}} \cdot 1=\frac{g \cdot \pi \cdot H}{\frac{g T^{2}}{2 \pi} \cdot \frac{\operatorname{senh} \frac{2 \pi h}{L}}{\cosh \frac{2 \pi h}{L}}} \cdot \frac{1}{\cosh \frac{2 \pi h}{L}}
$$

operando, se obtiene: 


$$
a_{\max }=\frac{2 \pi^{2} H}{T^{2}} \cdot \frac{1}{\operatorname{senh} \frac{2 \pi h}{L}}
$$

Por lo tanto, la máxima fuerza de inercia sobre el cilindro, a la profundidad del lecho marino es:

$$
\left.F_{i}\right|_{\max }=\rho \cdot \frac{\pi D^{2}}{4} \cdot C_{M} \cdot \frac{2 \pi^{2} H}{T^{2}} \cdot \frac{1}{\operatorname{senh} \frac{2 \pi h}{L}}
$$

Para calcular la fuerza de arrastre máxima, primero es necesario obtener la velocidad máxima, como se explicaba anteriormente. La velocidad viene determinada por la siguiente expresión:

$$
u=\frac{H}{2} \cdot \frac{g \cdot T}{L} \cdot \frac{\cosh \frac{2 \pi(h+z)}{L}}{\cosh \frac{2 \pi h}{L}} \cdot \cos \theta
$$

Si maximizamos el valor del campo de velocidades para la profundidad $z=-h$, se obtiene que:

$$
u_{\text {max }}=\frac{H}{2} \cdot \frac{g \cdot T}{\frac{g T^{2}}{2 \pi} \cdot \frac{\operatorname{senh} \frac{2 \pi h}{L}}{\cosh \frac{2 \pi h}{L}}} \cdot \frac{1}{\cosh \frac{2 \pi h}{L}}
$$

Operando, se obtiene:

$$
u_{\max }=\frac{\pi H}{T} \cdot \frac{1}{\operatorname{senh} \frac{2 \pi h}{L}}
$$

La fuerza máxima de arrastre será:

$$
\left.F_{D}\right|_{\text {max }}=\rho \cdot \frac{D}{2} \cdot C_{D} \cdot \frac{\pi H}{T} \cdot \frac{1}{\operatorname{senh} \frac{2 \pi h}{L}} \cdot\left|\frac{\pi H}{T} \cdot \frac{1}{\operatorname{senh} \frac{2 \pi h}{L}}\right|
$$

No es posible obtener la fuerza máxima como suma de las dos fuerzas máximas (inercia y arrastre), dado que ambas fuerzas tienen sus máximos desfasados 90․ No obstante, si 
podemos dividir ambas fuerzas para obtener el ratio que define cuál de las dos es la que domina el fenómeno:

$$
\frac{\left.F_{i}\right|_{\text {max }}}{\left.F_{D}\right|_{\text {max }}}=\frac{\rho \cdot \frac{\pi D^{2}}{4} \cdot C_{M} \cdot \frac{2 \pi^{2} H}{T^{2}} \cdot \frac{1}{\operatorname{senh} \frac{2 \pi h}{L}}}{\rho \cdot \frac{D}{2} \cdot C_{D} \cdot \frac{\pi H}{T} \cdot \frac{1}{\operatorname{senh} \frac{2 \pi h}{L}} \cdot\left|\frac{\pi H}{T} \cdot \frac{1}{\operatorname{senh} \frac{2 \pi h}{L}}\right|}
$$

Operando se obtiene:

$$
\frac{\left.F_{i}\right|_{\max }}{\left.F_{D}\right|_{\max }}=\pi^{2} \cdot \frac{C_{M}}{C_{D}} \cdot \frac{D}{T} \cdot \frac{1}{u_{\max }}=\pi^{2} \cdot \frac{C_{M}}{C_{D}} \cdot \frac{1}{K C}
$$

Tomando valores clásicos de los coeficientes de masa $\left(C_{M}=2,5\right)$ y arrastre $\left(C_{D}=1\right)$, como se mostraba en el punto 3.4.1.2, se obtiene:

$$
\frac{\left.F_{i}\right|_{\max }}{\left.F_{D}\right|_{\max }}=\frac{25}{K C}
$$

Por tanto, como queda demostrado, el fenómeno de la socavación debido a los efectos combinados de corriente y oleaje (como asegura Sumer y Fredsoe (2002), cuando KC toma valores entre 6 y 25) se encuentra dominado tanto por las fuerzas de inercia como por las fuerzas de arrastre. De este modo, para poder comprender este fenómeno, se llevará a cabo su caracterización, definiendo en detalle no sólo el campo de velocidades alrededor de la pila, sino también el campo de aceleraciones presente al pie de la estructura.

Cabe destacar también que si $\frac{H}{D}<2$ y KC $\leq 6$ la fuerza dominante que actúa es siempre la fuerza de inercia. Mientras que si $\frac{H}{D}>20$ y $K C>60$ las fuerzas que dominan serán las de arrastre. Estas comprobaciones son muy sencillas de realizar para tener un orden de magnitud sobre las variables que dominan el sistema.

No obstante, se obtendrán las fuerzas hidrodinámicas en fondo de acuerdo a los distintos rangos de cálculo definidos en este capítulo, incluyendo en el razonamiento anterior los 
Modelos de predicción de esfuerzos hidrodinámicos y socavación aplicados a ingeniería offshore

posibles efectos derivados de la no linealidad del oleaje, o la difracción para elementos de gran diámetro. 


\section{Capítulo 3}

METODOLOGÍA DE LA INVESTIGACIÓN 



\section{Capítulo 3}

\section{METODOLOGÍA DE LA INVESTIGACIÓN}

\section{Introducción}

En este capítulo se va a proceder a realizar la exposición de la metodología seguida durante el proceso investigador, desglosando cada una de las actividades desarrolladas, y el hilo de pensamiento lógico que motivó su realización.

\section{Metodología de la investigación}

La investigación comenzó con una revisión bibliográfica en profundidad sobre los modelos de mecánica de ondas, los modelos para el cálculo de fuerzas hidrodinámicas y sobre su aplicación en el análisis dinámico de estructuras offshore sometidas a la acción del oleaje. Por otro lado, también se realizó una exposición del estado del conocimiento en lo referente al estudio del fenómeno de socavación.

En esta primera fase, se observaron las principales variables que influyen en la socavación y se enfocó el análisis en aquellos autores que aportaron modelos para estudiar este fenómeno en condiciones de oleaje y corriente continua (Sumer et al., 2002; Rudolph y Bos, 2006; Raaijmakers y Rudolph, 2008).

Gracias a dicha revisión bibliográfica y a la información recopilada, en una segunda fase, se realizó un análisis crítico sobre las formulaciones y las recomendaciones existentes hasta la fecha, para la predicción de esfuerzos hidrodinámicos en parques eólicos marinos. Esta revisión crítica puso de manifiesto que existen multitud de teorías dentro de la mecánica de ondas para resolver diferentes casos de oleaje que se presentan en la naturaleza, y que además también existen multitud de teorías para realizar el posterior cálculo de las fuerzas 
hidrodinámicas. No obstante, hoy en día, pese a la gran evolución computacional, se ha observado que una gran multitud de parques eólicos offshore han sido diseñados siguiendo un criterio simplificado, que únicamente tiene en cuenta los modelos más sencillos de cálculo, entre los que se encontrarían la teoría lineal de ondas de Airy (1845), como modelo de mecánica de ondas, y la teoría de Morison et al. (1950), como modelo para caracterizar las fuerzas hidrodinámicas. Toda esta información se plasmó en el Capítulo 2. Estado del conocimiento.

Este análisis crítico permitió, en una tercera fase, el desarrollo de los métodos numéricos de cálculo para elaborar un nuevo método avanzado y novedoso de cálculo, que permitirá obtener una solución general para todos los diferentes dominios de cálculo, resueltos actualmente de forma parcial, por las distintas teorías.

Además, durante esta fase se analizaron los modelos de cálculo estructural dinámico, que tienen en cuenta la teoría de vibraciones, para predecir el comportamiento de la estructura, es decir, los movimientos y los esfuerzos que esta sufre, y se observaron limitaciones importantes en lo referente a la correcta caracterización del comportamiento estructural que tendrá la estructura sometida al efecto del oleaje y a la introducción en el cálculo del acoplamiento existente entre oleaje y estructura. Como conclusión de este análisis, se procedió a desarrollar un modelo numérico para realizar el cálculo estructural dinámico y otro modelo para tener en cuenta el efecto del acoplamiento entre oleaje y estructura, sobre los resultados finales.

Todos estos modelos fueron integrados en un único modelo numérico y fue ejecutado mediante el desarrollo de un software especializado programado completamente en código libre, que ha sido la herramienta específica para realizar todos los análisis, con una gran potencia de cálculo y con algoritmos de alta eficiencia. Esta herramienta pretende incorporar al cálculo todos los efectos existentes en el proceso entre los cuales se encuentran los efectos no lineales, la difracción, el uso de variables que nunca han sido explícitamente incluidas en el cálculo de fuerzas hidrodinámicas, como la temperatura y la salinidad del agua, los efectos de acoplamiento entre el movimiento de la estructura y el impacto del oleaje, y la incorporación explicita del campo de aceleraciones a las 
formulaciones utilizadas para estudiar el fenómeno de la socavación. La explicación detallada de cada uno de los aspectos de este modelo numérico, fue plasmada en el Capítulo 4. Desarrollo de la herramienta.

De este modo, en una cuarta fase se realizó un análisis de los resultados obtenidos mediante la utilización del método avanzado, propuesto en la presente Tesis Doctoral, en relación con los resultados obtenidos mediante el método tradicional. Observando por primera vez en un único modelo de cálculo, la influencia conjunta de variables como la temperatura y salinidad del agua, la difracción, los efectos no lineales, los efectos vibratorios de la estructura, el acoplamiento entre estructura y oleaje, y el efecto del campo de aceleraciones sobre la socavación de la cimentación. De esta forma, se pudo realizar un análisis de sensibilidad para entender el efecto producido por cada uno de los distintos factores intervinientes.

Para ello fue necesario recopilar información sobre el clima marítimo, las características técnicas y los datos sobre socavación de diferentes parques eólicos marinos europeos.

En una última fase, tras el análisis de los resultados, se llevó a cabo la exposición de las principales conclusiones para realizar el cálculo de fuerzas hidrodinámicas en estructuras offshore, aportando las principales desviaciones que se producen en dicho cálculo, cuando se utiliza el método tradicional, en vez del método avanzado.

A continuación, se muestra en la figura 1 el esquema metodológico seguido durante el proceso de investigación de la presente Tesis Doctoral: 


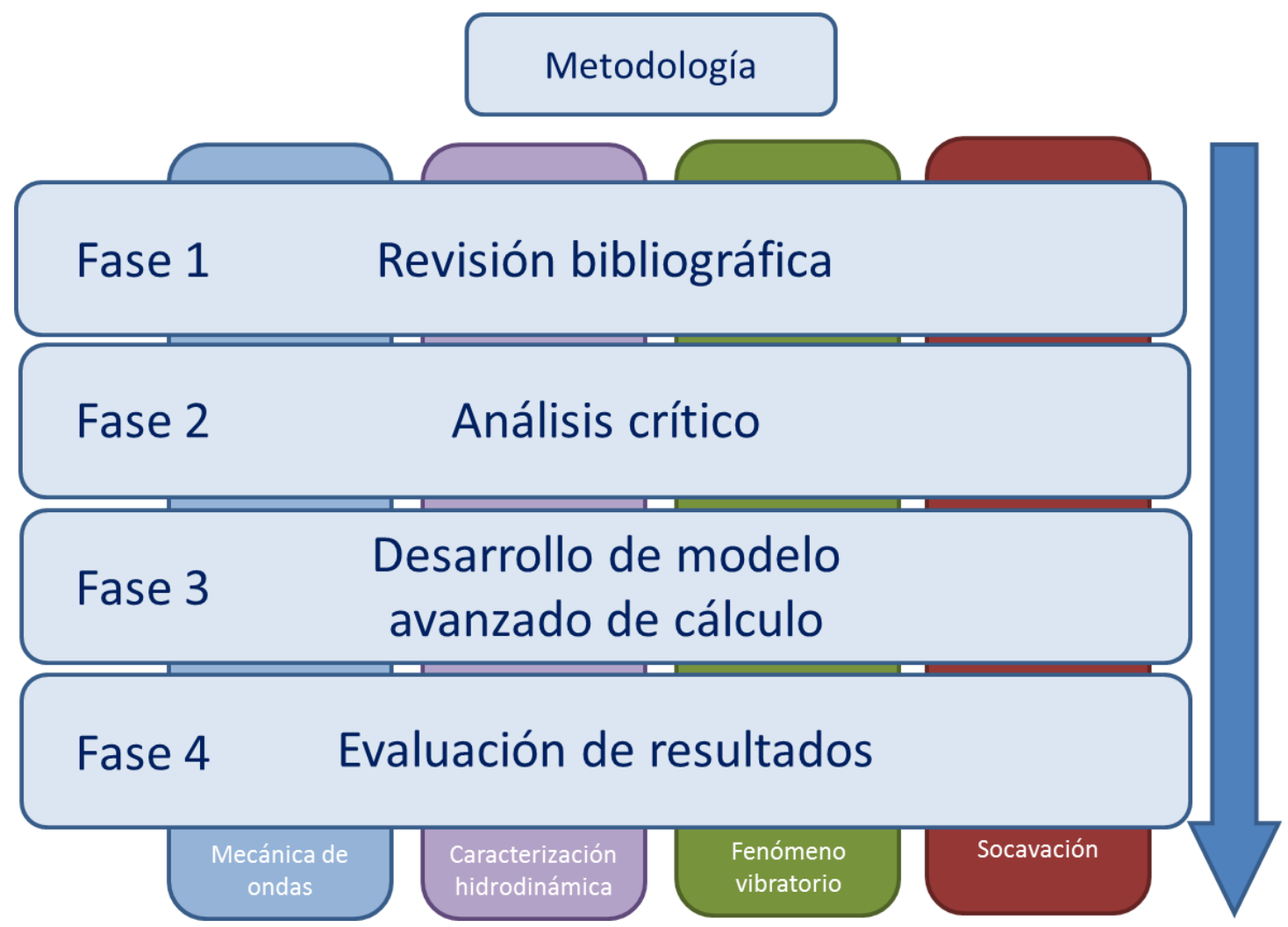

Figura 1. Metodología de la investigación (fuente: elaboración propia)

A modo de resumen, se puede concluir que los puntos que se van a desarrollar son:

- Proceso I: La mecánica de ondas será la herramienta fundamental que permitirá realizar un análisis del comportamiento del agua (velocidades y aceleraciones que experimenta el fluido), a partir de unas características iniciales (profundidad, altura y periodo del oleaje) que definirán el tipo de oleaje y, por lo tanto, la familia de formulaciones a utilizar.

- Proceso II: Las teorías de cálculo de fuerzas hidrodinámicas serán utilizadas, en segundo lugar, para calcular las presiones que genera el agua sobre un obstáculo de dimensiones definidas, permitiendo transformar los resultados obtenidos mediante la mecánica de ondas, en esfuerzos sobre una estructura.

- Procesos III y IV: La teoría de vibración estructural permitirá analizar cómo influyen las fuerzas hidrodinámicas sobre la estructura y qué movimientos se producirán en esta, pudiendo predecir los posibles problemas que sufrirá la estructura por fatiga y las sobretensiones generadas por determinados efectos de acoplamiento entre oleaje y estructura. 
- Proceso V: La introducción a las teorías de socavación, dado que al tener completamente caracterizado el comportamiento del agua, el comportamiento de la estructura y el comportamiento derivado de acoplar ambos mecanismos se podrá caracterizar el mecanismo de socavación que se producirá en la cimentación, incluyendo de forma explícita variables nunca antes incluidas en el estudio de este fenómeno como, por ejemplo, el campo de aceleraciones presente en el fluido. 


\section{Selección de casos reales}

Para la realización del análisis objeto de la presente Tesis Doctoral es necesaria la selección de datos procedentes de casos reales de parques eólicos offshore ya ejecutados en Europa, de los que se dispongan de medidas en campo.

\subsection{Fuentes de información disponible}

Durante la redacción de la presente Tesis Doctoral se ha llevado a cabo una recopilación de la información disponible sobre diferentes parques eólicos ejecutados en Europa. Las principales páginas web de las que se obtuvo dicha información son:

- www.marinedataexchange.co.uk/

- www.4coffshore.com

- Diversas webs pertenecientes a cada uno de los parques (www.nystedwindfarm.com, www.bowind.co.uk, www.hornsrev.dk, etc.)

También se utilizó la información disponible en los diferentes estudios realizados hasta la fecha como: Deutsche Wind Guard (2007), Louwersheimer (2007), Whitehouse et al. (2008), Harris et al. (2010), Carroll et al. (2010), Whitehouse et al. (2011), Bolle et al. (2012) y Matutano et al. (2015).

En la figura 2 se detalla la ubicación de los parques eólicos europeos identificados durante la primera etapa de análisis de esta investigación. Debido a la variada ubicación de los parques eólicos seleccionados, estos se encuentran situados a distintas profundidades y, por tanto, sometidos a diferentes climas marítimos. 


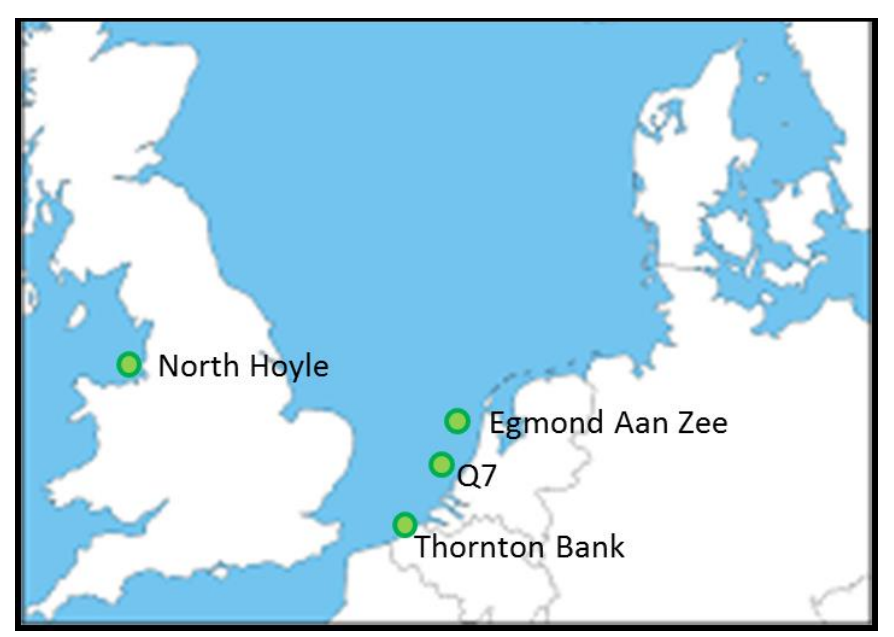

Figura 2. Localización de los parques eólicos offshore estudiados (fuente: elaboración propia)

En una segunda etapa se seleccionaron cuatro de estos parques en función de la información disponible y de su ubicación, con la intención de poder investigar así distintos emplazamientos a lo largo del territorio europeo (Reino Unido, Bélgica y Holanda).

En la tabla 1 se recogen los datos de los distintos parques eólicos offshore estudiados de acuerdo con los datos suministrados por Harris et al. (2010), Carroll et al. (2010), Whitehouse et al. (2011) y Matutano et al. (2015). En ella aparecen los nombres y los países donde se encuentran ubicados, los años en los que comenzaron la instalación de sus cimentaciones, los años de puesta en marcha y las tipologías de cimentaciones empleadas.

\begin{tabular}{|c|c|c|c|c|}
\hline Nombre & País & $\begin{array}{c}\text { Año de } \\
\text { instalación }\end{array}$ & $\begin{array}{c}\text { Año de puesta en } \\
\text { funcionamiento }\end{array}$ & $\begin{array}{c}\text { Tipo de } \\
\text { cimentación }\end{array}$ \\
\hline North Hoyle & Reino Unido & 2003 & 2004 & Monopila \\
\hline Egmond Aan Zee & Holanda & 2006 & 2006 & Monopila \\
\hline Princess Amalia Q7 & Holanda & 2006 & 2008 & Gravedad \\
\hline Thornton Bank & Bélgica & 2008 & 2009 & \\
\hline
\end{tabular}

Tabla 1. Parques eólicos instalados en Europa, estudiados en la presente Tesis (fuente: Harris et al., 2010, Carroll et al., 2010, Whitehouse et al., 2011, y Matutano et al., 2015) 
La información recopilada sobre dichos parques eólicos se encuentra resumida a continuación, clasificándose en función de los siguientes aspectos:

- Información sobre la cimentación.

- Características del clima marítimo.

La selección de los parques realizada para poder llevar a cabo el análisis estuvo condicionada por la escasa información disponible sobre diferentes parques eólicos marinos en Europa. Esta ausencia de información puede deberse en parte a la confidencialidad presente en la documentación asociada a este tipo de proyectos.

\subsection{Selección de datos}

Se ha observado que el $80,8 \%$ de los aerogeneradores instalados hasta la fecha se encuentran cimentados sobre estructuras de tipo monopila, según los datos del 2016, presentados por la EWEA. De acuerdo a esta información, la gran mayoría de parques eólicos fueron seleccionados en función de esta tipología, no obstante, también se seleccionó uno perteneciente a la tipología de cimentación por gravedad.

A continuación, se extracta en la tabla 2 la información principal para caracterizar las cimentaciones de cada uno de los parques eólicos offshore seleccionados: el tamaño del diámetro de las monopilas o de la estructura de gravedad y, la profundidad a la que se encuentran hincados en el lecho marino, salvo en el caso de la cimentación de gravedad.

\begin{tabular}{|c|c|c|c|c|}
\hline Nombre & País & $\begin{array}{c}\text { Tipo de } \\
\text { cimentación }\end{array}$ & $\begin{array}{c}\text { Diámetro } \\
\text { de la pila (m) }\end{array}$ & $\begin{array}{c}\text { Profundidad de la } \\
\text { hinca }(\mathbf{m})\end{array}$ \\
\hline Princess Amalia Q7 & Holanda & Monopila & 4 & - \\
\hline North Hoyle & Reino Unido & Monopila & 4 & 30 \\
\hline Egmond Aan Zee & Holanda & Monopila & 4,6 & Gravedad \\
\hline Thornton Bank & Bélgica & Gravedad & $6,5-17$ & \\
\hline
\end{tabular}

Tabla 2. Características de las cimentaciones (fuente: Harris et al., 2010, Carroll et al., 2010, Whitehouse et al., 2011, y 
Con respecto al clima marítimo, la tabla 3 recoge la información disponible sobre el clima marítimo de diseño en cada uno de los parques considerados a lo largo de la presente investigación, en todos ellos para un periodo de retorno de 50 años, tal y como establecen los criterios de diseño fijados por la DNV (2013) en su informe técnico OS-J101.

\begin{tabular}{|c|c|c|c|c|c|}
\hline Nombre & País & $\begin{array}{c}\text { Profundidad } \\
(\mathbf{m})\end{array}$ & $\begin{array}{c}\text { Altura de ola } \\
\text { significante, } \mathbf{H}_{\mathbf{s}} \\
(\mathbf{m})\end{array}$ & $\begin{array}{c}\text { Periodo de } \\
\text { Pico } \mathrm{T}_{\mathrm{p}}(\mathbf{s})\end{array}$ & $\begin{array}{c}\text { Velocidad de } \\
\text { corriente } \\
\text { (m/s) }\end{array}$ \\
\hline $\begin{array}{c}\text { Princess Amalia } \\
\text { Q7 }\end{array}$ & Holanda & $19-24$ & 7,7 & 9,7 & 1,30 \\
\hline North Hoyle & Reino & $7-11$ & 4,78 & 6,8 & 0,80 \\
\hline Egmond Aan Zee & Holanda & $16-22$ & 3,6 & 1,06 & 0,70 \\
\hline Thornton Bank & Bélgica & $10-24$ & 6,32 & & 1,20 \\
\hline
\end{tabular}

Tabla 3. Variables principales de clima marítimo para período de retorno de 50 años (fuente: Harris et al., 2010, Carroll et al., 2010, Whitehouse et al., 2011, y Matutano et al., 2015)

Como se puede observar en la tabla anterior, durante la investigación se ha detectado una gran carencia de información sobre las variables climáticas tenidas en cuenta durante el diseño, como la altura de ola significante $\left(H_{s}\right)$ o el periodo pico asociado $\left(T_{p}\right)$.

En este sentido, y como aconseja la experiencia, sería útil poder disponer y conocer la relación existente entre la altura de ola y periodo en cada emplazamiento:

$$
T_{p}=f\left(H_{s}\right)
$$

Relación que permitiría llevar a cabo el diseño de estas estructuras a través del conocimiento del clima marítimo, dado que $\mathrm{Hs}-\mathrm{Tp}$ son variables de estado. La caracterización de esta expresión no es objeto de este estudio, aunque sí se recogerá dentro de futuras líneas de investigación. 
Modelos de predicción de esfuerzos hidrodinámicos y socavación aplicados a ingeniería offshore

Para la realización de estos cálculos, se seleccionaron cuatro parques eólicos en Europa, de los que se tomaron las variables fundamentales de clima marítimo a tener en cuenta en el cálculo, tal y como muestra la tabla 4.

\begin{tabular}{|c|c|c|c|c|}
\hline Caso & $\begin{array}{c}\text { Princess Amalia } \\
\text { Q7 (Holanda) }\end{array}$ & $\begin{array}{c}\text { North Hoyle } \\
\text { (Reino Unido) }\end{array}$ & $\begin{array}{c}\text { Egmond Aan Zee } \\
\text { (Holanda) }\end{array}$ & $\begin{array}{c}\text { Thornton Bank } \\
\text { (Bélgica) }\end{array}$ \\
\hline $\begin{array}{c}\text { Altura de ola } \\
\text { significativa }\end{array}$ & $7,7 \mathrm{~m}$ & $4,78 \mathrm{~m}$ & $3,6 \mathrm{~m}$ & $16,0 \mathrm{~m}$ \\
\hline Profundidad & $24,0 \mathrm{~m}$ & $10,0 \mathrm{~m}$ & $22,0 \mathrm{~m}$ & $11,06 \mathrm{~s}$ \\
\hline Periodo de pico & $9,7 \mathrm{~s}$ & $6,8 \mathrm{~s}$ & $18,5 \mathrm{~s}$ & $1,20 \mathrm{~m} / \mathrm{s}$ \\
\hline Velocidad de & $1,30 \mathrm{~m} / \mathrm{s}$ & $0,80 \mathrm{~m} / \mathrm{s}$ & $0,70 \mathrm{~m} / \mathrm{s}$ & \\
\hline corriente & & & & \\
\hline
\end{tabular}

Tabla 4. Parques eólicos offshore analizados (fuente: Harris et al., 2010, Carroll et al., 2010, Whitehouse et al., 2011, y

Matutano et al., 2015) 


\section{Casos de estudio propuestos}

En este apartado, se procederá a enumerar los distintos objetivos comparativos que se van a analizar en cada uno de los procesos en relación con su proceso homólogo dentro del proceso tradicional de análisis explicado en el punto 2.1 del capítulo IV.

Además, se adjuntará la descripción de las variables fundamentales de los distintos casos a analizar, cuyos resultados se adjuntan en el capítulo V.

Cada caso de estudio será calculado mediante el método avanzado propuesto por la presente Tesis Doctoral, teniendo en cuenta todos los efectos de no linealidad, difracción, análisis dinámico de la estructura, efectos de acoplamiento entre el oleaje y la estructura, etc. Por otro lado, dicho caso de estudio será calculado nuevamente según el método tradicional, es decir, utilizando la teoría lineal de ondas, la fórmula lineal de flujo separado de Morison et al. (1950) y considerando un cálculo estático de la estructura, sin tener en cuenta los efectos de acoplamiento entre el oleaje y la estructura.

Por ello, el número total de casos a estudiar vendrá determinado por la combinatoria de los casos particulares definidos para los distintos procesos, tal y como muestra la tabla 5 :

\begin{tabular}{|c|c|}
\hline Proceso & Número de casos \\
\hline 1. Mecánica de ondas & 8 \\
\hline 2. Cálculo de fuerzas hidrodinámicas & 8 \\
\hline 3. Análisis dinámico & 2 \\
\hline 4. Acoplamiento & 2 \\
\hline Sub-total & $8 \cdot 8 \cdot 2 \cdot 2=256$ \\
\hline 5. Comportamiento de la cimentación & 16 \\
\hline Total & 272 \\
\hline
\end{tabular}

En los siguientes apartados se definen los distintos casos de estudio que se engloban dentro de cada uno de los procesos de cálculo. 
La comparación de los resultados aportados por el método avanzado con respecto a los generados por el método tradicional, será el foco central de análisis de la presente Tesis Doctoral.

\subsection{Proceso I: Mecánica de ondas}

Dentro del primer proceso de cálculo dedicado a la mecánica de ondas, los casos de análisis que se van a desarrollar son los siguientes:

- Caso A: resuelto mediante Stokes de 3o Orden

- Caso B: resuelto mediante Stokes de 5o Orden

- Caso C: resuelto mediante onda Cnoidal de 20 Orden

- Caso D: pertenecerá al dominio de no-linealidad total para ser resuelta mediante la Stream Function de Dean.

A continuación, se muestran en la tabla 6 las variables consideradas para caracterizar el oleaje en cada uno de los casos, obtenidas mediante los estudios realizados por Deutsche Wind Guard (2007), Louwersheimer (2007), Whitehouse et al. (2008), Harris et al. (2010), Carroll et al. (2010) y Whitehouse et al. (2011) y Matutano et al. (2015):

\begin{tabular}{|c|c|c|c|c|}
\hline Caso & A & B & C & D \\
\hline Nombre & Princess Amalia Q7 & North Hoyle & Egmond Aan Zee & Thornton Bank \\
\hline $\mathrm{H}_{\mathrm{s}}$ & $7,7 \mathrm{~m}$ & $4,78 \mathrm{~m}$ & $3,6 \mathrm{~m}$ & $6,32 \mathrm{~m}$ \\
\hline $\mathrm{h}$ & $24,0 \mathrm{~m}$ & $10,0 \mathrm{~m}$ & $22,0 \mathrm{~m}$ & $16,0 \mathrm{~m}$ \\
\hline $\mathrm{T}_{\mathrm{p}}$ & $9,7 \mathrm{~s}$ & $6,8 \mathrm{~s}$ & $18,5 \mathrm{~s}$ & $11,06 \mathrm{~s}$ \\
\hline
\end{tabular}

Tabla 6. Variables principales de caracterización del oleaje (fuente: elaboración propia a partir de los estudios referenciados en el párrafo anterior)

Se utilizará la altura de ola significativa $H_{s}$ y el periodo de pico asociado $T_{p}$, en régimen extremal para un periodo de retorno de 50 años, tal y como establecen los criterios de diseño fijados por la DNV (2013) en su informe técnico OS-J101.

El caso A se corresponde con los datos de oleaje correspondientes al parque eólico offshore Princess Amalia Q7 en Holanda, el caso B con los de North Hoyle en Reino Unido, el caso $\mathrm{C}$ con los de Egmond Aan Zee en Holanda y, finalmente, los del caso D con 
Thornton Bank en Bélgica. No se han podido encontrar datos acerca del periodo de pico existente para el caso del parque eólico de Egmond Aan Zee, por ello, ha sido necesario suponerlo. Dicho periodo de pico ha sido supuesto de forma que permita el uso de la teoría cnoidal, con la intención de que los resultados obtenidos de este estudio tengan un amplio espectro de situaciones posibles, al haber utilizado cuatro teorías diferentes de mecánica de ondas.

Cada uno de los casos mostrados en la tabla 6 será calculado, por un lado, teniendo en cuenta la teoría de ondas que corresponda de acuerdo a la clasificación de Le Méhauté (1976) y, por otro, según la teoría lineal de ondas. De esta forma, se podrá proceder a realizar un análisis comparativo, entre los resultados que contemplan mayores niveles de no linealidad y los resultados aportados por la teoría de Airy.

Los principales objetivos a analizar en este primer proceso serán los siguientes:

- Comparación de la variación del campo de velocidades y aceleraciones entre la teoría lineal y el resto de teorías.

- Análisis de las principales frecuencias generadas por el oleaje.

- Análisis sobre la variación de la amortiguación de la velocidad y la aceleración con la profundidad según las distintas teorías.

Además, se generarán gráficos de flujo para determinar cuál es el movimiento de cada una de las partículas de agua, tal y como se muestra en las figuras 3 y 4 :

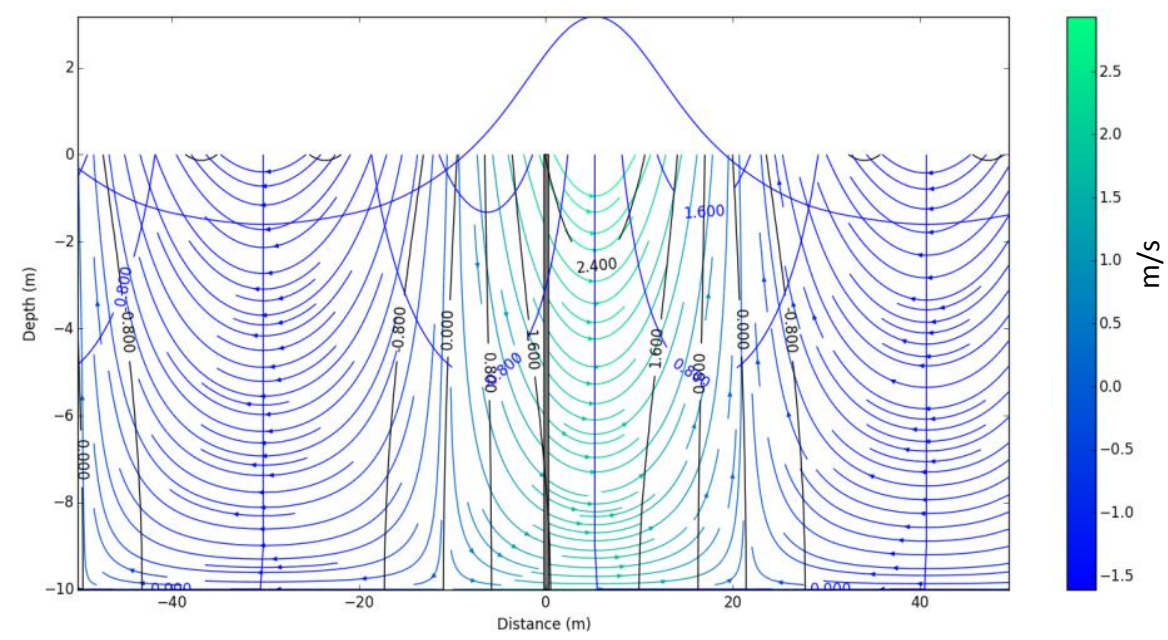

Figura 3. Ejemplo del flujo de velocidades calculado por el software (fuente: elaboración propia) 


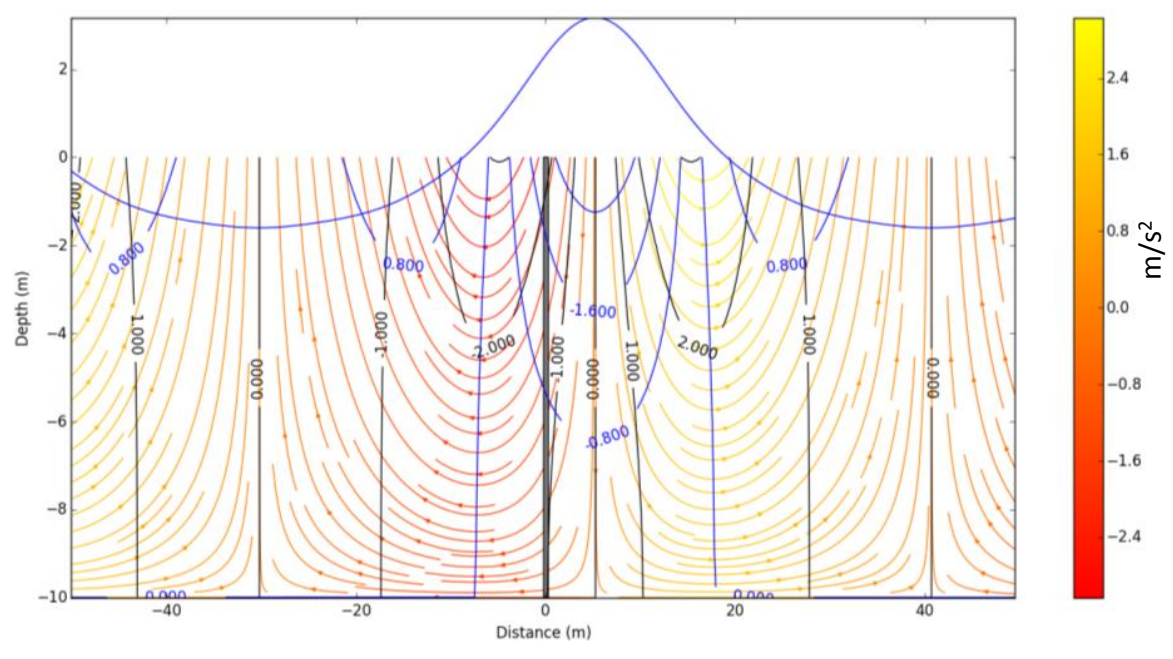

Figura 4. Ejemplo del flujo de aceleraciones calculado por el software (fuente: elaboración propia)

En los gráficos anteriores se pueden observar las flechas que muestran la dirección que siguen las velocidades y las aceleraciones de las partículas de agua para cada punto del espacio en un instante de tiempo determinado. Además, se puede observar que dichas flechas siguen un código de colores que especifica el valor de la velocidad o aceleración que tiene el fluido en un determinado punto. Este valor puede ser positivo o negativo. El valor negativo implica que la velocidad o la aceleración sigue, en dicho punto, un sentido inverso al que indica la flecha más cercana.

En el gráfico también se muestran líneas redondeadas azules y negras que unen los puntos de igual velocidad horizontal y vertical, respectivamente.

Estas figuras presentan una anomalía que es necesario explicar en detalle para evitar inducir a error. Como puede observarse el perfil de la ola se encuentra vacío en su cresta y relleno en su valle. Este hecho se debe únicamente a una limitación establecida por el propio código de programación que impide el poder realizar una imagen de salida que evite estos errores. No obstante, dichos efectos no afectan a los resultados del cálculo, dado que a nivel numérico si se ha podido enmendar dicho error con facilidad. 


\subsection{Proceso II: Cálculo de las fuerzas hidrodinámicas}

Dentro de este segundo proceso de cálculo se van a estudiar 4 diámetros de pilote diferentes para cada uno de los parques estudiados, para observar la influencia del diámetro sobre los resultados. Los diámetros a utilizar serán los siguientes:

- Caso 1: $\mathrm{D}=0,5 \mathrm{~m}$

- Caso 2: $\mathrm{D}=4,0 \mathrm{~m}$

- Caso 3: $\mathrm{D}=8,0 \mathrm{~m}$

- Caso 4: $\mathrm{D}=16,0 \mathrm{~m}$

En este proceso se comprobarán nuevamente los resultados obtenidos mediante el cálculo del método avanzado con los obtenidos mediante el denominado método tradicional.

Para la aplicación del método avanzado, se calculará cada uno de los casos definidos para este proceso mediante cada uno de los estados del mar calculados en el proceso I y se resolverá cada caso por medio de la teoría hidrodinámica que corresponda, de acuerdo a la clasificación mostrada en el punto 2.2.2 del capítulo IV.

Para la aplicación del método tradicional, se utilizarán los resultados de los casos analizados en el proceso anterior, que fueran resueltos mediante la teoría lineal de ondas y se aplicará la formulación correspondiente de la teoría lineal de flujo separado (fórmula de Morison et al.), para hallar las fuerzas hidrodinámicas.

Además, se analizará la influencia de la temperatura y de la salinidad del agua sobre las cargas hidrodinámicas calculadas mediante ambos métodos. Para ello, tanto en el método tradicional, como en el método avanzado, se compararán los valores de las cargas hidrodinámicas que genera un oleaje a temperatura de $1^{\circ} \mathrm{C}$ y salinidad del $0 \%$ con respecto a las generadas por un oleaje a temperatura de $22^{\circ} \mathrm{C}$ y salinidad del $35 \%$.

Los principales objetivos de este segundo proceso de cálculo, serán los siguientes:

- Comprobación de las fuerzas hidrodinámicas generadas por el método avanzado, con respecto a las generadas por el método tradicional para cada teoría de mecánica de ondas. 
- Análisis de la influencia de la temperatura y la salinidad sobre la distribución y magnitud de las cargas para cada teoría de mecánica de ondas.

- Análisis de la influencia de los efectos no lineales sobre la distribución y magnitud de las cargas, para cada teoría de mecánica de ondas.

- Análisis de los puntos que reciben las mayores cargas, para cada teoría de mecánica de ondas.

- Amortiguación de las distintas fuerzas con la profundidad.

- Variación del número de Reynolds.

- Variación del número de Keulegan-Carpenter.

- Variación del parámetro de Sarpkaya.

- Análisis de la influencia de los efectos de difracción sobre la distribución y magnitud de las cargas, para cada teoría de mecánica de ondas.

Además, se generarán gráficos de carga para determinar cuál es la magnitud y distribución de las fuerzas hidrodinámicas a lo largo de la pila, tal y como muestra la figura 5.

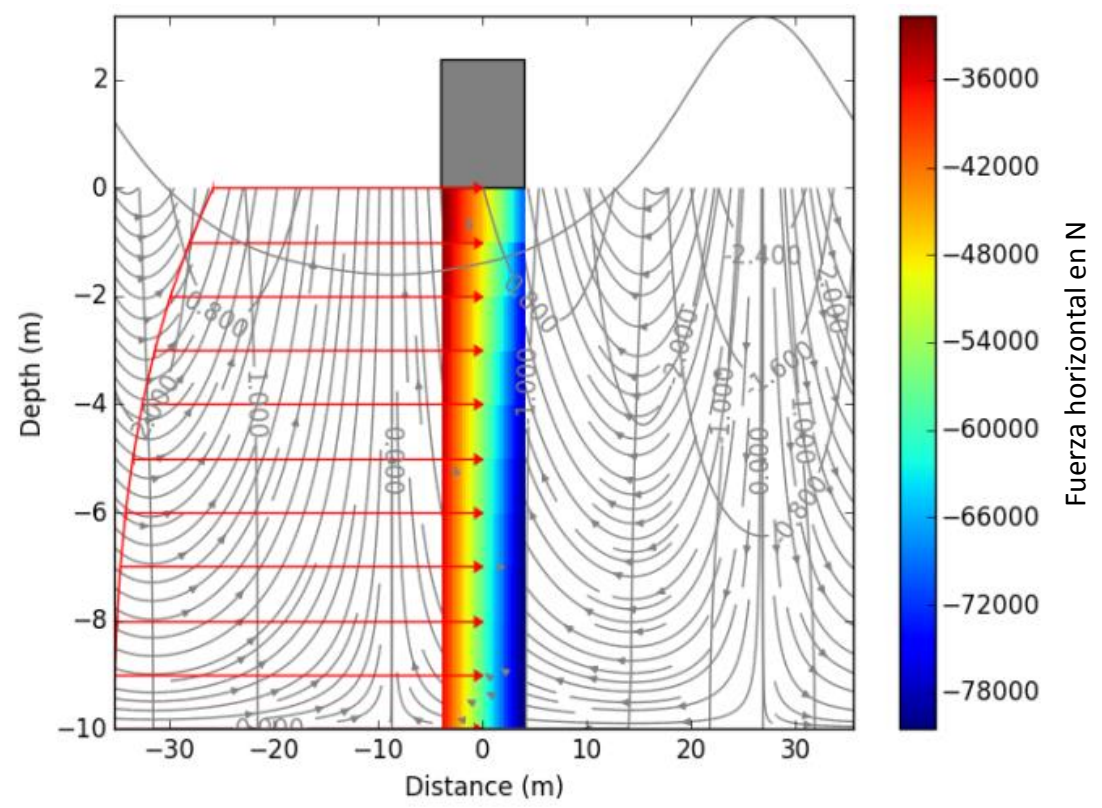

Figura 5. Distribución de fuerzas y localización de puntos pésimos (fuente: elaboración propia)

En la figura anterior se puede observar la ley de fuerzas que se genera sobre la estructura. Además, se incluye en escala de grises el diagrama de velocidades de las partículas del fluido y el perfil de la ola. 


\subsection{Proceso III: Análisis dinámico}

En este tercer proceso, se incluirán las variables principales que definirán el comportamiento estructural de la pila:

- Longitud de la pila(l).

- Área de la sección (A).

- Densidad del elemento estructural ( $\left.\rho_{\text {est }}\right)$.

- Momento de inercia de la sección (I).

- Momento estático de la sección $\left(m_{s}\right)$.

- Módulo de elasticidad de Young (E).

A estas variables se añadirá la variable diámetro, que ya fue incluida en el proceso anterior de cálculo.

En este proceso, en primer lugar, se hará el cálculo dinámico-vibratorio de la sección sometida a los distintos estados de carga del proceso anterior. En segundo lugar, se repetirá el cálculo para cada uno de los casos evaluados mediante el método tradicional, pero aplicando únicamente las fórmulas estáticas de resistencia de materiales.

De esta forma podrá comprobarse la influencia que tiene el análisis dinámico teniendo en cuenta los primeros 25 modos de vibración, sobre los estados tensionales que sufrirá el pilar frente a los resultados arrojados si se considera únicamente el cálculo estático.

Los principales objetivos de este tercer proceso son:

- Análisis de los esfuerzos generados en la cimentación entre los casos de cálculo tradicional y aquellos resueltos mediante el modelo optimizado.

- Análisis de movimientos que sufre la estructura comparando los generados por el modelo estático con los del modelo dinámico.

Además, el software generará los gráficos de movimiento de cada uno de los puntos de la viga resueltos mediante el método tradicional y el optimizado, tal y como muestra la figura 6: 

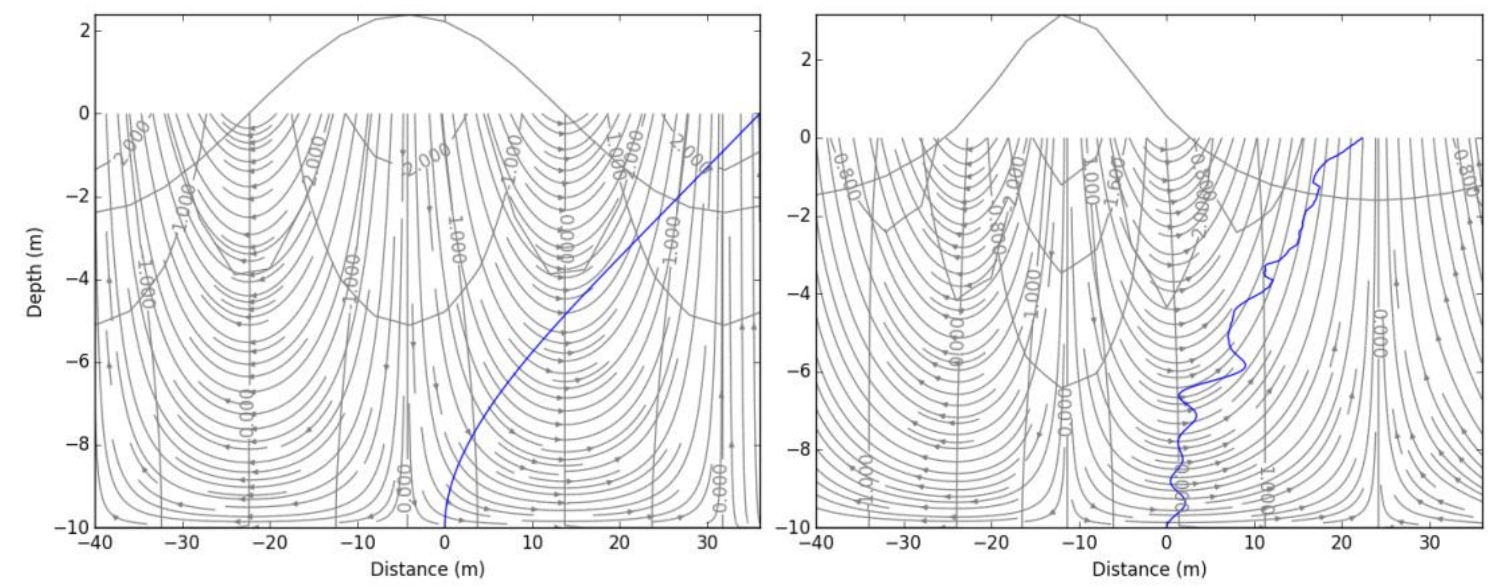

Figura 6. Movimiento de la estructura según el método tradicional con análisis estático y según el método avanzado con análisis

dinámico (fuente: elaboración propia)

Esta figura presenta otra anomalía que es necesario explicar en detalle para evitar inducir a error o confusión. El orden de magnitud de los movimientos horizontales mostrados para el fuste vertical aparentemente es de decenas de metros. Este hecho se debe a que se han escalado los movimientos horizontales que experimenta la estructura para poder visualizarlos sobre la propia imagen que muestra el perfil de la ola, aportando así una mejor visión del fenómeno al mostrar ambos fenómenos al mismo tiempo.

Este escalado no inducirá a confusión dado que se adjuntan los valores reales de cálculo del máximo desplazamiento horizontal de la estructura, en las tablas que se adjuntan debajo de cada una de las imágenes correspondientes a los distintos casos de cálculo.

\subsection{Proceso IV: Acoplamiento procesos II y III}

El proceso IV no incluirá ninguna variable adicional al cálculo. Este paso de cálculo está enfocado a aumentar la exactitud de los cálculos dinámicos al permitir introducir el acoplamiento entre el oleaje y la estructura en la herramienta de cálculo desarrollada mediante la presente Tesis Doctoral.

Se observará la diferencia entre los resultados obtenidos por el Proceso IV con aquellos que se obtuvieron en el proceso anterior, con la intención de poder determinar la influencia que supone la consideración del acoplamiento oleaje-estructura. 
Los objetivos perseguidos en este proceso son:

- Determinar la influencia que tiene el acoplamiento de la estructura con el oleaje, sobre el estado tensional de la misma.

- Determinar la influencia que tiene el acoplamiento de la estructura con el oleaje, sobre los desplazamientos que sufre la estructura.

- Determinar la influencia del acoplamiento frente a la aparición de posibles efectos de resonancia a ciertas frecuencias.

- Determinar cómo varía esta influencia dependiendo del diámetro y de la teoría hidrodinámica aplicada.

- Determinar cómo varía este acoplamiento con respecto a la teoría de ondas utilizada para caracterizar el oleaje.

Las salidas gráficas de este módulo serán similares a la generadas por el módulo de cálculo que realiza el proceso III.

\subsection{Proceso V: Comportamiento de la cimentación.}

Por último, en este proceso se a aplicar la fórmula modificada desarrollada mediante la presente Tesis Doctoral, que tiene como base la fórmula de Sumer et al. (2002), para definir las máximas profundidades de socavación estable, en cada uno de los casos estudiados.

Para ello, se van a utilizar los datos del campo de velocidades y aceleraciones que han sido calculados a lo largo de todo el proceso de análisis.

Estos resultados serán comparados con las mediciones obtenidas en campo por Deutsche Wind Guard (2007), Louwersheimer (2007), Whitehouse et al. (2008), Harris et al. (2010), Carroll et al. (2010), Whitehouse et al. (2011) y Matutano et al. (2015).

Los objetivos de este proceso son:

- Influencia de la teoría de ondas a aplicar, sobre la nueva formulación desarrollada para estimar la socavación. 
Modelos de predicción de esfuerzos hidrodinámicos y socavación aplicados a ingeniería offshore

- Influencia del diámetro, sobre la nueva formulación desarrollada para estimar la socavación.

- Influencia del campo de aceleraciones.

Este módulo no presentará ninguna salida gráfica y únicamente se limitará a proporcionar el resultado numérico. 


\section{Capítulo 4}

\section{DESARROLLO DE LA HERRAMIENTA}





\section{Capítulo 4}

\section{DESARROLLO DE LA HERRAMIENTA}

\section{Introducción}

En este capítulo se va a proceder a realizar la presentación en detalle de la herramienta de cálculo propuesta y desarrollada mediante la presente Tesis Doctoral, que se va a aplicar en los modelos de predicción de esfuerzos hidrodinámicos y de predicción de la socavación aplicados a ingeniería offshore. En el capítulo 2 se han definido en detalle los distintos mecanismos que influyen sobre la caracterización del impacto de una ola sobre un fuste vertical, en el mar, y sobre la afección que produce la socavación sobre la cimentación del mismo.

La línea principal del presente análisis pretende, por un lado, resolver de forma precisa el cálculo de los cuatro pilares fundamentales que definen el problema, y por otro, realizar una conexión entre estos cuatro mecanismos, que permita mediante un cálculo iterativo, que interactúen entre ellos y converjan hacia una solución que refleje mejor la realidad que los actuales modelos, que sectorizan el problema y tratan cada mecanismo de forma independiente. Tal y como muestra la figura 1, estos cuatro pilares son:

- La mecánica de ondas aplicada al oleaje.

- Cálculo de fuerzas hidrodinámicas.

- Cálculo de vibraciones aplicado a estructuras.

- Fenómeno de socavación.

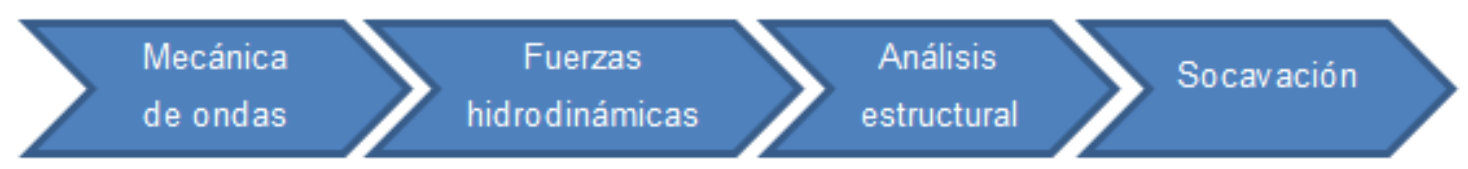

Figura 1. Los cuatro pilares que definen el problema estudiado (fuente: elaboración propia) 


\section{Esquema general del proceso}

En este apartado se procede a describir la metodología general para la predicción de esfuerzos hidrodinámicos y socavación aplicados a elementos estructurales offshore.

\subsection{Proceso tradicional de análisis}

Dada la complejidad del problema, a lo largo de las últimas décadas se ha tendido a utilizar importantes simplificaciones e hipótesis, para facilitar la caracterización de las fuerzas producidas por el oleaje al impactar este sobre una estructura.

Tradicionalmente se ha utilizado la teoría lineal de ondas (Airy, 1845), como formulación de base, para obtener el campo de velocidades y aceleraciones, para unas condiciones de oleaje dadas. Esta teoría permite caracterizar el oleaje y los movimientos que este genera, de una forma sencilla y compacta, sin necesidad de incurrir en sofisticados algoritmos que encuentren algún tipo de convergencia y sin un gran coste computacional. En la figura 2 se muestra la órbita que sigue una partícula de agua en aguas someras y en profundidades indefinidas, que respectivamente será elíptica o circular según los cálculos realizados mediante la teoría lineal de ondas.
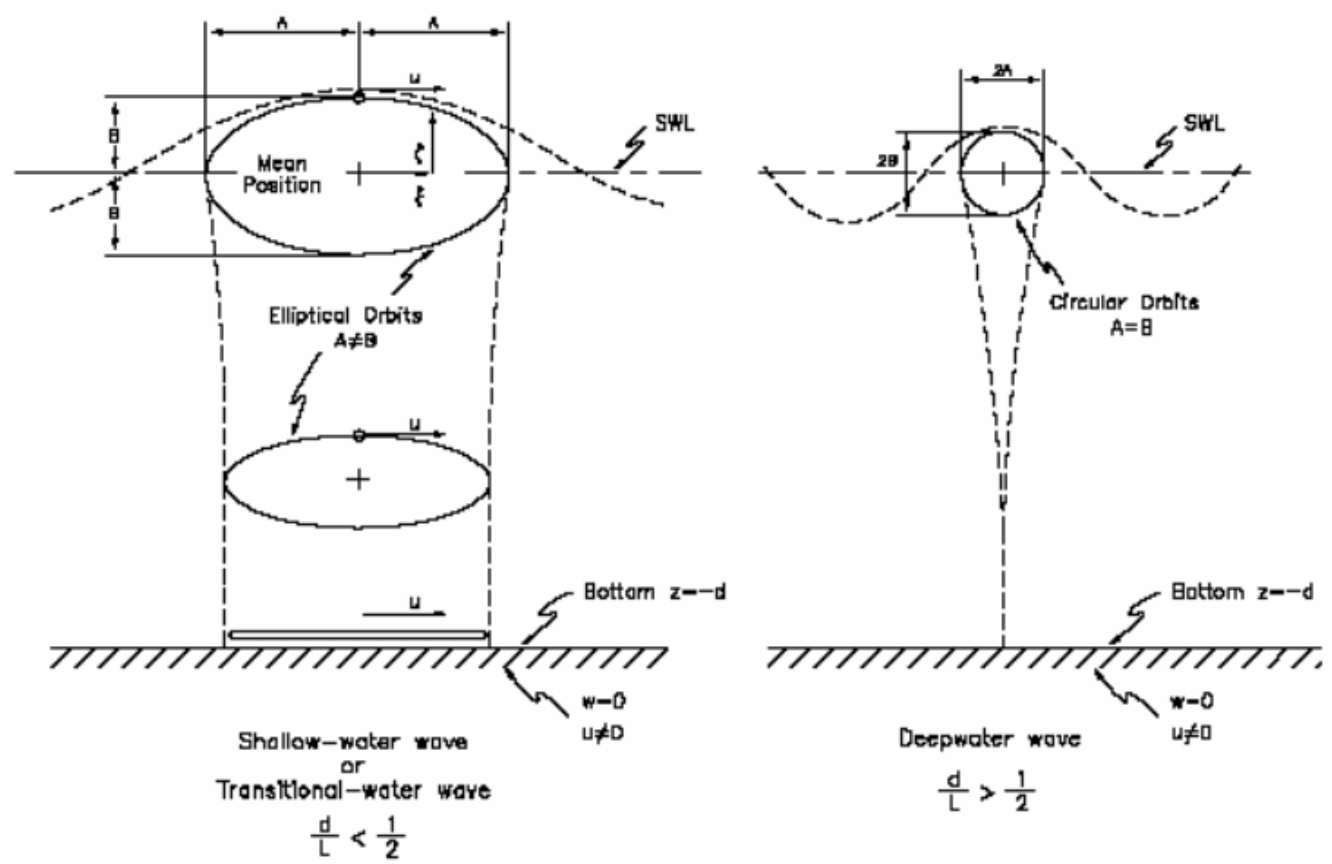

Figura 2. Movimiento de la partículas según la Teoría de Airy (fuente: Shore Protection Manual Vol. 1 p. 2-17) 
El uso de la teoría lineal de ondas genera un importante sesgo en los resultados, dado que no incluye en los cálculos los efectos no lineales que afectan al movimiento de las partículas de agua y, por lo tanto, a sus velocidades y aceleraciones, siendo válido su uso únicamente para la resolución de casos lineales. En la figura 3 se observan los distintos perfiles de elevación de la lámina libre de agua de acuerdo con las distintas teorías.

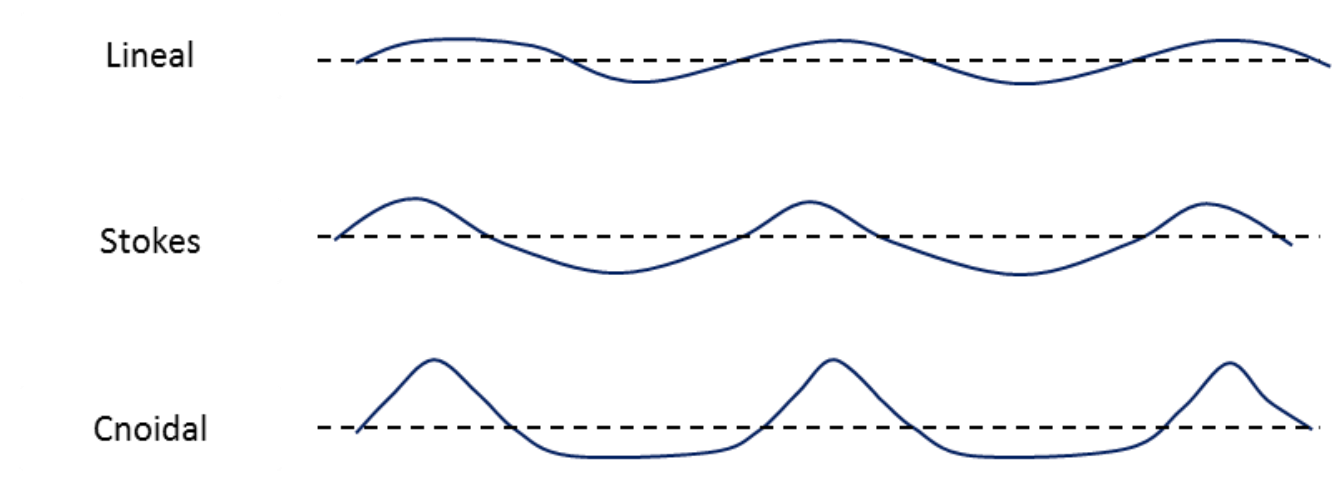

Figura 3. Perfiles de oleaje según distintas teorías de cálculo (fuente: elaboración propia)

Estos efectos no lineales tendrán una gran influencia en la distribución de presiones a lo largo del eje vertical, lo que podrá generar una desviación adicional en los resultados con respecto a los esfuerzos producidos sobre la cimentación. En la figura 4 se muestra de forma esquemática la distribución de fuerzas hidrodinámicas que se generan sobre una pila al paso de una ola.

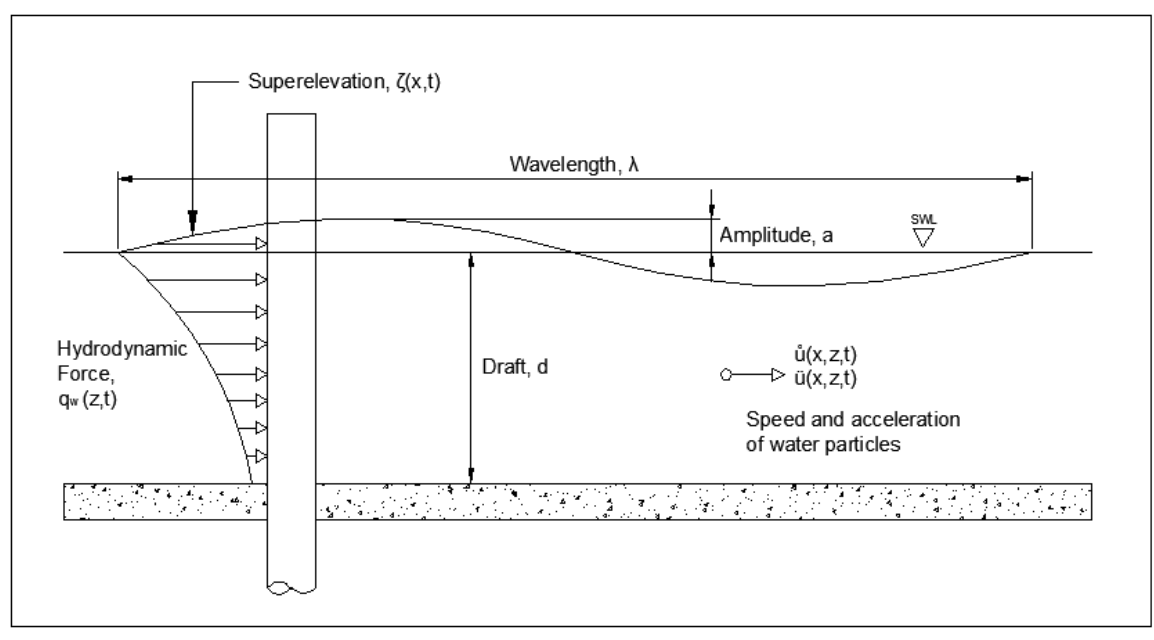

Figura 4. Esfuerzos hidrodinámicos producidos sobre la pila (fuente: elaboración propia) 
Por lo tanto, puede afirmarse que la teoría lineal de ondas, o teoría de Airy, solo deberá ser aplicada para aquellas partes del dominio de cálculo que así lo requieren de acuerdo con la clasificación de Le Méhauté (1976), como se observa en la figura 5.

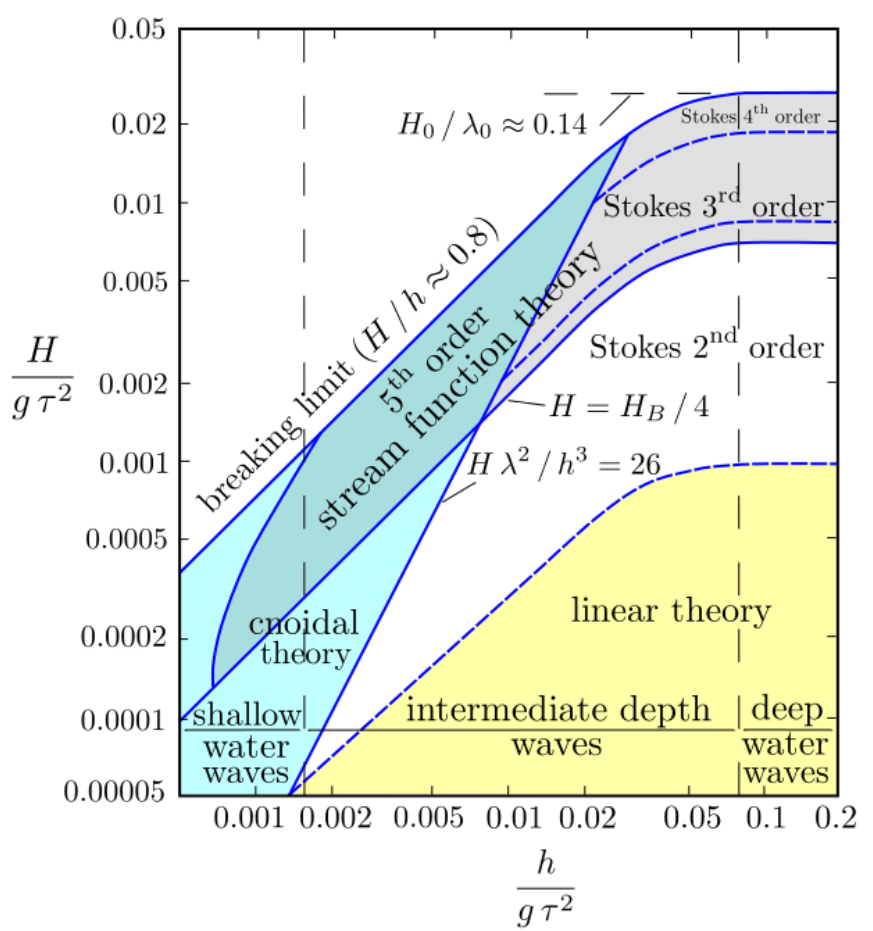

Figura 5. Clasificación de teorías de mecánica de ondas aplicadas a oleaje (fuente: Le Méhauté, 1976)

Por otro lado, la teoría utilizada para la obtención de las fuerzas hidrodinámicas en pilotes ha sido tradicionalmente la teoría de Morison et al (1950). Esto se debe también a su simplicidad y fácil aplicación, dado que dicha fórmula relaciona de forma directa el campo de aceleraciones y velocidades con las fuerzas hidrodinámicas generadas sobre la estructura.

$$
F=\frac{1}{2} \cdot \rho \cdot C_{D} \cdot D \cdot u \cdot|u|+\rho \cdot C_{M} \cdot A \cdot a_{x}=\rho \cdot \frac{D}{2} \cdot C_{D} \cdot u \cdot|u|+\rho \cdot \frac{\pi \cdot D^{2}}{4} \cdot C_{M} \cdot \frac{\partial u}{\partial t}
$$

No obstante, también se estará generando un importante error en los resultados de cálculo, dado que la aplicación de dicha fórmula no tiene en cuenta el potencial de difracción. Esto implica que la estructura no tiene ninguna influencia sobre el flujo de oleaje incidente, lo cual restringe el uso de esta teoría a estructuras esbeltas con un diámetro pequeño. En la figura 6 se muestran las distintas líneas de flujo que se generan 
alrededor de un obstáculo de pequeño diámetro en el que no se están produciendo efectos de difracción.

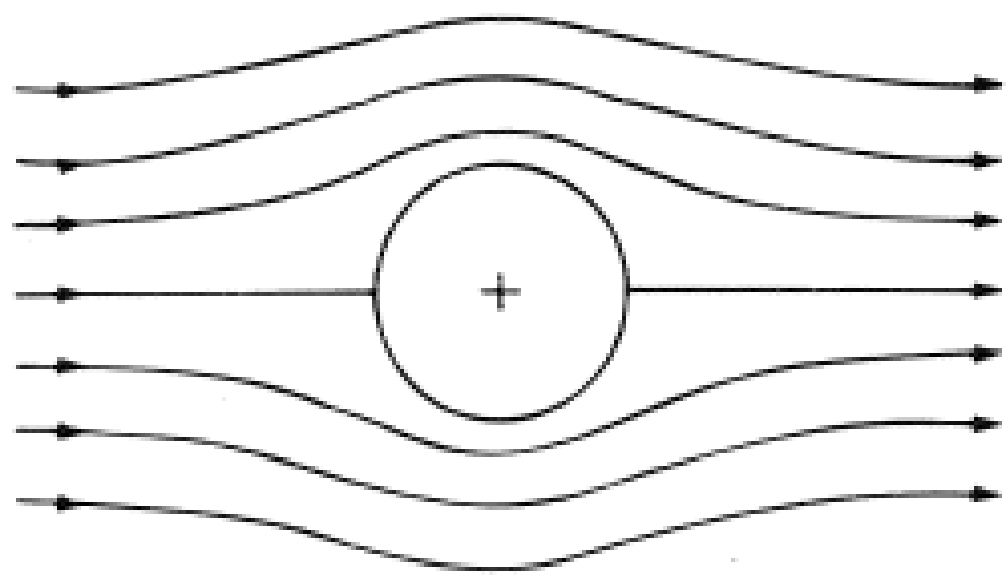

Figura 6. Estructura sin influencia sobre el flujo incidente (fuente: Bengt, 2011)

La fórmula de Morison et al., tradicionalmente, ha dado resultados muy favorables para pilotes con diámetros comprendidos entre 1 y 2 metros, habiendo sido ampliado su uso a diámetros de entre 4 y 8 metros. No obstante, hoy en día, muchos de los diámetros utilizados en parques eólicos offshore $(>8 \mathrm{~m}$ ) están comenzando a exceder en gran medida estos valores, lo que puede invalidar el uso de la teoría de Morison et al. En la figura 7 se observa una simulación del impacto de una ola sobre un cilindro vertical, en la cual se está teniendo en cuenta el efecto de difracción, dado que el diámetro del cilindro es lo suficiente grande como para generar una influencia sobre el oleaje incidente.

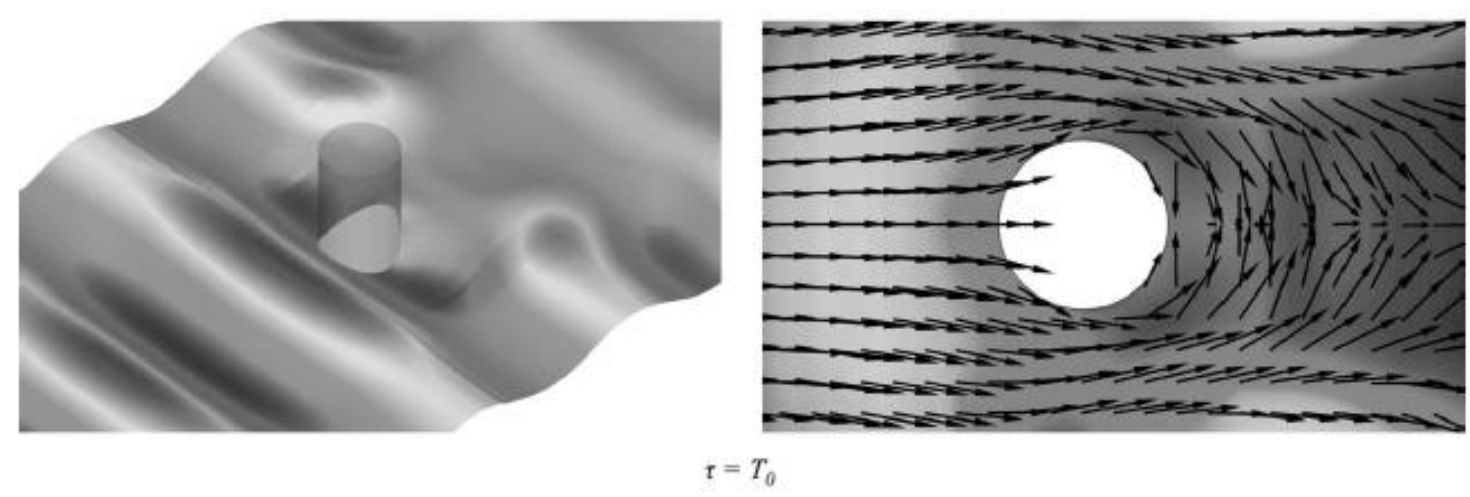

Figura 7. Estructura con influencia sobre el oleaje incidente (fuente: Han et al., 2015) 
El hecho de no tener en cuenta los efectos de dispersión energética producidos cuando una ola impacta sobre estructuras de gran diámetro implica que el uso de la fórmula de Morison et al. arrojará resultados que obligarán a sobredimensionar la estructura.

Posteriormente, tras obtener las fuerzas hidrodinámicas actuantes, se debe realizar un cálculo de los esfuerzos y movimientos que experimenta la estructura. Este cálculo se puede realizar mediante el uso de las fórmulas clásicas de resistencia de materiales para una estructura tipo viga ménsula, empotrada en el lecho marino, como se muestra en la figura 8.

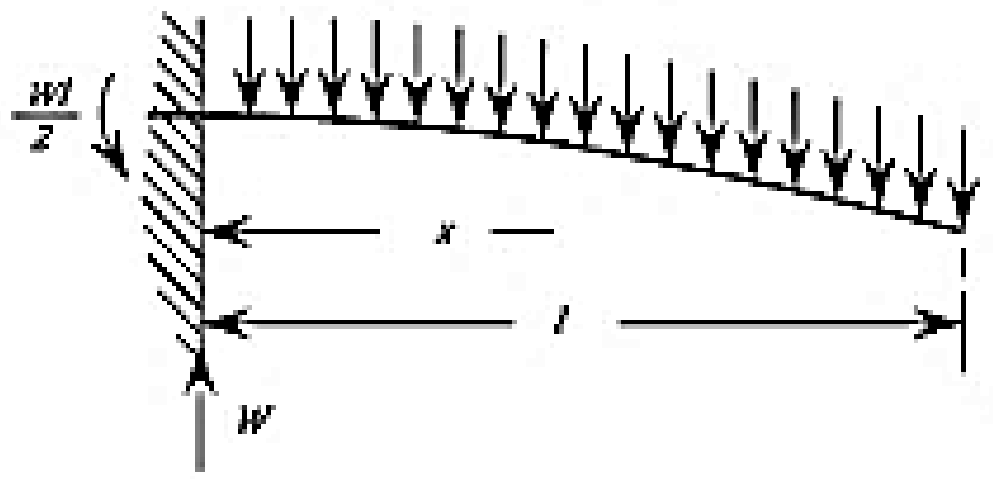

Figura 8. Análisis estático de viga empotrada (fuente: www.codecogs.com)

Este análisis también está generando un sesgo importante dado que no tiene en cuenta la naturaleza dinámica de las cargas actuantes $y$, por lo tanto, la posible acumulación energética que puede sufrir la estructura frente a determinadas frecuencias de carga. En la figura 9 se muestran los primeros cuatro modos de vibración que sufre una viga tipo ménsula.

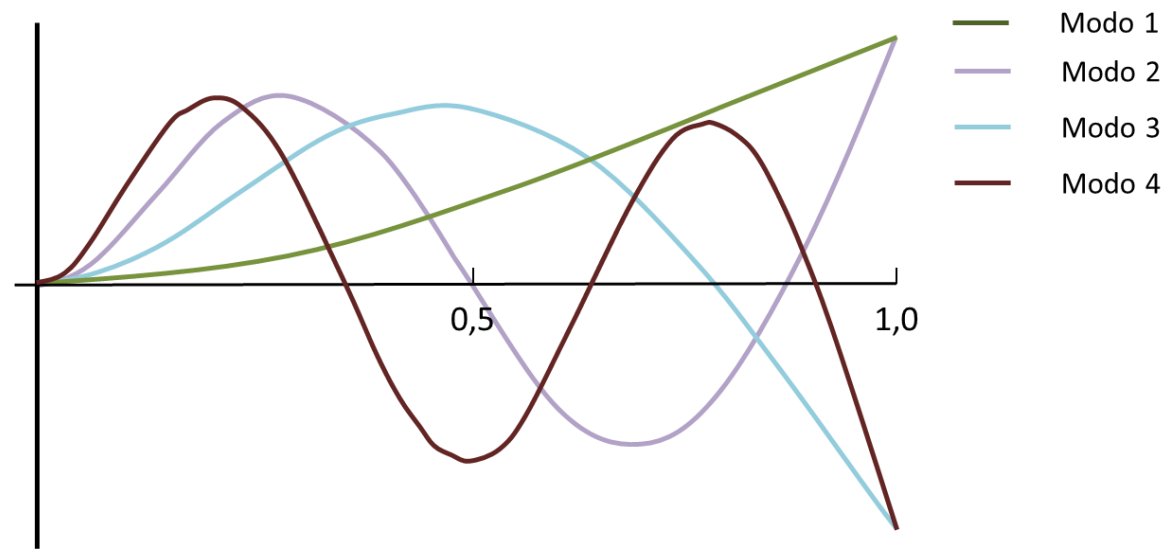

Figura 9. Modos de vibración de una viga empotrada(fuente: elaboración propia) 
El uso del análisis estático hace imposible realizar un estudio evolutivo que permita detectar los estados de carga pésimos que ocurrirán en la realidad sobre la estructura, pudiendo diferenciar qué frecuencias son las que generan un mayor daño estructural.

Por otro lado, tampoco se establece en el proceso de cálculo ningún tipo de relación de interacción entre los movimientos relativos de la estructura y el oleaje incidente, que permita acoplar ambos mecanismos y relacionar las cargas hidrodinámicas y las respuestas estructurales para cada instante temporal.

Por lo tanto, como conclusión de este apartado se puede observar en la figura 10, lo que se ha denominado como esquema tradicional de cálculo para pilas, de acuerdo con todo lo explicado en este punto.

\section{Método tradicional}

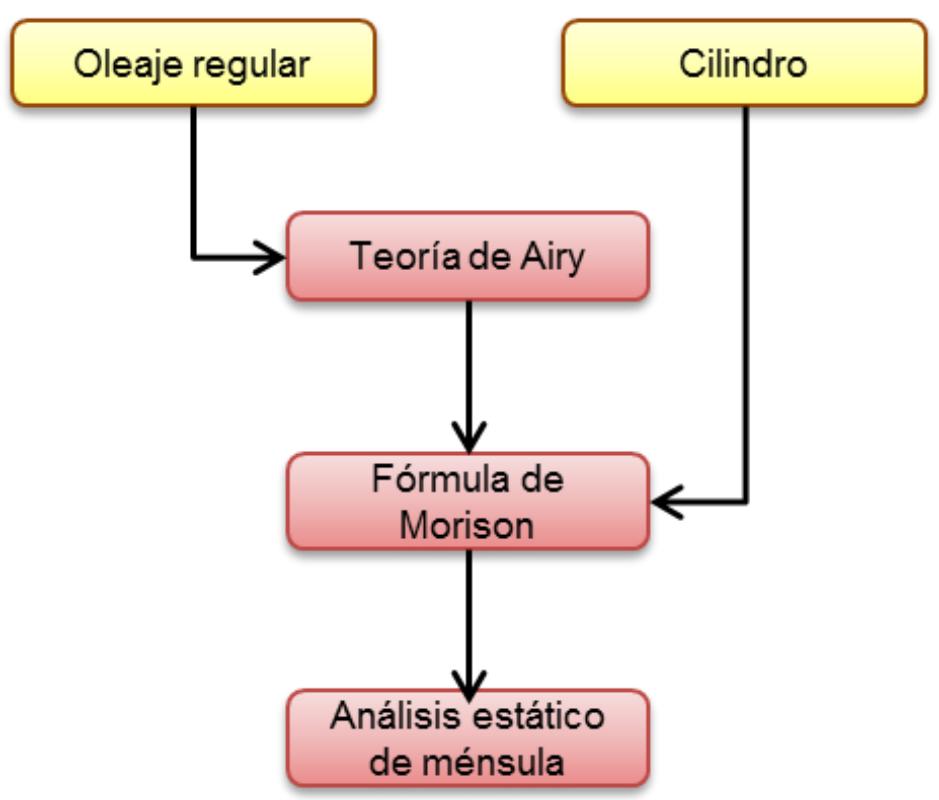

Figura 10. Esquema tradicional de cálculo para pilas (fuente: elaboración propia) 


\subsection{Proceso de cálculo propuesto}

A continuación, se describe la metodología propuesta mediante la presente Tesis Doctoral para resolver adecuadamente la interacción entre la estructura y el oleaje, y realizar una estimación más realista de los esfuerzos hidrodinámicos generados sobre una estructura offshore. Para ello, el problema se ha dividido en cinco procesos que se resolverán de forma independiente, como se muestra en la figura 11.

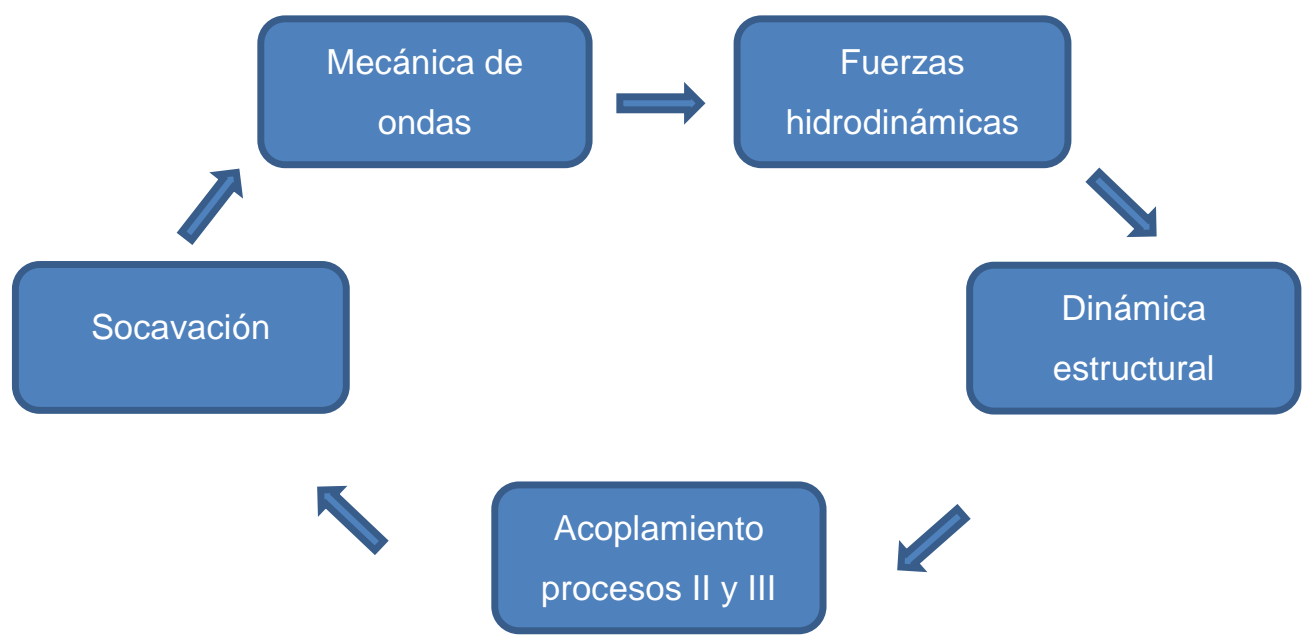

Figura 11. Procesos actuantes en el cálculo (fuente: elaboración propia)

Para la realización de los distintos cálculos se ha desarrollado un software específico que integra los cinco módulos de cálculo. Dicho software de análisis ha sido realizado mediante programación en Python 2.7, en código libre de alto nivel, utilizando librerías de optimización desarrolladas en $\mathrm{C}++$, que permiten la vectorización del cálculo, y por lo tanto, una gran velocidad en los procesos de análisis. Las variables de entrada son las siguientes:

- Malla de cálculo: se definirá el valor máximo y mínimo de $x, y, z, t$, así como la dimensión mínima de paso de malla $(d x, d y, d z, d t)$. De esta forma se definirá completamente el dominio sobre el que se va a realizar el cálculo. Cabe destacar que el software de cálculo resuelve las ecuaciones diferenciales, que definen los procesos I y II, de manera exacta, siendo la malla necesaria para los procesos III y IV que se resuelven mediante un modelo de diferencias finitas. 
- Datos del oleaje: Altura de ola significativa $\left(H_{s}\right)$, periodo de pico $\left(T_{p}\right)$ y profundidad (h). La profundidad coincidirá con el valor mínimo de z. Además también se añadirán los datos de temperatura del agua (Ta) y salinidad del agua (S).

- Estructura: por último se definirá el diámetro (D) de la estructura, su rigidez (EI) y su densidad ( $\left.\rho_{\text {est }}\right)$.

\subsubsection{Proceso I: Mecánica de ondas}

La primera parte del proceso tiene como variables de entrada las condiciones ambientales que definen el oleaje: la altura de ola significativa $\left(H_{s}\right)$, el periodo de pico $\left(T_{p}\right)$ y la profundidad (h). Estas serán las variables de primer orden que permitirán resolver la siguiente ecuación:

$$
\frac{\partial^{2} \phi}{\partial x^{2}}+\frac{\partial^{2} \phi}{\partial z^{2}}=0
$$

Teniendo en cuenta sus condiciones de contorno:

- Condición de contorno cinemática en la superficie libre.

$$
\frac{\partial \phi}{\partial z}=\frac{\partial \eta}{\partial t}+\frac{\partial \phi}{\partial x} \cdot \frac{\partial \eta}{\partial x} \longrightarrow w=\frac{\partial \eta}{\partial t}+u \cdot \frac{\partial \eta}{\partial x}
$$

- Condición de contorno dinámica en la superficie libre.

$$
\frac{\partial \vec{\phi}}{\partial t}+\frac{p_{a t m}}{\rho}+\frac{1}{2}(\nabla \phi)^{2}+g \eta=0
$$

- Condición de contorno por fondo.

$$
w=-u \cdot \frac{\partial h}{\partial x}=0
$$

- Condición de contorno lateral.

$$
\begin{aligned}
& \phi(x, t)=\phi(x+L, t) \\
& \phi(x, t)=\phi(x, t+T)
\end{aligned}
$$


A partir de las variables de entrada, la primera operación que realizará el software será la búsqueda del rango de profundidades en el que se encuentra el problema a estudiar.
i. Profundidades indefinidas:
$1 / 2<h / L$
ii. Profundidades de transición:
$1 / 25<h / L<1 / 2$
iii. Aguas someras:
$h / L<1 / 25$

Para ello se requiere la obtención de la longitud de onda asociada al oleaje. Se sigue un proceso de descarte, utilizando la teoría lineal de ondas, e iniciando el cálculo de la longitud de onda a partir de la expresión para aguas indefinidas:

$$
L_{0}=\frac{g T^{2}}{2 \pi}
$$

Si $1 / 2<h / L$, entonces el problema se encuentra en profundidades indefinidas. Si $1 / 2>$ $h / L$, entonces es necesario recalcular la longitud de onda del oleaje, utilizando la fórmula para aguas de transición:

$$
L=\frac{g T^{2}}{2 \pi} \cdot \tanh \left(\frac{2 \pi h}{L}\right)
$$

Para resolver la expresión anterior se requiere realizar un proceso iterativo, dado que no es posible extraer la longitud de onda (L) del operador tangente hiperbólica.

Una vez obtenida $\mathrm{L}$, si $1 / 25<h / L<1 / 2$, entonces el problema se encuentra en profundidades de transición. Si $1 / 25>h / L$, entonces es necesario recalcular la longitud de onda del oleaje, utilizando la fórmula para aguas someras:

$$
L=T \sqrt{g h}
$$

Posteriormente, se realiza el cálculo de los parámetros $H / g T^{2}$ y $h / g T^{2}$, que permitirá la clasificación del problema y la selección de la teoría de ondas adecuada que se debe aplicar. De acuerdo a la clasificación establecida en el gráfico de Le Méhauté (1976), 
durante el desarrollo de la presente Tesis Doctoral se han extraído los distintos rangos de aplicación de las teorías de mecánica de ondas, de una forma analítica:

Dominio I: $\quad h / g T^{2} \geq 0,03$

$$
H / g T^{2} \leq 0,001
$$

Dominio II: $\quad h / g T^{2} \geq 0,001$

$$
H / g T^{2} \leq 0,019476 \cdot\left(\frac{h}{g T^{2}}\right)^{0,89823}
$$

Dominio III:

$$
\begin{aligned}
& H / g T^{2} \leq 16,995657 \cdot\left(\frac{h}{g T^{2}}\right)^{1,925364} \\
& H / g T^{2} \leq 0,16388 \cdot\left(\frac{h}{g T^{2}}\right)^{0,97807} \\
& H / g T^{2} \leq 0,007
\end{aligned}
$$

Dominio IV:

$$
\begin{aligned}
& H / g T^{2} \leq 16,995657 \cdot\left(\frac{h}{g T^{2}}\right)^{1,925364} \\
& H / g T^{2} \leq 0,43 \cdot\left(\frac{h}{g T^{2}}\right)^{0,97807} \\
& H / g T^{2} \leq 0,02
\end{aligned}
$$

Dominio V:

$$
H / g T^{2} \leq 16,995657 \cdot\left(\frac{h}{g T^{2}}\right)^{1,925364}
$$$$
H / L \leq 0,142
$$

Dominio VI:

$$
\begin{aligned}
& H / g T^{2} \leq 0,16388 \cdot\left(\frac{h}{g T^{2}}\right)^{0,97807} \\
& H / g T^{2}>16,995657 \cdot\left(\frac{h}{g T^{2}}\right)^{1,925364}
\end{aligned}
$$

Dominio VII:

$$
\begin{aligned}
& H / g T^{2} \leq 0,61 \cdot\left(\frac{h}{g T^{2}}\right)^{0,97807} \\
& H / g T^{2} \leq 0,0265
\end{aligned}
$$


Por lo tanto, dependiendo de la altura de ola significativa $H_{s}$, su periodo de pico $T_{p}$ y la profundidad h, la solución del problema anterior vendrá dada por una de las teorías descritas en el capítulo 2: Airy, Stokes II, Stokes III, Stokes IV, Stokes V, Cnoidal o Stream Function. El software seleccionará automáticamente la teoría de ondas más adecuada dependiendo de las constantes $H / g T^{2}$ y $h / g T^{2}$, que situarán el problema en alguno de los dominios de cálculo definidos de forma analítica en el párrafo anterior. Es necesario que se cumplan de forma simultánea todas las reglas analíticas descritas anteriormente para un determinado dominio, para poder considerar que el problema se encuentra localizado dentro de dicho dominio. A continuación, se muestran los rangos de aplicación de cada una de las teorías, mostrando con el símbolo "U" la unión entre dos dominios y con el símbolo "-" a la sustracción de un dominio que se encuentre dentro de otro.

$\begin{array}{lll}\text { Airy } & = & D 1 \cup D 2 \\ \text { Stokes II } & = & D 3-D 2-D 1 \\ \text { Stokes III } & = & D 4-D 3-D 2-D 1 \\ \text { Stokes IV y V } & = & D 5-D 4-D 3-D 2-D 1 \\ \text { Cnoidal } & =D 6 \\ \text { Stream Function }= & D 7-D 6-D 5-D 4-D 3-D 2-D 1\end{array}$

Por otro lado, se tendrá también en cuenta el efecto de la temperatura (Ta) y la salinidad (S) en el cálculo. Ambos parámetros influirán de forma directa sobre la densidad del agua ( $\rho)$ a considerar en el análisis y sobre la viscosidad del agua. A continuación, se muestran en la tabla 1 dichos valores, de acuerdo a Chakravarti (2005):

\begin{tabular}{|c|c|c|c|c|}
\hline \multirow{2}{*}{$\begin{array}{c}\text { Temperatura } \\
\left({ }^{\circ} \mathrm{C}\right)\end{array}$} & \multicolumn{2}{|c|}{ Densidad del agua $\left(\mathrm{kg} / \mathrm{m}^{3}\right)$} & \multicolumn{2}{|c|}{ Viscosidad cinemática $\left(\mathrm{m}^{2} / \mathrm{s} \times 10^{-5}\right)$} \\
\hline & Agua dulce & Agua salada $35 \%$ & Agua dulce & Agua salada $35 \%$ \\
\hline 0 & $1.031,074$ & $1.031,952$ & 0,1792 & 0,1828 \\
\hline 1,11 & $1.031,076$ & $1.031,950$ & 0,1725 & 0,1763 \\
\hline 2,22 & $1.031,077$ & $1.031,947$ & 0,1661 & 0,1701 \\
\hline 3,33 & $1.031,077$ & $1.031,944$ & 0,1602 & 0,1643 \\
\hline 4,44 & $1.031,077$ & $1.031,941$ & 0,1546 & 0,1588 \\
\hline 5,56 & $1.031,077$ & $1.031,936$ & 0,1493 & 0,1539 \\
\hline 6,67 & $1.031,076$ & $1.031,931$ & 0,1443 & 0,149 \\
\hline 7,78 & $1.031,074$ & $1.031,926$ & 0,1395 & 0,1443 \\
\hline 8,89 & $1.031,073$ & $1.031,922$ & 0,1351 & 0,1398 \\
\hline
\end{tabular}




\begin{tabular}{|c|c|c|c|c|}
\hline 10 & $1.031,069$ & $1.031,915$ & 0,1308 & 0,1356 \\
\hline 11,11 & $1.031,066$ & $1.031,910$ & 0,1268 & 0,1316 \\
\hline 12,22 & $1.031,063$ & $1.031,904$ & 0,1229 & 0,1278 \\
\hline 13,33 & $1.031,058$ & $1.031,896$ & 0,1193 & 0,1242 \\
\hline 14,44 & $1.031,053$ & $1.031,890$ & 0,1158 & 0,1207 \\
\hline 15,56 & $1.031,049$ & $1.031,882$ & 0,1125 & 0,1174 \\
\hline 16,67 & $1.031,042$ & $1.031,874$ & 0,1093 & 0,1143 \\
\hline 17,78 & $1.031,036$ & $1.031,866$ & 0,1063 & 0,1113 \\
\hline 18,89 & $1.031,029$ & $1.031,858$ & 0,1034 & 0,1084 \\
\hline 20,00 & $1.031,023$ & $1.031,848$ & 0,1007 & 0,1056 \\
\hline 21,11 & $1.031,015$ & $1.031,838$ & 0,0980 & 0,1030 \\
\hline 22,22 & $1.031,009$ & $1.031,829$ & 0,0955 & 0,1005 \\
\hline
\end{tabular}

Tabla 1. Influencia de la salinidad y la temperatura sobre la densidad y la viscosidad del agua (fuente: elaboración propia a

partir de Chakravarti, 2005)

Para valores de temperatura o salinidad que se encuentren entre los valores mostrados por la tabla anterior, se procederá a obtener la densidad del agua, $\rho$, y la viscosidad cinemática, $v$, mediante interpolación lineal.

Por lo tanto, el software no solo seleccionará de forma automática la teoría de ondas más adecuada para resolver el problema, tal y como se ha expuesto anteriormente, sino que además calculará el valor de la densidad del agua y de su viscosidad de acuerdo con la tabla de Chakravarti (2005).

En conclusión, el software calculará automáticamente los valores de los parámetros $H / g T^{2}, h / g T^{2}, \rho$ y $v$, y procederá a aplicar la formulación perteneciente al dominio de cálculo que sea de aplicación, obteniendo como resultado la función de velocidad potencial $(\varphi)$ que permitirá la obtención de los siguientes datos, para cada punto del espacio definido, en un determinado instante temporal $(x, y, z, t)$ :

a) Perfil de elevación del oleaje

$$
\eta=\eta(x, y, z, t)
$$

b) Presiones

$$
P=P(x, y, z, t)
$$

c) Campo de desplazamientos horizontales y verticales

$$
\xi_{x}=\xi_{x}(x, y, z, t)
$$




$$
\begin{aligned}
& \xi_{y}=\xi_{y}(x, y, z, t) \\
& \xi_{z}=\xi_{z}(x, y, z, t)
\end{aligned}
$$

d) Campo de velocidades horizontales y verticales

$$
\begin{aligned}
u & =u(x, y, z, t) \\
v & =v(x, y, z, t) \\
w & =w(x, y, z, t)
\end{aligned}
$$

e) Campo de aceleraciones horizontales y verticales

$$
\begin{aligned}
& a_{x}=a_{x}(x, y, z, t) \\
& a_{y}=a_{y}(x, y, z, t) \\
& a_{z}=a_{z}(x, y, z, t)
\end{aligned}
$$

No obstante, todos los resultados extraídos de este primer análisis no tienen en cuenta la existencia de ningún tipo de estructura en mitad del dominio de cálculo, dado que se están calculando las características de un oleaje incidente sin tener en cuenta los posibles efectos generados por la difracción.

\subsubsection{Proceso II: Cálculo de las fuerzas hidrodinámicas}

Este segundo proceso toma del primero todas las variables que definen el movimiento, velocidad, aceleración y presión en cada uno de los puntos del dominio de cálculo, y procede a introducir la estructura, para hallar los efectos que produce el oleaje sobre ella.

En este análisis se toman como variables principales de entrada: el campo de velocidades (v) y el de aceleraciones (a), que fueron obtenidos como resultado del Proceso I de cálculo, $y$, además, se incluyen las dimensiones de la estructura, que en el caso de ser esta un cilindro, vendrán caracterizadas por su diámetro (D).

Como primer paso, el software necesitará realizar una clasificación del fenómeno para decidir qué teoría hidrodinámica debe aplicar. De acuerdo, con la relación $\mathrm{D} / \mathrm{L}$ y el número de Keulegan-Carpenter, el método de cálculo a seguir para calcular las fuerzas hidrodinámicas será seleccionado automáticamente de entre los seis tipos que definen el dominio global: 

a) Flujo separado lineal.
b) Flujo separado no lineal.
c) Inercial lineal.
d) Inercial no lineal.
e) Difracción lineal.
f) Difracción no lineal.

Para ello, el software realiza el cálculo de los siguientes ratios:

- $\mathrm{D} / \mathrm{L}$

- $K C=\frac{u_{\max T}}{D}$

- $\mathrm{KC}_{\max }$

El número de Keulegan-Carpenter (KC) define la influencia de las fuerzas viscosas en el problema. Para hacer la clasificación, se definirá KC para la superficie libre. Además, puede observarse que la máxima pendiente que puede sufrir una ola es:

$$
\frac{H}{L}=\frac{1}{7} \tanh k h
$$

De tal forma que para calcular $\mathrm{KC}_{\max }$ se procederá de la forma siguiente.

$$
K C_{\text {max }}=\frac{\pi(H / L)}{(D / L) \tanh k d}=\frac{\pi}{7} \cdot \frac{L}{D}
$$

A continuación, se muestra en la tabla 2 la clasificación de las teorías de cálculo para hallar

\begin{tabular}{|c|c|c|c|c|}
\hline Ratio & $\begin{array}{c}\mathrm{KC}<4 \\
\mathrm{KC} \leq 0,5 \cdot K C_{\max }\end{array}$ & $\begin{array}{c}\mathrm{KC}<4 \\
\mathrm{KC}>0,5 \cdot K C_{\max }\end{array}$ & $\begin{array}{c}\mathrm{KC}>4 \\
\mathrm{KC} \leq 0,5 \cdot K C_{\max }\end{array}$ & $\begin{array}{c}\mathrm{KC}>4 \\
\mathrm{KC}>0,5 \cdot K C_{\max }\end{array}$ \\
\hline $\mathrm{D} / \mathrm{L}<0,2$ & $\begin{array}{l}\text { Rango Inercial } \\
\text { Teoría lineal de } \\
\text { oleaje. } \\
\text { Fuerza: Froude- } \\
\text { Krylov. }\end{array}$ & $\begin{array}{l}\text { Rango Inercial } \\
\text { Teoría no lineal de } \\
\text { oleaje. } \\
\text { Fuerza: no lineal de } \\
\text { Froude-Krylov }\end{array}$ & $\begin{array}{l}\text { Rango Separación } \\
\text { Teoría lineal de oleaje } \\
\text { Fuerza: inercial más } \\
\text { arrastre. }\end{array}$ & $\begin{array}{l}\text { Rango Separación } \\
\text { Teoría no lineal de } \\
\text { oleaje } \\
\text { Fuerza: inercial más } \\
\text { arrastre, más términos } \\
\text { cuadráticos. }\end{array}$ \\
\hline
\end{tabular}
las fuerzas hidrodinámicas: 
Modelos de predicción de esfuerzos hidrodinámicos y socavación aplicados a ingeniería offshore

\begin{tabular}{|l|l|l|l|l|}
\hline \multirow{2}{*}{$\mathrm{D} / \mathrm{L}>0,2$} & Rango Difracción & Rango Difracción & & \\
& Teoría lineal de & Teoría no lineal de & & \\
& oleaje. & oleaje. & - & \\
& Fuerza: Teoría lineal & Fuerza: Teoría no lineal & & \\
& de difracción. & de difracción. & & \\
\hline
\end{tabular}

Tabla 2. Clasificación de fuerzas hidrodinámicas (fuente: elaboración propia)

La temperatura y la salinidad tendrán un peso importante en este segundo proceso, dado que definirán la viscosidad cinemática del agua y, por lo tanto, tendrán influencia sobre las fuerzas de arrastre. Por otro lado, definirán también la densidad del agua, modificando el valor absoluto de todas las fuerzas producidas.

También será necesario definir el número de Reynolds que domina el análisis para poder definir los parámetros $C_{M}$ y $C_{D}$. De acuerdo a las gráficas mostradas en el punto 5.6.1.2 del capítulo 2, los valores de $C_{M}$ y $C_{D}$ se hallan a partir del número de Reynolds (Shore Protection Manual, 1975; Achenbach, 1968):

$$
\begin{array}{ll}
C_{M}=2,0 & \text { cuando } \mathrm{R}_{\mathrm{e}}<2,5 \cdot 10^{5} \\
C_{M}=2,5-\frac{R_{e}}{5 \cdot 10^{5}} & \text { cuando } 2,5 \cdot 10^{5}<\mathrm{R}_{\mathrm{e}}<5 \cdot 10^{5} \\
C_{M}=1,5 & \text { cuando } \mathrm{R}_{\mathrm{e}}>5 \cdot 10^{5} \\
C_{D}=1,2 & \text { cuando } \mathrm{R}_{\mathrm{e}}<1 \cdot 10^{5} \\
C_{D}=1,2-\frac{1,2-0,7}{3 \cdot 10^{5}} \cdot\left(R_{e}-10^{5}\right) & \text { cuando } 1 \cdot 10^{5}<\mathrm{R}<4 \cdot 10^{5} \\
C_{D}=0,7 & \text { cuando } \mathrm{R}_{\mathrm{e}}>4 \cdot 10^{5}
\end{array}
$$

El número de Reynolds será hallado para cada instante del tiempo y para cada valor de la profundidad, de tal forma que $C_{M}$ y $C_{D}$ no serán constantes a lo largo del proceso.

$$
R e(z, t)=\frac{u(z, t) \cdot D}{v(T a, S)}
$$


Para los resultados que se encuentren dentro del rango inercial o de flujo separado, dado que no existe una modificación del oleaje incidente, el resultado de las fuerzas hidrodinámicas vendrá expresado por la fórmula de Morison et al.:

$$
F(z, t)=\rho \cdot \frac{\pi D^{2}}{4} \cdot C_{M} \cdot a_{x}+\rho \cdot \frac{D}{2} \cdot C_{D} \cdot u \cdot|u|
$$

No obstante, para los casos en que $D / L>2$, será necesario tener en cuenta el valor del potencial de difracción. La solución analítica al problema de difracción lineal fue obtenida por MacCamy y Fuchs (1954) para una profundidad general.

$$
F_{z}(z, t)=\frac{\pi}{8} \rho g H k D^{2} \frac{\cosh (k(z+h))}{\cosh (k h)} \frac{4 A(k a)}{\pi(k a)^{2}} \cos (\omega t-\theta)
$$

Sin embargo, se va a plantear el uso de un método numérico más sofisticado para poder resolver el potencial de difracción, que admita una mayor flexibilidad en el cálculo, permitiendo su uso para cualquier tipo de estructura, dado que la fórmula de MacCamy y Fuchs (1954) está desarrollada según la teoría lineal de ondas. La velocidad potencial puede ser descompuesta por separación de variables en:

$$
\phi(x, y, z, t)=\varphi(x, y) \cdot Z(z) \cdot e^{-i \omega t}
$$

Partiendo de la base de que la velocidad potencial será la suma del potencial generado por el oleaje incidente y el generado por el oleaje difractado:

$$
\phi=\phi_{I}+\phi_{D}
$$

Dado que $Z(z)$ y el exponencial serán iguales para ambos potenciales, la solución del problema puede limitarse únicamente a la solución de la superficie libre:

$$
\varphi(x, y)=\varphi_{I}(x, y)+\varphi_{D}(x, y)
$$


Para resolver el término de la difracción, la velocidad potencial $\varphi_{D}(x, y)$ está determinada por la bien conocida ecuación de Helmholtz:

$$
\nabla^{2} \varphi_{D}+k^{2} \varphi_{D}=0
$$

Además, la condición de contorno de Neumann y la denominada condición de Sommerfeld deben ser aplicadas respectivamente en la interfaz entre el fluido y la estructura, y la condición de radiación en el infinito:

$$
\begin{gathered}
\frac{\partial \varphi_{D}}{\partial \vec{n}}=-\frac{\partial \varphi_{I}}{\partial \vec{n}} \quad \text { para } \quad r=d \\
\lim _{k r \rightarrow \infty} \sqrt{k r}\left(\frac{\partial \varphi_{D}}{\partial r}-i k \varphi_{D}\right)=0
\end{gathered}
$$

siendo $r$ el eje radial y $n$ el vector unidad, normal a la superficie del cilindro.

La resolución del sistema se hará mediante el uso del método de elementos de contorno, en inglés Boundary Elements Method o BEM basándose en el método propuesto por Yang et al. (1992) y las posteriores mejoras aportadas por Bai et al. (2007) y Moghadaszadeh et al. (2015). Para ello, es necesario recurrir a la aplicación de la segunda identidad de Green, para hallar la función de velocidad potencial que satisface la ecuación de Laplace y que cumple con las condiciones de contorno definidas anteriormente en este apartado y en el apartado 2.2.1:

$$
\phi(x)=\frac{1}{2 \pi} \int_{S}\left[G(x, \xi) \frac{\partial \phi}{\partial n}(\xi)-\phi(\xi) \frac{\partial G}{\partial n}(x, \xi)\right] d s
$$

La función de Green elegida para resolver el problema es la siguiente:

$$
G(x, \xi)=\frac{1}{r}+\frac{1}{r^{\prime}}+\frac{1}{r^{\prime \prime}}+\frac{1}{r^{\prime \prime \prime}}
$$

donde: 


$$
\begin{aligned}
& r=|x-\xi|=\left[(x-\xi)^{2}+(y-\eta)^{2}+(z-\zeta)^{2}\right]^{1 / 2} \\
& r^{\prime}=\left|x-\xi^{\prime}\right|=\left[(x-\xi)^{2}+(y-\eta)^{2}+(z+\zeta+2 d)^{2}\right]^{1 / 2} \\
& r^{\prime \prime}=\left|x-\xi^{\prime \prime}\right|=\left[(x-\xi)^{2}+(y+\eta)^{2}+(z-\zeta)^{2}\right]^{1 / 2} \\
& r^{\prime \prime \prime}=\left|x-\xi^{\prime \prime \prime}\right|=\left[(x-\xi)^{2}+(y+\eta)^{2}+(z+\zeta+2 d)^{2}\right]^{1 / 2}
\end{aligned}
$$

Esta función de Green satisface la ecuación de Laplace para un dominio tridimensional y cumple todas las condiciones de contorno. Para resolverla será necesario discretizar el problema y sus contornos.

En la figura 12 se muestran las superficies de contorno que definen el problema:

- $\mathrm{S}_{\mathrm{C}}$ es el perímetro exterior del problema que va desde la superficie libre hasta el lecho marino.

- $S_{b}$ es la superficie de contorno de la estructura, que está en contacto con el agua.

- Ss es el plano de simetría que atraviesa la estructura por la mitad.

- $\mathrm{S}_{\mathrm{f}}$ es la superficie que representa la superficie libre.

- $S_{d}$ la superficie que representa el lecho marino.
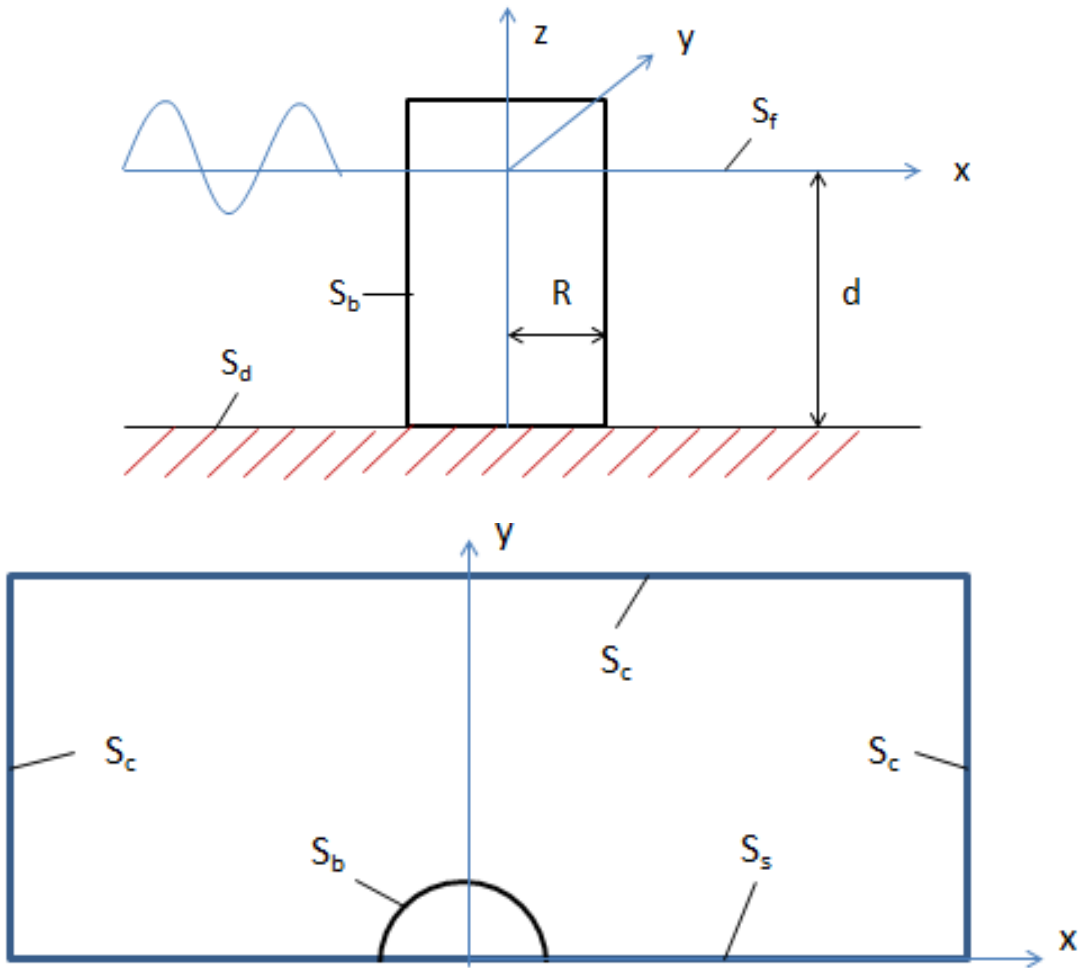

Figura 12. Representación de los contornos del problema (fuente: elaboración propia) 
Las superficies $S_{b}, S_{f}$ y $S_{c}$ son discretizadas en un número finito de elementos. El número de elementos en $S_{b}$ será $N_{b}$, en $S_{f}$ será $N_{f}-N_{b}$, en $S_{c}$ será $N_{c}-N_{f}$, siendo $N_{c}$ el número total de elementos. La superficies son utilizadas según el orden $S_{b}, S_{f}$ y $S_{c}$, con el índice j en el rango 1 a $N_{c}$. Siendo $x=x_{i}$ la coordenadas del punto central de cada elemento, la ecuación de Green puede reescribirse entonces como:

$$
\phi\left(x_{i}\right)=\frac{1}{2 \pi} \sum_{j=1}^{N_{c}}\left\{\int_{\Delta S_{j}} G\left(x_{i}, \xi\right) \frac{\partial \phi}{\partial n}(\xi) d S-\int_{\Delta S_{j}} \phi(\xi) \frac{\partial G}{\partial n}\left(x_{i}, \xi\right) d S\right\}
$$

donde $\Delta S_{j}$ es el área de cada elemento. Asumiendo que $\phi$ y $\partial \phi / \partial n$ pueden tomarse como constantes sobre cada $\Delta S_{j}$, la ecuación anterior puede ser transformada en conjunto de $\mathrm{N}_{c}$ ecuaciones lineales, dadas por:

$$
\sum_{j=1}^{N_{c}}\left[\alpha_{i j} \phi_{j}+b_{i j}\left(\frac{\partial \phi}{\partial n}\right)_{j}\right]=0, \quad i=1,2, \ldots, N_{c}
$$

donde los coeficientes vienen dados por:

$$
\begin{gathered}
\alpha_{i j}=\int_{\Delta S_{j}} \frac{\partial G}{\partial n}\left(x_{i}, \xi\right) d S+2 \pi \delta_{i j} \\
b_{i j}=-\int_{\Delta S_{j}} G\left(x_{i}, \xi\right) d S \\
\delta_{i j}=\begin{array}{ll}
1 & i=j \\
0 & i \neq j
\end{array}
\end{gathered}
$$

Considerando que hay $\mathrm{N}_{\mathrm{c}}$ incógnitas en las superficies de contorno, por ejemplo: $\phi_{j}=$ $\phi\left(x_{j}\right)$ en la superficie $\mathrm{S}_{\mathrm{b}} \mathrm{y}(\partial \phi / \partial n)_{j}=(\partial \phi / \partial n)\left(x_{j}\right)$ en $\mathrm{S}_{\mathrm{f}} \mathrm{y} \mathrm{S}_{c}$; la expresión anterior puede escribirse como:

$$
\sum_{j=1}^{N_{b}} A_{i j} \phi_{j}+\sum_{j=N_{b}+1}^{N_{f}} B_{i j}\left(\frac{\partial \phi}{\partial n}\right)_{j}+\sum_{j=N_{f}+1}^{N_{c}} C_{i j}\left(\frac{\partial \phi}{\partial n}\right)_{j}=D_{i}
$$


donde:

$$
\begin{array}{ll}
A_{i j}=a_{i j} & j=1,2, \ldots, N_{b} \\
B_{i j}=b_{i j} & j=N_{b}+1, N_{b}+2, \ldots, N_{f} \\
C_{i j}=b_{i j} & j=N_{f}+1, N_{f}+2, \ldots, N_{c} \\
D_{i}=-\sum_{j=1}^{N_{b}} b_{i j}\left(\frac{\partial \phi}{\partial n}\right)_{j}-\sum_{j=N_{b}+1}^{N_{c}} a_{i j} \phi_{j} & i=1,2, \ldots \ldots, N_{c}
\end{array}
$$

Teniendo en cuenta que las condiciones de contorno de la superficie libre pueden ser escritas como:

$$
\begin{gathered}
\frac{\partial \eta}{\partial t}=\frac{1}{n_{z}} \frac{\partial \phi}{\partial n} \\
\frac{\partial \phi}{\partial t}=-g \eta-\frac{1}{2} \nabla \phi \cdot \nabla \phi
\end{gathered}
$$

El método multipaso de cuarto orden de Adams-Bashford puede ser utilizado para discretizar las condiciones de contorno anteriores:

$$
\begin{aligned}
& \eta_{t+\Delta t}=\eta_{t}+\frac{1}{24} \cdot \Delta t \cdot\left[55 F_{t}^{1}-59 F_{t-\Delta t}^{1}+37 F_{t-2 \Delta t}^{1}-9 F_{t-3 \Delta t}^{1}\right] \\
& \phi_{t+\Delta t}=\phi_{t}+\frac{1}{24} \cdot \Delta t \cdot\left[55 F_{t}^{2}-59 F_{t-\Delta t}^{2}+37 F_{t-2 \Delta t}^{2}-9 F_{t-3 \Delta t}^{2}\right]
\end{aligned}
$$

donde:

$$
\begin{gathered}
F^{1}=\frac{1}{n_{z}} \frac{\partial \phi}{\partial n} \\
F^{2}=-g \eta-\frac{1}{2} \nabla \phi \cdot \nabla \phi
\end{gathered}
$$

Por otro lado, se debe satisfacer la condición de radiación de Sommerfeld, que puede expresarse como:

$$
\frac{\partial \phi_{D}}{\partial t}+C_{\phi_{D}} \frac{\partial \phi_{D}}{\partial l}=0 \quad \text { en } S_{c}
$$


siendo $l=|l|$ la dirección de propagación del oleaje difractado y $C_{\phi_{D}}$ la velocidad de fase, ambas desconocidas. Esta ecuación puede ser rescrita como:

$$
\frac{\partial \phi_{D}}{\partial t}+C_{\phi_{D}}\left(k_{x} \frac{\partial \phi_{D}}{\partial x}+k_{y} \frac{\partial \phi_{D}}{\partial y}\right)=0
$$

El método multipaso de cuarto orden de Adams-Bashford puede ser nuevamente utilizado para discretizar esta condición de contorno:

$$
\begin{aligned}
& \phi_{D_{t+\Delta t}}=\phi_{D_{t}}+\frac{1}{24} \cdot \Delta t \cdot\left[55 F_{t}^{3}-59 F_{t-\Delta t}^{3}+37 F_{t-2 \Delta t}^{3}-9 F_{t-3 \Delta t}^{3}\right] \\
& \eta_{D_{t+\Delta t}}=\eta_{D_{t}}+\frac{1}{24} \cdot \Delta t \cdot\left[55 F_{t}^{4}-59 F_{t-\Delta t}^{4}+37 F_{t-2 \Delta t}^{4}-9 F_{t-3 \Delta t}^{4}\right]
\end{aligned}
$$

donde:

$$
\begin{gathered}
F^{3}=-\alpha_{\phi_{D}} C_{\phi_{D}} \sqrt{\left(\frac{\partial \phi_{D}}{\partial x}\right)^{2}+\left(\frac{\partial \phi_{D}}{\partial y}\right)^{2}} \\
F^{4}=-\alpha_{\eta_{D}} C_{\eta_{D}} \sqrt{\left(\frac{\partial \eta_{D}}{\partial x}\right)^{2}+\left(\frac{\partial \eta_{D}}{\partial y}\right)^{2}}
\end{gathered}
$$

Mediante el método multipaso de cuarto orden de Adams-Bashford es posible ir obteniendo la solución total del sistema para cada paso temporal, teniendo en cuenta los efectos de difracción generados por la estructura.

En conclusión, el resultado de este segundo proceso serán las fuerzas generadas por el oleaje sobre la estructura para cada punto de la misma y cada diferencial de tiempo, teniendo en cuenta los posibles efectos de no linealidad y/o difracción.

No obstante, en este punto del proceso, el cálculo de las fuerzas está considerando a la estructura como un obstáculo infinitamente rígido que no sufre movimiento o desplazamiento alguno a causa de las fuerzas del oleaje. 


\subsubsection{Proceso III: Análisis dinámico}

En este tercer proceso se analiza el comportamiento estructural que sufrirá la estructura a causa de las fuerzas hidrodinámicas generadas por el oleaje. Para ello se supondrá que la monopila que sustenta el aerogenerador se comporta como una viga empotrada en el lecho marino y no se tendrá en cuenta el axil vertical generado por el peso del propio aerogenerador ni sus frecuencias $1 \mathrm{P}$ y $3 \mathrm{P}$, que serán objeto de futuras líneas de investigación.

De acuerdo con el desarrollo teórico mostrado en el capítulo 2, en primer lugar es necesario obtener las frecuencias propias de vibración de la ménsula. Esto se consigue hallando las soluciones posibles a la ecuación siguiente, que serán los autovalores del problema:

$$
\cos \alpha_{m} l \cosh \alpha_{m} l+1=0
$$

Por otro lado, una vez obtenidos los autovalores, los autovectores se obtienen de forma inmediata para cada uno de los modos de vibración, mediante la siguiente expresión:

$$
\frac{C_{3}}{C_{4}}=-\gamma_{m}=\frac{\cos \alpha_{m} l+\cosh \alpha_{m} l}{\operatorname{sen} \alpha_{m} l+\operatorname{senh} \alpha_{m} l}
$$

El software de cálculo utilizado en la presente Tesis Doctoral, incluye la solución de esta ecuación para los primeros 25 modos de vibración, tal y como muestra la tabla 3:

\begin{tabular}{|c|c|c|}
\hline Modos & Autovalores $\left(\boldsymbol{\alpha}_{\boldsymbol{m}} \boldsymbol{l}\right)$ & Autovectores $\left(\boldsymbol{\gamma}_{\mathrm{m}}\right)$ \\
\hline 1 & 1,87510410 & 0,7340955 \\
\hline 2 & 4,69409113 & 1,01846644 \\
\hline 3 & 7,85475743 & 0,99922450 \\
\hline 4 & 10,99554074 & 1,00003355 \\
\hline 5 & 14,13716839 & 0,99999855 \\
\hline 6 & 17,27875953 & 1,00000006 \\
\hline 7 & 20,4203522 & 1,00000000 \\
\hline 8 & 23,5619448 & 1,00000000 \\
\hline 9 & 26,7035375 & 1,00000000 \\
\hline 10 & 29,8451301 & 1,00000000 \\
\hline 11 & 32,9867228 & 1,00000000 \\
\hline 12 & 36,1283155 & 1,00000000 \\
\hline 13 & 39,2699081 & 1,00000000 \\
\hline 14 & 42,4115008 & 1,00000000 \\
\hline
\end{tabular}




\begin{tabular}{|c|c|c|}
\hline 15 & 45,55309341 & 1,00000000 \\
\hline 16 & 48,69468607 & 1,00000000 \\
\hline 17 & 51,83627872 & 1,00000000 \\
\hline 18 & 54,97787137 & 1,00000000 \\
\hline 19 & 58,11946403 & 1,00000000 \\
\hline 20 & 61,26105668 & 1,00000000 \\
\hline 21 & 64,40264933 & 1,00000000 \\
\hline 22 & 67,54424199 & 1,00000000 \\
\hline 23 & 70,68583464 & 1,00000000 \\
\hline 24 & 73,82742729 & 1,00000000 \\
\hline 25 & 76,96901995 & 1,00000000 \\
\hline
\end{tabular}

A partir de estos valores, que permanecen constantes para cualquier caso, sea cual sea la longitud de la ménsula, se obtienen las frecuencias de vibración. El software realiza este paso de cálculo automáticamente, tras aplicar la siguiente formula:

$$
\omega_{m}=\left(\alpha_{m} l\right)^{2} \sqrt{\frac{E I}{\rho A l^{4}}}
$$

donde:

- E es el módulo elástico de Young.

- I es el módulo de inercia de la sección.

- $\rho$ es la densidad del material con el que está hecho el pilar.

- A es el área de la sección.

- $\quad l$ es la longitud total.

También obtiene las frecuencias naturales de vibración, como:

$$
f(H z)=\frac{\omega_{m}}{2 \pi}
$$

La ecuación del movimiento viene determinada por:

$$
w(z, t)=\sum_{m=1}^{\infty} \mathrm{Z}_{\mathrm{m}}(z) \cdot\left(A_{m} \operatorname{sen} \omega_{m} t+B_{m} \cos \omega_{m} t\right)
$$


siendo $Z_{m}(z)$, la expresión hallada anteriormente mediante los autovalores y los autovectores:

$$
Z_{m}(z)=\left(\cosh \alpha_{m} z-\cos \alpha_{m} z\right)-\gamma_{m}\left(\operatorname{senh} \alpha_{m} z-\operatorname{sen} \alpha_{m} z\right)
$$

Que para el caso que se está resolviendo, será expresada como:

$$
\begin{aligned}
& Z_{m}(z)=\left(\cosh \frac{\left(\alpha_{m} l\right) z}{l}-\cos \frac{\left(\alpha_{m} l\right) z}{l}\right)-\gamma_{m}\left(\operatorname{senh} \frac{\left(\alpha_{m} l\right) z}{l}-\operatorname{sen} \frac{\left(\alpha_{m} l\right) z}{l}\right) \\
& m=1,2,3, \ldots .25
\end{aligned}
$$

Para hallar $A_{m}$ y $B_{m}$, la herramienta de cálculo necesita recurrir a las condiciones iniciales en las que se encuentra el pilar. Ambas condiciones vendrán representadas por las funciones $f(z)$ y $g(z)$, que representan respectivamente el desplazamiento inicial y la velocidad inicial, a lo largo de la pila para cada paso temporal $\left(t_{i}\right)$, y $F(z)$ que representará la fuerza ejercida por el oleaje en ese instante $t_{\mathrm{i}}$.

$$
\begin{gathered}
w\left(z, t=t_{i}\right)=f\left(z, t=t_{i}\right) \\
\frac{\partial w}{\partial t}\left(z, t=t_{i}\right)=g\left(z, t=t_{i}\right)
\end{gathered}
$$

Para cada instante de tiempo, el modelo de cálculo hallará la respuesta de la estructura frente a una deformación dada mediante un análisis estático.

$$
R\left(z, t=t_{i}\right)=-E I \cdot \frac{\partial^{4} f\left(z, t=t_{i}\right)}{\partial z^{4}}
$$

Posteriormente, restará esta respuesta estructural sobre la carga actuante $F\left(z, t=t_{i}\right)$ :

$$
\Delta F\left(z, t=t_{i}\right)=F\left(z, t=t_{i}\right)-R\left(z, t=t_{i}\right)
$$

A continuación, el software despeja los coeficientes $A_{m}$ y $B_{m}$, a partir de la aceleración inicial generada por el oleaje para ese instante $t_{i}$, $y$ la velocidad inicial $g(z)$ : 


$$
\begin{gathered}
g(z)=\sum_{m=1}^{\infty} A_{m} \omega_{m} Z_{m} \\
\frac{\Delta F(z)}{\rho_{e s t} A}=-\sum_{m=1}^{\infty} B_{m} \omega_{m}^{2} Z_{m}
\end{gathered}
$$

siendo:

- A el área del cilindro

- $\quad \rho_{\text {est }}$ densidad de la estructura

De esta forma se obtiene despejando, que $A_{m}$ y $B_{m}$ son respectivamente:

$$
\begin{gathered}
A_{m}=\frac{1}{\omega_{m}} \frac{\int_{0}^{l} g(z) Z_{m}(z) d z}{\int_{0}^{l} Z_{m}^{2}(z) d z} \\
B_{m}=\frac{1}{\omega_{m}^{2} \rho_{e s t} A} \frac{\int_{0}^{l} \Delta F(z) \cdot Z_{m}(z) d z}{\int_{0}^{l} Z_{m}^{2}(z) d z}
\end{gathered}
$$

Posteriormente, se hallan las funciones $f(z)$ y $g(z)$ para el siguiente instante temporal:

$$
\begin{gathered}
f\left(z, t=t_{i+1}\right)=\sum_{m=1}^{\infty} \mathrm{Z}_{\mathrm{m}}(z) \cdot\left(A_{m} \operatorname{sen} \omega_{m} t+B_{m} \cos \omega_{m} t\right) \\
g\left(z, t=t_{i+1}\right)=\sum_{m=1}^{\infty} \mathrm{Z}_{\mathrm{m}}(z) \cdot \omega_{m}\left(A_{m} \cos \omega_{m} t-B_{m} \operatorname{sen} \omega_{m} t\right)
\end{gathered}
$$

Mediante este método, se conocerá la deformación que sufre la estructura a lo largo del tiempo, así como su velocidad y aceleración instantánea. Además, se evaluará la respuesta estructural $R(z, t)$ generada para cada diferencial de tiempo.

Normalmente, en la mayor parte de los casos, solo es necesario resolver el análisis modal de los primeros 6 modos propios de vibración para obtener una solución acorde a la 
realidad. No obstante, en el presente estudio el software ha sido optimizado para aportar una gran potencia computacional, y por lo tanto se puede proceder a estudiar los primeros 25 modos de vibración sin un incremento sustancial del tiempo de cálculo.

En la figura 13, se muestra de forma esquemática el proceso de cálculo que realiza el software desarrollado mediante la presente Tesis Doctoral:

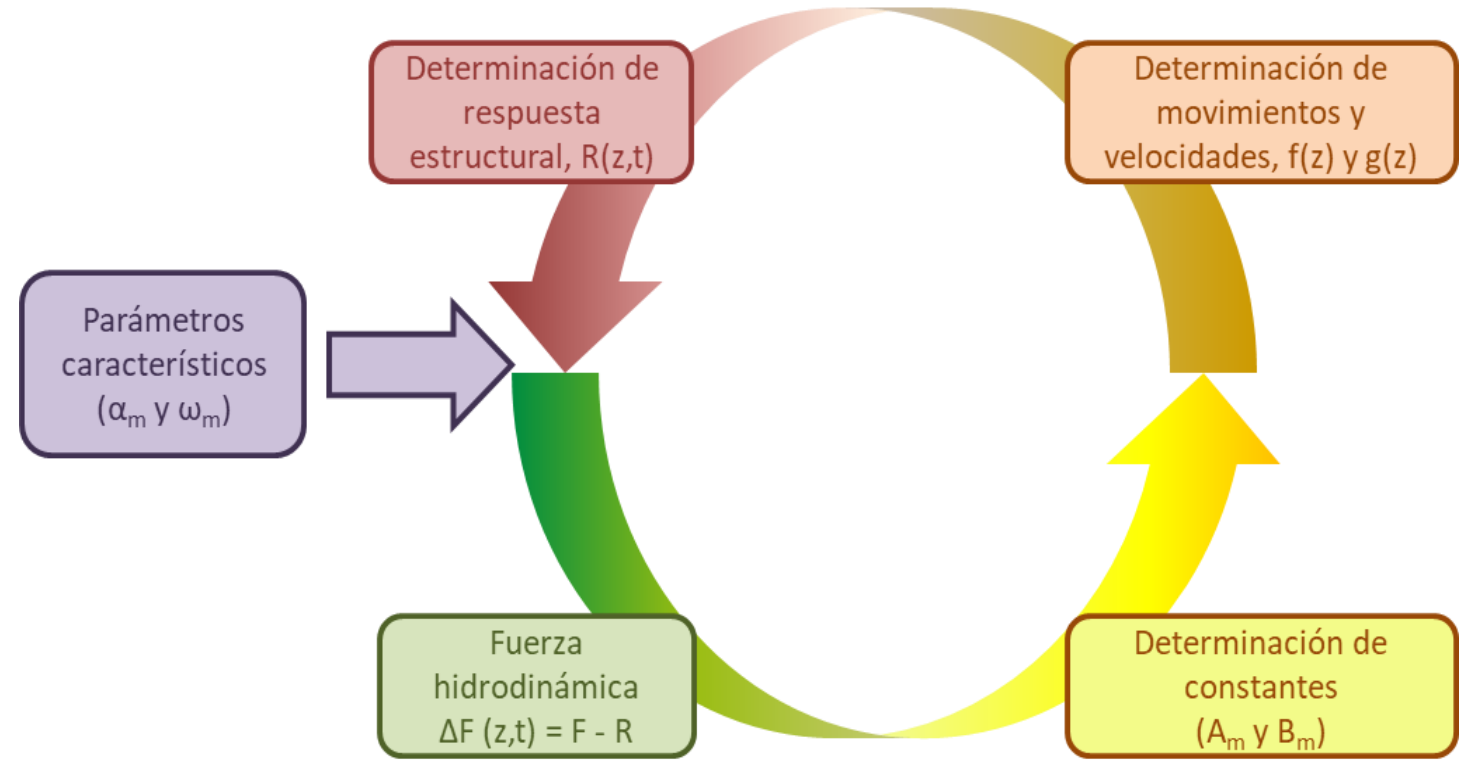

Figura 13. Proceso de cálculo (fuente: elaboración propia)

\subsubsection{Proceso IV: Acoplamiento procesos II y III}

A partir de los movimientos de la estructura hallados para cada instante temporal, y sus velocidades y aceleraciones instantáneas, se realizará un acoplamiento de los sistemas II y III. Este proceso tendrá un carácter iterativo hasta que se obtenga una convergencia de los resultados para todo el dominio de cálculo. De esta forma se podrán incluir en el cálculo los efectos derivados del acoplamiento entre el oleaje y la estructura.

En primer lugar, se introducen las aceleraciones y las velocidades de la estructura, en el proceso número II, para poder calcular las fuerzas hidrodinámicas teniendo en cuenta las velocidades y aceleraciones relativas, entre el fluido y la estructura. De esta forma se modifican las fuerzas hidrodinámicas halladas por dicho proceso, que calculaban las 
velocidades y aceleraciones del fluido, considerando a la estructura como un elemento infinitamente rígido.

$$
F=F_{\text {inercia }}\left(a_{\text {fluido }}-a_{\text {estr }}\right)+F_{\text {arrastre }}\left(v_{\text {fluido }}-v_{\text {estr }}\right)
$$

En segundo lugar, se vuelve a calcular cada uno de los estados de deformación de la estructura mediante el proceso de cálculo III, de acuerdo a las nuevas cargas hidrodinámicas calculadas mediante el proceso II.

El proceso se realiza 3 veces de forma inicial y, posteriormente, continúa iterando hasta que los resultados entre un paso de iteración y los dos pasos anteriores difieren en menos de un $0,1 \%$.

$$
\begin{aligned}
& \left|\frac{F_{i}-F_{i-2}}{F_{i}}\right| \leq 0,001 \\
& \left|\frac{F_{i}-F_{i-1}}{F_{i}}\right| \leq 0,001
\end{aligned}
$$

En la mayor parte de los ensayos realizados, la convergencia se consigue utilizando únicamente los tres primeros procesos de iteración. No obstante, existen situaciones en los que el oleaje puede sufrir acoplamiento con las frecuencias naturales de la estructura, lo que genera problemas de convergencia en los primeros pasos.

Posteriormente, tras obtener la convergencia de todos los estados de carga sufridos por la estructura para unas condiciones de oleaje dadas y para un determinado periodo de tiempo, el software procede a realizar el cálculo tensional y la distribución de presiones sobre la estructura, para detectar los instantes temporales en que se producen los casos pésimos de carga.

Para ello, se calcula el momento flector generado y el cortante para cada valor de $z$ y de $t$.

$$
M_{f}(z, t)=\int_{0}^{z} F(h, t) \cdot(z-h) \cdot d h
$$




$$
Q(z, t)=-\frac{\partial M_{f}}{\partial z}
$$

Posteriormente, se realiza su análisis de tensiones generadas por la flexión y por la compresión:

$$
\begin{gathered}
\sigma_{z}=\frac{M_{f}}{I_{y}} \cdot h_{x} \\
\sigma_{x}=\frac{3 F}{2 \pi} \cdot \frac{z^{3}}{\left(x^{2}+y^{2}+z^{2}\right)^{5 / 2}} \\
\tau_{x y}=\frac{Q \cdot m_{y}\left(h_{x}\right)}{I_{y} \cdot b\left(h_{x}\right)}
\end{gathered}
$$

De esta forma se analizarán:

- Los puntos críticos dentro de la estructura, que mostrarán donde se producirá el fallo de la misma a fatiga.

- Los puntos críticos de la cimentación, que implicarán la máxima tensión que soportará la cimentación de la estructura.

\subsubsection{Proceso V: Comportamiento de la cimentación.}

Una vez determinados los esfuerzos máximos, que sufrirá la cimentación, así como el campo de velocidades y aceleraciones verticales y horizontales del fluido, en su interfaz con el lecho marino, es posible caracterizar el fenómeno de socavación en el lecho marino, a largo plazo.

De acuerdo a la definición del perfil de velocidades del flujo marino definida por Nielsen (1992), la acción predominante en el fenómeno de socavación de un aerogenerador offshore, será el oleaje, debido a que la socavación estará localizada en la denominada zona inferior próxima al lecho (III).

Sumer et al. (1992) desarrollaron la siguiente fórmula adimensional, para el cálculo de la profundidad máxima de socavación en equilibrio, "Smax", alrededor de una pila expuesta simplemente a la acción del oleaje: 


$$
\frac{S_{\max }}{D}=1,3\left(1-e^{-0,03(K C-6)}\right) \quad \text { para } K C \geq 6
$$

Tal y como se demostró en el capítulo 2, a partir de la fórmula de Morison et al. es posible expresar el número de Keulegan-Carpenter, para el caso de un cilindro, como el ratio entre la máxima fuerza de inercia y la máxima fuerza de arrastre, de acuerdo con la siguiente expresión:

$$
\begin{gathered}
\frac{\left.F_{i}\right|_{\max }}{\left.F_{D}\right|_{\max }}=\pi^{2} \cdot \frac{C_{M}}{C_{D}} \cdot \frac{1}{K C} \\
K C=\frac{\left(U_{w} \cdot T\right)}{D}=\pi^{2} \cdot \frac{C_{M}}{C_{D}} \cdot \frac{\left.F_{D}\right|_{\max }}{\left.F_{i}\right|_{\max }} \\
K C=\pi^{2} \cdot \frac{C_{M}}{C_{D}} \cdot \frac{\rho \cdot \frac{D}{2} \cdot C_{D} \cdot u_{\max }^{2}}{\rho \cdot \frac{\pi D^{2}}{4} \cdot C_{M} \cdot a_{\max }}=\frac{2 \cdot \pi \cdot u_{\max }^{2}}{D \cdot a_{\max }}
\end{gathered}
$$

Este hecho permite reformular la expresión desarrollada por Sumer et al. (1992), incorporando el efecto de la aceleración del fluido sobre la socavación de la cimentación.

$$
\mathrm{S}_{\max }=1,3 \cdot \mathrm{D} \cdot\left(1-e^{-0,03\left(\frac{2 \cdot \pi \cdot u_{\max }^{2}}{D \cdot a_{\max }}-6\right)}\right)
$$

Se va calcular la profundidad máxima de socavación en equilibrio, S, para todos los casos analizados, mediante el uso de la formulación anterior. No obstante, para poder comparar los resultados con los datos medidos en campo, será necesario añadir el efecto de la corriente en el lecho marino, a la formula anterior. De esta forma se podrá predecir la profundidad máxima de socavación en equilibro, teniendo en cuenta de forma conjunta condiciones de oleaje y corriente. Para ello, se utilizará la fórmula modificada de Sumer et al. (2002) que tiene en cuenta condiciones de oleaje y corriente, de forma conjunta.

$$
\mathrm{S}_{\max }=1,3 \cdot \mathrm{D} \cdot\left(1-e^{-\mathrm{A}\left(\frac{2 \cdot \pi \cdot u_{\max }^{2}}{D \cdot a_{\max }}-\mathrm{B}\right)}\right)
$$


donde:

$$
\begin{gathered}
A=0,03+\frac{3}{4} U_{c w}^{2,6} \\
B=6 \mathrm{e}^{-4,7 \mathrm{U}_{c w}} \\
U_{c w}=\frac{U_{c}}{U_{c}+U_{m}}
\end{gathered}
$$

siendo $U_{c}$, la velocidad de la corriente y $U_{m}$ la velocidad del agua, debida al oleaje.

\subsubsection{Esquema de cálculo}

De acuerdo a la definición de los distintos procesos que se han descrito a lo largo de este capítulo, se propone el esquema de cálculo que se muestra en la figura 14:

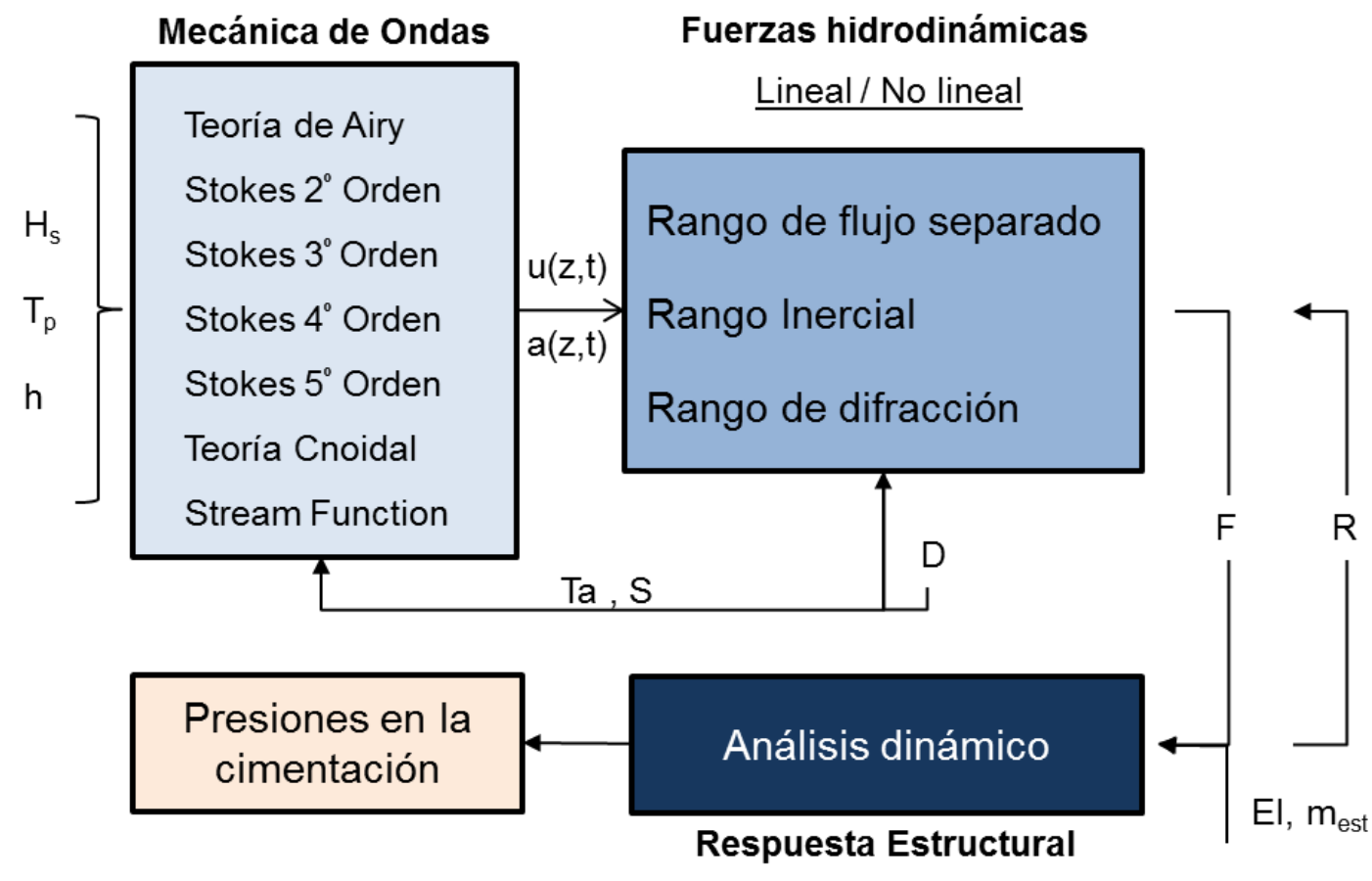

Figura 14. Proceso de cálculo general propuesto (fuente: elaboración propia)

Las variables representadas en el esquema anterior son:

- $\mathrm{H}_{\mathrm{s}}$ es la altura de ola significativa en metros para un periodo de retorno de 50 años. 
- $T_{p}$ es el periodo de pico asociado a $\mathrm{H}_{\mathrm{s}}$ en segundos.

- h es la profundidad en metros.

- Ta es la temperatura del agua en grados centígrados.

- S es la salinidad del agua en \%.

- D es el diámetro de la estructura en metros.

- El es la rigidez de la estructura en $\mathrm{Nm}^{2}$.

- $\mathrm{m}_{\text {est }}$ es la masa de la estructura en $\mathrm{kg} / \mathrm{m}$.

donde:

- $\mathrm{u}(\mathrm{z}, \mathrm{t})$ es el campo de velocidades calculado en $\mathrm{m} / \mathrm{s}$.

- $a(z, t)$ es el campo de aceleraciones calculado en $\mathrm{m} / \mathrm{s}^{2}$.

- F es la distribución de fuerzas hidrodinámicas calculadas en $\mathrm{N}$.

- $\mathrm{R}$ es la respuesta estructural calculada en $\mathrm{N}$.

Dicho esquema permite integrar todas las variables de cálculo de las que se carece en el método tradicional, descrito en el punto 2.1. 


\section{Capítulo 5}

APLICACIÓN DE LA HERRAMIENTA A LOS CASOS DE ESTUDIO 



\section{Capítulo 5}

\section{APLICACIÓN DE LA HERRAMIENTA A LOS CASOS DE ESTUDIO}

\section{Introducción}

A lo largo de este capítulo, se va a proceder a realizar una exposición completa y detallada de los diferentes resultados obtenidos mediante la utilización de la herramienta de cálculo desarrollada mediante la presente Tesis Doctoral. La metodología de cálculo y los casos a estudiar han sido descritos en detalle, en el "Capítulo III: Metodología de la Investigación".

Además, se va a realizar una discusión profunda de dichos resultados, que dará paso a las conclusiones principales en el siguiente capítulo.

Debido al gran número de casos de cálculo, los resultados parciales de las distintas simulaciones serán recopilados en el Apéndice 1 con objeto de no sobrecargar en exceso el cuerpo principal de la presente Tesis Doctoral.

El esquema que seguirá el capítulo para mostrar los diferentes resultados, estará dividido según los distintos procesos de cálculo que fueron expuestos en el capítulo anterior.

- Proceso I: Mecánica de ondas

- Caso A: cálculo según stokes de 3o Orden y cálculo según la teoría lineal.

- Caso B: cálculo según stokes de 50 Orden y cálculo según la teoría lineal.

- Caso C: cálculo según onda cnoidal de 2 o Orden y cálculo según la teoría lineal.

- Caso D: cálculo según Stream Function de Dean y cálculo según la teoría lineal. 
Modelos de predicción de esfuerzos hidrodinámicos y socavación aplicados a ingeniería offshore

- Proceso II: Cálculo de fuerzas hidrodinámicas

- Caso A: Diámetro igual a 0,5 m.

- Caso B: Diámetro igual a 4,0 m.

- Caso C: Diámetro igual a $8,0 \mathrm{~m}$.

- Caso D: Diámetro igual a $16,0 \mathrm{~m}$.

- Proceso III: Análisis dinámico

- Resultados de cálculo estático

- Resultados con 25 modos de vibración

- Proceso IV: Acoplamiento de procesos II y III

- Resultados tras acoplamiento de procesos

- Proceso V: Comportamiento de la cimentación 


\section{Exposición de resultados}

De acuerdo con todo lo expuesto en el anterior capítulo, se procede en este a realizar la exposición de los resultados.

\subsection{Proceso I: Mecánica de ondas}

De acuerdo con los resultados mostrados en el capítulo de los casos de estudio, es posible extraer las siguientes conclusiones a partir de los objetivos que se planteaban:

a. Comparación de la variación del campo de velocidades y aceleraciones entre la teoría lineal de Airy (1845) y el resto de teorías.

b. Análisis de principales frecuencias que componen el oleaje incidente.

c. Análisis sobre la variación de la amortiguación de la velocidad y la aceleración con la profundidad según las distintas teorías.

Se muestra a continuación, a modo de ejemplo, la salida gráfica del programa para uno de los casos estudiados, el caso A - Princess Amalia Q7 en Holanda, mostrando en las figuras 1 y 3 el cálculo según el modelo avanzado y en las figuras 2 y 4 el cálculo según la teoría lineal. La salida gráfica del programa para el resto de casos se adjunta en el apéndice 1 de la presente Tesis Doctoral.

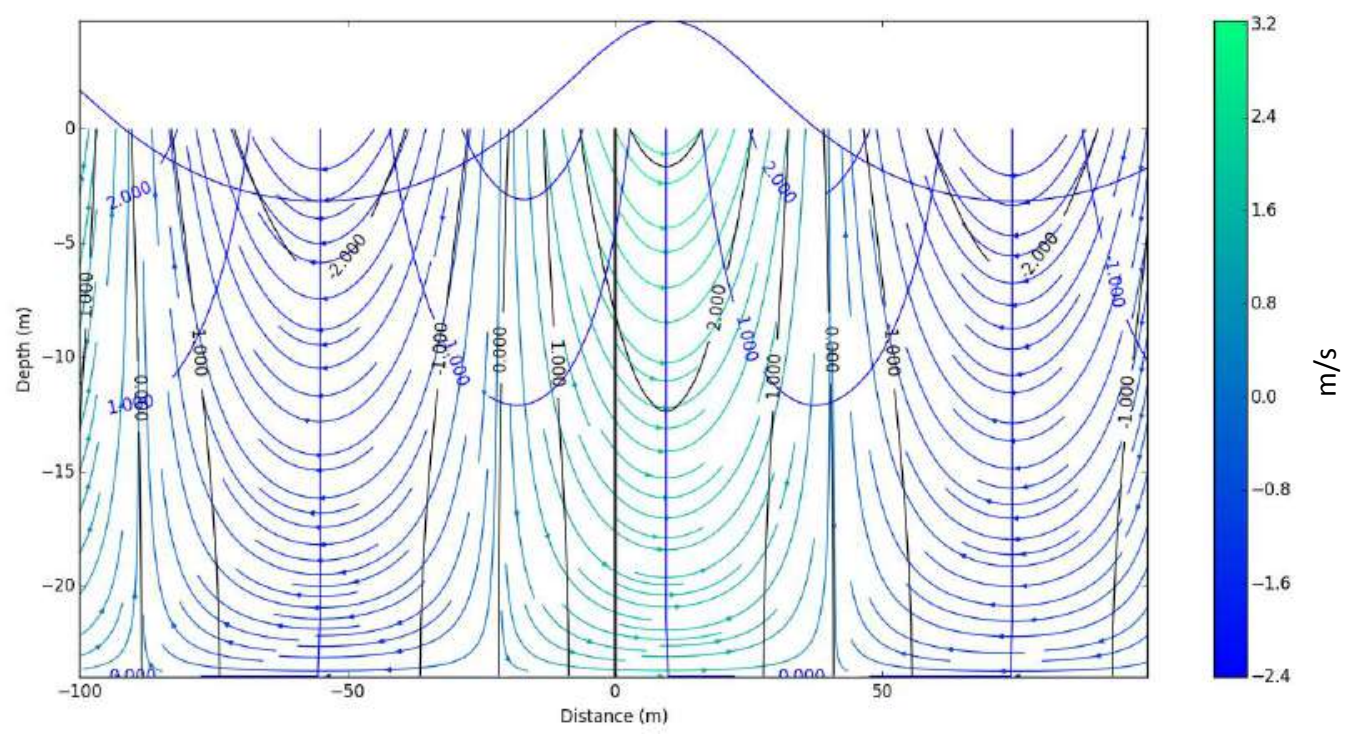

Figura 1. Velocidades para $H=7,7 m, h=24,0 m$ y $T=9,7 s$ según Stokes de 3O Orden 
Modelos de predicción de esfuerzos hidrodinámicos y socavación aplicados a ingeniería offshore

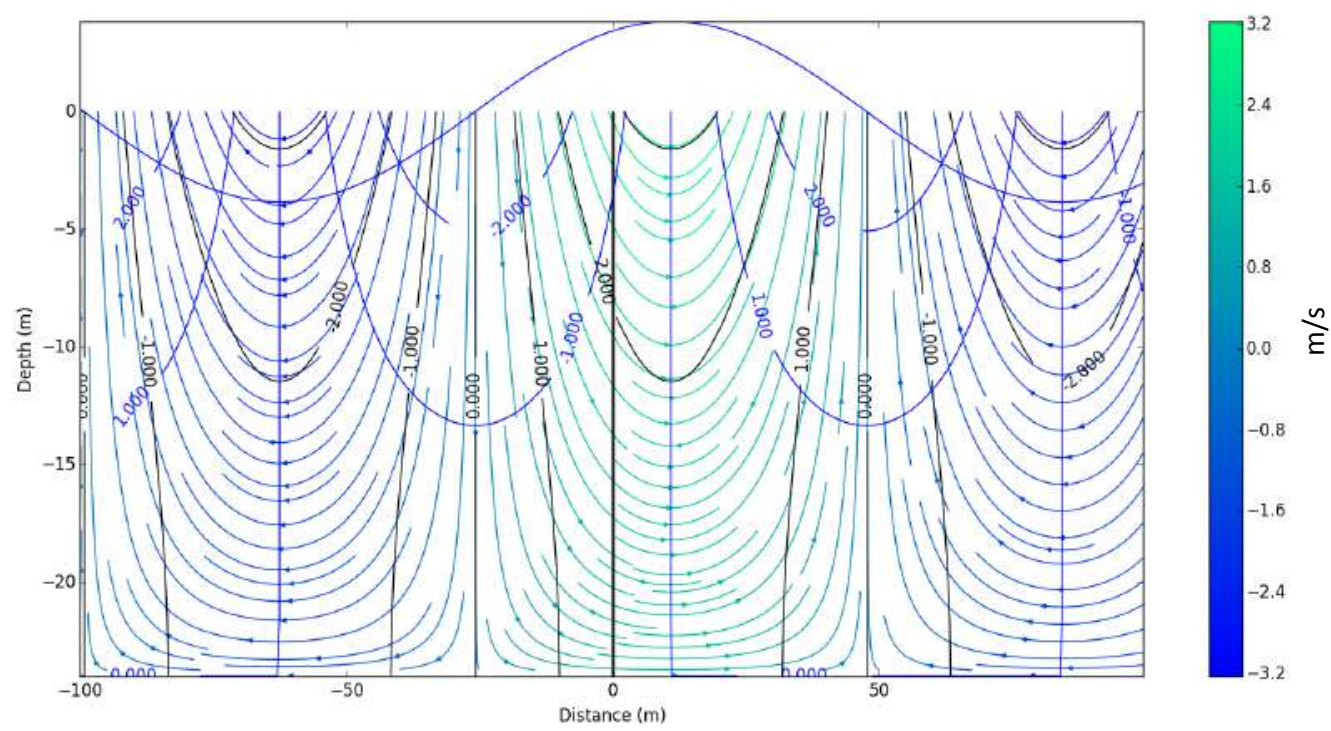

Figura 2. Velocidades para $H=7,7 m, h=24,0 m$ y $T=9,7 s$ según teoría lineal

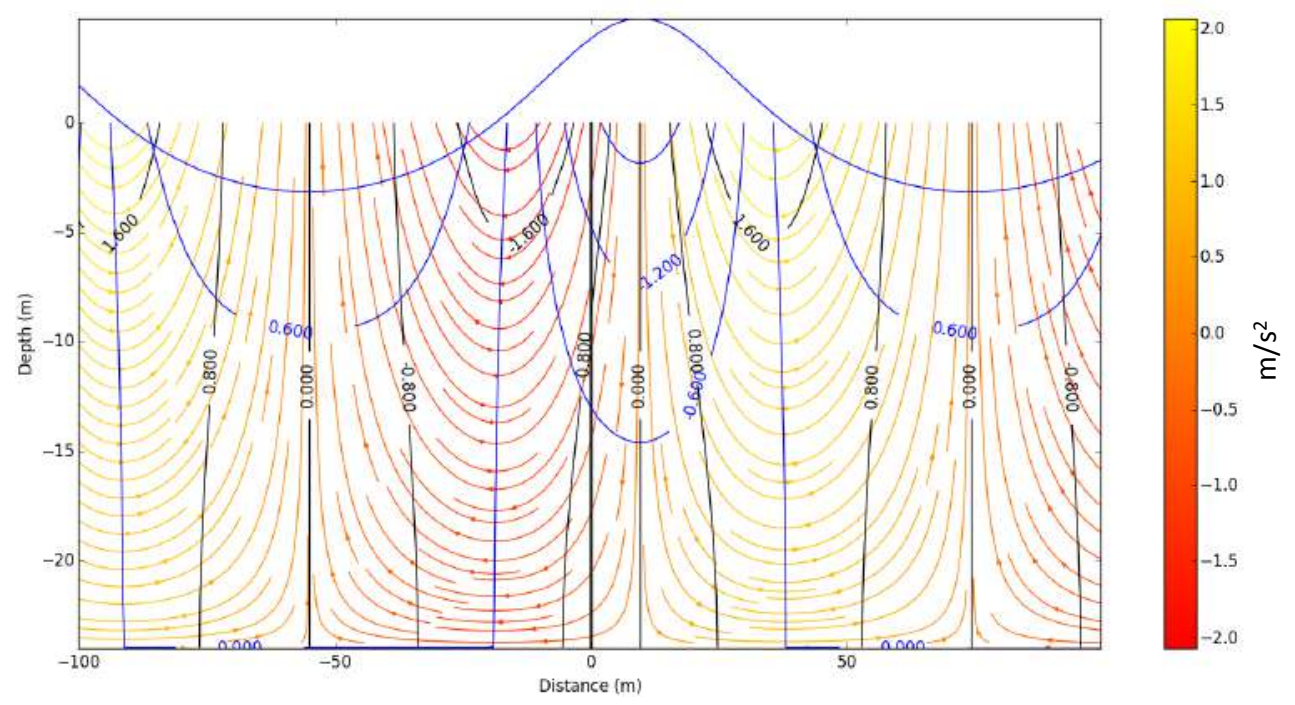

Figura 3. Aceleraciones para $H=7,7 m, h=24,0 m$ y $T=9,7 s$ según Stokes de 3O Orden

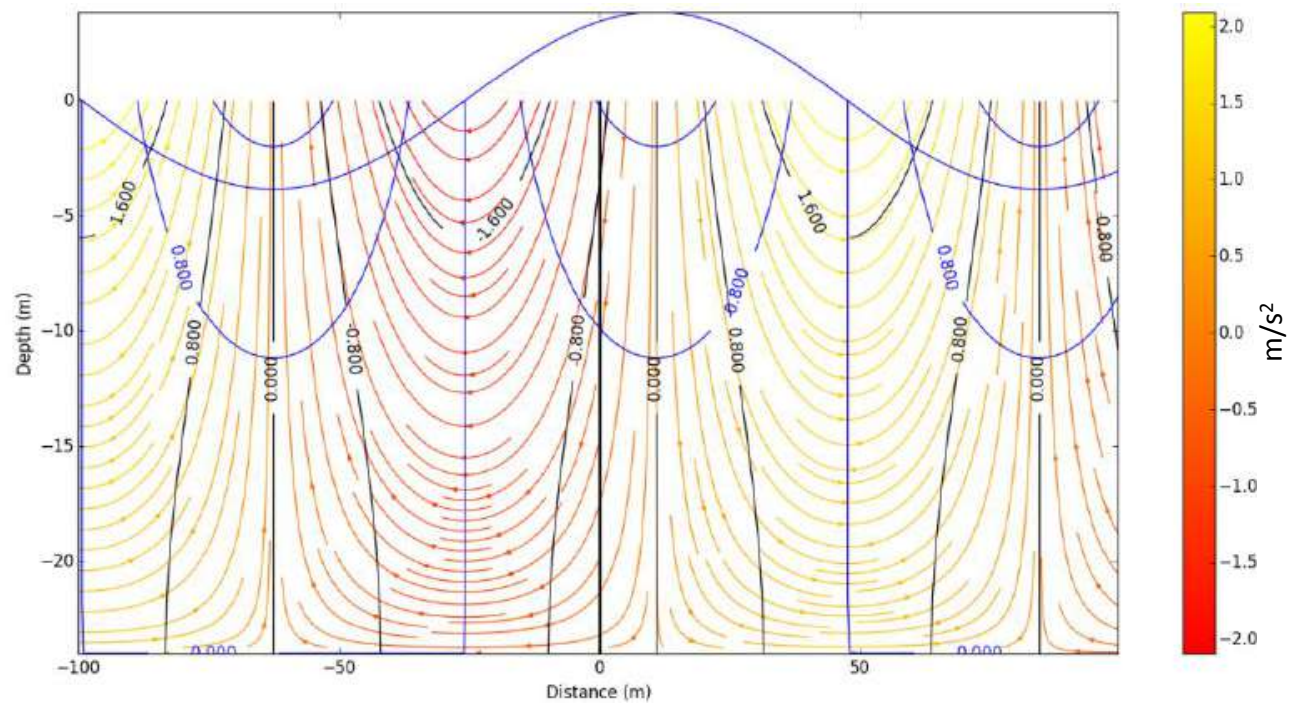

Figura 4. Aceleraciones para $H=7,7 m, h=24,0 m$ y $T=9,7 s$ según teoría lineal 
En primer lugar, el análisis de velocidades muestra una clara disminución de los valores correspondientes a las distintas teorías no lineales con respecto a los valores procedentes de la aplicación de la teoría lineal, prácticamente en todos los casos analizados. Esta variación afecta en cada uno de los casos de una forma independiente. En la tabla 1 se muestra el cálculo de las diferencias existentes entre los resultados aportados por las teorías no lineales con respecto a los valores resultantes de la aplicación de la teoría lineal:

$$
\text { Diferencia }=v_{\text {lineal }}-v_{\text {no lineal }}
$$

\begin{tabular}{|c|l|c|c|c|c|}
\hline Zona & \multicolumn{1}{|c|}{ Parámetro } & CASO A & CASO B & CASO C & CASO D \\
\hline \multirow{4}{*}{ Superficie } & Máxima velocidad horizontal (m/s) & $-0,0031$ & 0,2132 & 0,5826 & 0,5633 \\
\cline { 2 - 6 } & Máxima velocidad vertical (m/s) & 0,3707 & 0,7144 & 1,2137 & 1,0405 \\
\cline { 2 - 6 } & Media de velocidad horizontal (m/s) & 0,2446 & 0,5111 & 0,798 & 0,7789 \\
\cline { 2 - 6 } & Media de velocidad vertical (m/s) & 0,3072 & 0,6533 & 0,8425 & 0,8993 \\
\hline \multirow{4}{*}{ Fondo } & Máxima velocidad horizontal (m/s) & $-0,0824$ & $-0,1314$ & 0,1024 & 0,0589 \\
\cline { 2 - 6 } & Máxima velocidad vertical $(\mathrm{m} / \mathrm{s})$ & 0 & 0 & 0 & 0 \\
\cline { 2 - 6 } & Media de velocidad horizontal $(\mathrm{m} / \mathrm{s})$ & $-0,0022$ & 0,0501 & 0,3519 & 0,2318 \\
\cline { 2 - 6 } & Media de velocidad vertical $(\mathrm{m} / \mathrm{s})$ & 0 & 0 & 0 & 0 \\
\hline
\end{tabular}

Tabla 1. Error de los resultados de velocidades entre la teoría lineal y las no lineales

En la tabla 2 se muestran dichas variaciones calculadas a nivel relativo de acuerdo con la siguiente expresión:

$$
\% \text { Error }=\frac{v_{\text {lineal }}-v_{\text {no lineal }}}{v_{\text {no lineal }}}
$$

\begin{tabular}{|c|l|c|c|c|c|}
\hline Zona & \multicolumn{1}{|c|}{ Parámetro } & CASO A & CASO B & CASO C & CASO D \\
\hline \multirow{4}{*}{ Superficie } & Máxima velocidad horizontal (m/s) & $-0,10 \%$ & $7,26 \%$ & $31,78 \%$ & $17,85 \%$ \\
\cline { 2 - 6 } & Máxima velocidad vertical (m/s) & $15,23 \%$ & $36,68 \%$ & $187,24 \%$ & $54,06 \%$ \\
\cline { 2 - 6 } & Media de velocidad horizontal (m/s) & $13,51 \%$ & $34,24 \%$ & $107,85 \%$ & $49,01 \%$ \\
\cline { 2 - 6 } & Media de velocidad vertical (m/s) & $20,77 \%$ & $62,73 \%$ & $245,70 \%$ & $90,99 \%$ \\
\hline \multirow{3}{*}{ Fondo } & Máxima velocidad horizontal (m/s) & $-4,90 \%$ & $-7,25 \%$ & $7,13 \%$ & $2,69 \%$ \\
\cline { 2 - 6 } & Máxima velocidad vertical (m/s) & $0,00 \%$ & $0,00 \%$ & $0,00 \%$ & $0,00 \%$ \\
\cline { 2 - 6 } & Media de velocidad horizontal (m/s) & $-0,22 \%$ & $4,91 \%$ & $56,04 \%$ & $19,35 \%$ \\
\cline { 2 - 6 } & Media de velocidad vertical (m/s) & $0,00 \%$ & $0,00 \%$ & $0,00 \%$ & $0,00 \%$ \\
\hline
\end{tabular}

Tabla 2. Error relativo de los resultados de velocidades entre la teoría lineal y las no lineales

El máximo error se encuentra en el Caso C, correspondiente a la teoría cnoidal de 20 Orden, siendo la velocidad media vertical generada en superficie del orden de 3,5 veces más grande en el caso lineal que en el caso no lineal. 
Por otro lado, se observa que dichas variaciones en todos los casos tienen mucho más efecto en superficie que en fondo, lo cual demuestra que los efectos no lineales sufren una importante atenuación con la profundidad.

También se observa que, en todos los casos, dichas variaciones son mucho mayores en la dirección vertical con respecto a las variaciones mostradas en la dirección horizontal.

En segundo lugar, el análisis de aceleraciones muestra, de igual forma, una disminución general de los valores correspondientes a las distintas teorías no lineales con respecto a los valores procedentes de la teoría lineal. En la tabla 3 se muestra el cálculo de las diferencias existentes entre los resultados aportados por las teorías no lineales con respecto a los valores resultantes de la aplicación de la teoría lineal:

Diferencia $=a_{\text {lineal }}-a_{\text {no lineal }}$

\begin{tabular}{|c|l|c|c|c|c|}
\hline Zona & \multicolumn{1}{|c|}{ Parámetro } & CASO A & CASO B & CASO C & CASO D \\
\hline \multirow{4}{*}{ Superficie } & Máxima aceleración horizontal $\left(\mathrm{m} / \mathrm{s}^{2}\right)$ & 0,022 & $-0,1263$ & 0,2559 & 0,0671 \\
\cline { 2 - 6 } & Máxima aceleración vertical $\left(\mathrm{m} / \mathrm{s}^{2}\right)$ & 0,7839 & 1,2249 & 0,4883 & 0,9229 \\
\cline { 2 - 6 } & Media de aceleración horizontal $\left(\mathrm{m} / \mathrm{s}^{2}\right)$ & 0,1635 & 0,4617 & 0,2491 & 0,4265 \\
\cline { 2 - 6 } & Media de aceleración vertical $\left(\mathrm{m} / \mathrm{s}^{2}\right)$ & 0,1515 & 0,4142 & 0,2624 & 0,3774 \\
\hline \multirow{4}{*}{ Fondo } & Máxima aceleración horizontal $\left(\mathrm{m} / \mathrm{s}^{2}\right)$ & $-0,0199$ & $-0,0858$ & 0,1154 & 0,0373 \\
\cline { 2 - 6 } & Máxima aceleración vertical $\left(\mathrm{m} / \mathrm{s}^{2}\right)$ & 0 & 0 & 0 & 0 \\
\cline { 2 - 6 } & Media de aceleración horizontal $\left(\mathrm{m} / \mathrm{s}^{2}\right)$ & $-0,0015$ & 0,046 & 0,1134 & 0,1298 \\
\cline { 2 - 6 } & Media de aceleración vertical $\left(\mathrm{m} / \mathrm{s}^{2}\right)$ & 0 & 0 & 0 & 0 \\
\hline
\end{tabular}

En la tabla 4 se muestran dichas variaciones calculadas a nivel relativo de acuerdo con la siguiente expresión:

$$
\% \text { Error }=\frac{a_{\text {lineal }}-a_{\text {no lineal }}}{a_{\text {no lineal }}}
$$

\begin{tabular}{|c|l|c|c|c|c|}
\hline Zona & \multicolumn{1}{|c|}{ Parámetro } & CASO A & CASO B & CASO C & CASO D \\
\hline \multirow{4}{*}{ Superficie } & Máxima aceleración horizontal $\left(\mathrm{m} / \mathrm{s}^{2}\right)$ & $1,06 \%$ & $-4,16 \%$ & $45,32 \%$ & $3,28 \%$ \\
\cline { 2 - 6 } & Máxima aceleración vertical $\left(\mathrm{m} / \mathrm{s}^{2}\right)$ & $75,87 \%$ & $99,21 \%$ & $338,86 \%$ & $121,18 \%$ \\
\cline { 2 - 6 } & Media de aceleración horizontal $\left(\mathrm{m} / \mathrm{s}^{2}\right)$ & $13,99 \%$ & $33,22 \%$ & $91,18 \%$ & $46,42 \%$ \\
\cline { 2 - 6 } & Media de aceleración vertical $\left(\mathrm{m} / \mathrm{s}^{2}\right)$ & $15,08 \%$ & $35,96 \%$ & $187,16 \%$ & $54,30 \%$ \\
\hline
\end{tabular}




\begin{tabular}{|c|l|c|c|c|c|}
\hline Zona & \multicolumn{1}{|c|}{ Parámetro } & CASO A & CASO B & CASO C & CASO D \\
\hline \multirow{4}{*}{ Fondo } & Máxima aceleración horizontal $\left(\mathrm{m} / \mathrm{s}^{2}\right)$ & $-1,89 \%$ & $-5,24 \%$ & $28,33 \%$ & $3,01 \%$ \\
\cline { 2 - 6 } & Máxima aceleración vertical $\left(\mathrm{m} / \mathrm{s}^{2}\right)$ & $0,00 \%$ & $0,00 \%$ & $0,00 \%$ & $0,00 \%$ \\
\cline { 2 - 6 } & Media de aceleración horizontal $\left(\mathrm{m} / \mathrm{s}^{2}\right)$ & $-0,23 \%$ & $4,88 \%$ & $51,69 \%$ & $19,02 \%$ \\
\cline { 2 - 6 } & Media de aceleración vertical $\left(\mathrm{m} / \mathrm{s}^{2}\right)$ & $0,00 \%$ & $0,00 \%$ & $0,00 \%$ & $0,00 \%$ \\
\hline
\end{tabular}

Tabla 4. Error relativo de los resultados de aceleraciones entre la teoría lineal y las no lineales

En este caso, el máximo error se encuentra también en el Caso C, correspondiente a la teoría cnoidal de 20 Orden, siendo la aceleración media vertical generada en superficie del orden de 2,87 veces más grande en el caso lineal que en el caso no lineal.

Por otro lado, también se observa que en todos los casos, dichas variaciones tienen mucho más efecto en superficie que en fondo, como ocurría con las velocidades y que dichas variaciones son mayores en la dirección vertical con respecto a las variaciones mostradas en la dirección horizontal.

\subsection{Proceso II: Cálculo de las fuerzas hidrodinámicas}

El segundo proceso se corresponde con el cálculo de las acciones que generará el oleaje sobre la estructura a partir de las variables que definen el comportamiento de dicho oleaje, principalmente el campo de velocidades ( $v$ ) y el campo de aceleraciones (a). Los principales objetivos de este segundo proceso de cálculo son los siguientes:

- Comprobación de las fuerzas hidrodinámicas generadas por el método avanzado con respecto a las generadas por el método tradicional para cada teoría de mecánica de ondas.

- Análisis de la influencia de la temperatura y la salinidad sobre la distribución y magnitud de las cargas para cada teoría de mecánica de ondas.

- Análisis de la influencia de los efectos no lineales sobre la distribución y magnitud de las cargas para cada teoría de mecánica de ondas.

- Amortiguación de las distintas fuerzas con la profundidad.

Se muestra a continuación, a modo de ejemplo, la salida gráfica del programa para uno de los casos estudiados, el caso 1A - Princess Amalia Q7 en Holanda y diámetro de 0,5 metros, mostrando en la figura 5, el cálculo según el modelo avanzado y, en la figura 6, el cálculo 
según la teoría lineal. La salida gráfica del programa para el resto de casos se adjunta en el apéndice 1 de la presente Tesis Doctoral.

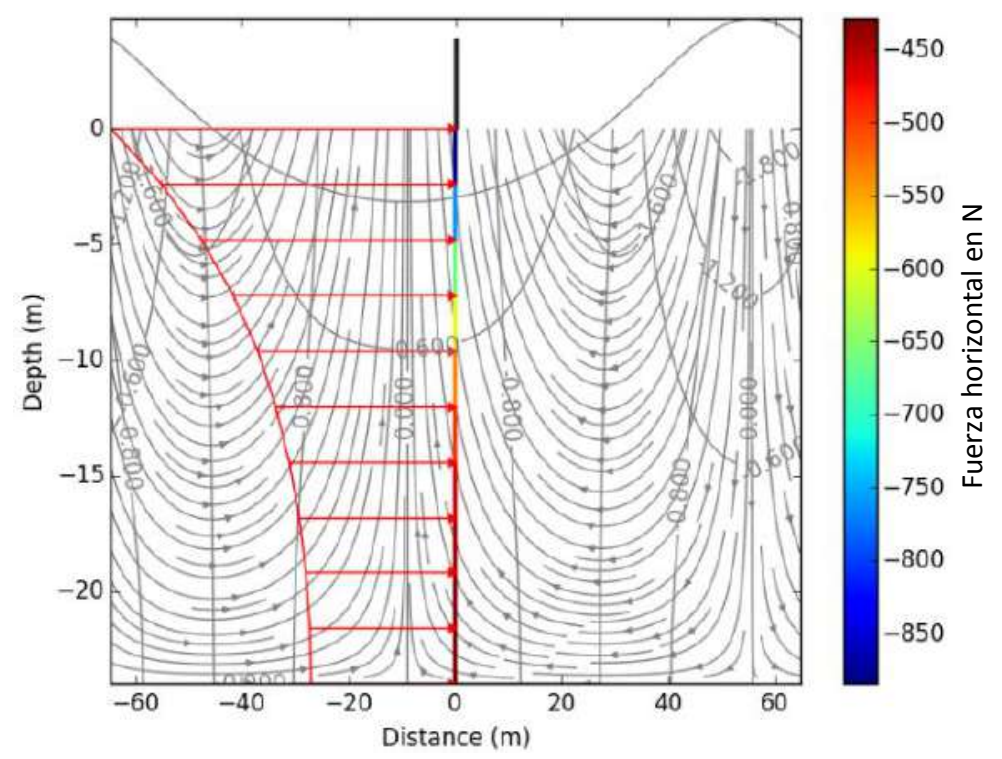

Figura 5. Momento flector máximo según método avanzado para $H=7,7 m, h=24,0 m, T=9,7 s$ y $D=0,5 m$

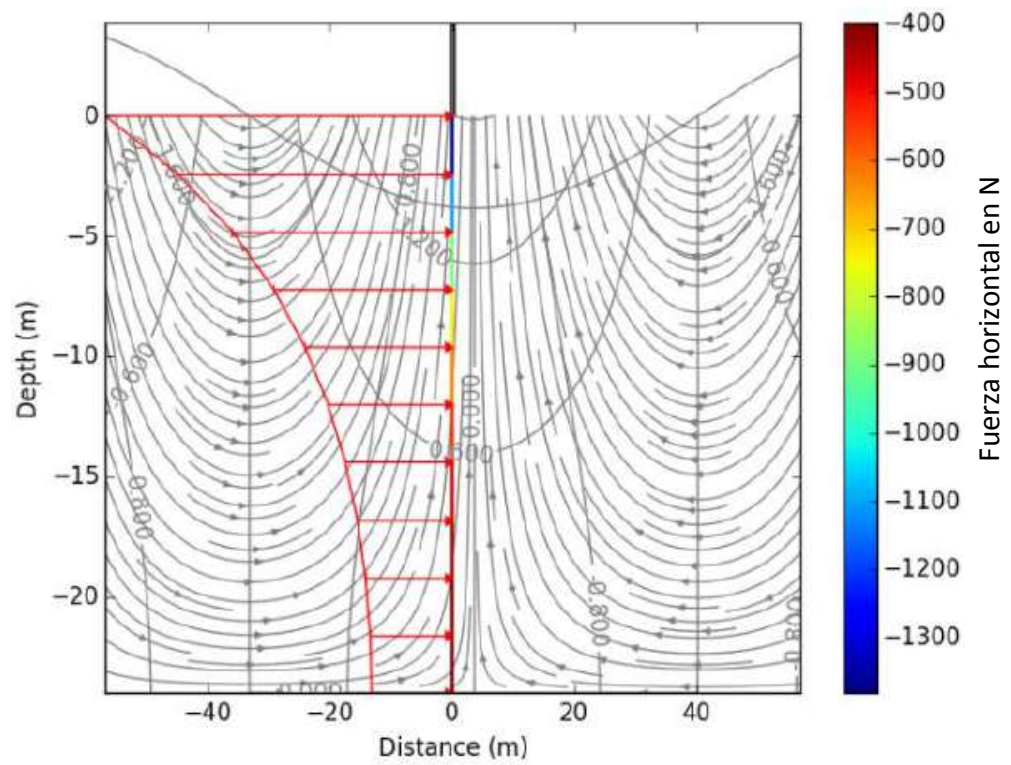

Figura 6. Momento flector máximo según método tradicional para $H=7,7 \mathrm{~m}, h=24,0 \mathrm{~m}, T=9,7 \mathrm{~s}$ y $D=0,5 \mathrm{~m}$

\subsubsection{Comparación entre método tradicional y método avanzado}

En este punto, se va a proceder a realizar el análisis comparativo entre los resultados obtenidos según el método tradicional, que aplica la teoría de Morison et al. (1950) y la teoría lineal de ondas, sin tener en cuenta los efectos no lineales ni de difracción y, los resultados obtenidos por el método avanzado, que como se explicó en el capítulo cuarto, tiene en cuenta todos los efectos no lineales, la difracción y la mecánica de ondas que mejor se ajusta al caso de estudio. 
El análisis de los resultados muestra un claro aumento de los valores procedentes del método tradicional con respecto a los procedentes del método avanzado. Esta variación afecta en cada uno de los casos de una forma independiente. En las tablas 5 y 6 se muestran dichas variaciones para el caso de temperatura de $1^{\circ} \mathrm{C}$ y salinidad del $0,0 \%$ y el caso de temperatura de $22^{\circ} \mathrm{C}$ y salinidad del $35 \%$, calculadas de acuerdo con la siguiente expresión:

$$
\% \text { Variación }=F_{\text {Tradicional }}-F_{\text {Avanzado }}
$$

\begin{tabular}{|c|c|c|c|c|c|}
\hline & & \multicolumn{4}{|c|}{ Temperatura de $1^{\circ} \mathrm{C}$ y Salinidad del 0,0\% } \\
\hline \multicolumn{6}{|c|}{ CASO A - Princess Amalia Q7 } \\
\hline & Parámetro & $\mathrm{D}=0,5 \mathrm{~m}$ & $\mathrm{D}=4,0 \mathrm{~m}$ & $\mathrm{D}=8,0 \mathrm{~m}$ & $\mathrm{D}=16,0 \mathrm{~m}$ \\
\hline \multirow{5}{*}{$\begin{array}{l}\frac{0}{U} \\
\dot{U} \frac{1}{d} \\
\frac{0}{J} \\
u\end{array}$} & Máxima fuerza de inercia (N) & 9,15 & $3.353,70$ & $15.634,90$ & $62.543,90$ \\
\hline & Máxima fuerza de arrastre $(\mathrm{N})$ & 758,28 & $6.066,20$ & $12.132,10$ & $24.264,80$ \\
\hline & Máxima fuerza no lineal (N) & $-10,83$ & $-668,30$ & $-2.373,20$ & $-4.691,00$ \\
\hline & Máx. fuerza de difracción (N) & 0,00 & 0,00 & 0,00 & 0,00 \\
\hline & Máxima fuerza total (N) & 636,10 & $2.743,70$ & $14.272,70$ & $59.955,50$ \\
\hline \multirow{5}{*}{$\begin{array}{l}\circ \\
\frac{0}{0} \\
\text { 온 }\end{array}$} & Máxima fuerza de inercia $(\mathrm{N})$ & $-8,08$ & $-425,60$ & $-1.254,70$ & $-4.294,30$ \\
\hline & Máxima fuerza de arrastre (N) & 98,26 & 786,00 & $1.572,00$ & $3.144,20$ \\
\hline & Máxima fuerza no lineal (N) & $-9,87$ & $-631,80$ & $-2.527,20$ & $-10.108,80$ \\
\hline & Máx. fuerza de difracción (N) & 0,00 & 0,00 & 0,00 & 0,00 \\
\hline & Máxima fuerza total (N) & 12,81 & $-336,10$ & $-936,60$ & $-3.150,60$ \\
\hline \multicolumn{6}{|c|}{ CASO B - North Hoyle } \\
\hline \multirow{5}{*}{$\begin{array}{l}\frac{0}{0} \\
\frac{0}{\frac{0}{d}} \\
\frac{0}{J} \\
v\end{array}$} & Máxima fuerza de inercia (N) & $-23,93$ & $9.957,90$ & $45.102,20$ & $193.319,10$ \\
\hline & Máxima fuerza de arrastre $(\mathrm{N})$ & 908,05 & $7.264,10$ & $14.528,30$ & $57.215,10$ \\
\hline & Máxima fuerza no lineal (N) & $-0,29$ & $-69,00$ & $-1.339,20$ & $-4.325,40$ \\
\hline & Máx. fuerza de difracción (N) & 0,00 & 0,00 & 0,00 & $-40.510,60$ \\
\hline & Máxima fuerza total (N) & 369,69 & $9.143,70$ & $44.229,70$ & $201.357,10$ \\
\hline \multirow{5}{*}{$\begin{array}{l}\text { 음 } \\
\text { ㅇ }\end{array}$} & Máxima fuerza de inercia (N) & $-34,74$ & $-208,70$ & $1.011,10$ & $10.682,70$ \\
\hline & Máxima fuerza de arrastre $(\mathrm{N})$ & 72,55 & 580,30 & $1.160,70$ & $16.301,90$ \\
\hline & Máxima fuerza no lineal (N) & $-21,72$ & $-1.390,20$ & $-5.561,30$ & $-7.587,60$ \\
\hline & Máx. fuerza de difracción (N) & 0,00 & 0,00 & 0,00 & $-30.307,70$ \\
\hline & Máxima fuerza total (N) & $-108,88$ & 146,50 & $2.568,90$ & $16.448,50$ \\
\hline \multicolumn{6}{|c|}{ CASO C - Egmond Aan Zee } \\
\hline \multirow{5}{*}{$\begin{array}{l}\frac{0}{0} \\
\frac{u}{\frac{U}{d}} \\
\frac{0}{J} \\
\bar{n}\end{array}$} & Máxima fuerza de inercia (N) & 104,51 & $10.287,10$ & $41.149,50$ & $164.593,90$ \\
\hline & Máxima fuerza de arrastre $(\mathrm{N})$ & 446,68 & $3.573,50$ & $7.147,00$ & $14.294,10$ \\
\hline & Máxima fuerza no lineal (N) & 0,00 & 0,00 & 0,00 & 0,00 \\
\hline & Máx. fuerza de difracción (N) & 0,00 & 0,00 & 0,00 & 0,00 \\
\hline & Máxima fuerza total (N) & 435,23 & $8.560,60$ & $38.121,40$ & $158.933,40$ \\
\hline \multirow{5}{*}{$\begin{array}{l}\text { 음 } \\
\text { 온 }\end{array}$} & Máxima fuerza de inercia (N) & 46,73 & $4.905,30$ & $20.976,00$ & $87.759,40$ \\
\hline & Máxima fuerza de arrastre (N) & 54,99 & 439,90 & 879,90 & $1.759,80$ \\
\hline & Máxima fuerza no lineal (N) & 0,00 & 0,00 & 0,00 & 0,00 \\
\hline & Máx. fuerza de difracción (N) & 0,00 & 0,00 & 0,00 & 0,00 \\
\hline & Máxima fuerza total (N) & 39,46 & $4.812,40$ & $20.964,30$ & $87.431,90$ \\
\hline
\end{tabular}


Modelos de predicción de esfuerzos hidrodinámicos y socavación aplicados a ingeniería offshore

CASO D - Thornton Bank

\begin{tabular}{|c|c|c|c|c|c|}
\hline \multicolumn{6}{|c|}{ CASO D - Thornton Bank } \\
\hline \multirow{5}{*}{ 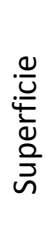 } & Máxima fuerza de inercia (N) & 71,20 & $13.384,60$ & $55.639,50$ & $232.726,50$ \\
\hline & Máxima fuerza de arrastre $(\mathrm{N})$ & 1915,15 & $15.320,80$ & $30.642,30$ & $61.284,70$ \\
\hline & Máxima fuerza no lineal $(\mathrm{N})$ & $-3,14$ & $-207,50$ & $-908,70$ & $-4.891,80$ \\
\hline & Máx. fuerza de difracción (N) & 0,00 & 0,00 & 0,00 & 0,00 \\
\hline & Máxima fuerza total (N) & 1546,59 & $12.873,80$ & $54.993,30$ & $232.906,30$ \\
\hline \multirow{5}{*}{$\begin{array}{l}\text { 음 } \\
\text { 은 } \\
\text { L }\end{array}$} & Máxima fuerza de inercia (N) & 15,08 & $3.587,60$ & $15.815,30$ & $66.789,50$ \\
\hline & Máxima fuerza de arrastre (N) & 558,97 & $4.471,60$ & $8.943,50$ & $17.887,00$ \\
\hline & Máxima fuerza no lineal (N) & $-9,12$ & $-583,40$ & $-2.333,60$ & $-9.334,20$ \\
\hline & Máx. fuerza de difracción (N) & 0,00 & 0,00 & 0,00 & 0,00 \\
\hline & Máxima fuerza total (N) & 319,24 & $3.646,60$ & $16.386,10$ & $69.456,20$ \\
\hline
\end{tabular}

Tabla 5. Comparación entre método avanzado y tradicional para $T^{\underline{a}}=1^{\circ} \mathrm{C}$ y $\mathrm{S}=0,0 \%$

Temperatura de $22^{\circ} \mathrm{C}$ y Salinidad del 35\%

\begin{tabular}{|c|c|c|c|c|c|}
\hline \multicolumn{6}{|c|}{ CASO A - Princess Amalia Q7 } \\
\hline & Parámetro & $D=0,5 m$ & $\mathrm{D}=4,0 \mathrm{~m}$ & $\mathrm{D}=8,0 \mathrm{~m}$ & $\mathrm{D}=16,0 \mathrm{~m}$ \\
\hline \multirow{5}{*}{ 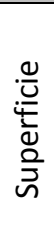 } & Máxima fuerza de inercia (N) & 20,99 & $3.613,90$ & $15.646,40$ & $88.809,90$ \\
\hline & Máxima fuerza de arrastre (N) & 758,84 & $6.070,60$ & $12.141,00$ & $24.282,60$ \\
\hline & Máxima fuerza no lineal (N) & $-10,84$ & $-668,80$ & $-2.374,90$ & $-4.694,40$ \\
\hline & Máx. fuerza de difracción (N) & 0,00 & 0,00 & 0,00 & 0,00 \\
\hline & Máxima fuerza total (N) & 636,57 & $3.078,50$ & $14.321,60$ & $86.440,20$ \\
\hline \multirow{5}{*}{$\begin{array}{l}\text { 음 } \\
\text { 운 }\end{array}$} & Máxima fuerza de inercia (N) & $-8,08$ & $-358,80$ & $-1.074,80$ & $-3.504,50$ \\
\hline & Máxima fuerza de arrastre $(\mathrm{N})$ & 98,33 & 786,60 & $1.573,20$ & $3.146,50$ \\
\hline & Máxima fuerza no lineal (N) & $-9,88$ & $-632,20$ & $-2.529,00$ & $-10.116,10$ \\
\hline & Máx. fuerza de difracción (N) & 0,00 & 0,00 & 0,00 & 0,00 \\
\hline & Máxima fuerza total (N) & 40,34 & $-280,00$ & $-806,60$ & $-2.591,20$ \\
\hline \multicolumn{6}{|c|}{ CASO B - North Hoyle } \\
\hline \multirow{5}{*}{$\begin{array}{l}\frac{0}{U} \\
\frac{i}{4} \\
\frac{0}{\nu} \\
\frac{0}{\nu} \\
\sim\end{array}$} & Máxima fuerza de inercia (N) & 44,85 & $11.550,90$ & $48.365,10$ & $167.584,80$ \\
\hline & Máxima fuerza de arrastre (N) & 908,71 & $7.269,30$ & $14.538,90$ & $57.256,90$ \\
\hline & Máxima fuerza no lineal $(\mathrm{N})$ & $-0,29$ & $-69,00$ & $-1.340,20$ & $-4.328,60$ \\
\hline & Máx. fuerza de difracción (N) & 0,00 & 0,00 & 0,00 & $-40.540,20$ \\
\hline & Máxima fuerza total (N) & 493,25 & $10.722,40$ & $47.354,40$ & $175.750,60$ \\
\hline \multirow{5}{*}{$\begin{array}{l}\text { 음 } \\
\text { ㅇ } \\
\text {. }\end{array}$} & Máxima fuerza de inercia (N) & $-34,22$ & 320,20 & $1.829,20$ & $10.690,60$ \\
\hline & Máxima fuerza de arrastre $(\mathrm{N})$ & 72,61 & 580,80 & $1.161,60$ & $16.313,80$ \\
\hline & Máxima fuerza no lineal (N) & $-21,74$ & $-1.391,30$ & $-5.565,40$ & $-7.593,10$ \\
\hline & Máx. fuerza de difracción (N) & 0,00 & 0,00 & 0,00 & $-30.329,90$ \\
\hline & Máxima fuerza total (N) & $-85,48$ & 624,50 & $3.294,10$ & $16.481,10$ \\
\hline \multicolumn{6}{|c|}{ CASO C - Egmond Aan Zee } \\
\hline \multirow{5}{*}{ 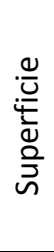 } & Máxima fuerza de inercia (N) & 123,91 & $10.294,60$ & $41.179,70$ & $164.714,10$ \\
\hline & Máxima fuerza de arrastre $(\mathrm{N})$ & 447,01 & $3.576,20$ & $7.152,20$ & $14.304,60$ \\
\hline & Máxima fuerza no lineal (N) & 0,00 & 0,00 & 0,00 & 0,00 \\
\hline & Máx. fuerza de difracción (N) & 0,00 & 0,00 & 0,00 & 0,00 \\
\hline & Máxima fuerza total (N) & 435,55 & $8.565,40$ & $38.149,30$ & $159.049,40$ \\
\hline \multirow{4}{*}{$\begin{array}{l}\text { 음 } \\
\text { 운 }\end{array}$} & Máxima fuerza de inercia (N) & 49,45 & $5.202,70$ & $21.550,70$ & $89.183,70$ \\
\hline & Máxima fuerza de arrastre (N) & 55,03 & 440,30 & 880,60 & $1.761,10$ \\
\hline & Máxima fuerza no lineal (N) & 0,00 & 0,00 & 0,00 & 0,00 \\
\hline & Máx. fuerza de difracción (N) & 0,00 & 0,00 & 0,00 & 0,00 \\
\hline
\end{tabular}


Modelos de predicción de esfuerzos hidrodinámicos y socavación aplicados a ingeniería offshore

\begin{tabular}{|c|c|c|c|c|c|}
\hline & Máxima fuerza total (N) & 51,15 & $4.815,90$ & $21.118,90$ & $87.495,90$ \\
\hline \multicolumn{6}{|c|}{ CASO D - Thornton Bank } \\
\hline \multirow{5}{*}{ 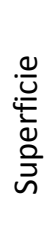 } & Máxima fuerza de inercia (N) & 128,73 & $13.955,20$ & $58.223,80$ & $232.896,50$ \\
\hline & Máxima fuerza de arrastre (N) & 1916,55 & $15.332,30$ & $30.664,70$ & $61.329,50$ \\
\hline & Máxima fuerza no lineal $(\mathrm{N})$ & $-3,14$ & $-207,70$ & $-909,40$ & $-4.895,30$ \\
\hline & Máx. fuerza de difracción (N) & 0,00 & 0,00 & 0,00 & 0,00 \\
\hline & Máxima fuerza total (N) & 1577,22 & $13.021,00$ & $56.626,50$ & $233.076,40$ \\
\hline \multirow{5}{*}{$\begin{array}{l}\text { 음 } \\
\text { 은 }\end{array}$} & Máxima fuerza de inercia (N) & 23,19 & $3.968,80$ & $16.709,50$ & $66.838,40$ \\
\hline & Máxima fuerza de arrastre (N) & 559,38 & $4.475,00$ & $8.950,00$ & $17.900,10$ \\
\hline & Máxima fuerza no lineal $(\mathrm{N})$ & $-9,12$ & $-583,80$ & $-2.335,30$ & $-9.341,00$ \\
\hline & Máx. fuerza de difracción (N) & 0,00 & 0,00 & 0,00 & 0,00 \\
\hline & Máxima fuerza total (N) & 391,01 & $4.028,30$ & $17.224,40$ & $69.583,70$ \\
\hline
\end{tabular}

Tabla 6. Comparación entre método avanzado y tradicional para $T^{a}=22^{\circ} \mathrm{C}$ y $\mathrm{S}=35,0 \%$

En las tablas 7 y 8 se muestran dichas variaciones a nivel relativo para el caso de temperatura de $1^{\circ} \mathrm{C}$ y salinidad del $0,0 \%$ y el caso de temperatura de $22^{\circ} \mathrm{C}$ y salinidad del 35\%, calculadas de acuerdo con la siguiente expresión:

$$
\% \text { Variación }=\frac{F_{\text {Tradicional }}-F_{\text {Avanzado }}}{F_{\text {Avanzado }}}
$$

\begin{tabular}{|c|c|c|c|c|c|}
\hline & & \multicolumn{4}{|c|}{ Temperatura de $1^{\circ} \mathrm{C}$ y Salinidad del $0,0 \%$} \\
\hline \multicolumn{6}{|c|}{ CASO A - Princess Amalia Q7 } \\
\hline & Parámetro & $\mathrm{D}=0,5 \mathrm{~m}$ & $\mathrm{D}=4,0 \mathrm{~m}$ & $\mathrm{D}=8,0 \mathrm{~m}$ & $\mathrm{D}=16,0 \mathrm{~m}$ \\
\hline \multirow{5}{*}{ 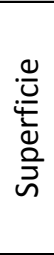 } & Máxima fuerza de inercia & $1,09 \%$ & $6,60 \%$ & $7,77 \%$ & $7,77 \%$ \\
\hline & Máxima fuerza de arrastre & $67,57 \%$ & $67,57 \%$ & $67,57 \%$ & $67,57 \%$ \\
\hline & Máxima fuerza no lineal & $-100,00 \%$ & $-100,00 \%$ & $-100,00 \%$ & $-100,00 \%$ \\
\hline & Máxima fuerza de difracción & - & - & - & - \\
\hline & Máxima fuerza total & $49,01 \%$ & $5,33 \%$ & $7,05 \%$ & $7,43 \%$ \\
\hline \multirow{5}{*}{$\begin{array}{l}\text { 음 } \\
\text { 인 }\end{array}$} & Máxima fuerza de inercia & $-1,89 \%$ & $-1,56 \%$ & $-1,16 \%$ & $-0,99 \%$ \\
\hline & Máxima fuerza de arrastre & $27,12 \%$ & $27,12 \%$ & $27,12 \%$ & $27,12 \%$ \\
\hline & Máxima fuerza no lineal & $-100,00 \%$ & $-100,00 \%$ & $-100,00 \%$ & $-100,00 \%$ \\
\hline & Máxima fuerza de difracción & - & - & - & - \\
\hline & Máxima fuerza total & $2,41 \%$ & $-1,24 \%$ & $-0,87 \%$ & $-0,73 \%$ \\
\hline \multicolumn{6}{|c|}{ CASO B - North Hoyle } \\
\hline \multirow{5}{*}{ 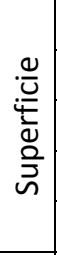 } & Máxima fuerza de inercia & $-1,99 \%$ & $15,22 \%$ & $17,59 \%$ & $19,09 \%$ \\
\hline & Máxima fuerza de arrastre & $103,19 \%$ & $103,19 \%$ & $103,19 \%$ & - \\
\hline & Máxima fuerza no lineal & $-100,00 \%$ & $-100,00 \%$ & $-100,00 \%$ & $-100,00 \%$ \\
\hline & Máxima fuerza de difracción & - & - & - & $-100,00 \%$ \\
\hline & Máxima fuerza total & $24,20 \%$ & $13,81 \%$ & $17,19 \%$ & $20,04 \%$ \\
\hline \multirow{4}{*}{$\begin{array}{l}\circ \\
\frac{0}{0} \\
\text { 온 } \\
\text {. }\end{array}$} & Máxima fuerza de inercia & $-5,24 \%$ & $-0,52 \%$ & $0,63 \%$ & $1,69 \%$ \\
\hline & Máxima fuerza de arrastre & $16,61 \%$ & $16,60 \%$ & $16,60 \%$ & - \\
\hline & Máxima fuerza no lineal & $-100,00 \%$ & $-100,00 \%$ & $-100,00 \%$ & $-100,00 \%$ \\
\hline & Máxima fuerza de difracción & - & - & - & $-100,00 \%$ \\
\hline
\end{tabular}


Modelos de predicción de esfuerzos hidrodinámicos y socavación aplicados a ingeniería offshore

\begin{tabular}{|c|c|c|c|c|c|}
\hline & Máxima fuerza total & $-13,10 \%$ & $0,37 \%$ & $1,62 \%$ & $2,62 \%$ \\
\hline \multicolumn{6}{|c|}{ CASO C - Egmond Aan Zee } \\
\hline \multirow{5}{*}{ 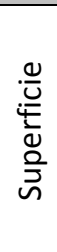 } & Máxima fuerza de inercia & $45,90 \%$ & $93,74 \%$ & $93,74 \%$ & $93,74 \%$ \\
\hline & Máxima fuerza de arrastre & $73,67 \%$ & $73,68 \%$ & $73,67 \%$ & $73,68 \%$ \\
\hline & Máxima fuerza no lineal & - & - & - & - \\
\hline & Máxima fuerza de difracción & - & - & - & - \\
\hline & Máxima fuerza total & $68,81 \%$ & $67,39 \%$ & $81,24 \%$ & $87,69 \%$ \\
\hline \multirow{5}{*}{$\begin{array}{l}\circ \\
\frac{0}{0} \\
\text { ㅇ }\end{array}$} & Máxima fuerza de inercia & $28,33 \%$ & $56,77 \%$ & $63,17 \%$ & $68,04 \%$ \\
\hline & Máxima fuerza de arrastre & $14,76 \%$ & $14,76 \%$ & $14,77 \%$ & $14,77 \%$ \\
\hline & Máxima fuerza no lineal & - & - & - & - \\
\hline & Máxima fuerza de difracción & - & - & - & - \\
\hline & Máxima fuerza total & $9,73 \%$ & $55,10 \%$ & $63,11 \%$ & $67,62 \%$ \\
\hline \multicolumn{6}{|c|}{ CASO D - Thornton Bank } \\
\hline \multirow{5}{*}{ 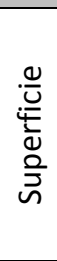 } & Máxima fuerza de inercia & $9,08 \%$ & $32,35 \%$ & $34,05 \%$ & $36,17 \%$ \\
\hline & Máxima fuerza de arrastre & $329,34 \%$ & $329,34 \%$ & $329,35 \%$ & $329,34 \%$ \\
\hline & Máxima fuerza no lineal & $-100,00 \%$ & $-100,00 \%$ & $-100,00 \%$ & $-100,00 \%$ \\
\hline & Máxima fuerza de difracción & - & - & - & - \\
\hline & Máxima fuerza total & $156,03 \%$ & $30,73 \%$ & $33,52 \%$ & $36,21 \%$ \\
\hline \multirow{5}{*}{$\begin{array}{l}\circ \\
\frac{0}{0} \\
\text { 인 }\end{array}$} & Máxima fuerza de inercia & $3,01 \%$ & $12,17 \%$ & $13,58 \%$ & $14,45 \%$ \\
\hline & Máxima fuerza de arrastre & $159,13 \%$ & $159,13 \%$ & $159,13 \%$ & $159,13 \%$ \\
\hline & Máxima fuerza no lineal & $-100,00 \%$ & $-100,00 \%$ & $-100,00 \%$ & $-100,00 \%$ \\
\hline & Máxima fuerza de difracción & - & - & - & - \\
\hline & Máxima fuerza total & $50,49 \%$ & $12,40 \%$ & $14,14 \%$ & $15,11 \%$ \\
\hline
\end{tabular}

Tabla 7. Comparación a nivel relativo entre método avanzado y tradicional para $T^{a}=1^{\circ} \mathrm{C}$ y $\mathrm{S}=0,0 \%$

Temperatura de $22^{\circ} \mathrm{C}$ y Salinidad del $35 \%$

CASO A - Princess Amalia Q7

\begin{tabular}{|c|c|c|c|c|c|}
\hline \multicolumn{2}{|r|}{ Parámetro } & $D=0,5 m$ & $\mathrm{D}=4,0 \mathrm{~m}$ & $\mathrm{D}=8,0 \mathrm{~m}$ & $\mathrm{D}=16,0 \mathrm{~m}$ \\
\hline \multirow{5}{*}{ 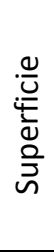 } & Máxima fuerza de inercia & $2,54 \%$ & $7,14 \%$ & $7,77 \%$ & $11,40 \%$ \\
\hline & Máxima fuerza de arrastre & $67,57 \%$ & $67,57 \%$ & $67,57 \%$ & $67,57 \%$ \\
\hline & Máxima fuerza no lineal & $-100,00 \%$ & $-100,00 \%$ & $-100,00 \%$ & $-100,00 \%$ \\
\hline & Máxima fuerza de difracción & - & - & - & - \\
\hline & Máxima fuerza total & $49,01 \%$ & $6,02 \%$ & $7,07 \%$ & $11,06 \%$ \\
\hline \multirow{5}{*}{$\begin{array}{l}\text { 음 } \\
\text { 인 }\end{array}$} & Máxima fuerza de inercia & $-1,89 \%$ & $-1,32 \%$ & $-0,99 \%$ & $-0,81 \%$ \\
\hline & Máxima fuerza de arrastre & $27,12 \%$ & $27,12 \%$ & $27,12 \%$ & $27,12 \%$ \\
\hline & Máxima fuerza no lineal & $-100,00 \%$ & $-100,00 \%$ & $-100,00 \%$ & $-100,00 \%$ \\
\hline & Máxima fuerza de difracción & - & - & - & - \\
\hline & Máxima fuerza total & $8,51 \%$ & $-1,03 \%$ & $-0,75 \%$ & $-0,60 \%$ \\
\hline \multicolumn{6}{|c|}{ CASO B - North Hoyle } \\
\hline \multirow{5}{*}{ 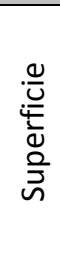 } & Máxima fuerza de inercia & $3,96 \%$ & $18,08 \%$ & $19,09 \%$ & $16,54 \%$ \\
\hline & Máxima fuerza de arrastre & $103,19 \%$ & $103,19 \%$ & $103,19 \%$ & - \\
\hline & Máxima fuerza no lineal & $-100,00 \%$ & $-100,00 \%$ & $-100,00 \%$ & $-100,00 \%$ \\
\hline & Máxima fuerza de difracción & - & - & - & $-100,00 \%$ \\
\hline & Máxima fuerza total & $35,10 \%$ & $16,57 \%$ & $18,62 \%$ & $17,48 \%$ \\
\hline \multirow{3}{*}{$\begin{array}{l}\text { 음 } \\
\text { 돈 }\end{array}$} & Máxima fuerza de inercia & $-5,16 \%$ & $0,80 \%$ & $1,15 \%$ & $1,69 \%$ \\
\hline & Máxima fuerza de arrastre & $16,61 \%$ & $16,61 \%$ & $16,61 \%$ & - \\
\hline & Máxima fuerza no lineal & $-100,00 \%$ & $-100,00 \%$ & $-100,00 \%$ & $-100,00 \%$ \\
\hline
\end{tabular}




\begin{tabular}{|c|c|c|c|c|c|}
\hline & Máxima fuerza de difracción & - & - & - & $-100,00 \%$ \\
\hline & Máxima fuerza total & $-11,44 \%$ & $1,58 \%$ & $2,09 \%$ & $2,63 \%$ \\
\hline \multicolumn{6}{|c|}{ CASO C - Egmond Aan Zee } \\
\hline \multirow{5}{*}{ 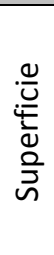 } & Máxima fuerza de inercia & $59,42 \%$ & $93,74 \%$ & $93,75 \%$ & $93,74 \%$ \\
\hline & Máxima fuerza de arrastre & $73,67 \%$ & $73,68 \%$ & $73,67 \%$ & $73,68 \%$ \\
\hline & Máxima fuerza no lineal & - & - & - & - \\
\hline & Máxima fuerza de difracción & - & - & - & - \\
\hline & Máxima fuerza total & $68,81 \%$ & $67,38 \%$ & $81,24 \%$ & $87,69 \%$ \\
\hline \multirow{5}{*}{$\begin{array}{l}\circ \\
\frac{0}{0} \\
\text { 인 }\end{array}$} & Máxima fuerza de inercia & $30,46 \%$ & $62,29 \%$ & $65,96 \%$ & $69,83 \%$ \\
\hline & Máxima fuerza de arrastre & $14,76 \%$ & $14,77 \%$ & $14,77 \%$ & $14,77 \%$ \\
\hline & Máxima fuerza no lineal & - & - & - & - \\
\hline & Máxima fuerza de difracción & - & - & - & - \\
\hline & Máxima fuerza total & $13,07 \%$ & $55,10 \%$ & $63,79 \%$ & $67,62 \%$ \\
\hline \multicolumn{6}{|c|}{ CASO D - Thornton Bank } \\
\hline \multirow{5}{*}{ 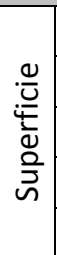 } & Máxima fuerza de inercia & $17,69 \%$ & $34,17 \%$ & $36,17 \%$ & $36,17 \%$ \\
\hline & Máxima fuerza de arrastre & $329,34 \%$ & $329,34 \%$ & $329,35 \%$ & $329,34 \%$ \\
\hline & Máxima fuerza no lineal & $-100,00 \%$ & $-100,00 \%$ & $-100,00 \%$ & $-100,00 \%$ \\
\hline & Máxima fuerza de difracción & - & - & - & - \\
\hline & Máxima fuerza total & $163,87 \%$ & $31,17 \%$ & $34,83 \%$ & $36,21 \%$ \\
\hline \multirow{5}{*}{$\begin{array}{l}\circ \\
\frac{0}{0} \\
\text { 운 }\end{array}$} & Máxima fuerza de inercia & $4,70 \%$ & $13,63 \%$ & $14,45 \%$ & $14,45 \%$ \\
\hline & Máxima fuerza de arrastre & $159,13 \%$ & $159,13 \%$ & $159,13 \%$ & $159,13 \%$ \\
\hline & Máxima fuerza no lineal & $-100,00 \%$ & $-100,00 \%$ & $-100,00 \%$ & $-100,00 \%$ \\
\hline & Máxima fuerza de difracción & - & - & - & - \\
\hline & Máxima fuerza total & $69,68 \%$ & $13,86 \%$ & $14,96 \%$ & $15,13 \%$ \\
\hline
\end{tabular}

Tabla 8. Comparación a nivel relativo entre método avanzado y tradicional para $T^{a}=22^{\circ} \mathrm{C}$ y $S=35,0 \%$

A partir de los resultados mostrados en las tablas anteriores, se puede observar que las máximas diferencias se producen en el caso de temperatura de $22^{\circ} \mathrm{C}$ y salinidad del $35 \%$ para el diámetro más pequeño, en el Caso D - Thornton Bank, en el cual la diferencia de la fuerza total máxima en superficie llega a ser del entorno de un $164 \%$ más elevada en el caso tradicional que la calculada según el método avanzado. Esto es debido a que las máximas diferencias entre el método tradicional y el método avanzado se producen en las fuerzas viscosas.

Por otro lado, se observa una segunda tendencia de crecimiento desde el diámetro de 4 metros hasta el diámetro de 16 metros, que viene causada por las fuerzas de inercia. Como puede observarse, la variación sufrida por las fuerzas de inercia experimenta un ligero crecimiento al mismo tiempo que crece el diámetro. 
También se observa que, generalmente, las fuerzas totales resultantes en fondo son superiores para el caso de cálculo tradicional a temperatura de $22^{\circ} \mathrm{C}$ y salinidad del $35 \%$, que para el caso de cálculo avanzado siendo la variación máxima del 69,68\%.

Además, se observa que todas las variaciones son mucho mayores en superficie que en fondo, como cabía esperar, de acuerdo con los resultados de mecánica de ondas mostrados en el Proceso I.

En la tablas 9 y 10 se muestran los valores de variación del máximo momento flector en el lecho marino.

\begin{tabular}{|l|c|c|c|c|}
\hline \multicolumn{5}{|c|}{ Temperatura de $\mathbf{1}^{\circ} \mathrm{C}$ y Salinidad del 0,0\% } \\
\hline \multicolumn{1}{|c|}{ Máximo momento flector } & $\mathrm{D}=0,5 \mathrm{~m}$ & $\mathrm{D}=4,0 \mathrm{~m}$ & $\mathrm{D}=8,0 \mathrm{~m}$ & $\mathrm{D}=16,0 \mathrm{~m}$ \\
\hline CASO A - Princess Amalia Q7 & $36,98 \%$ & $5,12 \%$ & $12,54 \%$ & $22,97 \%$ \\
\hline CASO B - North Hoyle & $3,81 \%$ & $15,20 \%$ & $27,10 \%$ & $32,14 \%$ \\
\hline CASO C - Egmond Aan Zee & $45,23 \%$ & $63,72 \%$ & $75,83 \%$ & $81,35 \%$ \\
\hline CASO D - Thornton Bank & $111,97 \%$ & $29,23 \%$ & $37,95 \%$ & $39,79 \%$ \\
\hline
\end{tabular}

\section{Temperatura de $22^{\circ} \mathrm{C}$ y Salinidad del $35 \%$}

\begin{tabular}{|l|c|c|c|c|}
\hline \multicolumn{1}{|c|}{ Máximo momento flector } & $\mathrm{D}=0,5 \mathrm{~m}$ & $\mathrm{D}=4,0 \mathrm{~m}$ & $\mathrm{D}=8,0 \mathrm{~m}$ & $\mathrm{D}=16,0 \mathrm{~m}$ \\
\hline CASO A - Princess Amalia Q7 & $37,13 \%$ & $9,84 \%$ & $22,52 \%$ & $30,78 \%$ \\
\hline CASO B - North Hoyle & $17,43 \%$ & $24,21 \%$ & $29,30 \%$ & $31,48 \%$ \\
\hline CASO C - Egmond Aan Zee & $45,76 \%$ & $63,72 \%$ & $75,83 \%$ & $81,35 \%$ \\
\hline CASO D - Thornton Bank & $129,71 \%$ & $34,56 \%$ & $37,95 \%$ & $39,79 \%$ \\
\hline
\end{tabular}

Tabla 10. Comparación entre método avanzado y tradicional para $T^{\underline{a}}=22^{\circ} \mathrm{C}$ y $\mathrm{S}=35,0 \%$

Con respecto a las variaciones del momento flector, se observa una tendencia creciente con el diámetro debida a las fuerzas de inercia, que en los casos B y C comienza en el diámetro de $0,5 \mathrm{~m}$ y, en los casos $\mathrm{A}$ y $\mathrm{D}$, comienza con el diámetro $4 \mathrm{~m}$, lo cual coincide con lo explicado en el punto anterior. Las variaciones experimentadas son generalmente mayores en el caso con temperatura de $22^{\circ} \mathrm{C}$ y salinidad del $35 \%$. Además, se observa que los mayores valores se dan para el menor diámetro, en el cual existe un predominio de las fuerzas viscosas siendo la variación máxima la del Caso D - Thornton Bank para el diámetro de $0,5 \mathrm{~m}$ con un $129,71 \%$. 


\subsubsection{Análisis de influencia de la temperatura y la salinidad}

En este punto, se va a proceder a realizar el análisis comparativo entre los resultados obtenidos en este segundo proceso de cálculo, para una temperatura de $1^{\circ} \mathrm{C}$ y salinidad del $0 \%$ con los obtenidos para una temperatura del agua de $22^{\circ} \mathrm{C}$ y salinidad del $35 \%$.

En primer lugar, el análisis de los resultados, calculados según el método avanzado, muestra un claro aumento de los valores correspondientes al caso de temperatura de $1^{\circ} \mathrm{C}$ y salinidad del $0 \%$ con respecto a los del segundo caso. Esta variación afecta en cada uno de los casos de una forma independiente. En la tabla 11 se muestran dichas variaciones en los cálculos realizados mediante el método avanzado calculadas, de acuerdo con la siguiente expresión:

$$
\% \text { Variación }=F_{T=1} \mathrm{C}, S=0 \%-F_{T=22^{\circ} \mathrm{C}}, \quad S=35 \%
$$

\begin{tabular}{|c|c|c|c|c|c|}
\hline & & \multicolumn{4}{|c|}{ Método Avanzado } \\
\hline \multicolumn{6}{|c|}{ CASO A - Princess Amalia Q7 } \\
\hline & Parámetro & $\mathrm{D}=0,5 \mathrm{~m}$ & $\mathrm{D}=4,0 \mathrm{~m}$ & $\mathrm{D}=8,0 \mathrm{~m}$ & $\mathrm{D}=16,0 \mathrm{~m}$ \\
\hline \multirow{5}{*}{ 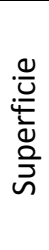 } & Máxima fuerza de inercia (N) & 11,23 & 220,60 & $-146,90$ & $25.632,60$ \\
\hline & Máxima fuerza de arrastre $(\mathrm{N})$ & $-0,82$ & $-6,60$ & $-13,10$ & $-26,20$ \\
\hline & Máxima fuerza no lineal (N) & $-0,01$ & $-0,50$ & $-1,70$ & $-3,40$ \\
\hline & Máx. fuerza de difracción $(\mathrm{N})$ & 0,00 & 0,00 & 0,00 & 0,00 \\
\hline & Máxima fuerza total (N) & $-0,95$ & 295,20 & $-109,50$ & $25.851,20$ \\
\hline \multirow{5}{*}{$\begin{array}{l}\text { 음 } \\
\text { 은 }\end{array}$} & Máxima fuerza de inercia (N) & $-0,31$ & 47,20 & 101,50 & 476,40 \\
\hline & Máxima fuerza de arrastre (N) & $-0,26$ & $-2,10$ & $-4,20$ & $-8,50$ \\
\hline & Máxima fuerza no lineal $(\mathrm{N})$ & $-0,01$ & $-0,40$ & $-1,80$ & $-7,30$ \\
\hline & Máx. fuerza de difracción (N) & 0,00 & 0,00 & 0,00 & 0,00 \\
\hline & Máxima fuerza total (N) & 57,50 & 36,50 & 51,60 & 246,00 \\
\hline \multicolumn{6}{|c|}{ CASO B - North Hoyle } \\
\hline \multirow{5}{*}{ 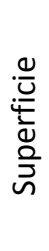 } & Máxima fuerza de inercia (N) & 67,91 & $1.538,00$ & $3.042,70$ & $-739,70$ \\
\hline & Máxima fuerza de arrastre $(\mathrm{N})$ & $-0,64$ & $-5,20$ & $-10,30$ & 0,00 \\
\hline & Máxima fuerza no lineal (N) & 0,00 & 0,00 & $-1,00$ & $-3,20$ \\
\hline & Máx. fuerza de difracción (N) & 0,00 & 0,00 & 0,00 & $-29,60$ \\
\hline & Máxima fuerza total $(\mathrm{N})$ & 122,17 & $1.523,60$ & $2.904,80$ & $-612,40$ \\
\hline \multirow{5}{*}{$\begin{array}{l}\text { 음 } \\
\text { 운 }\end{array}$} & Máxima fuerza de inercia (N) & 0,07 & 499,50 & 700,50 & $-462,40$ \\
\hline & Máxima fuerza de arrastre (N) & $-0,32$ & $-2,50$ & $-5,10$ & 0,00 \\
\hline & Máxima fuerza no lineal (N) & $-0,02$ & $-1,10$ & $-4,10$ & $-5,50$ \\
\hline & Máx. fuerza de difracción (N) & 0,00 & 0,00 & 0,00 & $-22,20$ \\
\hline & Máxima fuerza total (N) & 83,88 & 448,60 & 607,60 & $-437,70$ \\
\hline
\end{tabular}




\section{CASO C - Egmond Aan Zee}

\begin{tabular}{|c|c|c|c|c|c|}
\hline \multirow{5}{*}{ 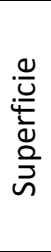 } & Máxima fuerza de inercia (N) & 19,16 & $-8,00$ & $-32,00$ & $-128,30$ \\
\hline & Máxima fuerza de arrastre $(\mathrm{N})$ & $-0,44$ & $-3,50$ & $-7,10$ & $-14,10$ \\
\hline & Máxima fuerza no lineal (N) & 0,00 & 0,00 & 0,00 & 0,00 \\
\hline & Máx. fuerza de difracción (N) & 0,00 & 0,00 & 0,00 & 0,00 \\
\hline & Máxima fuerza total $(\mathrm{N})$ & $-0,46$ & $-9,30$ & $-34,30$ & $-132,40$ \\
\hline \multirow{5}{*}{$\begin{array}{l}\text { 음 } \\
\text { 인 } \\
\text {. }\end{array}$} & Máxima fuerza de inercia (N) & 2,57 & 287,50 & 535,10 & $1.266,00$ \\
\hline & Máxima fuerza de arrastre (N) & $-0,27$ & $-2,10$ & $-4,30$ & $-8,70$ \\
\hline & Máxima fuerza no lineal (N) & 0,00 & 0,00 & 0,00 & 0,00 \\
\hline & Máx. fuerza de difracción (N) & 0,00 & 0,00 & 0,00 & 0,00 \\
\hline & Máxima fuerza total (N) & 14,03 & $-6,40$ & 115,00 & $-94,40$ \\
\hline \multicolumn{6}{|c|}{ CASO D - Thornton Bank } \\
\hline \multirow{5}{*}{ 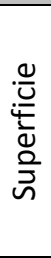 } & Máxima fuerza de inercia (N) & 56,91 & 530,10 & $2.424,30$ & $-470,00$ \\
\hline & Máxima fuerza de arrastre (N) & $-0,42$ & $-3,40$ & $-6,80$ & $-13,60$ \\
\hline & Máxima fuerza no lineal (N) & 0,00 & $-0,20$ & $-0,70$ & $-3,50$ \\
\hline & Máx. fuerza de difracción (N) & 0,00 & 0,00 & 0,00 & 0,00 \\
\hline & Máxima fuerza total (N) & 28,77 & 108,10 & $1.473,20$ & $-469,90$ \\
\hline \multirow{5}{*}{$\begin{array}{l}\text { 음 } \\
\text { 은 }\end{array}$} & Máxima fuerza de inercia (N) & 7,73 & 356,80 & 797,50 & $-337,60$ \\
\hline & Máxima fuerza de arrastre (N) & $-0,26$ & $-2,00$ & $-4,20$ & $-8,20$ \\
\hline & Máxima fuerza no lineal $(\mathrm{N})$ & $-0,01$ & $-0,40$ & $-1,70$ & $-6,80$ \\
\hline & Máx. fuerza de difracción (N) & 0,00 & 0,00 & 0,00 & 0,00 \\
\hline & Máxima fuerza total (N) & 71,08 & 357,60 & 741,70 & $-259,00$ \\
\hline
\end{tabular}

Tabla 11. Variación debida al cambio de temperatura y salinidad según el método avanzado

En la tabla 12 se muestran dichas variaciones a nivel relativo en los cálculos realizados mediante el método avanzado calculadas, de acuerdo con la siguiente expresión:

$$
\% \text { Variación }=\frac{F_{T=1 ㅇ ㅡ} C, S=0 \%-F_{T=22^{\circ} C, S=35 \%}}{F_{T=22^{\circ} C} C, S=35 \%}
$$

\begin{tabular}{|c|c|c|c|c|c|}
\hline & & \multicolumn{4}{|c|}{ Método Avanzado } \\
\hline \multicolumn{6}{|c|}{ CASO A - Princess Amalia Q7 } \\
\hline \multicolumn{2}{|r|}{ Parámetro } & $\mathrm{D}=0,5 \mathrm{~m}$ & $\mathrm{D}=4,0 \mathrm{~m}$ & $\mathrm{D}=8,0 \mathrm{~m}$ & $\mathrm{D}=16,0 \mathrm{~m}$ \\
\hline \multirow{5}{*}{ 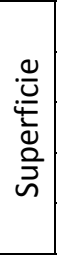 } & Máxima fuerza de inercia & $1,36 \%$ & $0,44 \%$ & $-0,07 \%$ & $3,29 \%$ \\
\hline & Máxima fuerza de arrastre & $-0,07 \%$ & $-0,07 \%$ & $-0,07 \%$ & $-0,07 \%$ \\
\hline & Máxima fuerza no lineal & $-0,07 \%$ & $-0,07 \%$ & $-0,07 \%$ & $-0,07 \%$ \\
\hline & Máxima fuerza de difracción & $0,00 \%$ & $0,00 \%$ & $0,00 \%$ & $0,00 \%$ \\
\hline & Máxima fuerza total & $-0,07 \%$ & $0,58 \%$ & $-0,05 \%$ & $3,31 \%$ \\
\hline \multirow{5}{*}{$\begin{array}{l}\text { 음 } \\
\text { 은 } \\
\text { ㄴ. }\end{array}$} & Máxima fuerza de inercia & $-0,07 \%$ & $0,17 \%$ & $0,09 \%$ & $0,11 \%$ \\
\hline & Máxima fuerza de arrastre & $-0,07 \%$ & $-0,07 \%$ & $-0,07 \%$ & $-0,07 \%$ \\
\hline & Máxima fuerza no lineal & $-0,07 \%$ & $-0,06 \%$ & $-0,07 \%$ & $-0,07 \%$ \\
\hline & Máxima fuerza de difracción & $0,00 \%$ & $0,00 \%$ & $0,00 \%$ & $0,00 \%$ \\
\hline & Máxima fuerza total & $12,13 \%$ & $0,13 \%$ & $0,05 \%$ & $0,06 \%$ \\
\hline
\end{tabular}


Modelos de predicción de esfuerzos hidrodinámicos y socavación aplicados a ingeniería offshore

\begin{tabular}{|c|c|c|c|c|c|}
\hline \multicolumn{6}{|c|}{ CASO B - North Hoyle } \\
\hline \multirow{5}{*}{ 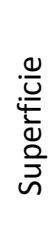 } & Máxima fuerza de inercia & $5,99 \%$ & $2,41 \%$ & $1,20 \%$ & $-0,07 \%$ \\
\hline & Máxima fuerza de arrastre & $-0,07 \%$ & $-0,07 \%$ & $-0,07 \%$ & $0,00 \%$ \\
\hline & Máxima fuerza no lineal & $-0,07 \%$ & $0,00 \%$ & $-0,07 \%$ & $-0,07 \%$ \\
\hline & Máxima fuerza de difracción & $0,00 \%$ & $0,00 \%$ & $0,00 \%$ & $-0,07 \%$ \\
\hline & Máxima fuerza total & $8,69 \%$ & $2,35 \%$ & $1,14 \%$ & $-0,06 \%$ \\
\hline \multirow{5}{*}{$\begin{array}{l}\text { 응 } \\
\text { 언 }\end{array}$} & Máxima fuerza de inercia & $0,01 \%$ & $1,25 \%$ & $0,44 \%$ & $-0,07 \%$ \\
\hline & Máxima fuerza de arrastre & $-0,07 \%$ & $-0,07 \%$ & $-0,07 \%$ & $0,00 \%$ \\
\hline & Máxima fuerza no lineal & $-0,07 \%$ & $-0,08 \%$ & $-0,07 \%$ & $-0,07 \%$ \\
\hline & Máxima fuerza de difracción & $0,00 \%$ & $0,00 \%$ & $0,00 \%$ & $-0,07 \%$ \\
\hline & Máxima fuerza total & $11,23 \%$ & $1,13 \%$ & $0,39 \%$ & $-0,07 \%$ \\
\hline \multicolumn{6}{|c|}{ CASO C - Egmond Aan Zee } \\
\hline \multirow{5}{*}{ 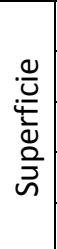 } & Máxima fuerza de inercia & $9,19 \%$ & $-0,07 \%$ & $-0,07 \%$ & $-0,07 \%$ \\
\hline & Máxima fuerza de arrastre & $-0,07 \%$ & $-0,07 \%$ & $-0,07 \%$ & $-0,07 \%$ \\
\hline & Máxima fuerza no lineal & $0,00 \%$ & $0,00 \%$ & $0,00 \%$ & $0,00 \%$ \\
\hline & Máxima fuerza de difracción & $0,00 \%$ & $0,00 \%$ & $0,00 \%$ & $0,00 \%$ \\
\hline & Máxima fuerza total & $-0,07 \%$ & $-0,07 \%$ & $-0,07 \%$ & $-0,07 \%$ \\
\hline \multirow{5}{*}{$\begin{array}{l}\circ \\
\frac{0}{0} \\
\text { 온 }\end{array}$} & Máxima fuerza de inercia & $1,58 \%$ & $3,44 \%$ & $1,64 \%$ & $0,99 \%$ \\
\hline & Máxima fuerza de arrastre & $-0,07 \%$ & $-0,07 \%$ & $-0,07 \%$ & $-0,07 \%$ \\
\hline & Máxima fuerza no lineal & $0,00 \%$ & $0,00 \%$ & $0,00 \%$ & $0,00 \%$ \\
\hline & Máxima fuerza de difracción & $0,00 \%$ & $0,00 \%$ & $0,00 \%$ & $0,00 \%$ \\
\hline & Máxima fuerza total & $3,59 \%$ & $-0,07 \%$ & $0,35 \%$ & $-0,07 \%$ \\
\hline \multicolumn{6}{|c|}{ CASO D - Thornton Bank } \\
\hline \multirow{5}{*}{ 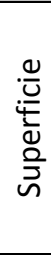 } & Máxima fuerza de inercia & $7,82 \%$ & $1,30 \%$ & $1,51 \%$ & $-0,07 \%$ \\
\hline & Máxima fuerza de arrastre & $-0,07 \%$ & $-0,07 \%$ & $-0,07 \%$ & $-0,07 \%$ \\
\hline & Máxima fuerza no lineal & $-0,07 \%$ & $-0,10 \%$ & $-0,08 \%$ & $-0,07 \%$ \\
\hline & Máxima fuerza de difracción & $0,00 \%$ & $0,00 \%$ & $0,00 \%$ & $0,00 \%$ \\
\hline & Máxima fuerza total & $2,99 \%$ & $0,26 \%$ & $0,91 \%$ & $-0,07 \%$ \\
\hline \multirow{5}{*}{$\begin{array}{l}\text { 음 } \\
\text { 인 }\end{array}$} & Máxima fuerza de inercia & $1,57 \%$ & $1,23 \%$ & $0,69 \%$ & $-0,07 \%$ \\
\hline & Máxima fuerza de arrastre & $-0,07 \%$ & $-0,07 \%$ & $-0,07 \%$ & $-0,07 \%$ \\
\hline & Máxima fuerza no lineal & $-0,07 \%$ & $-0,07 \%$ & $-0,07 \%$ & $-0,07 \%$ \\
\hline & Máxima fuerza de difracción & $0,00 \%$ & $0,00 \%$ & $0,00 \%$ & $0,00 \%$ \\
\hline & Máxima fuerza total & $12,67 \%$ & $1,23 \%$ & $0,64 \%$ & $-0,06 \%$ \\
\hline
\end{tabular}

Tabla 12. Variación relativa debida al cambio de temperatura y salinidad según el método avanzado

A partir de los resultados mostrados en la tabla anterior, se puede observar una clara tendencia que demuestra que las variaciones de salinidad y temperatura afectan de forma más significativa en los casos con diámetro más reducido. Este hecho implica que la variación más importante viene representada por la variación que sufren las fuerzas viscosas.

Las fuerzas viscosas tienen un carácter predominante en los diámetros de menor tamaño y, por lo tanto, se puede concluir que es la variación de la viscosidad del agua la que está 
generando estas diferencias. También pone de manifiesto, que la variación de la densidad del agua, que es la que influye sobre las fuerzas de inercia, no tiene una influencia tan significativa.

No obstante, esta segunda afirmación tiene una excepción en el valor obtenido para la fuerza total en superficie, para el caso de mayor diámetro, en el Caso A - Princess Amalia Q7, en el cual se observa un incremento de la fuerza de inercia superior al $3 \%$. Dicho incremento es producido por un resalto que aparece en el punto de máxima fuerza y que tiene su origen en el cambio del coeficiente de inercia $C_{M}$, que sufre un incremento debido a la variación del número de Reynolds. La fórmula que relaciona el número de Reynolds con el coeficiente de inercia $C_{M}$ es (Shore Protection Manual, 1975):

$$
\begin{array}{ll}
\mathrm{C}_{\mathrm{M}}=2,0 & \text { cuando } \mathrm{R}_{\mathrm{e}}<2,5 \cdot 10^{5} \\
\mathrm{C}_{\mathrm{M}}=2,5-\frac{R_{e}}{5 \cdot 10^{5}} & \text { cuando } 2,5 \cdot 10^{5}<\mathrm{R}_{\mathrm{e}}<5 \cdot 10^{5} \\
\mathrm{C}_{\mathrm{M}}=1,5 & \text { cuando } \mathrm{R}_{\mathrm{e}}>5 \cdot 10^{5}
\end{array}
$$

En la figura 7 se muestra la aparición del resalto en la zona de máxima fuerza de inercia:

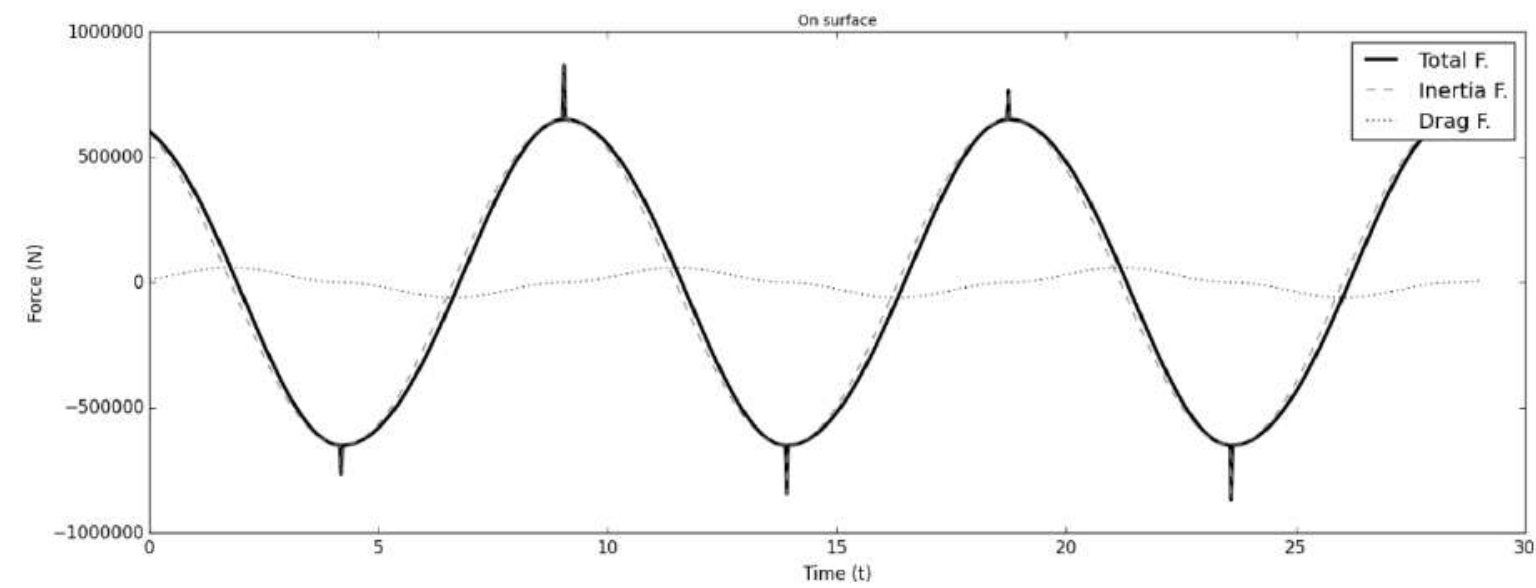

Figura 7. Fuerza en superficie, mostrada a lo largo del tiempo

También se observa que, generalmente, las fuerzas totales resultantes en fondo son superiores para el caso de temperatura de $1^{\circ} \mathrm{C}$ y salinidad del $0 \%$ que para el caso de temperatura de $22 \circ$ y salinidad del 35\%, siendo la variación máxima del $12,67 \%$. 
En la tabla 13 se muestran los valores de variación del máximo momento flector en el lecho marino.

\begin{tabular}{|l|c|c|c|c|}
\hline \multicolumn{5}{|c|}{ Método Avanzado } \\
\hline \multicolumn{1}{|c|}{ Máximo momento flector } & $\mathrm{D}=0,5 \mathrm{~m}$ & $\mathrm{D}=4,0 \mathrm{~m}$ & $\mathrm{D}=8,0 \mathrm{~m}$ & $\mathrm{D}=16,0 \mathrm{~m}$ \\
\hline CASO A - Princess Amalia Q7 & $0,05 \%$ & $4,42 \%$ & $8,79 \%$ & $6,27 \%$ \\
\hline CASO B - North Hoyle & $13,07 \%$ & $7,74 \%$ & $1,65 \%$ & $-0,07 \%$ \\
\hline CASO C - Egmond Aan Zee & $0,32 \%$ & $-0,07 \%$ & $-0,07 \%$ & $-0,07 \%$ \\
\hline CASO D - Thornton Bank & $8,29 \%$ & $4,05 \%$ & $-0,07 \%$ & $-0,07 \%$ \\
\hline
\end{tabular}

Con respecto a las variaciones del momento flector, se observa la misma tendencia que se describía en la tabla anterior. El momento generado es mayor para los casos de temperatura de $1^{\circ} \mathrm{C}$ y salinidad del $0 \%$, siendo la variación máxima del $13,07 \%$. No obstante, esta variación se va difuminando conforme aumenta el diámetro de la estructura.

Esta tendencia se muestra de forma contraria en los resultados mostrados para el oleaje del caso A. Este hecho se debe a la modificación de la ley de amortiguación de las fuerzas con respecto a la profundidad, lo cual demuestra que la teoría de ondas elegida para resolver el campo de aceleraciones y velocidades del oleaje tendrá una influencia importante sobre la predicción del efecto que tendrán las variaciones de temperatura y salinidad sobre el cálculo de las acciones generadas sobre la estructura.

\subsection{Proceso III: Análisis dinámico}

El tercer proceso se corresponde con el cálculo del comportamiento dinámico de la estructura, a partir de las fuerzas generadas por el oleaje, $F(z, t)$, para cada instante de tiempo, $\mathrm{t}_{\mathrm{i}}$.

Se va a evaluar el caso de pilotes de distintos diámetros mediante los cuatro casos de oleaje definidos en el apartado primero. También se van a utilizar las fuerzas hidrodinámicas calculadas según el método tradicional y según el método avanzado, de acuerdo con los resultados obtenidos en el segundo proceso de cálculo. Además, se introducirán los dos casos de temperatura del agua de $1^{\circ} \mathrm{C}$ y salinidad del $0 \%$ y el de temperatura de $22^{\circ} \mathrm{C}$ y salinidad del $35 \%$. 
Por último, como se explicó en el capítulo 4, el método tradicional tendrá en cuenta únicamente un cálculo estático, mientras que el método de cálculo avanzado introducirá además los efectos dinámicos que sufre la estructura.

En la figura 8 se muestran ambos métodos de cálculo de una forma esquemática:

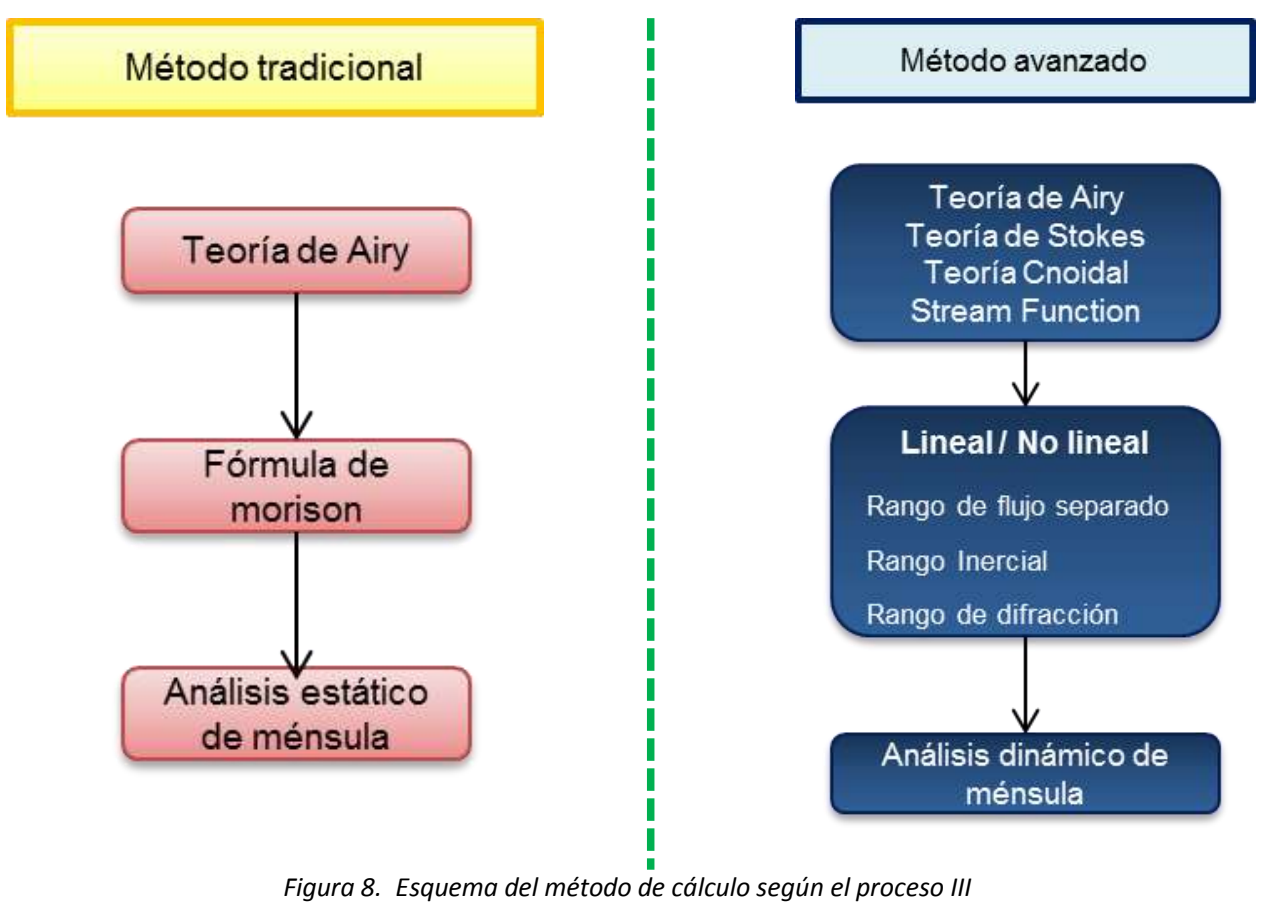

Las rigideces que se van a considerar para cada uno de los casos de estudio se muestran a continuación en la tabla 14:

\begin{tabular}{|c|c|c|c|}
\hline Diámetro & Descripción & Rigidez, El $\left(\mathrm{N} \cdot \mathrm{m}^{2}\right)$ & Croquis \\
\hline $0,5 \mathrm{~m}$ & $\begin{array}{l}\text { Se supone una pila hueca de } \\
\text { acero, con un espesor de } 20 \\
\text { centímetros. }\end{array}$ & $6,1261 \mathrm{E}+08$ & \\
\hline $4,0 \mathrm{~m}$ & $\begin{array}{l}\text { Se supone una pila hueca de } \\
\text { acero, con un espesor de } 30 \\
\text { centímetros. }\end{array}$ & $1,2013 E+12$ & \\
\hline
\end{tabular}




\begin{tabular}{|c|l|c|c|}
\hline $8,0 \mathrm{~m}$ & $\begin{array}{l}\text { Se supone una pila de } \\
\text { hormigón armado. }\end{array}$ & $5,6297 \mathrm{E}+12$ \\
$16,0 \mathrm{~m}$ & $\begin{array}{l}\text { Se supone una pila de } \\
\text { hormigón armado. }\end{array}$ & $9,0076 \mathrm{e}+13$
\end{tabular}

Tabla 14. Rigideces utilizadas en los casos de cálculo

Los diámetros de 8 y 16 metros se han definido como secciones de hormigón armado, tomando como ejemplo el parque eólico offshore de Thornton bank fase 1, que posee una estructura con cimentación de gravedad en forma de sección cónica de hormigón armado que va desde el diámetro de 17 metros hasta el diámetro de 6,5 metros.

\subsubsection{Resultados}

De acuerdo con los resultados mostrados en el apéndice 1 para el proceso III, es posible extraer las siguientes conclusiones a partir de los objetivos que se planteaban:

a) Los resultados generados mediante el proceso de cálculo tradicional, que utiliza un modelo estático para el cálculo de la estructura, son siempre del orden de entre un $20 \%$ y $100 \%$ mayores que los resultados obtenidos mediante el proceso de cálculo avanzado, que utiliza un modelo dinámico que tiene en cuenta las vibraciones que sufre la estructura.

b) Los máximos esfuerzos se producen en todos los casos en la sección correspondiente al empotramiento de la estructura en el lecho marino, tanto para el proceso de cálculo tradicional como para el proceso de cálculo avanzado.

c) Con respecto a los movimientos sufridos por la estructura, como cabía esperar, los máximos desplazamientos se producen en el extremo libre de la pila. Estos desplazamientos son menores al ser calculados mediante el proceso de cálculo estático, que los resultantes del proceso de cálculo dinámico.

\subsubsection{Comparación entre el método tradicional y el avanzado}

A continuación, se procede a mostrar los resultados del análisis comparativo entre la aplicación del método tradicional frente a la del método avanzado. 
El análisis de los resultados muestra un claro aumento de los valores procedentes del método tradicional con respecto a los procedentes del método avanzado. Esta variación afecta en cada uno de los casos de una forma independiente. En las tablas 15 y 16 se muestran dichas variaciones calculadas de acuerdo con la siguiente expresión:

$$
\% \text { Variación }=\frac{F_{\text {Tradicional }}-F_{\text {Avanzado }}}{F_{\text {Avanzado }}}
$$

\begin{tabular}{|c|c|c|c|c|}
\hline \multicolumn{5}{|c|}{ Temperatura de $1^{\circ} \mathrm{C}$ y Salinidad del $0,0 \%$} \\
\hline \multicolumn{5}{|c|}{ CASO A - Princess Amalia Q7 } \\
\hline Parámetro & $\mathrm{D}=0,5 \mathrm{~m}$ & $\mathrm{D}=4,0 \mathrm{~m}$ & $\mathrm{D}=8,0 \mathrm{~m}$ & $\mathrm{D}=16,0 \mathrm{~m}$ \\
\hline $\begin{array}{l}\text { Máximo desplazamiento en } \\
\text { superficie }(m)\end{array}$ & $-37,55 \%$ & $-45,26 \%$ & $-33,42 \%$ & $-41,62 \%$ \\
\hline $\begin{array}{l}\text { Máximo esfuerzo cortante en } \\
\text { cimentación (kN) }\end{array}$ & $24,67 \%$ & $3,45 \%$ & $13,28 \%$ & $18,06 \%$ \\
\hline $\begin{array}{l}\text { Máximo momento flector en } \\
\text { cimentación }(\mathrm{kN} \cdot \mathrm{m})\end{array}$ & $33,04 \%$ & $4,30 \%$ & $14,34 \%$ & $21,85 \%$ \\
\hline \multicolumn{5}{|c|}{ CASO B - North Hoyle } \\
\hline Parámetro & $\mathrm{D}=0,5 \mathrm{~m}$ & $\mathrm{D}=4,0 \mathrm{~m}$ & $\mathrm{D}=8,0 \mathrm{~m}$ & $\mathrm{D}=16,0 \mathrm{~m}$ \\
\hline $\begin{array}{l}\text { Máximo desplazamiento en } \\
\text { superficie }(\mathrm{m})\end{array}$ & $-43,87 \%$ & $-36,76 \%$ & $-38,96 \%$ & $-30,96 \%$ \\
\hline $\begin{array}{l}\text { Máximo esfuerzo cortante en } \\
\text { cimentación (kN) }\end{array}$ & $-11,47 \%$ & $13,40 \%$ & $17,10 \%$ & $32,43 \%$ \\
\hline $\begin{array}{l}\text { Máximo momento flector en } \\
\text { cimentación }(\mathrm{kN} \cdot \mathrm{m})\end{array}$ & $-7,14 \%$ & $12,09 \%$ & $19,79 \%$ & $29,20 \%$ \\
\hline \multicolumn{5}{|c|}{ CASO C - Egmond Aan Zee } \\
\hline Parámetro & $\mathrm{D}=0,5 \mathrm{~m}$ & $\mathrm{D}=4,0 \mathrm{~m}$ & $\mathrm{D}=8,0 \mathrm{~m}$ & $\mathrm{D}=16,0 \mathrm{~m}$ \\
\hline $\begin{array}{l}\text { Máximo desplazamiento en } \\
\text { superficie }(\mathrm{m})\end{array}$ & $-31,55 \%$ & $-17,66 \%$ & $-18,24 \%$ & $-5,21 \%$ \\
\hline $\begin{array}{l}\text { Máximo esfuerzo cortante en } \\
\text { cimentación }(\mathrm{kN})\end{array}$ & $26,43 \%$ & $62,28 \%$ & $72,41 \%$ & $76,83 \%$ \\
\hline $\begin{array}{l}\text { Máximo momento flector en } \\
\text { cimentación }(\mathrm{kN} \cdot \mathrm{m})\end{array}$ & $34,04 \%$ & $64,10 \%$ & $74,97 \%$ & $79,76 \%$ \\
\hline \multicolumn{5}{|c|}{ CASO D - Thornton Bank } \\
\hline Parámetro & $\mathrm{D}=0,5 \mathrm{~m}$ & $\mathrm{D}=4,0 \mathrm{~m}$ & $\mathrm{D}=8,0 \mathrm{~m}$ & $\mathrm{D}=16,0 \mathrm{~m}$ \\
\hline $\begin{array}{l}\text { Máximo desplazamiento en } \\
\text { superficie }(\mathrm{m})\end{array}$ & $23,36 \%$ & $-27,67 \%$ & $-31,44 \%$ & $-27,66 \%$ \\
\hline $\begin{array}{l}\text { Máximo esfuerzo cortante en } \\
\text { cimentación }(\mathrm{kN})\end{array}$ & $82,34 \%$ & $25,62 \%$ & $36,45 \%$ & $40,58 \%$ \\
\hline $\begin{array}{l}\text { Máximo momento flector en } \\
\text { cimentación }(\mathrm{kN} \cdot \mathrm{m})\end{array}$ & $96,31 \%$ & $25,85 \%$ & $36,20 \%$ & $40,48 \%$ \\
\hline
\end{tabular}

\begin{tabular}{|c|c|c|c|c|}
\hline \multicolumn{5}{|c|}{ Temperatura de $22^{\circ} \mathrm{C}$ y Salinidad del $35 \%$} \\
\hline \multicolumn{5}{|c|}{ CASO A - Princess Amalia Q7 } \\
\hline Parámetro & $D=0,5 m$ & $\mathrm{D}=4,0 \mathrm{~m}$ & $\mathrm{D}=8,0 \mathrm{~m}$ & $\mathrm{D}=16,0 \mathrm{~m}$ \\
\hline $\begin{array}{l}\text { Máximo desplazamiento en } \\
\text { superficie }(\mathrm{m})\end{array}$ & $-37,56 \%$ & $-40,07 \%$ & $-31,86 \%$ & $-38,04 \%$ \\
\hline $\begin{array}{l}\text { Máximo esfuerzo cortante en } \\
\text { cimentación }(\mathrm{kN})\end{array}$ & $28,55 \%$ & $12,00 \%$ & $17,12 \%$ & $22,02 \%$ \\
\hline $\begin{array}{l}\text { Máximo momento flector en } \\
\text { cimentación }(\mathrm{kN} \cdot \mathrm{m})\end{array}$ & $33,14 \%$ & $11,01 \%$ & $20,57 \%$ & $28,17 \%$ \\
\hline
\end{tabular}




\begin{tabular}{|c|c|c|c|c|}
\hline \multicolumn{5}{|c|}{ CASO B - North Hoyle } \\
\hline Parámetro & $D=0,5 m$ & $\mathrm{D}=4,0 \mathrm{~m}$ & $\mathrm{D}=8,0 \mathrm{~m}$ & $\mathrm{D}=16,0 \mathrm{~m}$ \\
\hline $\begin{array}{l}\text { Máximo desplazamiento en } \\
\text { superficie }(\mathrm{m})\end{array}$ & $-43,69 \%$ & $-36,77 \%$ & $-35,93 \%$ & $-29,69 \%$ \\
\hline $\begin{array}{l}\text { Máximo esfuerzo cortante en } \\
\text { cimentación }(\mathrm{kN})\end{array}$ & $-1,02 \%$ & $25,82 \%$ & $30,70 \%$ & $32,48 \%$ \\
\hline $\begin{array}{l}\text { Máximo momento flector en } \\
\text { cimentación }(\mathrm{kN} \cdot \mathrm{m})\end{array}$ & $4,84 \%$ & $21,92 \%$ & $30,88 \%$ & $32,95 \%$ \\
\hline \multicolumn{5}{|c|}{ CASO C - Egmond Aan Zee } \\
\hline Parámetro & $\mathrm{D}=0,5 \mathrm{~m}$ & $\mathrm{D}=4,0 \mathrm{~m}$ & $\mathrm{D}=8,0 \mathrm{~m}$ & $\mathrm{D}=16,0 \mathrm{~m}$ \\
\hline $\begin{array}{l}\text { Máximo desplazamiento en } \\
\text { superficie }(\mathrm{m})\end{array}$ & $-30,61 \%$ & $-17,67 \%$ & $-18,30 \%$ & $-5,21 \%$ \\
\hline $\begin{array}{l}\text { Máximo esfuerzo cortante en } \\
\text { cimentación }(\mathrm{kN})\end{array}$ & $27,16 \%$ & $62,28 \%$ & $72,38 \%$ & $76,83 \%$ \\
\hline $\begin{array}{l}\text { Máximo momento flector en } \\
\text { cimentación }(\mathrm{kN} \cdot \mathrm{m})\end{array}$ & $34,63 \%$ & $64,10 \%$ & $74,89 \%$ & $79,76 \%$ \\
\hline \multicolumn{5}{|c|}{ CASO D - Thornton Bank } \\
\hline Parámetro & $D=0,5 m$ & $\mathrm{D}=4,0 \mathrm{~m}$ & $\mathrm{D}=8,0 \mathrm{~m}$ & $\mathrm{D}=16,0 \mathrm{~m}$ \\
\hline $\begin{array}{l}\text { Máximo desplazamiento en } \\
\text { superficie }(\mathrm{m})\end{array}$ & $23,41 \%$ & $-27,67 \%$ & $-26,87 \%$ & $-27,66 \%$ \\
\hline $\begin{array}{l}\text { Máximo esfuerzo cortante en } \\
\text { cimentación }(\mathrm{kN})\end{array}$ & $105,44 \%$ & $34,77 \%$ & $39,06 \%$ & $40,58 \%$ \\
\hline $\begin{array}{l}\text { Máximo momento flector en } \\
\text { cimentación }(\mathrm{kN} \cdot \mathrm{m})\end{array}$ & $117,25 \%$ & $34,24 \%$ & $38,80 \%$ & $40,48 \%$ \\
\hline
\end{tabular}

Tabla 16. Comparación entre método avanzado y tradicional para Temperatura de $22^{\circ} \mathrm{C}$ y Salinidad del $35 \%$

A partir de los resultados mostrados en la tabla anterior, se puede observar que las máximas diferencias se producen en el caso de temperatura de $22^{\circ} \mathrm{C}$ y salinidad del $35 \%$ para el diámetro más pequeño. Esto es debido a que las máximas diferencias entre el método tradicional y el método avanzado se producen en las fuerzas viscosas. El máximo valor se encuentra en el Caso D - Thornton Bank, en el cual la diferencia del momento flector en la cimentación llega a ser del entorno de un 117,25\% más elevada en el caso tradicional que el calculado según el método avanzado.

Por otro lado, se observa una segunda tendencia de crecimiento desde el diámetro de 4 metros hasta el diámetro de 16 metros. Esto es debido a que conforme se va aumentando el diámetro de la estructura y las cargas sufridas por la misma, los efectos de vibración dinámica de la estructura también van siendo más importantes. Dicho efectos generan una mayor distribución de la ley de momentos flectores, a lo largo de toda la estructura, lo que genera que se incremente la magnitud de los movimientos medidos en el extremo libre, pero se reduzca el momento total que está sufriendo la cimentación. 
También se observa que, generalmente, las fuerzas totales resultantes en fondo son superiores para el caso de cálculo tradicional a temperatura de $22^{\circ} \mathrm{C}$ y salinidad del $35 \%$ que para el caso de cálculo avanzado siendo la variación máxima del 105,44\%.

Además, se observa que todas las variaciones son mucho mayores en superficie que en fondo, como cabía esperar, de acuerdo con los resultados obtenidos mediante el cálculo correspondiente a la mecánica de ondas, mostrados en el Proceso I.

\subsubsection{Análisis de influencia de la temperatura y la salinidad}

En este punto, se va a proceder a realizar el análisis comparativo entre los resultados obtenidos en este tercer proceso de cálculo para una temperatura de $1^{\circ} \mathrm{C}$ y salinidad del $0 \%$ y los obtenidos para una temperatura del agua de $22^{\circ} \mathrm{C}$ y salinidad del $35 \%$.

En primer lugar, el análisis de los resultados, calculados según el método avanzado, muestra, generalmente, un claro aumento de los valores correspondientes al caso de temperatura de $1^{\circ} \mathrm{C}$ y salinidad del $0 \%$ con respecto a los del segundo caso. Esta variación afecta en cada uno de los casos de una forma independiente. En la tabla 17 se muestran dichas variaciones calculadas de acuerdo con la siguiente expresión:

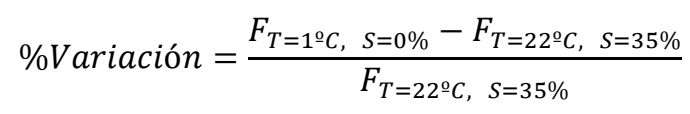

\begin{tabular}{|c|c|c|c|c|}
\hline \multicolumn{5}{|c|}{ Método avanzado } \\
\hline \multicolumn{5}{|c|}{ CASO A - Princess Amalia Q7 } \\
\hline Parámetro & $\mathrm{D}=0,5 \mathrm{~m}$ & $\mathrm{D}=4,0 \mathrm{~m}$ & $\mathrm{D}=8,0 \mathrm{~m}$ & $\mathrm{D}=16,0 \mathrm{~m}$ \\
\hline $\begin{array}{l}\text { Máximo desplazamiento en } \\
\text { superficie }(\mathrm{m})\end{array}$ & $-0,07 \%$ & $9,41 \%$ & $2,27 \%$ & $6,05 \%$ \\
\hline $\begin{array}{l}\text { Máximo esfuerzo cortante en } \\
\text { cimentación (kN) }\end{array}$ & $3,21 \%$ & $8,18 \%$ & $3,32 \%$ & $3,28 \%$ \\
\hline $\begin{array}{l}\text { Máximo momento flector en } \\
\text { cimentación }(\mathrm{kN} \cdot \mathrm{m})\end{array}$ & $0,04 \%$ & $6,36 \%$ & $5,37 \%$ & $5,11 \%$ \\
\hline \multicolumn{5}{|c|}{ CASO B - North Hoyle } \\
\hline Parámetro & $\mathrm{D}=0,5 \mathrm{~m}$ & $\mathrm{D}=4,0 \mathrm{~m}$ & $\mathrm{D}=8,0 \mathrm{~m}$ & $\mathrm{D}=16,0 \mathrm{~m}$ \\
\hline $\begin{array}{l}\text { Máximo desplazamiento en } \\
\text { superficie }(\mathrm{m})\end{array}$ & $0,26 \%$ & $-0,08 \%$ & $4,96 \%$ & $1,84 \%$ \\
\hline $\begin{array}{l}\text { Máximo esfuerzo cortante en } \\
\text { cimentación }(\mathrm{kN})\end{array}$ & $12,14 \%$ & $10,88 \%$ & $11,63 \%$ & $0,17 \%$ \\
\hline $\begin{array}{l}\text { Máximo momento flector en } \\
\text { cimentación }(\mathrm{kN} \cdot \mathrm{m})\end{array}$ & $12,94 \%$ & $8,69 \%$ & $9,27 \%$ & $3,01 \%$ \\
\hline
\end{tabular}




\begin{tabular}{|c|c|c|c|c|}
\hline \multicolumn{5}{|c|}{ CASO C - Egmond Aan Zee } \\
\hline Parámetro & $\mathrm{D}=0,5 \mathrm{~m}$ & $\mathrm{D}=4,0 \mathrm{~m}$ & $\mathrm{D}=8,0 \mathrm{~m}$ & $\mathrm{D}=16,0 \mathrm{~m}$ \\
\hline $\begin{array}{l}\text { Máximo desplazamiento en } \\
\text { superficie }(\mathrm{m})\end{array}$ & $1,30 \%$ & $-0,07 \%$ & $-0,07 \%$ & $-0,07 \%$ \\
\hline $\begin{array}{l}\text { Máximo esfuerzo cortante en } \\
\text { cimentación (kN) }\end{array}$ & $0,60 \%$ & $-0,07 \%$ & $-0,07 \%$ & $-0,07 \%$ \\
\hline $\begin{array}{l}\text { Máximo momento flector en } \\
\text { cimentación }(\mathrm{kN} \cdot \mathrm{m})\end{array}$ & $0,40 \%$ & $-0,07 \%$ & $-0,07 \%$ & $-0,07 \%$ \\
\hline \multicolumn{5}{|c|}{ CASO D - Thornton Bank } \\
\hline Parámetro & $\mathrm{D}=0,5 \mathrm{~m}$ & $\mathrm{D}=4,0 \mathrm{~m}$ & $\mathrm{D}=8,0 \mathrm{~m}$ & $\mathrm{D}=16,0 \mathrm{~m}$ \\
\hline $\begin{array}{l}\text { Máximo desplazamiento en } \\
\text { superficie }(\mathrm{m})\end{array}$ & $-0,04 \%$ & $-0,07 \%$ & $6,60 \%$ & $-0,08 \%$ \\
\hline $\begin{array}{l}\text { Máximo esfuerzo cortante en } \\
\text { cimentación (kN) }\end{array}$ & $12,59 \%$ & $7,21 \%$ & $1,84 \%$ & $-0,07 \%$ \\
\hline $\begin{array}{l}\text { Máximo momento flector en } \\
\text { cimentación }(\mathrm{kN} \cdot \mathrm{m})\end{array}$ & $10,59 \%$ & $6,60 \%$ & $1,83 \%$ & $-0,07 \%$ \\
\hline
\end{tabular}

Tabla 17. Comparación entre método avanzado y tradicional para Temperatura de $1^{\circ} \mathrm{C}$ y Salinidad del $0,0 \%$

A partir de los resultados mostrados en la tabla anterior, se puede observar una clara tendencia que demuestra que las variaciones de salinidad y temperatura afectan de forma más significativa en los casos con diámetro más reducido. Como se explicó previamente en el proceso II, este hecho implica que la variación más importante viene representada por la variación que sufren las fuerzas viscosas. Las fuerzas viscosas tienen un carácter predominante en los diámetros de menor tamaño y, por lo tanto, se puede concluir que es la variación de la viscosidad del agua la que está generando estas diferencias, tal y como se observó en los resultados mostrados en el Proceso II. También pone de manifiesto que la variación de la densidad del agua, que es la que influye sobre las fuerzas de inercia, tiene una influencia menor.

No obstante, esta segunda afirmación tiene una excepción en el valor obtenido para el máximo momento flector en cimentación para el caso de mayor diámetro en el Caso A Princess Amalia Q7, en el cual se observa un incremento superior al 5,11\% y en el Caso B North Hoyle, en el cual se observa un incremento del 3,01\%.

Dicho incremento es producido, en el primer caso, por un resalto que aparece en el punto de máxima fuerza y que tiene su origen en el cambio del coeficiente de inercia $C_{M}$, que sufre un incremento debido a la variación del número de Reynolds como se mostró en los resultados del Proceso II. En el segundo caso, se debe a una variación de la ley de esfuerzos, que genera un ligero incremento del momento flector resultante sobre la cimentación. 


\subsection{Proceso IV: Acoplamiento procesos II y III}

El cuarto proceso se corresponde con el cálculo del comportamiento dinámico de la estructura teniendo en cuenta la velocidad y la aceleración relativa que esta tiene en relación con la velocidad y aceleración del fluido que impacta sobre ella.

Nuevamente, se va a evaluar el caso de pilotes de distintos diámetros mediante los cuatro casos de oleaje definidos en el proceso primero de cálculo. Los resultados se van a comparar posteriormente con los esfuerzos estructurales obtenidos en el proceso tercero de cálculo. Además, se introducirán los dos casos relativos al estado del agua marina, el de temperatura del agua de $1{ }^{\circ} \mathrm{C}$ y salinidad del $0 \%$ y el de temperatura de $22^{\circ} \mathrm{C}$ y salinidad del $35 \%$.

Por último, como se explicó en el capítulo 4, el esquema de cálculo iterativo que se va a seguir a continuación, es el mostrado en la figura 9:

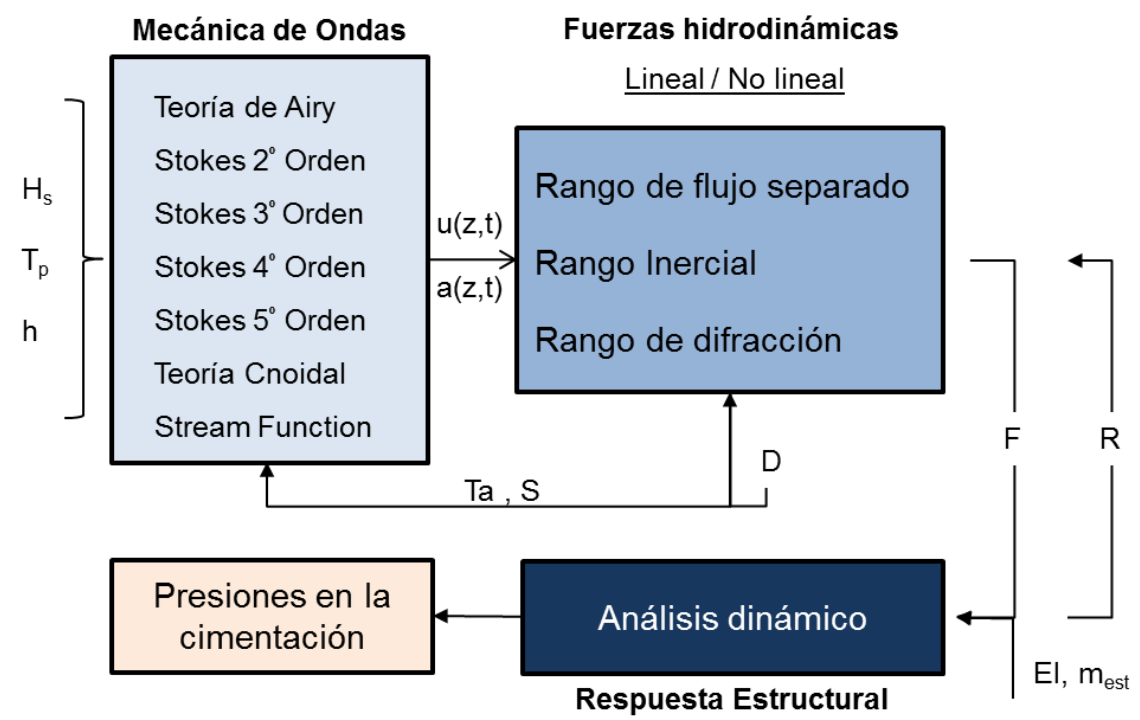

Figura 9. Proceso de cálculo general propuesto

Los objetivos perseguidos en este proceso son:

- Determinar la influencia que tiene el acoplamiento de la estructura con el oleaje sobre los esfuerzos que se generan sobre la estructura. 
- Determinar cómo varía esta influencia dependiendo del diámetro y de la teoría hidrodinámica aplicada.

- Determinar cómo varía este acoplamiento con respecto a la teoría de ondas utilizada para caracterizar el oleaje.

\subsubsection{Análisis de influencia del acoplamiento}

El proceso IV no incluirá ninguna variable adicional al cálculo. Este paso de cálculo está enfocado a aumentar la exactitud de los cálculos dinámicos al permitir el acoplamiento del oleaje con la estructura.

No obstante, sí se observará la diferencia entre los resultados obtenidos por el Proceso IV con aquellos que se obtuvieron en el proceso anterior con la intención de poder determinar la influencia que supone la consideración del acoplamiento de ambos procesos.

A continuación, se procede a mostrar la tabla de análisis comparativo entre los resultados generados mediante el método avanzado sin acoplamiento frente a los resultados obtenidos mediante el método avanzado con acoplamiento.

El análisis de los resultados muestra con carácter general una reducción de los valores procedentes del método avanzado con acoplamiento con respecto a los procedentes del método avanzado sin acoplamiento. Esta variación afecta en cada uno de los casos de una forma independiente. En las tablas 18 y 19 se muestran dichas variaciones calculadas de acuerdo con la siguiente expresión:

$$
\% \text { Variación }=\frac{F_{\text {Acoplamiento }}-F_{\text {No Acoplamiento }}}{F_{\text {No Acoplamiento }}}
$$

\begin{tabular}{|l|r|r|r|r|}
\hline \multicolumn{5}{|c|}{ Temperatura de $1^{\circ} \mathrm{C}$ y Salinidad del 0,0\% } \\
\hline \multicolumn{4}{|c|}{ CASO A - Princess Amalia Q7 } \\
\hline Parámetro & $\mathrm{D}=0,5 \mathrm{~m}$ & $\mathrm{D}=4,0 \mathrm{~m}$ & $\mathrm{D}=8,0 \mathrm{~m}$ & $\mathrm{D}=16,0 \mathrm{~m}$ \\
\hline $\begin{array}{l}\text { Máximo desplazamiento en } \\
\text { superficie (m) }\end{array}$ & $65,36 \%$ & $-0,26 \%$ & $-3,04 \%$ & $-18,64 \%$ \\
\hline $\begin{array}{l}\text { Máximo esfuerzo cortante en } \\
\text { cimentación (kN) }\end{array}$ & $122,78 \%$ & $-1,34 \%$ & $1,88 \%$ & $-0,03 \%$ \\
\hline $\begin{array}{l}\text { Máximo momento flector en } \\
\text { cimentación }(\mathrm{kN} \cdot \mathrm{m} \text { ) }\end{array}$ & $152,97 \%$ & $-3,30 \%$ & $-3,56 \%$ & $-5,23 \%$ \\
\hline
\end{tabular}


Modelos de predicción de esfuerzos hidrodinámicos y socavación aplicados a ingeniería offshore

\begin{tabular}{|c|c|c|c|c|}
\hline \multicolumn{5}{|c|}{ CASO B - North Hoyle } \\
\hline Parámetro & $\mathrm{D}=0,5 \mathrm{~m}$ & $\mathrm{D}=4,0 \mathrm{~m}$ & $\mathrm{D}=8,0 \mathrm{~m}$ & $\mathrm{D}=16,0 \mathrm{~m}$ \\
\hline $\begin{array}{l}\text { Máximo desplazamiento en } \\
\text { superficie }(\mathrm{m})\end{array}$ & $1,40 \%$ & $-3,28 \%$ & $-4,40 \%$ & $2,98 \%$ \\
\hline $\begin{array}{l}\text { Máximo esfuerzo cortante en } \\
\text { cimentación }(\mathrm{kN})\end{array}$ & $1,29 \%$ & $5,30 \%$ & $-0,86 \%$ & $-0,28 \%$ \\
\hline $\begin{array}{l}\text { Máximo momento flector en } \\
\text { cimentación }(\mathrm{kN} \cdot \mathrm{m})\end{array}$ & $1,74 \%$ & $2,61 \%$ & $-1,10 \%$ & $-2,97 \%$ \\
\hline \multicolumn{5}{|c|}{ CASO C - Egmond Aan Zee } \\
\hline Parámetro & $D=0,5 m$ & $\mathrm{D}=4,0 \mathrm{~m}$ & $\mathrm{D}=8,0 \mathrm{~m}$ & $\mathrm{D}=16,0 \mathrm{~m}$ \\
\hline $\begin{array}{l}\text { Máximo desplazamiento en } \\
\text { superficie }(\mathrm{m})\end{array}$ & $-22,00 \%$ & $-7,85 \%$ & $-1,11 \%$ & $-5,62 \%$ \\
\hline $\begin{array}{l}\text { Máximo esfuerzo cortante en } \\
\text { cimentación (kN) }\end{array}$ & $22,70 \%$ & $-0,68 \%$ & $-0,63 \%$ & $-0,64 \%$ \\
\hline $\begin{array}{l}\text { Máximo momento flector en } \\
\text { cimentación }(\mathrm{kN} \cdot \mathrm{m})\end{array}$ & $32,34 \%$ & $-0,98 \%$ & $-0,76 \%$ & $-0,69 \%$ \\
\hline \multicolumn{5}{|c|}{ CASO D - Thornton Bank } \\
\hline Parámetro & $\mathrm{D}=0,5 \mathrm{~m}$ & $\mathrm{D}=4,0 \mathrm{~m}$ & $\mathrm{D}=8,0 \mathrm{~m}$ & $\mathrm{D}=16,0 \mathrm{~m}$ \\
\hline $\begin{array}{l}\text { Máximo desplazamiento en } \\
\text { superficie }(\mathrm{m})\end{array}$ & $-20,87 \%$ & $-7,65 \%$ & $-10,60 \%$ & $-13,09 \%$ \\
\hline $\begin{array}{l}\text { Máximo esfuerzo cortante en } \\
\text { cimentación }(\mathrm{kN})\end{array}$ & $16,93 \%$ & $-2,74 \%$ & $0,24 \%$ & $-0,23 \%$ \\
\hline $\begin{array}{l}\text { Máximo momento flector en } \\
\text { cimentación }(\mathrm{kN} \cdot \mathrm{m})\end{array}$ & $22,20 \%$ & $-7,15 \%$ & $-1,39 \%$ & $0,09 \%$ \\
\hline
\end{tabular}

Tabla 18. Comparación entre método avanzado con acoplamiento y sin acoplamiento para Temperatura de $1^{\circ} \mathrm{C}$ y Salinidad del $0,0 \%$

\begin{tabular}{|c|c|c|c|c|}
\hline \multicolumn{5}{|c|}{ Temperatura de $22^{\circ} \mathrm{C}$ y Salinidad del $35 \%$} \\
\hline \multicolumn{5}{|c|}{ CASO A - Princess Amalia Q7 } \\
\hline Parámetro & $\mathrm{D}=0,5 \mathrm{~m}$ & $\mathrm{D}=4,0 \mathrm{~m}$ & $\mathrm{D}=8,0 \mathrm{~m}$ & $\mathrm{D}=16,0 \mathrm{~m}$ \\
\hline $\begin{array}{l}\text { Máximo desplazamiento en } \\
\text { superficie }(\mathrm{m})\end{array}$ & $23,56 \%$ & $-0,43 \%$ & $-0,78 \%$ & $-13,68 \%$ \\
\hline $\begin{array}{l}\text { Máximo esfuerzo cortante en } \\
\text { cimentación }(\mathrm{kN})\end{array}$ & $109,77 \%$ & $2,08 \%$ & $1,70 \%$ & $0,14 \%$ \\
\hline $\begin{array}{l}\text { Máximo momento flector en } \\
\text { cimentación }(\mathrm{kN} \cdot \mathrm{m})\end{array}$ & $131,22 \%$ & $-4,51 \%$ & $-3,03 \%$ & $-2,79 \%$ \\
\hline \multicolumn{5}{|c|}{ CASO B - North Hoyle } \\
\hline Parámetro & $\mathrm{D}=0,5 \mathrm{~m}$ & $\mathrm{D}=4,0 \mathrm{~m}$ & $\mathrm{D}=8,0 \mathrm{~m}$ & $\mathrm{D}=16,0 \mathrm{~m}$ \\
\hline $\begin{array}{l}\text { Máximo desplazamiento en } \\
\text { superficie }(\mathrm{m})\end{array}$ & $1,49 \%$ & $-3,29 \%$ & $-1,73 \%$ & $4,95 \%$ \\
\hline $\begin{array}{l}\text { Máximo esfuerzo cortante en } \\
\text { cimentación }(\mathrm{kN})\end{array}$ & $2,87 \%$ & $10,08 \%$ & $-0,05 \%$ & $-0,04 \%$ \\
\hline $\begin{array}{l}\text { Máximo momento flector en } \\
\text { cimentación }(\mathrm{kN} \cdot \mathrm{m})\end{array}$ & $3,94 \%$ & $3,52 \%$ & $0,03 \%$ & $0,03 \%$ \\
\hline \multicolumn{5}{|c|}{ CASO C - Egmond Aan Zee } \\
\hline Parámetro & $\mathrm{D}=0,5 \mathrm{~m}$ & $\mathrm{D}=4,0 \mathrm{~m}$ & $\mathrm{D}=8,0 \mathrm{~m}$ & $\mathrm{D}=16,0 \mathrm{~m}$ \\
\hline $\begin{array}{l}\text { Máximo desplazamiento en } \\
\text { superficie }(\mathrm{m})\end{array}$ & $-23,30 \%$ & $-7,85 \%$ & $-1,11 \%$ & $-5,61 \%$ \\
\hline $\begin{array}{l}\text { Máximo esfuerzo cortante en } \\
\text { cimentación }(\mathrm{kN})\end{array}$ & $16,85 \%$ & $-0,68 \%$ & $-0,63 \%$ & $-0,64 \%$ \\
\hline $\begin{array}{l}\text { Máximo momento flector en } \\
\text { cimentación }(\mathrm{kN} \cdot \mathrm{m})\end{array}$ & $19,24 \%$ & $-0,98 \%$ & $-0,76 \%$ & $-0,69 \%$ \\
\hline \multicolumn{5}{|c|}{ CASO D - Thornton Bank } \\
\hline Parámetro & $\mathrm{D}=0,5 \mathrm{~m}$ & $\mathrm{D}=4,0 \mathrm{~m}$ & $\mathrm{D}=8,0 \mathrm{~m}$ & $\mathrm{D}=16,0 \mathrm{~m}$ \\
\hline $\begin{array}{l}\text { Máximo desplazamiento en } \\
\text { superficie }(\mathrm{m})\end{array}$ & $-20,84 \%$ & $-7,65 \%$ & $-4,63 \%$ & $-13,09 \%$ \\
\hline
\end{tabular}




\begin{tabular}{|l|r|r|r|r|}
\hline $\begin{array}{l}\text { Máximo esfuerzo cortante en } \\
\text { cimentación }(\mathrm{kN})\end{array}$ & $15,31 \%$ & $0,02 \%$ & $0,18 \%$ & $-0,23 \%$ \\
\hline $\begin{array}{l}\text { Máximo momento flector en } \\
\text { cimentación }(\mathrm{kN} \cdot \mathrm{m})\end{array}$ & $19,93 \%$ & $-0,95 \%$ & $0,49 \%$ & $0,09 \%$ \\
\hline
\end{tabular}

A partir de los resultados mostrados en la tabla anterior, se puede observar que las máximas diferencias se producen en el caso de temperatura de $1^{\circ} \mathrm{C}$ y salinidad del $0 \%$ para el diámetro más pequeño. Esto es debido a que la excitación producida por el oleaje sobre la estructura se localiza en frecuencias cercanas a las frecuencias de resonancia de la estructura. El máximo valor se encuentra en el Caso A - Princess Amalia Q7, en el cual la diferencia del momento flector en la cimentación llega a ser del entorno de un 152,97\% más elevada en el caso con acoplamiento, que la calculada según el método avanzado sin acoplamiento.

No obstante, se puede observar que para el resto de casos con diámetros mayores a 0,5 metros, con carácter general, el cálculo, que tiene en cuenta el acoplamiento, arroja resultados con valores para el momento flector menores, dado que al tener en cuenta el movimiento relativo de la estructura, esta presenta una cierta flexibilidad frente al impacto de la onda, que le permite distribuir mejor los esfuerzos a lo largo del tiempo, generando que los máximos esfuerzos sufridos sean menores. Como cabría esperar, este efecto va teniendo menor influencia conforme se aumenta el diámetro de la estructura dado que cuanto más aumenta el diámetro más aumenta la rigidez y la estructura tiene menos capacidad para comportarse de forma flexible y reducir los esfuerzos totales generados por el oleaje sobre ella.

\subsection{Proceso V: Comportamiento de la cimentación.}

El quinto proceso se corresponde con la simulación del fenómeno de socavación en el lecho de la cimentación, teniendo en cuenta las aceleraciones y velocidades halladas en el fondo marino mediante los procesos de cálculo anteriores.

Las variables de cálculo que se van a utilizar en este quinto proceso incorporan el efecto de la corriente para que puedan ser posteriormente comparadas con las mediciones en campo. Es necesario incorporar este efecto porque apenas existen ejemplos de 
mediciones de socavación en parques eólicos offshore, que únicamente estén sometidos al efecto del oleaje. A continuación, se muestra en la tabla 20 los valores utilizados para la simulación.

\begin{tabular}{|c|c|c|c|c|}
\hline Caso & $\begin{array}{c}\text { Altura de ola } \\
\text { significativa }\end{array}$ & Profundidad & $\begin{array}{c}\text { Periodo de } \\
\text { pico }\end{array}$ & Corriente \\
\hline A & $7,70 \mathrm{~m}$ & $24 \mathrm{~m}$ & $9,7 \mathrm{~s}$ & $1,30 \mathrm{~m} / \mathrm{s}$ \\
\hline B & $4,78 \mathrm{~m}$ & $10 \mathrm{~m}$ & $6,8 \mathrm{~s}$ & $0,80 \mathrm{~m} / \mathrm{s}$ \\
\hline C & $3,60 \mathrm{~m}$ & $22 \mathrm{~m}$ & $18,5 \mathrm{~s}$ & $0,7 \mathrm{~m} / \mathrm{s}$ \\
\hline D & $6,32 \mathrm{~m}$ & $16 \mathrm{~m}$ & $11,06 \mathrm{~s}$ & $1,2 \mathrm{~m} / \mathrm{s}$ \\
\hline \multicolumn{4}{|r|}{ Tabla 20. Variables para el cálculo de la socavación } \\
\hline
\end{tabular}

El método tradicional utilizará únicamente la formulación del número de KeuleganCarpenter, que se muestra a continuación:

$$
K C=\frac{\left(\mathrm{u}_{\max } \cdot T\right)}{D}
$$

El método avanzado utilizará la formulación modificada del número de KeuleganCarpenter, que incorpora explícitamente el efecto del campo de aceleraciones:

$$
K C=\frac{2 \cdot \pi \cdot u_{\max }^{2}}{D \cdot a_{\max }}
$$

Se van a definir tres casos de cálculo: el primero será el método avanzado aplicando la teoría de ondas que corresponda y la fórmula modificada del número de KeuleganCarpenter, el segundo tendrá en cuenta el modelo avanzado de teoría de ondas y la fórmula tradicional para hallar el número de Keulegan-Carpenter y, por último, un caso con teoría lineal de ondas y la fórmula tradicional del número de Keulegan-Carpenter.

Los objetivos de este proceso son:

- Influencia del campo de aceleraciones sobre la socavación.

- Influencia de la teoría de ondas a aplicar sobre la socavación.

- Influencia del diámetro sobre la socavación. 


\subsubsection{Análisis de influencia del campo de aceleraciones sobre la socavación}

El proceso $\mathrm{V}$ incluye a la velocidad de corriente en el fondo marino como variable adicional de cálculo. La introducción de esta nueva variable en el modelo permitirá validar los resultados de la simulación con los valores medidos en campo.

Se observará la diferencia entre los resultados obtenidos mediante la formulación avanzada desarrollada a lo largo de la presente Tesis Doctoral con aquellos obtenidos mediante la expresión tradicional del número de Keulegan-Carpenter, con y sin aplicación de los modelos avanzados de mecánica de ondas.

En primer lugar, el análisis de los resultados muestra con carácter general una ligera reducción de los valores procedentes del método avanzado de teoría de ondas con la formulación tradicional para el número de Keulegan-Carpenter con respecto a los procedentes del método tradicional. Esta variación afecta en cada uno de los casos de una forma independiente, siendo despreciable en la mayor parte de ellos. En la tabla 21 se muestran dichas variaciones calculadas de acuerdo con la siguiente expresión:

$$
\% \text { Variación }=\frac{S_{\text {avanzado }, K C \text { tradicional }}-S_{\text {tradicional }}}{S_{\text {tradicional }}}
$$

\begin{tabular}{|c|c|c|c|c|}
\hline \multicolumn{5}{|c|}{ CASO A - Princess Amalia Q7 } \\
\hline Parámetro & $\mathrm{D}=0,5 \mathrm{~m}$ & $\mathrm{D}=4,0 \mathrm{~m}$ & $\mathrm{D}=8,0 \mathrm{~m}$ & $\mathrm{D}=16,0 \mathrm{~m}$ \\
\hline $\begin{array}{l}\text { Profundidad de socavación } \\
\text { máxima en equilibrio }(\mathrm{m})\end{array}$ & $0,00 \%$ & $-0,48 \%$ & $-1,89 \%$ & $-4,26 \%$ \\
\hline \multicolumn{5}{|c|}{ CASO B - North Hoyle } \\
\hline Parámetro & $D=0,5 m$ & $\mathrm{D}=4,0 \mathrm{~m}$ & $\mathrm{D}=8,0 \mathrm{~m}$ & $\mathrm{D}=16,0 \mathrm{~m}$ \\
\hline $\begin{array}{l}\text { Profundidad de socavación } \\
\text { máxima en equilibrio }(\mathrm{m})\end{array}$ & $0,00 \%$ & $0,00 \%$ & $-0,16 \%$ & $\mathrm{~N} / \mathrm{A}$ \\
\hline \multicolumn{5}{|c|}{ CASO C - Egmond Aan Zee } \\
\hline Parámetro & $\mathrm{D}=0,5 \mathrm{~m}$ & $\mathrm{D}=4,0 \mathrm{~m}$ & $\mathrm{D}=8,0 \mathrm{~m}$ & $\mathrm{D}=16,0 \mathrm{~m}$ \\
\hline $\begin{array}{l}\text { Profundidad de socavación } \\
\text { máxima en equilibrio }(\mathrm{m})\end{array}$ & $0,00 \%$ & $0,00 \%$ & $0,16 \%$ & $0,32 \%$ \\
\hline \multicolumn{5}{|c|}{ CASO D - Thornton Bank } \\
\hline Parámetro & $D=0,5 \mathrm{~m}$ & $\mathrm{D}=4,0 \mathrm{~m}$ & $\mathrm{D}=8,0 \mathrm{~m}$ & $\mathrm{D}=16,0 \mathrm{~m}$ \\
\hline $\begin{array}{l}\text { Profundidad de socavación } \\
\text { máxima en equilibrio }(\mathrm{m})\end{array}$ & $0,16 \%$ & $0,18 \%$ & $0,47 \%$ & $0,79 \%$ \\
\hline
\end{tabular}

Tabla 21. Comparación entre el método avanzado con KC según fórmula tradicional y el método tradicional 
A partir de los resultados mostrados en la tabla anterior, se puede observar que las máximas diferencias se producen en el caso del parque eólico offshore Princess Amalia Q7 en Holanda para el diámetro más grande. Como puede observarse, estas variaciones son distintas para cada una de las teorías de mecánica de ondas aplicadas a cada uno de los casos. En el caso del parque Princess Amalia Q7, se ha utilizado la teoría de Stokes de tercer orden, siendo los resultados obtenidos menores que los de la teoría lineal de ondas. En el caso del parque de North Hoyle, la teoría utilizada es Stokes de quinto orden, observándose que apenas existen diferencias entre los resultados aportados por esta teoría y los aportados por la teoría lineal. En el caso del parque Egmond Ann Zee, la teoría utilizada es la cnoidal de segundo orden, y como puede observarse, en este caso dicha teoría presenta un reducido incremento de los valores correspondientes a la profundidad de socavación con respecto a los aportados por la teoría lineal. Por último, en el caso del parque de Thornton Bank, se ha aplicado la teoría de Stream Function de Dean y se observa, nuevamente, que los valores obtenidos mediante esta teoría de ondas son superiores a los aportados por la teoría lineal.

Por otro lado, se observa una tendencia de crecimiento con respecto al diámetro, que magnifica las diferencias entre las distintas teorías y la teoría lineal conforme aumenta el diámetro de la pila. Esto es debido a que la variable diámetro se encuentra multiplicando en el denominador de la fórmula del número de Keulegan-Carpenter y, por ello, cuanto más aumenta el diámetro más aumentan las diferencias, tanto en sentido positivo como negativo. Este hecho también ocurre a nivel numérico en el caso del parque de North Hoyle, lo que ocurre es que el efecto es tan reducido que, debido al redondeo de los valores, la diferencia queda redondeada a cero.

En segundo lugar, el análisis de los resultados muestra con carácter general una reducción de los valores procedentes del método avanzado con el número de Keulegan-Carpenter calculado según el método tradicional con respecto a los procedentes del método avanzado utilizando la nueva formulación desarrollada para calcular el número de Keulegan-Carpenter. Esta variación afecta en cada uno de los casos de una forma independiente. En la tabla 22 se muestran dichas variaciones calculadas de acuerdo con la siguiente expresión: 


$$
\% \text { Variación }=\frac{S_{\text {avanzado }}-S_{\text {avanzado,KC tradicional }}}{S_{\text {avanzado,KC tradicional }}}
$$

\begin{tabular}{|c|c|c|c|c|}
\hline \multicolumn{5}{|c|}{ CASO A - Princess Amalia Q7 } \\
\hline Parámetro & $D=0,5 m$ & $\mathrm{D}=4,0 \mathrm{~m}$ & $D=8,0 m$ & $\mathrm{D}=16,0 \mathrm{~m}$ \\
\hline $\begin{array}{l}\text { Profundidad de socavación } \\
\text { máxima en equilibrio (m) }\end{array}$ & $0,32 \%$ & $3,13 \%$ & $4,69 \%$ & $12,88 \%$ \\
\hline \multicolumn{5}{|c|}{ CASO B - North Hoyle } \\
\hline Parámetro & $D=0,5 m$ & $\mathrm{D}=4,0 \mathrm{~m}$ & $D=8,0 m$ & $\mathrm{D}=16,0 \mathrm{~m}$ \\
\hline $\begin{array}{l}\text { Profundidad de socavación } \\
\text { máxima en equilibrio }(\mathrm{m})\end{array}$ & $0,99 \%$ & $3,79 \%$ & $27,85 \%$ & $\mathrm{~N} / \mathrm{A}$ \\
\hline \multicolumn{5}{|c|}{ CASO C - Egmond Aan Zee } \\
\hline Parámetro & $D=0,5 m$ & $\mathrm{D}=4,0 \mathrm{~m}$ & $D=8,0 m$ & $\mathrm{D}=16,0 \mathrm{~m}$ \\
\hline $\begin{array}{l}\text { Profundidad de socavación } \\
\text { máxima en equilibrio }(\mathrm{m})\end{array}$ & $1,26 \%$ & $19,28 \%$ & $29,37 \%$ & $85,74 \%$ \\
\hline \multicolumn{5}{|c|}{ CASO D - Thornton Bank } \\
\hline Parámetro & $D=0,5 m$ & $D=4,0 m$ & $D=8,0 m$ & $\mathrm{D}=16,0 \mathrm{~m}$ \\
\hline $\begin{array}{l}\text { Profundidad de socavación } \\
\text { máxima en equilibrio }(\mathrm{m})\end{array}$ & $0,00 \%$ & $0,29 \%$ & $0,48 \%$ & $1,13 \%$ \\
\hline
\end{tabular}

Tabla 22. Comparación entre método avanzado de socavación y el método avanzado con KC según fórmula tradicional

A partir de los resultados mostrados en la tabla anterior, se puede observar que las máximas diferencias se producen, en este caso, para el parque eólico offshore Egmond Aan Zee para el diámetro más grande. Como puede observarse, estas variaciones son distintas para cada una de las teorías de ondas aplicadas a cada uno de los casos, pero son siempre positivas.

Estas diferencias permiten reflejar que la introducción explícita del efecto del campo de aceleraciones genera un aumento en los fenómenos de socavación de la cimentación. Estas diferencias no son despreciables, dado que oscilan en un rango variado dependiendo de la teoría de ondas aplicada.

Para el caso del parque Princess Amalia Q7, con teoría de Stokes de tercer orden, se observa que dichas variaciones no superan el 13\%. En el caso del parque de North Hoyle, con Stokes de quinto orden, dichas diferencias alcanza valores alrededor de un $28 \%$. En el caso del parque Egmond Ann Zee, con teoría cnoidal de segundo orden, como puede observarse, se alcanzan diferencias máximas del entorno del $86 \%$. Por último, en el caso 
del parque de Thornton Bank, con teoría de Stream Function de Dean, se observa una reducida variación que alcanza el 1,13\% en su valor máximo.

Por otro lado, se observa, nuevamente, una tendencia de crecimiento con respecto al diámetro que magnifica las diferencias entre la formulación que tiene en cuenta el efecto del campo de aceleraciones y la formulación tradicional. Esto es debido a que la variable diámetro se encuentra multiplicando en el denominador de la fórmula del número de Keulegan-Carpenter y, por ello, cuanto más aumenta el diámetro más aumentan las diferencias. 


\section{Análisis general de los resultados y discusión}

En este apartado se va a proceder a realizar un análisis general de los resultados aportados por cada uno de los procesos. Además, se desarrollará una discusión profunda de los mismos, que permita, posteriormente, realizar una exposición detallada de las principales conclusiones extraídas de cada uno de los procesos de cálculo.

\subsection{Proceso I: Mecánica de ondas}

En primer lugar, este análisis pretendía observar las diferencias existentes entre el campo de velocidades y el de aceleraciones para cada una de las distintas teorías planteadas en relación con los resultados obtenidos mediante la teoría lineal de ondas. A continuación, se muestran las principales diferencias en la figura 10 con respecto al campo de velocidades:
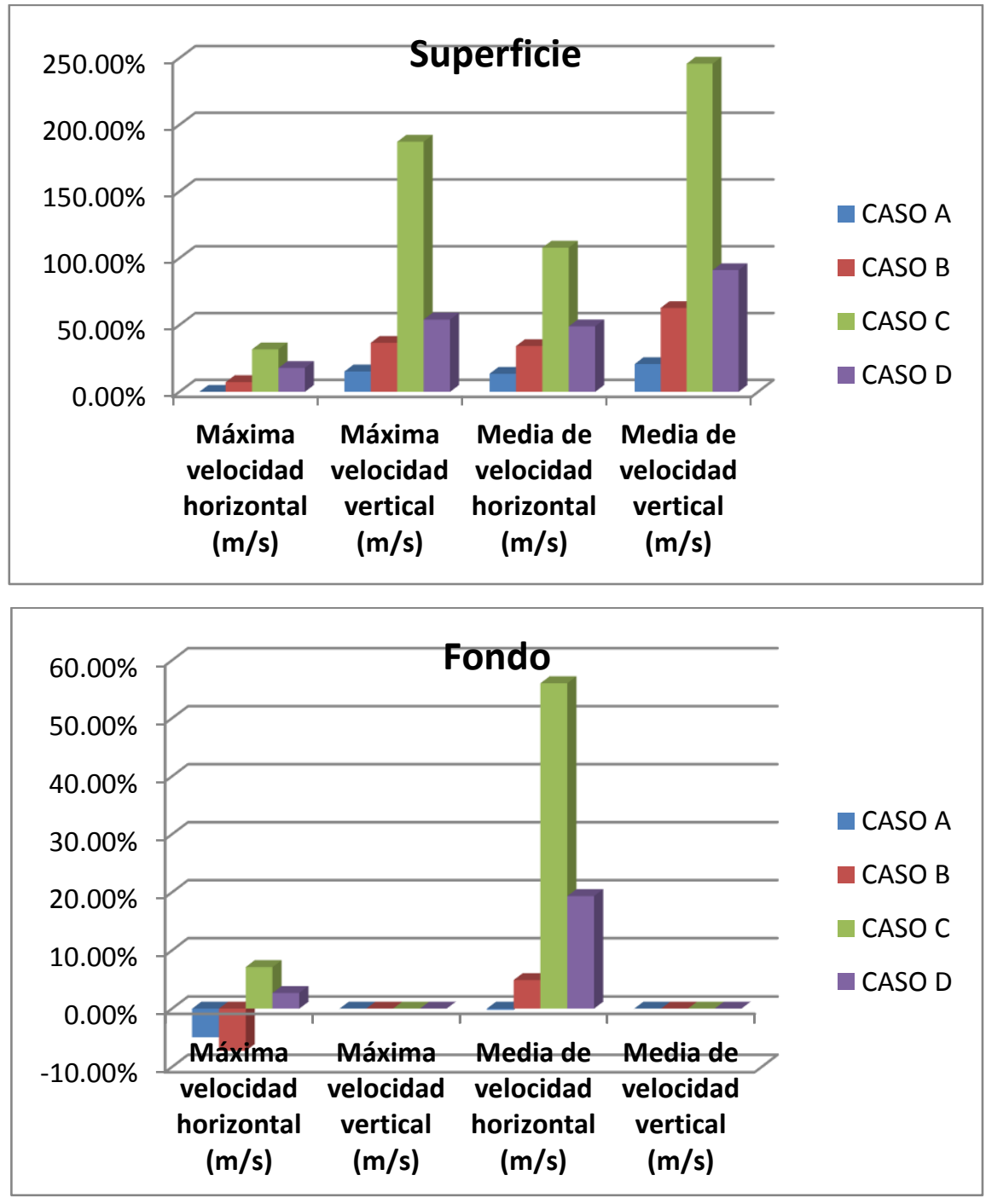

Figura 10. Comparación de velocidades entre teorías de ondas 
Las variaciones han sido calculadas de acuerdo con la siguiente expresión:

$$
\% \text { Error }=\frac{v_{\text {lineal }}-v_{\text {no lineal }}}{v_{\text {no lineal }}}
$$

Como puede observarse en la figura anterior, todas las teorías de ondas no lineales presentan valores de velocidad mucho más reducidos, que los obtenidos mediante la teoría lineal. Esto implicará que en los modelos de cálculo se estarán sobrestimando las fuerzas de arrastre y, por lo tanto, para estructuras que posean un diámetro reducido se estará sobredimensionando la capacidad resistente de las mismas.

Además, se observa que este sobredimensionamiento puede llegar a ser del orden de hasta 2 veces el requerido, como muestra el resultado de la teoría cnoidal de $2 \circ$ orden, en sus valores de variación de velocidad horizontal. Con respecto al campo de aceleraciones, se muestran las variaciones en la figura 11 calculadas de acuerdo con la siguiente expresión:

$$
\% \text { Error }=\frac{a_{\text {lineal }}-a_{\text {no lineal }}}{a_{\text {no lineal }}}
$$

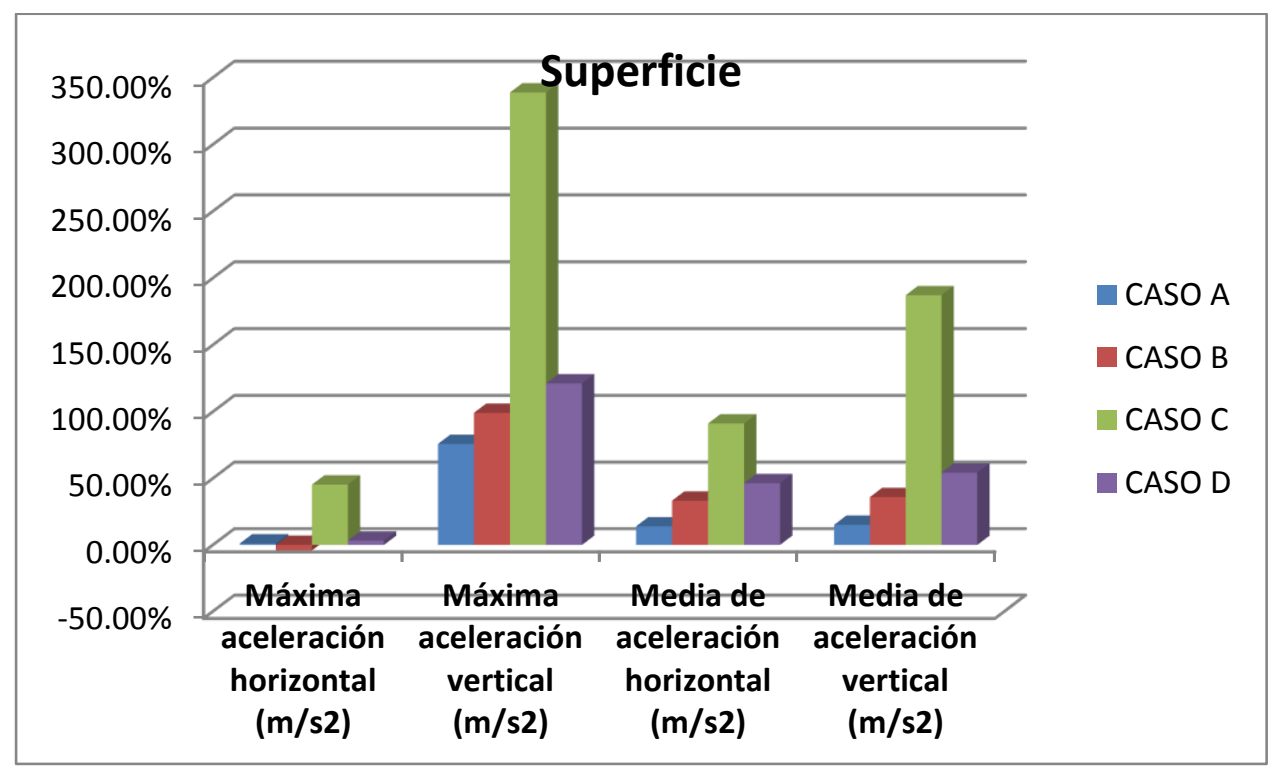




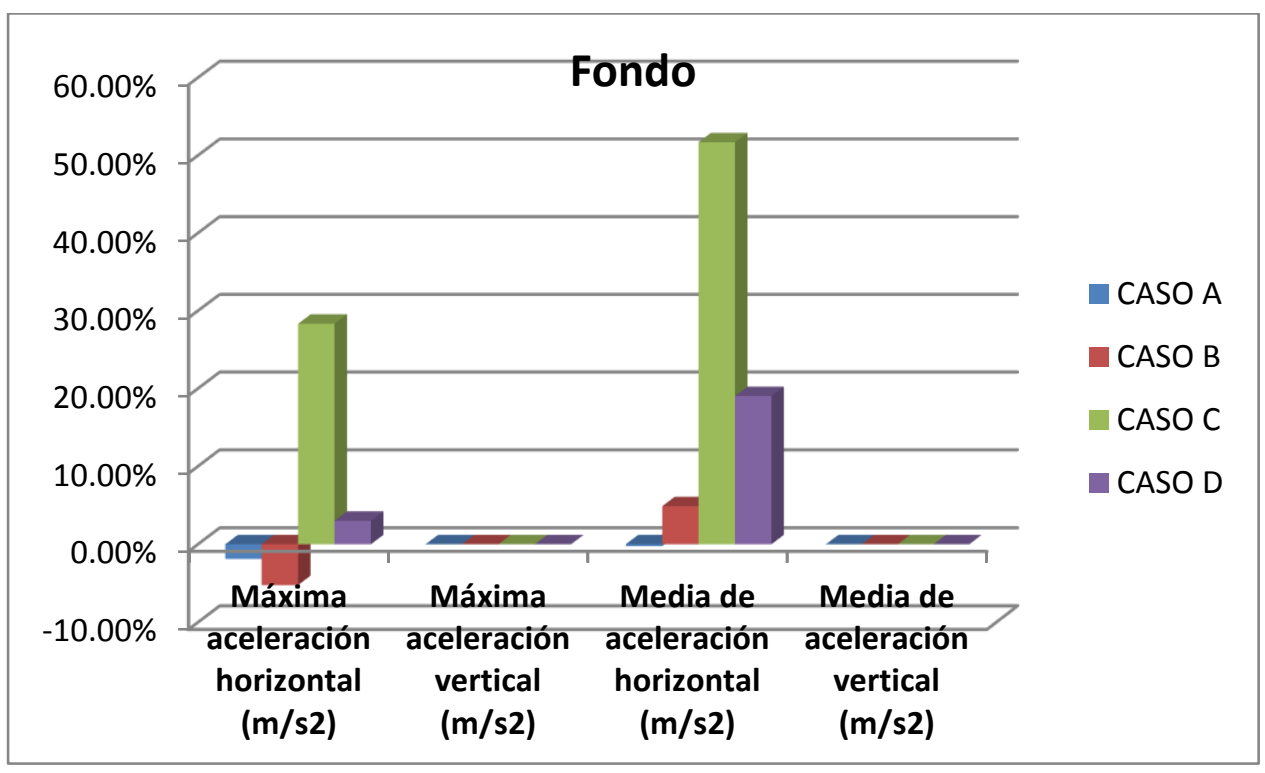

Figura 11. Comparación de aceleraciones entre teorías de ondas

Puede observarse, nuevamente, que, con carácter general, las teorías de ondas no lineales presentan valores de aceleración mucho más reducidos que los obtenidos mediante la teoría lineal. Esto implicará que en los modelos de cálculo se estarán sobrestimando las fuerzas de inercia y, por lo tanto, para estructuras que posean un diámetro importante, se estará sobredimensionando la capacidad resistente de las mismas.

Además, se observa que este sobredimensionamiento puede llegar a ser del orden de hasta 2 veces el requerido, como muestra el resultado de la teoría cnoidal de 20 orden, en sus valores de variación de aceleración horizontal.

No obstante, tanto para el campo de velocidades como para el campo de aceleraciones, existe una excepción con respecto a los valores aportados por el caso A y el caso B en el fondo marino para los diámetros más pequeños, donde se observa que las teorías no lineales de Stokes de tercer orden y Stokes de quinto orden, respectivamente, aportan valores mayores de velocidad y aceleración que los aportados por la teoría lineal.

Por ello, en estos casos, pese a que la solicitación de la estructura será menor, de acuerdo con los resultados del modelo avanzado de mecánica de ondas, los posibles valores de socavación de la cimentación serán mayores que los estimados mediante la teoría lineal. Por otro lado, se observa que dichas variaciones, en todos los casos, tienen mucho más efecto en superficie que en fondo, lo cual demuestra que los efectos no lineales se ven 
fuertemente atenuados con la profundidad. También se observa que, en todos los casos, dichas variaciones son mucho mayores en la dirección vertical con respecto a las variaciones mostradas en la dirección horizontal.

Con respecto al análisis de frecuencias, tal y como puede observarse en los gráficos de resultados incluidos en el "Apéndice 1", se observa que las teorías no lineales, como cabría esperar, reparten su espectro entre un mayor número de frecuencias, mientras que la teoría lineal lo concentra todo en una única frecuencia. En este caso, la teoría cnoidal de 2 - Orden, utilizada para calcular el caso $\mathrm{C}$, es la que genera un mayor reparto frecuencial. Este efecto será tenido en cuenta durante los apartados siguientes para observar la influencia de las distintas frecuencias sobre el comportamiento dinámico de la estructura.

Por último, con respecto a los valores de amortiguación, tal y como puede observarse en los gráficos de resultados incluidos en el "Apéndice 1", se observa también que la teoría lineal tiene una amortiguación mayor que las teorías no lineales, tanto en el campo de velocidades como en el campo de aceleraciones. Este efecto también tendrá una gran influencia sobre el comportamiento de la estructura y será analizado en los apartados siguientes.

\subsection{Proceso II: Cálculo de fuerzas hidrodinámicas}

Este segundo análisis pretendía identificar, en primer lugar, las diferencias existentes entre el método tradicional, que no tiene en cuenta efectos no lineales ni de difracción y el método avanzado, que tiene en cuenta todos los efectos no lineales, la difracción y la mecánica de ondas que mejor se ajuste al caso de estudio. A continuación, se muestran en la figura 12 las principales variaciones de la fuerza total, en superficie y en fondo, para cada uno de los parques estudiados en relación con el diámetro para el caso de temperatura de $22^{\circ} \mathrm{C}$ y salinidad del $35 \%$, dado que este caso ofrece las mayores variaciones. 


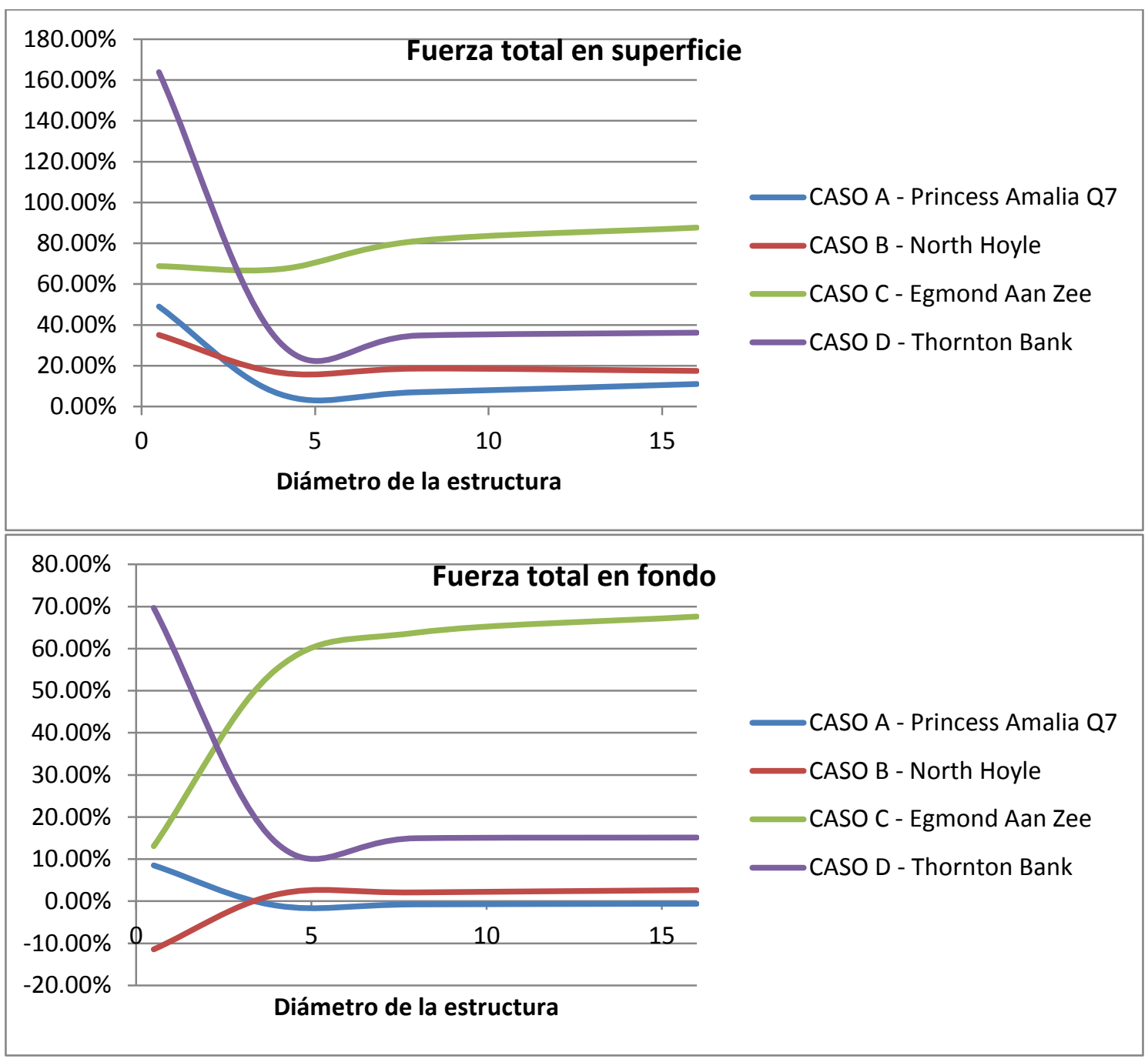

Figura 12. Comparación de fuerza total entre método avanzado y tradicional, en relación al diámetro

Estas variaciones han sido calculadas de acuerdo con la siguiente expresión:

$$
\% \text { Variación }=\frac{F_{\text {Tradicional }}-F_{\text {Avanzado }}}{F_{\text {Avanzado }}}
$$

El análisis de los resultados muestra un claro aumento de los valores procedentes del método tradicional con respecto a los procedentes del método avanzado. El máximo valor se encuentra en el Caso D - Thornton Bank, en el cual la diferencia de la fuerza total máxima en superficie llega a ser del entorno de un $163 \%$ más elevada en el caso tradicional que la calculada según el método avanzado para el diámetro más pequeño y alcanza un valor de entorno al 36\% para el diámetro más grande. En el Caso A - Princess Amalia Q7, estos valores alcanzan un valor máximo de un $49 \%$ para el diámetro más pequeño y un valor del $11 \%$ para los diámetros mayores. En el Caso B - North Hoyle, 
dichos valores alcanzan un $35 \%$ para el menor diámetro y valores de un $18 \%$ para los diámetros mayores. Por último, en el caso C - Egmond Aan Zee, el máximo valor alcanza el $87 \%$ para los casos de mayor diámetro.

A partir de los resultados mostrados en la figura anterior, se puede observar que las máximas diferencias se producen en el caso del diámetro más pequeño, es decir, 0,5 metros. Esto es debido a que las máximas diferencias entre el método tradicional y el método avanzado se producen en las fuerzas viscosas, y estas son las fuerzas predominantes en los diámetros en que $D / L<0,2$. A continuación, se muestra la variación que sufren las fuerzas de arrastre en la figura 13.
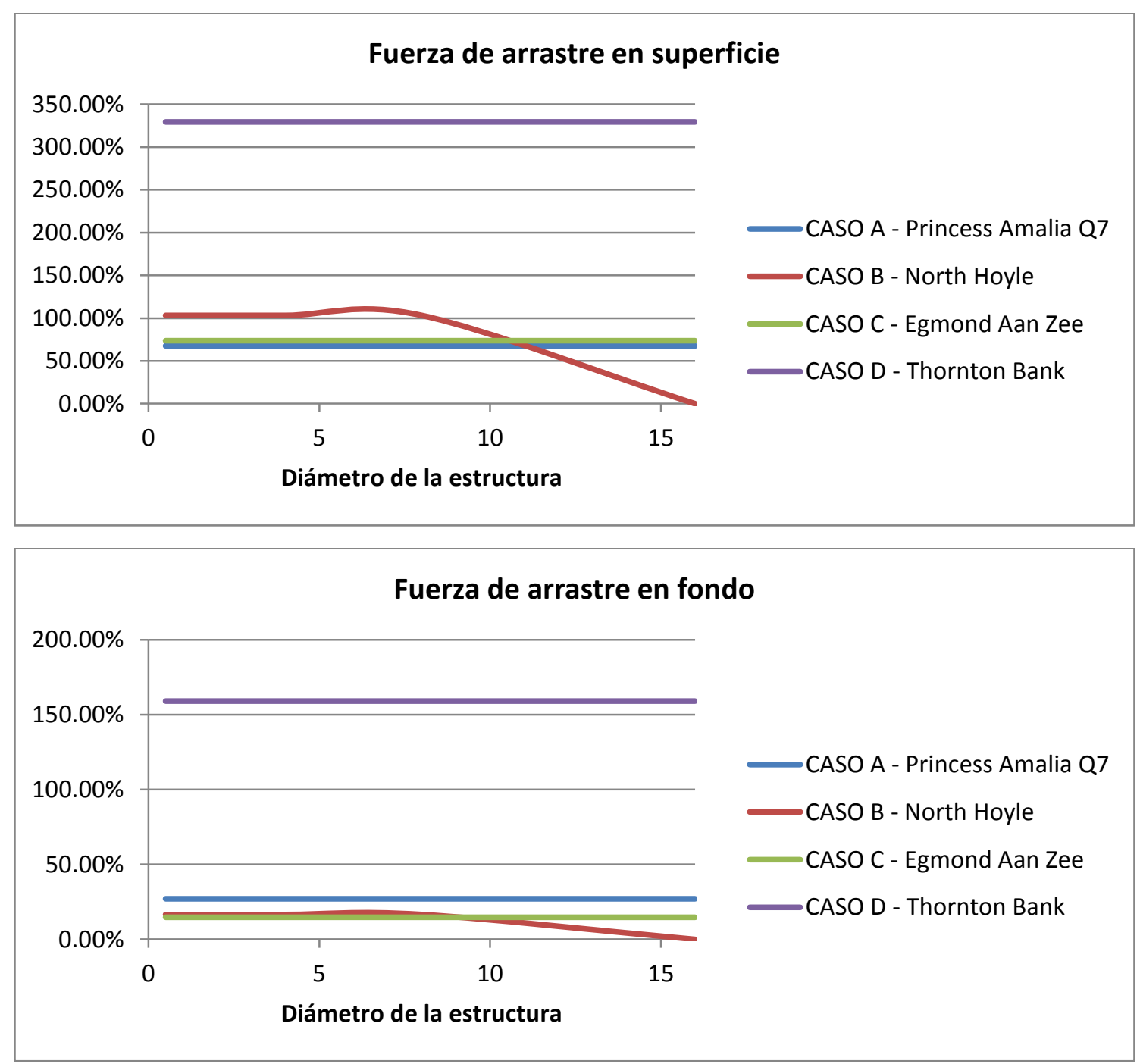

Figura 13. Comparación de fuerzas de arrastre entre método avanzado y tradicional, en relación al diámetro 
Como puede observarse, la variación que sufren las fuerzas de arrastre se mantiene constante con el diámetro. Por lo tanto, pese a que tienen un gran peso sobre la fuerza total para los diámetros pequeños, conforme aumenta el diámetro, su influencia desaparece. La pendiente decreciente que se observa en el Caso B - North Hoyle es debida a que para el diámetro de 16 metros, la herramienta de cálculo detecta la aparición de efectos debidos a la difracción, y debido a este hecho, los valores experimentados por las fuerzas de arrastre son tan pequeños en comparación con la fuerza total resultante que la herramienta de cálculo los considera despreciables y considera que su valor es cero. Por este motivo, la variación producida se muestra como cero en la figura 13.

Por otro lado, se observa una segunda tendencia de crecimiento desde el diámetro de 4 metros hasta el diámetro de 16 metros, que viene causada por las fuerzas de inercia, tal y como se muestra en la figura 14. A continuación, se muestran en la figura 14 las variaciones que experimentan las fuerzas de inercia.

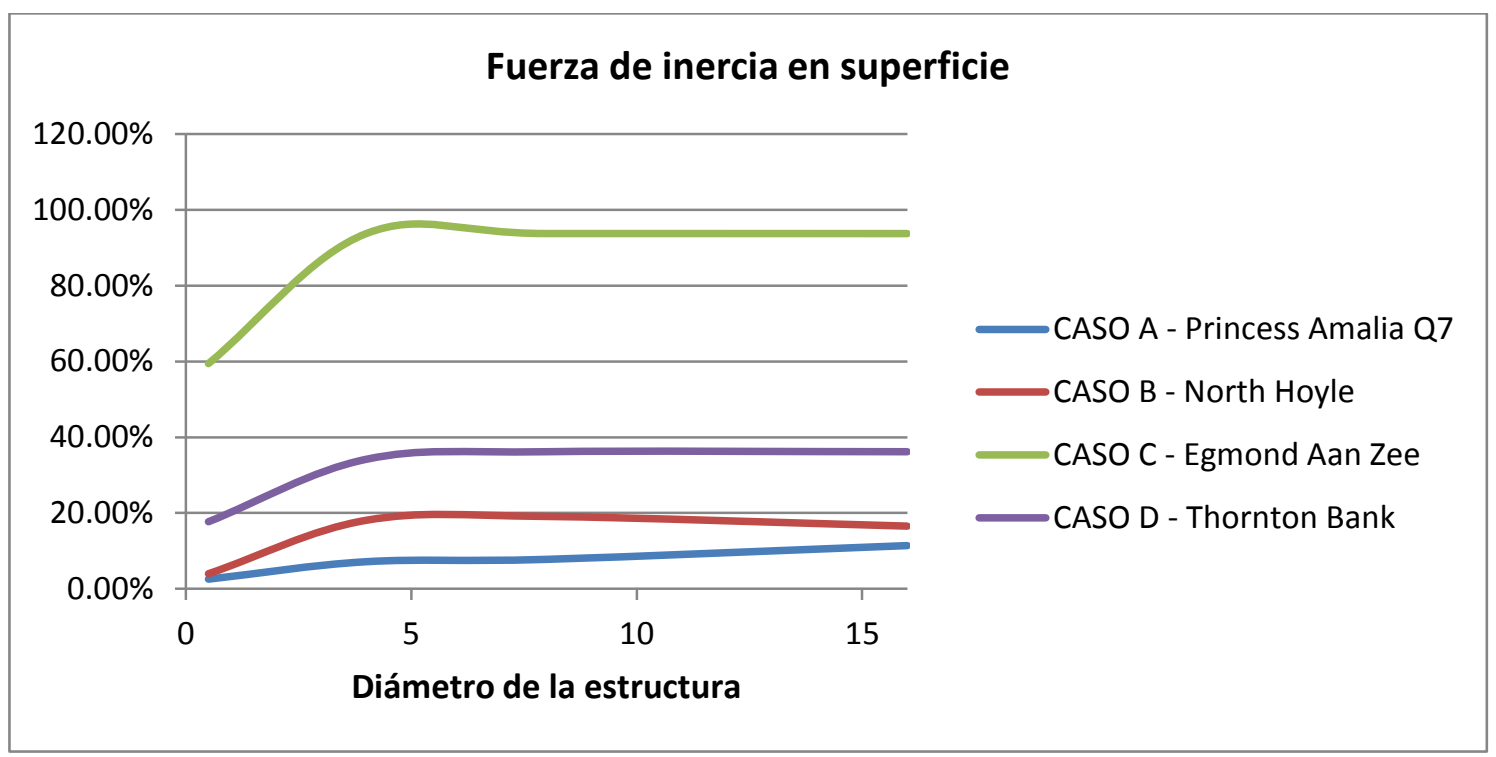




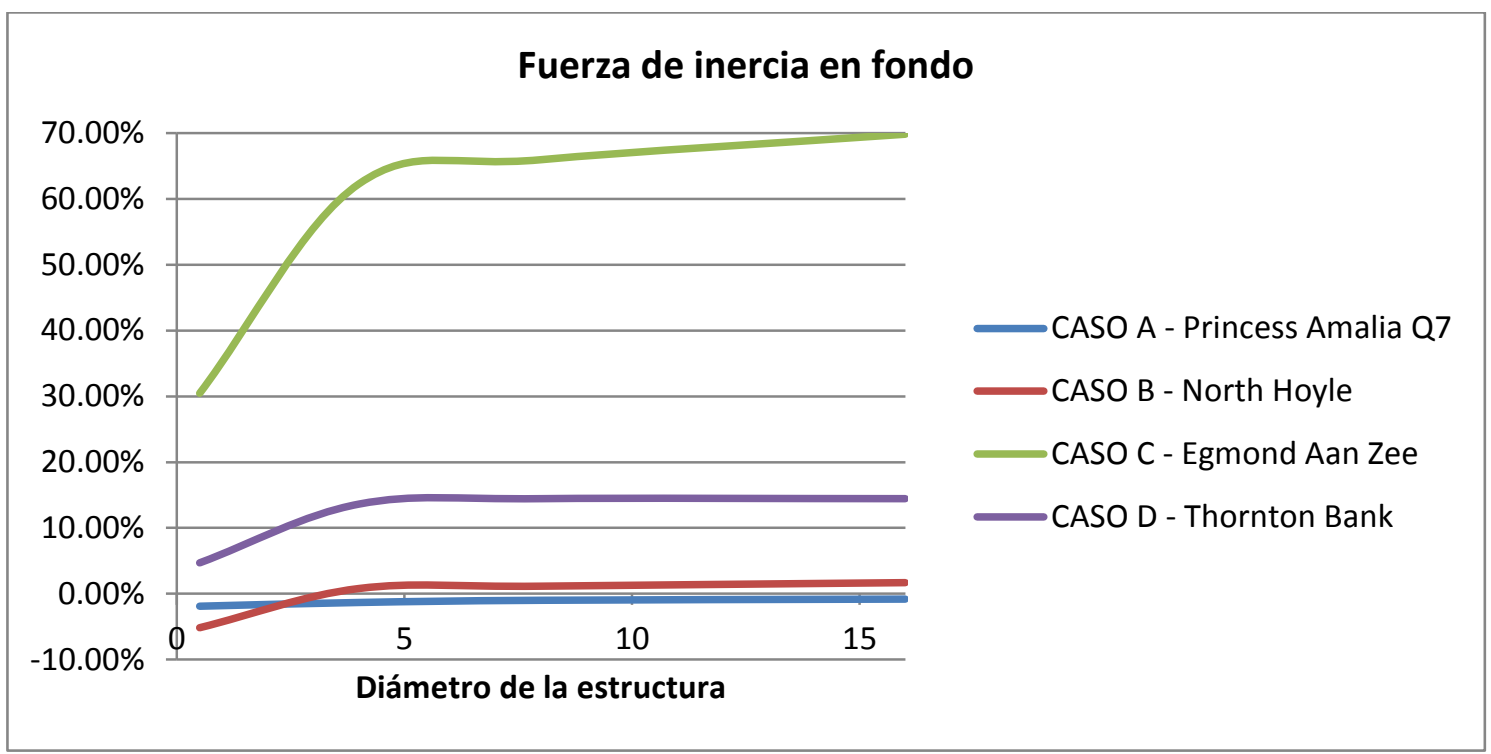

Figura 14. Comparación de fuerzas de inercia entre método avanzado y tradicional, en relación al diámetro

Como puede observarse, la variación sufrida por las fuerzas de inercia experimenta un ligero crecimiento, al mismo tiempo que crece el diámetro. La variación de la fuerza total para los diámetros mayores vendrá influenciada de forma predominante por la variación sufrida por las fuerzas de inercia.

Por todo ello, se puede señalar que la pendiente de la función que define la variación de la fuerza total entre el método tradicional y el avanzado en el intervalo de diámetros entre 0,5 y 4 metros viene definida por la pérdida de influencia que sufren las fuerzas de arrastre, pese a que mantienen su valor de variación prácticamente constante aunque varíe el diámetro. En el intervalo entre 4 y 16 metros de diámetro, la pendiente tiende asintóticamente a imitar a la pendiente que experimenta la función de crecimiento de las fuerzas de inercia cuando aumenta el diámetro.

Además, se observa que todas las variaciones son mucho mayores en superficie que en fondo, como cabía esperar, de acuerdo con los resultados de mecánica de ondas mostrados en el Proceso I.

Con respecto a la influencia de la salinidad y la temperatura, se observa que en la mayor parte de los casos la fuerza hidrodinámica total es mayor para el caso de temperatura del agua a $1^{\circ} \mathrm{C}$ y salinidad del $0 \%$ que para el caso de temperatura a $22^{\circ} \mathrm{C}$ y salinidad del $35 \%$. A continuación, se muestra en la figura 15 la variación de las fuerzas hidrodinámicas con la 
temperatura y la salinidad, siendo estas variaciones calculadas de acuerdo con la siguiente expresión:

$$
\% \text { Variación }=\frac{F_{T=11^{\circ} C,} S=0 \%{ }^{\circ}-F_{T=22^{\circ} C, S=35 \%}}{F_{T=22^{\circ} C} C, S=35 \%}
$$
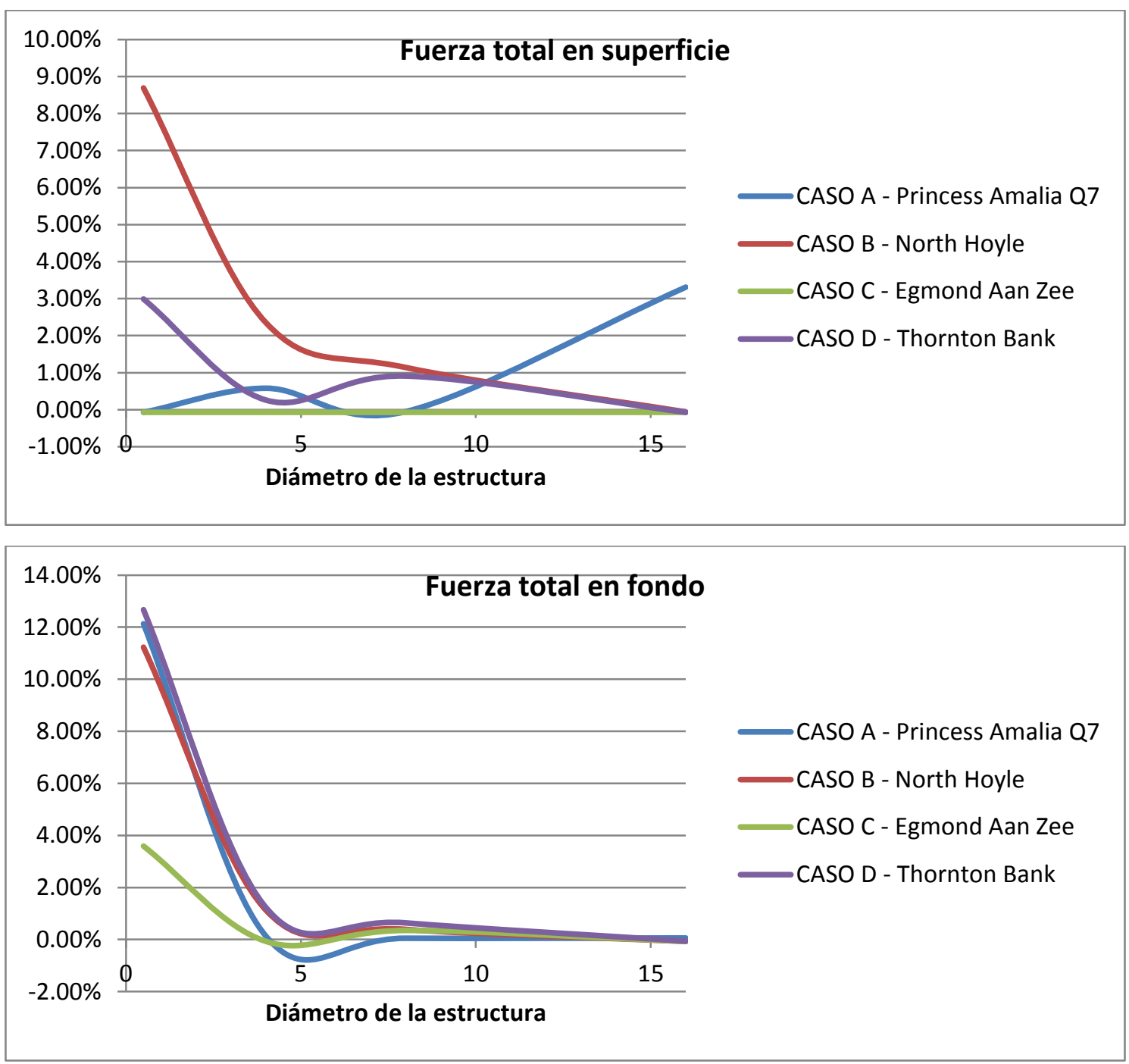

Figura 15. Variación de fuerza total para variaciones de temperatura y salinidad, en relación al diámetro

Dicha variación puede alcanzar valores de hasta un $9 \%$ en superficie y un $13 \%$ en el lecho marino. Se observa que, generalmente, esta variación se está produciendo para el diámetro menor y que, posteriormente, tiende a cero conforme aumenta el diámetro. Este hecho pone de manifiesto que la variación de la salinidad y la temperatura tendrá predominantemente influencia sobre las fuerzas de arrastre $y$, por ello, induce variaciones máximas de la fuerza total en los diámetros más pequeños $\mathrm{y}$, posteriormente, va tendiendo asintóticamente a cero. Esta influencia sobre las fuerzas de arrastre, en 
realidad, actúa como una variación de la fuerza de arrastre media, dado que, como se puede observar en las tablas de resultados, la fuerza máxima de arrastre no solo no aumenta sino que se ve reducida un $0,07 \%$. Por lo tanto, se puede concluir que es la variación de la viscosidad del agua la que está generando estas diferencias. También pone de manifiesto que la variación de la densidad del agua, que es la que influye sobre las fuerzas de inercia, no tiene una influencia muy significativa. No obstante, esta segunda afirmación tiene una excepción en el valor obtenido para la fuerza total en superficie para el caso de mayor diámetro, en el Caso A - Princess Amalia Q7, en el cual se observa un incremento superior al $3 \%$ de la fuerza de inercia.

Por todo ello, puede afirmarse que un incremento de la temperatura y la salinidad del agua tendrá el mismo efecto que una reducción virtual del diámetro de cálculo.

La figura 15 también pone de manifiesto que, si bien la influencia de la temperatura y la salinidad en el fondo marino presenta una tendencia constante para todos los casos estudiados, en superficie, dicha influencia dependerá mucho de la teoría de ondas que se esté aplicando, dado que se podrán tener casos con una tendencia totalmente contraria, como en el caso A - Princess Amalia Q7, o casos en los que la influencia es despreciable, como en el Caso C - Egmond Aan Zee.

\subsection{Proceso III: Análisis dinámico de la estructura}

El tercer análisis se corresponde con el cálculo del comportamiento dinámico de la estructura a partir de las fuerzas hidrodinámicas generadas por el oleaje, realizándose una comparación entre el método tradicional, que tendrá en cuenta únicamente un cálculo estático, y el método de cálculo avanzado que introducirá los efectos dinámicos que sufre la estructura. De acuerdo con los resultados mostrados en este apartado, es posible extraer las siguientes conclusiones a partir de los objetivos que se planteaban.

Los resultados generados mediante el proceso de cálculo tradicional, que utiliza un modelo estático para el cálculo de la estructura, son siempre del orden de entre un $20 \%$ y $100 \%$ mayores que los resultados obtenidos mediante el proceso de cálculo avanzado, que 
utiliza un modelo dinámico que tiene en cuenta las vibraciones que sufre la estructura. A continuación, se muestra la variación de los esfuerzos en el lecho marino en la figura 16.

Estas variaciones son calculadas de acuerdo con la siguiente expresión:

$$
\% \text { Variación }=\frac{F_{\text {Tradicional }}-F_{\text {Avanzado }}}{F_{\text {Avanzado }}}
$$
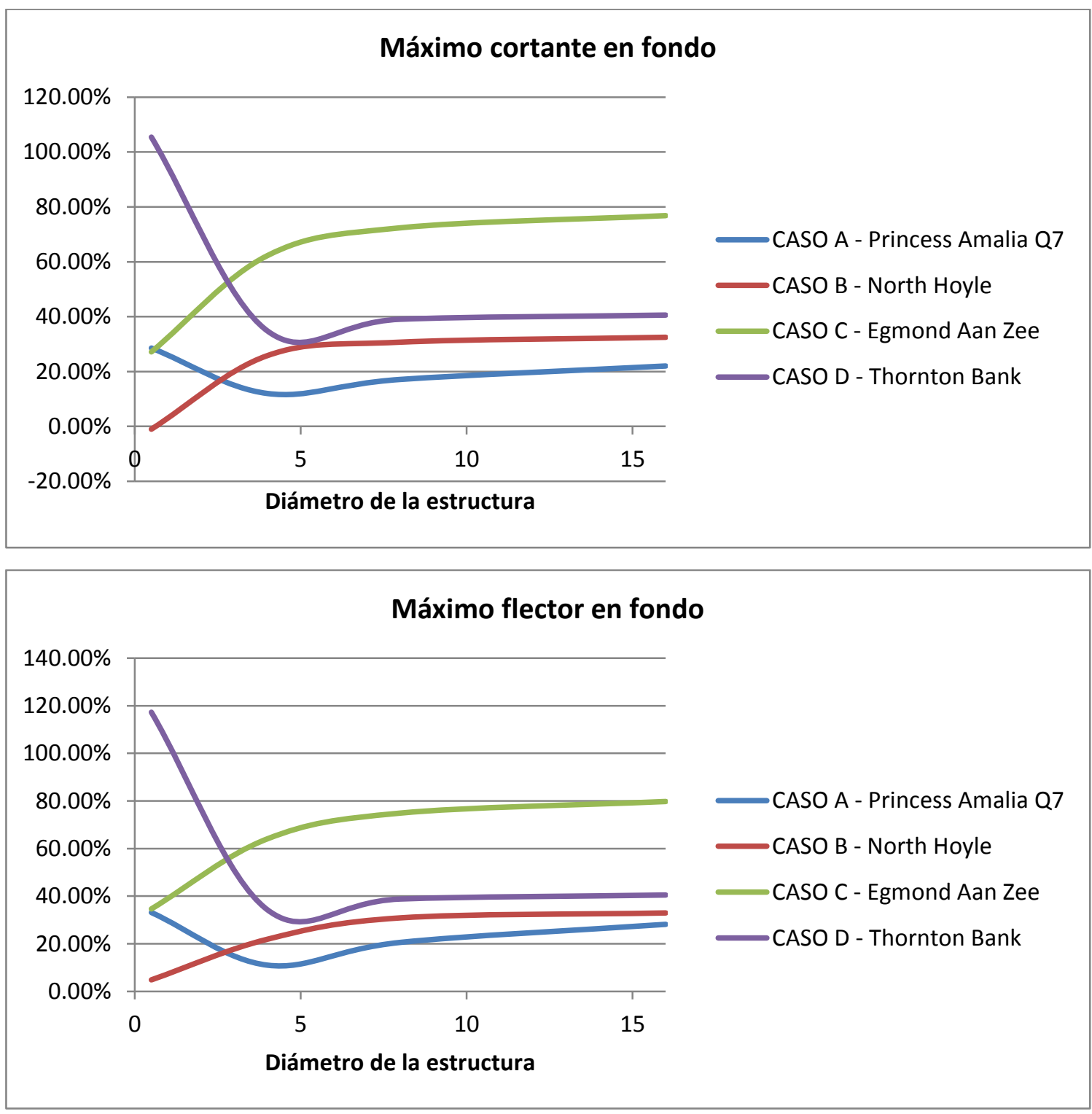

Figura 16. Variación de los esfuerzos en el lecho marino, en relación al diámetro

A partir de los resultados mostrados en la figura anterior, se puede observar que, dependiendo de la teoría de ondas utilizada, se van a encontrar las máximas diferencias para el diámetro más pequeño o para el diámetro más grande. Por un lado, en los casos $\mathrm{A}$ 
y D las máximas diferencias entre el método tradicional y el método avanzado se producen en el diámetro más pequeño y, por lo tanto, en las fuerzas viscosas. Por otro, en los casos B y C, las máximas diferencias se producen en el caso del diámetro más grande y, por lo tanto, ponen de manifiesto que en estos casos son las fuerzas de inercia las que están influyendo en estas variaciones de forma predominante.

El máximo valor se encuentra en el Caso D - Thornton Bank, en el cual la diferencia del momento flector en la cimentación llega a ser del entorno de un 117,25\% más elevada en el caso tradicional que la calculada según el método avanzado.

No obstante, los cuatro casos tienen en común una segunda tendencia de crecimiento en la diferencia entre los resultados generados por el método tradicional frente a los obtenidos mediante el método avanzado desde el diámetro de 4 metros hasta el diámetro de 16 metros. Esto es debido a que, conforme se va aumentando el diámetro de la estructura y las cargas sufridas por la misma, los efectos de vibración dinámica de la estructura también van siendo más importantes. Dicho efectos generan una mayor distribución de la ley de momentos flectores a largo de toda la estructura, lo que genera que se incremente la magnitud de los movimientos medidos en el extremo libre, pero se reduzca el momento total que está sufriendo la cimentación.

También se observa que, generalmente, las fuerzas totales resultantes en fondo son superiores para el caso de cálculo tradicional a temperatura de $22^{\circ} \mathrm{C}$ y salinidad del $35 \%$ que para el caso de cálculo avanzado siendo la variación máxima del 105,44\%.

Con respecto a los movimientos sufridos por la estructura, como cabía esperar, los máximos desplazamientos se producen en el extremo libre de la pila. Estos desplazamientos son menores al ser calculados mediante el proceso de cálculo estático que los resultantes del proceso de cálculo dinámico, tal y como muestra la figura 17. 


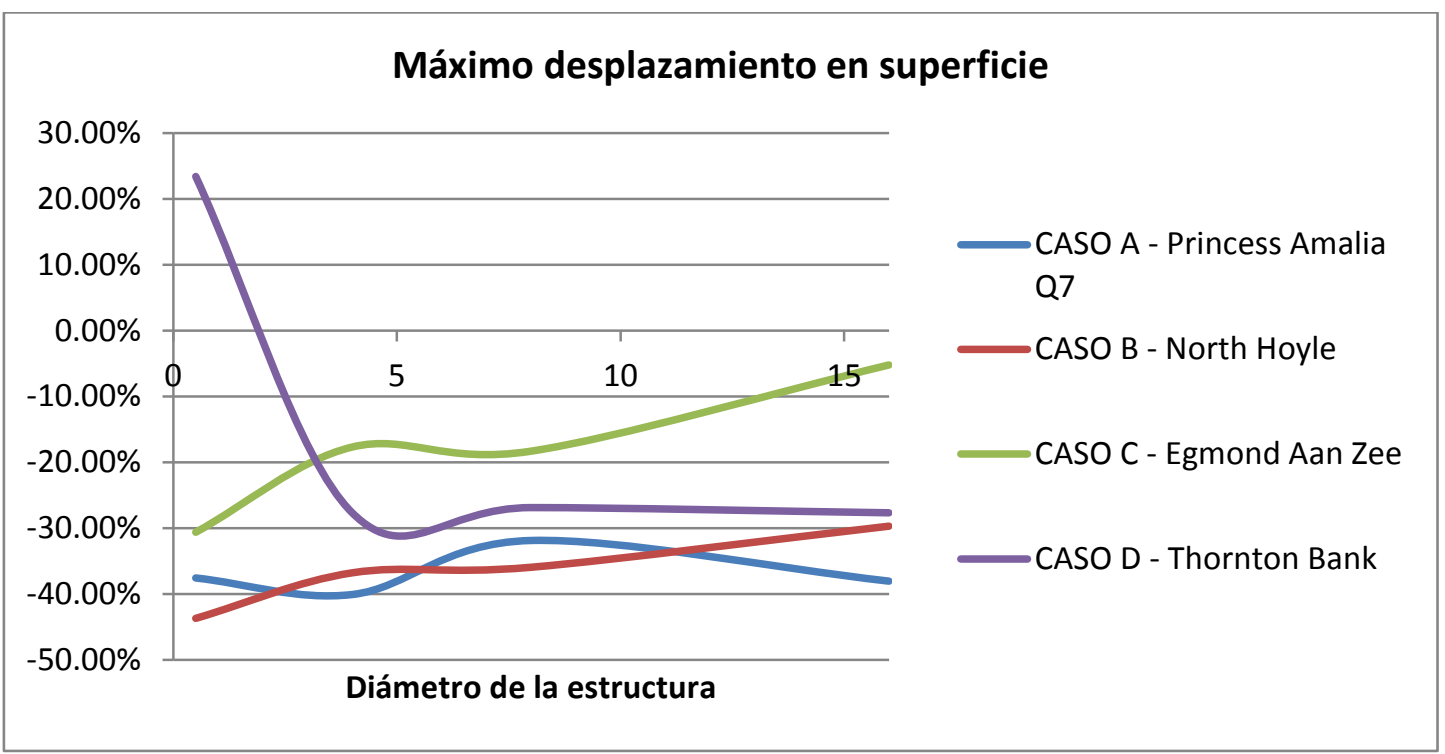

Figura 17. Variación de los desplazamientos en superficie, en relación al diámetro

Las variaciones que se producen en el cálculo debido a la influencia de la temperatura y la salinidad se muestran a continuación en la figura 18. Dichas variaciones son calculadas de acuerdo con la siguiente expresión:

$$
\% \text { Variación }=\frac{F_{T=1{ }^{\circ} \mathrm{C},}, S=0 \%{ }^{\circ}-F_{T=22^{\circ} \mathrm{C}}, S=35 \%}{F_{T=22^{\circ} \mathrm{C}}, S=35 \%}
$$

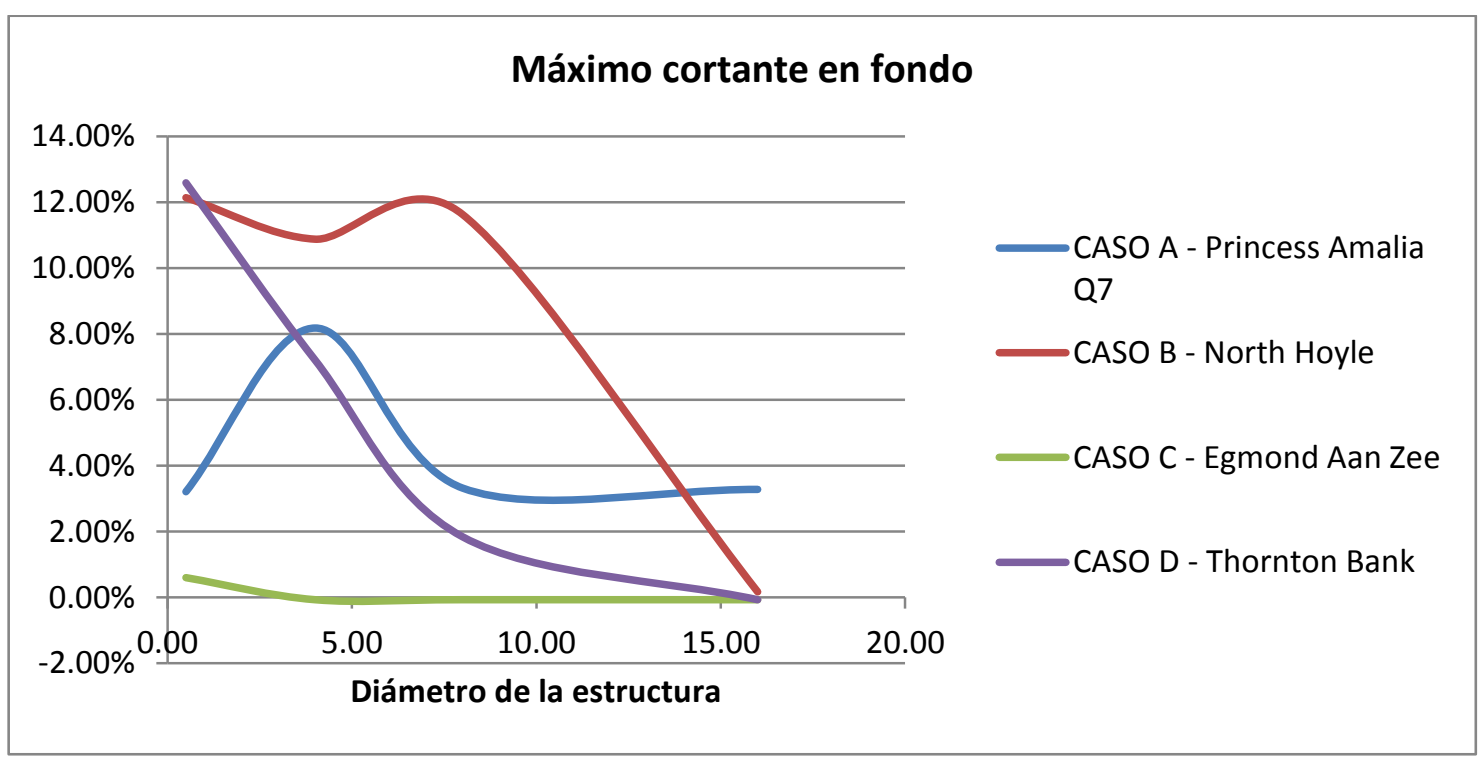




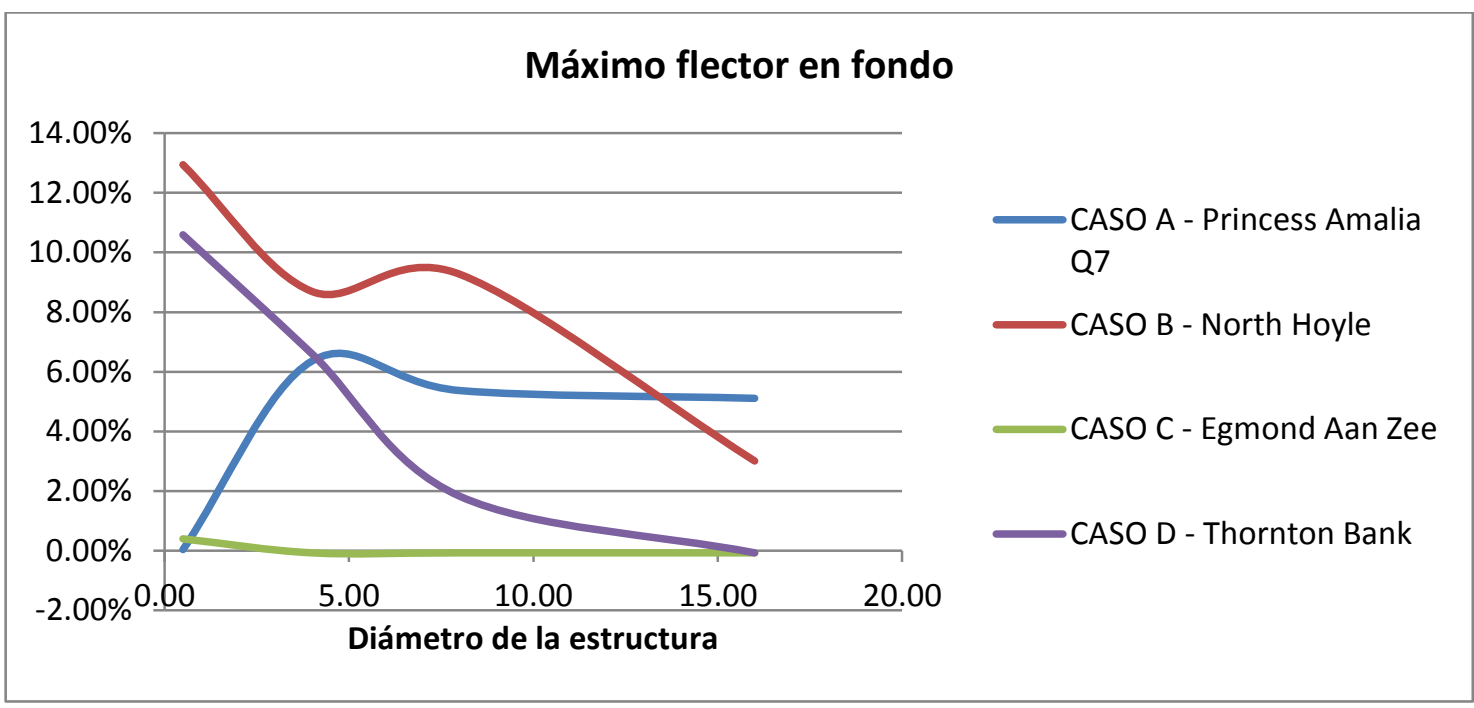

Figura 18. Variación de los esfuerzos en la cimentación debido a la temperatura y la salinidad, en relación al diámetro

Se puede observar una clara tendencia que demuestra que las variaciones de salinidad y temperatura afectan de forma más significativa en los casos con diámetro más reducido con carácter general. Nuevamente, este hecho implica que la variación más importante viene representada por la variación que sufren las fuerzas viscosas. También pone de manifiesto que la variación de la densidad del agua, que es la que influye sobre las fuerzas de inercia, tiene una influencia menor siendo estas conclusiones totalmente coherentes con las obtenidas en el proceso II.

Las variaciones de los movimientos de la estructura debidas a modificaciones de la temperatura y la salinidad se muestran a continuación en la figura 19.

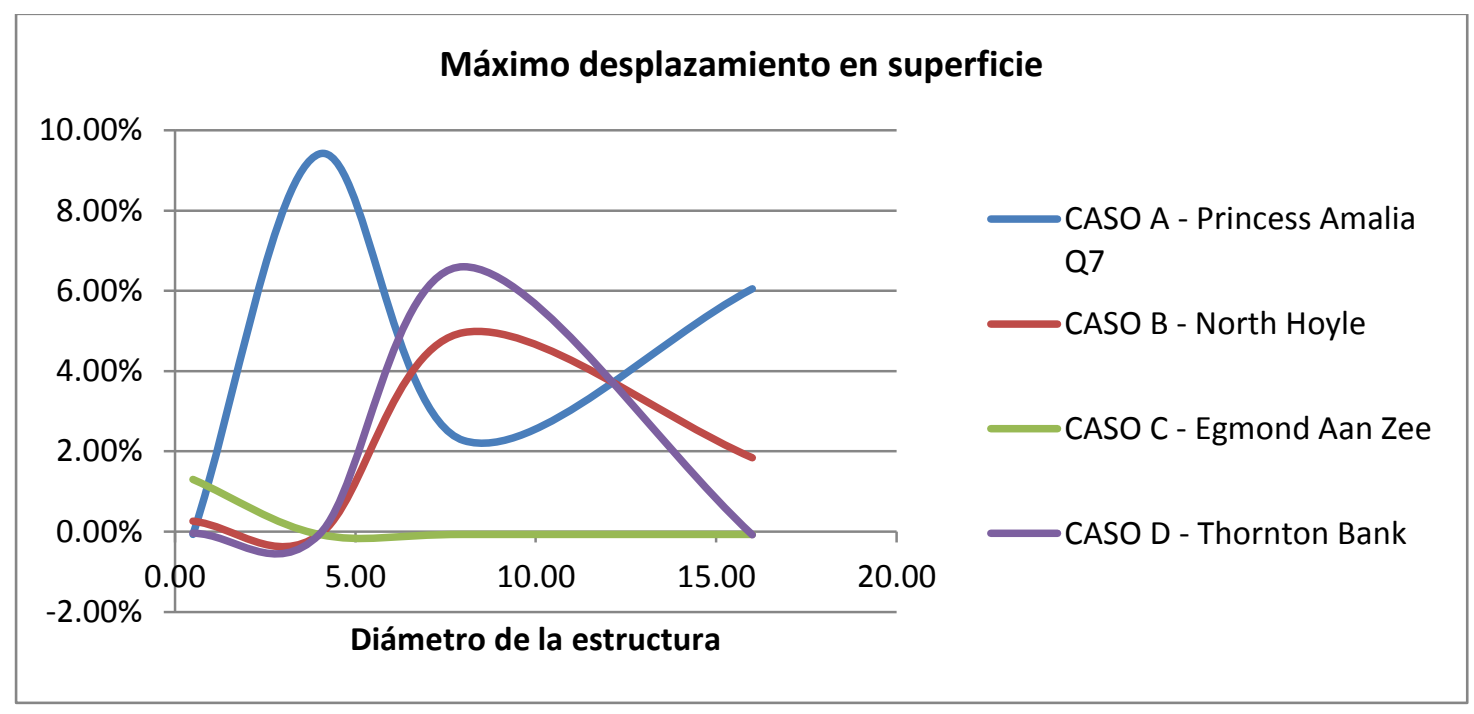

Figura 19. Variación de los movimientos de la estructura debido a la temperatura y la salinidad, en relación al diámetro 
En la figura 19 se observa que los movimientos de la estructura serán mayores para el caso de temperatura de $1^{\circ} \mathrm{C}$ y salinidad del $0 \%$. Este hecho se debe a que, tal y como se definió en el apartado anterior, un incremento de la temperatura y la salinidad del agua tendrá el mismo efecto que una reducción virtual del diámetro de cálculo en lo referente a las cargas hidrodinámicas y, por lo tanto, al mantenerse la rigidez de la estructura constante se producen mayores movimientos para valores de salinidad y temperatura bajos.

\subsection{Proceso IV: Acoplamiento de procesos II y III}

El cuarto análisis se corresponde con el cálculo del comportamiento dinámico de la estructura teniendo en cuenta la velocidad y la aceleración relativa que esta tiene en relación con la velocidad y aceleración del fluido que impacta sobre ella. Los resultados se van a comparar con las fuerzas hidrodinámicas generadas en el proceso III calculadas por el método avanzado. A continuación, se muestran en la figura 20 las principales diferencias generadas por el acoplamiento para el caso de temperatura de $1^{\circ} \mathrm{C}$ y salinidad del $0 \%$, dado que es el caso en el que se generan las mayores diferencias.

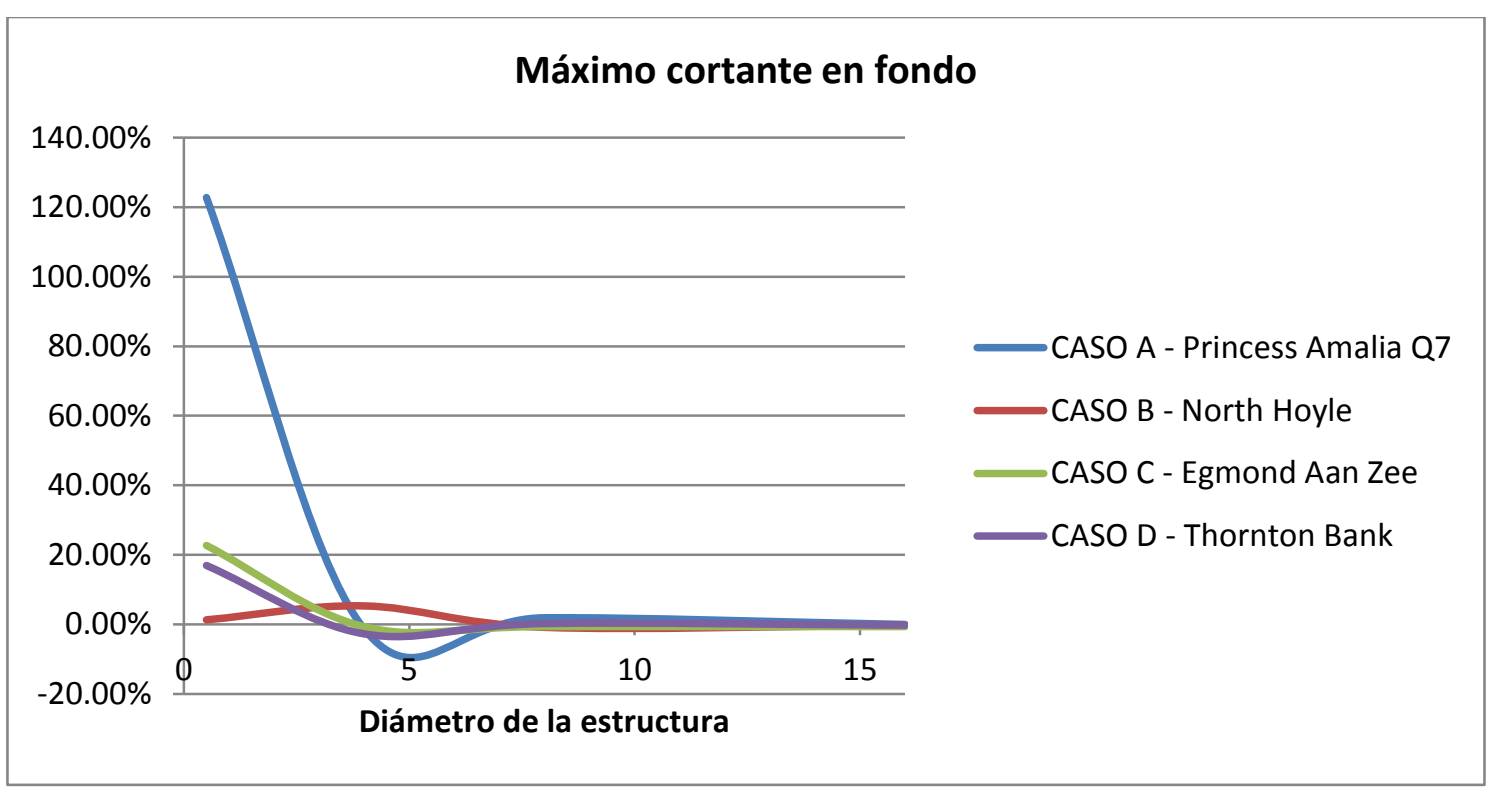




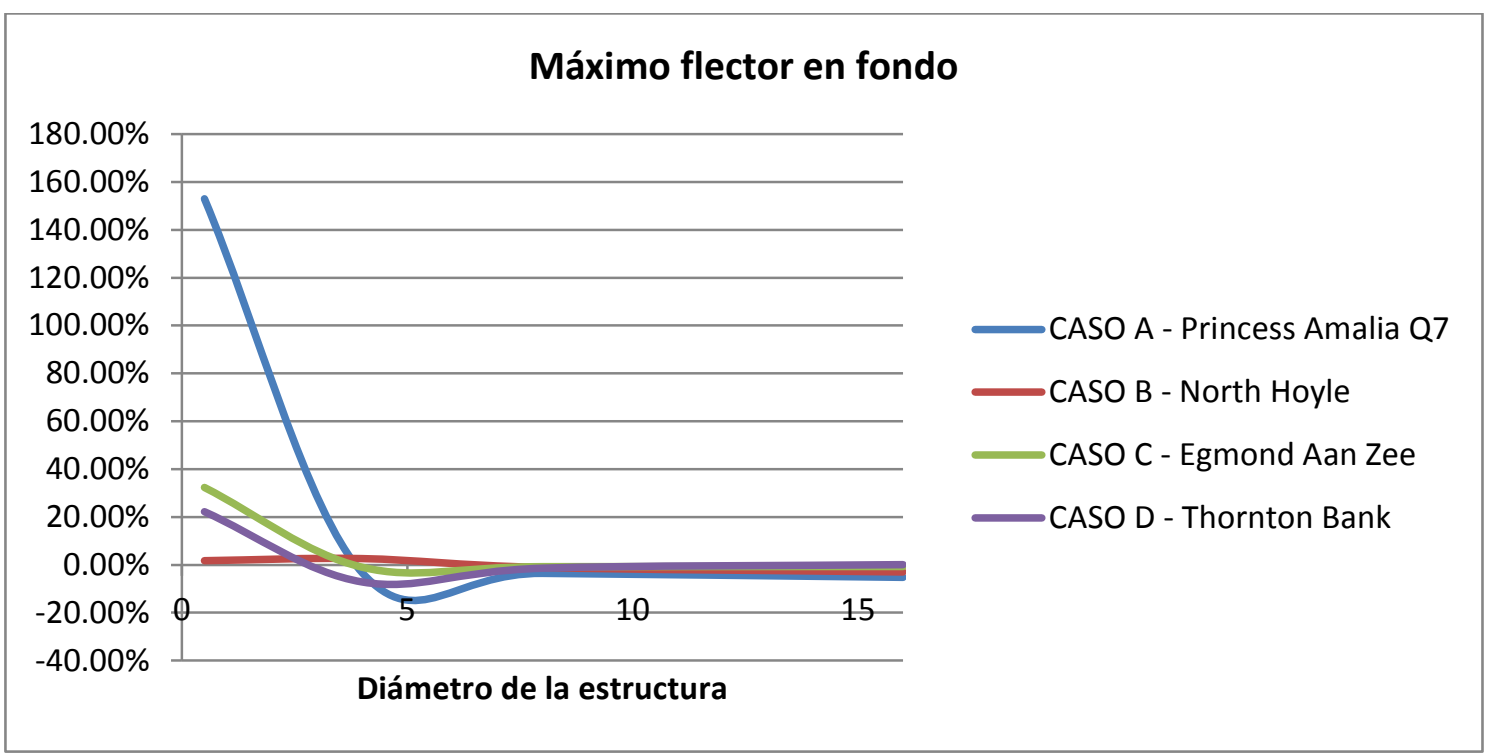

Figura 20. Variación de los esfuerzos en la cimentación debido a la consideración del acoplamiento, en relación al diámetro

Dichas diferencias han sido calculadas de acuerdo con la siguiente expresión:

$$
\% \text { Variación }=\frac{F_{\text {Acoplamiento }}-F_{\text {No Acoplamiento }}}{F_{\text {No Acoplamiento }}}
$$

Se puede observar que las máximas diferencias se producen en el caso del diámetro más pequeño. Esto es debido a que la excitación producida por el oleaje sobre la estructura se localiza en frecuencias cercanas a las frecuencias de resonancia de la estructura. Para los diámetros mayores, la estructura tiene una rigidez tan grande que su frecuencia de resonancia se aleja completamente de las frecuencias de excitación pésimas, que genera el oleaje. Además, se puede observar que para el resto de casos con diámetros mayores a 0,5 metros, con carácter general, el cálculo que tiene en cuenta el acoplamiento arroja resultados con valores menores para el momento flector, dado que al tener en cuenta el movimiento relativo de la estructura, esta presenta una cierta flexibilidad frente al impacto de la onda que le permite distribuir mejor los esfuerzos a lo largo del tiempo generando que los máximos esfuerzos sufridos sean menores. Como cabría esperar, este efecto va teniendo menor influencia conforme se aumenta el diámetro de la estructura, dado que cuanto más aumenta el diámetro más aumenta la rigidez y la estructura tiene menos capacidad para comportarse de forma flexible y reducir los esfuerzos totales generados por el oleaje sobre ella. 
El máximo valor se encuentra en el Caso A - Princess Amalia Q7, en el cual la diferencia del momento flector en la cimentación llega a ser del entorno de un 152,97\% más elevada en el caso con acoplamiento que la calculada según el método avanzado sin acoplamiento, debido a que la frecuencia de resonancia correspondientes al diámetro de 0,5 metros se encuentra muy próxima a la frecuencia de excitación generada por el oleaje.

\subsection{Proceso V: Comportamiento de la cimentación}

El quinto análisis se corresponde con la simulación del fenómeno de socavación en el lecho de la cimentación, teniendo en cuenta las aceleraciones y velocidades halladas en el fondo marino. En este caso, el método tradicional únicamente utiliza la formulación tradicional del número de Keulegan-Carpenter, mientras que el método avanzado utiliza la formulación modificada del número de Keulegan-Carpenter que incorpora el efecto del campo de aceleraciones. A continuación, la figura 21 va a mostrar las variaciones existentes entre el método avanzado y el tradicional cuando ambos utilizan la formulación clásica de Keulegan-Carpenter. Dichas variaciones serán calculadas de acuerdo con la siguiente expresión:

$$
\% \text { Variación }=\frac{S_{\text {avanzado }, K C \text { tradicional }}-S_{\text {tradicional }}}{S_{\text {tradicional }}}
$$

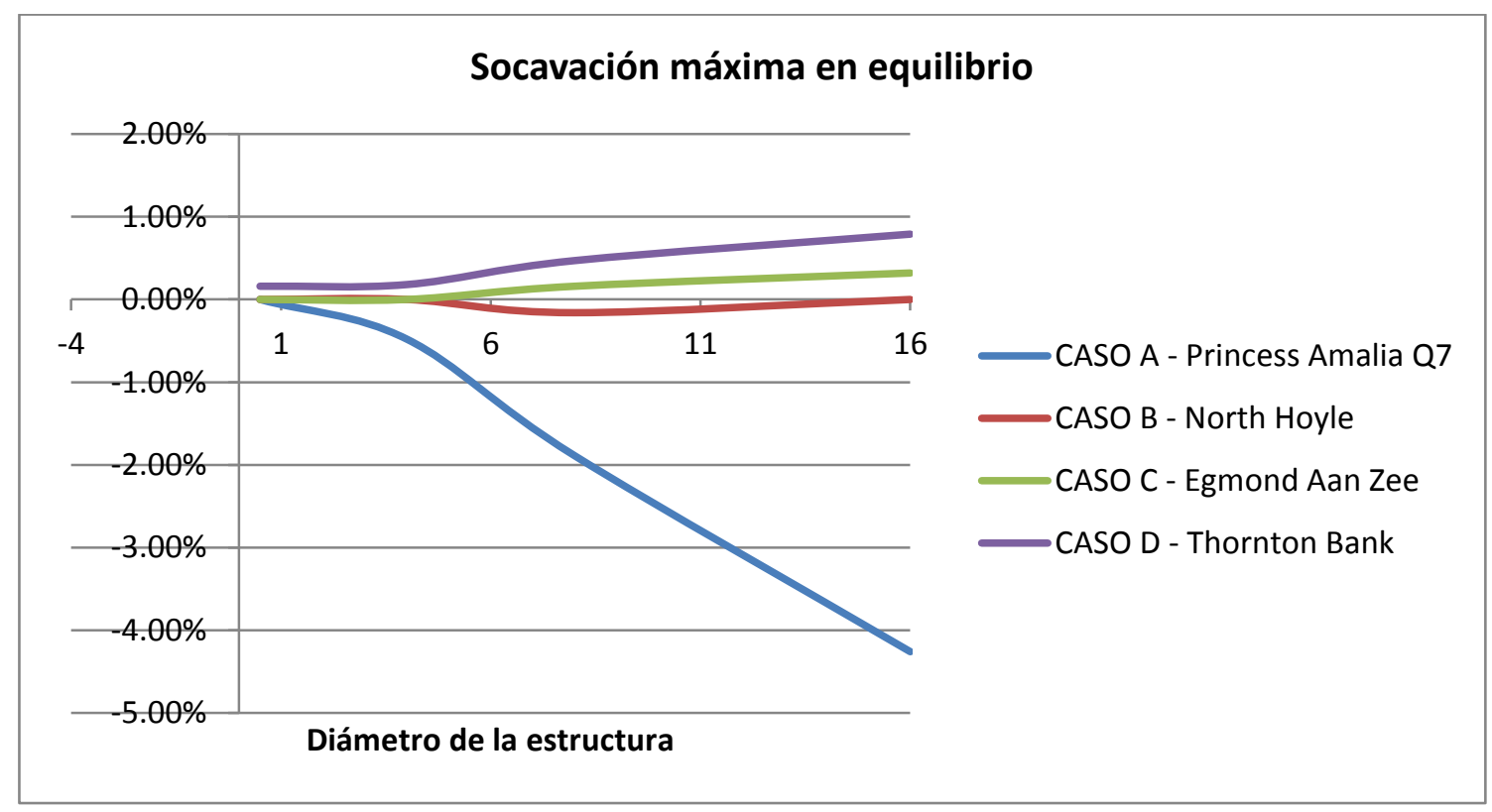

Figura 21. Variación de la socavación entre el método avanzado y el método tradicional ambos con KC tradicional 
Se observa una tendencia de crecimiento con respecto al diámetro que magnifica las diferencias entre las distintas teorías y la teoría lineal conforme aumenta el diámetro de la pila. Esto es debido a que la variable diámetro se encuentra multiplicando en el denominador de la fórmula del número de Keulegan-Carpenter y, por ello, cuanto más aumenta el diámetro más aumentan las diferencias, tanto en sentido positivo como negativo.

Estas variaciones son distintas para cada una de las teorías de ondas aplicadas a cada uno de los casos.

En el caso del parque Princess Amalia Q7, se ha utilizado la teoría de Stokes de tercer orden, siendo los resultados obtenidos menores que los de la teoría lineal de ondas.

En el caso del parque de North Hoyle, la teoría utilizada es Stokes de quinto orden, observándose que apenas existen diferencias entre los resultados aportados por esta teoría y los aportados por la teoría lineal.

En el caso del parque Egmond Ann Zee, la teoría utilizada es la cnoidal de segundo orden, y como puede observarse, en este caso dicha teoría presenta un reducido incremento de los valores correspondientes a la profundidad de socavación con respecto a los aportados por la teoría lineal.

Por último, en el caso del parque de Thornton Bank, se ha aplicado la teoría de Stream Function de Dean, y se observa nuevamente que los valores obtenidos mediante esta teoría de ondas son superiores a los aportados por la teoría lineal.

Se puede observar que las máximas diferencias se producen en el caso del parque eólico offshore Princess Amalia Q7 en Holanda, para el diámetro más grande, siendo estas diferencias superiores al $4,2 \%$.

En segundo lugar, se han comparado los valores de socavación obtenidos mediante el método avanzado, pero utilizando en un caso la fórmula clásica para el número de 
Keulegan - Carpenter y en el otro caso la formulación avanzada desarrollada en la presente Tesis Doctoral que incorpora de forma explícita el efecto del campo de aceleraciones. A continuación, se procede a mostrar los resultados de esta comparativa en la figura 22. Estas variaciones han sido calculadas de acuerdo con la siguiente expresión:

$$
\% \text { Variación }=\frac{S_{\text {avanzado }}-S_{\text {avanzado,KC tradicional }}}{S_{\text {avanzado }, K C \text { tradicional }}}
$$

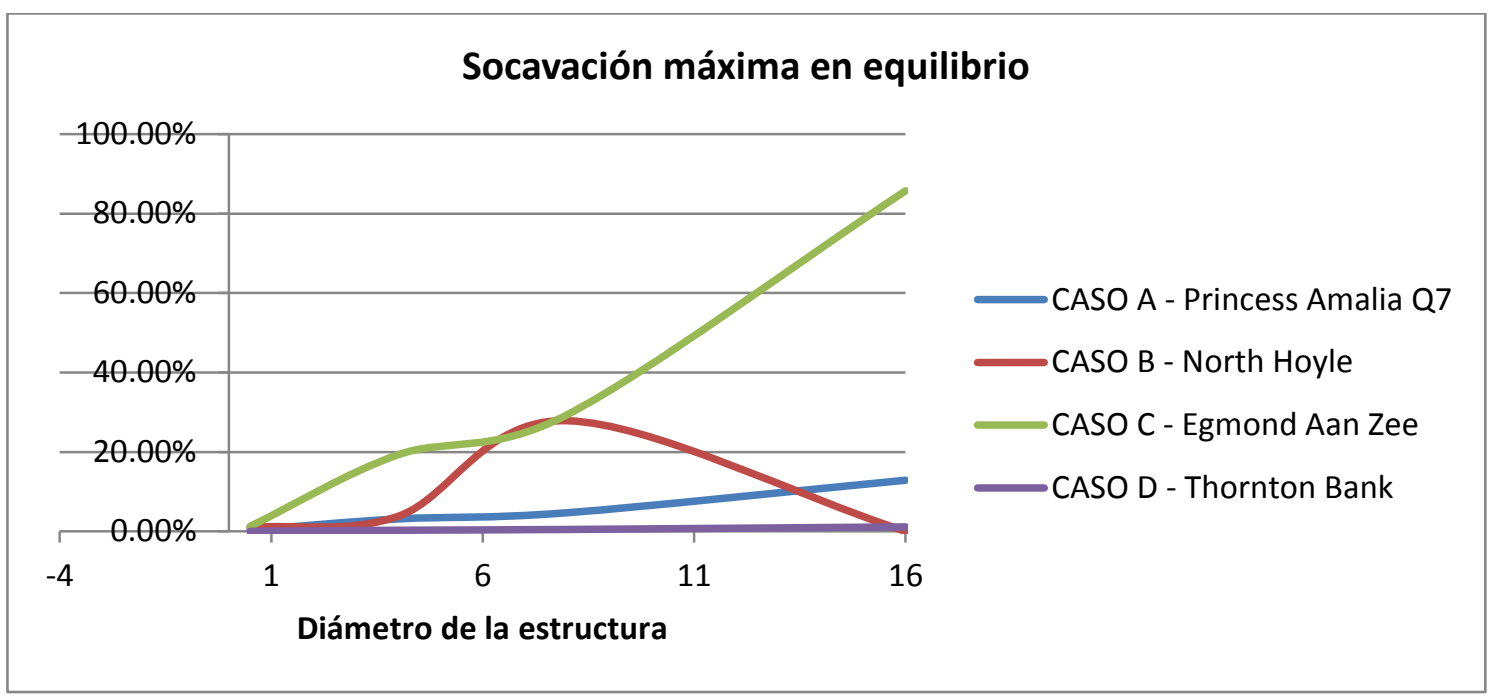

Figura 22. Variación de la socavación entre el método avanzado utilizando KC tradicional y KC modificado

A partir de los resultados mostrados en la figura anterior, se puede observar que las máximas diferencias se producen, en este caso, para el parque eólico offshore Egmond Aan Zee para el diámetro más grande. Como puede observarse, estas variaciones son distintas para cada una de las teorías de ondas aplicadas a cada uno de los casos, pero son siempre positivas.

Estas diferencias permiten reflejar que la introducción explícita del efecto del campo de aceleraciones genera un aumento en los fenómenos de socavación de la cimentación. Estas diferencias no son despreciables, dado que oscilan en un rango variado dependiendo de la teoría de ondas aplicada.

Para el caso del parque Princess Amalia Q7, con teoría de Stokes de tercer orden, se observa que dichas variaciones no superan el $13 \%$. En el caso del parque de North Hoyle, 
con Stokes de quinto orden, dichas diferencias alcanzan valores alrededor de un $28 \%$. En el caso del parque Egmond Ann Zee, con teoría cnoidal de segundo orden, y como puede observarse, se alcanzan diferencias máximas del entorno del $86 \%$. Por último, en el caso del parque de Thornton Bank, con teoría de Stream Function de Dean, se observa una reducida variación que alcanza el 1,13\% en su valor máximo.

Por otro lado, se observa nuevamente una tendencia de crecimiento con respecto al diámetro que magnifica las diferencias entre la formulación que tiene en cuenta el efecto del campo de aceleraciones y la formulación tradicional. Esto es debido a que la variable diámetro se encuentra multiplicando en el denominador de la fórmula del número de Keulegan-Carpenter y, por ello, cuanto más aumenta el diámetro más aumentan las diferencias.

Por último, se va a realizar una comparación entre los valores calculados mediante el método avanzado, utilizando la fórmula clásica de Keulegan-Carpenter y la fórmula modificada, y los valores reales medidos en campo por Deutsche Wind Guard (2007), Louwersheimer (2007), Whitehouse et al. (2008), Harris et al. (2010), Carroll et al. (2010), Whitehouse et al. (2011) y Matutano et al. (2015), para poder estimar si la formulación modificada se ajusta mejor a la realidad. A continuación, se muestran en la figura 23 estos valores:

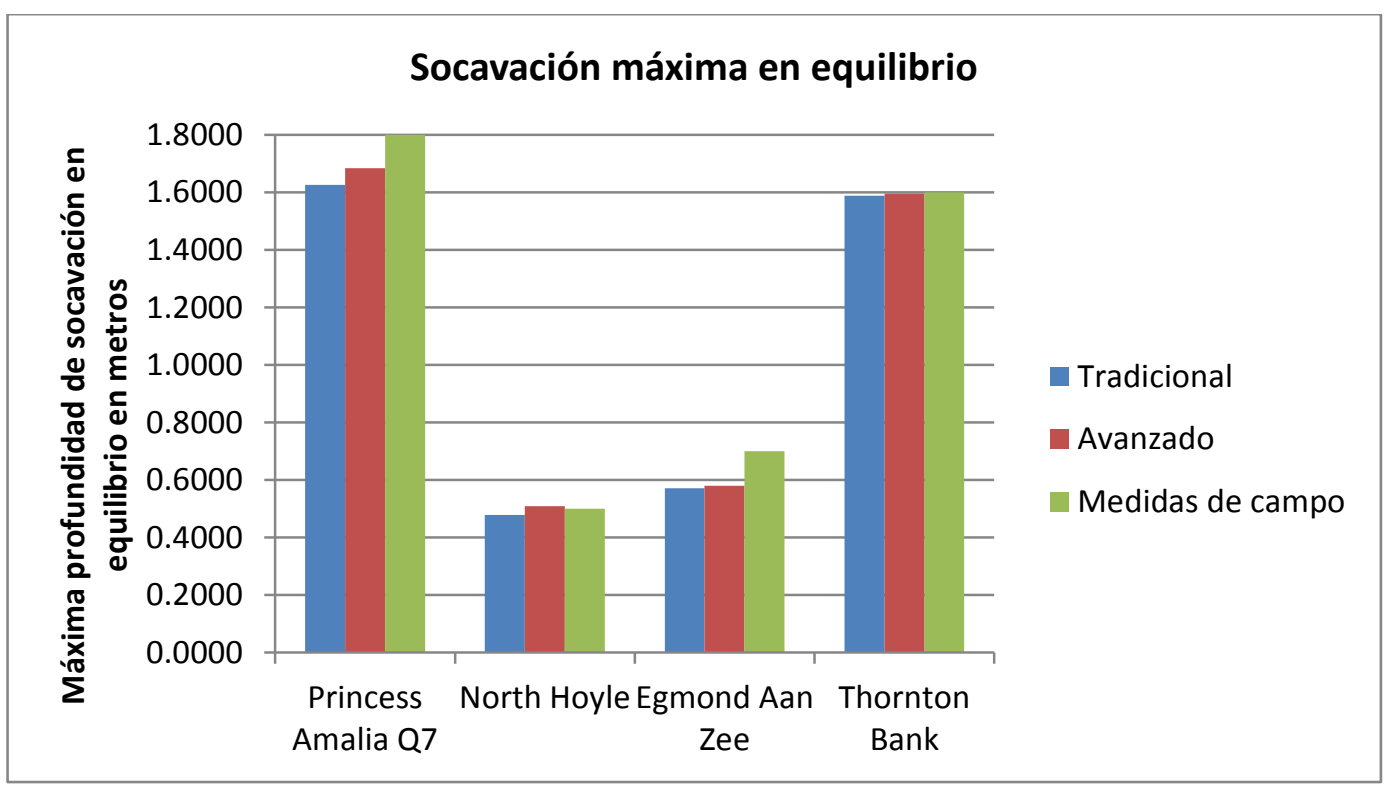

Figura 23. Comparación de la socavación máxima en equilibrio 
De acuerdo con los valores mostrados en la figura 23 , se observa que los resultados obtenidos mediante la formulación modificada se ajustan mejor a las medidas reales tomadas en campo. En la figura 24 se va a mostrar el error cometido por cada una de las dos formulaciones con respecto a los valores reales.

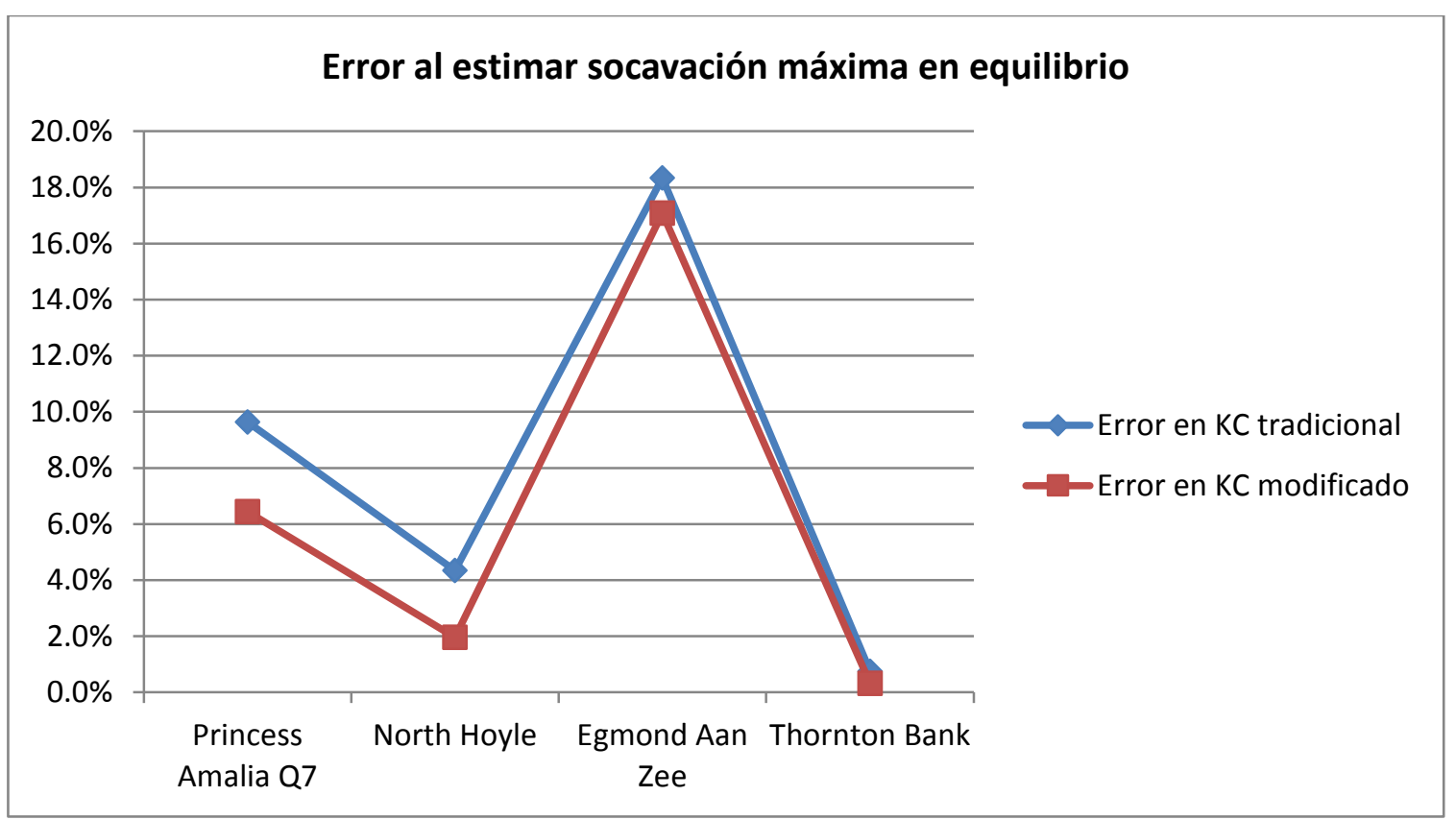

Figura 24. Error generado en cada uno de las dos formulaciones de cálculo

Se observa que el error máximo cometido oscila entre un 0,3\% y un $18,3 \%$ siendo siempre menor en la fórmula modificada, lo que permite afirmar que la formulación desarrollada en la presente Tesis Doctoral permite estimar de una forma más precisa los valores de socavación máxima en equilibrio. 

Capítulo 6

CONCLUSIONES 



\section{Capítulo 6}

\section{CONCLUSIONES}

\section{Consideraciones previas}

En este capítulo se va a proceder a realizar el resumen final de las conclusiones extraídas a lo largo de los distintos capítulos que componen la presente Tesis Doctoral, así como las principales aportaciones originales que se han realizado al campo objeto de estudio y las futuras líneas de investigación que se plantean, como siguiente paso a los análisis desarrollados.

A lo largo de la presente Tesis Doctoral se ha demostrado que la implantación de la energía eólica a nivel mundial ha experimentado un crecimiento exponencial a lo largo de los últimos años, teniendo un importante impacto sobre los parques eólicos offshore, dado que permiten la generación de una mayor cantidad de energía que sus equivalentes onshore. No obstante, también se ha destacado que estos parques offshore presentan importantes limitaciones desde el punto de vista del diseño, la construcción, el mantenimiento y la posterior operación, lo que conlleva a un importante incremento de costes debido a la dificultad de posicionar una estructura de este tipo, en medio del mar en vez de en tierra firme.

Por lo tanto, se ha destacado la imperiosa necesidad de desarrollar nuevos modelos de cálculo más avanzados que permitan aumentar la precisión con la que se estiman las acciones que genera el oleaje sobre las estructuras offshore y sus cimentaciones, y por lo tanto permitan realizar mejores diseños y que la energía eólica offshore pueda continuar creciendo, con la instalación de nuevos parques en zonas más profundas y alejadas de la costa, donde la producción energética será mayor. 
Se han identificado dos factores como las principales barreras que impiden que los actuales modelos puedan alcanzar una mayor precisión. En primer lugar, la dificultad intrínseca en la realización del propio cálculo para estimar las fuerzas hidrodinámicas, debido a la complejidad del fenómeno, y, por lo tanto, a la complejidad de la formulación asociada a la resolución del mismo. En segundo lugar, la escasez de datos fiables, necesarios para caracterizar correctamente la mecánica de ondas que modelizará el oleaje, de una forma totalmente precisa.

En la presente Tesis Doctoral se ha procedido a desarrollar una herramienta de cálculo que, como primer objetivo, permitiera resolver de forma eficiente los complejos mecanismos que definen el fenómeno físico; y como objetivos secundarios, permitiera incluir en el análisis variables nunca antes utilizadas como: la salinidad o la temperatura, en el proceso general de cálculo de la estructura; el campo de aceleraciones, en el fenómeno de la socavación; o tener en cuenta la interacción oleaje-estructura de una forma eficiente y precisa.

En conclusión, el método desarrollado permitirá aumentar la precisión en el diseño estructural de parques eólicos offshore, haciendo que se reduzcan las limitaciones que sufre este campo, debido a las diversas incertidumbres que afectan al diseño, y permitiendo también que se puedan ajustar más los costes asociados a la construcción de estas estructuras. 


\section{Conclusiones}

A continuación, se procede a realizar la exposición de las distintas conclusiones alcanzadas a lo largo de la presente Tesis Doctoral.

Existen multitud de tipologías estructurales dependiendo principalmente de la profundidad a la que se va a implantar la estructura offshore y del objeto o función principal para la que han sido diseñadas, siendo el rendimiento financiero que la explotación sea capaz de generar, la capacidad portante del lecho marino y las condiciones medioambientales del lugar de instalación, tres de las variables principales que definirán en gran medida, el tipo de estructura a utilizar. Actualmente, la tipología estructural más utilizada en parques eólicos offshore es la estructura con cimentación tipo monopila, perteneciendo a esta tipología el 80,8\% de todas las unidades instaladas en Europa.

En ingeniería offshore aplicada a la generación de energía eléctrica a partir del aprovechamiento de la energía eólica, actualmente, las estructuras dejan de ser rentables en profundidades superiores a los 50 metros. A partir de esta profundidad, la elección de una de las tres tipologías flotantes frente al uso de estructuras tipo jacket, atiende a otros condicionantes como: la capacidad portante del lecho marino, los condicionantes de la operación o mantenimiento que exigen pocas amplitudes de movimiento de la estructura, la rentabilidad económica, etc.

La acción del oleaje es una de las principales causas de fallo en las estructuras offshore, y por lo tanto la correcta caracterización de las acciones climáticas es uno de los aspectos clave en el diseño de estas estructuras. Para ello, será necesario realizar: en primer lugar, un exhaustivo cálculo de la mecánica de ondas, que definirá el comportamiento del oleaje; en segundo lugar, una detallada aplicación de la teoría hidrodinámica, para obtener las acciones que dicho oleaje genera sobre una estructura que se encuentra en medio del flujo de corriente; en tercer lugar, la aplicación de las teorías vibratorias, para caracterizar el comportamiento dinámico que experimentará la estructura, y para poder tener en cuenta los efectos de interrelación y acoplamiento entre el oleaje y la estructura y, por último, el estudio del fenómeno de socavación que se producirá sobre dicha estructura. 
Con respecto al cálculo de la mecánica de ondas que define el comportamiento del oleaje, es necesario tener en cuenta que el proceso de propagación de oleaje, a través de un fluido, es no lineal y se ve influenciado por un gran número de variables y efectos, cuya interacción es complicada de estudiar desde un punto de vista teórico. Por este motivo, diversos autores han sido capaces de proponer y demostrar distintas soluciones, en los diferentes dominios que definen el medio físico, para poder plantear una solución analítica del mismo que permita obtener el campo de velocidades o de aceleraciones de una forma precisa.

A pesar de la multitud de formulaciones que existen para caracterizar el oleaje, la teoría de Stokes y la teoría cnoidal han demostrado ser las que mejor se adaptan a la realidad, siendo su error, con respecto a la condición de contorno no lineal que parametriza la superficie libre, el más pequeño de todas las teorías desarrolladas. La teoría de Stokes será válida para aguas relativamente profundas y la teoría cnoidal será válida para aguas relativamente someras. Por lo tanto, de acuerdo con el gráfico de clasificación de Le Méhauté (1976), la aplicación de la teoría de Stokes junto con la teoría cnoidal y la Stream Function de Dean hace posible la resolución de las ecuaciones que definen el oleaje en todo el dominio de cálculo.

La gran dificultad para la realización del cálculo de la teoría de Stokes, la teoría cnoidal y la Stream Function de Dean obligan al desarrollo de potentes herramientas de cálculo con base en la informática para poder hallar el campo de velocidades y aceleraciones de un fluido sometido a la acción del oleaje en un determinado dominio.

Con respecto a la correcta aplicación de la teoría hidrodinámica, en primer lugar, se debe tener presente que la acción que ejerce un fluido sobre un cuerpo sumergido, dependerá, principalmente, de la fuerza de inercia, debida al campo de aceleraciones y de la fuerza de arrastre, debida al campo de velocidades, siempre y cuando, la longitud de onda del oleaje incidente cumpla la relación $\frac{D}{L} \leq 0,2$. Si no se cumple esta relación, las fuerzas de arrastre son despreciables y las fuerzas de inercia serán minoradas a causa del potencial de difracción generado por el propio cuerpo sumergido que sí generará influencia sobre el flujo de oleaje que incide sobre él. 
De acuerdo a los estudios realizados por multitud de autores, el número de Reynolds, Re, tendrá una influencia muy importante en la caracterización de las fuerzas hidrodinámicas, generando diferencias de hasta un $80 \%$ en las fuerzas de inercia, y diferencias de hasta un $85 \%$ en el caso de las fuerzas de arrastre; convirtiéndose, por lo tanto, en un parámetro fundamental para estudiar el fenómeno físico, junto con el número de KeuleganCarpenter.

El dominio de cálculo para resolver la caracterización de las fuerzas hidrodinámicas que se producen sobre un cuerpo sometido a la acción del oleaje, puede ser dividido en seis subdominios, que dependerán fundamentalmente de la relación $\frac{D}{L}$, de la relación $\frac{H}{L}$ y del número de Keulegan-Carpenter, KC.

La socavación es un fenómeno físico que se encuentra directamente relacionado con el movimiento de los sedimentos que componen el lecho marino. La presencia de cualquier obstáculo en el fondo marino, provoca cambios en las condiciones del flujo del agua, produciendo un incremento en la capacidad de transporte de sedimentos, y provocando así el proceso de la socavación en las proximidades del obstáculo.

El fenómeno de socavación que se produce en el mar, es mucho más complejo que el desarrollado en el medio fluvial, dado que en el medio marítimo el oleaje es la acción predominante y la acción de la corriente no puede ser despreciada, y en los ríos, el fenómeno se produce bajo condiciones de corriente continua. Por lo tanto, la formulación aplicada a la caracterización de la socavación máxima en cimentaciones de estructuras offshore deberá tener en cuenta la acción del oleaje y de la corriente, de forma conjunta.

El movimiento de sedimentos se define mediante la distinción de tres etapas: una etapa primera de erosión, una segunda etapa de transporte y una etapa final de deposición.

Las formulaciones principales que tienen en cuenta la acción conjunta del oleaje y la corriente, incluyen el número de Keulegan-Carpenter y, la relación entre la velocidad generada en el fondo marino por el oleaje y la velocidad generada por la corriente, como parámetros principales para la realización del cálculo de la máxima profundidad de 
socavación estable. Este hecho implica que solo el campo de velocidades tiene influencia de forma explícita sobre la profundidad máxima de socavación y que, por lo tanto, son las fuerzas de arrastre las que más influirán en este fenómeno.

A partir de la fórmula de Morison et al. es posible realizar un desarrollo teórico, que permite introducir de forma explícita la influencia del campo de aceleraciones sobre el proceso de la socavación, permitiendo así el estudio de la influencia generada por las fuerzas de inercia sobre la socavación.

Debido a la extensa cantidad de líneas de investigación presentes en el campo objeto de estudio de la presente Tesis Doctoral, se ha considerado imprescindible el realizar una detallada y meticulosa revisión bibliográfica, con el fin de poder discernir los principales modelos de cálculo utilizados para: la mecánica de ondas, la caracterización hidrodinámica, el estudio del comportamiento dinámico estructural y la socavación; que aportan una mayor precisión en el cálculo y permiten comprender el fenómeno físico en su conjunto.

Como fruto de esta revisión bibliográfica se ha observado que gran parte de los diseños que se realizan hoy en día, simplifican de forma importante los cálculos pertenecientes a uno o varios de los mecanismos que definen el proceso completo de cálculo, permitiendo denominar como método de cálculo simplificado, definido en la presente Tesis Doctoral como método tradicional, a aquel compuesto por: la teoría de Airy o Stokes de primer orden, la teoría de Morison et al., el cálculo estático de la estructura, y la aplicación de la teoría de socavación de Sumer et al. (2002).

Este método tradicional de cálculo presenta importantes limitaciones, al no tener en cuenta la influencia de: los efectos no lineales; de la difracción; de variables nunca antes incluidas en este tipo de modelos, como la temperatura y la salinidad; de los efectos dinámicos generados en la estructura; de los mecanismos de acoplamiento entre el oleaje y la estructura; y, el efecto del campo de aceleraciones sobre el fenómeno de socavación. Estas limitaciones pueden introducir errores en el dimensionamiento de las estructuras offshore, que se destacan aún más hoy en día, si cabe, debido fundamentalmente a que se 
están incrementando de forma importante los diámetros utilizados en estas estructuras marinas tipo monopila, y se están localizando las mismas en zonas mucho más alejadas de la costa, haciendo que los esquemas tradicionales y simplificados, de cálculo y diseño, dejen de ser válidos.

Por lo tanto, es necesaria la implantación de un método de cálculo avanzado, que permita establecer una solución general que no presente las limitaciones existentes en el método tradicional, y que aporte una solución que tenga en cuenta todas las particularidades existentes en los diseños que se están desarrollando hoy en día, en el campo de la ingeniería offshore.

Debido a la gran complejidad inherente a los cálculos relacionados con la mecánica de ondas, con la caracterización de la fuerzas hidrodinámicas, con la caracterización del comportamiento dinámico de la estructura y con la simulación de los efectos de acoplamiento entre oleaje y estructura; se ha establecido como objetivo necesario el desarrollo de una potente herramienta de cálculo, con base informática, que permita integrar todos estos importantes condicionantes de una forma compacta, eficiente y precisa.

Por último, la revisión bibliográfica también dejó patente la importante falta de información disponible en lo referente a mediciones reales en campo de las fuerzas hidrodinámicas generadas por el mar sobre estructuras de soporte de aerogeneradores offshore, así como la ausencia de mediciones de los movimientos y tensiones sufridos por la estructura, y los posibles efectos de acoplamiento del oleaje. La información existente en este campo es, en su mayor parte, de carácter confidencial, y, por lo tanto, de muy difícil acceso. No obstante, sí se ha encontrado información en relación con la medición en campo del fenómeno de la socavación, en varios parques eólicos offshore europeos. Por lo tanto, esta información ha sido recopilada y evaluada, seleccionando los 4 parques más idóneos, para poder comparar los resultados de sus mediciones tomadas en campo, con los resultados obtenidos mediante el modelo de cálculo desarrollado durante la presente Tesis Doctoral. 
La herramienta de software que se ha desarrollado, debe sectorizar todo el árbol de procesos en cinco módulos de cálculo independientes, como son: los procesos de mecánica de ondas, los procesos de caracterización de fuerzas hidrodinámicas, los procesos de cálculo estructural, los procesos de acoplamiento oleaje - estructura y los procesos de simulación del fenómeno de socavación. De esta forma, es posible tener un control completo de las variables que intervienen en el cálculo, y permite implantar algoritmos independientes de alta eficiencia.

La herramienta de cálculo se ha desarrollado mediante Python 2.7 en código libre de alto nivel, y utilizando librerías de optimización desarrolladas en $\mathrm{C}++$, que permiten la vectorización del cálculo, y, por lo tanto, una gran velocidad en los procesos de análisis.

Con respecto a los procesos englobados dentro de la mecánica de ondas, es importante destacar que los modelos que únicamente utilizan teoría lineal de ondas, generan un importante sesgo en los resultados, dado que no incluyen en los cálculos, los efectos no lineales. Estos efectos no lineales tendrán una afección importante en los resultados obtenidos, con respecto al movimiento de las partículas de agua, y, por lo tanto, con respecto al campo de velocidades y aceleraciones instantáneas, haciendo que el uso de la teoría lineal sea válido únicamente para la resolución de casos puramente lineales. Además, estos efectos no lineales tendrán influencia en la distribución de fuerzas hidrodinámicas, a lo largo del eje vertical, lo que podrá generar una desviación adicional en los resultados con respecto a los esfuerzos producidos sobre la cimentación, debido a la variación de la ley de esfuerzos cortantes y flectores que sufre la estructura. Por otro lado, el uso adecuado de las teorías no lineales permitirá caracterizar la realidad de una manera más fidedigna, aumentando el espectro de frecuencias con las que el oleaje excitará a la estructura.

Para realizar la selección de la teoría de ondas no lineal adecuada, que deberá ser utilizada en el cálculo, se ha realizado una tabla de clasificación que sectoriza todo el domino físico, para las distintas teorías de mecánica de ondas. 
Además, se ha introducido de forma innovadora en el cálculo, el efecto de la salinidad del agua y de la temperatura, mediante su influencia sobre la viscosidad y la densidad, con la intención de poder observar su influencia sobre el proceso global de cálculo.

Con respecto a la utilización de la teoría de Morison et al., como fórmula de base para la caracterización de las fuerzas hidrodinámicas, es necesario indicar que también se introducirá un sesgo importante en los resultados, debido a que no tiene en cuenta los efectos no lineales ni los efectos debidos al potencial de difracción. Este hecho restringe el uso de dicha teoría a estructuras esbeltas, y que se encuentren en un rango de cálculo lineal. Dicha teoría ha sido analizada por multitud de autores relevantes dentro del campo de la investigación offshore, y se ha demostrado que arroja valores muy fiables para pilotes cuyo diámetro esté comprendido entre 0,5 y 2,0 metros. No obstante, hoy en día las nuevas estructuras offshore poseen diámetros que pueden alcanzar en algunos casos valores entre los 8 y los 16 metros, lo que puede invalidar el uso de dicha teoría, dado que arroja valores muy conservadores que obligarán a sobredimensionar la estructura.

Por todo ello, se han establecido seis subdominios diferentes de cálculo que permiten definir las fuerzas hidrodinámicas, sean cuales sean las características del oleaje y de la estructura, dentro del domino completo de cálculo. La relación diámetro entre longitud de onda, $\mathrm{D} / \mathrm{L}$, el número de Keulegan-Carpenter $\mathrm{KC}$ y la relación del número $\mathrm{KC}$, con respecto al máximo KC, son los parámetros que definen cuál será el subdominio de cálculo, de aplicación, y si existen o no, efectos no lineales o de difracción.

Desde un punto de vista del análisis estructural, se ha observado que la utilización de un análisis estructural estático no representará fielmente la realidad, y estará obviando procesos vibratorios de acumulación energética que tendrán una gran influencia en el diseño de la estructura. La utilización de modelos estructurales dinámicos permite tener en cuenta la naturaleza dinámica de la carga inducida por el oleaje, haciendo posible la predicción de posibles efectos de resonancia en la estructura, o la elección de la rigidez adecuada para la misma, que permita reducir de forma razonable el efecto de amplificación dinámica, que puedan sufrir las cargas. De esta forma, se realizará un estudio evolutivo que permita detectar los estados de carga pésimos que ocurrirán en la 
realidad sobre la estructura, pudiendo diferenciar qué frecuencias son las que generan un mayor daño estructural, y además establecer una simulación de los procesos de acoplamiento oleaje - estructura.

Para ello, se han resuelto las ecuaciones diferenciales que definen el problema de un fuste vertical empotrado en un extremo, y sometido a la acción variable de una ley de fuerzas a lo largo de su eje vertical y se ha establecido una solución general que permite conocer las tensiones y el movimiento de la estructura para cada instante temporal, teniendo en cuenta los primeros 25 modos de vibración.

Se ha introducido en el cálculo el efecto de acoplamiento oleaje - estructura, al conectar de forma iterativa el proceso de cálculo de las fuerzas hidrodinámicas, con el proceso de cálculo estructural dinámico.

Con respecto al estudio del fenómeno de socavación, se ha procedido a realizar un nuevo desarrollo del número de Keulegan - Carpenter, con el fin de poder proponer una nueva formulación, a partir de la expresión de Sumer et al. (2002), introduciendo de forma explícita el efecto del campo de aceleraciones, sobre el valor predicho para la profundidad máxima de socavación en equilibrio.

El análisis de velocidades muestra que las distintas teorías no lineales aportan valores menores que los resultantes de la teoría lineal. El error obtenido en la máxima velocidad horizontal alcanza valores de hasta el $32 \%$, lo que implica que, dado que las fuerzas de arrastre se calculan con el cuadrado de la velocidad, el error generado entre el método avanzado y el método tradicional, podrá ser de hasta el 52\%, es decir, que las fuerzas de arrastre calculadas mediante el método tradicional serán hasta 2 veces superiores a las calculadas mediante el método avanzado, lo que habrá generado un sobredimensionamiento de la estructura. No obstante, también se observa un caso en que dicha regla no se cumple, y que presenta valores de velocidad que son un $1 \%$ superiores en el cálculo no lineal. Por otro lado, se observa que dichas variaciones en todos los casos, tienen mucho más efecto en superficie que en fondo, y que dichas variaciones son mucho mayores en la dirección vertical. 
El análisis de aceleraciones muestra también que las distintas teorías no lineales aportan valores menores que los resultantes de la teoría lineal. El error obtenido en la máxima aceleración horizontal alcanza valores de hasta el $45 \%$, lo que implica que, dado que las fuerzas de inercia son lineales con la aceleración, las fuerzas de inercia calculadas mediante el método tradicional serán hasta 1,81 veces superiores a las calculadas mediante el método avanzado, lo que habrá generado nuevamente un sobredimensionamiento de la estructura. No obstante, también se observa un caso en que dicha regla no se cumple, y que presenta valores de aceleración que son un $5 \%$ superiores en el cálculo no lineal. Por otro lado, se observa que dichas variaciones en todos los casos, tienen mucho más efecto en superficie que en fondo, y que dichas variaciones son mucho mayores en la dirección vertical.

Con respecto al análisis de frecuencias, tal y como puede observarse en el Apéndice 1, las teorías no lineales distribuyen su energía en un mayor número de frecuencias de mayor magnitud, mientras que la teoría lineal se concentra fundamentalmente en una única frecuencia. Este hecho deberá ser tenido en cuenta durante la elección de la rigidez de la estructura, para evitar sufrir posibles efectos de resonancia en la estructura.

Con respecto a la amortiguación con la profundidad, del campo de velocidades y aceleraciones, se ha observado que es ligeramente mayor en el caso lineal que en el caso no lineal, lo que implica que la ley de momentos flectores será diferente, y por lo tanto el momento último que debe resistir la cimentación, también lo será.

El análisis de las fuerzas hidrodinámicas muestra que los resultados obtenidos por la teoría lineal y la fórmula de Morison et al., alcanzan valores hasta un 163\% superiores, con respecto a los resultados calculados según el método avanzado, propuesto en la presente Tesis Doctoral.

Las máximas diferencias se producen en el caso del diámetro más pequeño, es decir, se producen en las fuerzas viscosas. Como puede observarse esta variación sufrida por las fuerzas de arrastre se mantiene constante con el diámetro. Por lo tanto, pese a que tienen 
un gran peso sobre la fuerza total para los diámetros pequeños, conforme aumenta el diámetro, su influencia desaparece.

Generalmente se observa una tendencia de crecimiento a partir del diámetro de 4,0 metros. Esta variación es debida a la tendencia de crecimiento en la variación que experimentan las fuerzas de inercia, según aumenta el diámetro de la estructura. Por lo tanto, la variación de la fuerza total, según aumenta el diámetro, estará fundamentalmente causada por la variación que sufren las fuerzas de inercia.

Por ello, se puede señalar que generalmente las fuerzas calculadas por el método tradicional son siempre superiores a las calculadas por el método avanzado, ocasionando el sobredimensionamiento de la estructura. Esta variación alcanza valores de hasta un 161\% para el diámetro de menor, y posteriormente la función que define la variación de la fuerza total, decae profundamente en el intervalo de diámetros entre 0,5 y 4 metros, a causa de la pérdida de influencia que sufren las fuerzas de arrastre; y en el intervalo entre 4 y 16 metros, la pendiente se vuelve a tornar creciente, tendiendo asintóticamente a imitar a la pendiente que experimenta la función de crecimiento de las fuerzas de inercia cuando aumenta el diámetro, pudiendo alcanzar valores de variación de hasta el 87\%.

Con respecto a la influencia de la salinidad y la temperatura, se observa que la fuerza hidrodinámica total es mayor para el caso de temperatura del agua a $1^{\circ} \mathrm{C}$ y salinidad del $0 \%$, que para el caso de temperatura a $22^{\circ} \mathrm{C}$ y salinidad del $35 \%$. Dicha variación, puede alcanzar valores de hasta un $9 \%$ en superficie y un $13 \%$ en el lecho marino. Se observa que generalmente, esta variación se está produciendo para el diámetro menor, y que posteriormente tiende a cero, conforme aumenta el diámetro. Este hecho pone de manifiesto que la variación de la salinidad y la temperatura, tendrá predominantemente influencia sobre las fuerzas de arrastre, y por ello induce variaciones máximas de la fuerza total, en los diámetros más pequeños, y posteriormente va tendiendo asintóticamente a cero. 
Se observa, tras analizar los resultados obtenidos, que un incremento de la temperatura y la salinidad del agua, tendrá el mismo efecto que una reducción virtual del diámetro de cálculo.

Con respecto a los resultados aportados por el modelo de cálculo estructural, se observa que los resultados generados mediante el proceso de cálculo tradicional, que utiliza un modelo estático para el cálculo de la estructura, son siempre del orden de entre un $20 \%$ y $100 \%$ mayores que los resultados obtenidos mediante el proceso de cálculo avanzado, que utiliza un modelo dinámico que tiene en cuenta las vibraciones que sufre la estructura. Este hecho se debe fundamentalmente, a que como se observó en los puntos anteriores, las fuerzas calculadas según el método tradicional son del orden de entre un $40 \%$ y un $161 \%$ superiores, a las calculadas según el método avanzado. Por ello, se puede observar que el método de cálculo dinámico está teniendo una capacidad de acumulación de la energía de deformación, que en realidad aportará resultados entre un $20 \%$ y un $61 \%$ mayores, que los resultados aportados por el método estático.

Esta variación de resultados tiene su máximo valor en los diámetros de menor tamaño, debido a que la rigidez de la estructura crece mucho más rápido con el diámetro, que el nivel de cargas actuante, y, por lo tanto, conforme aumenta el diámetro, la estructura se vuelve tan rígida que los efectos dinámicos van perdiendo relevancia y el análisis estático aporta resultados muy similares a los del análisis dinámico.

Este mismo efecto ocurre con respecto a la influencia que tiene la temperatura y la salinidad, sobre el análisis dinámico, que presenta con carácter general sus máximos valores en los diámetros menores, tal y como se describía anteriormente en el apartado de fuerzas hidrodinámicas. Teniendo como excepción el caso de cálculo correspondiente al parque eólico de Princess Amalia Q7, que experimenta un pico en el diámetro de 4,0 metros, debido a efectos de resonancia.

No obstante, este efecto de acumulación energético que presenta el método dinámico frente al estático, desaparece y además generalmente tendrá el efecto contrario, si se consideran los efectos de acoplamiento entre el oleaje y la estructura. La incorporación de 
estos efectos permite que la estructura pueda dejar de ser entendida como un acumulador de energía de deformación, y comience a comportarse con un elemento estructural que está aumentando su flexibilidad, y que reduce el nivel de carga hidrodinámica recibida por el oleaje, debido a que presenta un movimiento de acoplamiento con él, reduciendo el valor de las aceleraciones y velocidades relativas que ejerce el flujo de agua con respecto a la estructura.

Se observa que las diferencias generadas al tener en cuenta el efecto del acoplamiento alcanzan valores de hasta un $152 \%$, pero que desaparecen rápidamente con el incremento del diámetro, haciendo que los valores aportados por el análisis dinámico con acoplamiento, y los aportados por el análisis dinámico sin acoplamiento, posean diferencias prácticamente despreciables, al incrementarse la rigidez de la estructura.

Por último, el análisis del fenómeno de socavación demuestra que el método de cálculo avanzado propuesto por la presente Tesis Doctoral aporta valores mayores en lo referente a la máxima profundidad de socavación en equilibrio, prevista para cada uno de los casos, en relación a los valores aportados por el método tradicional.

Se observa que el error cometido entre los valores de cálculo y las medidas reales obtenidas en campo, se reduce del orden de hasta un $3,2 \%$, si se utiliza la nueva formulación propuesta por la presente Tesis Doctoral, en lo referente a la estimación de la máxima profundidad de socavación en equilibrio. 


\section{Aportaciones originales de la investigación}

La investigación desarrollada durante la presente Tesis Doctoral tiene como principal aportación, la realización de una herramienta avanzada de cálculo que ha permitido integrar todos los elementos intervinientes en el diseño de estructuras offshore frente a cargas hidrodinámicas, en un único modelo numérico de gran eficiencia y potencia, estableciendo así una solución general, válida para el dominio físico al completo. En la elaboración de esta herramienta se ha realizado una profunda revisión del estado del conocimiento, que ha permitido extraer las principales teorías actuantes para caracterizar cada uno de los fenómenos que intervienen en el proceso, y ha permitido detectar las deficiencias y debilidades que presentan cada una de ellas, definiendo así sus rangos válidos de aplicación. Además, la utilización de esta herramienta ha permitido posteriormente, la realización de una profunda reflexión comparativa, para detectar los principales errores y las limitaciones que presenta el método de cálculo más simplificado y tradicional, así como la afección que dichas limitaciones presentan sobre las estructuras construidas hoy en día, con mayores diámetros y a mayores profundidades, y sus implicaciones derivadas, a nivel de diseño.

Por otro lado, como segunda aportación de la presente Tesis Doctoral, cabe destacar que debido a la gran desagregación de procesos que se ha llevado a cabo para establecer esta solución general, ha sido posible el realizar la introducción de variables, nunca antes incluidas de forma explícita en este tipo de cálculos, como la temperatura y la salinidad del agua. Se ha observado la influencia de ambas sobre los distintos procesos de cálculo, y se ha realizado un análisis de sensibilidad con respecto al diámetro y a la teoría de mecánica de ondas utilizada.

Además, como tercera aportación de la presente Tesis Doctoral, se ha desarrollado una nueva formulación de cálculo para incluir los efectos dinámicos vibratorios en el cálculo del comportamiento estructural, de la estructura offshore. Esta nueva formulación a integrado la teoría de mecánica vibratoria en el cálculo, permitiendo analizar el comportamiento estructural, para los primeros 25 modos de vibración, incorporando el 
uso de algoritmos de alta eficiencia, que han permitido realizar un cálculo rápido y preciso, de todo el proceso.

Esta nueva formulación y su implantación mediante algoritmos de alta eficiencia ha permitido realizar la cuarta aportación de la presente Tesis Doctoral, mediante la propuesta de un método de cálculo que permita incorporar en el análisis los efectos de acoplamiento entre el oleaje y la estructura, sin dilatar el tiempo de cálculo de una forma excesiva y sin perder potencia o precisión en los resultados obtenidos.

Por último, se ha propuesto una modificación de una de las teorías más utilizadas y contrastadas para la evaluación de la máxima profundidad de socavación en equilibrio y se ha demostrado que dicha aportación ha reducido el error existente entre los valores teóricos de cálculo, y los valores procedentes de las mediciones reales realizadas en campo. 


\section{Futuras líneas de investigación}

Debido al fuerte carácter teórico que ha acompañado a parte de las principales aportaciones que ha realizado la presente Tesis Doctoral, será necesario la realización de modelos físicos y de la toma de un mayor número de medidas en campo, que permitan mejorar las formulaciones y desarrollos teóricos, y aplicar los factores de corrección pertinentes que se puedan establecer tras el análisis de estos resultados procedentes de la experimentación.

En conclusión, se proponen las siguientes líneas de investigación:

- La mejora del conocimiento en lo referente a las variables que definen el oleaje en cada uno de los parques eólicos estudiados, sobre todo, en relación con la fórmula que relaciona la altura de ola significativa con el periodo de pico.

- La verificación de los valores obtenidos para el campo de velocidades y aceleraciones, en todo el dominio de cálculo utilizado para la caracterización de las fuerzas hidrodinámicas.

- La medición en modelos físicos o en campo, de las fuerzas hidrodinámicas generadas por el oleaje, para comprobar la validez de las expresiones teóricas utilizadas, y ajustar o modificar sus rangos de aplicación.

- La mejora del conocimiento, en lo referente a la influencia de las variables de temperatura y salinidad del agua, y la verificación de los valores proporcionados, en cuanto a la sensibilidad de la influencia de estas variables, frente a la teoría de mecánica de ondas utilizada y el diámetro de la estructura.

- La medición en modelos físicos o en campo, de los valores de tensión experimentados por las estructuras, para verificar el error cometido por los nuevos métodos de análisis dinámico, que incluyen el efecto de acoplamiento oleaje estructura.

- La aplicación del método avanzado de cálculo a otros tipos de estructuras: de sección cónica, en celosía, flotantes, etc.

- La introducción en el modelo de cálculo el peso y las cargas generadas por el aerogenerador. 
- La realización del análisis teniendo en cuenta las frecuencias naturales nominales del rotor $1 \mathrm{P}$ y $3 \mathrm{P}$.

- Por último, el desarrollo de nuevos algoritmos de cálculo e ingeniería de software, que permitan aumentar el número de modos de vibración considerados en el cálculo, así como disminuir el tamaño de la malla o rejilla de cálculo, utilizada en el análisis. 


\section{Capítulo 7}

REFERENCIAS BIBLIOGRÁFICAS 



\section{Capítulo 7}

\section{REFERENCIAS BIBLIOGRÁFICAS}

\section{Capítulo 1.}

BOE, (2.007). "Real Decreto 1028/2007, de 20 de julio, por el que se establece el procedimiento administrativo para la tramitación de las solicitudes de autorización de instalaciones de generación eléctrica en el mar territorial”. Publicación Oficial en el Boletín del Estado, número 183, pp. 33.171 - 33.179.

Dong-Sheng, J. (2008). "Offshore Wind Energy: the next North Sea Oil". Sea Technology Magazine, pp. 33-35.

Espejo Martín, Cayetano (2004). “La Energía Eólica en España”. Investigaciones Geográficas n. 35, pp. 45-65.

Esteban Pérez, María Dolores (2009). "Propuesta de una metodología para la implantación de parques eólicos offshore". Tesis Doctoral de la Universidad Politécnica de Madrid, $550 \mathrm{pp}$.

EWEA, (2017). "The European offshore wind industry. Key trends and statistics 2016". Informe anual publicado por la EWEA en Enero de 2017, 24 pp.

EWEA, (2017). " Wind in power. 2016 European statistics". Informe anual publicado por la EWEA en Febrero de 2017, 37 pp. 
Modelos de predicción de esfuerzos hidrodinámicos y socavación aplicados a ingeniería offshore

Fernández Díez, Pedro (1993). “Energía Eólica”. Libro del Departamento de Ingeniería Eléctrica y Energética de la Universidad de Cantabria, Editorial: Universidad de Cantabria, 136 pp.

GWEC, (2016). "Global wind energy report. Annual market update 2015". Informe anual publicado por GWEC en Abril de 2016, 76 pp.

Naciones Unidas (1998). "Protocolo de Kyoto de la Convención Marco de las Naciones Unidas sobre el Cambio Climático". Documento técnico, 24 pp.

Negro, V., López-Gutiérrez, J.S., Esteban, M.D. y Matutano, C. (2014). "Uncertainties in the design of support structures and foundation for offshore wind turbines", Renewable Energy Journal, Elsevier 63, pp. 125-132.

www.ewea.org (Página web de European Wind Energy Association)

www.gwec.net (Página web del Global Wind Energy Council)

www.thewindpower.net (Página Web de Wind Power) 


\section{Capítulo 2.}

Achenbach, E. (1968). "Distribution of local pressure and skin friction around a circular cylinder in cross-flow up to $\operatorname{Re}=5 \times 10^{6}$ ". Journal Fluid Mechanics, Vol. 34, pp. 625-639.

Airy, G.B. (1845). "On tides and waves". Encyclopedia Metropolitana, vol. 5 (mixed sciences), London, pp. 241-396.

Amate, J.; Víctor, L.; Martín, D.D.; García, L.; Pablo, M.; Alonso, G. (2014). "TLPWIND A smart way to drive costs down". Ponencia realizada en la EWEA 2014 Scientific, Fira de Barcelona Gran Via, Spain, 10-13 March 2014.

Ashuri, T. y Zaaijer, M. B. (2007). "Review of design concept, methods and considerations of offshore wind turbines". Ponencia de OWEC (European Offshore Wind Conference), 10 pp., Alemania.

Bettess, P. y Zienkiewicz, O.C. (1977). "Diffraction and refraction of surface waves using finite and infinite elements". International Journal for Numerical Methods in Engineering, Vol. 11 (8), pp 1271-1290.

Brorsen, M. (2007). "Non-linear Waves". Aalborg: Department of Civil Engineering, Aalborg University. DCE Lecture Notes, No. 9.

Brown, D.T. y Patel, M.H. (1985). "An investigation of wave induced inertia and drag forces on semisubmersible vessels". RINA Trans. 127, pp. 1-11.

Butterfield, S., Musial, W. y Jonkman, J. (2005). "Engineering Challenges for Floating Offshore Wind Turbines". Offshore Wind Conference, 13 pp. Copenague, Dinamarca.

Byrne, B. W. y Houlsby, G. T. (2003). "Foundations for offshore wind turbines". Philosophical Transactions of the Royal Society A, 361, 1.813, pp 2.909-2.930. 
Carroll, B., Cooper, B., Dewey, N., Whitehead, P., Dolphin, T., Rees, J., Judd, A., Whitehouse, R., y Harris, J. (2010). "A further review of sediment monitoring data". Cowrie ScourSed-09, Southampton, UK, 106 pp.

Chakrabarti, S.K. (1972). "Nonlinear wave forces on vertical Cylinder". J. Hydraulics Div., ASCE 98, No. HYII, 1895-1909.

Chakrabarti, S.K. (1990). "Nonlinear Methods in Offshore Engineering". Elsevier Science, New York.

Chakrabarti, S.K. (2005). "Handbook of Offshore Engineering". Elsevier Science, New York.

Chakrabarti, S.K., Tam, W.A. and Wolbert, A.L. (1975). "Wave forces on a randomly oriented tube". In Proceedings of the 7th Offshore Technology Conference, OTC 2190, Houston, Texas, pp. 433-447.

Chaplin, J.R. (1988). "Non-linear forces on horizontal cylinders in the inertia regime in waves at high Reynolds numbers". In Proceedings of the International Conference on the Behaviour of Offshore Structures, BOSS'88, Vol. 2, Trondheim, pp. 505519.

Chappelear, J. E. (1962). "Shallow-water waves". Journal of Geophysical Research, 67, pp. 4693-4704.

Clauss, G.; Lehmann, E.; Östergaard, C. (1992). "Offshore Structures. Volume I: Conceptual Design and Hydromechanics". Libro publicado por Springer-Verlag London, 1992, $342 \mathrm{pp}$.

Cook, G.R. y Simiu, E. (1989). "Hydrodynamic forces in vertical cylinders and the Lighthill correction". Ocean Egineering, Vol. 16, pp. 355-372. 
Costa, M. (2015). " Offshore wind industry review of GBSs. Identifying the key barriers to large scale commercialisation of Gravity Based Structures (GBSs) in the Offshore Wind Industry". Informe técnico publicado por Carbon Trust, Reino Unido, Noviembre 2015, 54 pp.

De Vos, L., (2008). "Optimisation of scour protection design for monopiles and quantification of wave run-up". Ph. D. Thesis, Universiteit Gent, Belgium.

De Vries, W. (2011). "Support Structure Concepts for Deep Water". Final Report WP4.2, in WP4: Offshore Foundations and Support Structures. Delft University of Technology, Delft, The Netherlands, 210 pp.

Dean, R. G. (1965). "Stream function representation of nonlinear ocean waves". Journal of Geophysical Research, Vol. 70, No. 18, p. 4561-4572.

Dean, E.T.R. (2010). "Offshore Geotechnical Engineering - Principles and Practice". Libro publicado por Thomas Telford, Reston, VA, U.S.A., 520 pp.

Djidrov, M., Gavriloski, V., y Jovanova, J. (2014). "Vibration analysis of cantilever beam for damage detection". FME Transactions, Vol. 42, No. 4, pp. 311-316.

DNV (2013). "Offshore Standard DNV-OS-J101. Design of Offshore Wind Turbine Structures". Documento técnico.

Dongfeng, M., Chao Z., Laibin Z., Gui C. (2015). "Dynamic response of offshore jacket platform including foundation degradation under cyclic loadings". Ocean Engineering, Vol. 100, pp. 35-45.

Eatock Taylor, R. y Zietsman, J. (1982). "Hydrodynamic loading on multi component bodies". In Proceedings of the International Conference on the Behaviour of Offshore Structures, BOSS'82, pp. 424-445. 
EWEA (2013). "Deep water. The next step for offshore wind energy". Informe publicado por European Wind Energy Association en Julio de 2013, 51 pp.

Faltinsen, O.M. (1990a). "Sea Loads on Ships and Offshore Structures". Cambridge University Press, Cambridge.

Faltinsen, O.M. (1990b). "Wave loads on offshore structures". Ann. Rev. Fluid Mech. 22, pp. 35-56.

Feng, A., Chen, Z., y Price, W., (2015). "A desingularized Rankine source method for nonlinear wave-body interaction problems". Ocean Engineering, Vol. 101, pp. 131-141.

Fenton, J.D. (1985). "A Fifth-Order Stokes Theory for Steady Waves". Journal of Waterway, Port, Coastal and Ocean Engineering, Vol. 111, No. 2, March 1985. Fe de erratas publicada en la misma revista en, Vol. 113, No. 4, 1987.

Fenton, J. D. (1990). "Nonlinear wave theories". The Sea - Ocean Engineering Science, Part A, B. Le Méhauté \& D. M. Hanes (eds), Vol. 9, Wiley, New York, pp. 3-25.

Fredsøe, J. y Deigaard, R. (1992). "Mechanics of Coastal Sediment Transport". Advanced series on Ocean Engineering, Vol. 3. World Scientific.

Garrett, C. J. R. (1971). "Wave forces on a circular dock". Journal of Fluid Mechanics, Vol. 46 , pp. $129-139$.

Goda, Y. y Yoshimura, T. (1972). "Wave force on a vessel tied at off-shore dolphins". Proceedings of the 13th Coastal Engineering Conference, Vol. III, ch. 96, pp. 1723-1742. 
Graham, J.M.R. (1979). "Analytical methods of representing wave-induced forces on cylinders". In Proceedings in Mechanics of Wave-induced Forces on Cylinders, T.L. Shaw (ed.), pp. 133-151. Pitman, London.

Haskind, M.D. (1957). "The exciting forces and wetting of ships in waves". Izvestia Akademii Nauk S.S.S.R, Otdelenie Tekhnicheskikh Nauk, 7, 65-79. (David Taylor Model Basin Translation №.307, March 1962).

Hau, E. (2013). "Wind Turbines. Fundamentals, Technologies, Application, Economics". Libro publicado por Springer Berlin Heidelberg, 2013, 879 pp.

Havelock, T.H. (1940). "The pressure of water waves upon a fixed obstacle". Proc. Roy. Soc London, A 175. pp. 409-421.

Hereman, W. (2011). " Shallow Water Waves and Solitary Waves". Mathematics of Complexity and Dynamical Systems, Springer, New York, pp. 1520-1532.

Hogben, N. (1976). "Wave loads on structures". In Proceedings of the First International Conference on Behaviour of Offshore Structures, BOSS'76, Vol. 1, pp. 187-219.

Hooft, J.P. (1971). "A mathematical method of determining hydrodynamically induced forces on a semisubmersible". SNAME Trans. Vol. 79, pp. 28-70.

Hooft, J.P. (1982). "Advanced Dynamics of Marine Structures". John Wiley, New York.

Horikawa, K. (1988). "Nearshore dynamics and coastal processes". University of Tokyo press, Japan.

Houston, J.R. (1981). "Combined refraction and diffraction of short waves using the finite element method". Appl. Ocean Res., 3 (4), pp. 163-170. 
Isaacson, M., Premasirl, S., y Yang, G., (1998). "Wave interaction with vertical slotted barrier". Journal of Waterway Port Coastal and Ocean Engineering, 124 (3), pp. $118-126$.

Isobe, M., Nishimura, H., y Horikawa, K. (1978). "Expressions of perturbation solutions for conservative waves by using wave height". Proc. 33rd Annu. Conf. of JSCE, II, pp. 760-761.

Isobe, M. (1988). "Waves and nearshore currents". Chapter 2, Basic wave theories, in K. Horikawa Ed., Nearshore Dynamics and Coastal Processes-Theory, Measurement and Predictive Model, Univ. of Tokyo Press, pp. 30-32.

Iversen, H. W., Balent, R. (1951). "A Correlating Modulus for Fluid Resistance in Accelerated Motion". Journal of Applied Physics, Vol. 22, Issue 3, pp. 324-328.

Iwagaki, Y. (1968). "Hyperbolic waves and their shoaling". Proceedings, 11th International Conference of Coastal Engineering, London, Vol. 1, pp. 124-144.

Karimirad, Madjid (2014). "Offshore Energy Structures for Wind Power, Wave Energy and Hybrid Marine Platforms". Libro publicado por Springer International Publishing, Switzerland, 2014, $301 \mathrm{pp}$.

Khalkar, V., y Ramachandran, S. (2017). "Vibration analysis of a cantilever beam for oblique cracks". ARPN Journal of Engineering and Applied Sciences, Vol. 12, No. 4, pp. $1144-1151$.

Kirby, J. T. (2003). "Boussinesq models and applications to nearshore wave propagation, surfzone processes and wave-induced currents". In Advances in Coastal Modeling, V. C. Lakhan (ed), Elsevier, 41 pp. 
Korteweg, D. J., y de Vries, G. (1895). "On the change of form of long waves advancing in a rectangular canal, and on a new type of long stationary waves". Phil. Mag. (5) 39, 422-443.

Koterayama, W. y Nakamura, M. (1988). Wave forces acting on a moving cylinder. ASME Trans. J. Offshore Mech. Arctic Engng, Vol. 110, pp. 315-319.

Koutandos, E.V., (2009). "Hydrodynamics of vertical semi-immersed slotted barrier". WSEAS Transactions on Fluid Mechanics, Vol. 4 (3), pp. 85-96.

Krolis, V. D., van der Tempel, J. y de Vries, W. (2007). "Evaluation of foundation design for monopile support structures for offshore wind turbines". Ponencia en OWEC (European Offshore Wind Conference), 7 pp., Alemania.

Laitone, E. V. (1960). "The second approximation to cnoidal and solitary waves". Journal of Fluid Mechanics, Vol. 9, pp. 430 - 444.

Laitone, E.V. (1965). "Series solution for shallow water waves". Journal of Geophysical Research, Vol. 70, No. 4, pp. 995-998.

Le Méhauté, B. (1976). "An introduction to hydrodynamics and water waves". SpringerVerlag, New York, 323 pp.

Lighthill, J. (1979). "Waves and hydrodynamic loading". In Proceedings of the 2nd International Conference on the Behaviour of Offshore Structures, BOSS'79, London, pp. 1-40.

Lighthill, J. (1986). "Fundamentals concerning wave loading on offshore structures". Journal of Fluid Mechanics, Vol. 173, 667-681.

MacCamy, R.C. y Fuchs, R.A. (1954). "Wave forces on piles: a diffraction theory". Technical Memorandum No. 69, Beach Erosion Board, Corps of Engineers. 
Mathis, H. (2007). "Offshore foundation in concrete. Cost reduction by serial production". Ponencia en OWEC (European Offshore Wind Conference), 5 pp., Alemania.

Matutano, C. (2013). "Caracterización de los sistemas de protección basados en materiales naturales destinados al control de la socavación en obras marítimas presentes en instalaciones eólicas marinas". Tesis Doctoral de la Universidad Politécnica de Madrid, 515 pp.

Molin, B. (1979). "Second-order diffraction loads on three-dimensional bodies". Applied Ocean Research, Vol. 1, pp. 197-202.

Morison, J.R., O'Brien, M.P., Johnson, J.W. y Schaaf, S.A. (1950). "The force exerted by surface waves on piles". Petrol. Trans. AIME 189, 149-154. Muir Wood, A.M. 1969. Coastal Hydraulics. Macmillan, New York.

Nishimura, H., Isobe, M., y Horikawa, K. (1977). "Higher Order Solutions of Stokes and Cnoidal Waves". Journal of the Faculty of Engineering, Univ. of Tokyo, Series B, Vol. 34, pp. 267-293.

Ogilvie, T.F. (1963). First and Second Order Forces on a Cylinder Submerged Under a Free Surface. Journal of Fluid Mechanics, Vol. 16, pp. 451-472.

Omer, G. C., y Hall, H. H. (1949). "The scattering of a tsunami by a cylindrical island." J. Seismological Soc. Amer., 39(4), pp. 257-260.

Ostergaard, C. y Schellin, T.E. (1987). "Comparison of experimental and theoretical wave on floating and compliant offshore structures". Applied Ocean Research, Vol. 9, pp. 192-213.

Paulling, J.R. (1970). "Wave induced forces and motions of tubular structures". In 8th Symposium of Naval Hydrodynamics, Pasadena, pp. 1083-1110. 
Quallen, S. y Xing, T. (2016). "CFD simulation of a floating offshore wind turbine system using a variable-speed generator-torque controller". Renewable Energy, Vol. 97, pp. 230-242.

Raaijmakers, T. C. y Rudolph, D. (2008). "Time-dependent scour development under combined current and waves conditions - laboratory experiments with online monitoring technique". Paper presented at the Fourth International Conference on Scour and Erosion, Tokyo, Japan.

Rainey, R.C.T. (1989). "A new equation for calculating wave loads on offshore structures". Journal of Fluid Mechanics, Vol. 204, pp. 295-324.

Repetto, C.E., Roatta, A., y Welti, R.J. (2012). "Forced vibrations of a cantilever beam". European Journal of Physics Vol.33(5), pp.1187-1195

Rudolph, D. y Bos. K.J. (2006). "Scour around a monopile under combined wave current conditions and low KC-numbers". Proceeding of the International Conference on Scour and Erosion (ICSE), Amsterdam.

Rudolph, D., Raaijmakers, T. C., Stam, C. J. M. y Op den Velde, W. (2007). "Evaluation of scour development around offshore monopoles based on measurements in the Q7 windpark". Ponencia en OWEC (European Offshore Wind Conference), 10 pp., Alemania.

Sarpkaya, T. e Isaacson, M. (1981). "Mechanics of Wave Forces on Offshore Structures". Van Nostrand-Reinhold, New York, 651 pp.

Sarpkaya, T. (2001). "On the force decompositions of Lighthill and Morison". Journal of Fluids and Structures, Vol. 15, pp. 227-233. 
Sedighi, H.M., Reza, A., y Zare, J. (2012). "Analytical approximation of nonlinear frequency of cantilever beam vibrations". Journal of Vibroengineering, Vol. 14(1), pp. 270279.

Seidel, M. (2007). "Jacket substructures for the Repower 5M wind turbine". Ponencia en OWEC (European Offshore Wind Conference), 8 pp., Alemania.

Shields, A. (1936). "Application of similarity principles and turbulence research to bed-load movement". Pasadena Institute of Technology, California, USA.

Skjelbreia, L. y Hendrickson, J. (1960). "Fifth Order Gravity Wave Theory". Proceedings, 7th Conference Coastal Engineering, Vol. I, pp. 184-196.

Sonalla, M.I. (1989). "Vibrations of cantilever beams with various initial conditions". Master of Science, Ohio State University, Mechanical Engineering, 120 pp.

Sotelo, G. (1980). "Hidráulica General". Volumen 1. Editorial Limusa. 1980.

Soulsby, R., (1997). "Dynamics of Marine Sands: A Manual for Practical Applications". Thomas Telford.

Stokes, G.G. (1847). "On the Theory of Oscillatory Waves," Trans. Cambridge Phil. Soc., Vol. 8, and Supplement, Scia Papers, Vol. I, 1847.

Sumer, B.M., Fredsøe, J. y Christiansen, N. (1992). "Scour around vertical pile in waves". Journal of Waterway, Port, Coastal and Ocean Engineering, Vol. 118(1), pp. 15-31.

Sumer, B.M. y Fredsøe, J. (2002). "The mechanics of scour in the marine environment". World Scientific Press, Advanced Series on Ocean Engineering, Vol. 17, Singapore.

Svendsen, I. A. y Jonsson, I. G., (1980). "Hydrodynamics of Coastal Regions". Den Private Ingeniørfond, Technical University of Denmark, 2nd edition. 
Svendsen, I. A. (1985). "Steep water waves". Lecture notes for a course in Advanced Ocean Engineering, ISVA, 65 pp.

Takagi, M., Arai, S-I., Takezawa, S., Tanaka, K. y Takarada, N. (1985). "A comparison of methods for calculating the motion of a semi-submersible". Ocean Engineering, Vol. 12, pp. 45-97.

Thiagarajan, K. P., y Dagher, H. J. (2014). "A Review of Floating Platform Concepts for Offshore Wind Energy Generation". Journal of offshore mechanics and Arctic engineering, Vol. 136, No. 2, 021906.

Tsay, T.K. y Liu, P.L.F. (1983). "A finite element model for wave refraction and diffraction". Applied Ocean Research, 5 ( 1 ).

Tsay, T. K., Zhu, W., Liu, P. L. F. (1989). "A Finite-Element Model for Wave Refraction, Diffraction, Reflection and Dissipation". Applied Ocean Research, Vol. 11, pp. $33-38$.

Tsuchiya, Y. y Yasuda, T. (1985). "Cnoidal waves in shallow water and their mass transport". Advances in Nonlinear Waves, Vol. 2, Research Notes in Mathematics, Pitman Press, p. 352.

U.S. Army Corps of Engineers (1984). "Shore Protection Manual - Volume I". Coastal Engineering Research Center, Department of the Army, 337 pp.

U.S. Army Corps of Engineers (1975). "Shore Protection Manual - Volume II". Coastal Engineering Research Center, Department of the Army.

Vannucci, P. (1993). "Forze d'onda su cilindri verticali immersi". 7o Colloquio A.I.O.M. (Associazione d'Ingegneria Offshore e Marina), p. 47-53. Pisa. 
Vannucci, P. (2006). "Wave actions on immersed bodies". Cours de Mécanique pour I'Industrie du Pétrole - Offshore Engineering. Université de Versailles et Saint Quentin-en-Yvelines.

Whitehouse, R.J.S. (1998). "Scour at marine structures: a manual for practical applications". Thomas Telford, London, UK.

www.4coffshore.com (Página web de 4C Offshore)

www.areva.com (Página web de Areva)

www.foundocean.com (Página web de Found Ocean)

www.lorc.dk (Página web de Lind $\varnothing$ Offshore Renewables Center)

www.nrel.gov (Página web de National Renewable Energy Laboratory, NREL)

www.offshorewind.biz (Página web de Offshore Wind)

www.offshorewindenergy.org (Página web de Offshore Wind Energy, OWE)

www.statoil.com (Página web de Statoil)

Yasuda, T. (1978). "Study on finite amplitude wave theories in shallow water and their validity ranges". Tesis Doctoral, Universidad de Kyoto, 145 pp.

Zanke, U. (1982). "Scours at piles in steady flow and under the influence of waves". Technical Communication 54, Univ. Hannover (Germany).

Zheng, S. y Zhang, Y., (2015). "Wave diffraction from a truncated cylinder in front of a vertical wall". Ocean Engineering, 104, pp. 329-343. 
Modelos de predicción de esfuerzos hidrodinámicos y socavación aplicados a ingeniería offshore

Zhu, D., (2013). "Full wave solution for hydrodynamic behaviors of pile breakwater". China Ocean Engineering, 27(3), pp. 323-334. 


\section{Capítulo 3.}

Airy, G.B. (1845). "On tides and waves". Encyclopedia Metropolitana, vol. 5 (mixed sciences), London, pp. 241-396.

Bolle, A., De Winter, J., Goossens, W., Haerens, P., y Dewaele, G. (2012). "Scour monitoring around offshore jackets and gravity based foundations". Proceedings Sixth International Conference on Scour and Erosion, Paris, Agosto 27-31, pp. 799-806.

Carroll, B., Cooper, B., Dewey, N., Whitehead, P., Dolphin, T., Rees, J., Judd, A., Whitehouse, R., y Harris, J. (2010). "A further review of sediment monitoring data". Cowrie ScourSed-09, Southampton, UK, 106 pp.

Deutsche WindGuard GmbH, Deutsche Energie-Agentur GmbH, University of Groningen, (2007). Case study: European Offshore Wind Farms- a survey for the Analysis of the Experiences and Lessons Learnt by Developers of Offshore Wind Farms, Final Report.

DNV (2013). "Offshore Standard DNV-OS-J101. Design of Offshore Wind Turbine Structures". Documento técnico.

EWEA, (2017). "The European offshore wind industry. Key trends and statistics 2016". Informe anual publicado por la EWEA en Enero de 2017, 24 pp.

Harris, J.M., Whitehouse, R.J.S. y Sutherland, J. (2010). "Scour assessment in complex marine soils- an evaluation through case examples". Proceedings of the 5th International Conference on Scour and Erosion (ICSE-5), San Francisco, CA, USA.

Louwersheimer W.F. (2007). "Scour around an offshore wind turbine". MSc Thesis, Delft University of Technology, The Netherlands. 
Matutano, C., Negro, V., López, J.S., Esteban, M.D., (2015). Design of scour Protection Systems in Offshore Wind Farms. Journal of Energy Resources Technology. 137 (n. 5). 1-5.

Morison, J.R., O'Brien, M.P., Johnson, J.W. y Schaaf, S.A. (1950). "The force exerted by surface waves on piles". Petrol. Trans. AIME 189, 149-154. Muir Wood, A.M. 1969. Coastal Hydraulics. Macmillan, New York.

Raaijmakers, T. C. y Rudolph, D. (2008). "Time-dependent scour development under combined current and waves conditions - laboratory experiments with online monitoring technique". Paper presented at the Fourth International Conference on Scour and Erosion, Tokyo, Japan.

Rudolph, D. y Bos. K.J. (2006). "Scour around a monopile under combined wave current conditions and low KC-numbers". Proceeding of the International Conference on Scour and Erosion (ICSE), Amsterdam.

Sumer, B.M. y Fredsøe, J. (2002). "The mechanics of scour in the marine environment". World Scientific Press, Advanced Series on Ocean Engineering, Vol. 17, Singapore.

Whitehouse, R.J.S., Harris, J.M., Rees J. (2008). "Dynamics of scour pits and scour protection - Synthesis report and recommendations (Milestones 2 and 3)". Department of energy and climate change.

Whitehouse, R.J.S., Harris, J.M., Sutherland, J. y Rees, J. (2011). "The nature of scour development and scour protection at offshore wind farm foundations". Marine Pollution Bulletin 62, pp. 73-88.

www.4coffshore.com (Página web de 4C Offshore Limited)

www.bowind.co.uk, (Página Web del parque eólico marino Barrow) 
Modelos de predicción de esfuerzos hidrodinámicos y socavación aplicados a ingeniería offshore

www.hornsrev.dk, (Página Web del parque eólico marino Horns Rev)

www.marinedataexchange.co.uk/ (Página web para la obtención de datos de parques eólicos offshore, perteneciente a The Crown Estate, Reino unido)

www.nystedwindfarm.com (Página web del parque eólico Nysted) 


\section{Capítulo 4.}

Achenbach, E. (1968). "Distribution of local pressure and skin friction around a circular cylinder in cross-flow up to $\operatorname{Re}=5 \times 10^{6}$ ". Journal Fluid Mechanics, Vol. 34, pp. 625-639.

Airy, G.B. (1845). "On tides and waves". Encyclopedia Metropolitana, vol. 5 (mixed sciences), London, pp. 241-396.

Bai, W., y Taylor, R.E (2007). "Numerical simulation of fully nonlinear regular and focused wave diffraction around a vertical cylinder using domain decomposition". Applied Ocean Research, Vol. 29, pp. 55-71

Bengt, S. (2011). "Tubes, Crossflow over". Thermopedia, DOI: 10.1615 / AtoZ.t.tubes_crossflow_over.

Carroll, B., Cooper, B., Dewey, N., Whitehead, P., Dolphin, T., Rees, J., Judd, A., Whitehouse, R., y Harris, J. (2010). "A further review of sediment monitoring data". Cowrie ScourSed-09, Southampton, UK, 106 pp.

Chakrabarti, S.K. (2005). "Handbook of Offshore Engineering". Elsevier Science, New York.

Deutsche WindGuard GmbH, Deutsche Energie-Agentur GmbH, University of Groningen, (2007). Case study: European Offshore Wind Farms- a survey for the Analysis of the Experiences and Lessons Learnt by Developers of Offshore Wind Farms, Final Report.

Han, Y., Zhan, J.M., Su, W., Li, Y.S., y Zhou, Q. (2015). "Numerical Simulation of In-Line Response of a Vertical Cylinder in Regular Waves". Journal of Coastal Research: Volume 31, Issue 4: pp. $879-891$. 
Harris, J.M., Whitehouse, R.J.S. y Sutherland, J. (2010). "Scour assessment in complex marine soils- an evaluation through case examples". Proceedings of the 5th International Conference on Scour and Erosion (ICSE-5), San Francisco, CA, USA.

Le Méhauté, B. (1976). "An introduction to hydrodynamics and water waves". SpringerVerlag, New York, 323 pp.

Louwersheimer W.F. (2007). "Scour around an offshore wind turbine". MSc Thesis, Delft University of Technology, The Netherlands.

MacCamy, R.C. y Fuchs, R.A. (1954). "Wave forces on piles: a diffraction theory". Technical Memorandum No. 69, Beach Erosion Board, Corps of Engineers.

Matutano, C., Negro, V., López, J.S., Esteban, M.D., (2015). Design of scour Protection Systems in Offshore Wind Farms. Journal of Energy Resources Technology. 137 (n. 5). $1-5$.

Moghadaszadeh, S.O., y Khaji, N., (2015). "Development and application of a semianalytical method with diagonal coefficient matrices for analysis of wave diffraction around vertical cylinders of arbitrary cross-sections". Ocean Engineering, Vol. 110, pp. 292-302

Morison, J.R., O'Brien, M.P., Johnson, J.W. y Schaaf, S.A. (1950). "The force exerted by surface waves on piles". Petrol. Trans. AIME 189, 149-154. Muir Wood, A.M. 1969. Coastal Hydraulics. Macmillan, New York.

Nielsen, P. (1992). "Coastal Bottom Boundary Layers and Sediment Transport". Advanced Series on Ocean Engineering, Vol. 4. World Scientific.

Sonalla, M.I. (1989). "Vibrations of cantilever beams with various initial conditions". Master of Science, Ohio State University, Mechanical Engineering, 120 pp. 
Sumer, B.M., Fredsøe, J. y Christiansen, N. (1992). "Scour around vertical pile in waves". Journal of Waterway, Port, Coastal and Ocean Engineering, Vol. 118(1), pp. 15-31.

Sumer, B.M. y Fredsøe, J. (2002). "The mechanics of scour in the marine environment". World Scientific Press, Advanced Series on Ocean Engineering, Vol. 17, Singapore.

U.S. Army Corps of Engineers (1984). "Shore Protection Manual - Volume I". Coastal Engineering Research Center, Department of the Army, 337 pp.

U.S. Army Corps of Engineers (1975). "Shore Protection Manual - Volume II". Coastal Engineering Research Center, Department of the Army.

Whitehouse, R.J.S., Harris, J.M., Rees J. (2008). "Dynamics of scour pits and scour protection - Synthesis report and recommendations (Milestones 2 and 3)". Department of energy and climate change.

Whitehouse, R.J.S., Harris, J.M., Sutherland, J. y Rees, J. (2011). "The nature of scour development and scour protection at offshore wind farm foundations". Marine Pollution Bulletin 62, pp. 73-88.

www.codecogs.com (Página web de codecogs)

Yang, C., y Ertekin, R. C., (1992). "Numerical Simulation of Nonlinear Wave Diffraction by a Vertical Cylinder". Journal of Offshore Mechanics and Arctic Engineering, Vol. 114(1), pp. 36-44 


\section{Capítulo 5.}

Airy, G.B. (1845). "On tides and waves". Encyclopedia Metropolitana, vol. 5 (mixed sciences), London, pp. 241-396.

Carroll, B., Cooper, B., Dewey, N., Whitehead, P., Dolphin, T., Rees, J., Judd, A., Whitehouse, R., y Harris, J. (2010). "A further review of sediment monitoring data". Cowrie ScourSed-09, Southampton, UK, 106 pp.

Deutsche WindGuard GmbH, Deutsche Energie-Agentur GmbH, University of Groningen, (2007). Case study: European Offshore Wind Farms- a survey for the Analysis of the Experiences and Lessons Learnt by Developers of Offshore Wind Farms, Final Report.

Harris, J.M., Whitehouse, R.J.S. y Sutherland, J. (2010). "Scour assessment in complex marine soils- an evaluation through case examples". Proceedings of the 5th International Conference on Scour and Erosion (ICSE-5), San Francisco, CA, USA.

Louwersheimer W.F. (2007). "Scour around an offshore wind turbine". MSc Thesis, Delft University of Technology, The Netherlands.

Matutano, C., Negro, V., López, J.S., Esteban, M.D., (2015). Design of scour Protection Systems in Offshore Wind Farms. Journal of Energy Resources Technology. 137 (n. 5). $1-5$.

Morison, J.R., O'Brien, M.P., Johnson, J.W. y Schaaf, S.A. (1950). "The force exerted by surface waves on piles". Petrol. Trans. AIME 189, 149-154. Muir Wood, A.M. 1969. Coastal Hydraulics. Macmillan, New York.

U.S. Army Corps of Engineers (1975). "Shore Protection Manual - Volume II". Coastal Engineering Research Center, Department of the Army. 
Modelos de predicción de esfuerzos hidrodinámicos y socavación aplicados a ingeniería offshore

Whitehouse, R.J.S., Harris, J.M., Rees J. (2008). "Dynamics of scour pits and scour protection - Synthesis report and recommendations (Milestones 2 and 3)". Department of energy and climate change.

Whitehouse, R.J.S., Harris, J.M., Sutherland, J. y Rees, J. (2011). "The nature of scour development and scour protection at offshore wind farm foundations". Marine Pollution Bulletin 62, pp. 73-88. 

Apéndice 1

RESULTADOS DE LOS CASOS DE ESTUDIO 



\section{Apéndice 1}

\section{RESULTADOS DE LOS CASOS DE ESTUDIO}

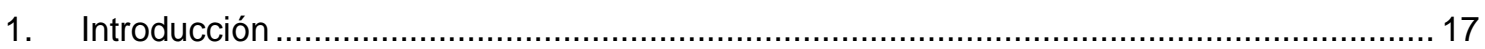

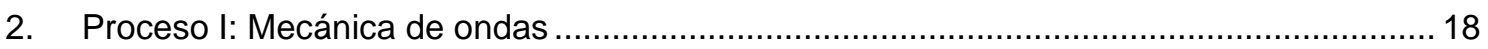

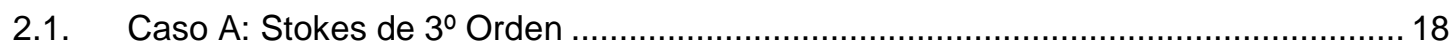

2.1.1. Comparación entre el campo de velocidades y aceleraciones, con la teoría lineal.

2.1.2. Análisis de principales frecuencias generadas por el oleaje.............................21

2.1.3. Análisis sobre la amortiguación de la velocidad y la aceleración con la profundidad

2.2. Caso B: Stokes de $5^{\circ}$ Orden 23

2.2.1. Comparación entre el campo de velocidades y aceleraciones, con la teoría lineal. 23

2.2.2. Análisis de principales frecuencias generadas por el oleaje............................. 26

2.2.3. Análisis sobre la amortiguación de la velocidad y la aceleración con la profundidad 27

2.3. Caso C: Onda Cnoidal de $2^{\circ}$ Orden 28

2.3.1. Comparación entre el campo de velocidades y aceleraciones, con la teoría lineal. 28

2.3.2. Análisis de principales frecuencias generadas por el oleaje. 31

2.3.3. Análisis sobre la amortiguación de la velocidad y la aceleración con la profundidad 32

2.4. Caso D: Stream Function de Dean. .33

2.4.1. Comparación entre el campo de velocidades y aceleraciones, con la teoría lineal.

2.4.2. Análisis de principales frecuencias generadas por el oleaje. 36

2.4.3. Análisis sobre la amortiguación de la velocidad y la aceleración con la profundidad 37

3. Proceso II: Cálculo de las fuerzas hidrodinámicas 38 


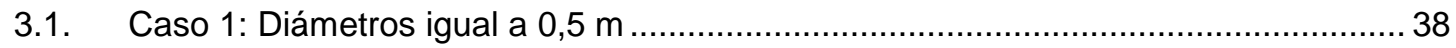

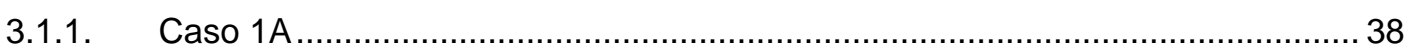

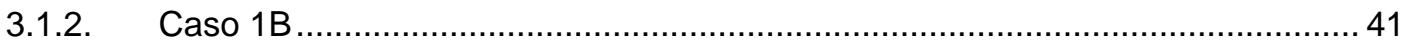

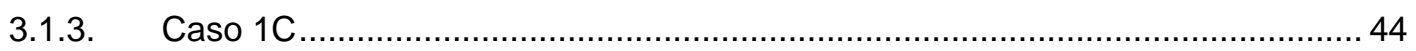

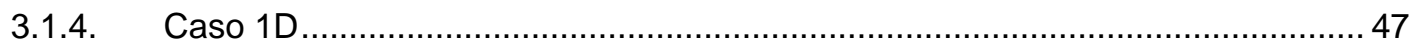

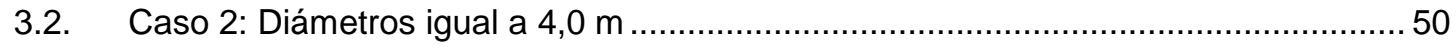

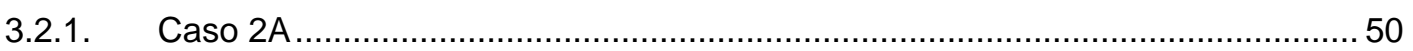

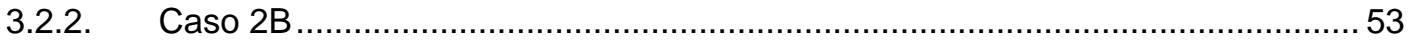

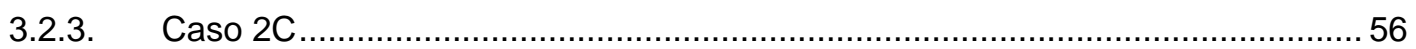

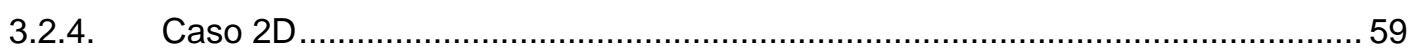

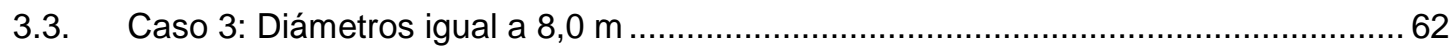

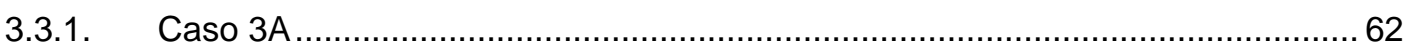

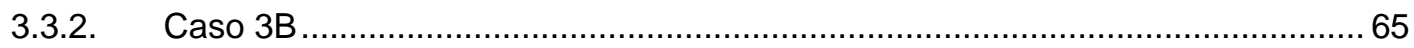

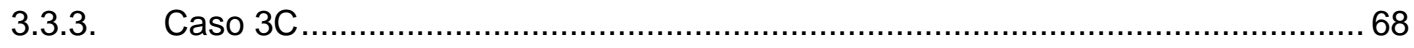

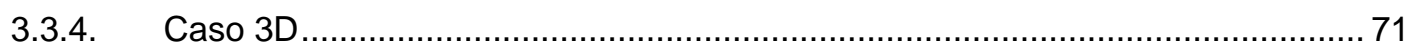

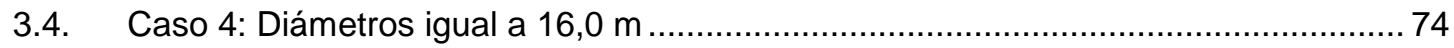

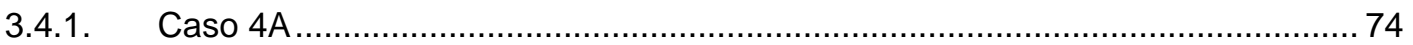

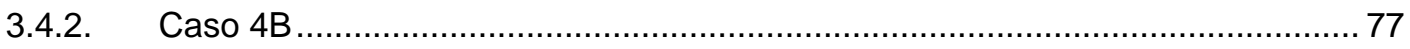

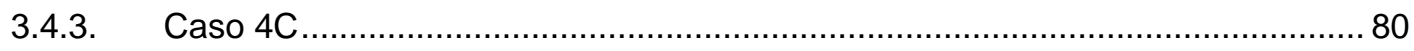

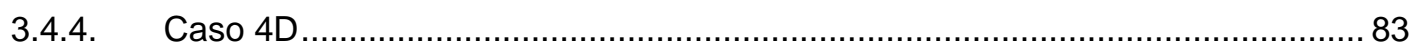

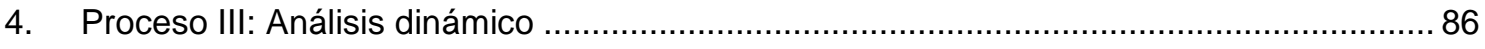

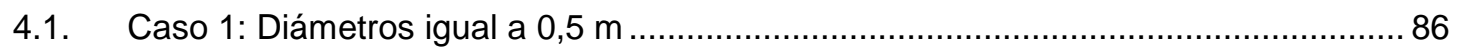

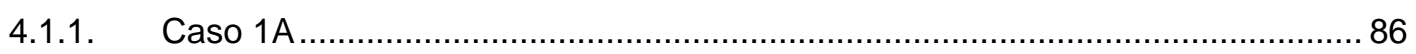

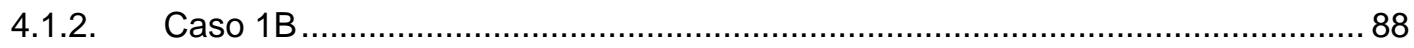

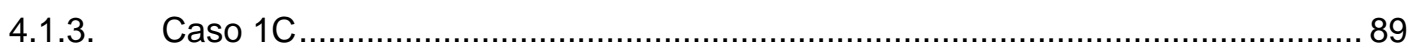

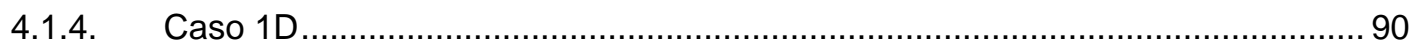

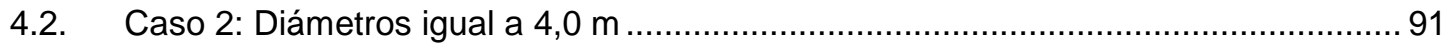

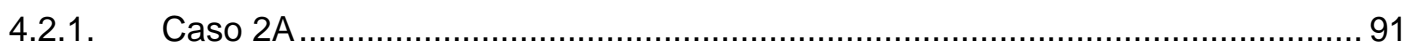

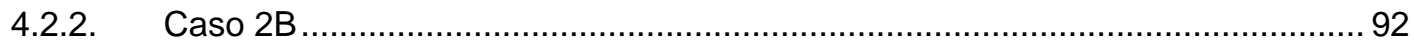

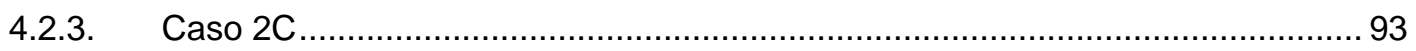

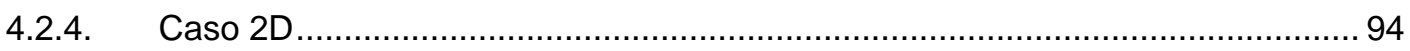




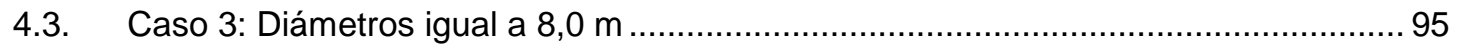

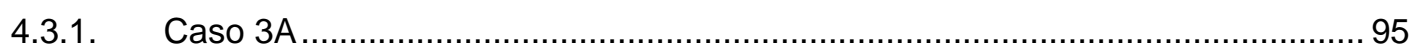

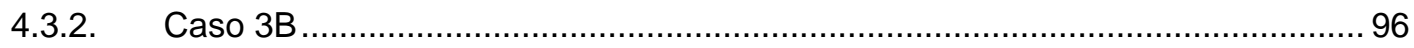

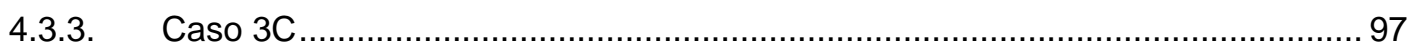

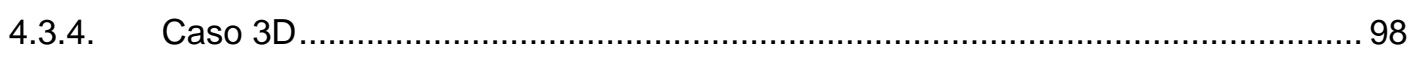

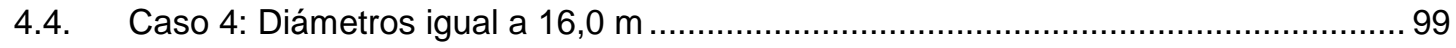

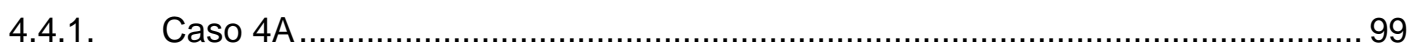

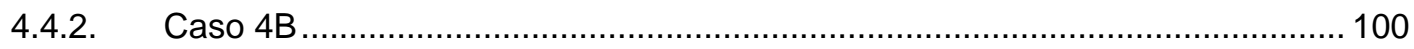

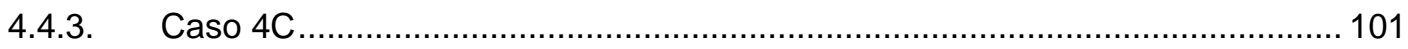

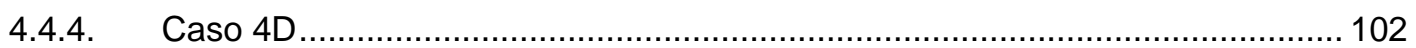

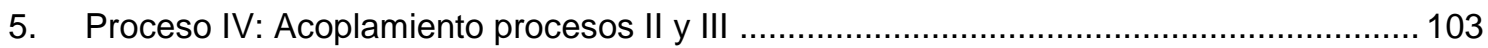

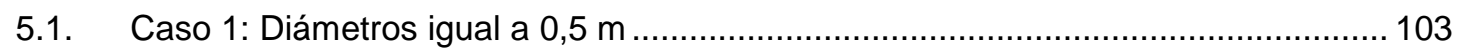

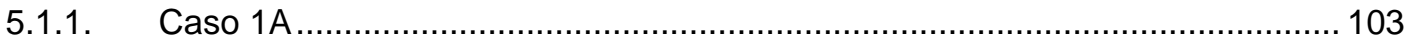

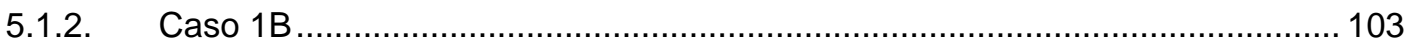

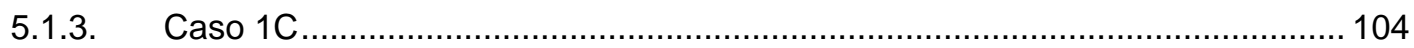

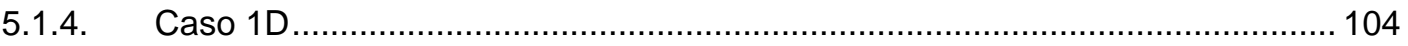

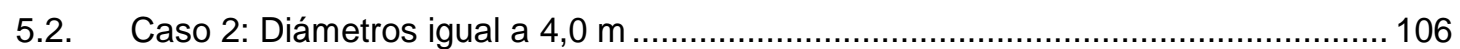

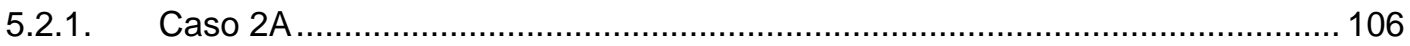

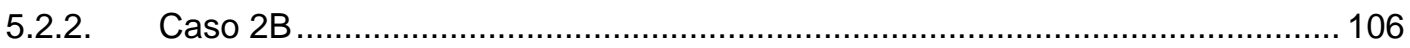

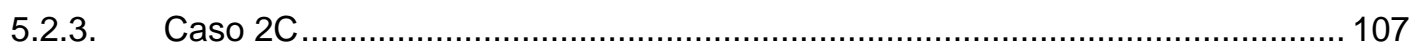

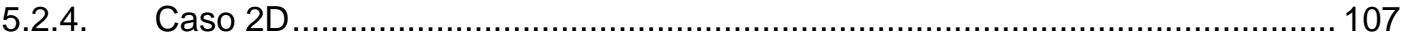

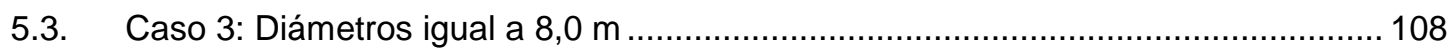

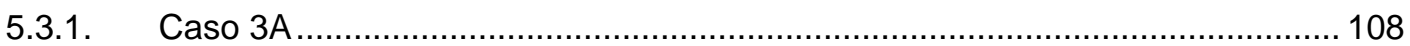

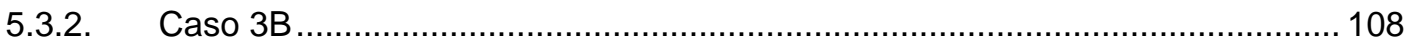

5.3.3. Caso 3C

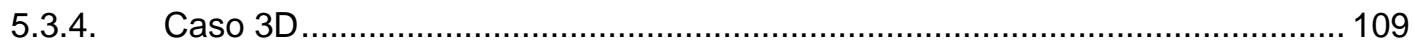

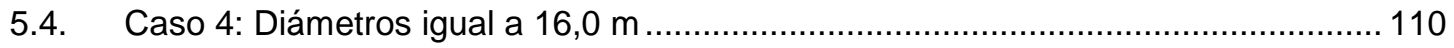

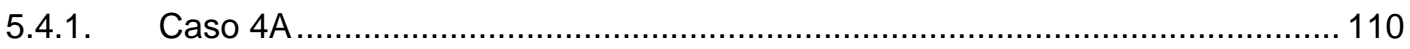

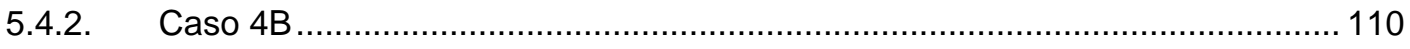

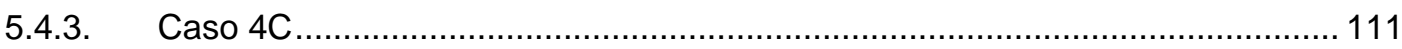

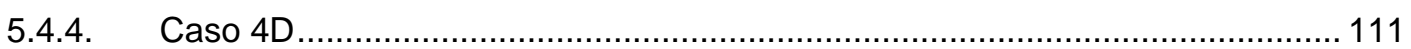




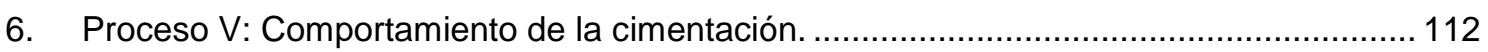

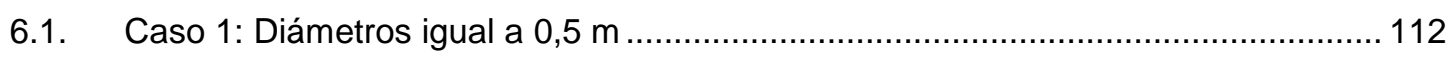

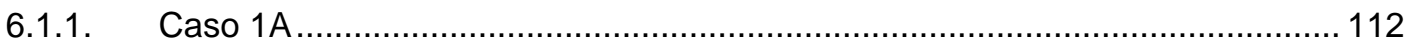

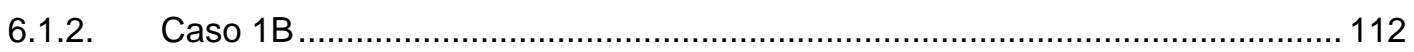

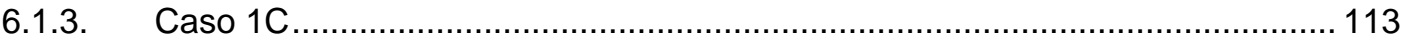

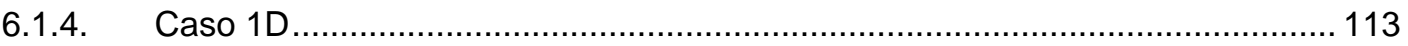

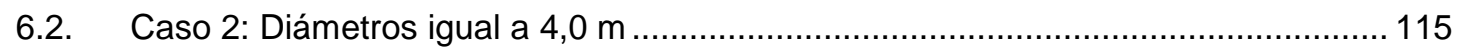

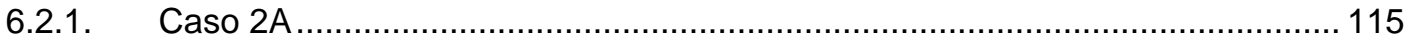

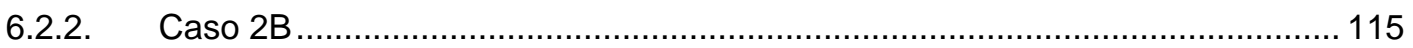

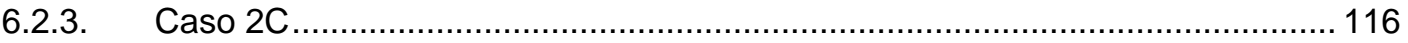

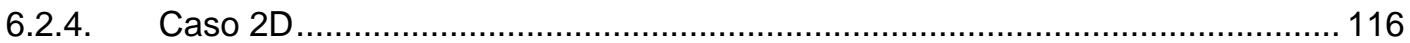

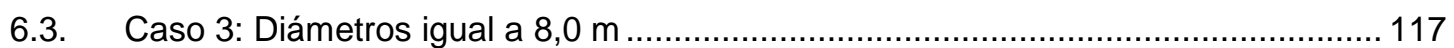

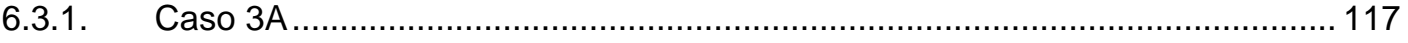

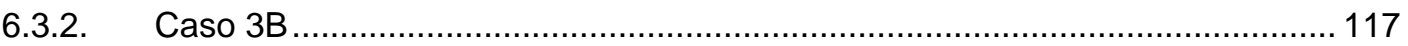

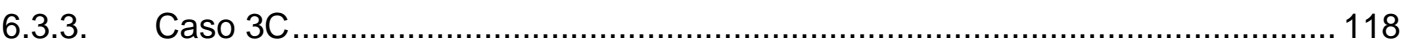

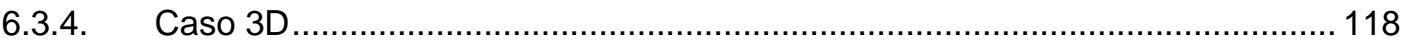

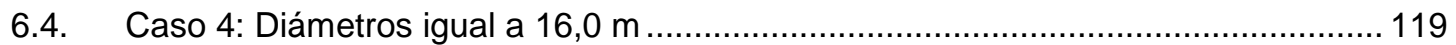

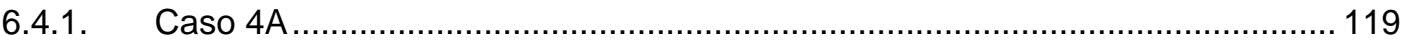

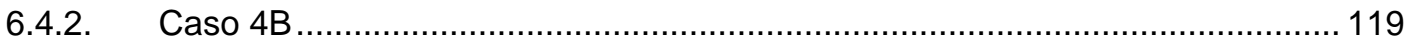

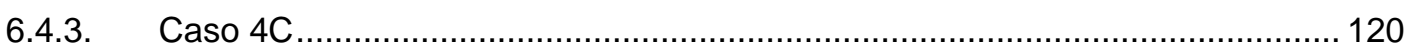

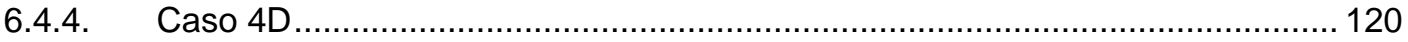




\section{Apéndice 1}

\section{ÍNDICE DE FIGURAS}

Figura 1. Velocidades para $\mathrm{H}=7,7 \mathrm{~m}, \mathrm{~h}=24,0 \mathrm{~m}$ y $\mathrm{T}=9,7 \mathrm{~s}$ según Stokes de 3o Orden ............. 19

Figura 2. Velocidades para $\mathrm{H}=7,7 \mathrm{~m}, \mathrm{~h}=24,0 \mathrm{~m}$ y $\mathrm{T}=9,7 \mathrm{~s}$ según teoría lineal ........................ 19

Figura 3. Aceleraciones para $\mathrm{H}=7,7 \mathrm{~m}, \mathrm{~h}=24,0 \mathrm{~m}$ y $\mathrm{T}=9,7$ s según Stokes de $3^{\circ}$ Orden ......... 20

Figura 4. Aceleraciones para $\mathrm{H}=7,7 \mathrm{~m}, \mathrm{~h}=24,0 \mathrm{~m}$ y $\mathrm{T}=9,7 \mathrm{~s}$ según teoría lineal ..................... 20

Figura 5. Velocidades: Al a izquierda resultados de la teoría lineal, a la derecha de Stokes de

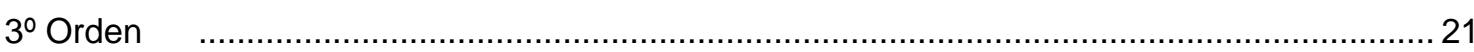

Figura 6. Aceleraciones: Al a izquierda resultados de la teoría lineal, a la derecha de Stokes de 3 o Orden

Figura 7. Amortiguamiento de velocidades, a la izquierda teoría lineal, a la derecha Stokes de 3o Orden

Figura 8. Amortiguamiento de aceleraciones, a la izquierda teoría lineal, a la derecha Stokes de 3 o Orden

Figura 9. Velocidades para $\mathrm{H}=4,78 \mathrm{~m}, \mathrm{~h}=10,0 \mathrm{~m}$ y $\mathrm{T}=6,8 \mathrm{~s}$ según Stokes de $5^{\circ}$ Orden …....... 23

Figura 10. Velocidades para $\mathrm{H}=4,78 \mathrm{~m}, \mathrm{~h}=10,0 \mathrm{~m}$ y $\mathrm{T}=6,8 \mathrm{~s}$ según teoría lineal .24

Figura 11. Aceleraciones para $\mathrm{H}=4,78 \mathrm{~m}, \mathrm{~h}=10,0 \mathrm{~m}$ y $\mathrm{T}=6,8$ s según Stokes de $5^{\circ}$ Orden $\ldots 25$

Figura 12. Aceleraciones para $\mathrm{H}=4,78 \mathrm{~m}, \mathrm{~h}=10,0 \mathrm{~m}$ y $\mathrm{T}=6,8$ s según teoría lineal 25

Figura 13. Velocidades: Al a izquierda resultados de la teoría lineal, a la derecha de Stokes de $5^{\circ}$ Orden

Figura 14. Aceleraciones: Al a izquierda resultados de la teoría lineal, a la derecha de Stokes de $5^{\circ}$ Orden

Figura 15. Amortiguamiento de velocidades, a la izquierda teoría lineal, a la derecha Stokes de $5^{\circ}$ Orden

Figura 16. Amortiguamiento de aceleraciones, a la izquierda teoría lineal, a la derecha Stokes de $5^{\circ}$ Orden 27

Figura 17. Velocidades para $\mathrm{H}=3,6 \mathrm{~m}, \mathrm{~h}=22,0 \mathrm{~m}$ y $\mathrm{T}=18,5$ s según Cnoidal de $2^{\circ}$ Orden ...... 28

Figura 18. Velocidades para $\mathrm{H}=3,6 \mathrm{~m}, \mathrm{~h}=22,0 \mathrm{~m}$ y $\mathrm{T}=18,5$ s según teoría lineal .....................29

Figura 19. Aceleraciones para $\mathrm{H}=3,6 \mathrm{~m}, \mathrm{~h}=22,0 \mathrm{~m}$ y $\mathrm{T}=18,5$ s según Cnoidal de $2^{\circ}$ Orden .. 30 
Figura 20. Aceleraciones para $\mathrm{H}=3,6 \mathrm{~m}, \mathrm{~h}=22,0 \mathrm{~m}$ y $\mathrm{T}=18,5$ s según teoría lineal 30

Figura 21. Velocidades: Al a izquierda resultados de la teoría lineal, a la derecha de Cnoidal de $2^{\circ}$ Orden

Figura 22. Aceleraciones: Al a izquierda resultados de la teoría lineal, a la derecha de Cnoidal de $2^{\circ}$ Orden

Figura 23. Amortiguamiento de velocidades, a la izquierda teoría lineal, a la derecha Cnoidal de $2^{\circ}$ Orden 32

Figura 24. Amortiguamiento de aceleraciones, a la izquierda teoría lineal, a la derecha Cnoidal de $2^{\circ}$ Orden 32

Figura 25. Velocidades para $\mathrm{H}=6,32 \mathrm{~m}, \mathrm{~h}=16,0 \mathrm{~m}$ y $\mathrm{T}=11,06 \mathrm{~s}$ según Stream Function de $5^{\circ}$ Orden 33

Figura 26. Velocidades para $\mathrm{H}=6,32 \mathrm{~m}, \mathrm{~h}=16,0 \mathrm{~m}$ y $\mathrm{T}=11,06$ s según teoría lineal 34

Figura 27. Aceleraciones para $\mathrm{H}=6,32 \mathrm{~m}, \mathrm{~h}=16,0 \mathrm{~m}$ y $\mathrm{T}=11,06$ s según Stream Function de $5^{\circ}$

Orden 35

Figura 28. Aceleraciones para $\mathrm{H}=6,32 \mathrm{~m}, \mathrm{~h}=16,0 \mathrm{~m}$ y $\mathrm{T}=11,06$ s según teoría lineal 35

Figura 29. Velocidades: Al a izquierda resultados de la teoría lineal, a la derecha de Stream Function de 5을 36

Figura 30. Aceleraciones: Al a izquierda resultados de la teoría lineal, a la derecha de Stream Function de 5을 36

Figura 31. Amortiguamiento de velocidades, a la izquierda teoría lineal, a la derecha Stream Function de 5을 37

Figura 32. Amortiguamiento de aceleraciones, a la izquierda teoría lineal, a la derecha Stream Function de $5^{\circ}$ Orden 37

Figura 33. Momento flector máximo para $H=7,7 \mathrm{~m}, \mathrm{~h}=24,0 \mathrm{~m}, \mathrm{~T}=9,7 \mathrm{~s}$ y $\mathrm{D}=0,5 \mathrm{~m} \ldots \ldots \ldots \ldots . . . . .38$

Figura 34. Momento flector máximo para $\mathrm{H}=4,78 \mathrm{~m}, \mathrm{~h}=10,0 \mathrm{~m}, \mathrm{~T}=6,8 \mathrm{~s}$ y $\mathrm{D}=0,5 \mathrm{~m} \ldots \ldots \ldots \ldots \ldots . . . .11$

Figura 35. Momento flector máximo para $H=3,6 \mathrm{~m}, \mathrm{~h}=22,0 \mathrm{~m}, \mathrm{~T}=18,5$ s y $\mathrm{D}=0,5 \mathrm{~m} \ldots \ldots \ldots \ldots . . . . .44$

Figura 36. Momento flector máximo para $H=6,32 \mathrm{~m}, \mathrm{~h}=16 \mathrm{~m}, \mathrm{~T}=11,06$ s y $\mathrm{D}=0,5 \mathrm{~m} \ldots \ldots \ldots \ldots . . .47$

Figura 37. Momento flector máximo para $\mathrm{H}=7,7 \mathrm{~m}, \mathrm{~h}=24,0 \mathrm{~m}, \mathrm{~T}=9,7 \mathrm{~s}$ y $\mathrm{D}=4,0 \mathrm{~m} \ldots \ldots \ldots \ldots \ldots . . . . . .50$

Figura 38. Momento flector máximo para $H=4,78 m, h=10,0 m, T=6,8$ s y $D=4,0 m \ldots \ldots \ldots \ldots . . .53$

Figura 39. Momento flector máximo para $\mathrm{H}=3,6 \mathrm{~m}, \mathrm{~h}=22,0 \mathrm{~m}, \mathrm{~T}=18,5$ s y $\mathrm{D}=4,0 \mathrm{~m} \ldots \ldots \ldots \ldots . . . . .56$

Figura 40. Momento flector máximo para $\mathrm{H}=6,32 \mathrm{~m}, \mathrm{~h}=16 \mathrm{~m}, \mathrm{~T}=11,06 \mathrm{~s}$ y $\mathrm{D}=4,0 \mathrm{~m} \ldots \ldots \ldots \ldots . . . . .59$

Figura 41. Momento flector máximo para $\mathrm{H}=7,7 \mathrm{~m}, \mathrm{~h}=24,0 \mathrm{~m}, \mathrm{~T}=9,7 \mathrm{~s}$ y $\mathrm{D}=8,0 \mathrm{~m}$................. 62

Figura 42. Momento flector máximo para $\mathrm{H}=4,78 \mathrm{~m}, \mathrm{~h}=10,0 \mathrm{~m}, \mathrm{~T}=6,8$ s y $\mathrm{D}=8,0 \mathrm{~m}$................65 
Figura 43. Momento flector máximo para $\mathrm{H}=3,6 \mathrm{~m}, \mathrm{~h}=22,0 \mathrm{~m}, \mathrm{~T}=18,5 \mathrm{~s}$ y $\mathrm{D}=8,0 \mathrm{~m}$ .68

Figura 44. Momento flector máximo para $\mathrm{H}=6,32 \mathrm{~m}, \mathrm{~h}=16 \mathrm{~m}, \mathrm{~T}=11,06 \mathrm{~s}$ y $\mathrm{D}=8,0 \mathrm{~m}$.............. 71

Figura 45. Momento flector máximo para $H=7,7 \mathrm{~m}, \mathrm{~h}=24,0 \mathrm{~m}, \mathrm{~T}=9,7 \mathrm{~s}$ y $\mathrm{D}=16,0 \mathrm{~m}$ .74

Figura 46. Momento flector máximo para $H=4,78 \mathrm{~m}, \mathrm{~h}=10,0 \mathrm{~m}, \mathrm{~T}=6,8 \mathrm{~s}$ y $\mathrm{D}=16,0 \mathrm{~m}$ 77

Figura 47. Momento flector máximo para $H=3,6 \mathrm{~m}, \mathrm{~h}=22,0 \mathrm{~m}, \mathrm{~T}=18,5 \mathrm{~s}$ y $\mathrm{D}=16,0 \mathrm{~m}$ 80

Figura 48. Momento flector máximo para $\mathrm{H}=6,32 \mathrm{~m}, \mathrm{~h}=16 \mathrm{~m}, \mathrm{~T}=11,06$ s y $\mathrm{D}=16,0 \mathrm{~m}$ .83

Figura 49. Deformada en el instante de momento flector máximo para $\mathrm{H}=7,7 \mathrm{~m}, \mathrm{~h}=24,0 \mathrm{~m}$, $T=9,7 \mathrm{~s}$ y $\mathrm{D}=0,5 \mathrm{~m}$ .. 86

Figura 50. Deformada en el instante de momento flector máximo para $\mathrm{H}=4,78 \mathrm{~m}, \mathrm{~h}=10,0 \mathrm{~m}$, $T=6,8 \mathrm{~s}$ y $\mathrm{D}=0,5 \mathrm{~m}$ .88

Figura 51. Deformada en el instante de momento flector máximo para $\mathrm{H}=3,6 \mathrm{~m}, \mathrm{~h}=22,0 \mathrm{~m}$, $T=18,5$ s y $D=0,5 m$.

Figura 52. Deformada en el instante de momento flector máximo para $H=6,32 m, h=16,0 m$, $T=11,06$ s y $D=0,5 m$. .90

Figura 53. Deformada en el instante de momento flector máximo para $\mathrm{H}=7,7 \mathrm{~m}, \mathrm{~h}=24,0 \mathrm{~m}$, $T=9,7 \mathrm{~s}$ y $\mathrm{D}=4,0 \mathrm{~m}$ 91

Figura 54. Deformada en el instante de momento flector máximo para $H=4,78 m, h=10,0 m$, $\mathrm{T}=6,8 \mathrm{~s}$ y $\mathrm{D}=4,0 \mathrm{~m}$ .92

Figura 55. Deformada en el instante de momento flector máximo para $\mathrm{H}=3,6 \mathrm{~m}, \mathrm{~h}=22,0 \mathrm{~m}$, $\mathrm{T}=18,5 \mathrm{~s}$ y $\mathrm{D}=4,0 \mathrm{~m}$. .93

Figura 56. Deformada en el instante de momento flector máximo para $H=6,32 m, h=16,0 m$, $T=11,06 s$ y $D=4,0 m$. .94

Figura 57. Deformada en el instante de momento flector máximo para $H=7,7 m, h=24,0 m$, $T=9,7 \mathrm{~s}$ y $\mathrm{D}=8,0 \mathrm{~m}$ .95

Figura 58. Deformada en el instante de momento flector máximo para $H=4,78 m, h=10,0 m$, $T=6,8 \mathrm{~s}$ y $\mathrm{D}=8,0 \mathrm{~m}$ .96

Figura 59. Deformada en el instante de momento flector máximo para $\mathrm{H}=3,6 \mathrm{~m}, \mathrm{~h}=22,0 \mathrm{~m}$, $T=18,5$ s y $D=8,0 m$.

Figura 60. Deformada en el instante de momento flector máximo para $H=6,32 m, h=16,0 m$, $T=11,06$ s y $D=8,0 m$. .98

Figura 61. Deformada en el instante de momento flector máximo para $\mathrm{H}=7,7 \mathrm{~m}, \mathrm{~h}=24,0 \mathrm{~m}$, $T=9,7 \mathrm{~s}$ y $\mathrm{D}=16,0 \mathrm{~m}$. .99

Figura 62. Deformada en el instante de momento flector máximo para $H=4,78 m, h=10,0 m$, $T=6,8 \mathrm{~s}$ y $\mathrm{D}=16,0 \mathrm{~m}$. 100 
Modelos de predicción de esfuerzos hidrodinámicos y socavación aplicados a ingeniería offshore

Figura 63. Deformada en el instante de momento flector máximo para $\mathrm{H}=3,6 \mathrm{~m}, \mathrm{~h}=22,0 \mathrm{~m}$, $T=18,5$ s y $D=16,0 \mathrm{~m}$. 101

Figura 64. Deformada en el instante de momento flector máximo para $H=6,32 m, h=16,0 m$, $\mathrm{T}=11,06 \mathrm{~s}$ y $\mathrm{D}=16,0 \mathrm{~m}$ 102 


\section{Apéndice 1}

\section{INDICE DE TABLAS}

Tabla 1. Comparación de campo de velocidades caso A …................................................. 20

Tabla 2. Comparación de campo de aceleraciones caso A ................................................. 21

Tabla 3. Comparación de campo de velocidades caso B ...................................................... 24

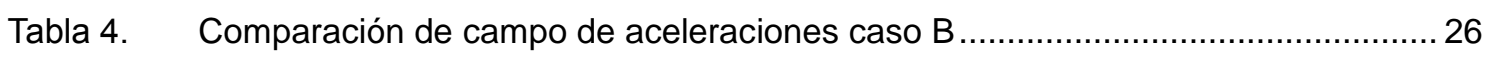

Tabla 5. Comparación de campo de velocidades caso C ...................................................29

Tabla 6. Comparación de campo de aceleraciones caso C ..................................................... 31

Tabla 7. Comparación de campo de velocidades caso D ………....................................... 34

Tabla 8. Comparación de campo de aceleraciones caso D................................................. 36

Tabla 9. Fuerzas hidrodinámicas para el caso de $H=7,7 \mathrm{~m}, \mathrm{~h}=24,0 \mathrm{~m}, \mathrm{~T}=9,7 \mathrm{~s}$ y $\mathrm{D}=0,5 \mathrm{~m}$..... 39

Tabla 10. Fuerzas hidrodinámicas para momento flector máximo, para $\mathrm{H}=7,7 \mathrm{~m}, \mathrm{~h}=24,0 \mathrm{~m}$,

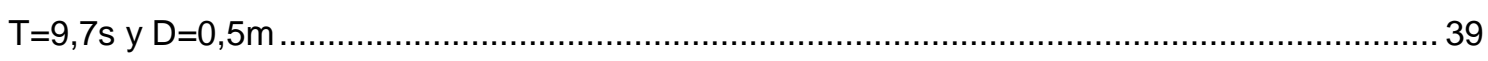

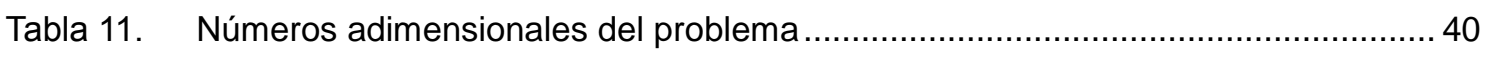

Tabla 12. Fuerzas hidrodinámicas para el caso de $H=4,78 m, h=10,0 m, T=6,8$ s y $D=0,5 m \ldots 42$

Tabla 13. Fuerzas hidrodinámicas para momento flector máximo, para $\mathrm{H}=4,78 \mathrm{~m}, \mathrm{~h}=10,0 \mathrm{~m}$,

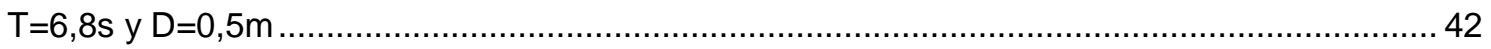

Tabla 14. Números adimensionales del problema .......................................................... 43

Tabla 15. Fuerzas hidrodinámicas para el caso de $\mathrm{H}=3,6 \mathrm{~m}, \mathrm{~h}=22,0 \mathrm{~m}, \mathrm{~T}=18,5$ s y $\mathrm{D}=0,5 \mathrm{~m} \ldots 45$

Tabla 16. Fuerzas hidrodinámicas para momento flector máximo, para $\mathrm{H}=3,6 \mathrm{~m}, \mathrm{~h}=22,0 \mathrm{~m}$,

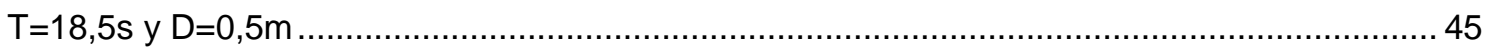

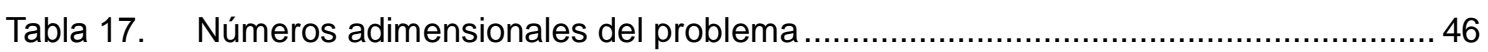

Tabla 18. Fuerzas hidrodinámicas para el caso de $\mathrm{H}=6,32 \mathrm{~m}, \mathrm{~h}=16 \mathrm{~m}, \mathrm{~T}=11,06$ s y $\mathrm{D}=0,5 \mathrm{~m}$.. 48 Tabla 19. Fuerzas hidrodinámicas para momento flector máximo, para $H=6,32 m, h=16 m$,

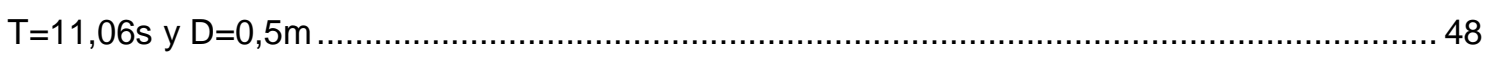

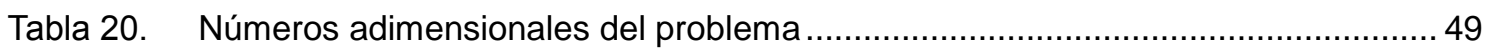

Tabla 21. Fuerzas hidrodinámicas para el caso de $\mathrm{H}=7,7 \mathrm{~m}, \mathrm{~h}=24,0 \mathrm{~m}, \mathrm{~T}=9,7 \mathrm{~s}$ y $\mathrm{D}=4,0 \mathrm{~m} \ldots . .51$ 
Tabla 22. Fuerzas hidrodinámicas para momento flector máximo, para $\mathrm{H}=7,7 \mathrm{~m}, \mathrm{~h}=24,0 \mathrm{~m}$, $T=9,7 \mathrm{~s}$ y $\mathrm{D}=4,0 \mathrm{~m}$. 51

Tabla 23. Números adimensionales del problema .52

Tabla 24. Fuerzas hidrodinámicas para el caso de $\mathrm{H}=4,78 \mathrm{~m}, \mathrm{~h}=10,0 \mathrm{~m}, \mathrm{~T}=6,8 \mathrm{~s}$ y $\mathrm{D}=4,0 \mathrm{~m} \ldots 54$

Tabla 25. Fuerzas hidrodinámicas para momento flector máximo, para $H=4,78 m, h=10,0 m$, $\mathrm{T}=6,8 \mathrm{~s}$ y $\mathrm{D}=4,0 \mathrm{~m}$. 54

Tabla 26. Números adimensionales del problema .55

Tabla 27. Fuerzas hidrodinámicas para el caso de $\mathrm{H}=3,6 \mathrm{~m}, \mathrm{~h}=22,0 \mathrm{~m}, \mathrm{~T}=18,5 \mathrm{~s}$ y $\mathrm{D}=4,0 \mathrm{~m} \ldots 57$

Tabla 28. Fuerzas hidrodinámicas para momento flector máximo, para $\mathrm{H}=3,6 \mathrm{~m}, \mathrm{~h}=22,0 \mathrm{~m}$, $\mathrm{T}=18,5 \mathrm{~s}$ y $\mathrm{D}=4,0 \mathrm{~m}$. .57

Tabla 29. Números adimensionales del problema ........................................................... 58

Tabla 30. Fuerzas hidrodinámicas para el caso de $\mathrm{H}=6,32 \mathrm{~m}, \mathrm{~h}=16 \mathrm{~m}, \mathrm{~T}=11,06$ s y $\mathrm{D}=4,0 \mathrm{~m} . .60$

Tabla 31. Fuerzas hidrodinámicas para momento flector máximo, para $\mathrm{H}=6,32 \mathrm{~m}, \mathrm{~h}=16 \mathrm{~m}$, $\mathrm{T}=11,06 \mathrm{~s}$ y $\mathrm{D}=4,0 \mathrm{~m}$. 60

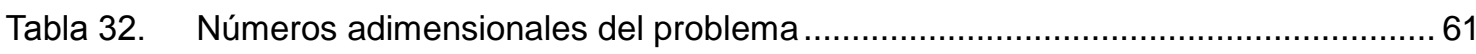

Tabla 33. Fuerzas hidrodinámicas para el caso de $\mathrm{H}=7,7 \mathrm{~m}, \mathrm{~h}=24,0 \mathrm{~m}, \mathrm{~T}=9,7 \mathrm{~s}$ y $\mathrm{D}=8,0 \mathrm{~m} \ldots . .63$

Tabla 34. Fuerzas hidrodinámicas para momento flector máximo, para $\mathrm{H}=7,7 \mathrm{~m}, \mathrm{~h}=24,0 \mathrm{~m}$, $T=9,7 \mathrm{~s}$ y $\mathrm{D}=8,0 \mathrm{~m}$. 63

Tabla 35. Números adimensionales del problema. .64

Tabla 36. Fuerzas hidrodinámicas para el caso de $\mathrm{H}=4,78 \mathrm{~m}, \mathrm{~h}=10,0 \mathrm{~m}, \mathrm{~T}=6,8 \mathrm{~s}$ y $\mathrm{D}=8,0 \mathrm{~m} \ldots 66$

Tabla 37. Fuerzas hidrodinámicas para momento flector máximo, para $\mathrm{H}=4,78 \mathrm{~m}, \mathrm{~h}=10,0 \mathrm{~m}$, $\mathrm{T}=6,8 \mathrm{~s}$ y $\mathrm{D}=8,0 \mathrm{~m}$. 66

Tabla 38. Números adimensionales del problema 67

Tabla 39. Fuerzas hidrodinámicas para el caso de $\mathrm{H}=3,6 \mathrm{~m}, \mathrm{~h}=22,0 \mathrm{~m}, \mathrm{~T}=18,5$ s y $\mathrm{D}=8,0 \mathrm{~m} \ldots 69$

Tabla 40. Fuerzas hidrodinámicas para momento flector máximo, para $\mathrm{H}=3,6 \mathrm{~m}, \mathrm{~h}=22,0 \mathrm{~m}$, $\mathrm{T}=18,5 \mathrm{~s}$ y $\mathrm{D}=8,0 \mathrm{~m}$. 69

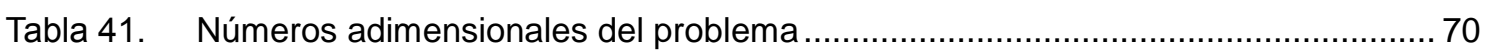

Tabla 42. Fuerzas hidrodinámicas para el caso de $\mathrm{H}=6,32 \mathrm{~m}, \mathrm{~h}=16 \mathrm{~m}, \mathrm{~T}=11,06$ s y $\mathrm{D}=8,0 \mathrm{~m} . .72$

Tabla 43. Fuerzas hidrodinámicas para momento flector máximo, para $\mathrm{H}=6,32 \mathrm{~m}, \mathrm{~h}=16 \mathrm{~m}$, $\mathrm{T}=11,06 \mathrm{~s}$ y $\mathrm{D}=8,0 \mathrm{~m}$. .72

Tabla 44. Números adimensionales del problema. .73

Tabla 45. Fuerzas hidrodinámicas para el caso de $\mathrm{H}=7,7 \mathrm{~m}, \mathrm{~h}=24,0 \mathrm{~m}, \mathrm{~T}=9,7 \mathrm{~s}$ y $\mathrm{D}=16,0 \mathrm{~m} \ldots 75$ 
Tabla 46. Fuerzas hidrodinámicas para momento flector máximo, para $\mathrm{H}=7,7 \mathrm{~m}, \mathrm{~h}=24,0 \mathrm{~m}$, $\mathrm{T}=9,7 \mathrm{~s}$ y $\mathrm{D}=16,0 \mathrm{~m}$ .75

Tabla 47. Números adimensionales del problema .76

Tabla 48. Fuerzas hidrodinámicas para el caso de $H=4,78 m, h=10,0 m, T=6,8$ s y $D=16,0 m .78$

Tabla 49. Fuerzas hidrodinámicas para momento flector máximo, para $H=4,78 m, h=10,0 m$, $\mathrm{T}=6,8 \mathrm{~s}$ y $\mathrm{D}=16,0 \mathrm{~m}$..... .78

Tabla 50. Números adimensionales del problema .79

Tabla 51. Fuerzas hidrodinámicas para el caso de $H=3,6 m, h=22,0 m, T=18,5$ s y $D=16,0 m$. 81

Tabla 52. Fuerzas hidrodinámicas para momento flector máximo, para $\mathrm{H}=3,6 \mathrm{~m}, \mathrm{~h}=22,0 \mathrm{~m}$, $\mathrm{T}=18,5$ s y $\mathrm{D}=16,0 \mathrm{~m}$. 81

Tabla 53. Números adimensionales del problema..... .82

Tabla 54. Fuerzas hidrodinámicas para el caso de $\mathrm{H}=6,32 \mathrm{~m}, \mathrm{~h}=16 \mathrm{~m}, \mathrm{~T}=11,06$ s y $\mathrm{D}=16,0 \mathrm{~m} 84$

Tabla 55. Fuerzas hidrodinámicas para momento flector máximo, para $\mathrm{H}=6,32 \mathrm{~m}, \mathrm{~h}=16 \mathrm{~m}$, $\mathrm{T}=11,06 \mathrm{~s}$ y $\mathrm{D}=16,0 \mathrm{~m}$ 84

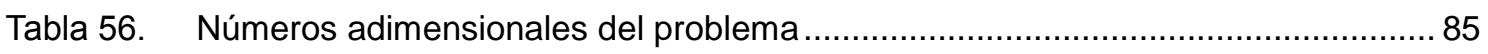

Tabla 57. Comportamiento de la estructura para el caso de $\mathrm{H}=7,7 \mathrm{~m}, \mathrm{~h}=24,0 \mathrm{~m}, \mathrm{~T}=9,7 \mathrm{~s}$ y $\mathrm{D}=0,5 \mathrm{~m}$

Tabla 58. Comportamiento de la estructura para el caso de $H=4,78 m, h=10,0 m, T=6,8$ s y $\mathrm{D}=0,5 \mathrm{~m}$ 88

Tabla 59. Comportamiento de la estructura para el caso de $H=3,6 m, h=22,0 m, T=18,5$ s y $\mathrm{D}=0,5 \mathrm{~m}$ .89

Tabla 60. Comportamiento de la estructura para el caso de $H=6,32 m, h=16,0 m, T=11,06$ s y $\mathrm{D}=0,5 \mathrm{~m}$ 90

Tabla 61. Comportamiento de la estructura para el caso de $H=7,7 m, h=24,0 m, T=9,7 s$ y $\mathrm{D}=4,0 \mathrm{~m}$ 91

Tabla 62. Comportamiento de la estructura para el caso de $H=4,78 m, h=10,0 m, T=6,8$ s y $\mathrm{D}=4,0 \mathrm{~m}$ 92

Tabla 63. Comportamiento de la estructura para el caso de $\mathrm{H}=3,6 \mathrm{~m}, \mathrm{~h}=22,0 \mathrm{~m}, \mathrm{~T}=18,5 \mathrm{~s}$ y $\mathrm{D}=4,0 \mathrm{~m}$ .93

Tabla 64. Comportamiento de la estructura para el caso de $H=6,32 m, h=16,0 m, T=11,06$ s y $\mathrm{D}=4,0 \mathrm{~m}$

Tabla 65. Comportamiento de la estructura para el caso de $\mathrm{H}=7,7 \mathrm{~m}, \mathrm{~h}=24,0 \mathrm{~m}, \mathrm{~T}=9,7 \mathrm{~s}$ y $\mathrm{D}=8,0 \mathrm{~m}$ 
Tabla 66. Comportamiento de la estructura para el caso de $H=4,78 m, h=10,0 m, T=6,8$ s y

$\mathrm{D}=8,0 \mathrm{~m}$

Tabla 67. Comportamiento de la estructura para el caso de $H=3,6 m, h=22,0 m, T=18,5$ s y

$\mathrm{D}=8,0 \mathrm{~m}$

Tabla 68. Comportamiento de la estructura para el caso de $H=6,32 m, h=16,0 m, T=11,06$ s y

$\mathrm{D}=8,0 \mathrm{~m}$ .98

Tabla 69. Comportamiento de la estructura para el caso de $\mathrm{H}=7,7 \mathrm{~m}, \mathrm{~h}=24,0 \mathrm{~m}, \mathrm{~T}=9,7 \mathrm{~s}$ y

$\mathrm{D}=16,0 \mathrm{~m}$ 99

Tabla 70. Comportamiento de la estructura para el caso de $\mathrm{H}=4,78 \mathrm{~m}, \mathrm{~h}=10,0 \mathrm{~m}, \mathrm{~T}=6,8 \mathrm{~s}$ y

$\mathrm{D}=16,0 \mathrm{~m}$ 100

Tabla 71. Comportamiento de la estructura para el caso de $\mathrm{H}=3,6 \mathrm{~m}, \mathrm{~h}=22,0 \mathrm{~m}, \mathrm{~T}=18,5 \mathrm{~s}$ y

$\mathrm{D}=16,0 \mathrm{~m}$ 101

Tabla 72. Comportamiento de la estructura para el caso de $H=6,32 m, h=16,0 m, T=11,06$ s y

$\mathrm{D}=16,0 \mathrm{~m}$ 102

Tabla 73. Comportamiento de la estructura para el caso de $H=7,7 m, h=24,0 m, T=9,7 \mathrm{~s}$ y $\mathrm{D}=0,5 \mathrm{~m}$ 103

Tabla 74. Comportamiento de la estructura para el caso de $H=4,78 m, h=10,0 m, T=6,8$ s y $\mathrm{D}=0,5 \mathrm{~m}$ 104

Tabla 75. Comportamiento de la estructura para el caso de $H=3,6 m, h=22,0 m, T=18,5$ s y $\mathrm{D}=0,5 \mathrm{~m}$ 104

Tabla 76. Comportamiento de la estructura para el caso de $H=6,32 m, h=16,0 m, T=11,06$ s y $\mathrm{D}=0,5 \mathrm{~m}$ 105

Tabla 77. Comportamiento de la estructura para el caso de $H=7,7 m, h=24,0 m, T=9,7 \mathrm{~s}$ y $\mathrm{D}=4,0 \mathrm{~m}$ 106

Tabla 78. Comportamiento de la estructura para el caso de $H=4,78 m, h=10,0 m, T=6,8$ s y $\mathrm{D}=4,0 \mathrm{~m}$ 106

Tabla 79. Comportamiento de la estructura para el caso de $H=3,6 m, h=22,0 m, T=18,5$ s y $\mathrm{D}=4,0 \mathrm{~m}$ 107

Tabla 80. Comportamiento de la estructura para el caso de $H=6,32 m, h=16,0 m, T=11,06$ s y $\mathrm{D}=4,0 \mathrm{~m}$ 107

Tabla 81. Comportamiento de la estructura para el caso de $H=7,7 m, h=24,0 m, T=9,7 \mathrm{~s}$ y $\mathrm{D}=8,0 \mathrm{~m}$ 108

Tabla 82. Comportamiento de la estructura para el caso de $\mathrm{H}=4,78 \mathrm{~m}, \mathrm{~h}=10,0 \mathrm{~m}, \mathrm{~T}=6,8 \mathrm{~s}$ y $\mathrm{D}=8,0 \mathrm{~m}$ 108 
Tabla 83. Comportamiento de la estructura para el caso de $\mathrm{H}=3,6 \mathrm{~m}, \mathrm{~h}=22,0 \mathrm{~m}, \mathrm{~T}=18,5 \mathrm{~s}$ y

$\mathrm{D}=8,0 \mathrm{~m}$ 109

Tabla 84. Comportamiento de la estructura para el caso de $H=6,32 m, h=16,0 m, T=11,06$ s y

$\mathrm{D}=8,0 \mathrm{~m}$ 109

Tabla 85. Comportamiento de la estructura para el caso de $H=7,7 m, h=24,0 m, T=9,7 s$ y

$\mathrm{D}=16,0 \mathrm{~m}$ 110

Tabla 86. Comportamiento de la estructura para el caso de $H=4,78 m, h=10,0 m, T=6,8$ s y

$\mathrm{D}=16,0 \mathrm{~m}$ 110

Tabla 87. Comportamiento de la estructura para el caso de $H=3,6 m, h=22,0 m, T=18,5$ s y

$\mathrm{D}=16,0 \mathrm{~m}$

Tabla 88. Comportamiento de la estructura para el caso de $H=6,32 m, h=16,0 m, T=11,06$ s y

$\mathrm{D}=16,0 \mathrm{~m}$

Tabla 89. Fenómeno de socavación para el caso de $H=7,7 \mathrm{~m}, \mathrm{~h}=24,0 \mathrm{~m}, \mathrm{~T}=9,7 \mathrm{~s}$, $\mathrm{uc}=1,3 \mathrm{~m} / \mathrm{s}$ y $\mathrm{D}=0,5 \mathrm{~m}$

Tabla 90. Fenómeno de socavación para el caso de $\mathrm{H}=4,78 \mathrm{~m}, \mathrm{~h}=10,0 \mathrm{~m}, \mathrm{~T}=6,8 \mathrm{~s}, \mathrm{uc}=0,8 \mathrm{~m} / \mathrm{s}$ y $\mathrm{D}=0,5 \mathrm{~m}$

Tabla 91. Fenómeno de socavación para el caso de $\mathrm{H}=3,6 \mathrm{~m}, \mathrm{~h}=22,0 \mathrm{~m}, \mathrm{~T}=18,5 \mathrm{~s}$, uc=0,7m/s y $\mathrm{D}=0,5 \mathrm{~m}$

Tabla 92. Fenómeno de socavación para el caso de $\mathrm{H}=6,32 \mathrm{~m}, \mathrm{~h}=16,0 \mathrm{~m}, \mathrm{~T}=11,06 \mathrm{~s}, \mathrm{uc}=1,2 \mathrm{~m} / \mathrm{s}$ y $D=0,5 m$

Tabla 93. Fenómeno de socavación para el caso de $H=7,7 \mathrm{~m}, \mathrm{~h}=24,0 \mathrm{~m}, \mathrm{~T}=9,7 \mathrm{~s}$, uc=1,3m/s y $\mathrm{D}=4,0 \mathrm{~m}$

Tabla 94. Fenómeno de socavación para el caso de $\mathrm{H}=4,78 \mathrm{~m}, \mathrm{~h}=10,0 \mathrm{~m}, \mathrm{~T}=6,8 \mathrm{~s}, \mathrm{uc}=0,8 \mathrm{~m} / \mathrm{s}$ y $\mathrm{D}=4,0 \mathrm{~m}$

Tabla 95. Fenómeno de socavación para el caso de $\mathrm{H}=3,6 \mathrm{~m}, \mathrm{~h}=22,0 \mathrm{~m}, \mathrm{~T}=18,5 \mathrm{~s}, \mathrm{uc}=0,7 \mathrm{~m} / \mathrm{s}$ y $\mathrm{D}=4,0 \mathrm{~m}$

Tabla 96. Fenómeno de socavación para el caso de $\mathrm{H}=6,32 \mathrm{~m}, \mathrm{~h}=16,0 \mathrm{~m}, \mathrm{~T}=11,06 \mathrm{~s}, \mathrm{uc}=1,2 \mathrm{~m} / \mathrm{s}$ y $D=4,0 m$

Tabla 97. Fenómeno de socavación para el caso de $\mathrm{H}=7,7 \mathrm{~m}, \mathrm{~h}=24,0 \mathrm{~m}, \mathrm{~T}=9,7 \mathrm{~s}$, $\mathrm{uc}=1,3 \mathrm{~m} / \mathrm{s}$ y $\mathrm{D}=8,0 \mathrm{~m}$

Tabla 98. Fenómeno de socavación para el caso de $\mathrm{H}=4,78 \mathrm{~m}, \mathrm{~h}=10,0 \mathrm{~m}, \mathrm{~T}=6,8 \mathrm{~s}$, $\mathrm{uc}=0,8 \mathrm{~m} / \mathrm{s}$ y $\mathrm{D}=8,0 \mathrm{~m}$

Tabla 99. Fenómeno de socavación para el caso de $\mathrm{H}=3,6 \mathrm{~m}, \mathrm{~h}=22,0 \mathrm{~m}, \mathrm{~T}=18,5 \mathrm{~s}$, uc=0,7m/s y $\mathrm{D}=8,0 \mathrm{~m}$ 118 
Tabla 100. Fenómeno de socavación para el caso de $H=6,32 m, h=16,0 m, T=11,06 s$, $\mathrm{uc}=1,2 \mathrm{~m} / \mathrm{s}$ y $\mathrm{D}=8,0 \mathrm{~m}$

Tabla 101. Fenómeno de socavación para el caso de $\mathrm{H}=7,7 \mathrm{~m}, \mathrm{~h}=24,0 \mathrm{~m}, \mathrm{~T}=9,7 \mathrm{~s}, \mathrm{uc}=1,3 \mathrm{~m} / \mathrm{s}$ y $D=16,0 m$

Tabla 102. Fenómeno de socavación para el caso de $\mathrm{H}=4,78 \mathrm{~m}, \mathrm{~h}=10,0 \mathrm{~m}, \mathrm{~T}=6,8 \mathrm{~s}, \mathrm{uc}=0,8 \mathrm{~m} / \mathrm{s}$ y $D=16,0 m$

Tabla 103. Fenómeno de socavación para el caso de $\mathrm{H}=3,6 \mathrm{~m}, \mathrm{~h}=22,0 \mathrm{~m}, \mathrm{~T}=18,5 \mathrm{~s}, \mathrm{uc}=0,7 \mathrm{~m} / \mathrm{s}$ y $\mathrm{D}=16,0 \mathrm{~m}$ 120

Tabla 104. Fenómeno de socavación para el caso de $\mathrm{H}=6,32 \mathrm{~m}, \mathrm{~h}=16,0 \mathrm{~m}, \mathrm{~T}=11,06 \mathrm{~s}$, $\mathrm{uc}=1,2 \mathrm{~m} / \mathrm{s}$ y $\mathrm{D}=16,0 \mathrm{~m}$ 120 


\section{Apéndice 1}

\section{RESULTADOS DE LOS CASOS DE ESTUDIO}

\section{Introducción}

En este apéndice se procede a realizar la exposición de los resultados obtenidos por la herramienta desarrollada mediante la presente Tesis Doctoral, para cada uno de los casos de análisis definidos.

El documento se ha dividido en 5 capítulos, conteniendo cada uno de ellos los resultados pertenecientes a uno de los 5 procesos de cálculo definidos dentro de la herramienta de informática. 


\section{Proceso I: Mecánica de ondas}

El primer proceso se corresponde con el cálculo, de las variables que definirán el comportamiento del oleaje, a partir de las variables climáticas de altura de ola significativa $(H)$, periodo de pico del oleaje $(T)$ y profundidad $(h)$.

\subsection{Caso A: Stokes de $3^{\circ}$ Orden}

Los datos de entrada se corresponden con el caso de cálculo para el parque eólico offshore Princess Amalia Q7 en Holanda, y son los siguientes:

- $\quad$ Altura de ola significativa: 7,7 m

- $\quad$ Profundidad: $24,0 \mathrm{~m}$

- $\quad$ Periodo de pico: 9,7 s

De acuerdo con estos datos, la teoría de ondas a utilizar será la de Stokes de 3o orden, en profundidades de transición.

$$
\begin{gathered}
\delta_{1}=\frac{H}{g T^{2}}=0,00834 \quad \delta_{2}=\frac{h}{g T^{2}}=0,026 \\
\frac{d}{L}=\frac{24}{129,36} \rightarrow 0,04<0,186<0,5 \rightarrow \text { Profundidades de transición }
\end{gathered}
$$

\subsubsection{Comparación entre el campo de velocidades y aceleraciones, con la teoría lineal.}

A continuación, se muestra en la figura 1 el diagrama de velocidades procedente del cálculo mediante la teoría no lineal, y en la figura 2 el mismo cálculo pero realizado según la teoría lineal. 


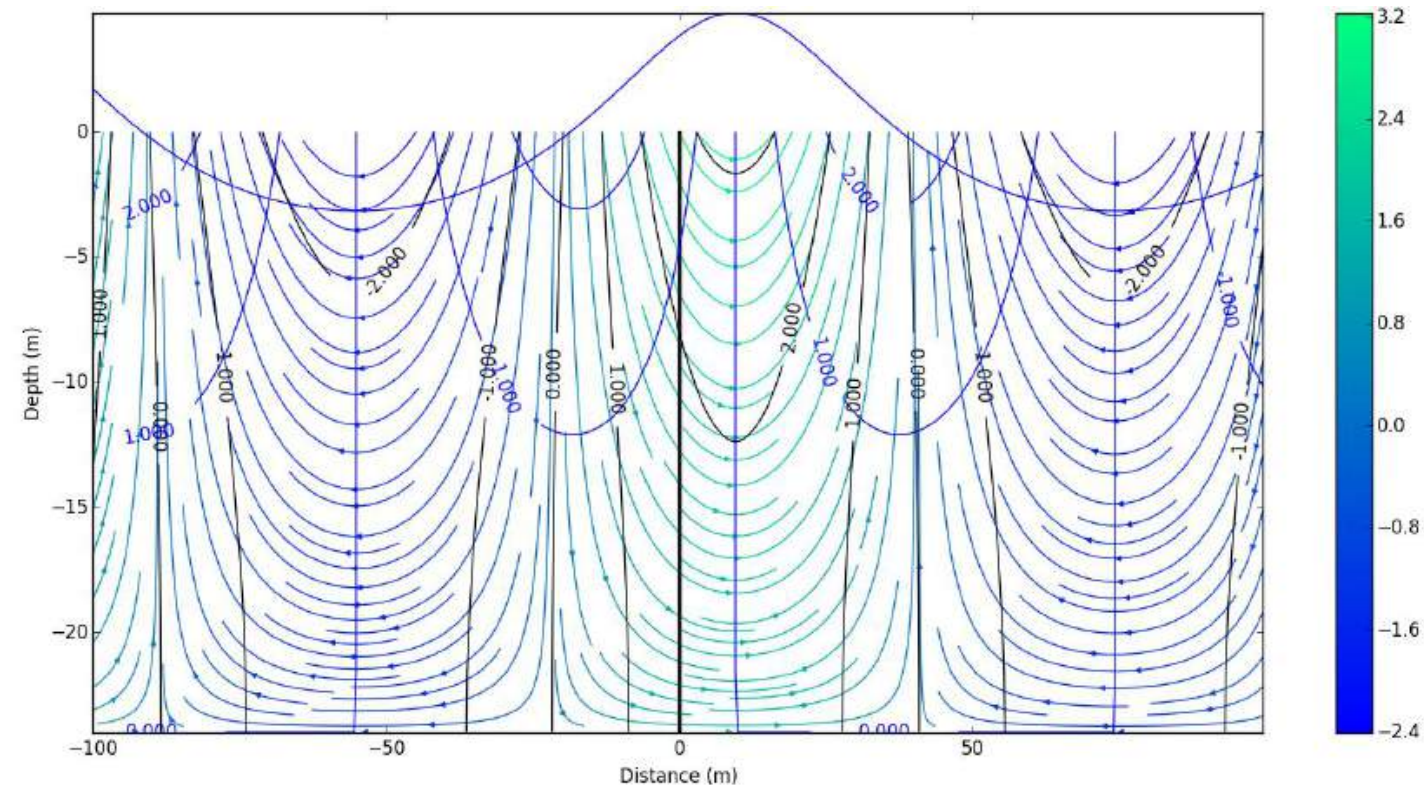

Figura 1. Velocidades para $H=7,7 m, h=24,0 m$ y $T=9,7 s$ según Stokes de 3Orden

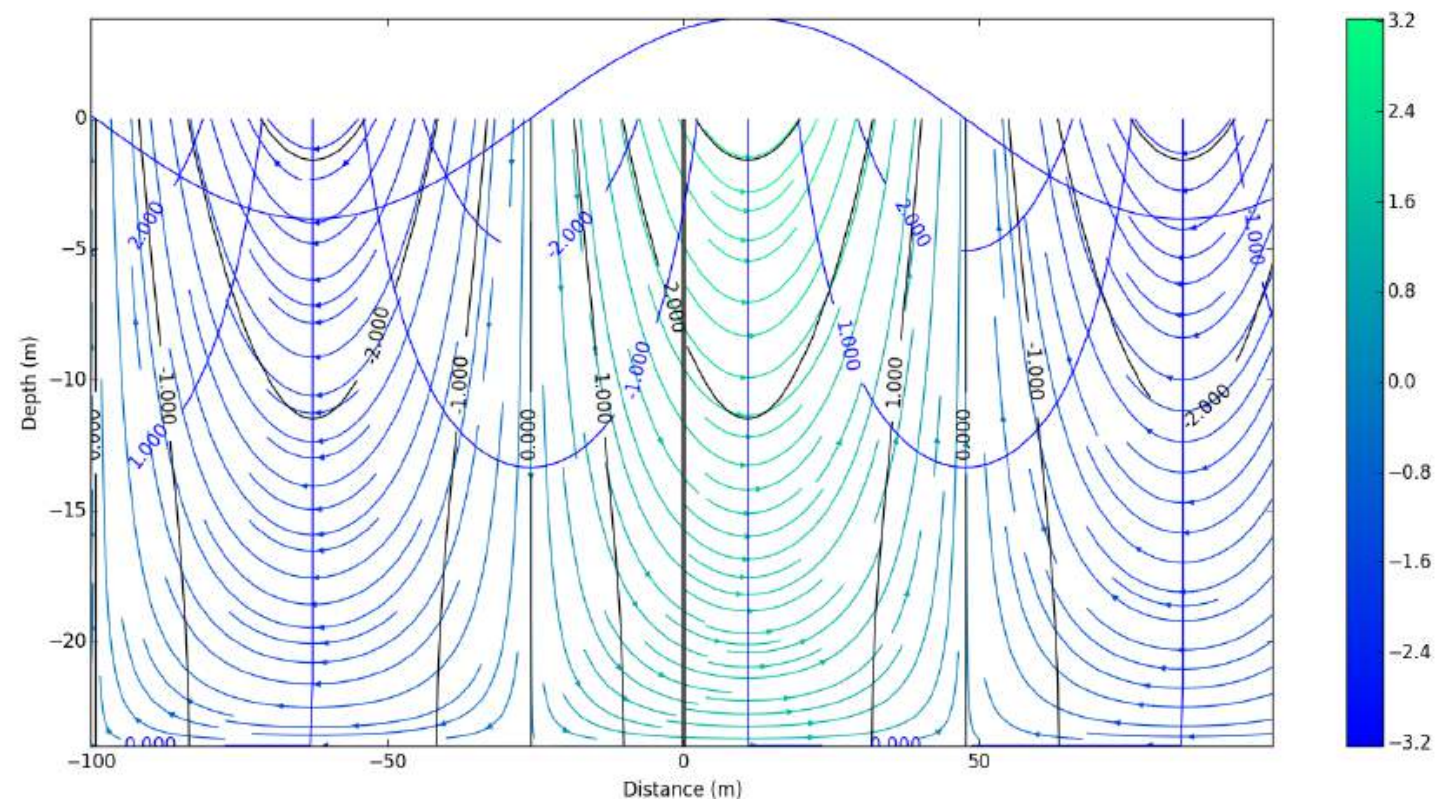

Figura 2. Velocidades para $H=7,7 m, h=24,0 m$ y $T=9,7$ s según teoría lineal

Los valores más característicos de la simulación, tanto para el caso lineal como para el caso no lineal, se muestran a continuación en la tabla 1:

\begin{tabular}{|c|l|c|c|}
\hline Zona & \multicolumn{1}{|c|}{ Parámetro } & CASO A & Teoría lineal \\
\hline \multirow{4}{*}{ Superficie } & Máxima velocidad horizontal $(\mathrm{m} / \mathrm{s})$ & 3,2313 & 3,2282 \\
\cline { 2 - 4 } & Máxima velocidad vertical $(\mathrm{m} / \mathrm{s})$ & 2,4345 & 2,8052 \\
\cline { 2 - 4 } & Media de velocidad horizontal $(\mathrm{m} / \mathrm{s})$ & 1,8099 & 2,0545 \\
\cline { 2 - 4 } & Media de velocidad vertical $(\mathrm{m} / \mathrm{s})$ & 1,4794 & 1,7866 \\
\hline Fondo & Máxima velocidad horizontal $(\mathrm{m} / \mathrm{s})$ & 1,68 & 1,5976 \\
\hline
\end{tabular}




\begin{tabular}{|l|l|c|c|}
\hline & Máxima velocidad vertical $(\mathrm{m} / \mathrm{s})$ & 0,0 & 0,0 \\
\cline { 2 - 4 } & Media de velocidad horizontal $(\mathrm{m} / \mathrm{s})$ & 1,019 & 1,0168 \\
\cline { 2 - 4 } & Media de velocidad vertical $(\mathrm{m} / \mathrm{s})$ & 0,0 & 0,0 \\
\hline
\end{tabular}

Tabla 1. Comparación de campo de velocidades caso $A$

En segundo lugar, se muestra en la figura 3 el diagrama de aceleraciones procedente del cálculo mediante la teoría no lineal, y en la figura 4 el mismo cálculo pero realizado según la teoría lineal.

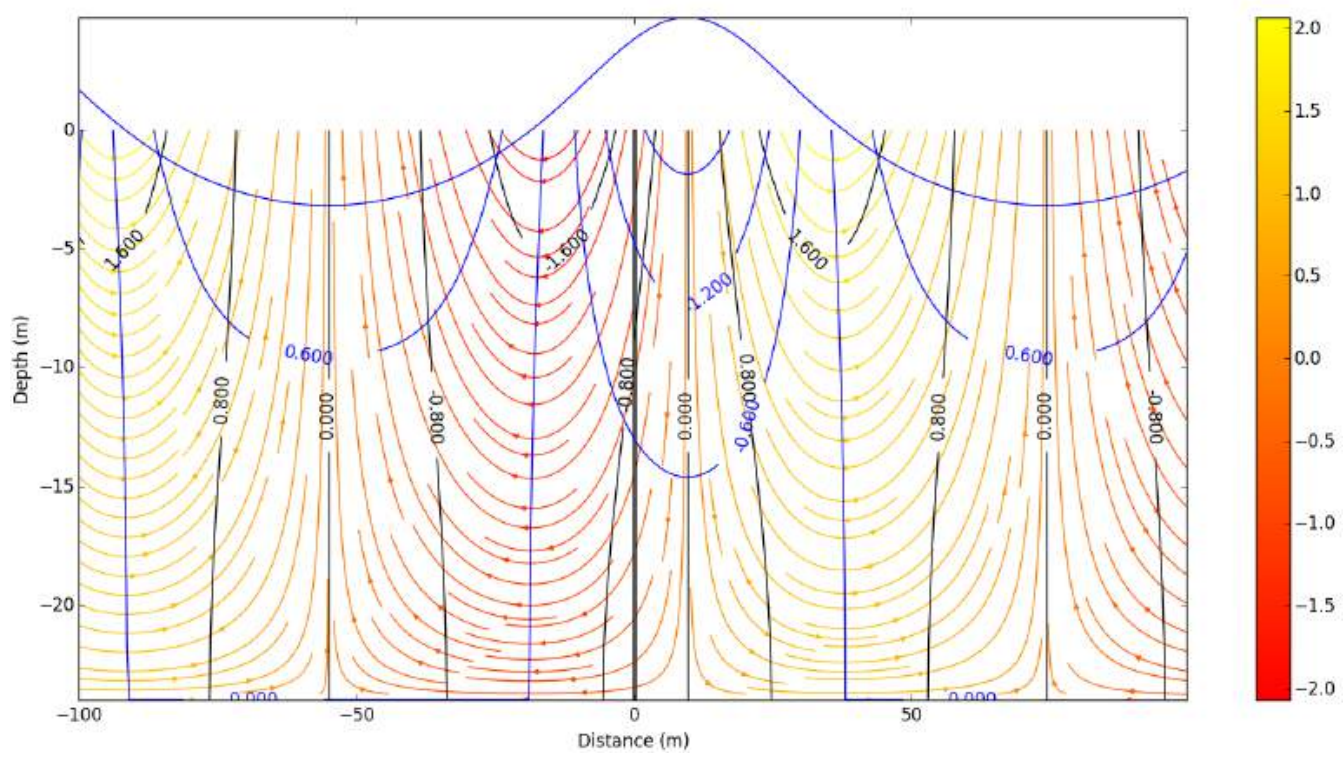

Figura 3. Aceleraciones para $H=7,7 m, h=24,0 m$ y $T=9,7$ s según Stokes de 3Oden

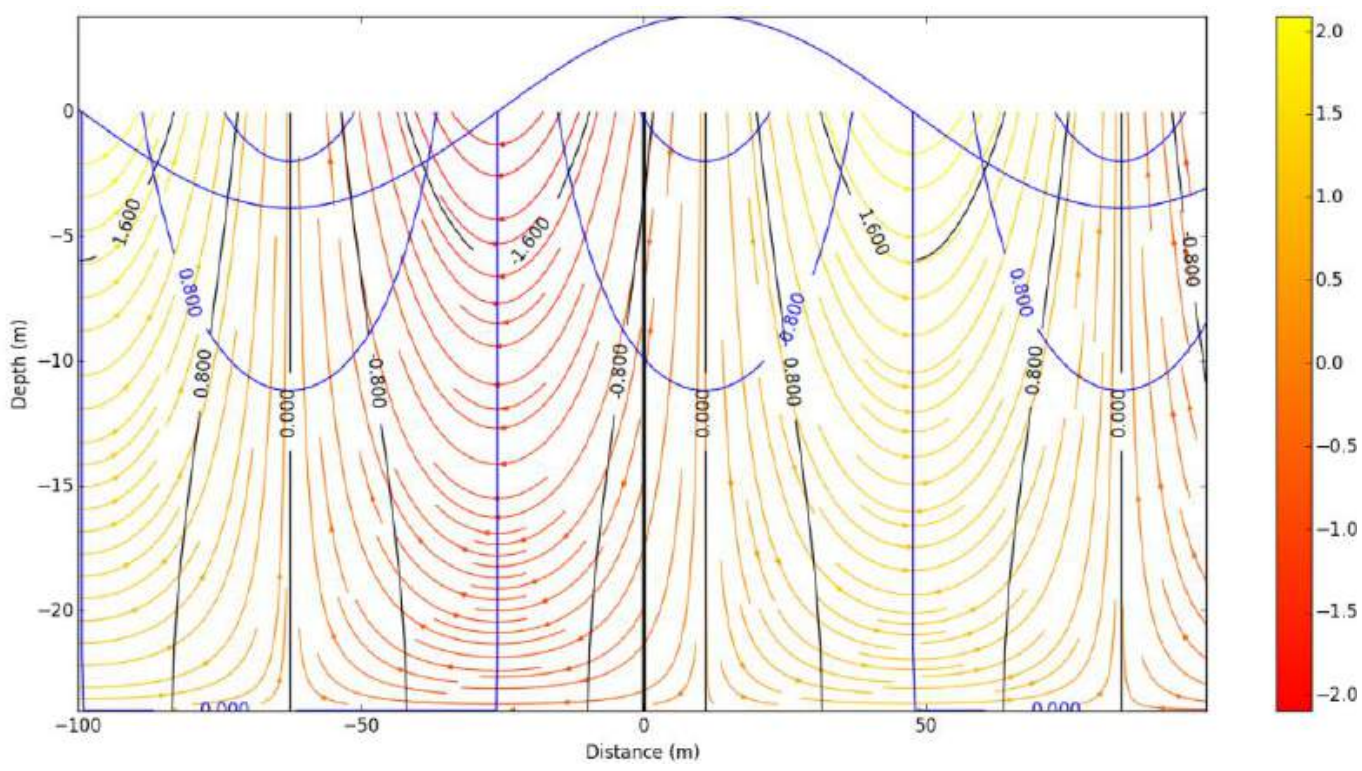

Figura 4. Aceleraciones para $H=7,7 m, h=24,0 m$ y $T=9,7$ s según teoría lineal

Los valores más característicos de la simulación, tanto para el caso lineal como para el caso no lineal, se muestran a continuación en la tabla 2: 


\begin{tabular}{|c|l|c|c|}
\hline Zona & \multicolumn{1}{|c|}{ Parámetro } & CASO A & Teoría clásica \\
\hline \multirow{4}{*}{ Superficie } & Máxima aceleración horizontal $\left(\mathrm{m} / \mathrm{s}^{2}\right)$ & 2,0691 & 2,0911 \\
\cline { 2 - 4 } & Máxima aceleración vertical $\left(\mathrm{m} / \mathrm{s}^{2}\right)$ & 1,0332 & 1,8171 \\
\cline { 2 - 4 } & Media de aceleración horizontal $\left(\mathrm{m} / \mathrm{s}^{2}\right)$ & 1,1683 & 1,3318 \\
\cline { 2 - 4 } & Media de aceleración vertical $\left(\mathrm{m} / \mathrm{s}^{2}\right)$ & 1,0049 & 1,1564 \\
\hline \multirow{4}{*}{ Fondo } & Máxima aceleración horizontal $\left(\mathrm{m} / \mathrm{s}^{2}\right)$ & 1,0548 & 1,0349 \\
\cline { 2 - 4 } & Máxima aceleración vertical $\left(\mathrm{m} / \mathrm{s}^{2}\right)$ & 0,0 & 0,0 \\
\cline { 2 - 4 } & Media de aceleración horizontal $\left(\mathrm{m} / \mathrm{s}^{2}\right)$ & 0,6606 & 0,6591 \\
\cline { 2 - 4 } & Media de aceleración vertical $\left(\mathrm{m} / \mathrm{s}^{2}\right)$ & 0,0 & 0,0 \\
\hline
\end{tabular}

Tabla 2. Comparación de campo de aceleraciones caso $A$

\subsubsection{Análisis de principales frecuencias generadas por el oleaje}

También se procede a realizar una transformada de Fourier para los resultados en superficie, para observar la variación generada en la distribución de la velocidad (figura 5) y la aceleración (figura 6) en frecuencias:
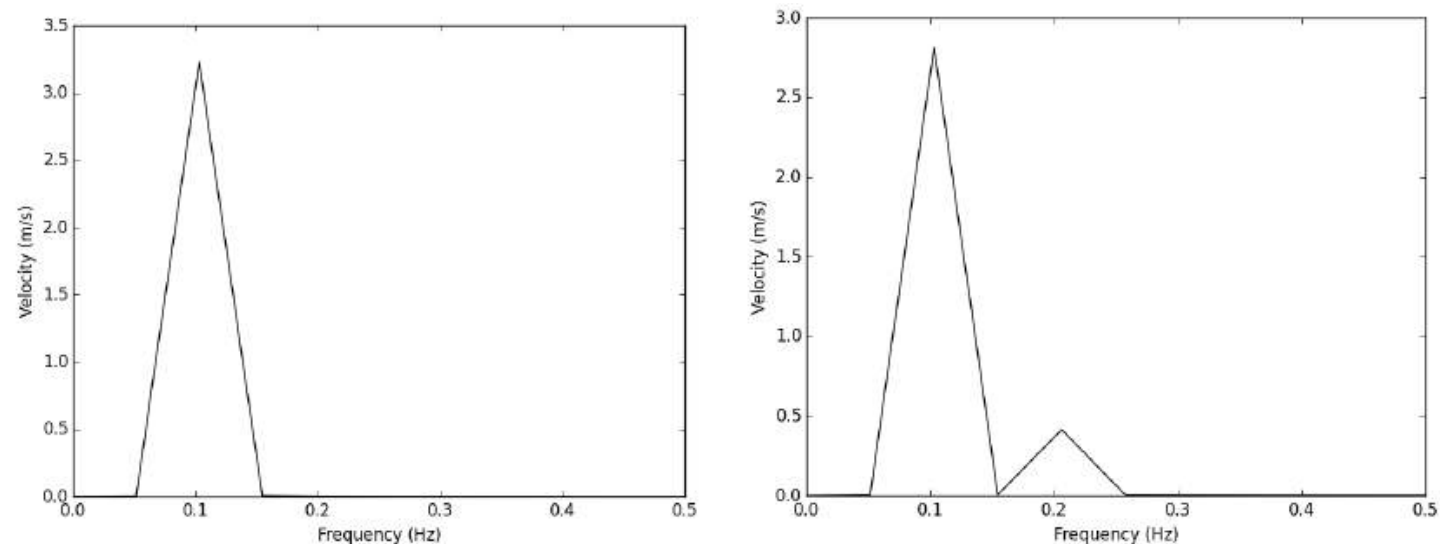

Figura 5. Velocidades: Al a izquierda resultados de la teoría lineal, a la derecha de Stokes de $3^{\circ}$ Orden
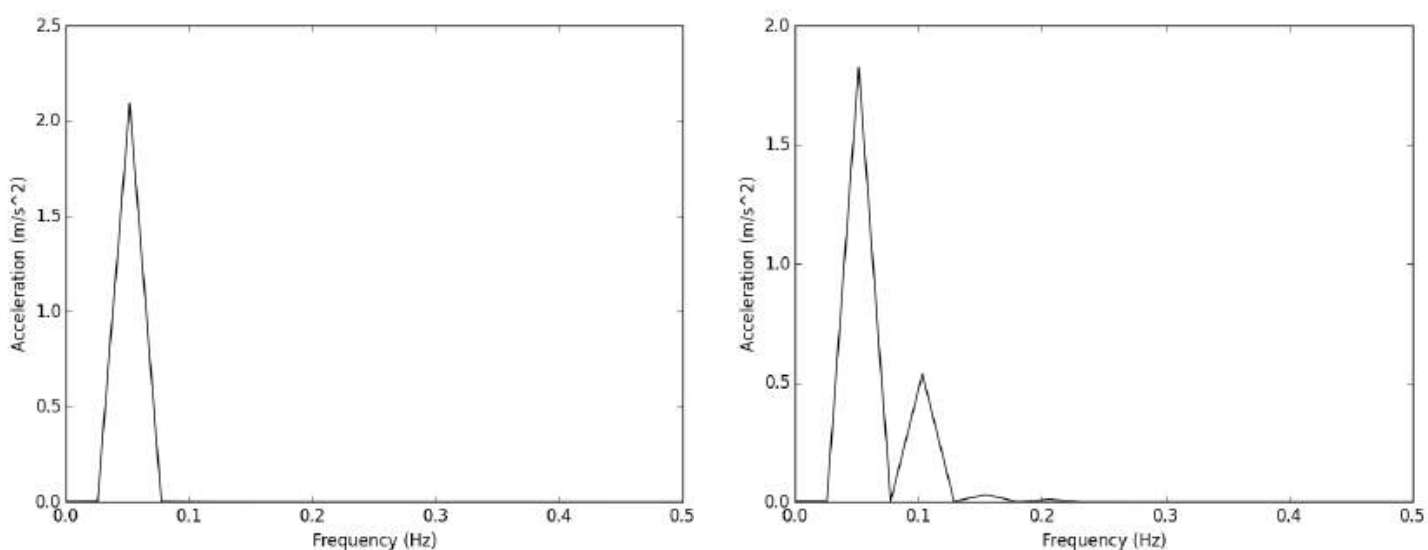

Figura 6. Aceleraciones: Al a izquierda resultados de la teoría lineal, a la derecha de Stokes de 3oㅡㅁon 


\subsubsection{Análisis sobre la amortiguación de la velocidad y la aceleración con la profundidad}

También se muestran los valores máximos de velocidad (figura 7) y aceleración (figura 8) a distintas profundidades para observar la variación que sufre el amortiguamiento con la profundidad, entre la teoría lineal, y el caso en estudio.
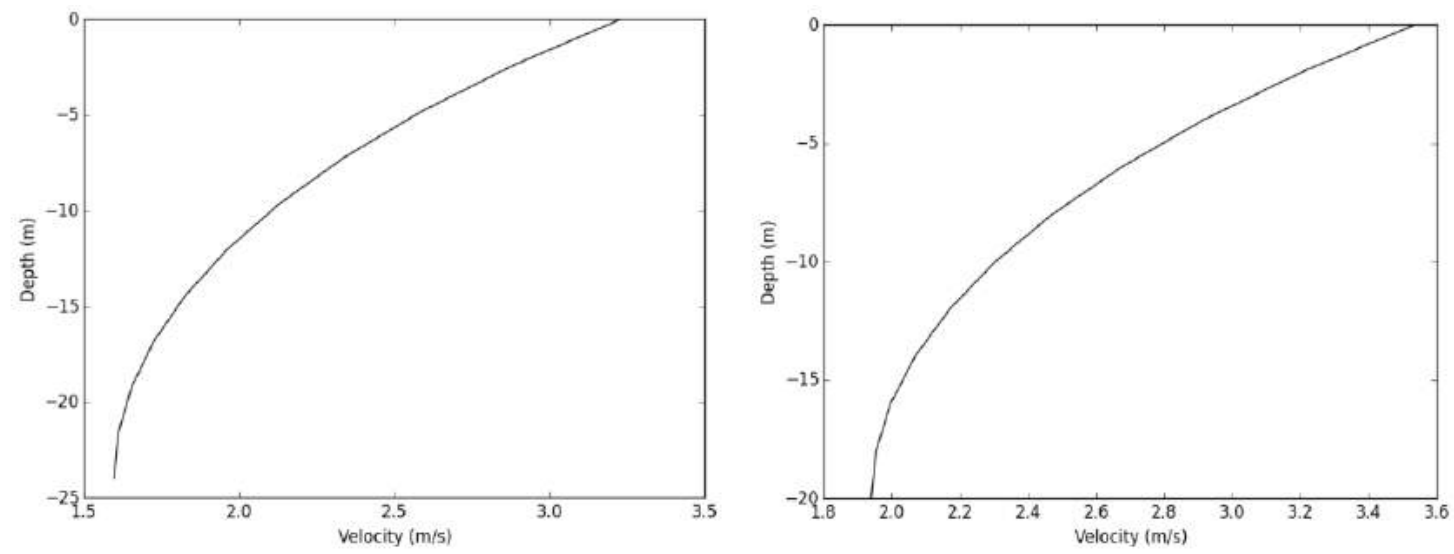

Figura 7. Amortiguamiento de velocidades, a la izquierda teoría lineal, a la derecha Stokes de $3^{\circ}$ Orden
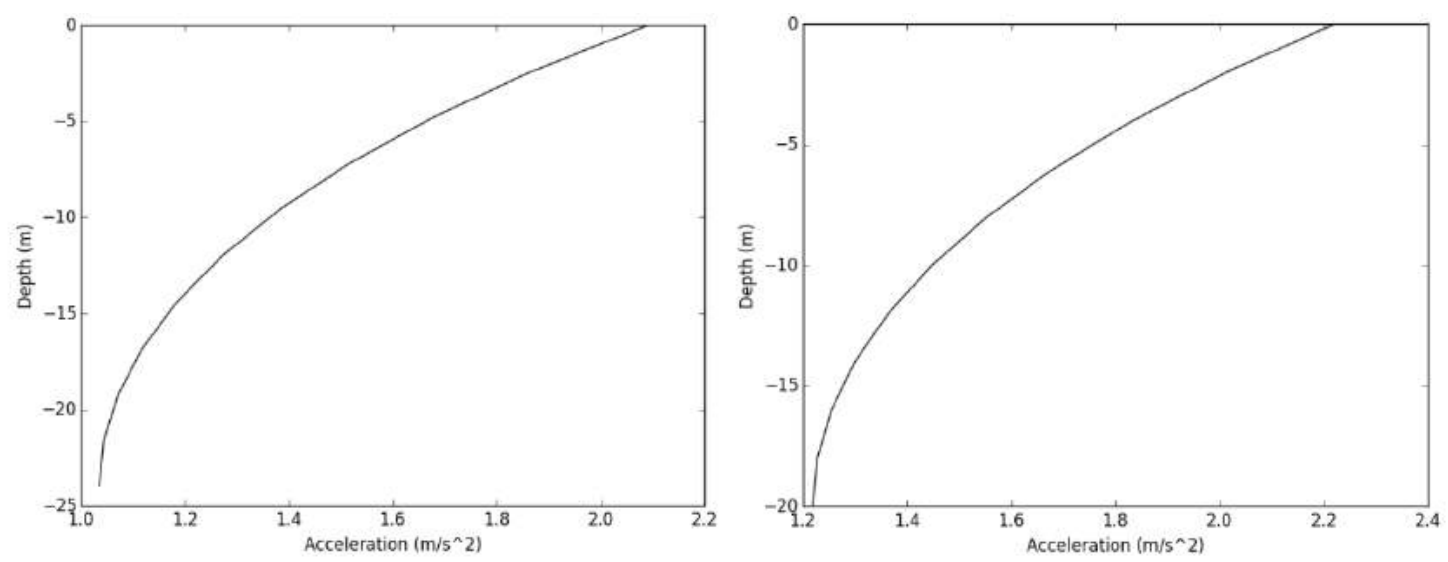

Figura 8. Amortiguamiento de aceleraciones, a la izquierda teoría lineal, a la derecha Stokes de 3ํㅡㅁㅏen 


\subsection{Caso B: Stokes de 5o Orden}

Los datos de entrada se corresponden con el caso de cálculo para el parque eólico offshore North Hoyle en Reino Unido, y son los siguientes:

- $\quad$ Altura de ola significativa: $4,78 \mathrm{~m}$

- $\quad$ Profundidad: $10,0 \mathrm{~m}$

- $\quad$ Periodo de pico: $6,8 \mathrm{~s}$

De acuerdo con estos datos, la teoría de ondas a utilizar será la de Stokes de 5o orden, en profundidades de transición.

$$
\begin{gathered}
\delta_{1}=\frac{H}{g T^{2}}=0,01054 \quad \delta_{2}=\frac{h}{g T^{2}}=0,02204 \\
\frac{d}{L}=\frac{10}{70,88} \rightarrow 0,04<0,141<0,5 \rightarrow \text { Profundidades de transición }
\end{gathered}
$$

\subsubsection{Comparación entre el campo de velocidades y aceleraciones, con la teoría lineal.}

A continuación, se muestra en la figura 9 el diagrama de velocidades procedente del cálculo mediante la teoría no lineal, y en la figura 10 el mismo cálculo pero realizado según la teoría lineal.

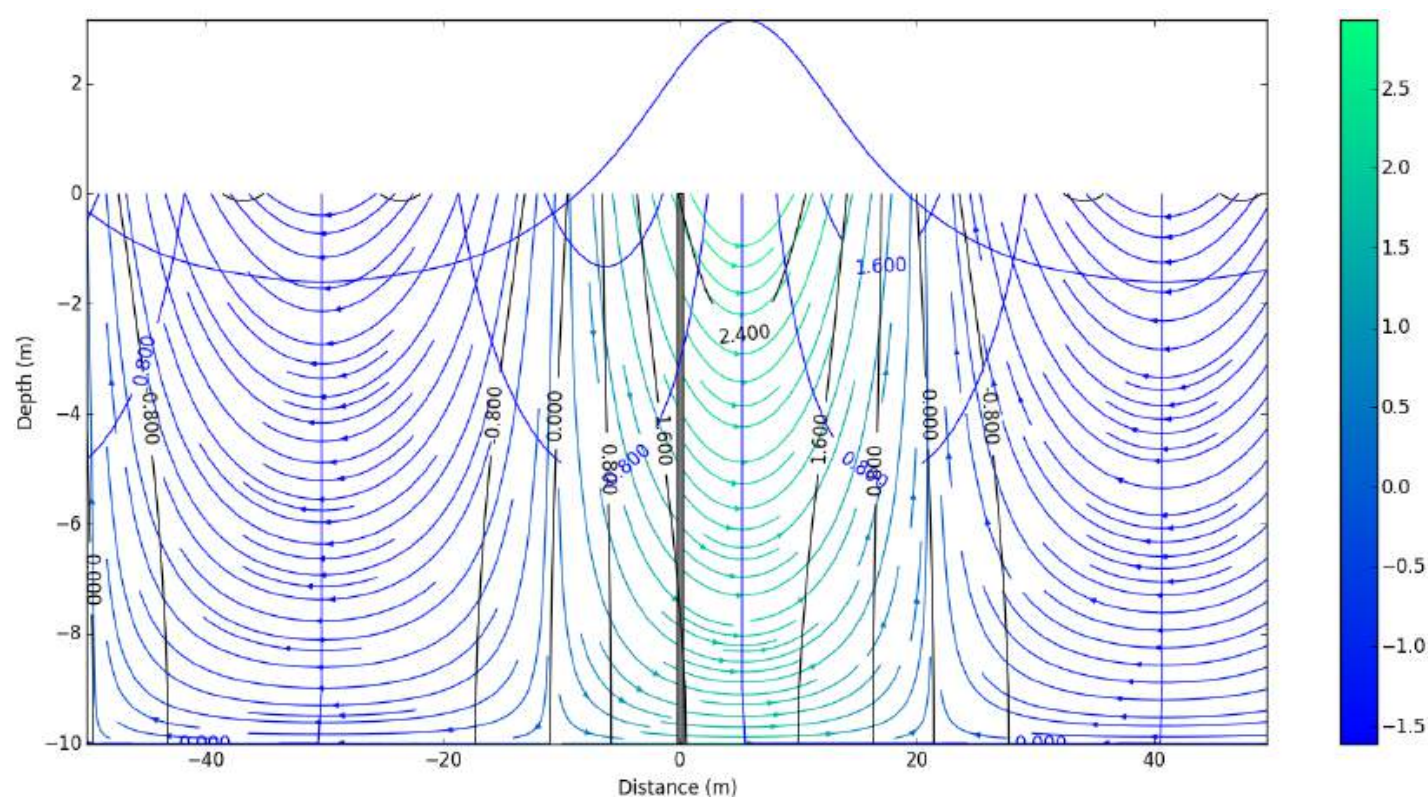

Figura 9. Velocidades para $H=4,78 m, h=10,0 m$ y $T=6,8$ s según Stokes de 5Oㅁen 


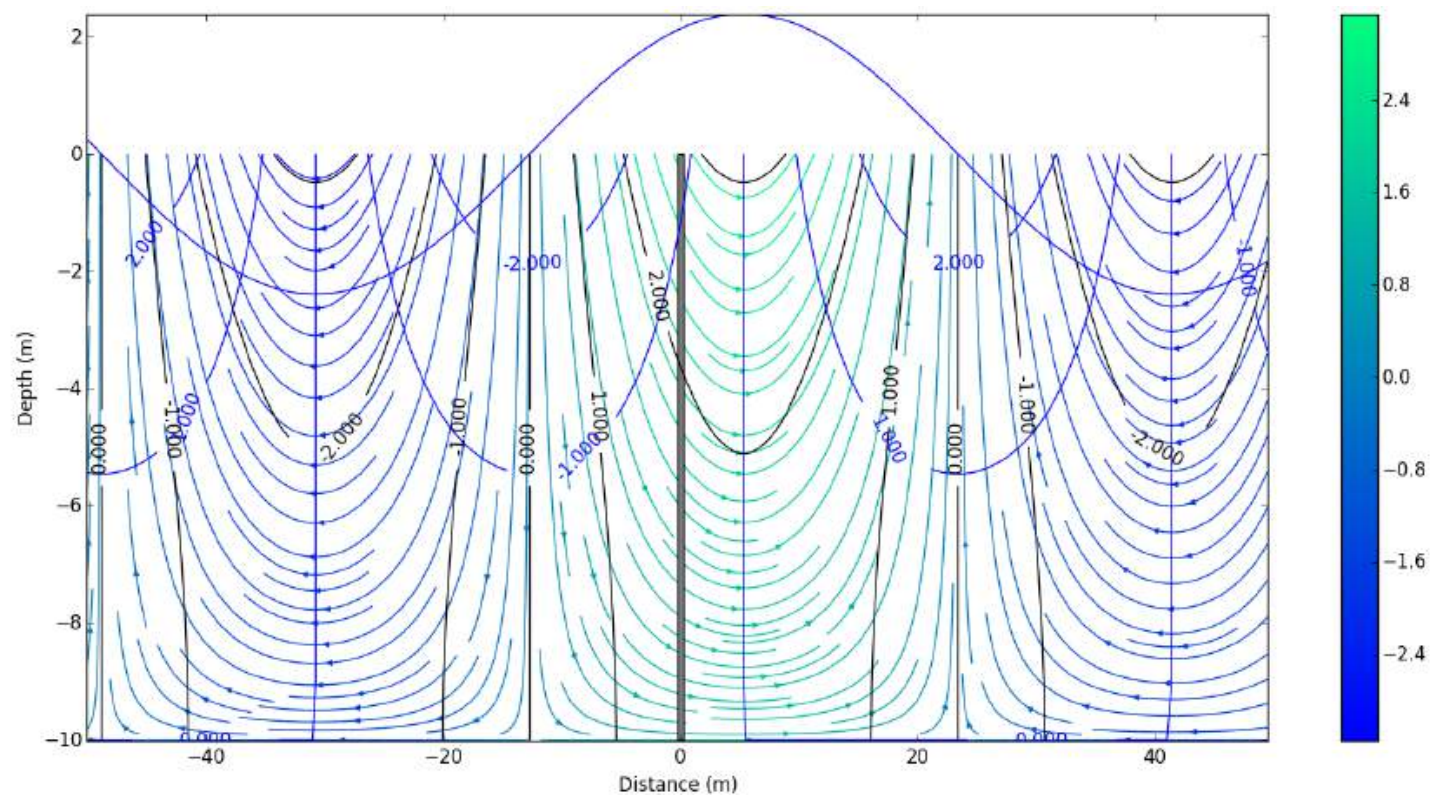

Figura 10. Velocidades para $H=4,78 m, h=10,0 m$ y $T=6,8$ s según teoría lineal

Los valores más característicos de la simulación, tanto para el caso lineal como para el caso no lineal, se muestran a continuación en la tabla 3:

\begin{tabular}{|c|l|c|c|}
\hline Zona & \multicolumn{1}{|c|}{ Parámetro } & CASO B & Teoría lineal \\
\hline \multirow{4}{*}{ Superficie } & Máxima velocidad horizontal $(\mathrm{m} / \mathrm{s})$ & 2,9347 & 3,1479 \\
\cline { 2 - 4 } & Máxima velocidad vertical $(\mathrm{m} / \mathrm{s})$ & 1,9475 & 2,6619 \\
\cline { 2 - 4 } & Media de velocidad horizontal $(\mathrm{m} / \mathrm{s})$ & 1,4929 & 2,004 \\
\cline { 2 - 4 } & Media de velocidad vertical $(\mathrm{m} / \mathrm{s})$ & 1,0414 & 1,6947 \\
\hline \multirow{4}{*}{ Fondo } & Máxima velocidad horizontal $(\mathrm{m} / \mathrm{s})$ & 1,8117 & 1,6803 \\
\cline { 2 - 4 } & Máxima velocidad vertical $(\mathrm{m} / \mathrm{s})$ & 0,0 & $-0,0$ \\
\cline { 2 - 4 } & Media de velocidad horizontal $(\mathrm{m} / \mathrm{s})$ & 1,0196 & 1,0697 \\
\cline { 2 - 4 } & Media de velocidad vertical $(\mathrm{m} / \mathrm{s})$ & 0,0 & 0,0 \\
\hline
\end{tabular}

En segundo lugar, se muestra en la figura 11 el diagrama de aceleraciones procedente del cálculo mediante la teoría no lineal, y en la figura 12 el mismo cálculo pero realizado según la teoría lineal. 


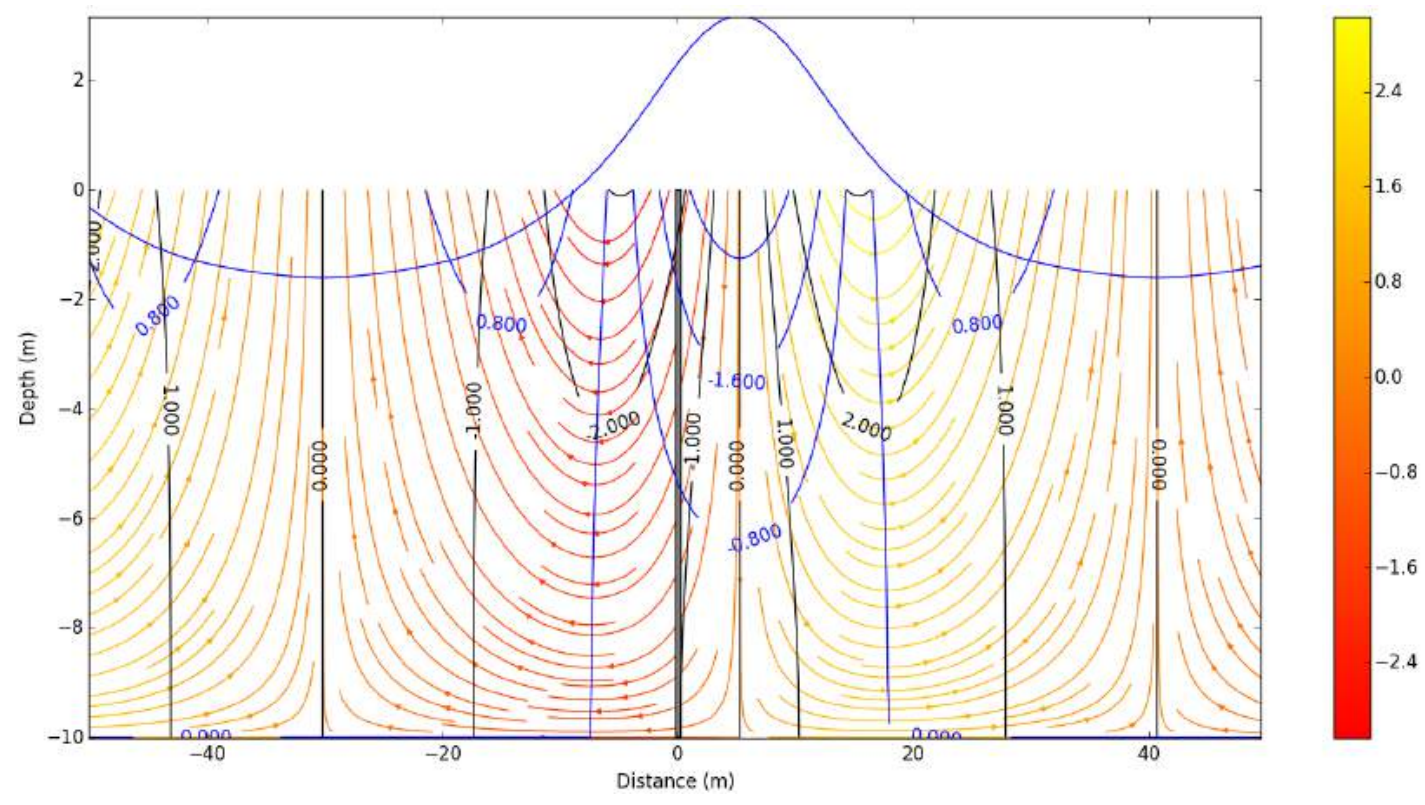

Figura 11. Aceleraciones para $H=4,78 m, h=10,0 m$ y $T=6,8$ s según Stokes de $5^{\circ}$ Orden

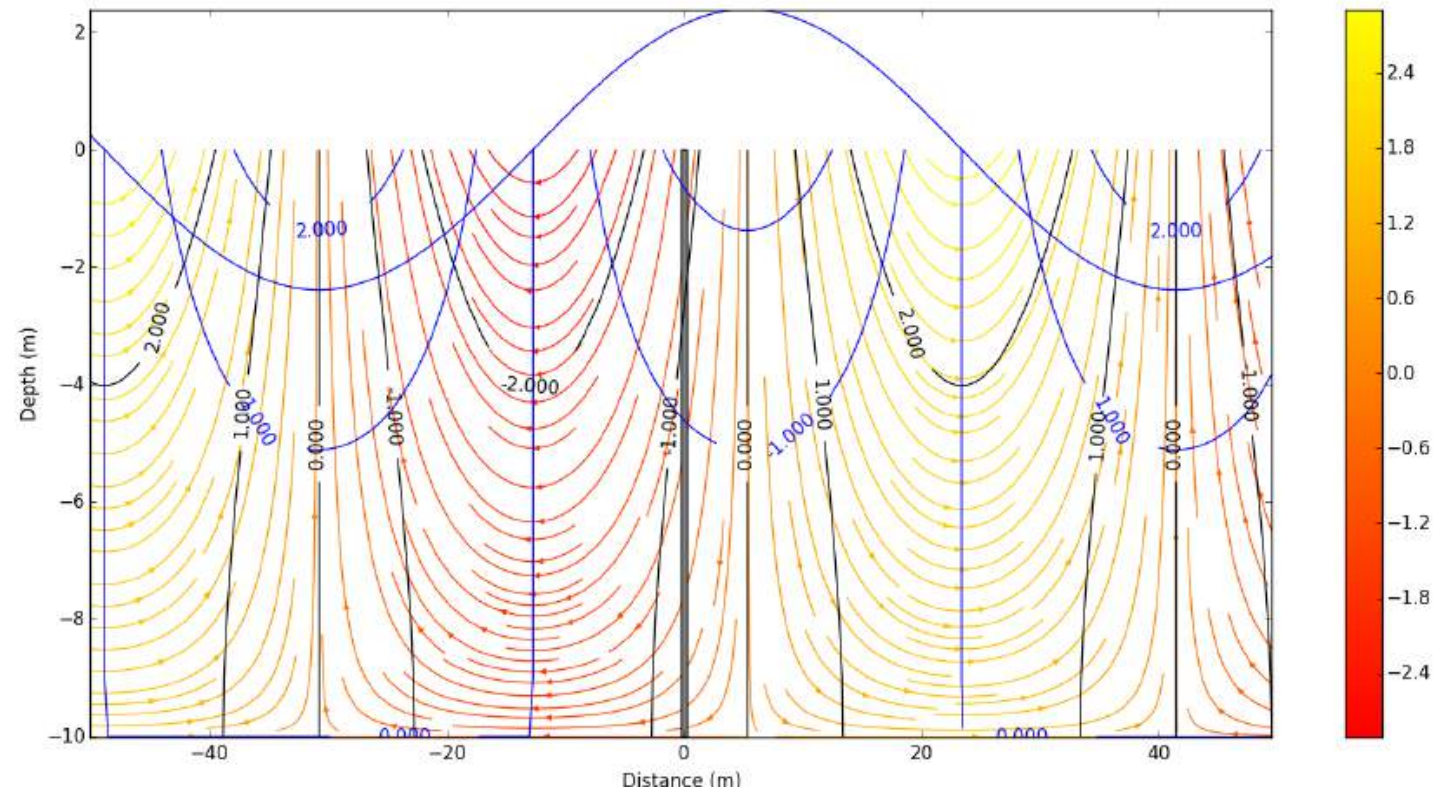

Figura 12. Aceleraciones para $H=4,78 m, h=10,0 m$ y $T=6,8$ s según teoría lineal

Los valores más característicos de la simulación, tanto para el caso lineal como para el caso no lineal, se muestran a continuación en la tabla 4:

\begin{tabular}{|c|l|c|c|}
\hline Zona & \multicolumn{1}{|c|}{ Parámetro } & CASO B & Teoría clásica \\
\hline \multirow{4}{*}{ Superficie } & Máxima aceleración horizontal $\left(\mathrm{m} / \mathrm{s}^{2}\right)$ & 3,0349 & 2,9086 \\
\cline { 2 - 4 } & Máxima aceleración vertical $\left(\mathrm{m} / \mathrm{s}^{2}\right)$ & 1,2347 & 2,4596 \\
\cline { 2 - 4 } & Media de aceleración horizontal $\left(\mathrm{m} / \mathrm{s}^{2}\right)$ & 1,39 & 1,8517 \\
\cline { 2 - 4 } & Media de aceleración vertical $\left(\mathrm{m} / \mathrm{s}^{2}\right)$ & 1,1517 & 1,5659 \\
\hline
\end{tabular}




\begin{tabular}{|l|l|c|c|}
\hline \multirow{4}{*}{ Fondo } & Máxima aceleración horizontal $\left(\mathrm{m} / \mathrm{s}^{2}\right)$ & 1,6384 & 1,5526 \\
\cline { 2 - 4 } & Máxima aceleración vertical $\left(\mathrm{m} / \mathrm{s}^{2}\right)$ & $-0,0$ & 0,0 \\
\cline { 2 - 4 } & Media de aceleración horizontal $\left(\mathrm{m} / \mathrm{s}^{2}\right)$ & 0,9424 & 0,9884 \\
\cline { 2 - 4 } & Media de aceleración vertical $\left(\mathrm{m} / \mathrm{s}^{2}\right)$ & 0,0 & 0,0 \\
\hline
\end{tabular}

Tabla 4. Comparación de campo de aceleraciones caso $B$

\subsubsection{Análisis de principales frecuencias generadas por el oleaje}

También se procede a realizar una transformada de Fourier para los resultados en superficie, para observar la variación generada en la distribución de la velocidad (figura 13) y la aceleración (figura 14), en frecuencias:
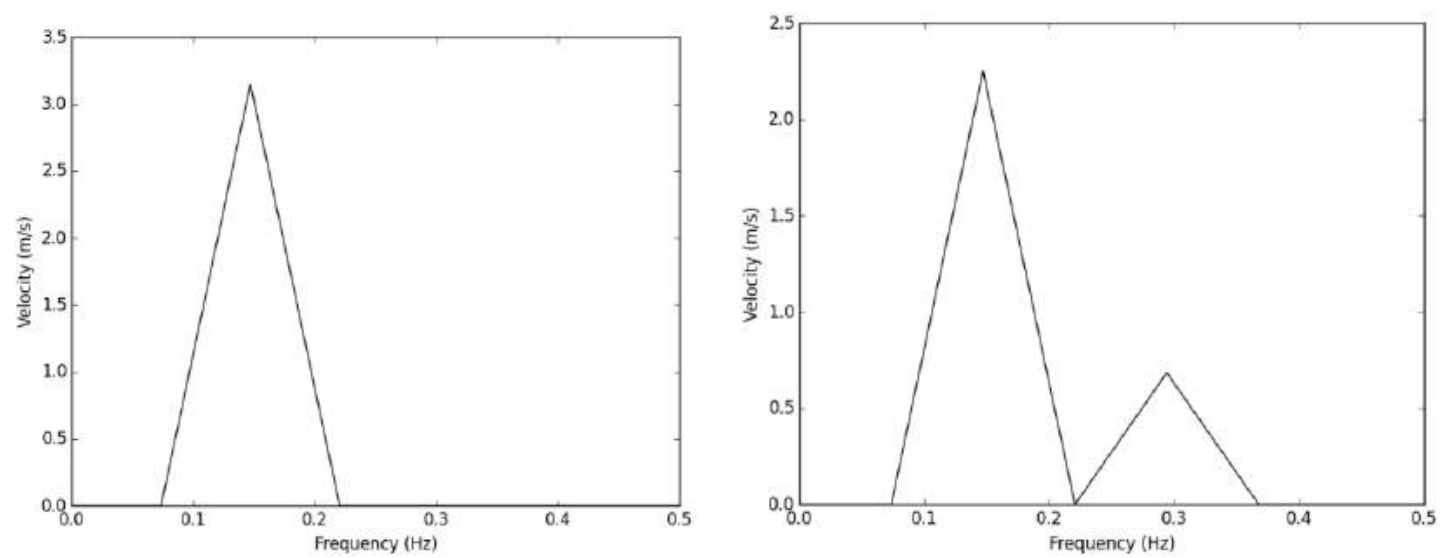

Figura 13. Velocidades: Al a izquierda resultados de la teoría lineal, a la derecha de Stokes de 5o Orden
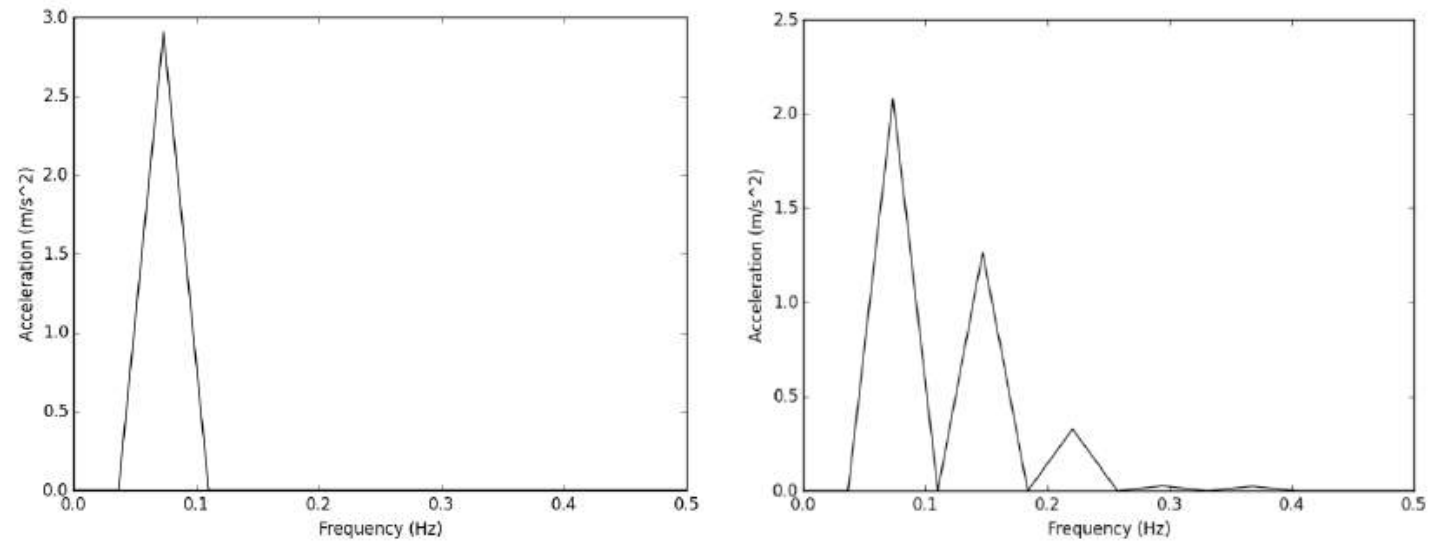

Figura 14. Aceleraciones: Al a izquierda resultados de la teoría lineal, a la derecha de Stokes de 5ºrden 


\subsubsection{Análisis sobre la amortiguación de la velocidad y la aceleración con la profundidad}

También se muestran los valores máximos de velocidad (figura 15) y aceleración (figura 16) a distintas profundidades para observar la variación que sufre el amortiguamiento con la profundidad, entre la teoría lineal, y el caso en estudio.
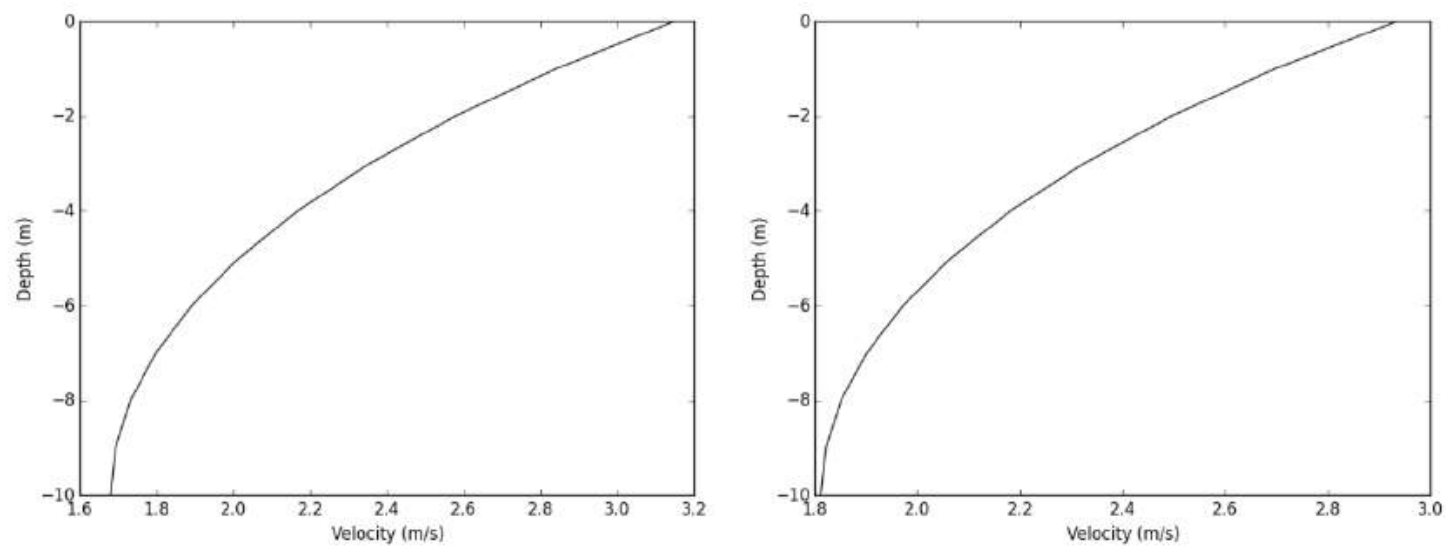

Figura 15. Amortiguamiento de velocidades, a la izquierda teoría lineal, a la derecha Stokes de 5o Orden
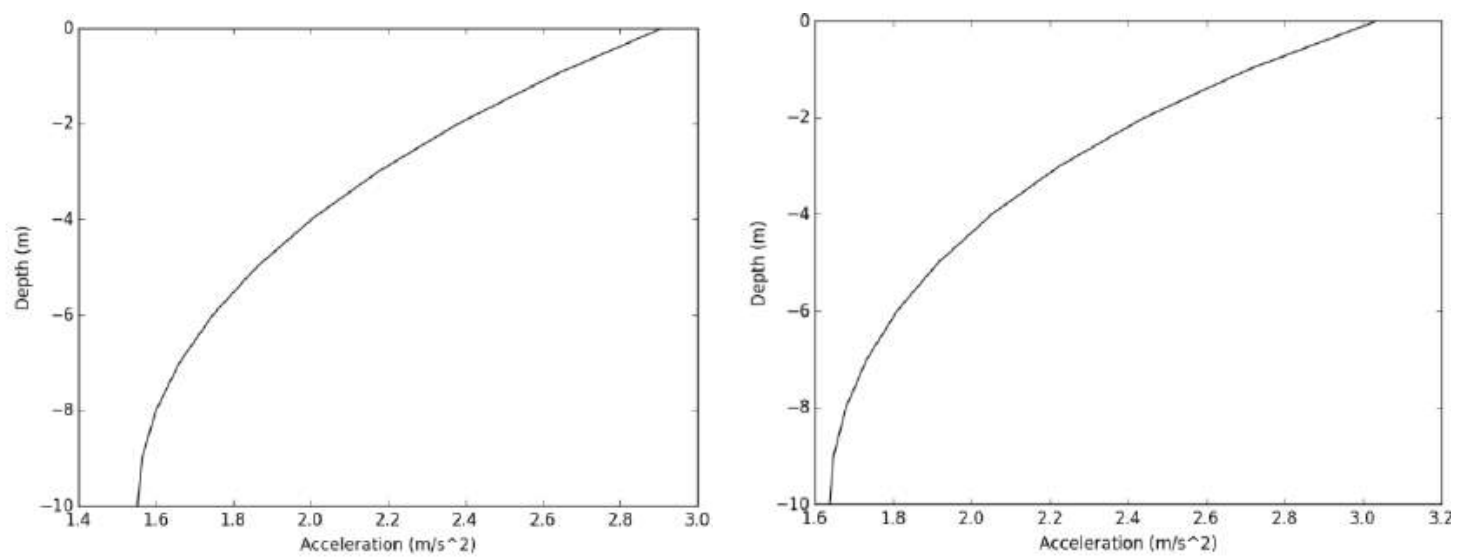

Figura 16. Amortiguamiento de aceleraciones, a la izquierda teoría lineal, a la derecha Stokes de 5o Orden 


\subsection{Caso C: Onda Cnoidal de $2^{\circ}$ Orden}

Los datos de entrada se corresponden con el caso de cálculo para el parque eólico offshore Egmond Aan Zee en Holanda, y son los siguientes:

- $\quad$ Altura de ola significativa: 3,6 m

- $\quad$ Profundidad: $22,0 \mathrm{~m}$

- $\quad$ Periodo de pico: $18,5 \mathrm{~s}$

De acuerdo con estos datos, la teoría de ondas a utilizar será la teoría Cnoidal de 2oorden, en profundidades de transición.

$$
\begin{gathered}
\delta_{1}=\frac{H}{g T^{2}}=0,00107 \quad \delta_{2}=\frac{h}{g T^{2}}=0,00655 \\
\frac{d}{L}=\frac{22}{268,22} \rightarrow 0,04<0,082<0,5 \rightarrow \text { Profundidades de transición }
\end{gathered}
$$

\subsubsection{Comparación entre el campo de velocidades y aceleraciones, con la teoría lineal.}

A continuación, se muestra en la figura 17 el diagrama de velocidades procedente del cálculo mediante la teoría no lineal, y en la figura 18 el mismo cálculo pero realizado según la teoría lineal.

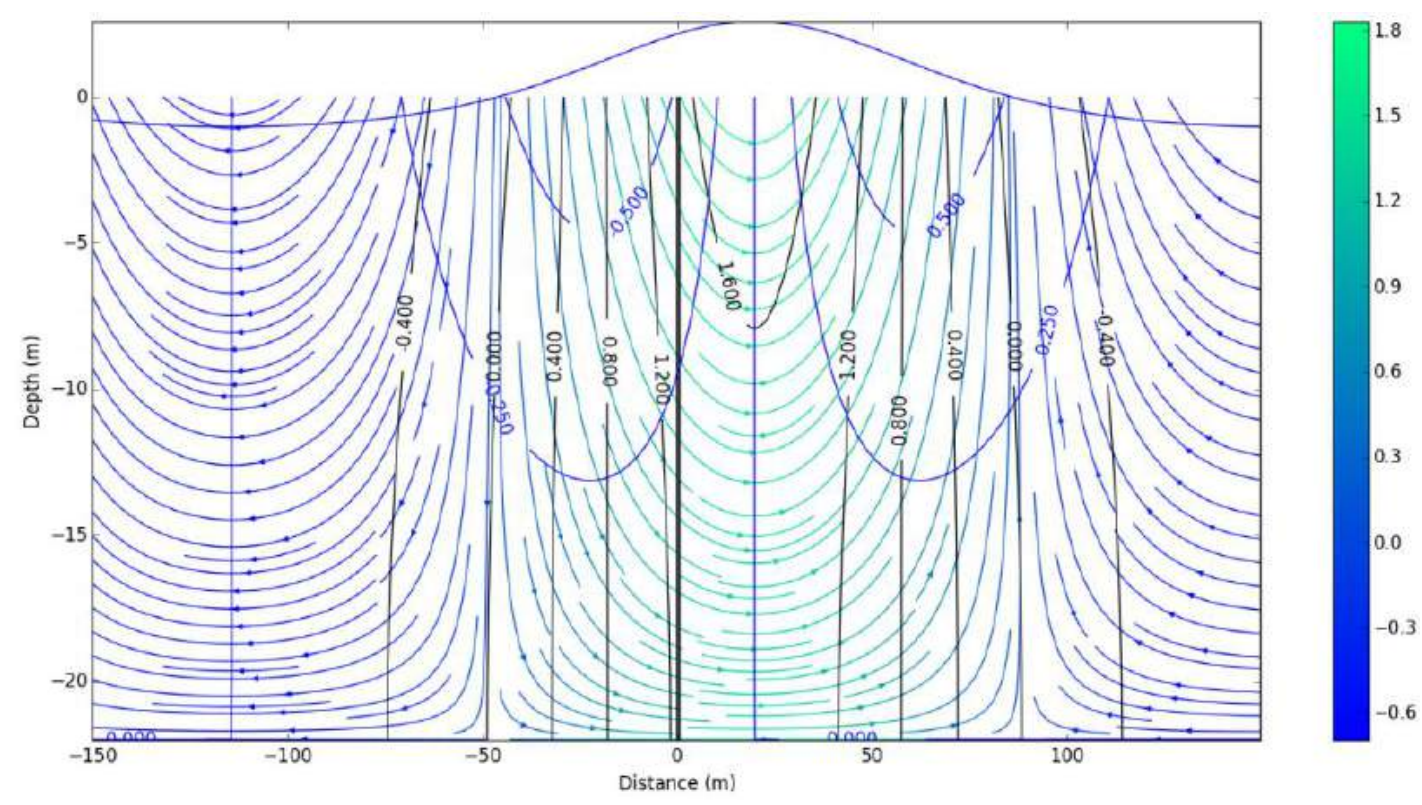

Figura 17. Velocidades para $H=3,6 m, h=22,0 m$ y $T=18,5$ s según Cnoidal de 2º Orden 


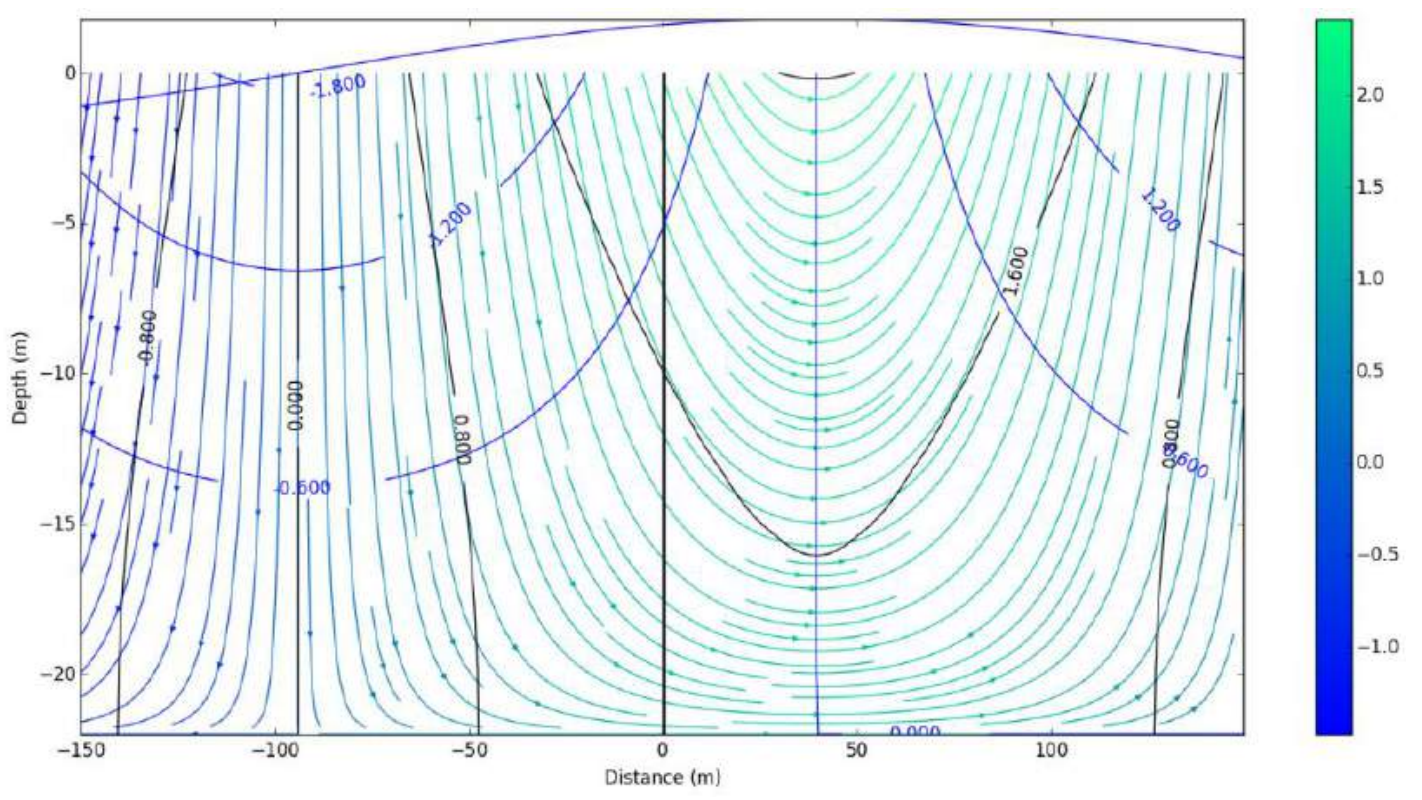

Figura 18. Velocidades para $H=3,6 m, h=22,0 m$ y $T=18,5$ s según teoría lineal

Los valores más característicos de la simulación, tanto para el caso lineal como para el caso no lineal, se muestran a continuación en la tabla 5:

\begin{tabular}{|c|l|c|c|}
\hline Zona & \multicolumn{1}{|c|}{ Parámetro } & CASO C & Teoría lineal \\
\hline \multirow{4}{*}{ Superficie } & Máxima velocidad horizontal $(\mathrm{m} / \mathrm{s})$ & 1,8331 & 2,4157 \\
\cline { 2 - 4 } & Máxima velocidad vertical $(\mathrm{m} / \mathrm{s})$ & 0,6482 & 1,8619 \\
\cline { 2 - 4 } & Media de velocidad horizontal $(\mathrm{m} / \mathrm{s})$ & 0,7399 & 1,5379 \\
\cline { 2 - 4 } & Media de velocidad vertical $(\mathrm{m} / \mathrm{s})$ & 0,3429 & 1,1854 \\
\hline \multirow{4}{*}{ Fondo } & Máxima velocidad horizontal $(\mathrm{m} / \mathrm{s})$ & 1,4367 & 1,5391 \\
\cline { 2 - 4 } & Máxima velocidad vertical $(\mathrm{m} / \mathrm{s})$ & 0,0 & $-0,0$ \\
\cline { 2 - 4 } & Media de velocidad horizontal $(\mathrm{m} / \mathrm{s})$ & 0,6279 & 0,9798 \\
\cline { 2 - 4 } & Media de velocidad vertical $(\mathrm{m} / \mathrm{s})$ & 0,0 & 0,0 \\
\hline
\end{tabular}

Tabla 5. Comparación de campo de velocidades caso $\mathrm{C}$

En segundo lugar, se muestra en la figura 19 el diagrama de aceleraciones procedente del cálculo mediante la teoría no lineal, y en la figura 20 el mismo cálculo pero realizado según la teoría lineal. 


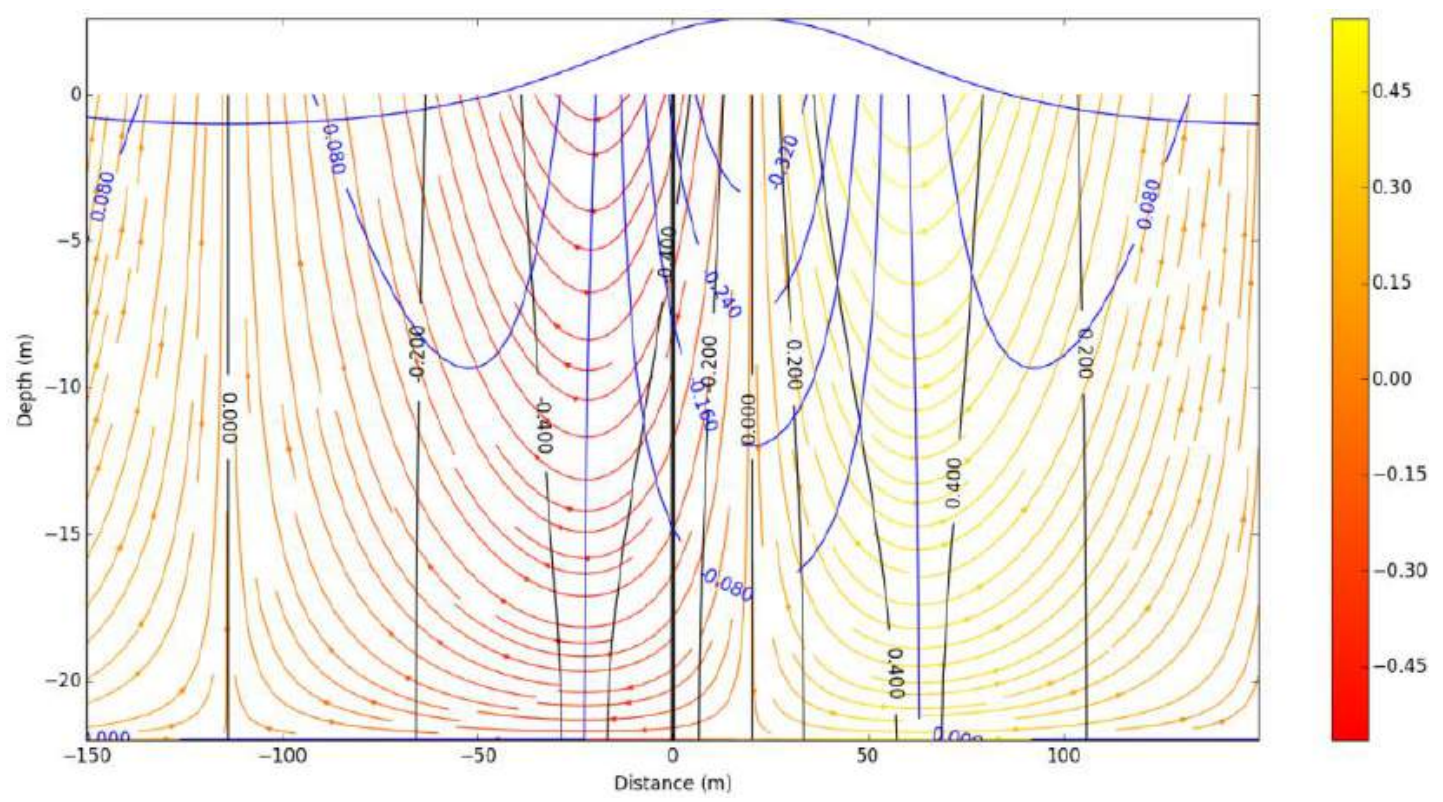

Figura 19. Aceleraciones para $\mathrm{H}=3,6 m, h=22,0 m$ y $T=18,5$ s según Cnoidal de 2º Orden

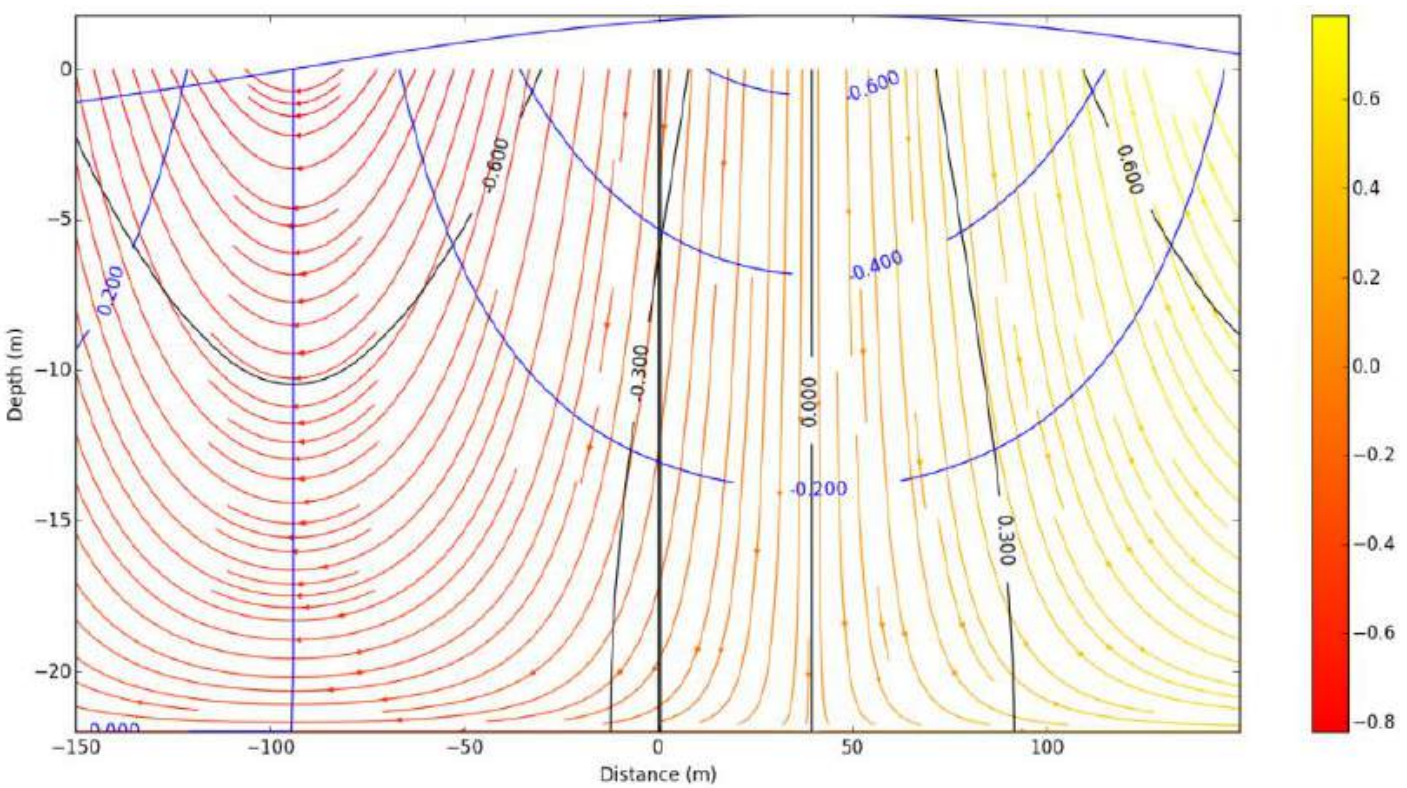

Figura 20. Aceleraciones para $\mathrm{H}=3,6 \mathrm{~m}, \mathrm{~h}=22,0 \mathrm{~m}$ y $\mathrm{T}=18,5$ s según teoría lineal

Los valores más característicos de la simulación, tanto para el caso lineal como para el caso no lineal, se muestran a continuación en la tabla 6:

\begin{tabular}{|c|l|c|c|}
\hline Zona & \multicolumn{1}{|c|}{ Parámetro } & CASO C & Teoría clásica \\
\hline \multirow{4}{*}{ Superficie } & Máxima aceleración horizontal $\left(\mathrm{m} / \mathrm{s}^{2}\right)$ & 0,5646 & 0,8205 \\
\cline { 2 - 4 } & Máxima aceleración vertical $\left(\mathrm{m} / \mathrm{s}^{2}\right)$ & 0,1441 & 0,6324 \\
\cline { 2 - 4 } & Media de aceleración horizontal $\left(\mathrm{m} / \mathrm{s}^{2}\right)$ & 0,2732 & 0,5223 \\
\cline { 2 - 4 } & Media de aceleración vertical $\left(\mathrm{m} / \mathrm{s}^{2}\right)$ & 0,1402 & 0,4026 \\
\hline
\end{tabular}




\begin{tabular}{|l|l|c|c|}
\hline \multirow{4}{*}{ Fondo } & Máxima aceleración horizontal $\left(\mathrm{m} / \mathrm{s}^{2}\right)$ & 0,4073 & 0,5227 \\
\cline { 2 - 4 } & Máxima aceleración vertical $\left(\mathrm{m} / \mathrm{s}^{2}\right)$ & 0,0 & 0,0 \\
\cline { 2 - 4 } & Media de aceleración horizontal $\left(\mathrm{m} / \mathrm{s}^{2}\right)$ & 0,2194 & 0,3328 \\
\cline { 2 - 4 } & Media de aceleración vertical $\left(\mathrm{m} / \mathrm{s}^{2}\right)$ & 0,0 & 0,0 \\
\hline
\end{tabular}

Tabla 6. Comparación de campo de aceleraciones caso $C$

\subsubsection{Análisis de principales frecuencias generadas por el oleaje}

También se procede a realizar una transformada de Fourier para los resultados en superficie, para observar la variación generada en la distribución de la velocidad (figura 21) y la aceleración (figura 22), en frecuencias:
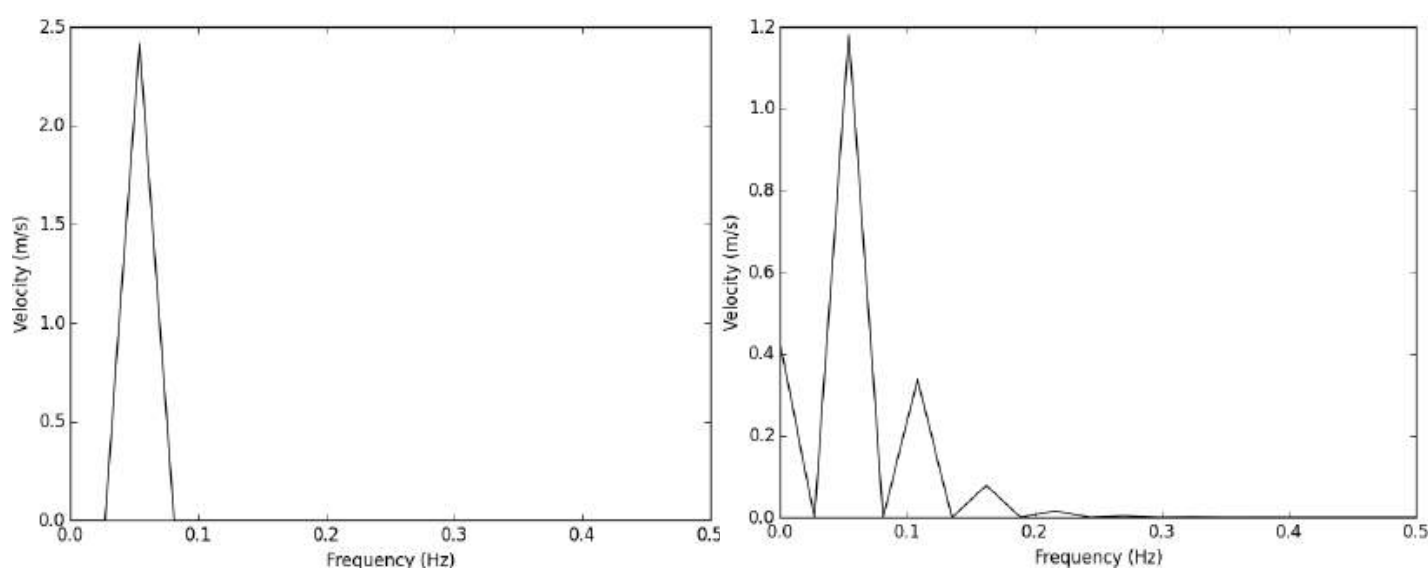

Figura 21. Velocidades: Al a izquierda resultados de la teoría lineal, a la derecha de Cnoidal de 2º Orden
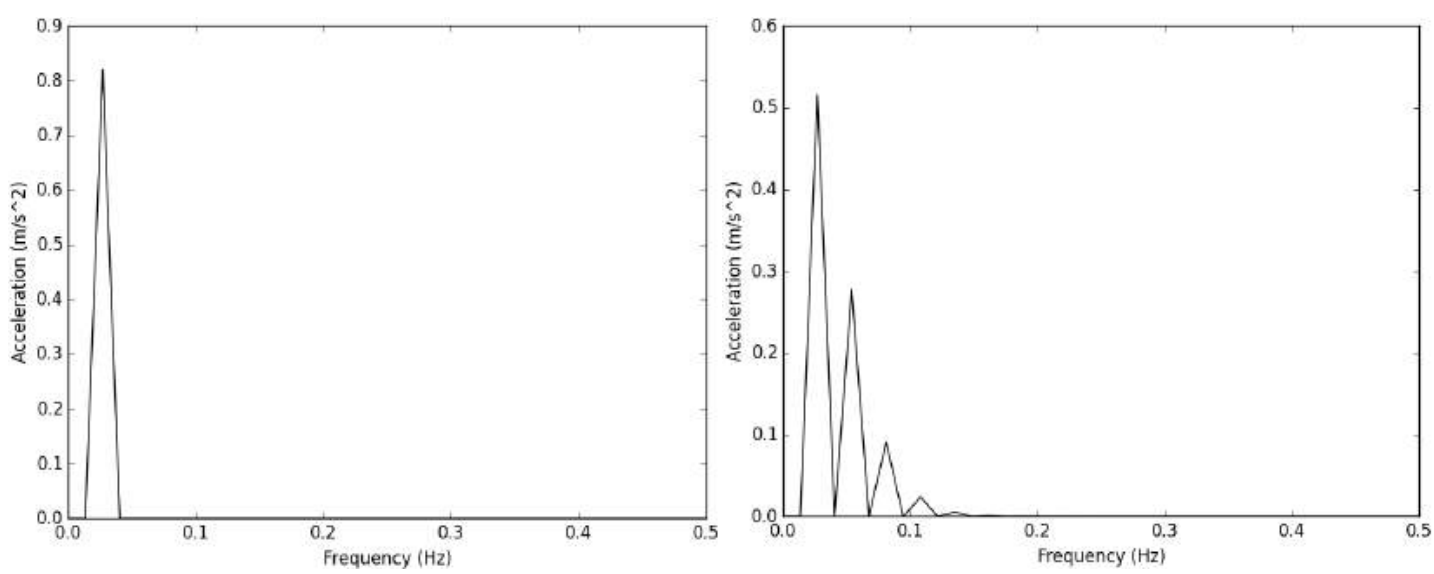

Figura 22. Aceleraciones: Al a izquierda resultados de la teoría lineal, a la derecha de Cnoidal de 2o Orden 


\subsubsection{Análisis sobre la amortiguación de la velocidad y la aceleración con la profundidad}

También se muestran los valores máximos de velocidad (figura 23) y aceleración (figura 24) a distintas profundidades para observar la variación que sufre el amortiguamiento con la profundidad, entre la teoría lineal, y el caso en estudio.
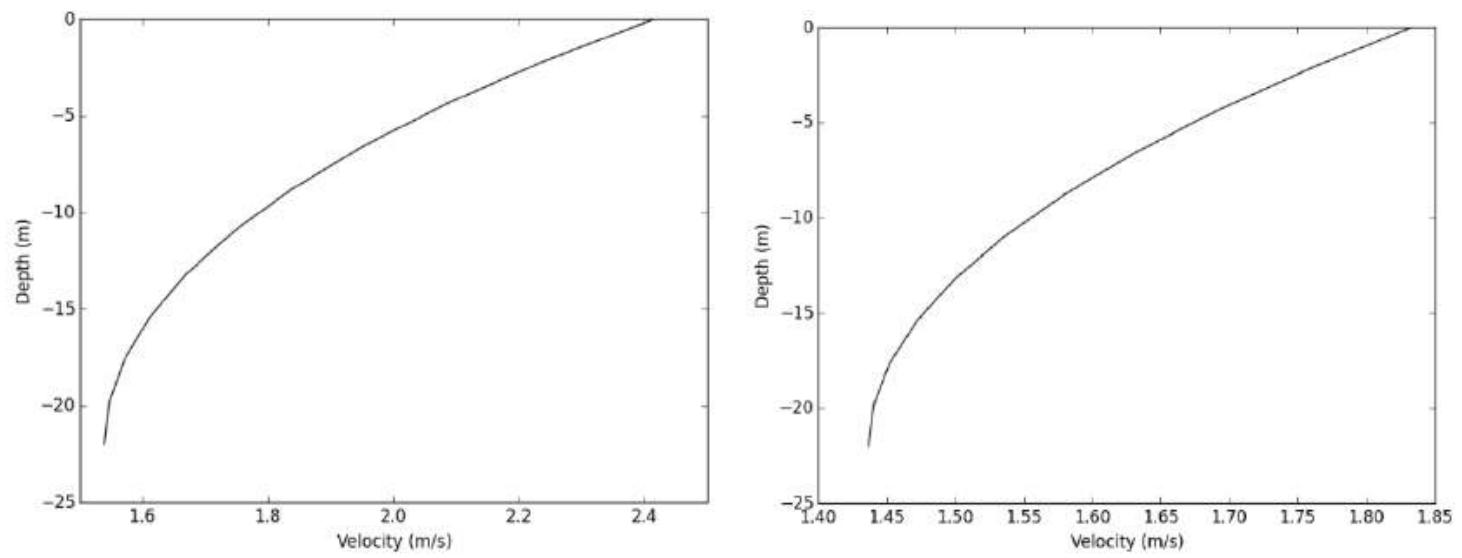

Figura 23. Amortiguamiento de velocidades, a la izquierda teoría lineal, a la derecha Cnoidal de $2^{\circ}$ Orden
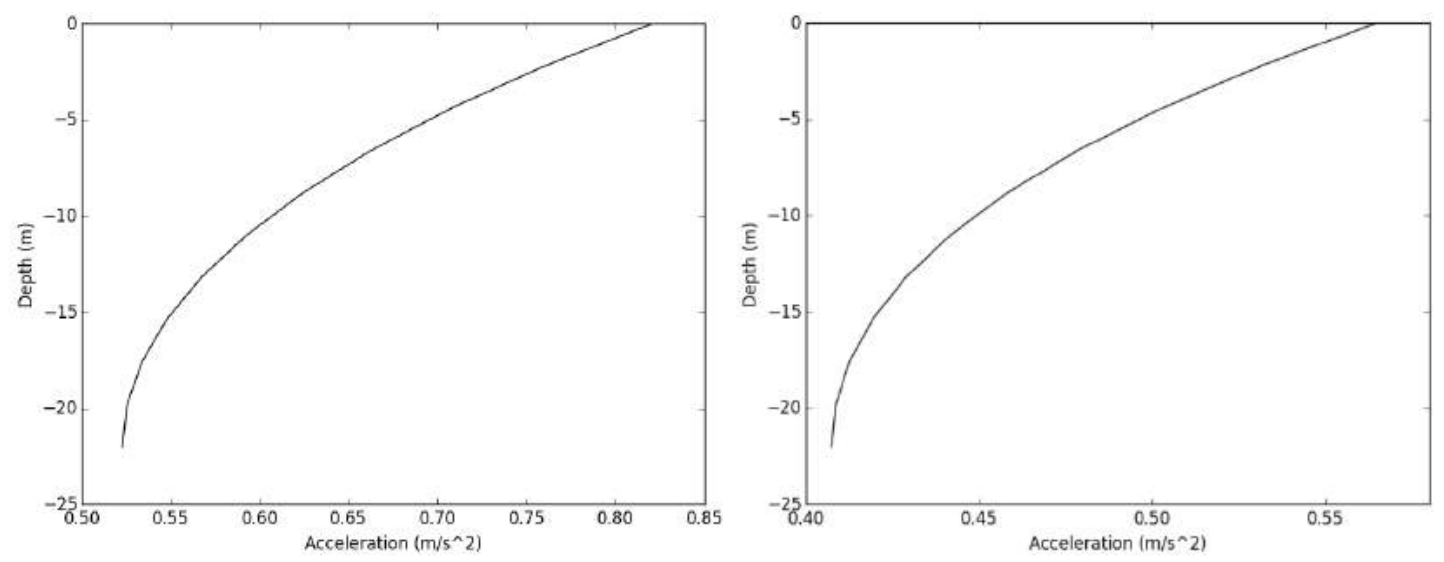

Figura 24. Amortiguamiento de aceleraciones, a la izquierda teoría lineal, a la derecha Cnoidal de 2ºrden 


\subsection{Caso D: Stream Function de Dean.}

Los datos de entrada se corresponden con el caso de cálculo para el parque eólico offshore Thornton Bank en Bélgica, y son los siguientes:

- $\quad$ Altura de ola significativa: 6,32 m

- $\quad$ Profundidad: $16,0 \mathrm{~m}$

- $\quad$ Periodo de pico: 11,06 s

De acuerdo con estos datos, la teoría de ondas a utilizar será la Stream Function de Dean de 5o orden, en profundidades de transición.

$$
\begin{gathered}
\delta_{1}=\frac{H}{g T^{2}}=0,00527 \quad \delta_{2}=\frac{h}{g T^{2}}=0,01333 \\
\frac{d}{L}=\frac{16}{91,53} \rightarrow 0,04<0,174<0,5 \rightarrow \text { Profundidades de transición }
\end{gathered}
$$

\subsubsection{Comparación entre el campo de velocidades y aceleraciones, con la teoría lineal.}

A continuación, se muestra en la figura 25 el diagrama de velocidades procedente del cálculo mediante la teoría no lineal, y en la figura 26 el mismo cálculo pero realizado según la teoría lineal.

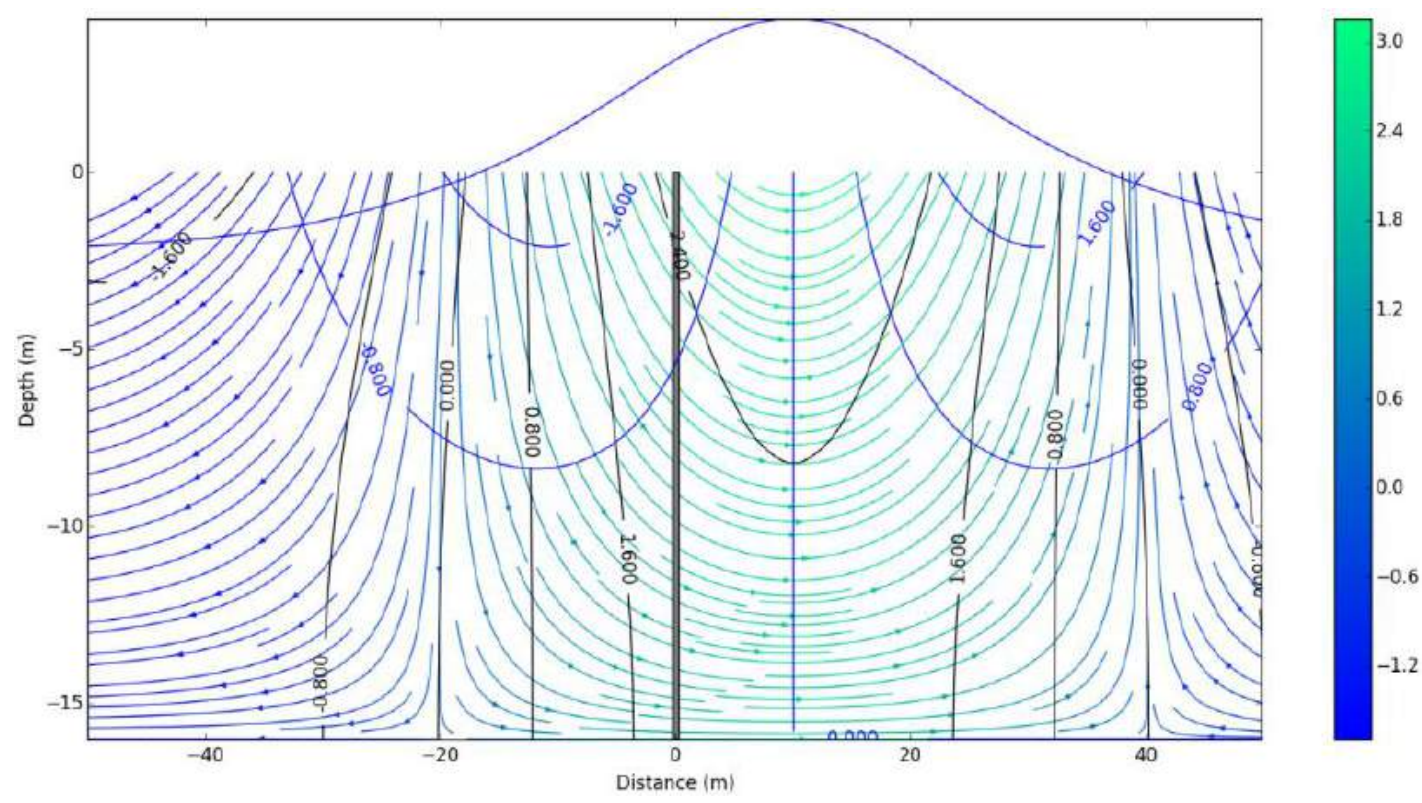

Figura 25. Velocidades para $H=6,32 m, h=16,0 m$ y $T=11,06$ s según Stream Function de 5o Orden 


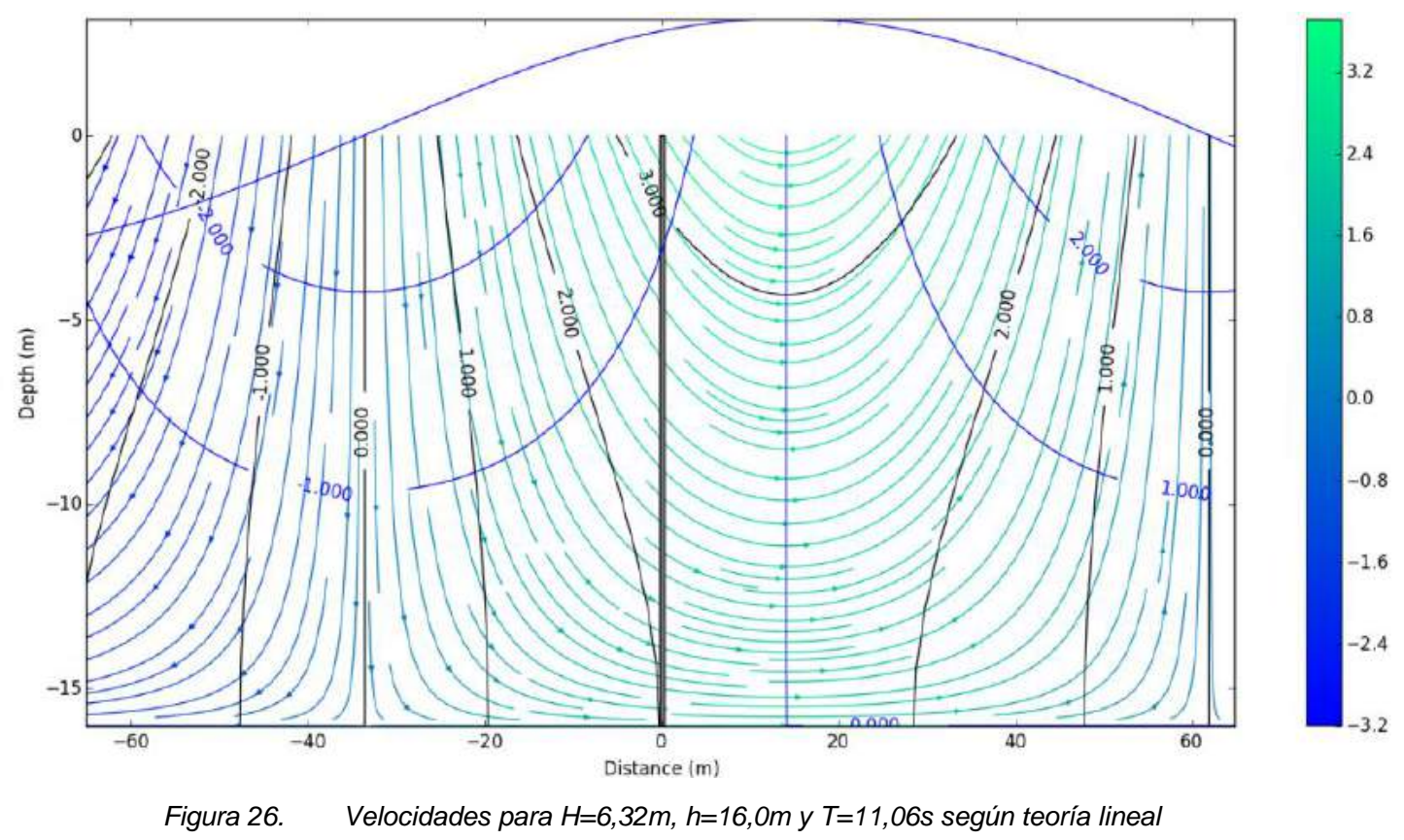

Los valores más característicos de la simulación, tanto para el caso lineal como para el caso no lineal, se muestran a continuación en la tabla 7:

\begin{tabular}{|c|l|c|c|}
\hline Zona & \multicolumn{1}{|c|}{ Parámetro } & CASO D & Teoría lineal \\
\hline \multirow{4}{*}{ Superficie } & Máxima velocidad horizontal $(\mathrm{m} / \mathrm{s})$ & 3,1565 & 3,7198 \\
\cline { 2 - 4 } & Máxima velocidad vertical $(\mathrm{m} / \mathrm{s})$ & 1,9246 & 2,9651 \\
\cline { 2 - 4 } & Media de velocidad horizontal $(\mathrm{m} / \mathrm{s})$ & 1,5892 & 2,3681 \\
\cline { 2 - 4 } & Media de velocidad vertical $(\mathrm{m} / \mathrm{s})$ & 0,9884 & 1,8877 \\
\hline \multirow{3}{*}{ Fondo } & Máxima velocidad horizontal $(\mathrm{m} / \mathrm{s})$ & 2,1871 & 2,246 \\
\cline { 2 - 4 } & Máxima velocidad vertical $(\mathrm{m} / \mathrm{s})$ & 0,0 & $-0,0$ \\
\cline { 2 - 4 } & Media de velocidad horizontal $(\mathrm{m} / \mathrm{s})$ & 1,1981 & 1,4299 \\
\cline { 2 - 4 } & Media de velocidad vertical $(\mathrm{m} / \mathrm{s})$ & 0,0 & 0,0 \\
\hline
\end{tabular}

En segundo lugar, se muestra en la figura 27 el diagrama de aceleraciones procedente del cálculo mediante la teoría no lineal, y en la figura 28 el mismo cálculo pero realizado según la teoría lineal. 


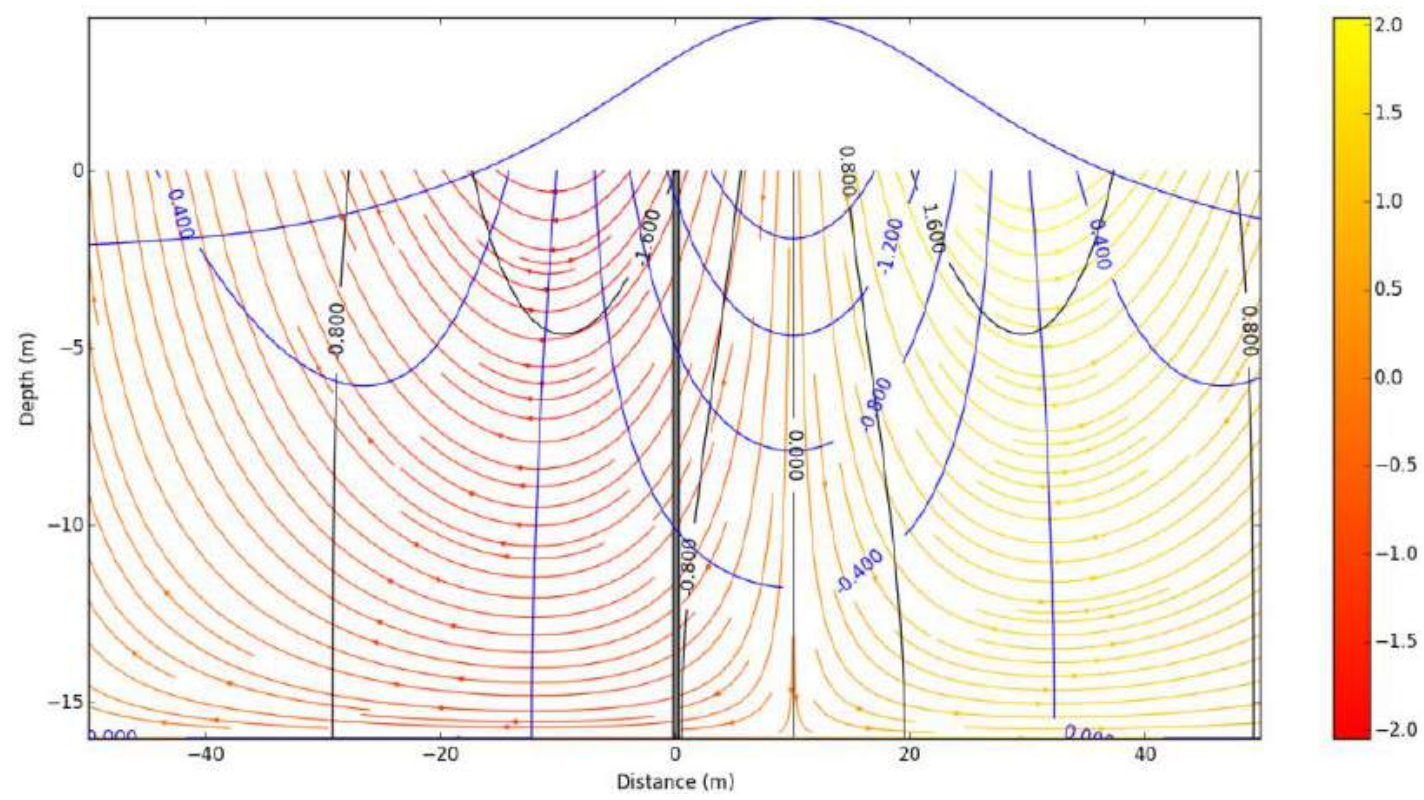

Figura 27. Aceleraciones para $H=6,32 m, h=16,0 m$ y $T=11,06$ s según Stream Function de $5^{\circ}$ Orden

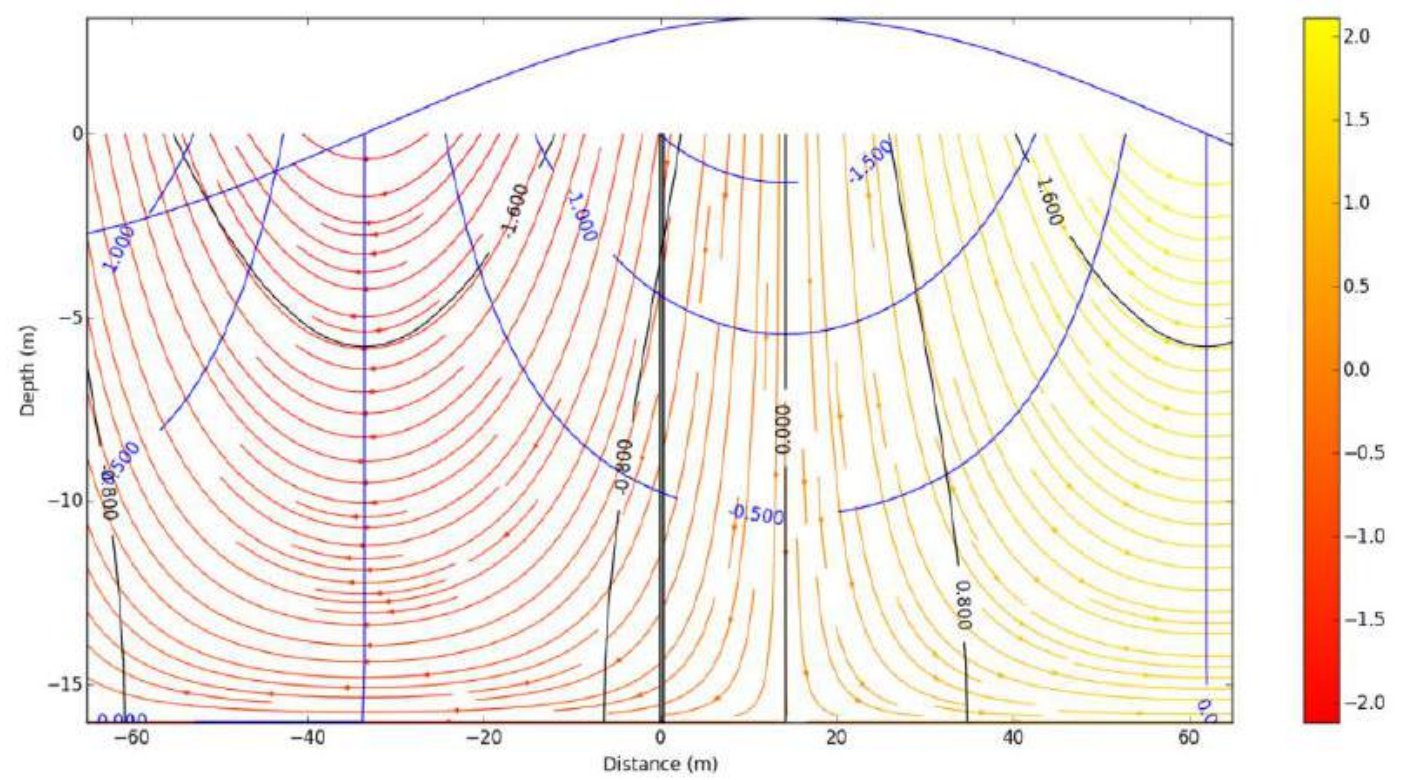

Figura 28. Aceleraciones para $H=6,32 m, h=16,0 m$ y $T=11,06$ s según teoría lineal

Los valores más característicos de la simulación, tanto para el caso lineal como para el caso no lineal, se muestran a continuación en la tabla 8:

\begin{tabular}{|c|l|c|c|}
\hline Zona & \multicolumn{1}{|c|}{ Parámetro } & CASO D & Teoría clásica \\
\hline \multirow{4}{*}{ Superficie } & Máxima aceleración horizontal $\left(\mathrm{m} / \mathrm{s}^{2}\right)$ & 2,0461 & 2,1132 \\
\cline { 2 - 4 } & Máxima aceleración vertical $\left(\mathrm{m} / \mathrm{s}^{2}\right)$ & 0,7616 & 1,6845 \\
\cline { 2 - 4 } & Media de aceleración horizontal $\left(\mathrm{m} / \mathrm{s}^{2}\right)$ & 0,9188 & 1,3453 \\
\cline { 2 - 4 } & Media de aceleración vertical $\left(\mathrm{m} / \mathrm{s}^{2}\right)$ & 0,695 & 1,0724 \\
\hline
\end{tabular}




\begin{tabular}{|l|l|c|c|}
\hline \multirow{4}{*}{ Fondo } & Máxima aceleración horizontal $\left(\mathrm{m} / \mathrm{s}^{2}\right)$ & 1,2387 & 1,276 \\
\cline { 2 - 4 } & Máxima aceleración vertical $\left(\mathrm{m} / \mathrm{s}^{2}\right)$ & $-0,0$ & 0,0 \\
\cline { 2 - 4 } & Media de aceleración horizontal $\left(\mathrm{m} / \mathrm{s}^{2}\right)$ & 0,6825 & 0,8123 \\
\cline { 2 - 4 } & Media de aceleración vertical $\left(\mathrm{m} / \mathrm{s}^{2}\right)$ & 0,0 & 0,0 \\
\hline
\end{tabular}

Tabla 8. Comparación de campo de aceleraciones caso $D$

\subsubsection{Análisis de principales frecuencias generadas por el oleaje}

También se procede a realizar una transformada de Fourier para los resultados en superficie, para observar la variación generada en la distribución de la velocidad (figura 29) y la aceleración (figura 30), en frecuencias:
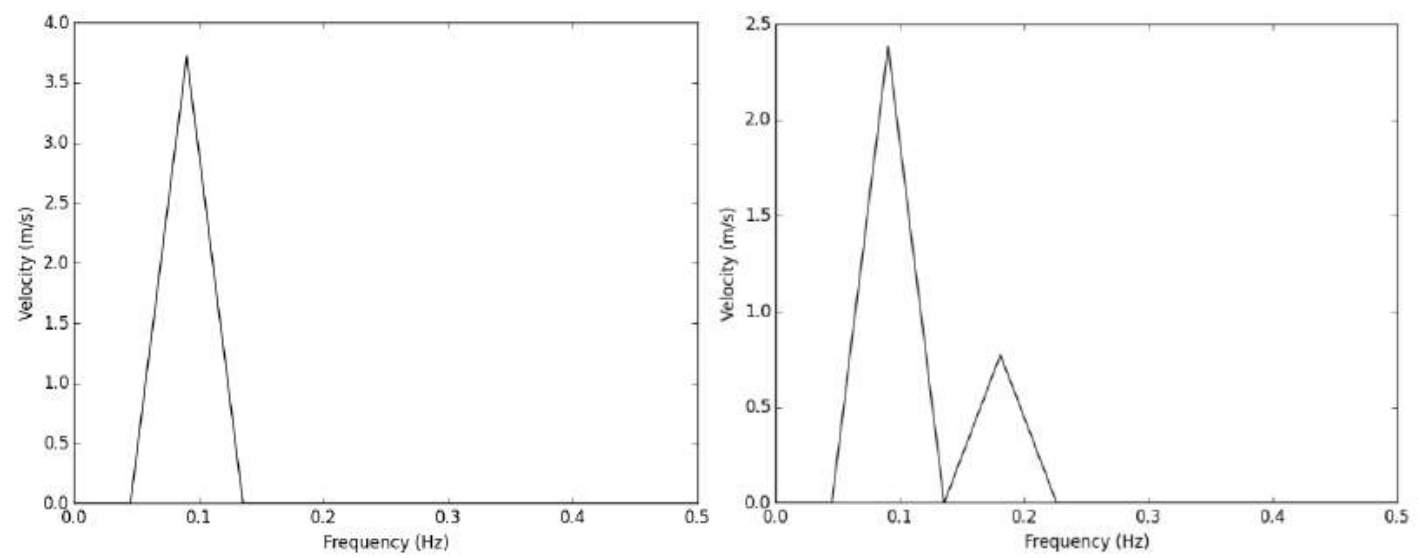

Figura 29. Velocidades: Al a izquierda resultados de la teoría lineal, a la derecha de Stream Function de 5o Orden
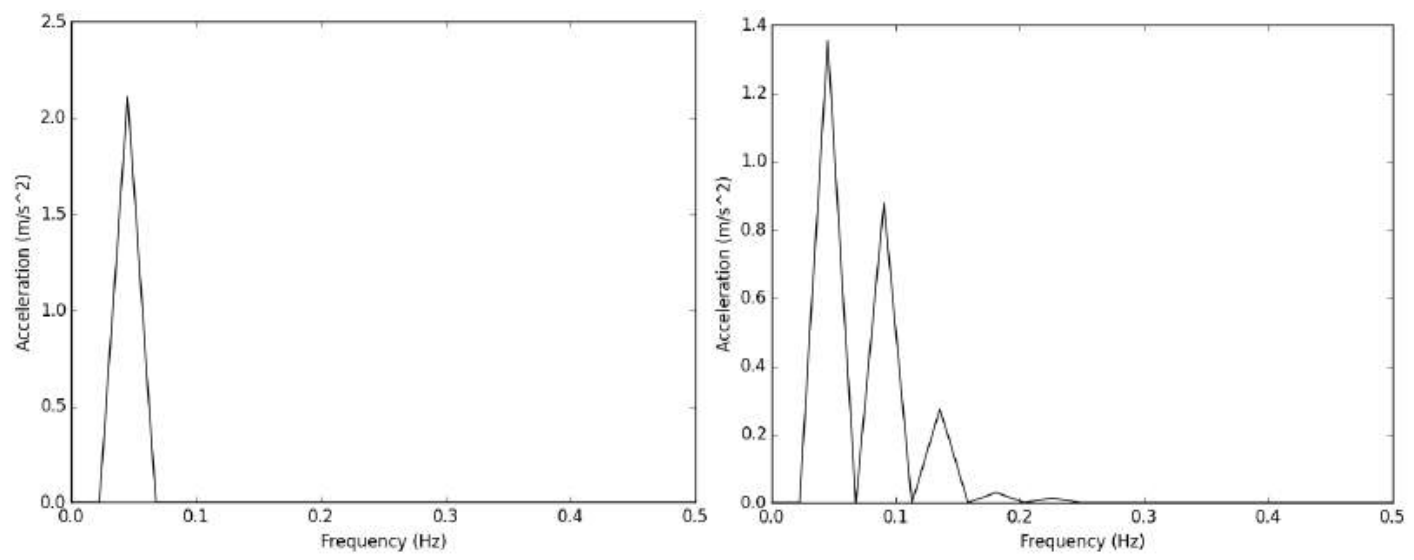

Figura 30. Aceleraciones: Al a izquierda resultados de la teoría lineal, a la derecha de Stream Function de 5O Orden 


\subsubsection{Análisis sobre la amortiguación de la velocidad y la aceleración con la profundidad}

También se muestran los valores máximos de velocidad (figura 31) y aceleración (figura 32) a distintas profundidades para observar la variación que sufre el amortiguamiento con la profundidad, entre la teoría lineal, y el caso en estudio.
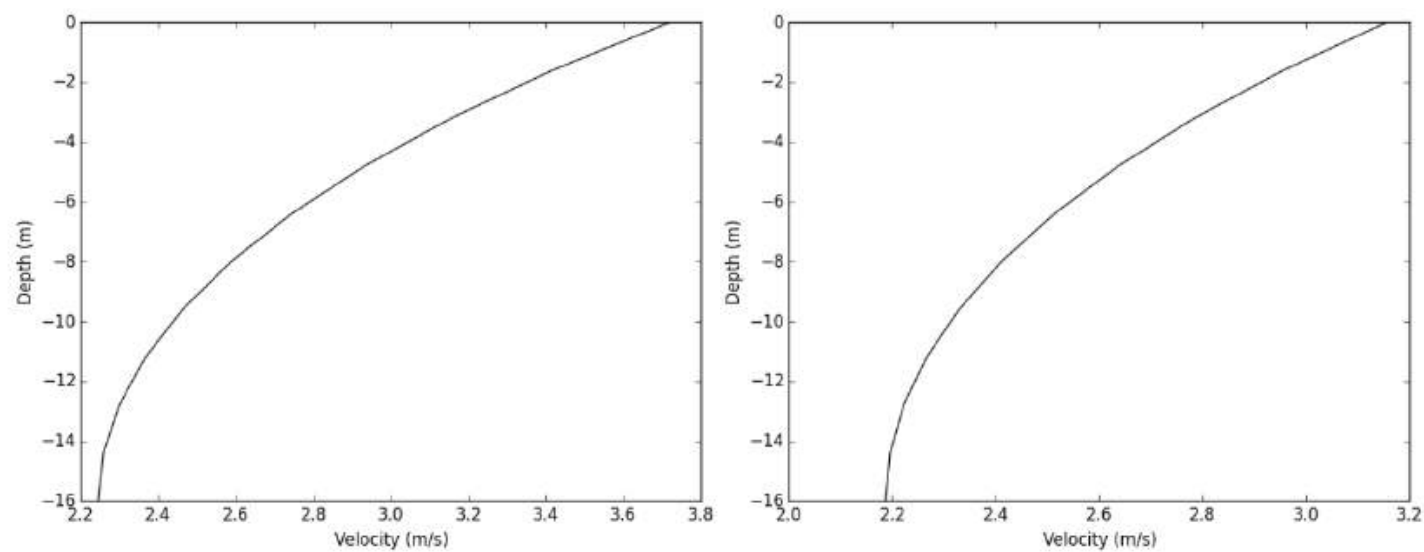

Figura 31. Amortiguamiento de velocidades, a la izquierda teoría lineal, a la derecha Stream Function de 5o Orden
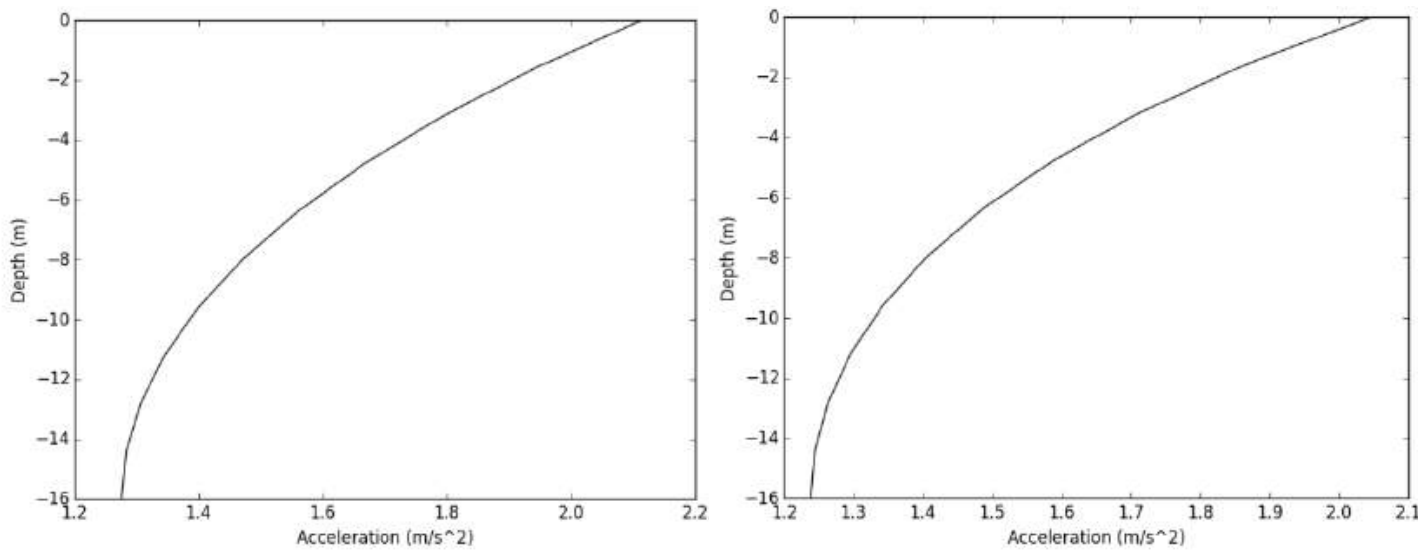

Figura 32. Amortiguamiento de aceleraciones, a la izquierda teoría lineal, a la derecha Stream Function de 5Oden 


\section{Proceso II: Cálculo de las fuerzas hidrodinámicas}

El segundo proceso se corresponde con el cálculo, de las acciones que generará el oleaje sobre la estructura, a partir de las variables que definen el comportamiento de dicho oleaje, principalmente el campo de velocidades ( $v$ ) y el campo de aceleraciones (a).

\subsection{Caso 1: Diámetros igual a $0,5 \mathrm{~m}$}

Se va a evaluar el caso de pilotes de 0,5 m de diámetro mediante los cuatro casos de oleaje definidos en el apartado anterior, calculados según la teoría lineal y según las teorías no lineales, tanto para el caso de una temperatura del agua de 1 으 $\mathrm{C}$ salinidad del $0 \%$, como para el caso de una temperatura de 22 ㅇ C y salinidad del $35 \%$.

\subsubsection{Caso $1 \mathrm{~A}$}

Los datos de entrada se corresponden con el caso de cálculo para el parque eólico offshore Princess Amalia Q7 en Holanda: altura de ola significativa 7,7 m, profundidad 24,0 m y periodo de pico 9,7 s. De acuerdo con estos datos, en la figura 33 se muestra la distribución de fuerzas hidrodinámicas, generadas por el oleaje, en el instante en que producen el máximo momento flector, sobre la base del pilote, mostrando el cálculo según el método avanzado a la izquierda y según el método tradicional, a la derecha.
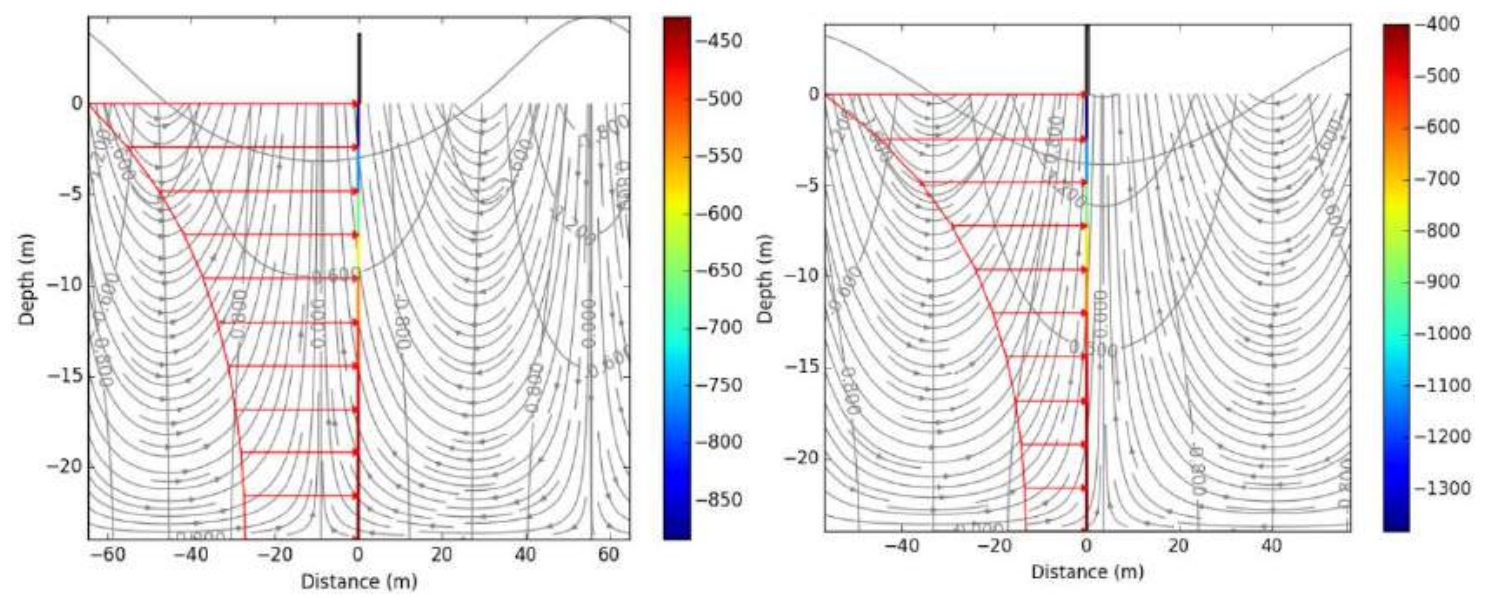

Figura 33. Momento flector máximo para $H=7,7 m, h=24,0 m, T=9,7 s$ y $D=0,5 m$

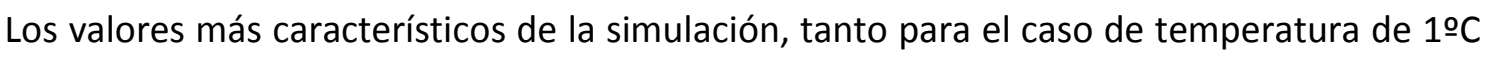
y salinidad de $0 \%$, como para el caso de temperatura de $22{ }^{\circ} \mathrm{C}$ y salinidad del $35 \%$, se muestran, a continuación, en la tabla 9: 


\begin{tabular}{|c|c|c|c|c|c|}
\hline & \multirow[b]{2}{*}{ Parámetro } & \multicolumn{2}{|c|}{ Método Avanzado } & \multicolumn{2}{|c|}{ Método tradicional } \\
\hline & & $\begin{array}{c}\mathrm{T}^{\mathrm{a}}=19 \mathrm{C}, \\
\mathrm{S}=0 \%\end{array}$ & $\begin{array}{c}\mathrm{T}^{\mathrm{a}}=22{ }^{\circ} \mathrm{C}, \\
\mathrm{S}=35 \%\end{array}$ & 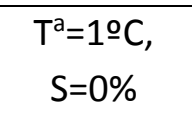 & $\begin{array}{c}\mathrm{T}^{\mathrm{a}}=22^{\circ} \mathrm{C} \\
\mathrm{S}=35 \%\end{array}$ \\
\hline \multirow{5}{*}{$\begin{array}{l}\frac{0}{U} \\
\frac{i}{4} \\
\frac{0}{2} \\
0 \\
0\end{array}$} & $\begin{array}{l}\text { Máxima fuerza de inercia } \\
\text { (N) }\end{array}$ & 837,5477 & 826,3223 & 846,6948 & 847,3133 \\
\hline & $\begin{array}{l}\text { Máxima fuerza de arrastre } \\
\text { (N) }\end{array}$ & 1122,1801 & 1122,9998 & 1880,4649 & 1881,8386 \\
\hline & Máxima fuerza no lineal (N) & 10,8273 & 10,8352 & 0,0 & 0,0 \\
\hline & $\begin{array}{l}\text { Máxima fuerza de } \\
\text { difracción }(\mathrm{N})\end{array}$ & 0,0 & 0,0 & 0,0 & 0,0 \\
\hline & Máxima fuerza total (N) & 1297,9681 & 1298,9163 & 1934,0714 & 1935,4843 \\
\hline \multirow{5}{*}{ 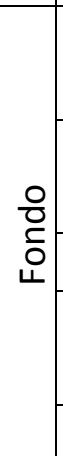 } & $\begin{array}{l}\text { Máxima fuerza de inercia } \\
\text { (N) }\end{array}$ & 427,0978 & 427,4098 & 419,0204 & 419,3265 \\
\hline & $\begin{array}{l}\text { Máxima fuerza de arrastre } \\
\text { (N) }\end{array}$ & 362,2967 & 362,5613 & 460,5553 & 460,8918 \\
\hline & Máxima fuerza no lineal (N) & 9,8718 & 9,8791 & 0,0 & 0,0 \\
\hline & $\begin{array}{l}\text { Máxima fuerza de } \\
\text { difracción }(\mathrm{N})\end{array}$ & 0,0 & 0,0 & 0,0 & 0,0 \\
\hline & Máxima fuerza total (N) & 531,7132 & 474,21 & 544,5214 & 514,5453 \\
\hline
\end{tabular}

Los valores de dichas fuerzas en el fondo marino, en el instante en que producen el máximo momento flector sobre la cimentación, se muestran en la tabla 10:

\begin{tabular}{|c|c|c|c|c|c|}
\hline & \multirow[b]{2}{*}{ Parámetro } & \multicolumn{2}{|c|}{ Método Avanzado } & \multicolumn{2}{|c|}{ Método tradicional } \\
\hline & & $\begin{array}{l}\mathrm{T}^{\mathrm{a}}=10 \mathrm{C} \\
\mathrm{S}=0 \%\end{array}$ & $\begin{array}{c}\mathrm{T}^{\mathrm{a}}=22^{\circ} \mathrm{C}, \\
\mathrm{S}=35 \%\end{array}$ & $\begin{array}{c}\mathrm{T}^{\mathrm{a}}=19 \mathrm{C} \\
\mathrm{S}=0 \%\end{array}$ & $\begin{array}{c}\mathrm{T}^{\mathrm{a}}=22{ }^{\circ} \mathrm{C}, \\
\mathrm{S}=35 \%\end{array}$ \\
\hline \multirow{6}{*}{$\begin{array}{l}\text { 응 } \\
\text { 인 } \\
\text { 인 }\end{array}$} & $\begin{array}{l}\text { Máximo momento flector } \\
(\mathrm{kN} \cdot \mathrm{m})\end{array}$ & 114,959 & 114,897 & 157,472 & 157,554 \\
\hline & Máxima fuerza de inercia (N) & 166,6857 & 149,5932 & 70,0103 & 65,7736 \\
\hline & $\begin{array}{l}\text { Máxima fuerza de arrastre } \\
\text { (N) }\end{array}$ & 300,8977 & 304,4979 & 440,4148 & 440,7365 \\
\hline & Máxima fuerza no lineal (N) & $-7,4075$ & $-7,2464$ & 0,0 & 0,0 \\
\hline & $\begin{array}{l}\text { Máxima fuerza de difracción } \\
\text { (N) }\end{array}$ & 0,0 & 0,0 & 0,0 & 0,0 \\
\hline & Máxima fuerza total (N) & 460,1758 & 446,8447 & 510,4251 & 506,5101 \\
\hline
\end{tabular}


Por último, se adjuntan en la tabla 11, los valores característicos del número de KeuleganCarpenter $(\mathrm{KC})$, del número de Reynolds $(\mathrm{Re})$ y el parámetro beta de Sarpkaya $(\beta)$ :

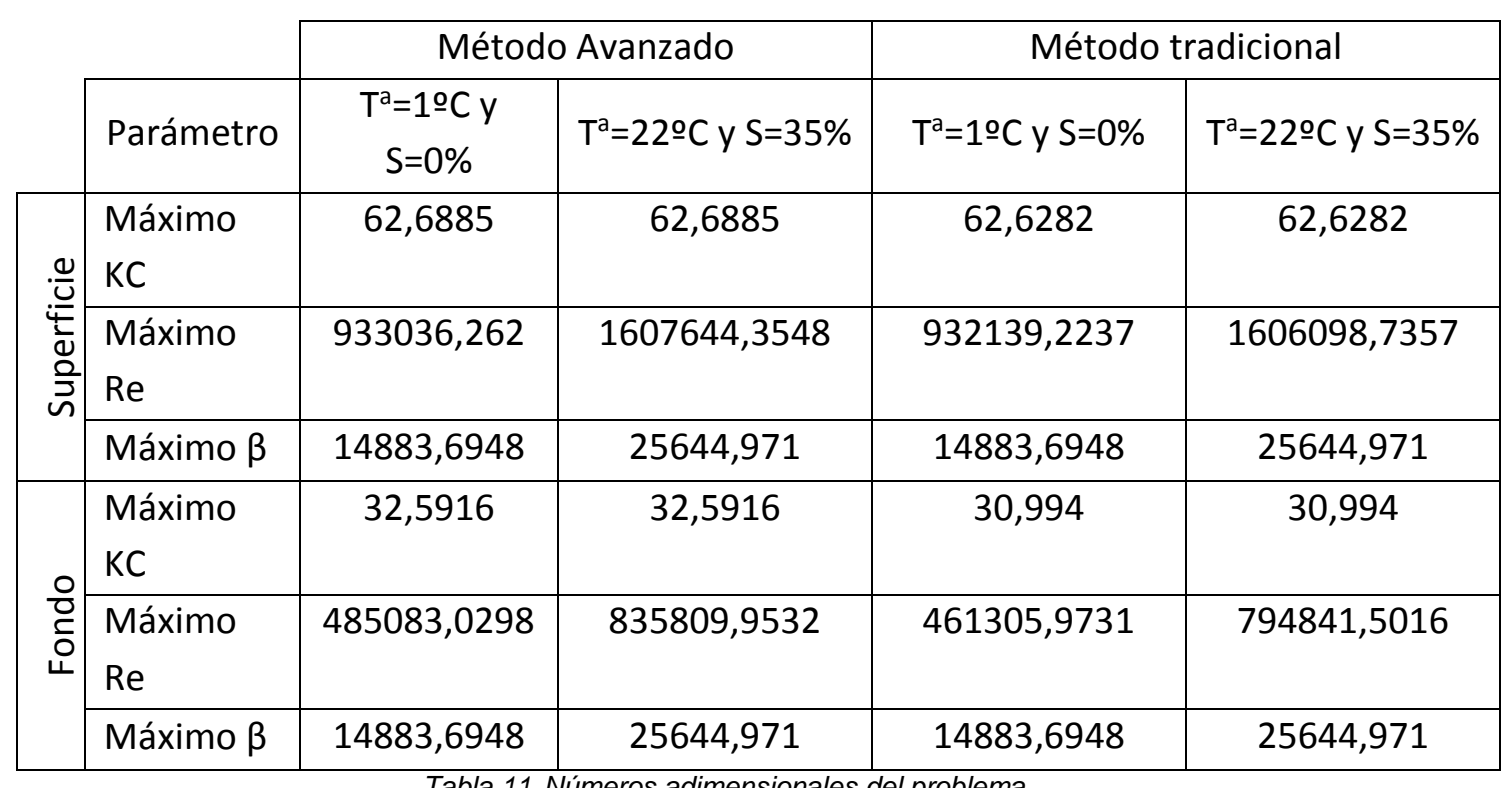




\subsubsection{Caso 1B}

Los datos de entrada se corresponden con el caso de cálculo para el parque eólico offshore North Hoyle en Reino Unido: altura de ola significativa $4,78 \mathrm{~m}$, profundidad $10,0 \mathrm{~m}$ y periodo de pico 6,8 s. De acuerdo con estos datos, en la figura 34 se muestra la distribución de fuerzas hidrodinámicas, generadas por el oleaje, en el instante en que producen el máximo momento flector, sobre la base del pilote, mostrando el cálculo según el método avanzado a la izquierda y según el método tradicional, a la derecha.
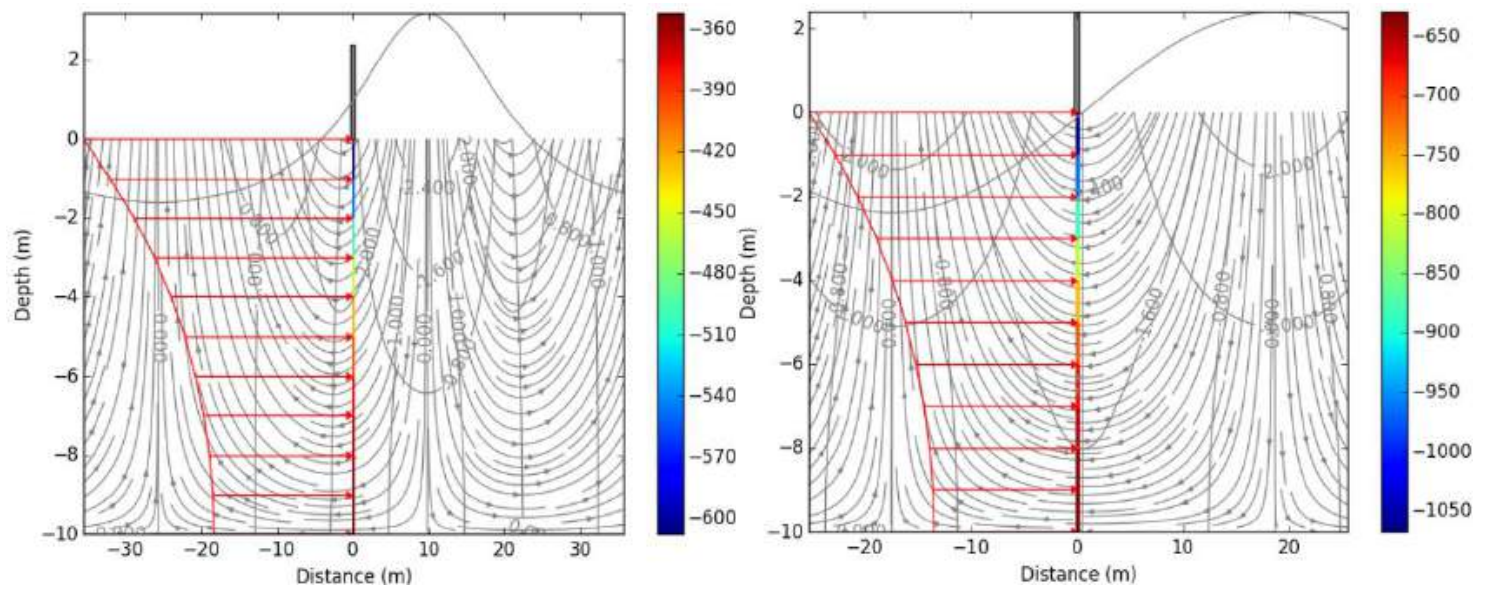

Figura 34. Momento flector máximo para $\mathrm{H}=4,78 \mathrm{~m}, h=10,0 \mathrm{~m}, \mathrm{~T}=6,8 \mathrm{~s}$ y $\mathrm{D}=0,5 \mathrm{~m}$

Los valores más característicos de la simulación, tanto para el caso de temperatura de 1 으 y salinidad de $0 \%$, como para el caso de temperatura de $22{ }^{\circ} \mathrm{C}$ y salinidad del $35 \%$, se muestran, a continuación, en la tabla 12:

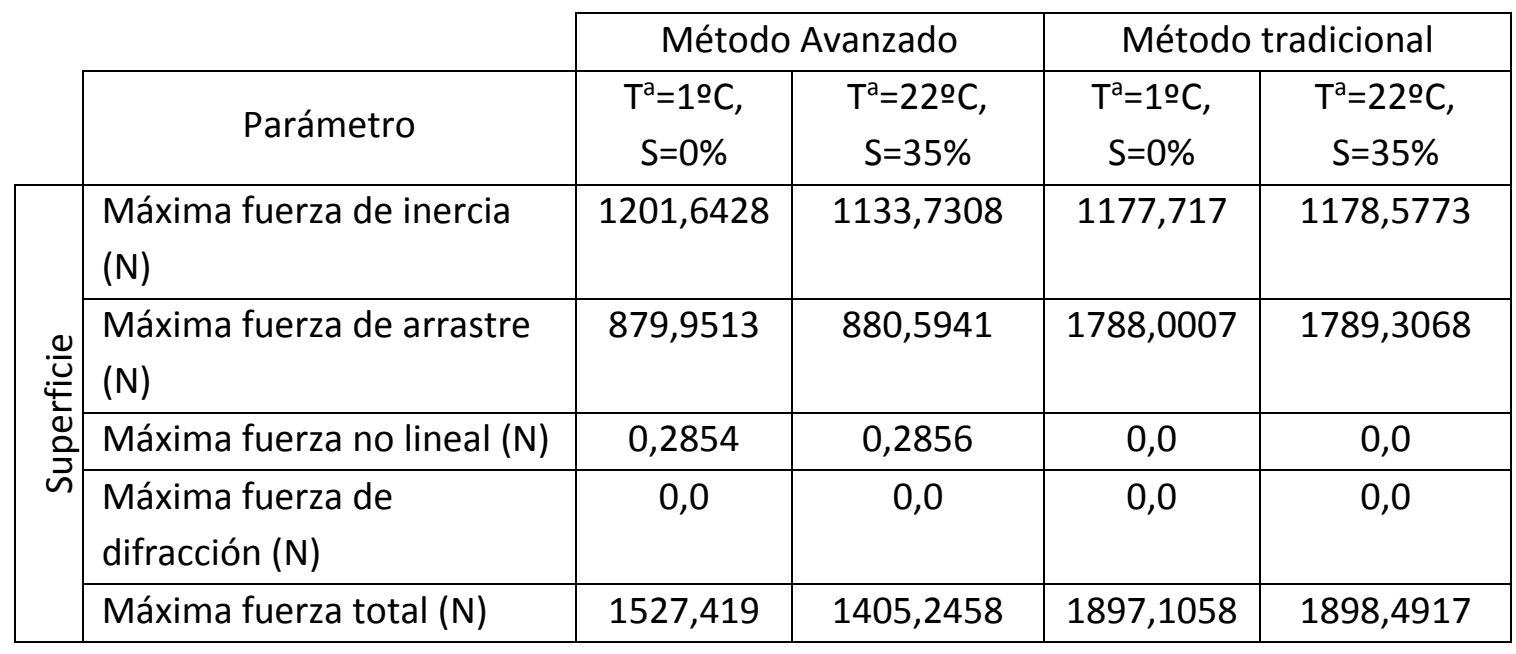




\begin{tabular}{|c|c|c|c|c|c|}
\hline \multirow{5}{*}{$\begin{array}{l}\frac{0}{0} \\
\text { 둥 }\end{array}$} & $\begin{array}{l}\text { Máxima fuerza de inercia } \\
\text { (N) }\end{array}$ & 663,3856 & 663,3183 & 628,644 & 629,1032 \\
\hline & $\begin{array}{l}\text { Máxima fuerza de arrastre } \\
\text { (N) }\end{array}$ & 436,8887 & 437,2078 & 509,4427 & 509,8148 \\
\hline & Máxima fuerza no lineal (N) & 21,7239 & 21,7398 & 0,0 & 0,0 \\
\hline & $\begin{array}{l}\text { Máxima fuerza de } \\
\text { difracción (N) }\end{array}$ & 0,0 & 0,0 & 0,0 & 0,0 \\
\hline & Máxima fuerza total (N) & 831,0377 & 747,1541 & 722,1532 & 661,6697 \\
\hline
\end{tabular}

Los valores de dichas fuerzas en el fondo marino, en el instante en que producen el máximo momento flector sobre la cimentación, se muestran en la tabla 13:

\begin{tabular}{|c|c|c|c|c|c|}
\hline & & \multicolumn{2}{|c|}{ Método Avanzado } & \multicolumn{2}{|c|}{ Método tradicional } \\
\hline & Parámetro & $\begin{aligned} \mathrm{T}^{\mathrm{a}}=1 \text { 으, } \\
\mathrm{S}=0 \%\end{aligned}$ & $\begin{array}{c}\mathrm{T}^{\mathrm{a}}=22 \mathrm{o} \mathrm{C}, \\
\mathrm{S}=35 \%\end{array}$ & $\begin{array}{c}\mathrm{T}^{\mathrm{a}}=1 \mathrm{o} \mathrm{C} \\
\mathrm{S}=0 \%\end{array}$ & $\begin{array}{c}\mathrm{T}^{\mathrm{a}}=22 \mathrm{o} \mathrm{C}, \\
\mathrm{S}=35 \%\end{array}$ \\
\hline \multirow{6}{*}{$\begin{array}{l}\text { 응 } \\
\text { 운 }\end{array}$} & $\begin{array}{l}\text { Máximo momento flector } \\
(\mathrm{kN} \cdot \mathrm{m})\end{array}$ & 65,574 & 57,996 & 68,075 & 68,105 \\
\hline & Máxima fuerza de inercia (N) & 654,1357 & 663,3183 & 151,4012 & 144,1984 \\
\hline & $\begin{array}{l}\text { Máxima fuerza de arrastre } \\
\text { (N) }\end{array}$ & 188,5407 & 100,1125 & 461,8664 & 462,2038 \\
\hline & Máxima fuerza no lineal (N) & $-20,0263$ & $-16,2766$ & 0,0 & 0,0 \\
\hline & $\begin{array}{l}\text { Máxima fuerza de difracción } \\
\text { (N) }\end{array}$ & 0,0 & 0,0 & 0,0 & 0,0 \\
\hline & Máxima fuerza total (N) & 822,65 & 747,1541 & 613,2675 & 606,4022 \\
\hline
\end{tabular}

Por último, se adjuntan en la tabla 14, los valores característicos del número de KeuleganCarpenter (KC), del número de Reynolds (Re) y el parámetro beta de Sarpkaya $(\beta)$ : 
Modelos de predicción de esfuerzos hidrodinámicos y socavación aplicados a ingeniería offshore

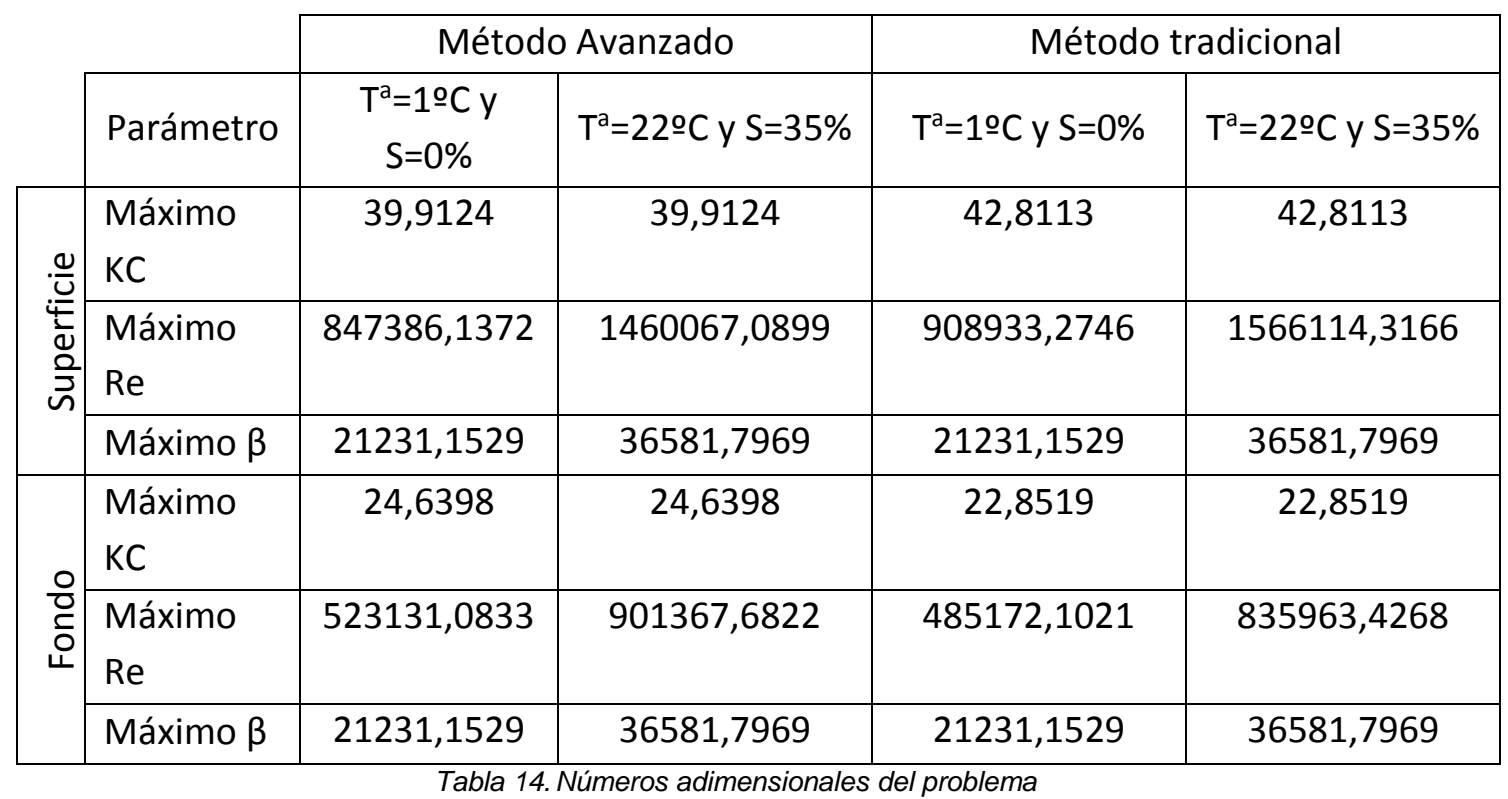




\subsubsection{Caso $1 \mathrm{C}$}

Los datos de entrada se corresponden con el caso de cálculo para el parque eólico offshore Egmond Aan Zee en Holanda: altura de ola significativa 3,6 m, profundidad 22,0 m y periodo de pico 18,5 s. De acuerdo con estos datos, en la figura 35 se muestra la distribución de fuerzas hidrodinámicas, generadas por el oleaje, en el instante en que producen el máximo momento flector, sobre la base del pilote, mostrando el cálculo según el método avanzado a la izquierda y según el método tradicional, a la derecha.
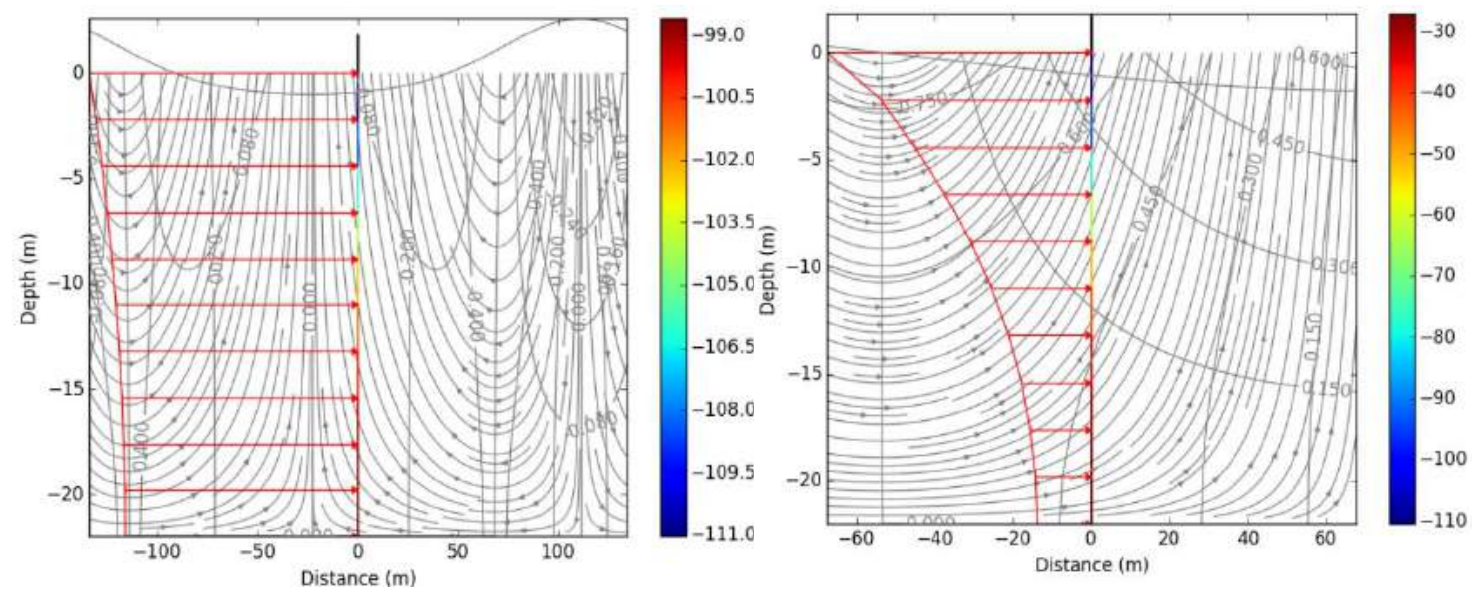

Figura 35. Momento flector máximo para $\mathrm{H}=3,6 \mathrm{~m}, h=22,0 \mathrm{~m}, \mathrm{~T}=18,5$ s y $D=0,5 \mathrm{~m}$

Los valores más característicos de la simulación, tanto para el caso de temperatura de 1 으 y salinidad de $0 \%$, como para el caso de temperatura de $22{ }^{\circ} \mathrm{C}$ y salinidad del $35 \%$, se muestran, a continuación, en la tabla 15:

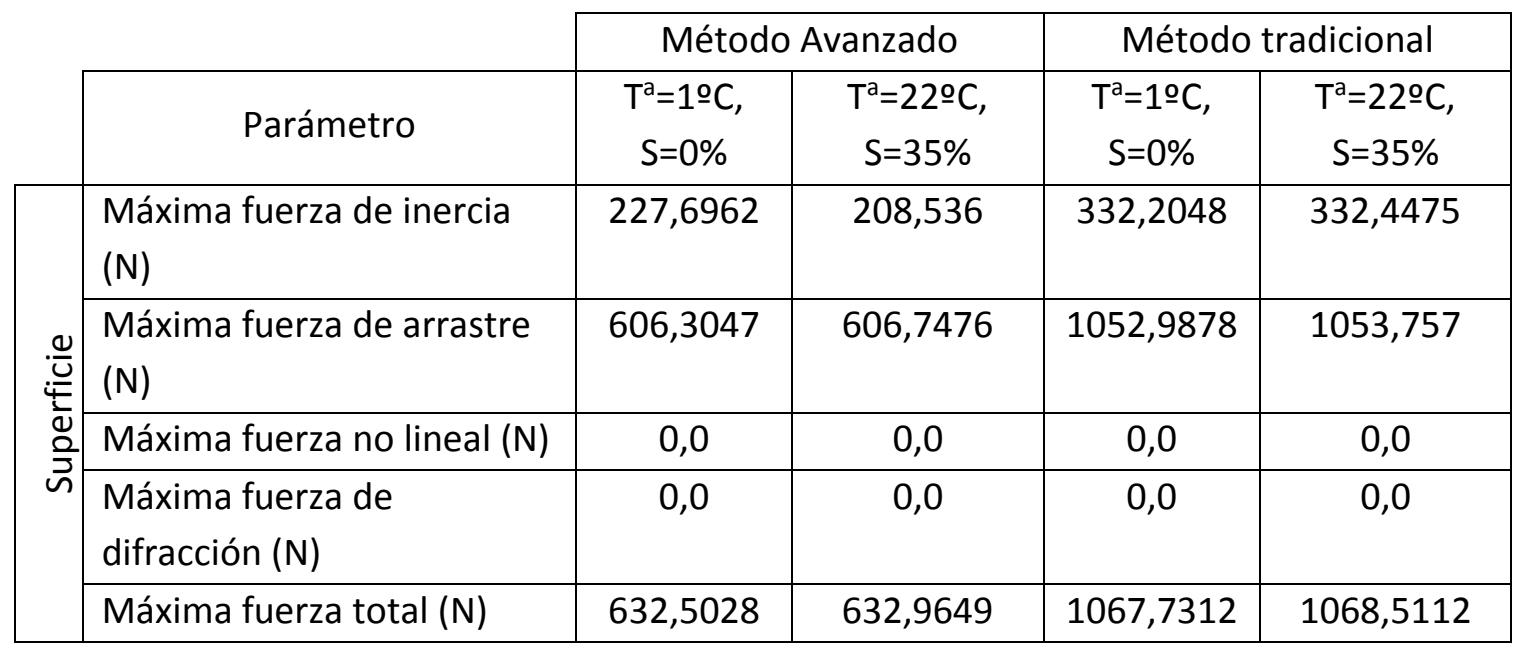




\begin{tabular}{|c|c|c|c|c|c|}
\hline \multirow{5}{*}{ 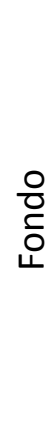 } & $\begin{array}{l}\text { Máxima fuerza de inercia } \\
\text { (N) }\end{array}$ & 164,9282 & 162,3572 & 211,6546 & 211,8092 \\
\hline & $\begin{array}{l}\text { Máxima fuerza de arrastre } \\
\text { (N) }\end{array}$ & 372,4412 & 372,7132 & 427,4319 & 427,7442 \\
\hline & Máxima fuerza no lineal $(\mathrm{N})$ & 0,0 & 0,0 & 0,0 & 0,0 \\
\hline & $\begin{array}{l}\text { Máxima fuerza de } \\
\text { difracción (N) }\end{array}$ & 0,0 & 0,0 & 0,0 & 0,0 \\
\hline & Máxima fuerza total (N) & 405,3718 & 391,3375 & 444,8321 & 442,4878 \\
\hline
\end{tabular}

Los valores de dichas fuerzas en el fondo marino, en el instante en que producen el máximo momento flector sobre la cimentación, se muestran en la tabla 16:

\begin{tabular}{|c|c|c|c|c|c|}
\hline & & \multicolumn{2}{|c|}{ Método Avanzado } & \multicolumn{2}{|c|}{ Método tradicional } \\
\hline & Parámetro & $\begin{array}{c}\mathrm{T}^{\mathrm{a}}=1 \mathrm{O} \mathrm{C} \\
\mathrm{S}=0 \%\end{array}$ & $\begin{array}{c}\mathrm{T}^{\mathrm{a}}=22{ }^{\circ} \mathrm{C}, \\
\mathrm{S}=35 \%\end{array}$ & $\begin{array}{c}\mathrm{T}^{\mathrm{a}}=1 \mathrm{O} \mathrm{C} \\
\mathrm{S}=0 \%\end{array}$ & $\begin{array}{c}\mathrm{T}^{\mathrm{a}}=22{ }^{\circ} \mathrm{C}, \\
\mathrm{S}=35 \%\end{array}$ \\
\hline \multirow{6}{*}{$\begin{array}{l}0 \\
\frac{0}{0} \\
\text { 인 } \\
\text { ㄴ. }\end{array}$} & $\begin{array}{l}\text { Máximo momento flector } \\
(\mathrm{kN} \cdot \mathrm{m})\end{array}$ & 63,139 & 62,938 & 91,696 & 91,738 \\
\hline & Máxima fuerza de inercia $(\mathrm{N})$ & 37,418 & 33,2916 & 24,1617 & 22,3876 \\
\hline & $\begin{array}{l}\text { Máxima fuerza de arrastre } \\
\text { (N) }\end{array}$ & 357,4864 & 357,7475 & 418,947 & 419,253 \\
\hline & Máxima fuerza no lineal (N) & 0,0 & 0,0 & 0,0 & 0,0 \\
\hline & $\begin{array}{l}\text { Máxima fuerza de difracción } \\
\text { (N) }\end{array}$ & 0,0 & 0,0 & 0,0 & 0,0 \\
\hline & Máxima fuerza total (N) & 394,9044 & 391,0391 & 443,1087 & 441,6406 \\
\hline
\end{tabular}

Por último, se adjuntan en la tabla 17, los valores característicos del número de KeuleganCarpenter (KC), del número de Reynolds (Re) y el parámetro beta de Sarpkaya $(\beta)$ : 
Modelos de predicción de esfuerzos hidrodinámicos y socavación aplicados a ingeniería offshore

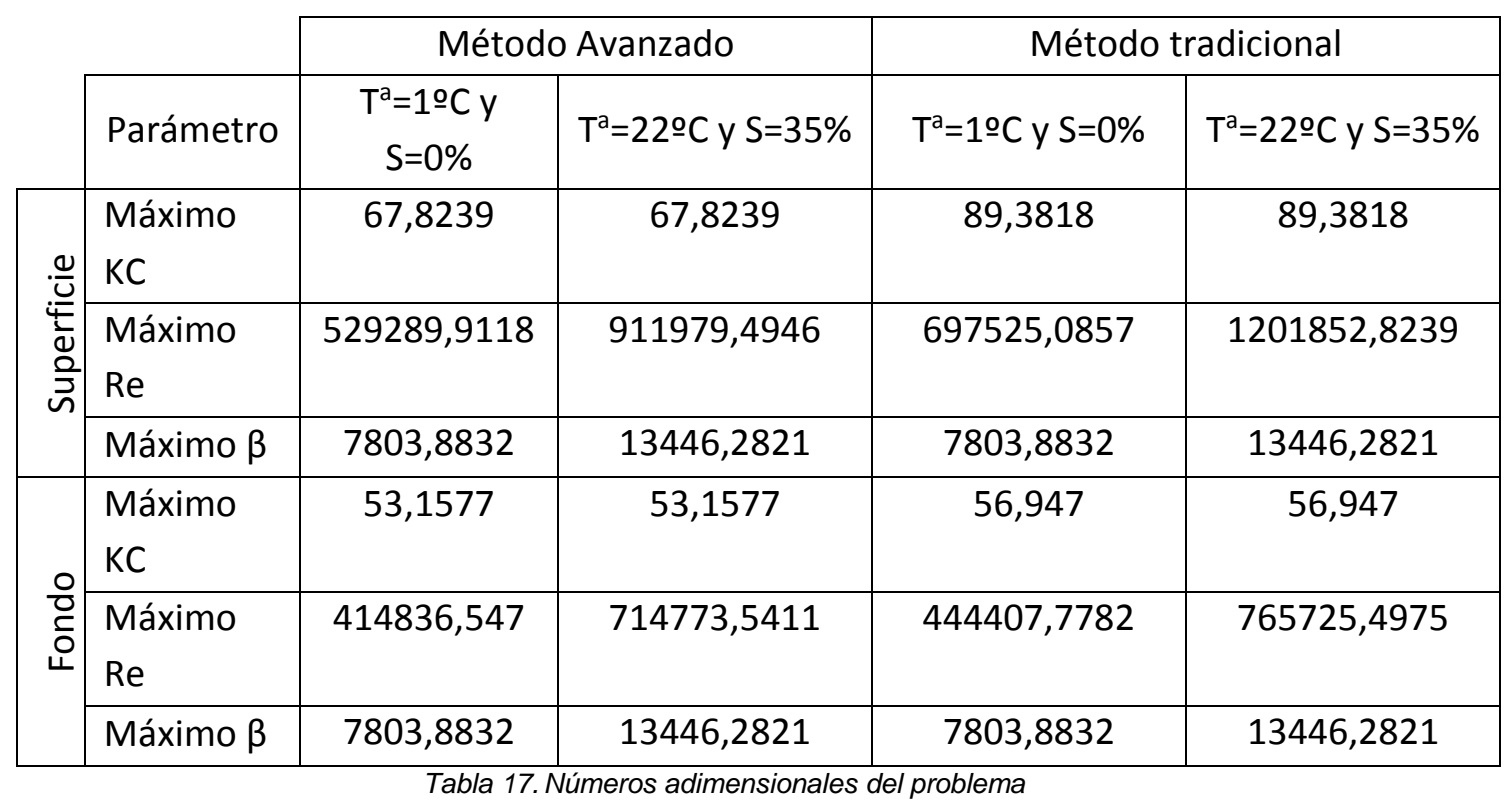




\subsubsection{Caso 1D}

Los datos de entrada se corresponden con el caso de cálculo para el parque eólico offshore Thornton Bank en Bélgica: altura de ola significativa $6,32 \mathrm{~m}$, profundidad $16,0 \mathrm{~m}$ y periodo de pico 11,06 s. De acuerdo con estos datos, en la figura 36 se muestra la distribución de fuerzas hidrodinámicas, generadas por el oleaje, en el instante en que producen el máximo momento flector, sobre la base del pilote, mostrando el cálculo según el método avanzado a la izquierda y según el método tradicional, a la derecha.
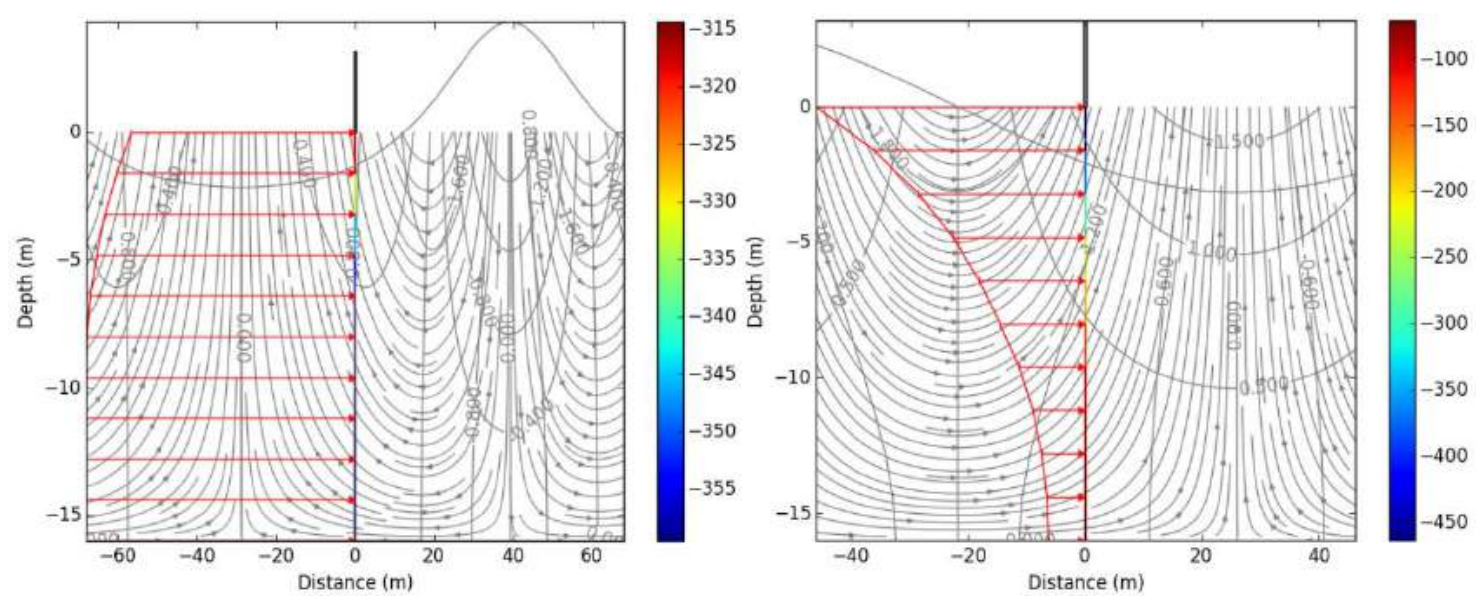

Figura 36. Momento flector máximo para $H=6,32 m, h=16 \mathrm{~m}, T=11,06$ s y $D=0,5 \mathrm{~m}$

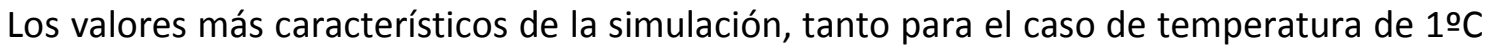
y salinidad de $0 \%$, como para el caso de temperatura de $22^{\circ} \mathrm{C}$ y salinidad del $35 \%$, se muestran, a continuación, en la tabla 18:

\begin{tabular}{|c|c|c|c|c|c|}
\hline & \multirow[b]{3}{*}{ Parámetro } & \multirow{2}{*}{\multicolumn{2}{|c|}{ Método Avanzado }} & \multirow{2}{*}{\multicolumn{2}{|c|}{ Método tradicional }} \\
\hline & & & & & \\
\hline & & 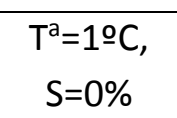 & $\begin{array}{c}\mathrm{T}^{\mathrm{a}}=22^{\circ} \mathrm{C} \\
\mathrm{S}=35 \%\end{array}$ & $\begin{array}{c}\mathrm{T}^{\mathrm{a}}=19 \mathrm{C} \\
\mathrm{S}=0 \%\end{array}$ & $\begin{array}{c}\mathrm{T}^{\mathrm{a}}=22^{\circ} \mathrm{C} \\
\mathrm{S}=35 \%\end{array}$ \\
\hline \multirow{5}{*}{ 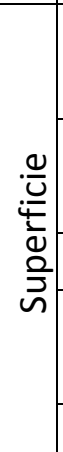 } & $\begin{array}{l}\text { Máxima fuerza de inercia } \\
\text { (N) }\end{array}$ & 784,4409 & 727,5313 & 855,6372 & 856,2622 \\
\hline & $\begin{array}{l}\text { Máxima fuerza de arrastre } \\
\text { (N) }\end{array}$ & 581,5026 & 581,9274 & 2496,6491 & 2498,4729 \\
\hline & Máxima fuerza no lineal (N) & 3,142 & 3,1443 & 0,0 & 0,0 \\
\hline & $\begin{array}{l}\text { Máxima fuerza de } \\
\text { difracción }(\mathrm{N})\end{array}$ & 0,0 & 0,0 & 0,0 & 0,0 \\
\hline & Máxima fuerza total (N) & 991,234 & 962,4601 & 2537,8269 & 2539,6808 \\
\hline
\end{tabular}




\begin{tabular}{|c|c|c|c|c|c|}
\hline \multirow{5}{*}{$\begin{array}{l}\frac{0}{0} \\
\text { 둥 }\end{array}$} & $\begin{array}{l}\text { Máxima fuerza de inercia } \\
\text { (N) }\end{array}$ & 501,5597 & 493,8266 & 516,6377 & 517,0151 \\
\hline & $\begin{array}{l}\text { Máxima fuerza de arrastre } \\
\text { (N) }\end{array}$ & 351,2595 & 351,5161 & 910,2284 & 910,8933 \\
\hline & Máxima fuerza no lineal (N) & 9,1154 & 9,122 & 0,0 & 0,0 \\
\hline & $\begin{array}{l}\text { Máxima fuerza de } \\
\text { difracción }(\mathrm{N})\end{array}$ & 0,0 & 0,0 & 0,0 & 0,0 \\
\hline & Máxima fuerza total (N) & 632,2247 & 561,1435 & 951,4615 & 952,1565 \\
\hline
\end{tabular}

Los valores de dichas fuerzas en el fondo marino, en el instante en que producen el máximo momento flector sobre la cimentación, se muestran en la tabla 19:

\begin{tabular}{|c|c|c|c|c|c|}
\hline & & \multicolumn{2}{|c|}{ Método Avanzado } & \multicolumn{2}{|c|}{ Método tradicional } \\
\hline & Parámetro & $\begin{array}{l}\mathrm{T}^{\mathrm{a}}=1 \mathrm{o} \mathrm{C} \\
\mathrm{S}=0 \%\end{array}$ & $\begin{array}{c}\mathrm{T}^{\mathrm{a}}=22{ }^{\circ} \mathrm{C}, \\
\mathrm{S}=35 \%\end{array}$ & $\begin{array}{c}\mathrm{T}^{\mathrm{a}}=1 \mathrm{O} \mathrm{C}, \\
\mathrm{S}=0 \%\end{array}$ & $\begin{array}{c}\mathrm{T}^{\mathrm{a}}=22{ }^{\circ} \mathrm{C}, \\
\mathrm{S}=35 \%\end{array}$ \\
\hline \multirow{6}{*}{$\begin{array}{l}\text { 응 } \\
\text { 운 }\end{array}$} & $\begin{array}{l}\text { Máximo momento flector } \\
(\mathrm{kN} \cdot \mathrm{m})\end{array}$ & 72,343 & 66,803 & 153,344 & 153,456 \\
\hline & Máxima fuerza de inercia (N) & 501,2522 & 298,3257 & 57,389 & 57,4309 \\
\hline & $\begin{array}{l}\text { Máxima fuerza de arrastre } \\
\text { (N) }\end{array}$ & 138,266 & 246,9521 & 890,2656 & 890,9159 \\
\hline & Máxima fuerza no lineal (N) & $-8,2574$ & $-8,3406$ & 0,0 & 0,0 \\
\hline & $\begin{array}{l}\text { Máxima fuerza de difracción } \\
\text { (N) }\end{array}$ & 0,0 & 0,0 & 0,0 & 0,0 \\
\hline & Máxima fuerza total (N) & 631,2608 & 536,9372 & 947,6545 & 948,3468 \\
\hline
\end{tabular}

Por último, se adjuntan en la tabla 20, los valores característicos del número de KeuleganCarpenter $(\mathrm{KC})$, del número de Reynolds $(\mathrm{Re})$ y el parámetro beta de Sarpkaya $(\beta)$ : 


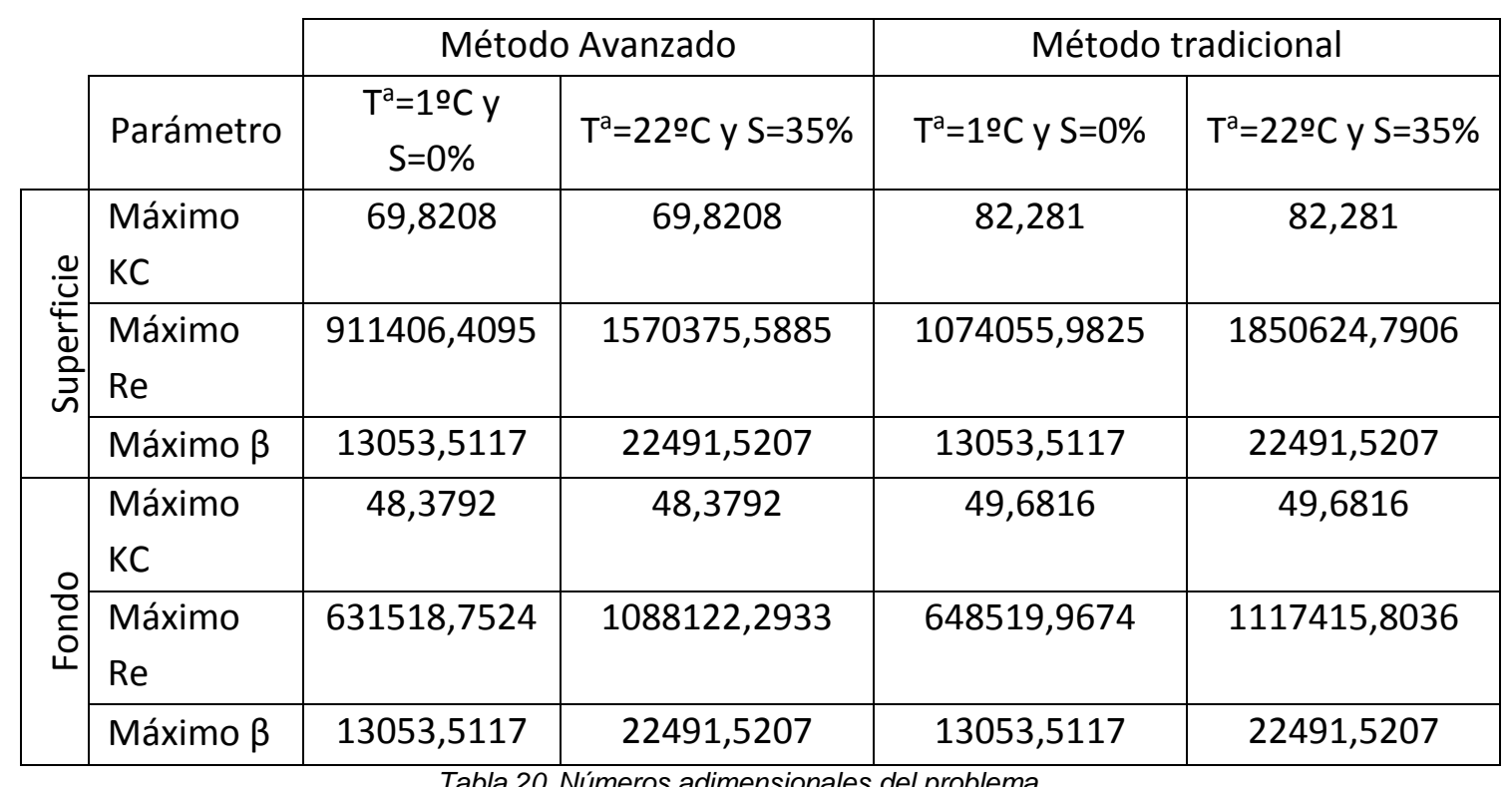

Tabla 20. Números adimensionales del problema 


\subsection{Caso 2: Diámetros igual a 4,0 m}

Se va a evaluar el caso de pilotes de 4,0 m de diámetro mediante los cuatro casos de oleaje definidos en el apartado anterior, calculados según la teoría lineal y según las teorías no lineales, tanto para el caso de una temperatura del agua de 1 으 $\mathrm{C}$ y salinidad del $0 \%$, como para el caso de una temperatura de 22 ㅇ C y salinidad del $35 \%$.

\subsubsection{Caso $2 \mathrm{~A}$}

Los datos de entrada se corresponden con el caso de cálculo para el parque eólico offshore Princess Amalia Q7 en Holanda: altura de ola significativa 7,7 m, profundidad 24,0 m y periodo de pico 9,7 s. De acuerdo con estos datos, en la figura 37 se muestra la distribución de fuerzas hidrodinámicas, generadas por el oleaje, en el instante en que producen el máximo momento flector, sobre la base del pilote, mostrando el cálculo según el método avanzado a la izquierda y según el método tradicional, a la derecha.
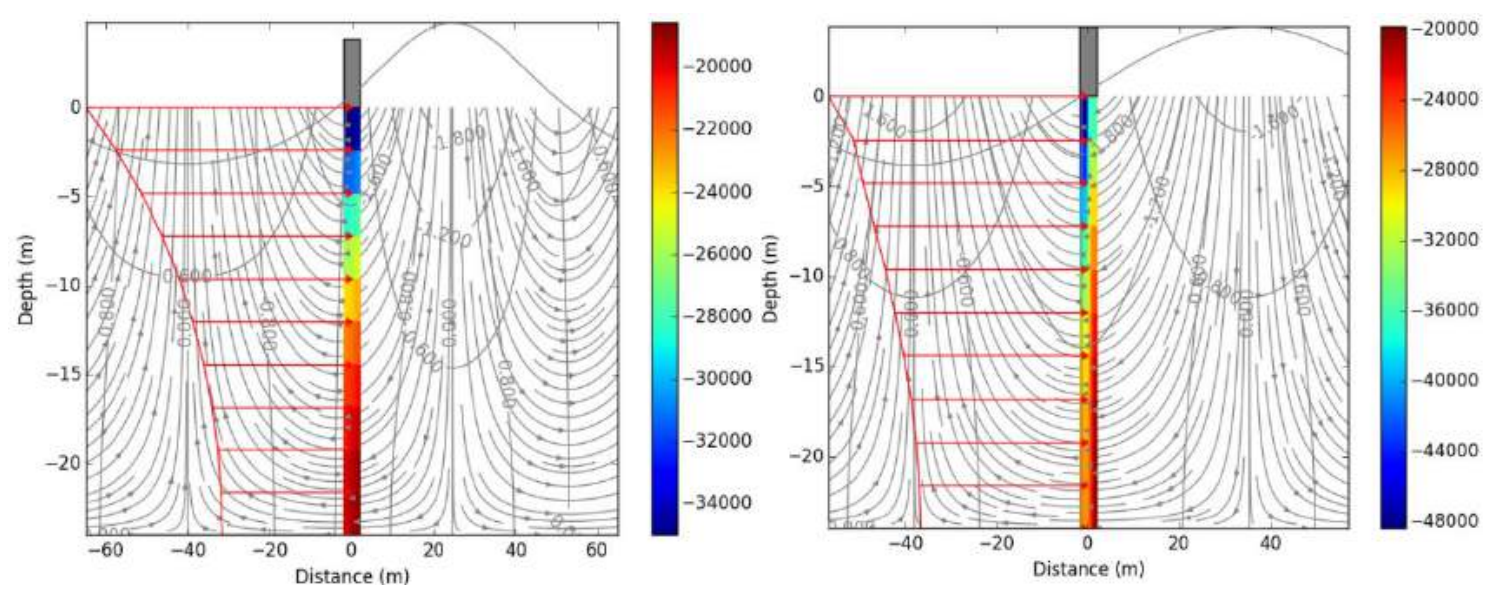

Figura 37. Momento flector máximo para $\mathrm{H}=7,7 \mathrm{~m}, \mathrm{~h}=24,0 \mathrm{~m}, \mathrm{~T}=9,7 \mathrm{~s}$ y $\mathrm{D}=4,0 \mathrm{~m}$

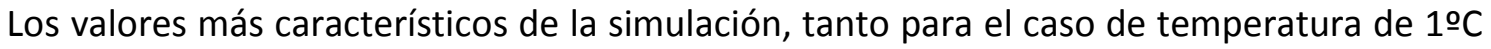
y salinidad de $0 \%$, como para el caso de temperatura de $22{ }^{\circ} \mathrm{C}$ y salinidad del $35 \%$, se muestran, a continuación, en la tabla 21: 


\begin{tabular}{|c|c|c|c|c|c|}
\hline & \multirow[b]{2}{*}{ Parámetro } & \multicolumn{2}{|c|}{ Método Avanzado } & \multicolumn{2}{|c|}{ Método tradicional } \\
\hline & & $\begin{array}{c}\mathrm{T}^{\mathrm{a}}=1 \mathrm{O} \mathrm{C} \\
\mathrm{S}=0 \%\end{array}$ & $\begin{array}{c}\mathrm{T}^{\mathrm{a}}=22 \mathrm{o} C, \\
\mathrm{~S}=35 \%\end{array}$ & $\begin{array}{c}\mathrm{T}^{\mathrm{a}}=1 \mathrm{O} \mathrm{C}, \\
\mathrm{S}=0 \%\end{array}$ & $\begin{array}{c}\mathrm{T}^{\mathrm{a}}=22 \mathrm{o} C, \\
\mathrm{~S}=35 \%\end{array}$ \\
\hline \multirow{5}{*}{ 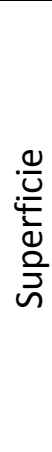 } & $\begin{array}{l}\text { Máxima fuerza de inercia } \\
(\mathrm{kN})\end{array}$ & 50,8347 & 50,6141 & 54,1884 & 54,228 \\
\hline & $\begin{array}{l}\text { Máxima fuerza de arrastre } \\
(\mathrm{kN})\end{array}$ & 8,9775 & 8,9841 & 15,0437 & 15,0547 \\
\hline & Máxima fuerza no lineal (kN) & 0,6683 & 0,6688 & 0,0 & 0,0 \\
\hline & $\begin{array}{l}\text { Máxima fuerza de difracción } \\
(\mathrm{kN})\end{array}$ & 0,0 & 0,0 & 0,0 & 0,0 \\
\hline & Máxima fuerza total (kN) & 51,445 & 51,1498 & 54,1887 & 54,2283 \\
\hline \multirow{5}{*}{ 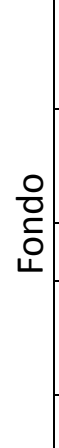 } & $\begin{array}{l}\text { Máxima fuerza de inercia } \\
(\mathrm{kN})\end{array}$ & 27,2429 & 27,1957 & 26,8173 & 26,8369 \\
\hline & $\begin{array}{l}\text { Máxima fuerza de arrastre } \\
(\mathrm{kN})\end{array}$ & 2,8984 & 2,9005 & 3,6844 & 3,6871 \\
\hline & Máxima fuerza no lineal (kN) & 0,6318 & 0,6322 & 0,0 & 0,0 \\
\hline & $\begin{array}{l}\text { Máxima fuerza de difracción } \\
(\mathrm{kN})\end{array}$ & 0,0 & 0,0 & 0,0 & 0,0 \\
\hline & Máxima fuerza total (kN) & 27,1534 & 27,1169 & 26,8173 & 26,8369 \\
\hline
\end{tabular}

Los valores de dichas fuerzas en el fondo marino, en el instante en que producen el máximo momento flector sobre la cimentación, se muestran en la tabla 22:

\begin{tabular}{|c|c|c|c|c|c|}
\hline & \multirow[b]{2}{*}{ Parámetro } & \multicolumn{2}{|c|}{ Método Avanzado } & \multicolumn{2}{|c|}{ Método tradicional } \\
\hline & & $\begin{array}{c}\mathrm{T}^{\mathrm{a}}=1 \mathrm{o} \mathrm{C} \\
\mathrm{S}=0 \%\end{array}$ & $\begin{array}{c}\mathrm{T}^{\mathrm{a}}=22{ }^{\circ} \mathrm{C}, \\
\mathrm{S}=35 \%\end{array}$ & $\begin{array}{c}\mathrm{T}^{\mathrm{a}}=1 \mathrm{o} \mathrm{C}, \\
\mathrm{S}=0 \%\end{array}$ & $\begin{array}{c}\mathrm{T}^{\mathrm{a}}=22{ }^{\circ} \mathrm{C}, \\
\mathrm{S}=35 \%\end{array}$ \\
\hline \multirow{6}{*}{$\begin{array}{l}\text { 응 } \\
\text { 운 }\end{array}$} & $\begin{array}{l}\text { Máximo momento flector } \\
(\mathrm{kN} \cdot \mathrm{m})\end{array}$ & 5187,079 & 4967,646 & 5452,54 & 5456,523 \\
\hline & $\begin{array}{l}\text { Máxima fuerza de inercia } \\
(\mathrm{kN})\end{array}$ & 26,4796 & 20,9946 & 26,8173 & 26,8369 \\
\hline & $\begin{array}{l}\text { Máxima fuerza de arrastre } \\
(\mathrm{kN})\end{array}$ & 0,0573 & 0,0445 & 0,0001 & 0,0001 \\
\hline & Máxima fuerza no lineal (kN) & $-0,1554$ & $-0,1555$ & 0,0 & 0,0 \\
\hline & $\begin{array}{l}\text { Máxima fuerza de difracción } \\
(\mathrm{kN})\end{array}$ & 0,0 & 0,0 & 0,0 & 0,0 \\
\hline & Máxima fuerza total (kN) & 26,3816 & 20,8836 & 26,8173 & 26,8369 \\
\hline
\end{tabular}

Tabla 22. Fuerzas hidrodinámicas para momento flector máximo, para $\mathrm{H}=7,7 \mathrm{~m}, h=24,0 \mathrm{~m}, T=9,7$ s y $D=4,0 \mathrm{~m}$ 
Por último, se adjuntan en la tabla 23, los valores característicos del número de KeuleganCarpenter (KC), del número de Reynolds (Re) y el parámetro beta de Sarpkaya $(\beta)$ :

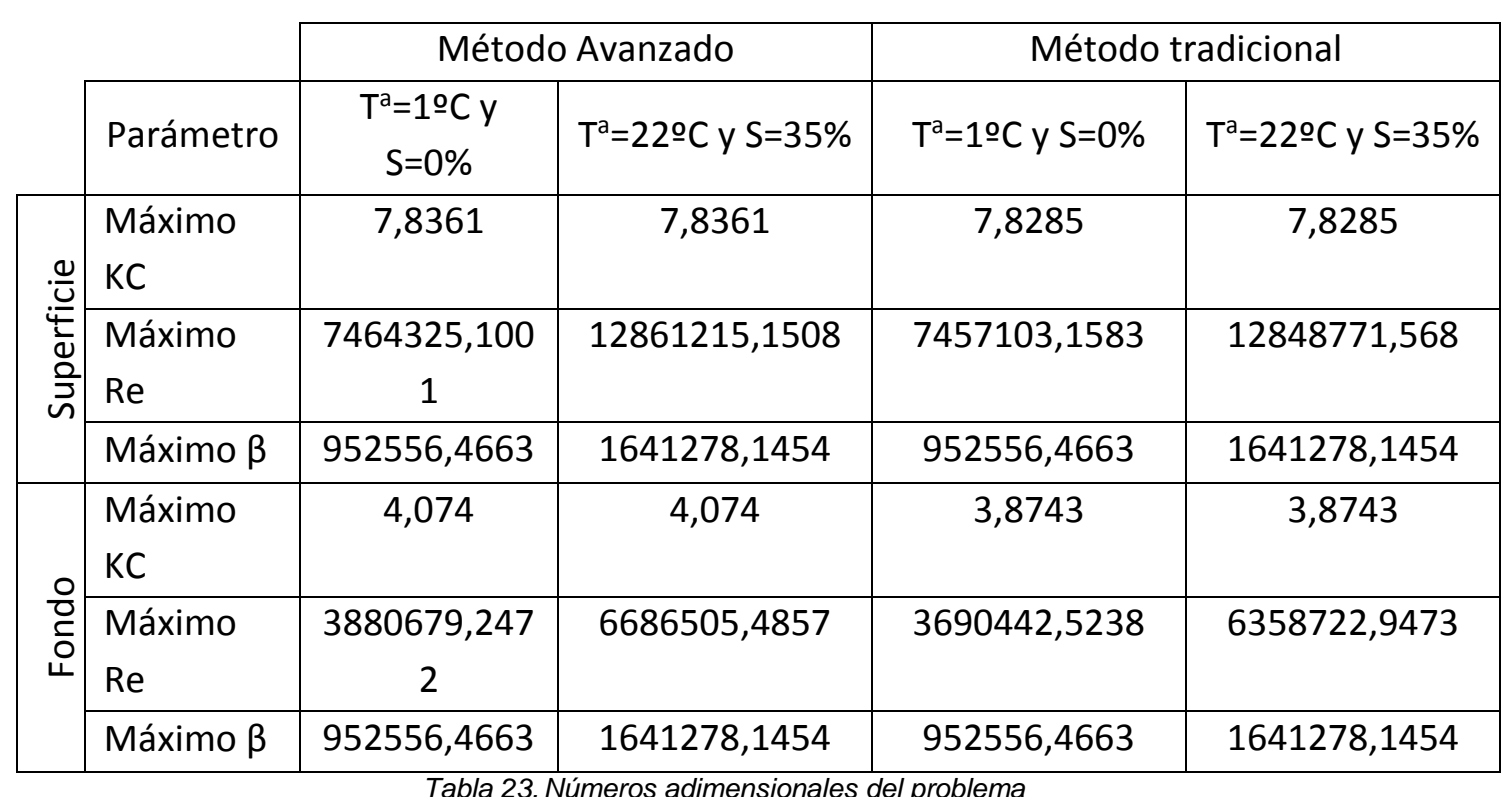




\subsubsection{Caso 2B}

Los datos de entrada se corresponden con el caso de cálculo para el parque eólico offshore North Hoyle en Reino Unido: altura de ola significativa $4,78 \mathrm{~m}$, profundidad $10,0 \mathrm{~m}$ y periodo de pico 6,8 s. De acuerdo con estos datos, en la figura 38 se muestra la distribución de fuerzas hidrodinámicas, generadas por el oleaje, en el instante en que producen el máximo momento flector, sobre la base del pilote, mostrando el cálculo según el método avanzado a la izquierda y según el método tradicional, a la derecha.
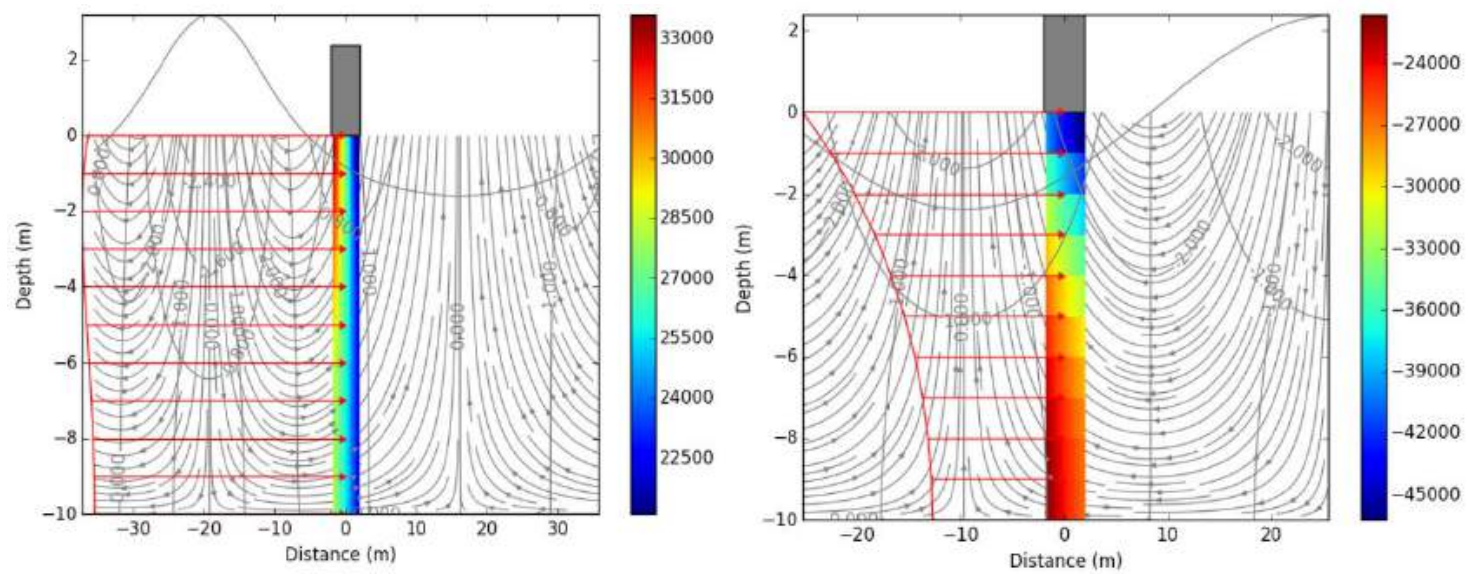

Figura 38. Momento flector máximo para $H=4,78 m, h=10,0 m, T=6,8$ s y $D=4,0 m$

Los valores más característicos de la simulación, tanto para el caso de temperatura de 1 으 y salinidad de $0 \%$, como para el caso de temperatura de $22{ }^{\circ} \mathrm{C}$ y salinidad del $35 \%$, se muestran, a continuación, en la tabla 24:

\begin{tabular}{|c|c|c|c|c|c|}
\hline & \multirow[b]{2}{*}{ Parámetro } & \multicolumn{2}{|c|}{ Método Avanzado } & \multicolumn{2}{|c|}{ Método tradicional } \\
\hline & & $\begin{array}{c}\mathrm{T}^{\mathrm{a}}=1 \mathrm{o} \mathrm{C} \\
\mathrm{S}=0 \%\end{array}$ & $\begin{array}{c}\mathrm{T}^{\mathrm{a}}=22 \mathrm{o} \mathrm{C}, \\
\mathrm{S}=35 \%\end{array}$ & $\begin{array}{c}\mathrm{T}^{\mathrm{a}}=1 \stackrel{\mathrm{o}}{ } \mathrm{C} \\
\mathrm{S}=0 \%\end{array}$ & $\begin{array}{c}\mathrm{T}^{\mathrm{a}}=22 \mathrm{o} \mathrm{C}, \\
\mathrm{S}=35 \%\end{array}$ \\
\hline \multirow{5}{*}{$\begin{array}{l}\frac{0}{u} \\
\frac{0}{2} \\
\frac{0}{3} \\
\tilde{n}\end{array}$} & $\begin{array}{l}\text { Máxima fuerza de inercia } \\
(\mathrm{kN})\end{array}$ & 65,4154 & 63,8774 & 75,3733 & 75,4283 \\
\hline & $\begin{array}{l}\text { Máxima fuerza de arrastre } \\
(\mathrm{kN})\end{array}$ & 7,0397 & 7,0449 & 14,3038 & 14,3142 \\
\hline & Máxima fuerza no lineal (kN) & 0,069 & 0,069 & 0,0 & 0,0 \\
\hline & $\begin{array}{l}\text { Máxima fuerza de difracción } \\
(\mathrm{kN})\end{array}$ & 0,0 & 0,0 & 0,0 & 0,0 \\
\hline & Máxima fuerza total (kN) & 66,23 & 64,7064 & 75,3737 & 75,4288 \\
\hline
\end{tabular}




\begin{tabular}{|c|l|c|c|c|c|}
\hline \multirow{2}{*}{$\begin{array}{l}\text { Máxima fuerza de inercia } \\
(\mathrm{kN})\end{array}$} & 40,4416 & 39,9421 & 40,2329 & 40,2623 \\
\cline { 2 - 5 } & $\begin{array}{l}\text { Máxima fuerza de arrastre } \\
\text { 을 }\end{array}$ & 3,4952 & 3,4977 & 4,0755 & 4,0785 \\
\hline \multirow{4}{*}{+} & Máxima fuerza no lineal (kN) & 1,3902 & 1,3913 & 0,0 & 0,0 \\
\cline { 2 - 6 } & $\begin{array}{l}\text { Máxima fuerza de difracción } \\
\text { (kN) }\end{array}$ & 0,0 & 0,0 & 0,0 & 0,0 \\
\cline { 2 - 6 } & Máxima fuerza total (kN) & 40,0863 & 39,6377 & 40,2328 & 40,2622 \\
\hline
\end{tabular}

Tabla 24. Fuerzas hidrodinámicas para el caso de $\mathrm{H}=4,78 \mathrm{~m}, h=10,0 \mathrm{~m}, T=6,8 \mathrm{~s}$ y $\mathrm{D}=4,0 \mathrm{~m}$

Los valores de dichas fuerzas en el fondo marino, en el instante en que producen el máximo momento flector sobre la cimentación, se muestran en la tabla 25:

\begin{tabular}{|c|c|c|c|c|c|}
\hline & \multirow[b]{2}{*}{ Parámetro } & \multicolumn{2}{|c|}{ Método Avanzado } & \multicolumn{2}{|c|}{ Método tradicional } \\
\hline & & $\begin{array}{c}\mathrm{T}^{\mathrm{a}}=1 \stackrel{\circ}{ } \mathrm{C}, \\
\mathrm{S}=0 \%\end{array}$ & $\begin{array}{c}\mathrm{T}^{\mathrm{a}}=22{ }^{\circ} \mathrm{C}, \\
\mathrm{S}=35 \%\end{array}$ & $\begin{array}{c}\mathrm{T}^{\mathrm{a}}=10 \mathrm{O}, \\
\mathrm{S}=0 \%\end{array}$ & $\begin{array}{c}\mathrm{T}^{\mathrm{a}}=22{ }^{\circ} \mathrm{C}, \\
\mathrm{S}=35 \%\end{array}$ \\
\hline \multirow{6}{*}{$\begin{array}{l}\text { 응 } \\
\frac{0}{0} \\
\text { 이 }\end{array}$} & $\begin{array}{l}\text { Máximo momento flector } \\
(\mathrm{kN} \cdot \mathrm{m})\end{array}$ & 2813,292 & 2611,092 & 3240,82 & 3243,188 \\
\hline & $\begin{array}{l}\text { Máxima fuerza de inercia } \\
(\mathrm{kN})\end{array}$ & 36,6727 & 30,7762 & 40,2324 & 40,2618 \\
\hline & $\begin{array}{l}\text { Máxima fuerza de arrastre } \\
(\mathrm{kN})\end{array}$ & 0,1958 & 0,1956 & 0,0004 & 0,0004 \\
\hline & Máxima fuerza no lineal (kN) & $-0,6077$ & $-0,6394$ & 0,0 & 0,0 \\
\hline & $\begin{array}{l}\text { Máxima fuerza de difracción } \\
(\mathrm{kN})\end{array}$ & 0,0 & 0,0 & 0,0 & 0,0 \\
\hline & Máxima fuerza total (kN) & 36,2608 & 30,3324 & 40,2328 & 40,2622 \\
\hline
\end{tabular}

Tabla 25. Fuerzas hidrodinámicas para momento flector máximo, para $H=4,78 m, h=10,0 m, T=6,8$ s y $D=4,0 m$

Por último, se adjuntan en la tabla 26, los valores característicos del número de KeuleganCarpenter $(\mathrm{KC})$, del número de Reynolds $(\mathrm{Re})$ y el parámetro beta de Sarpkaya $(\beta)$ : 
Modelos de predicción de esfuerzos hidrodinámicos y socavación aplicados a ingeniería offshore

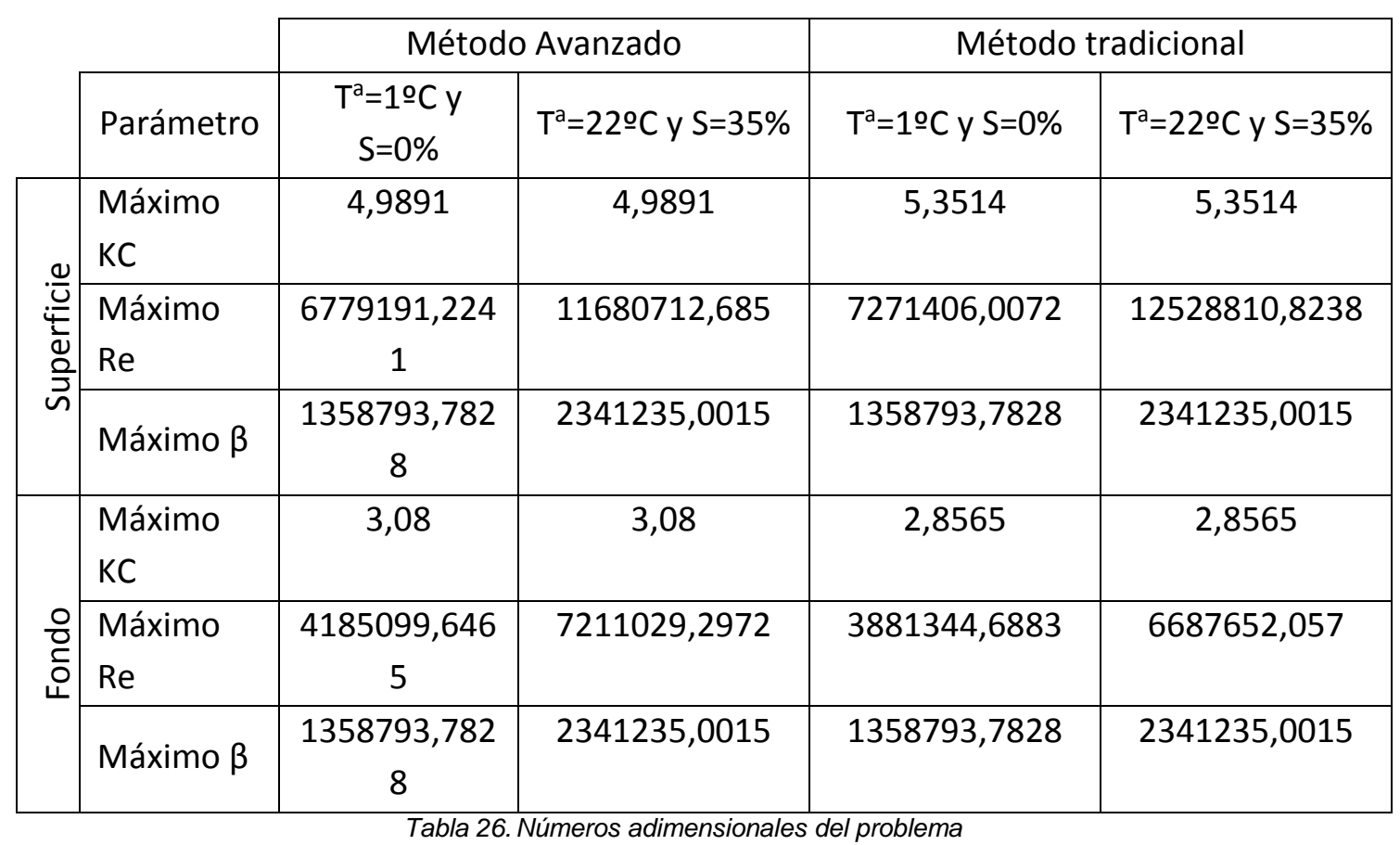




\subsubsection{Caso $2 \mathrm{C}$}

Los datos de entrada se corresponden con el caso de cálculo para el parque eólico offshore Egmond Aan Zee en Holanda: altura de ola significativa 3,6 m, profundidad 22,0 m y periodo de pico 18,5 s. De acuerdo con estos datos, en la figura 39 se muestra la distribución de fuerzas hidrodinámicas, generadas por el oleaje, en el instante en que producen el máximo momento flector, sobre la base del pilote, mostrando el cálculo según el método avanzado a la izquierda y según el método tradicional, a la derecha.
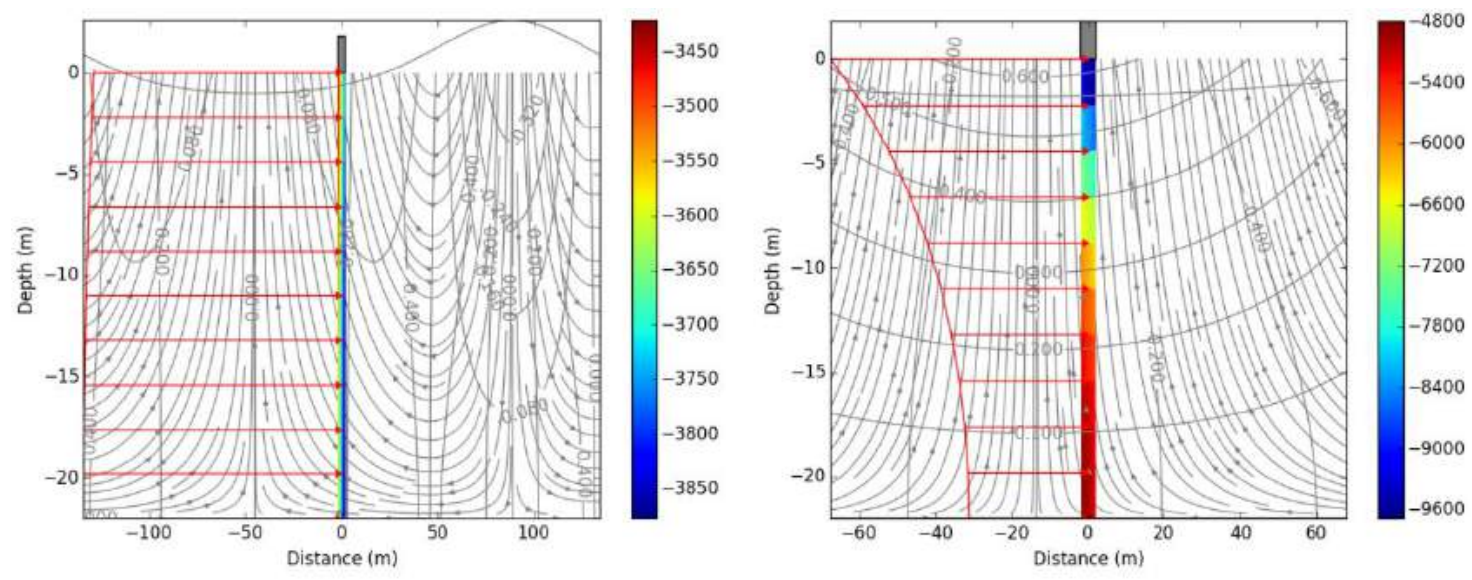

Figura 39. Momento flector máximo para $H=3,6 m, h=22,0 m, T=18,5$ s y $D=4,0 m$

Los valores más característicos de la simulación, tanto para el caso de temperatura de 1 ํㅡ y salinidad de $0 \%$, como para el caso de temperatura de $22{ }^{\circ} \mathrm{C}$ y salinidad del $35 \%$, se muestran, a continuación, en la tabla 27:

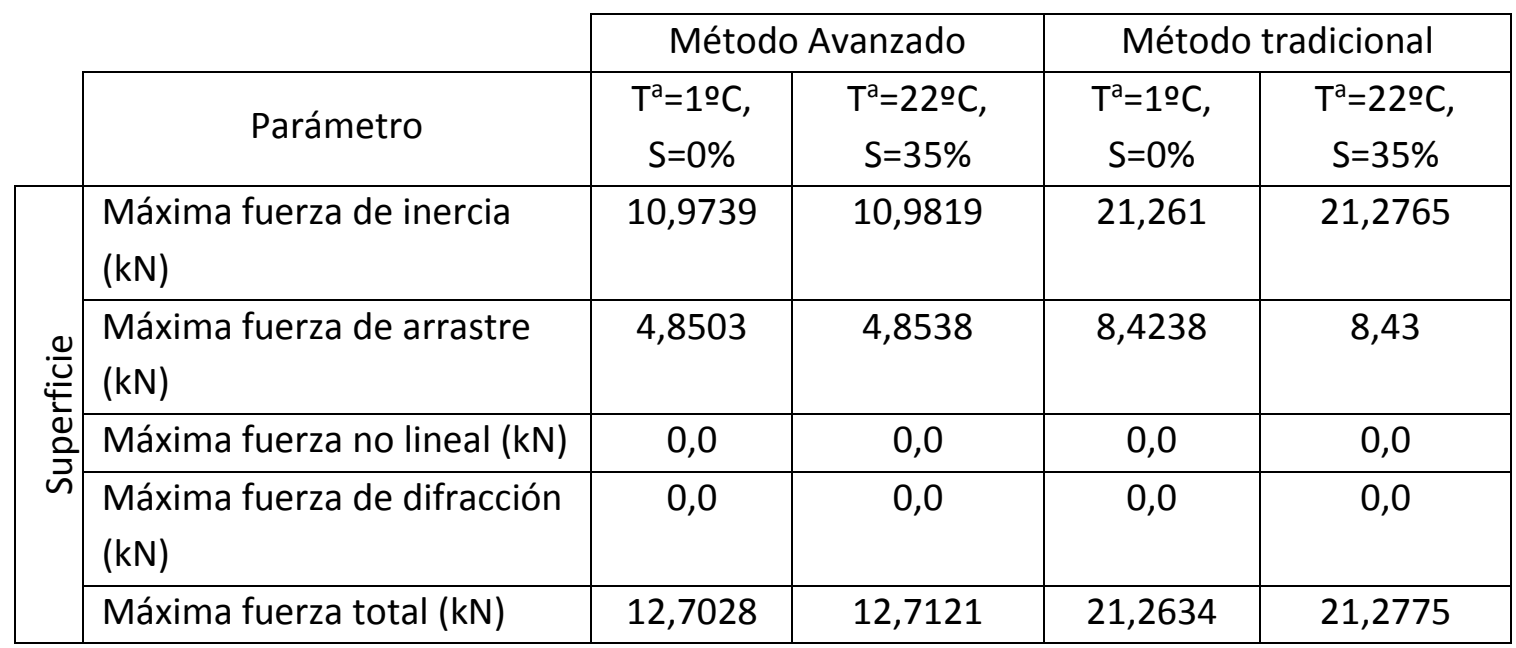




\begin{tabular}{|c|c|c|c|c|c|}
\hline \multirow{5}{*}{$\begin{array}{l}\text { 음 } \\
\text { 은 }\end{array}$} & $\begin{array}{l}\text { Máxima fuerza de inercia } \\
(\mathrm{kN})\end{array}$ & 8,6405 & 8,353 & 13,5458 & 13,5557 \\
\hline & $\begin{array}{l}\text { Máxima fuerza de arrastre } \\
(\mathrm{kN})\end{array}$ & 2,9795 & 2,9816 & 3,4194 & 3,4219 \\
\hline & Máxima fuerza no lineal (kN) & 0,0 & 0,0 & 0,0 & 0,0 \\
\hline & $\begin{array}{l}\text { Máxima fuerza de difracción } \\
(\mathrm{kN})\end{array}$ & 0,0 & 0,0 & 0,0 & 0,0 \\
\hline & Máxima fuerza total (kN) & 8,7335 & 8,7399 & 13,5459 & 13,5558 \\
\hline
\end{tabular}

Tabla 27. Fuerzas hidrodinámicas para el caso de $\mathrm{H}=3,6 \mathrm{~m}, \mathrm{~h}=22,0 \mathrm{~m}, \mathrm{~T}=18,5 \mathrm{~s}$ y $\mathrm{D}=4,0 \mathrm{~m}$

Los valores de dichas fuerzas en el fondo marino, en el instante en que producen el máximo momento flector sobre la cimentación, se muestran en la tabla 28:

\begin{tabular}{|c|c|c|c|c|c|}
\hline & & \multicolumn{2}{|c|}{ Método Avanzado } & \multicolumn{2}{|c|}{ Método tradicional } \\
\hline & Parámetro & $\begin{array}{c}\mathrm{T}^{\mathrm{a}}=1 \mathrm{O} \mathrm{C}, \\
\mathrm{S}=0 \%\end{array}$ & $\begin{array}{c}\mathrm{T}^{\mathrm{a}}=22{ }^{\circ} \mathrm{C}, \\
\mathrm{S}=35 \%\end{array}$ & $\begin{array}{c}\mathrm{T}^{\mathrm{a}}=1 \mathrm{O} \mathrm{C}, \\
\mathrm{S}=0 \%\end{array}$ & $\begin{array}{c}\mathrm{T}^{\mathrm{a}}=22{ }^{\circ} \mathrm{C}, \\
\mathrm{S}=35 \%\end{array}$ \\
\hline \multirow{6}{*}{$\begin{array}{l}\frac{0}{0} \\
\frac{1}{0} \\
\text { 닌 }\end{array}$} & $\begin{array}{l}\text { Máximo momento flector } \\
(\mathrm{kN} \cdot \mathrm{m})\end{array}$ & 1307,638 & 1308,593 & 2140,93 & 2142,442 \\
\hline & $\begin{array}{l}\text { Máxima fuerza de inercia } \\
(\mathrm{kN})\end{array}$ & 7,0956 & 7,1008 & 13,54 & 13,5539 \\
\hline & $\begin{array}{l}\text { Máxima fuerza de arrastre } \\
(\mathrm{kN})\end{array}$ & 1,4811 & 1,4822 & 0,0051 & 0,0017 \\
\hline & Máxima fuerza no lineal (kN) & 0,0 & 0,0 & 0,0 & 0,0 \\
\hline & $\begin{array}{l}\text { Máxima fuerza de difracción } \\
(\mathrm{kN})\end{array}$ & 0,0 & 0,0 & 0,0 & 0,0 \\
\hline & Máxima fuerza total (kN) & 8,5767 & 8,583 & 13,5451 & 13,5556 \\
\hline
\end{tabular}

Tabla 28. Fuerzas hidrodinámicas para momento flector máximo, para $H=3,6 m, h=22,0 m, T=18,5$ s y $D=4,0 m$

Por último, se adjuntan en la tabla 29, los valores característicos del número de KeuleganCarpenter $(\mathrm{KC})$, del número de Reynolds $(\mathrm{Re})$ y el parámetro beta de Sarpkaya $(\beta)$ : 
Modelos de predicción de esfuerzos hidrodinámicos y socavación aplicados a ingeniería offshore

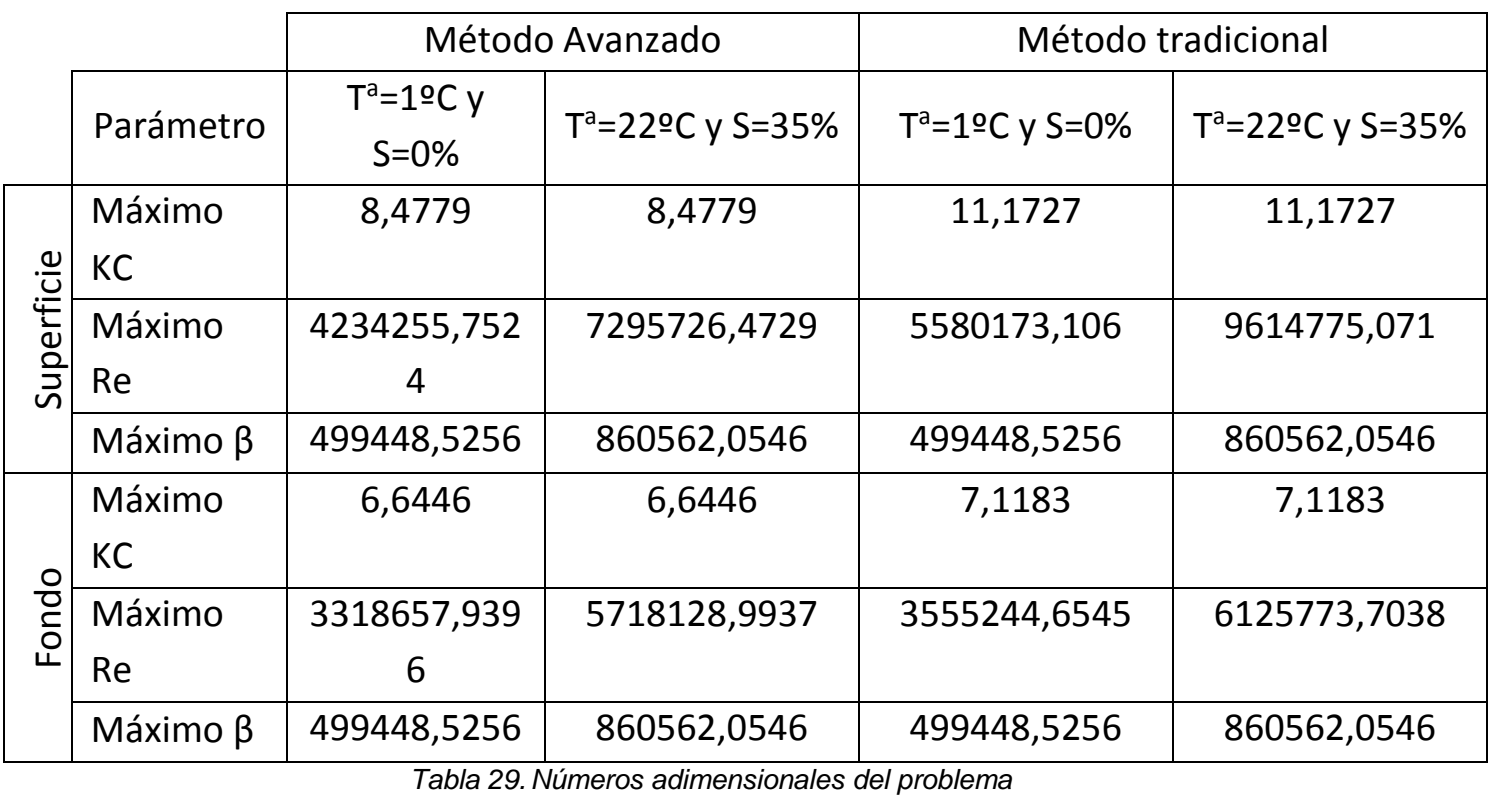




\subsubsection{Caso 2D}

Los datos de entrada se corresponden con el caso de cálculo para el parque eólico offshore Thornton Bank en Bélgica: altura de ola significativa $6,32 \mathrm{~m}$, profundidad $16,0 \mathrm{~m}$ y periodo de pico 11,06 s. De acuerdo con estos datos, en la figura 40 se muestra la distribución de fuerzas hidrodinámicas, generadas por el oleaje, en el instante en que producen el máximo momento flector, sobre la base del pilote, mostrando el cálculo según el método avanzado a la izquierda y según el método tradicional, a la derecha.
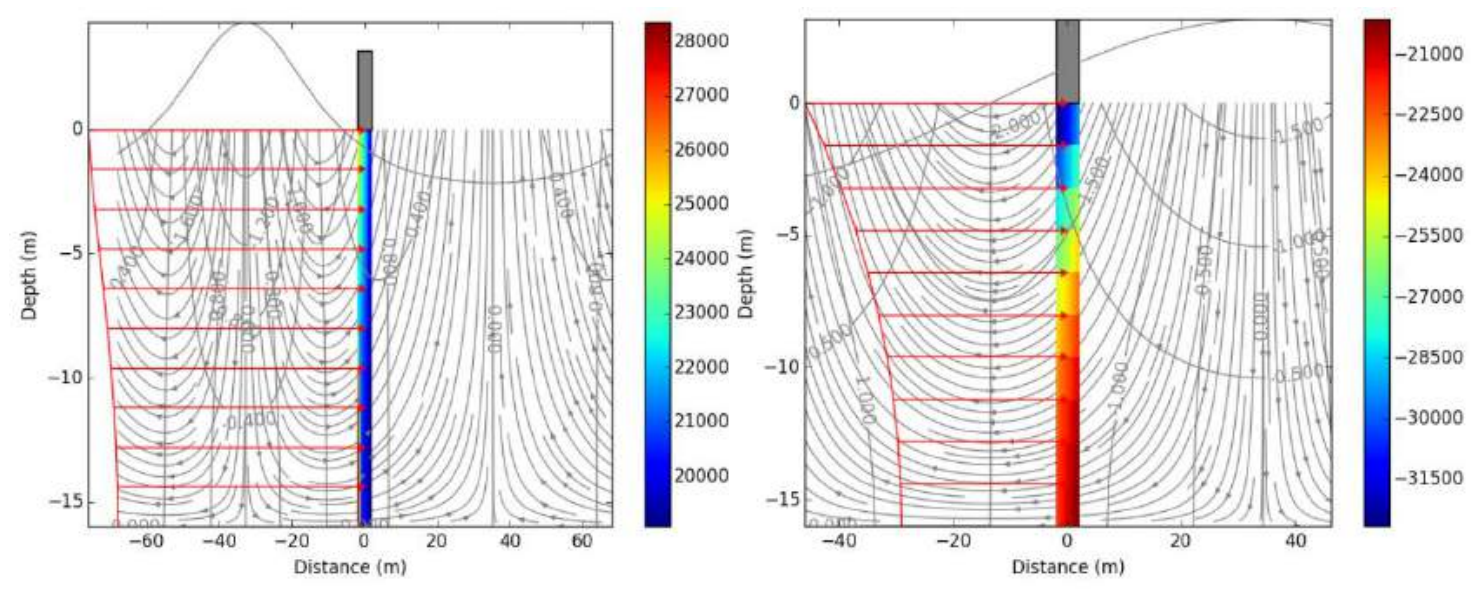

Figura 40. Momento flector máximo para $H=6,32 \mathrm{~m}, h=16 \mathrm{~m}, T=11,06$ s y $D=4,0 \mathrm{~m}$

Los valores más característicos de la simulación, tanto para el caso de temperatura de 1 으 y salinidad de $0 \%$, como para el caso de temperatura de $22{ }^{\circ} \mathrm{C}$ y salinidad del $35 \%$, se muestran, a continuación, en la tabla 30:

\begin{tabular}{|c|c|c|c|c|c|}
\hline & & \multicolumn{2}{|c|}{ Método Avanzado } & \multicolumn{2}{|c|}{ Método tradicional } \\
\hline & Parámetro & $\begin{array}{c}\mathrm{T}^{\mathrm{a}}=19 \mathrm{C} \\
\mathrm{S}=0 \%\end{array}$ & $\begin{array}{c}\mathrm{T}^{\mathrm{a}}=22 \mathrm{o} \mathrm{C}, \\
\mathrm{S}=35 \%\end{array}$ & $\begin{array}{c}\mathrm{T}^{\mathrm{a}}=19 \mathrm{C} \\
\mathrm{S}=0 \%\end{array}$ & $\begin{array}{c}\mathrm{T}^{\mathrm{a}}=22{ }^{\circ} \mathrm{C}, \\
\mathrm{S}=35 \%\end{array}$ \\
\hline \multirow{5}{*}{ 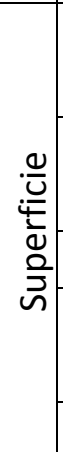 } & $\begin{array}{l}\text { Máxima fuerza de inercia } \\
(\mathrm{kN})\end{array}$ & 41,3756 & 40,8455 & 54,7602 & 54,8007 \\
\hline & $\begin{array}{l}\text { Máxima fuerza de arrastre } \\
(\mathrm{kN})\end{array}$ & 4,652 & 4,6554 & 19,9728 & 19,9877 \\
\hline & Máxima fuerza no lineal (kN) & 0,2075 & 0,2077 & 0,0 & 0,0 \\
\hline & $\begin{array}{l}\text { Máxima fuerza de difracción } \\
(\mathrm{kN})\end{array}$ & 0,0 & 0,0 & 0,0 & 0,0 \\
\hline & Máxima fuerza total (kN) & 41,8885 & 41,7804 & 54,7623 & 54,8014 \\
\hline
\end{tabular}




\begin{tabular}{|c|c|c|c|c|c|}
\hline \multirow{5}{*}{$\begin{array}{l}\text { 윰 } \\
\text { 은 } \\
\text { 난 }\end{array}$} & $\begin{array}{l}\text { Máxima fuerza de inercia } \\
(\mathrm{kN})\end{array}$ & 29,4769 & 29,1201 & 33,0645 & 33,0889 \\
\hline & $\begin{array}{l}\text { Máxima fuerza de arrastre } \\
(\mathrm{kN})\end{array}$ & 2,8101 & 2,8121 & 7,2817 & 7,2871 \\
\hline & Máxima fuerza no lineal (kN) & 0,5834 & 0,5838 & 0,0 & 0,0 \\
\hline & $\begin{array}{l}\text { Máxima fuerza de difracción } \\
(\mathrm{kN})\end{array}$ & 0,0 & 0,0 & 0,0 & 0,0 \\
\hline & Máxima fuerza total (kN) & 29,4182 & 29,0606 & 33,0648 & 33,0889 \\
\hline
\end{tabular}

Tabla 30. Fuerzas hidrodinámicas para el caso de $H=6,32 \mathrm{~m}, h=16 \mathrm{~m}, T=11,06$ s y $D=4,0 \mathrm{~m}$

Los valores de dichas fuerzas en el fondo marino, en el instante en que producen el máximo momento flector sobre la cimentación, se muestran en la tabla 31:

\begin{tabular}{|c|c|c|c|c|c|}
\hline & & \multicolumn{2}{|c|}{ Método Avanzado } & \multicolumn{2}{|c|}{ Método tradicional } \\
\hline & Parámetro & $\begin{array}{c}\mathrm{T}^{\mathrm{a}}=1 \mathrm{o} \mathrm{C} \\
\mathrm{S}=0 \%\end{array}$ & $\begin{array}{c}\mathrm{T}^{\mathrm{a}}=22 \mathrm{o} \mathrm{C}, \\
\mathrm{S}=35 \%\end{array}$ & $\begin{array}{c}\mathrm{T}^{\mathrm{a}}=1 \mathrm{o} \mathrm{C} \\
\mathrm{S}=0 \%\end{array}$ & $\begin{array}{c}\mathrm{T}^{\mathrm{a}}=22 \mathrm{o} \mathrm{C}, \\
\mathrm{S}=35 \%\end{array}$ \\
\hline \multirow{6}{*}{$\begin{array}{l}\text { 음 } \\
\text { 운 }\end{array}$} & $\begin{array}{l}\text { Máximo momento flector } \\
(\mathrm{kN} \cdot \mathrm{m})\end{array}$ & 3043,122 & 2924,796 & 3932,777 & 3935,648 \\
\hline & $\begin{array}{l}\text { Máxima fuerza de inercia } \\
(\mathrm{kN})\end{array}$ & 25,5257 & 23,3597 & 33,0645 & 33,0874 \\
\hline & $\begin{array}{l}\text { Máxima fuerza de arrastre } \\
(\mathrm{kN})\end{array}$ & 0,1773 & 1,2189 & 0,0003 & 0,0013 \\
\hline & Máxima fuerza no lineal (kN) & $-0,2835$ & $-0,5786$ & 0,0 & 0,0 \\
\hline & $\begin{array}{l}\text { Máxima fuerza de difracción } \\
\text { (kN) }\end{array}$ & 0,0 & 0,0 & 0,0 & 0,0 \\
\hline & Máxima fuerza total (kN) & 25,4195 & 24,0 & 33,0648 & 33,0886 \\
\hline
\end{tabular}

Tabla 31. Fuerzas hidrodinámicas para momento flector máximo, para $H=6,32 m, h=16 m, T=11,06$ s y $D=4,0 m$

Por último, se adjuntan en la tabla 32, los valores característicos del número de KeuleganCarpenter $(\mathrm{KC})$, del número de Reynolds $(\mathrm{Re})$ y el parámetro beta de Sarpkaya $(\beta)$ : 

aplicados a ingeniería offshore

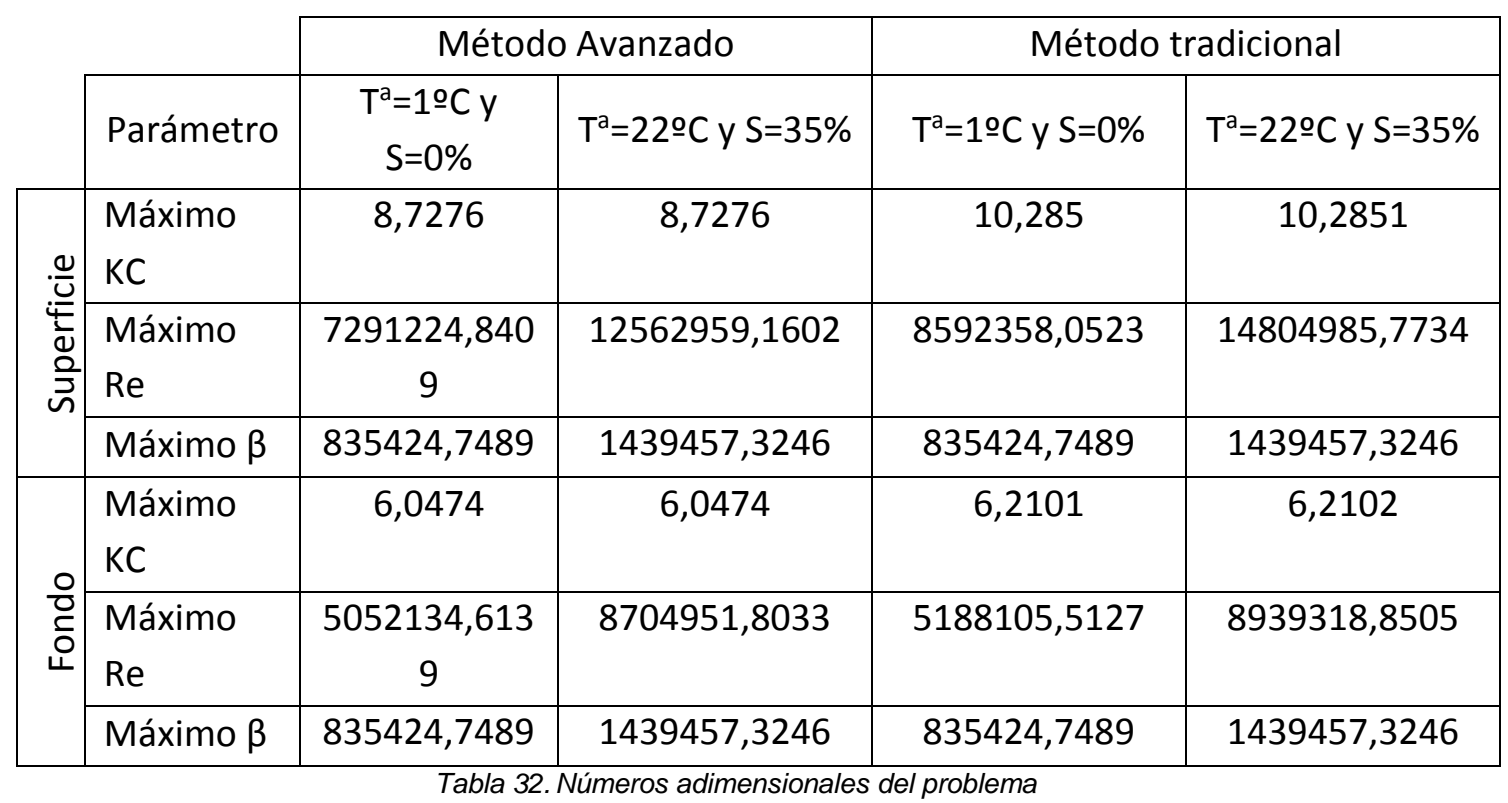




\subsection{Caso 3: Diámetros igual a $8,0 \mathrm{~m}$}

Se va a evaluar el caso de pilotes de 8,0 m de diámetro mediante los cuatro casos de oleaje definidos en el apartado anterior, calculados según la teoría lineal y según las teorías no lineales, tanto para el caso de una temperatura del agua de 1 으 $\mathrm{C}$ y salinidad del $0 \%$, como para el caso de una temperatura de 22 ㅇ C y salinidad del $35 \%$.

\subsubsection{Caso $3 \mathrm{~A}$}

Los datos de entrada se corresponden con el caso de cálculo para el parque eólico offshore Princess Amalia Q7 en Holanda: altura de ola significativa 7,7 m, profundidad 24,0 m y periodo de pico 9,7 s. De acuerdo con estos datos, en la figura 41 se muestra la distribución de fuerzas hidrodinámicas, generadas por el oleaje, en el instante en que producen el máximo momento flector, sobre la base del pilote, mostrando el cálculo según el método avanzado a la izquierda y según el método tradicional, a la derecha.
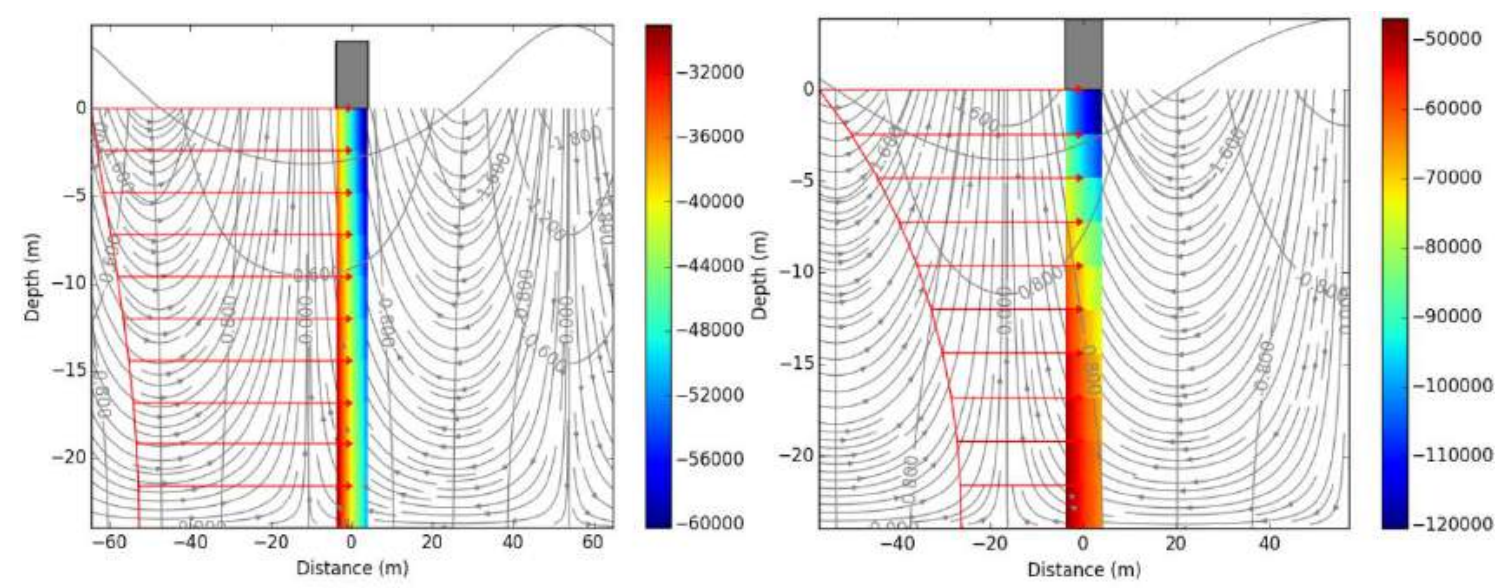

Figura 41. Momento flector máximo para $H=7,7 \mathrm{~m}, h=24,0 \mathrm{~m}, T=9,7 \mathrm{~s}$ y $D=8,0 \mathrm{~m}$

Los valores más característicos de la simulación, tanto para el caso de temperatura de 1 ํㅡ y salinidad de $0 \%$, como para el caso de temperatura de $22^{\circ} \mathrm{C}$ y salinidad del $35 \%$, se muestran, a continuación, en la tabla 33: 


\begin{tabular}{|c|c|c|c|c|c|}
\hline & \multirow[b]{2}{*}{ Parámetro } & \multicolumn{2}{|c|}{ Método Avanzado } & \multicolumn{2}{|c|}{ Método tradicional } \\
\hline & & $\begin{array}{c}\mathrm{T}^{\mathrm{a}}=1 \mathrm{o} \mathrm{C}, \\
\mathrm{S}=0 \%\end{array}$ & $\begin{array}{c}\mathrm{T}^{\mathrm{a}}=22 \mathrm{o} C \\
\mathrm{~S}=35 \%\end{array}$ & $\begin{array}{c}\mathrm{T}^{\mathrm{a}}=1 \mathrm{o} \mathrm{C} \\
\mathrm{S}=0 \%\end{array}$ & $\begin{array}{c}\mathrm{T}^{\mathrm{a}}=22 \mathrm{o} C, \\
\mathrm{~S}=35 \%\end{array}$ \\
\hline \multirow{5}{*}{ 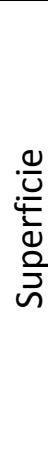 } & $\begin{array}{l}\text { Máxima fuerza de inercia } \\
(\mathrm{kN})\end{array}$ & 201,1175 & 201,2644 & 216,7524 & 216,9108 \\
\hline & $\begin{array}{l}\text { Máxima fuerza de arrastre } \\
(\mathrm{kN})\end{array}$ & 17,9549 & 17,968 & 30,087 & 30,109 \\
\hline & Máxima fuerza no lineal (kN) & 2,3732 & 2,3749 & 0,0 & 0,0 \\
\hline & $\begin{array}{l}\text { Máxima fuerza de difracción } \\
(\mathrm{kN})\end{array}$ & 0,0 & 0,0 & 0,0 & 0,0 \\
\hline & Máxima fuerza total (kN) & 202,4786 & 202,5881 & 216,7513 & 216,9097 \\
\hline \multirow{5}{*}{ 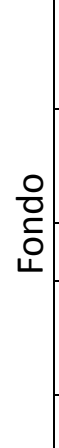 } & $\begin{array}{l}\text { Máxima fuerza de inercia } \\
(\mathrm{kN})\end{array}$ & 108,5232 & 108,4217 & 107,2685 & 107,3469 \\
\hline & $\begin{array}{l}\text { Máxima fuerza de arrastre } \\
(\mathrm{kN})\end{array}$ & 5,7968 & 5,801 & 7,3688 & 7,3742 \\
\hline & Máxima fuerza no lineal (kN) & 2,5272 & 2,529 & 0,0 & 0,0 \\
\hline & $\begin{array}{l}\text { Máxima fuerza de difracción } \\
(\mathrm{kN})\end{array}$ & 0,0 & 0,0 & 0,0 & 0,0 \\
\hline & Máxima fuerza total (kN) & 108,2048 & 108,1532 & 107,2682 & 107,3466 \\
\hline
\end{tabular}

Los valores de dichas fuerzas en el fondo marino, en el instante en que producen el máximo momento flector sobre la cimentación, se muestran en la tabla 34:

\begin{tabular}{|c|c|c|c|c|c|}
\hline & & \multicolumn{2}{|c|}{ Método Avanzado } & \multicolumn{2}{|c|}{ Método tradicional } \\
\hline & Parámetro & $\begin{array}{c}\mathrm{T}^{\mathrm{a}}=1 \stackrel{\mathrm{o}}{ } \mathrm{C} \\
\mathrm{S}=0 \%\end{array}$ & $\begin{array}{c}\mathrm{T}^{\mathrm{a}}=22{ }^{\circ} \mathrm{C} \\
\mathrm{S}=35 \%\end{array}$ & $\begin{array}{c}\mathrm{T}^{\mathrm{a}}=19 \mathrm{O}, \\
\mathrm{S}=0 \%\end{array}$ & $\begin{array}{c}\mathrm{T}^{\mathrm{a}}=22{ }^{\circ} \mathrm{C} \\
\mathrm{S}=35 \%\end{array}$ \\
\hline \multirow{6}{*}{$\begin{array}{l}\circ \\
\frac{0}{0} \\
\text { 인 }\end{array}$} & $\begin{array}{l}\text { Máximo momento flector } \\
(\mathrm{kN} \cdot \mathrm{m})\end{array}$ & 19379,495 & 17814,123 & 21809,864 & 21825,797 \\
\hline & $\begin{array}{l}\text { Máxima fuerza de inercia } \\
(\mathrm{kN})\end{array}$ & 81,8006 & 81,9285 & 107,2685 & 107,3469 \\
\hline & $\begin{array}{l}\text { Máxima fuerza de arrastre } \\
(\mathrm{kN})\end{array}$ & 0,087 & 0,1057 & $-0,0003$ & $-0,0003$ \\
\hline & Máxima fuerza no lineal (kN) & $-0,6146$ & $-0,6765$ & 0,0 & 0,0 \\
\hline & $\begin{array}{l}\text { Máxima fuerza de difracción } \\
(\mathrm{kN})\end{array}$ & 0,0 & 0,0 & 0,0 & 0,0 \\
\hline & Máxima fuerza total (kN) & 81,273 & 81,3576 & 107,2682 & 107,3466 \\
\hline
\end{tabular}

Tabla 34. Fuerzas hidrodinámicas para momento flector máximo, para $H=7,7 m, h=24,0 m, T=9,7 s$ y $D=8,0 m$ 
Por último, se adjuntan en la tabla 35, los valores característicos del número de KeuleganCarpenter (KC), del número de Reynolds (Re) y el parámetro beta de Sarpkaya $(\beta)$ :

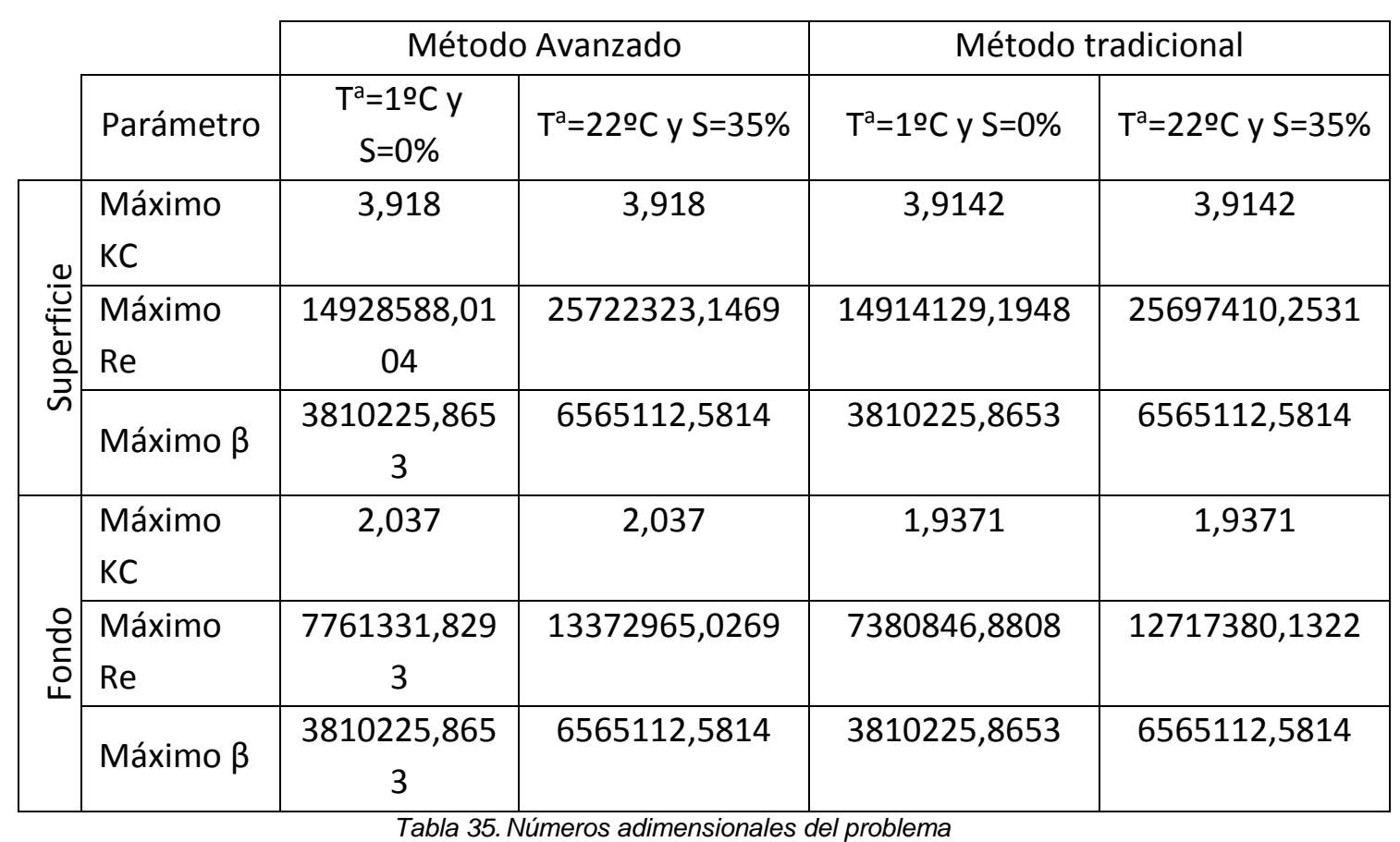




\subsubsection{Caso 3B}

Los datos de entrada se corresponden con el caso de cálculo para el parque eólico offshore North Hoyle en Reino Unido: altura de ola significativa $4,78 \mathrm{~m}$, profundidad $10,0 \mathrm{~m}$ y periodo de pico 6,8 s. De acuerdo con estos datos, en la figura 42 se muestra la distribución de fuerzas hidrodinámicas, generadas por el oleaje, en el instante en que producen el máximo momento flector, sobre la base del pilote, mostrando el cálculo según el método avanzado a la izquierda y según el método tradicional, a la derecha.
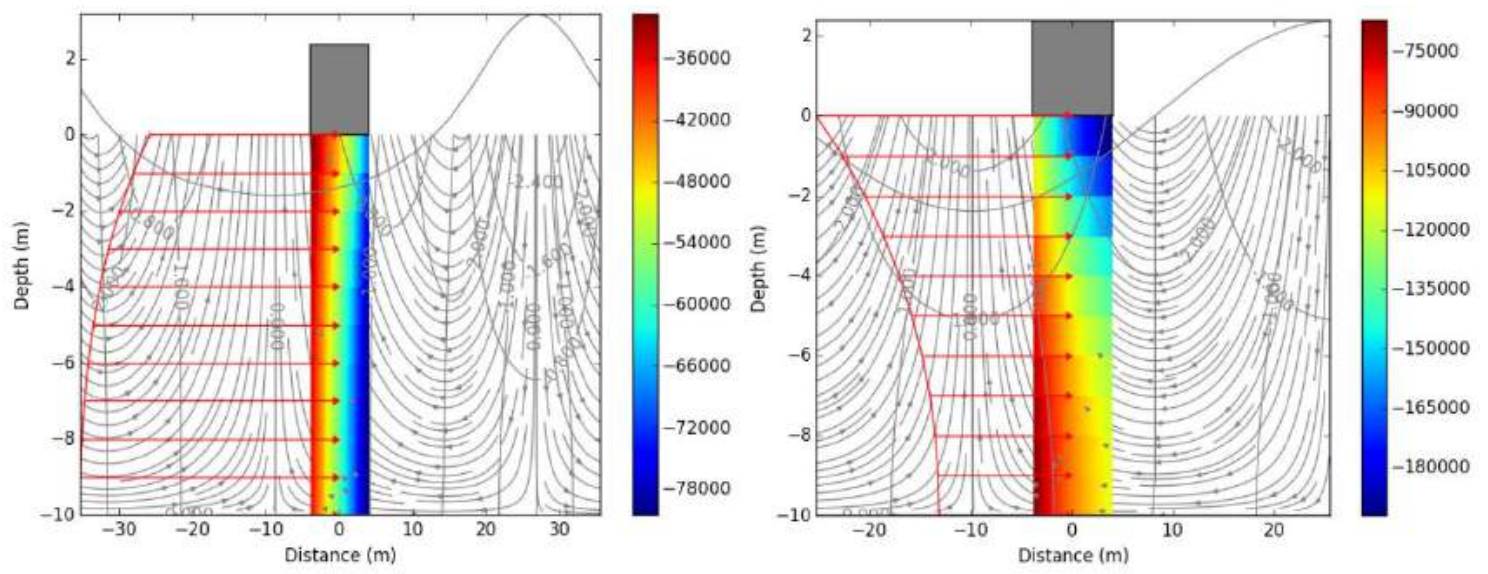

Figura 42. Momento flector máximo para $H=4,78 m, h=10,0 m, T=6,8 s$ y $D=8,0 m$

Los valores más característicos de la simulación, tanto para el caso de temperatura de 1 ํㅡ y salinidad de $0 \%$, como para el caso de temperatura de $22{ }^{\circ} \mathrm{C}$ y salinidad del $35 \%$, se muestran, a continuación, en la tabla 36:

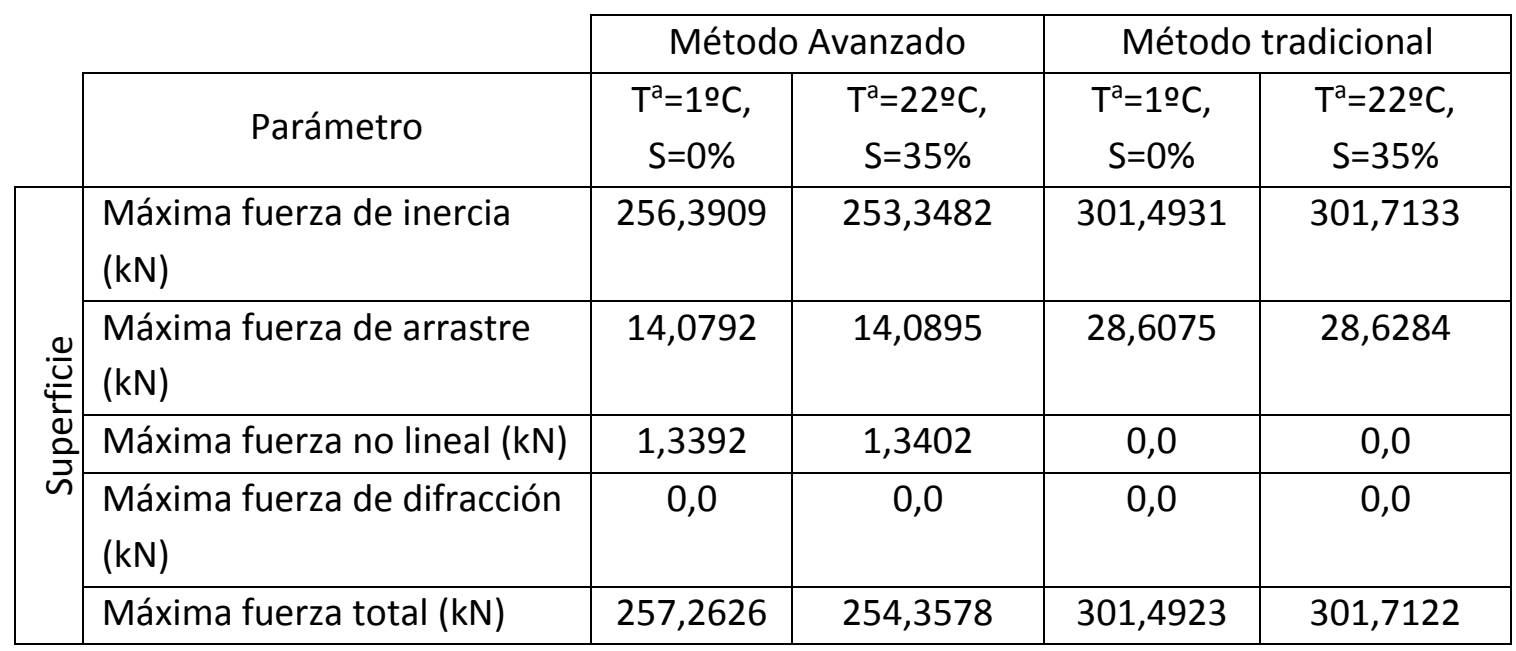




\begin{tabular}{|c|c|c|c|c|c|}
\hline \multirow{5}{*}{$\begin{array}{l}\frac{0}{0} \\
\text { 둥 }\end{array}$} & $\begin{array}{l}\text { Máxima fuerza de inercia } \\
(\mathrm{kN})\end{array}$ & 159,9204 & 159,2199 & 160,9315 & 161,0491 \\
\hline & $\begin{array}{l}\text { Máxima fuerza de arrastre } \\
(\mathrm{kN})\end{array}$ & 6,9902 & 6,9953 & 8,1509 & 8,1569 \\
\hline & Máxima fuerza no lineal (kN) & 5,5613 & 5,5654 & 0,0 & 0,0 \\
\hline & $\begin{array}{l}\text { Máxima fuerza de difracción } \\
(\mathrm{kN})\end{array}$ & 0,0 & 0,0 & 0,0 & 0,0 \\
\hline & Máxima fuerza total (kN) & 158,3622 & 157,7546 & 160,9311 & 161,0487 \\
\hline
\end{tabular}

Los valores de dichas fuerzas en el fondo marino, en el instante en que producen el máximo momento flector sobre la cimentación, se muestran en la tabla 37:

\begin{tabular}{|c|c|c|c|c|c|}
\hline & & \multicolumn{2}{|c|}{ Método Avanzado } & \multicolumn{2}{|c|}{ Método tradicional } \\
\hline & Parámetro & $\begin{array}{c}\mathrm{T}^{\mathrm{a}}=1 \stackrel{\circ}{ } \mathrm{C} \\
\mathrm{S}=0 \%\end{array}$ & $\begin{array}{c}\mathrm{T}^{\mathrm{a}}=22{ }^{\circ} \mathrm{C} \\
\mathrm{S}=35 \%\end{array}$ & $\begin{array}{c}\mathrm{T}^{\mathrm{a}}=1 \stackrel{\circ}{ } \mathrm{C} \\
\mathrm{S}=0 \%\end{array}$ & $\begin{array}{c}\mathrm{T}^{\mathrm{a}}=22{ }^{\circ} \mathrm{C}, \\
\mathrm{S}=35 \%\end{array}$ \\
\hline \multirow{6}{*}{$\begin{array}{l}0 \\
0 \\
0 \\
0 \\
ㅇ\end{array}$} & $\begin{array}{l}\text { Máximo momento flector } \\
(\mathrm{kN} \cdot \mathrm{m})\end{array}$ & 10199,184 & 10033,338 & 12963,198 & 12972,669 \\
\hline & $\begin{array}{l}\text { Máxima fuerza de inercia } \\
(\mathrm{kN})\end{array}$ & 123,173 & 124,1681 & 160,9315 & 161,0491 \\
\hline & $\begin{array}{l}\text { Máxima fuerza de arrastre } \\
(\mathrm{kN})\end{array}$ & 0,4044 & 2,4445 & $-0,0004$ & $-0,0004$ \\
\hline & Máxima fuerza no lineal (kN) & $-2,5968$ & $-5,3071$ & 0,0 & 0,0 \\
\hline & $\begin{array}{l}\text { Máxima fuerza de difracción } \\
(\mathrm{kN})\end{array}$ & 0,0 & 0,0 & 0,0 & 0,0 \\
\hline & Máxima fuerza total (kN) & 120,9807 & 121,3055 & 160,9311 & 161,0487 \\
\hline
\end{tabular}

Tabla 37. Fuerzas hidrodinámicas para momento flector máximo, para $H=4,78 m, h=10,0 m, T=6,8 s$ y $D=8,0 m$

Por último, se adjuntan en la tabla 38, los valores característicos del número de KeuleganCarpenter $(\mathrm{KC})$, del número de Reynolds $(\mathrm{Re})$ y el parámetro beta de Sarpkaya $(\beta)$ : 
Modelos de predicción de esfuerzos hidrodinámicos y socavación aplicados a ingeniería offshore

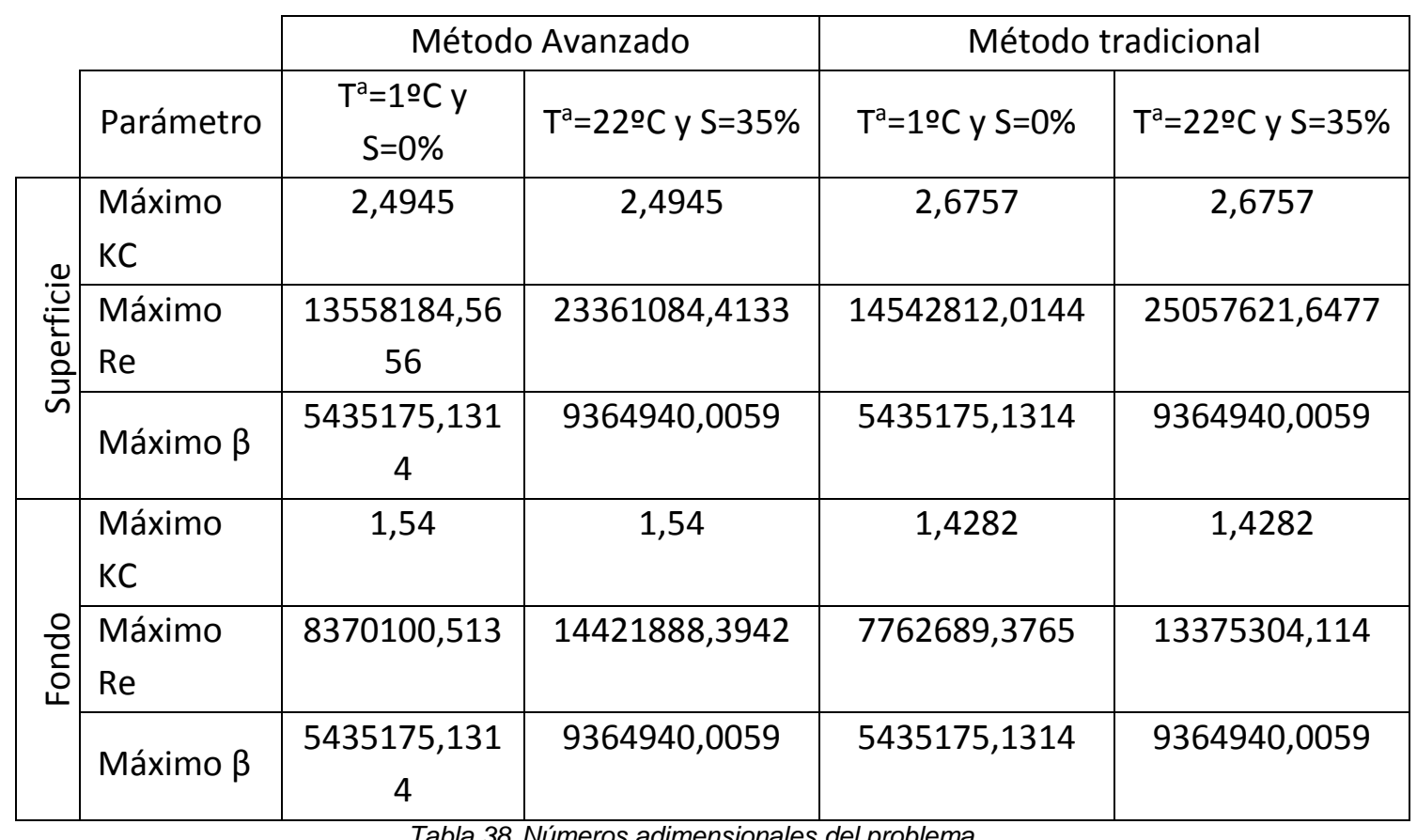




\subsubsection{Caso $3 \mathrm{C}$}

Los datos de entrada se corresponden con el caso de cálculo para el parque eólico offshore Egmond Aan Zee en Holanda: altura de ola significativa 3,6 m, profundidad 22,0 m y periodo de pico 18,5 s. De acuerdo con estos datos, en la figura 43 se muestra la distribución de fuerzas hidrodinámicas, generadas por el oleaje, en el instante en que producen el máximo momento flector, sobre la base del pilote, mostrando el cálculo según el método avanzado a la izquierda y según el método tradicional, a la derecha.
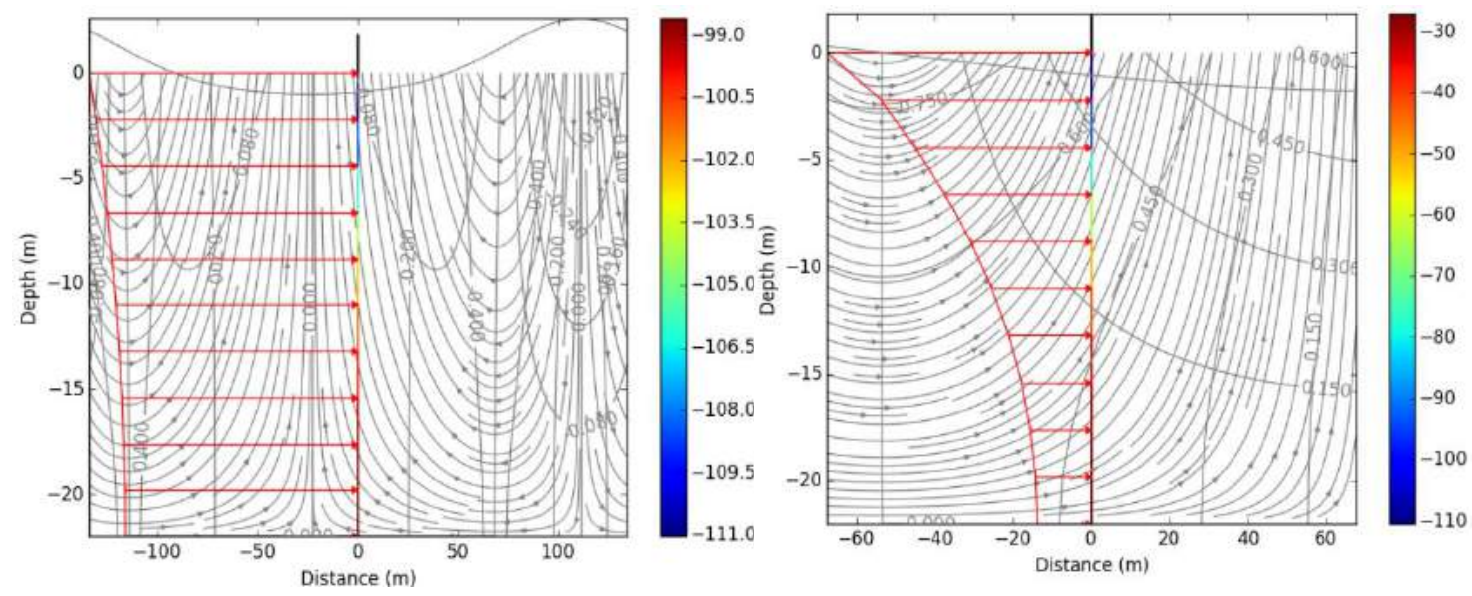

Figura 43. Momento flector máximo para $\mathrm{H}=3,6 \mathrm{~m}, h=22,0 \mathrm{~m}, \mathrm{~T}=18,5$ s y $D=8,0 \mathrm{~m}$

Los valores más característicos de la simulación, tanto para el caso de temperatura de 1 으 y salinidad de $0 \%$, como para el caso de temperatura de $22{ }^{\circ} \mathrm{C}$ y salinidad del $35 \%$, se muestran, a continuación, en la tabla 39:

\begin{tabular}{|c|c|c|c|c|c|}
\hline & \multirow[b]{2}{*}{ Parámetro } & \multicolumn{2}{|c|}{ Método Avanzado } & \multicolumn{2}{|c|}{ Método tradicional } \\
\hline & & $\begin{array}{c}\mathrm{T}^{\mathrm{a}}=1{ }^{\circ} \mathrm{C} \\
\mathrm{S}=0 \%\end{array}$ & $\begin{array}{c}\mathrm{T}^{\mathrm{a}}=22 \mathrm{o} \mathrm{C}, \\
\mathrm{S}=35 \%\end{array}$ & $\begin{array}{c}\mathrm{T}^{\mathrm{a}}=1 \stackrel{\mathrm{o}}{ } \mathrm{C} \\
\mathrm{S}=0 \%\end{array}$ & $\begin{array}{c}\mathrm{T}^{\mathrm{a}}=22 \mathrm{o} \mathrm{C}, \\
\mathrm{S}=35 \%\end{array}$ \\
\hline \multirow{5}{*}{$\begin{array}{l}\frac{0}{u} \\
\frac{0}{2} \\
\frac{0}{3} \\
\tilde{n}\end{array}$} & $\begin{array}{l}\text { Máxima fuerza de inercia } \\
(\mathrm{kN})\end{array}$ & 43,8952 & 43,9272 & 85,0447 & 85,1069 \\
\hline & $\begin{array}{l}\text { Máxima fuerza de arrastre } \\
(\mathrm{kN})\end{array}$ & 9,7009 & 9,708 & 16,8479 & 16,8602 \\
\hline & Máxima fuerza no lineal (kN) & 0,0 & 0,0 & 0,0 & 0,0 \\
\hline & $\begin{array}{l}\text { Máxima fuerza de difracción } \\
(\mathrm{kN})\end{array}$ & 0,0 & 0,0 & 0,0 & 0,0 \\
\hline & Máxima fuerza total (kN) & 46,9234 & 46,9577 & 85,0448 & 85,107 \\
\hline
\end{tabular}




\begin{tabular}{|c|c|c|c|c|c|}
\hline \multirow{5}{*}{$\begin{array}{l}\frac{0}{0} \\
\frac{1}{0} \\
\text { 广 }\end{array}$} & $\begin{array}{l}\text { Máxima fuerza de inercia } \\
(\mathrm{kN})\end{array}$ & 33,2078 & 32,6727 & 54,1838 & 54,2234 \\
\hline & $\begin{array}{l}\text { Máxima fuerza de arrastre } \\
(\mathrm{kN})\end{array}$ & 5,9591 & 5,9634 & 6,839 & 6,844 \\
\hline & Máxima fuerza no lineal (kN) & 0,0 & 0,0 & 0,0 & 0,0 \\
\hline & $\begin{array}{l}\text { Máxima fuerza de difracción } \\
\text { (kN) }\end{array}$ & 0,0 & 0,0 & 0,0 & 0,0 \\
\hline & Máxima fuerza total (kN) & 33,2195 & 33,1045 & 54,1838 & 54,2234 \\
\hline
\end{tabular}

Los valores de dichas fuerzas en el fondo marino, en el instante en que producen el máximo momento flector sobre la cimentación, se muestran en la tabla 40:

\begin{tabular}{|c|c|c|c|c|c|}
\hline & \multirow[b]{2}{*}{ Parámetro } & \multicolumn{2}{|c|}{ Método Avanzado } & \multicolumn{2}{|c|}{ Método tradicional } \\
\hline & & $\begin{array}{c}\mathrm{T}^{\mathrm{a}}=1 \mathrm{o} \mathrm{C} \\
\mathrm{S}=0 \%\end{array}$ & $\begin{array}{c}\mathrm{T}^{\mathrm{a}}=22 \mathrm{o} \mathrm{C}, \\
\mathrm{S}=35 \%\end{array}$ & $\begin{array}{c}\mathrm{T}^{\mathrm{a}}=1 \stackrel{\mathrm{o}}{ } \mathrm{C} \\
\mathrm{S}=0 \%\end{array}$ & $\begin{array}{c}\mathrm{T}^{\mathrm{a}}=22{ }^{\circ} \mathrm{C}, \\
\mathrm{S}=35 \%\end{array}$ \\
\hline \multirow{6}{*}{$\begin{array}{l}\text { 응 } \\
\text { 은 }\end{array}$} & $\begin{array}{l}\text { Máximo momento flector } \\
(\mathrm{kN} \cdot \mathrm{m})\end{array}$ & 4870,276 & 4873,834 & 8563,399 & 8569,655 \\
\hline & $\begin{array}{l}\text { Máxima fuerza de inercia } \\
(\mathrm{kN})\end{array}$ & 29,9743 & 29,9962 & 54,1838 & 54,2234 \\
\hline & $\begin{array}{l}\text { Máxima fuerza de arrastre } \\
(\mathrm{kN})\end{array}$ & 2,4334 & 2,4351 & 0,0 & 0,0 \\
\hline & Máxima fuerza no lineal (kN) & 0,0 & 0,0 & 0,0 & 0,0 \\
\hline & $\begin{array}{l}\text { Máxima fuerza de difracción } \\
(\mathrm{kN})\end{array}$ & 0,0 & 0,0 & 0,0 & 0,0 \\
\hline & Máxima fuerza total (kN) & 32,4077 & 32,4313 & 54,1838 & 54,2234 \\
\hline
\end{tabular}

Por último, se adjuntan en la tabla 41, los valores característicos del número de KeuleganCarpenter $(\mathrm{KC})$, del número de Reynolds (Re) y el parámetro beta de Sarpkaya $(\beta)$ : 
Modelos de predicción de esfuerzos hidrodinámicos y socavación aplicados a ingeniería offshore

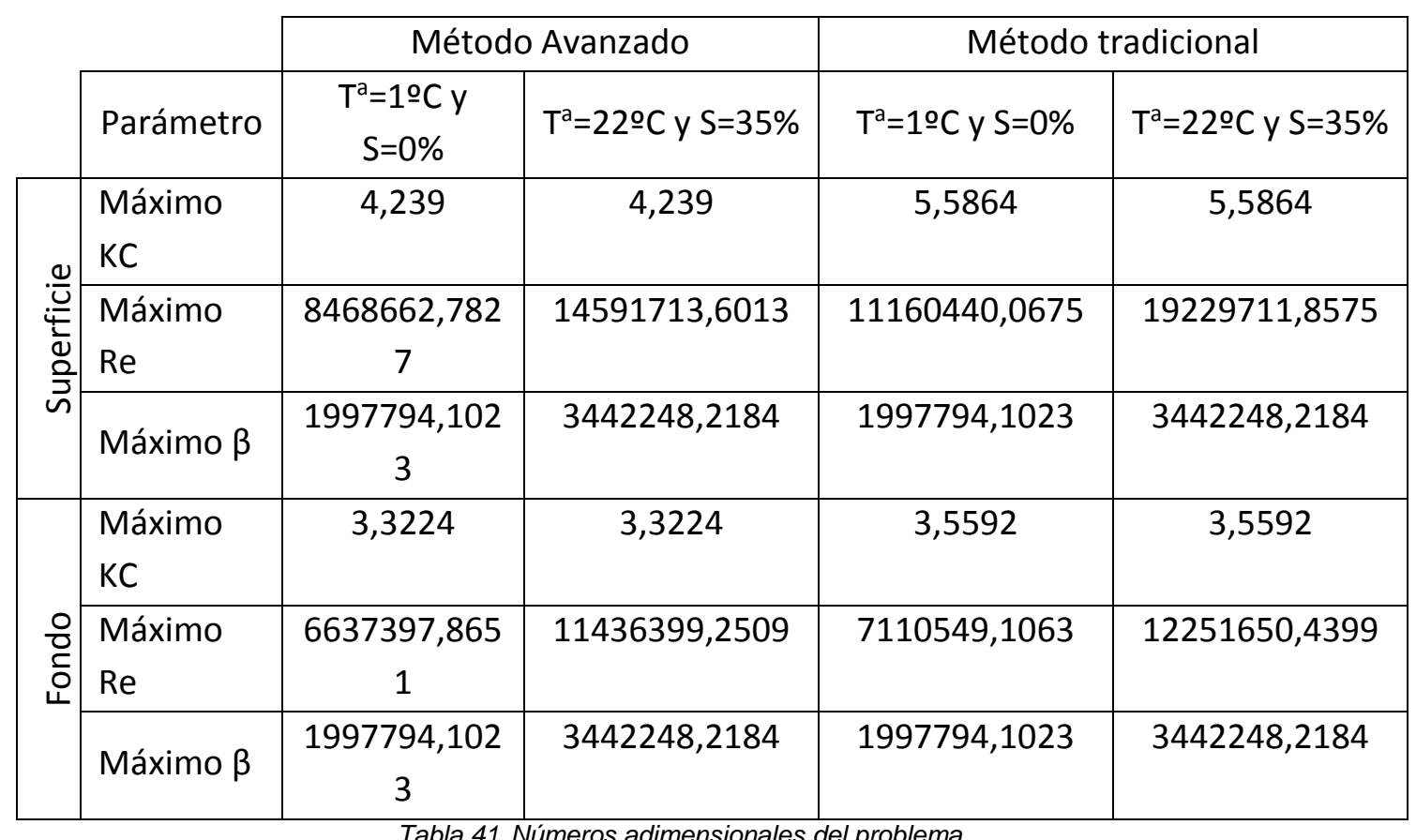




\subsubsection{Caso 3D}

Los datos de entrada se corresponden con el caso de cálculo para el parque eólico offshore Thornton Bank en Bélgica: altura de ola significativa $6,32 \mathrm{~m}$, profundidad $16,0 \mathrm{~m}$ y periodo de pico 11,06 s. De acuerdo con estos datos, en la figura 44 se muestra la distribución de fuerzas hidrodinámicas, generadas por el oleaje, en el instante en que producen el máximo momento flector, sobre la base del pilote, mostrando el cálculo según el método avanzado a la izquierda y según el método tradicional, a la derecha.
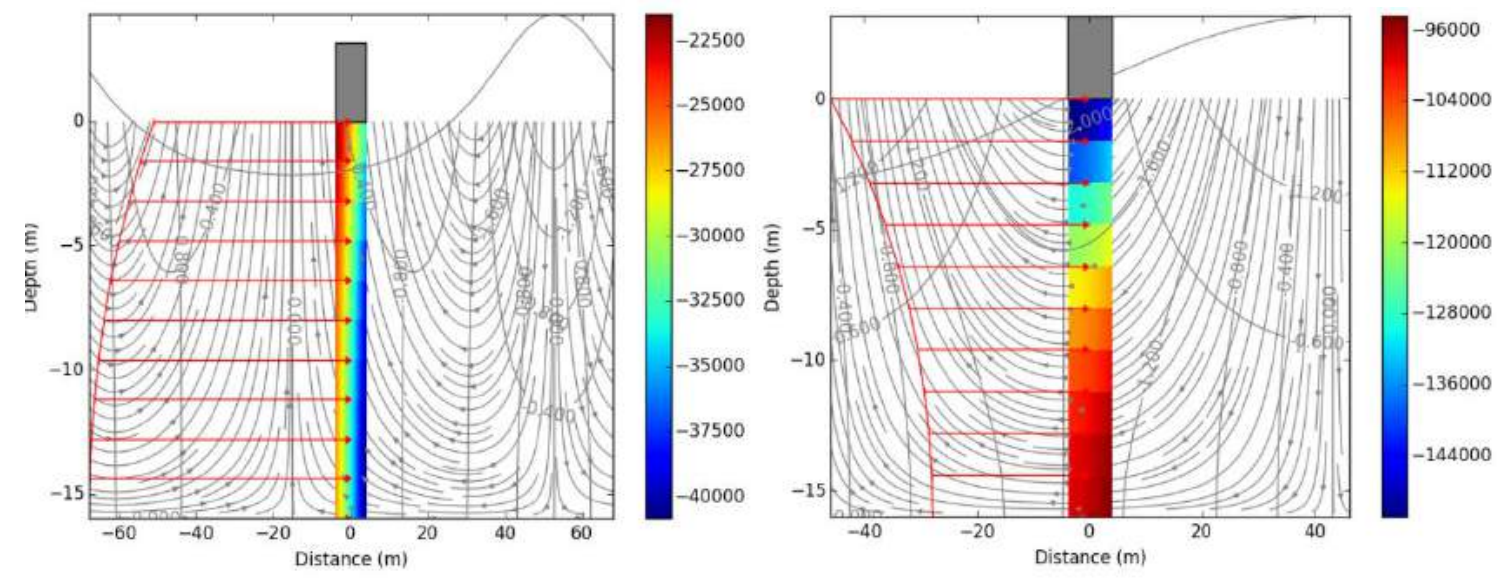

Figura 44. Momento flector máximo para $H=6,32 m, h=16 \mathrm{~m}, T=11,06$ s y $D=8,0 \mathrm{~m}$

Los valores más característicos de la simulación, tanto para el caso de temperatura de 1 으 y salinidad de $0 \%$, como para el caso de temperatura de $22{ }^{\circ} \mathrm{C}$ y salinidad del $35 \%$, se muestran, a continuación, en la tabla 42:

\begin{tabular}{|c|c|c|c|c|c|}
\hline & \multirow[b]{2}{*}{ Parámetro } & \multicolumn{2}{|c|}{ Método Avanzado } & \multicolumn{2}{|c|}{ Método tradicional } \\
\hline & & $\begin{array}{l}\mathrm{T}^{\mathrm{a}}=1 \mathrm{O} \mathrm{C} \\
\mathrm{S}=0 \%\end{array}$ & $\begin{array}{c}\mathrm{T}^{\mathrm{a}}=22 \mathrm{o} \mathrm{C}, \\
\mathrm{S}=35 \%\end{array}$ & $\begin{array}{c}\mathrm{T}^{\mathrm{a}}=1 \stackrel{\mathrm{o} C}{ } \\
\mathrm{~S}=0 \%\end{array}$ & $\begin{array}{c}\mathrm{T}^{\mathrm{a}}=22 \mathrm{o} \mathrm{C}, \\
\mathrm{S}=35 \%\end{array}$ \\
\hline \multirow{5}{*}{ 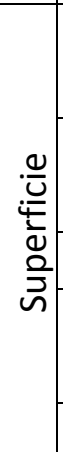 } & $\begin{array}{l}\text { Máxima fuerza de inercia } \\
(\mathrm{kN})\end{array}$ & 163,4034 & 160,9791 & 219,0429 & 219,2029 \\
\hline & $\begin{array}{l}\text { Máxima fuerza de arrastre } \\
(\mathrm{kN})\end{array}$ & 9,304 & 9,3108 & 39,9463 & 39,9755 \\
\hline & Máxima fuerza no lineal (kN) & 0,9087 & 0,9094 & 0,0 & 0,0 \\
\hline & $\begin{array}{l}\text { Máxima fuerza de difracción } \\
(\mathrm{kN})\end{array}$ & 0,0 & 0,0 & 0,0 & 0,0 \\
\hline & Máxima fuerza total (kN) & 164,0492 & 162,576 & 219,0425 & 219,2025 \\
\hline
\end{tabular}




\begin{tabular}{|c|c|c|c|c|c|}
\hline \multirow{5}{*}{$\begin{array}{l}\text { 윰 } \\
\text { 은 } \\
\text { 난 }\end{array}$} & $\begin{array}{l}\text { Máxima fuerza de inercia } \\
(\mathrm{kN})\end{array}$ & 116,4438 & 115,6463 & 132,2591 & 132,3558 \\
\hline & $\begin{array}{l}\text { Máxima fuerza de arrastre } \\
(\mathrm{kN})\end{array}$ & 5,6201 & 5,6243 & 14,5636 & 14,5743 \\
\hline & Máxima fuerza no lineal (kN) & 2,3336 & 2,3353 & 0,0 & 0,0 \\
\hline & $\begin{array}{l}\text { Máxima fuerza de difracción } \\
(\mathrm{kN})\end{array}$ & 0,0 & 0,0 & 0,0 & 0,0 \\
\hline & Máxima fuerza total (kN) & 115,8729 & 115,1312 & 132,259 & 132,3556 \\
\hline
\end{tabular}

Tabla 42. Fuerzas hidrodinámicas para el caso de $H=6,32 \mathrm{~m}, h=16 \mathrm{~m}, T=11,06$ s y $D=8,0 \mathrm{~m}$

Los valores de dichas fuerzas en el fondo marino, en el instante en que producen el máximo momento flector sobre la cimentación, se muestran en la tabla 43:

\begin{tabular}{|c|c|c|c|c|c|}
\hline & & \multicolumn{2}{|c|}{ Método Avanzado } & \multicolumn{2}{|c|}{ Método tradicional } \\
\hline & Parámetro & $\begin{aligned} \mathrm{T}^{\mathrm{a}} & =10 \mathrm{C}, \\
\mathrm{S} & =0 \%\end{aligned}$ & $\begin{array}{c}\mathrm{T}^{\mathrm{a}}=22 \mathrm{o} \mathrm{C} \\
\mathrm{S}=35 \%\end{array}$ & $\begin{array}{c}\mathrm{T}^{\mathrm{a}}=1 \mathrm{o} \mathrm{C}, \\
\mathrm{S}=0 \%\end{array}$ & $\begin{array}{c}\mathrm{T}^{\mathrm{a}}=22{ }^{\circ} \mathrm{C} \\
\mathrm{S}=35 \%\end{array}$ \\
\hline \multirow{6}{*}{$\begin{array}{l}\text { 음 } \\
\text { 운 }\end{array}$} & $\begin{array}{l}\text { Máximo momento flector } \\
(\mathrm{kN} \cdot \mathrm{m})\end{array}$ & 11403,162 & 11411,689 & 15731,022 & 15742,514 \\
\hline & $\begin{array}{l}\text { Máxima fuerza de inercia } \\
(\mathrm{kN})\end{array}$ & 94,1326 & 93,8719 & 132,2591 & 132,3558 \\
\hline & $\begin{array}{l}\text { Máxima fuerza de arrastre } \\
(\mathrm{kN})\end{array}$ & 2,3009 & 2,3635 & $-0,0002$ & $-0,0002$ \\
\hline & Máxima fuerza no lineal (kN) & $-2,295$ & $-2,3054$ & 0,0 & 0,0 \\
\hline & $\begin{array}{l}\text { Máxima fuerza de difracción } \\
\text { (kN) }\end{array}$ & 0,0 & 0,0 & 0,0 & 0,0 \\
\hline & Máxima fuerza total (kN) & 94,1385 & 93,9299 & 132,259 & 132,3556 \\
\hline
\end{tabular}

Tabla 43. Fuerzas hidrodinámicas para momento flector máximo, para $H=6,32 m, h=16 m, T=11,06 s$ y $D=8,0 m$

Por último, se adjuntan en la tabla 44, los valores característicos del número de KeuleganCarpenter $(\mathrm{KC})$, del número de Reynolds $(\mathrm{Re})$ y el parámetro beta de Sarpkaya $(\beta)$ : 
Modelos de predicción de esfuerzos hidrodinámicos y socavación aplicados a ingeniería offshore

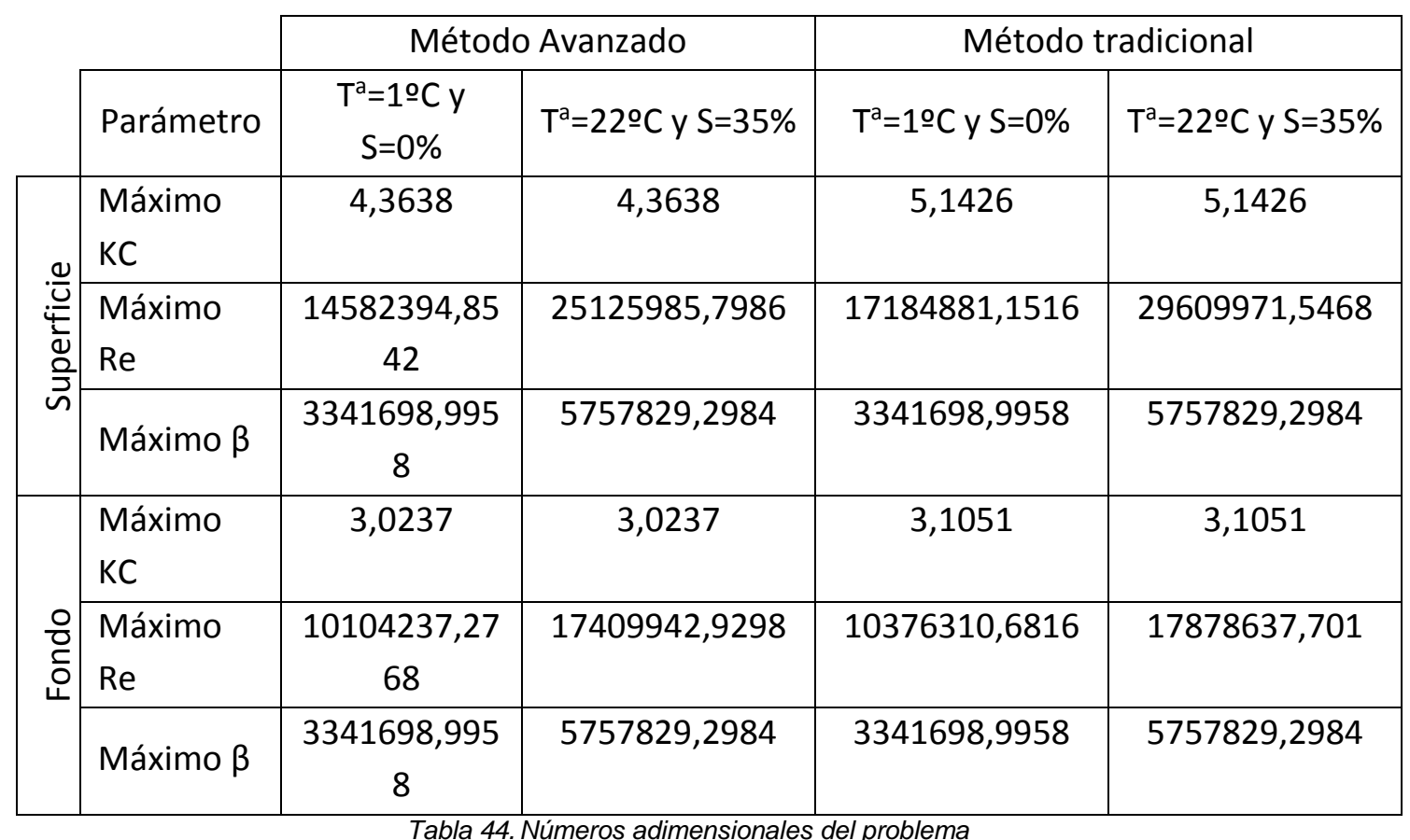




\subsection{Caso 4: Diámetros igual a 16,0 m}

Se va a evaluar el caso de pilotes de 16,0 m de diámetro mediante los cuatro casos de oleaje definidos en el apartado anterior, calculados según la teoría lineal y según las teorías no lineales, tanto para el caso de una temperatura del agua de 1 으 $\mathrm{C}$ y salinidad del $0 \%$, como para el caso de una temperatura de $22{ }^{\circ} \mathrm{C}$ y salinidad del $35 \%$.

\subsubsection{Caso $4 \mathrm{~A}$}

Los datos de entrada se corresponden con el caso de cálculo para el parque eólico offshore Princess Amalia Q7 en Holanda: altura de ola significativa 7,7 m, profundidad 24,0 m y periodo de pico 9,7 s. De acuerdo con estos datos, en la figura 45 se muestra la distribución de fuerzas hidrodinámicas, generadas por el oleaje, en el instante en que producen el máximo momento flector, sobre la base del pilote, mostrando el cálculo según el método avanzado a la izquierda y según el método tradicional, a la derecha.
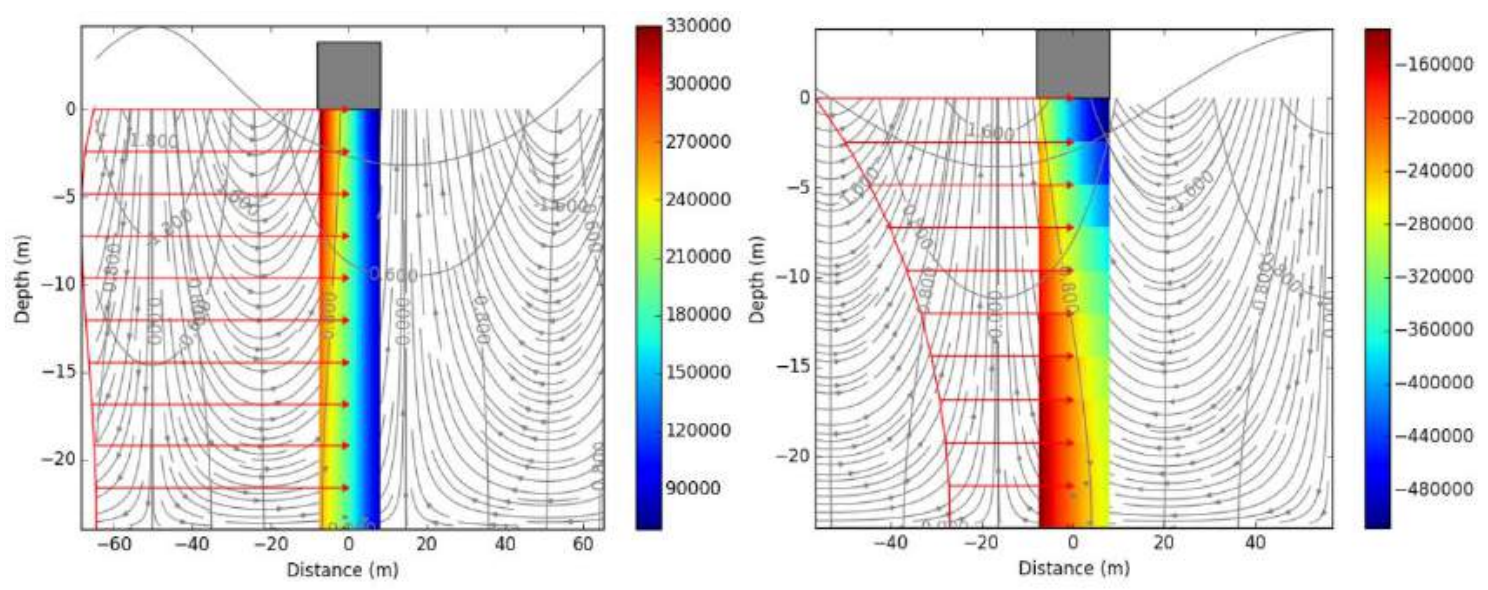

Figura 45. Momento flector máximo para $H=7,7 \mathrm{~m}, h=24,0 \mathrm{~m}, T=9,7$ s y $D=16,0 \mathrm{~m}$

Los valores más característicos de la simulación, tanto para el caso de temperatura de 1 ํㅡ y salinidad de $0 \%$, como para el caso de temperatura de $22^{\circ} \mathrm{C}$ y salinidad del $35 \%$, se muestran, a continuación, en la tabla 45: 


\begin{tabular}{|c|c|c|c|c|c|}
\hline & \multirow[b]{2}{*}{ Parámetro } & \multicolumn{2}{|c|}{ Método Avanzado } & \multicolumn{2}{|c|}{ Método tradicional } \\
\hline & & $\begin{aligned} \mathrm{T}^{\mathrm{a}}=10 \mathrm{C} \\
\mathrm{S}=0 \%\end{aligned}$ & $\begin{array}{c}\mathrm{T}^{\mathrm{a}}=22^{\circ} \mathrm{C}, \\
\mathrm{S}=35 \%\end{array}$ & $\begin{aligned} \mathrm{T}^{\mathrm{a}} & =1 \mathrm{O} \mathrm{C}, \\
\mathrm{S} & =0 \%\end{aligned}$ & $\begin{array}{c}\mathrm{T}^{\mathrm{a}}=22^{\circ} \mathrm{C}, \\
\mathrm{S}=35 \%\end{array}$ \\
\hline \multirow{5}{*}{ 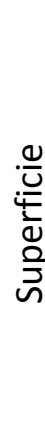 } & $\begin{array}{l}\text { Máxima fuerza de inercia } \\
(\mathrm{kN})\end{array}$ & 804,4699 & 778,8373 & 867,0138 & 867,6472 \\
\hline & $\begin{array}{l}\text { Máxima fuerza de arrastre } \\
(\mathrm{kN})\end{array}$ & 35,9098 & 35,936 & 60,1746 & 60,2186 \\
\hline & Máxima fuerza no lineal (kN) & 4,691 & 4,6944 & 0,0 & 0,0 \\
\hline & $\begin{array}{l}\text { Máxima fuerza de difracción } \\
(\mathrm{kN})\end{array}$ & 0,0 & 0,0 & 0,0 & 0,0 \\
\hline & Máxima fuerza total (kN) & 807,057 & 781,2058 & 867,0125 & 867,646 \\
\hline \multirow{5}{*}{$\begin{array}{l}0 \\
\frac{0}{0} \\
0 \\
\text { ㄴ. }\end{array}$} & $\begin{array}{l}\text { Máxima fuerza de inercia } \\
(\mathrm{kN})\end{array}$ & 433,3704 & 432,894 & 429,0761 & 429,3895 \\
\hline & $\begin{array}{l}\text { Máxima fuerza de arrastre } \\
(\mathrm{kN})\end{array}$ & 11,5935 & 11,602 & 14,7377 & 14,7485 \\
\hline & Máxima fuerza no lineal (kN) & 10,1088 & 10,1161 & 0,0 & 0,0 \\
\hline & $\begin{array}{l}\text { Máxima fuerza de difracción } \\
(\mathrm{kN})\end{array}$ & 0,0 & 0,0 & 0,0 & 0,0 \\
\hline & Máxima fuerza total (kN) & 432,2264 & 431,9804 & 429,0758 & 429,3892 \\
\hline
\end{tabular}

Tabla 45. Fuerzas hidrodinámicas para el caso de $H=7,7 m, h=24,0 m, T=9,7$ s y $D=16,0 m$

Los valores de dichas fuerzas en el fondo marino, en el instante en que producen el máximo momento flector sobre la cimentación, se muestran en la tabla 46:

\begin{tabular}{|c|c|c|c|c|c|}
\hline & & \multicolumn{2}{|c|}{ Método Avanzado } & \multicolumn{2}{|c|}{ Método tradicional } \\
\hline & Parámetro & $\begin{aligned} \mathrm{T}^{\mathrm{a}} & =10 \mathrm{C}, \\
\mathrm{S} & =0 \%\end{aligned}$ & $\begin{array}{c}\mathrm{T}^{\mathrm{a}}=22{ }^{\circ} \mathrm{C} \\
\mathrm{S}=35 \%\end{array}$ & $\begin{array}{c}\mathrm{T}^{\mathrm{a}}=19 \mathrm{O}, \\
\mathrm{S}=0 \%\end{array}$ & $\begin{array}{c}\mathrm{T}^{\mathrm{a}}=22{ }^{\circ} \mathrm{C} \\
\mathrm{S}=35 \%\end{array}$ \\
\hline \multirow{6}{*}{$\begin{array}{l}0 \\
\text { 은 } \\
\text { 인 }\end{array}$} & $\begin{array}{l}\text { Máximo momento flector } \\
(\mathrm{kN} \cdot \mathrm{m})\end{array}$ & 70943,102 & 66755,033 & 87240,116 & 87303,852 \\
\hline & $\begin{array}{l}\text { Máxima fuerza de inercia } \\
(\mathrm{kN})\end{array}$ & 327,4747 & 327,7139 & 429,0761 & 429,3895 \\
\hline & $\begin{array}{l}\text { Máxima fuerza de arrastre } \\
(\mathrm{kN})\end{array}$ & 0,2112 & 0,2114 & $-0,0003$ & $-0,0003$ \\
\hline & Máxima fuerza no lineal (kN) & $-2,7042$ & $-2,7061$ & 0,0 & 0,0 \\
\hline & $\begin{array}{l}\text { Máxima fuerza de difracción } \\
(\mathrm{kN})\end{array}$ & 0,0 & 0,0 & 0,0 & 0,0 \\
\hline & Máxima fuerza total (kN) & 324,9818 & 325,2192 & 429,0758 & 429,3892 \\
\hline
\end{tabular}

Tabla 46. Fuerzas hidrodinámicas para momento flector máximo, para $H=7,7 m, h=24,0 m, T=9,7$ s y $D=16,0 m$ 
Por último, se adjuntan en la tabla 47, los valores característicos del número de KeuleganCarpenter (KC), del número de Reynolds (Re) y el parámetro beta de Sarpkaya $(\beta)$ :

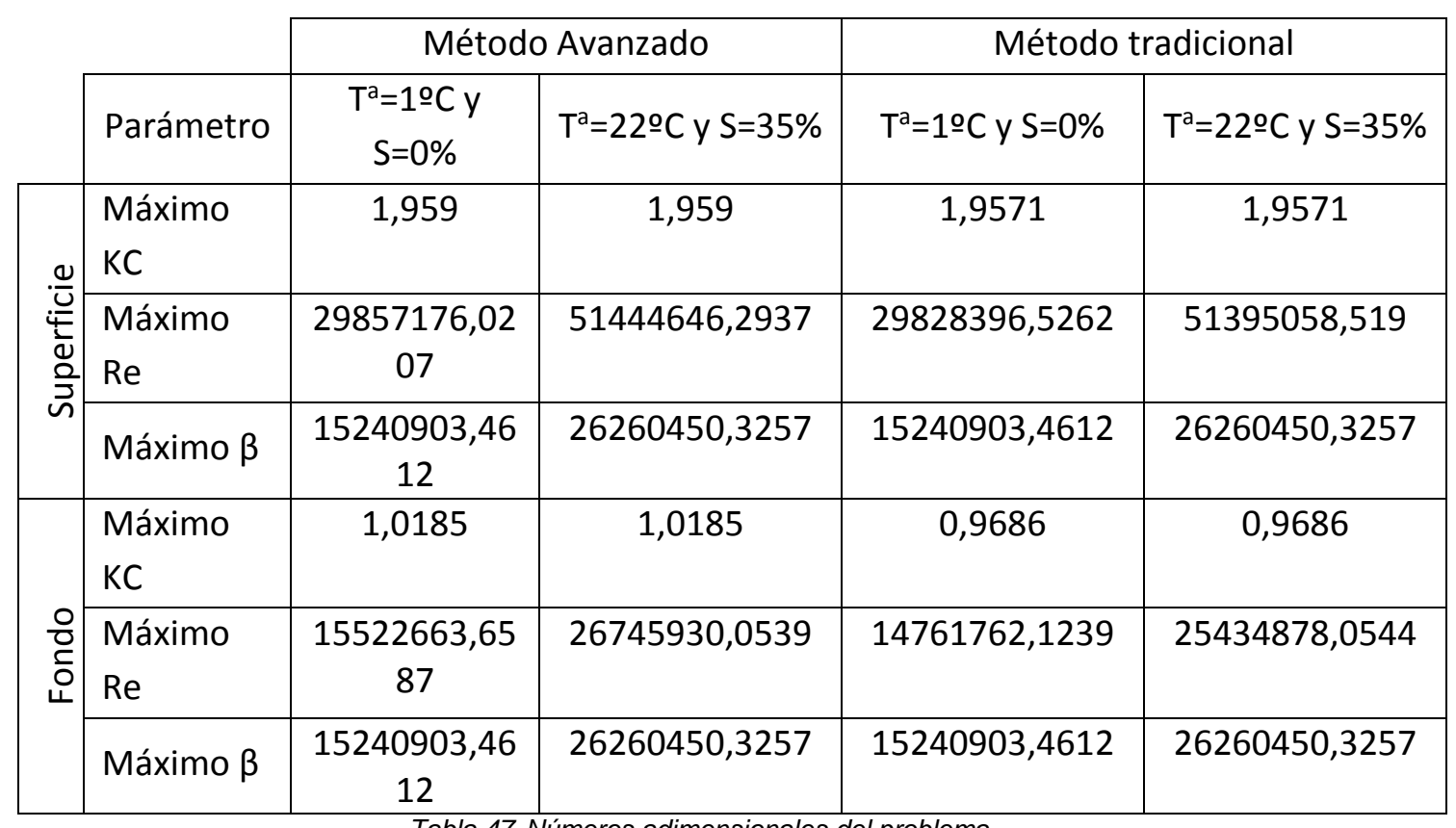




\subsubsection{Caso 4B}

Los datos de entrada se corresponden con el caso de cálculo para el parque eólico offshore North Hoyle en Reino Unido: altura de ola significativa $4,78 \mathrm{~m}$, profundidad $10,0 \mathrm{~m}$ y periodo de pico 6,8 s. De acuerdo con estos datos, en la figura 46 se muestra la distribución de fuerzas hidrodinámicas, generadas por el oleaje, en el instante en que producen el máximo momento flector, sobre la base del pilote, mostrando el cálculo según el método avanzado a la izquierda y según el método tradicional, a la derecha.
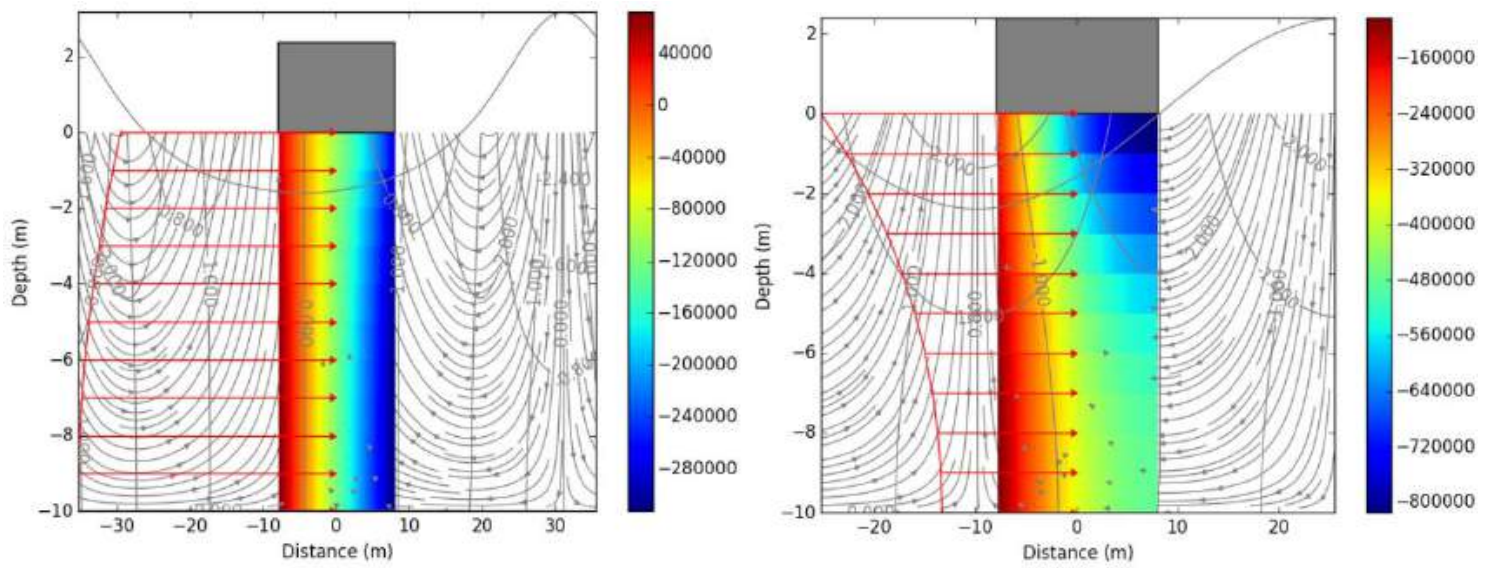

Figura 46. Momento flector máximo para $H=4,78 m, h=10,0 m, T=6,8$ s y $D=16,0 m$

Los valores más característicos de la simulación, tanto para el caso de temperatura de 1 ํㅡ y salinidad de $0 \%$, como para el caso de temperatura de $22{ }^{\circ} \mathrm{C}$ y salinidad del $35 \%$, se muestran, a continuación, en la tabla 48:

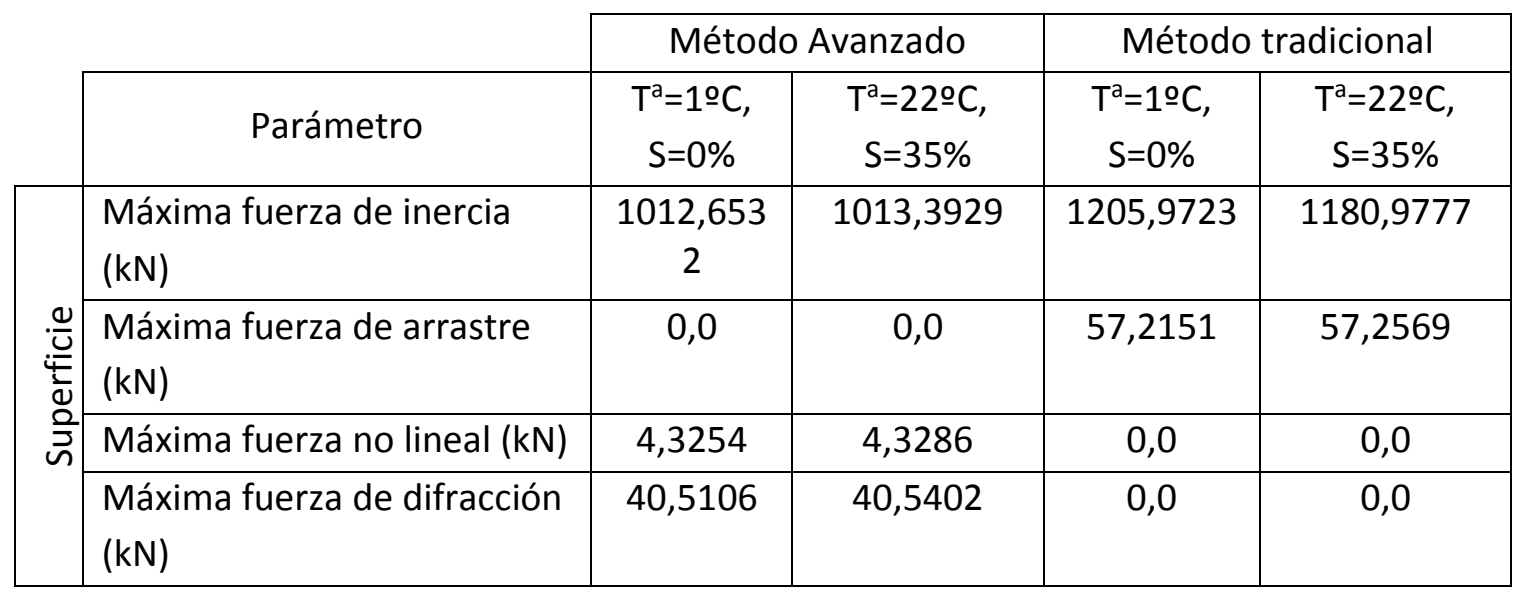




\begin{tabular}{|c|c|c|c|c|c|}
\hline & Máxima fuerza total (kN) & $\begin{array}{c}1004,612 \\
5\end{array}$ & 1005,2249 & 1205,9696 & 1180,9755 \\
\hline \multirow{5}{*}{$\begin{array}{l}\text { 융 } \\
\text { 은 } \\
\text { 인 }\end{array}$} & $\begin{array}{l}\text { Máxima fuerza de inercia } \\
(\mathrm{kN})\end{array}$ & 633,0434 & 633,5058 & 643,7261 & 644,1964 \\
\hline & $\begin{array}{l}\text { Máxima fuerza de arrastre } \\
(\mathrm{kN})\end{array}$ & 0,0 & 0,0 & 16,3019 & 16,3138 \\
\hline & Máxima fuerza no lineal (kN) & 7,5876 & 7,5931 & 0,0 & 0,0 \\
\hline & $\begin{array}{l}\text { Máxima fuerza de difracción } \\
(\mathrm{kN})\end{array}$ & 30,3077 & 30,3299 & 0,0 & 0,0 \\
\hline & Máxima fuerza total (kN) & 627,2768 & 627,7145 & 643,7253 & 644,1956 \\
\hline
\end{tabular}

Los valores de dichas fuerzas en el fondo marino, en el instante en que producen el máximo momento flector sobre la cimentación, se muestran en la tabla 49:

\begin{tabular}{|c|c|c|c|c|c|}
\hline & \multirow[b]{2}{*}{ Parámetro } & \multicolumn{2}{|c|}{ Método Avanzado } & \multicolumn{2}{|c|}{ Método tradicional } \\
\hline & & $\begin{aligned} \mathrm{T}^{\mathrm{a}} & =10 \mathrm{C} \\
\mathrm{S} & =0 \%\end{aligned}$ & $\begin{array}{c}\mathrm{T}^{\mathrm{a}}=22^{\circ} \mathrm{C}, \\
\mathrm{S}=35 \%\end{array}$ & $\begin{array}{c}\mathrm{T}^{\mathrm{a}}=1 \mathrm{O} \mathrm{C} \\
\mathrm{S}=0 \%\end{array}$ & $\begin{array}{c}\mathrm{T}^{\mathrm{a}}=22{ }^{\circ} \mathrm{C}, \\
\mathrm{S}=35 \%\end{array}$ \\
\hline \multirow{6}{*}{ 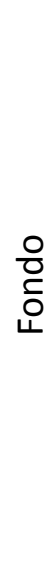 } & $\begin{array}{l}\text { Máximo momento flector } \\
(\mathrm{kN} \cdot \mathrm{m})\end{array}$ & 39240,739 & 39269,404 & 51852,897 & 51632,033 \\
\hline & $\begin{array}{l}\text { Máxima fuerza de inercia } \\
(\mathrm{kN})\end{array}$ & 498,1554 & 498,5193 & 643,7261 & 644,1964 \\
\hline & $\begin{array}{l}\text { Máxima fuerza de arrastre } \\
(\mathrm{kN})\end{array}$ & 0,0 & 0,0 & $-0,0008$ & $-0,0008$ \\
\hline & Máxima fuerza no lineal (kN) & 7,5619 & 7,5674 & 0,0 & 0,0 \\
\hline & $\begin{array}{l}\text { Máxima fuerza de difracción } \\
(\mathrm{kN})\end{array}$ & 16,3194 & 16,3313 & 0,0 & 0,0 \\
\hline & Máxima fuerza total (kN) & 481,836 & 482,188 & 643,7253 & 644,1956 \\
\hline
\end{tabular}

Tabla 49. Fuerzas hidrodinámicas para momento flector máximo, para $H=4,78 \mathrm{~m}, h=10,0 \mathrm{~m}, \mathrm{~T}=6,8 \mathrm{~s}$ y $\mathrm{D}=16,0 \mathrm{~m}$

Por último, se adjuntan en la tabla 50, los valores característicos del número de KeuleganCarpenter $(\mathrm{KC})$, del número de Reynolds $(\mathrm{Re})$ y el parámetro beta de Sarpkaya $(\beta)$ : 
Modelos de predicción de esfuerzos hidrodinámicos y socavación aplicados a ingeniería offshore

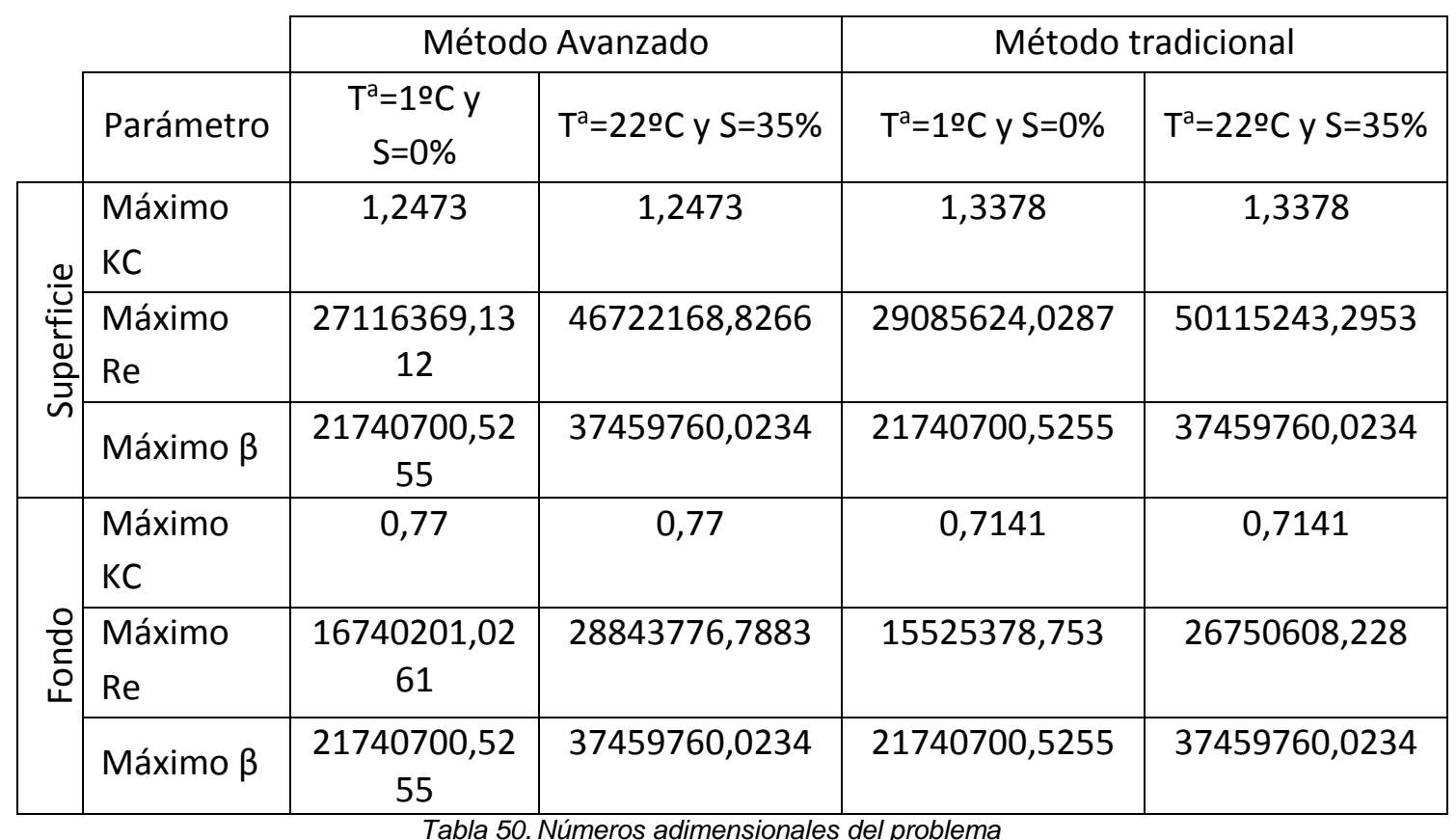




\subsubsection{Caso 4C}

Los datos de entrada se corresponden con el caso de cálculo para el parque eólico offshore Egmond Aan Zee en Holanda: altura de ola significativa 3,6 m, profundidad 22,0 m y periodo de pico 18,5 s. De acuerdo con estos datos, en la figura 47 se muestra la distribución de fuerzas hidrodinámicas, generadas por el oleaje, en el instante en que producen el máximo momento flector, sobre la base del pilote, mostrando el cálculo según el método avanzado a la izquierda y según el método tradicional, a la derecha.
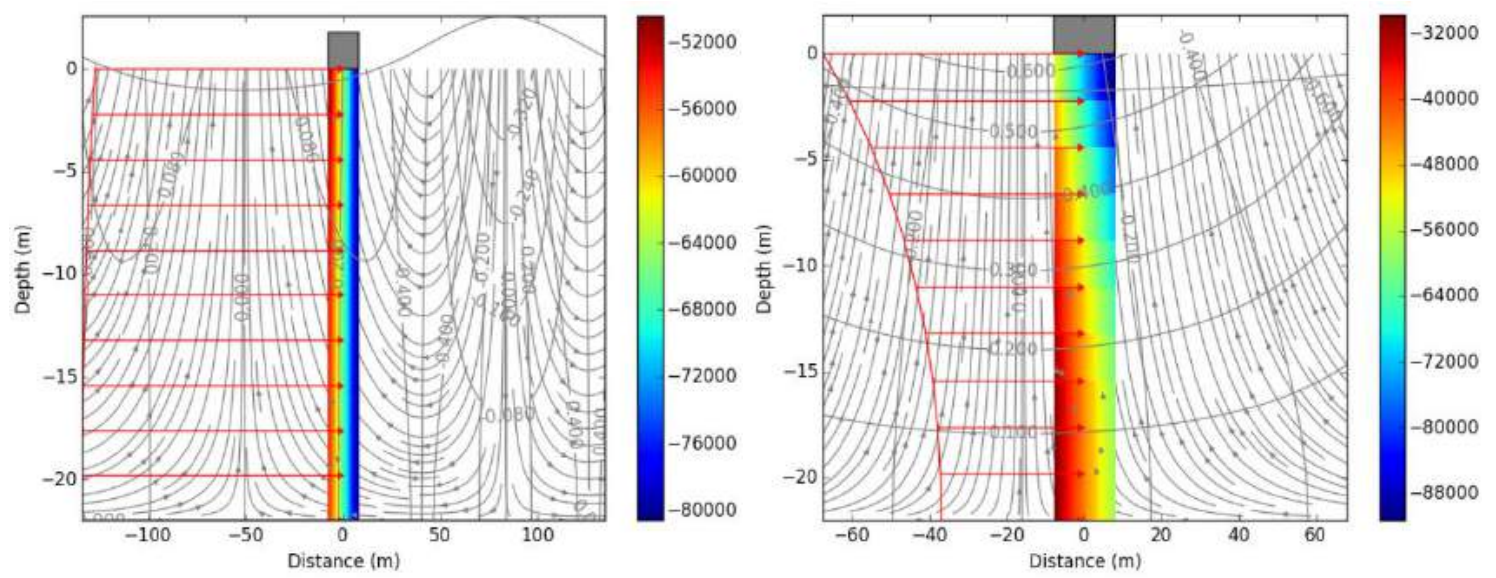

Figura 47. Momento flector máximo para $H=3,6 m, h=22,0 m, T=18,5$ s y $D=16,0 m$

Los valores más característicos de la simulación, tanto para el caso de temperatura de 1 으 y salinidad de $0 \%$, como para el caso de temperatura de $22{ }^{\circ} \mathrm{C}$ y salinidad del $35 \%$, se muestran, a continuación, en la tabla 51:

\begin{tabular}{|c|c|c|c|c|c|}
\hline & \multirow[b]{2}{*}{ Parámetro } & \multicolumn{2}{|c|}{ Método Avanzado } & \multicolumn{2}{|c|}{ Método tradicional } \\
\hline & & $\begin{array}{c}\mathrm{T}^{\mathrm{a}}=1 \mathrm{o} \mathrm{C}, \\
\mathrm{S}=0 \%\end{array}$ & $\begin{array}{c}\mathrm{T}^{\mathrm{a}}=22 \mathrm{o} \mathrm{C}, \\
\mathrm{S}=35 \%\end{array}$ & $\begin{array}{c}\mathrm{T}^{\mathrm{a}}=1 \mathrm{o} \mathrm{C} \\
\mathrm{S}=0 \%\end{array}$ & $\begin{array}{c}\mathrm{T}^{\mathrm{a}}=22 \mathrm{o} C, \\
\mathrm{~S}=35 \%\end{array}$ \\
\hline \multirow{5}{*}{$\begin{array}{l}\frac{0}{u} \\
\frac{0}{2} \\
\frac{0}{3} \\
\tilde{n}\end{array}$} & $\begin{array}{l}\text { Máxima fuerza de inercia } \\
(\mathrm{kN})\end{array}$ & 175,5822 & 175,7105 & 340,1761 & 340,4246 \\
\hline & $\begin{array}{l}\text { Máxima fuerza de arrastre } \\
(\mathrm{kN})\end{array}$ & 19,4012 & 19,4153 & 33,6953 & 33,7199 \\
\hline & Máxima fuerza no lineal (kN) & 0,0 & 0,0 & 0,0 & 0,0 \\
\hline & $\begin{array}{l}\text { Máxima fuerza de difracción } \\
(\mathrm{kN})\end{array}$ & 0,0 & 0,0 & 0,0 & 0,0 \\
\hline & Máxima fuerza total (kN) & 181,2438 & 181,3762 & 340,1772 & 340,4256 \\
\hline
\end{tabular}




\begin{tabular}{|c|c|c|c|c|c|}
\hline \multirow{5}{*}{$\begin{array}{l}\frac{0}{0} \\
\text { 둥 }\end{array}$} & $\begin{array}{l}\text { Máxima fuerza de inercia } \\
(\mathrm{kN})\end{array}$ & 128,9739 & 127,7079 & 216,7333 & 216,8916 \\
\hline & $\begin{array}{l}\text { Máxima fuerza de arrastre } \\
(\mathrm{kN})\end{array}$ & 11,9179 & 11,9266 & 13,6777 & 13,6877 \\
\hline & Máxima fuerza no lineal (kN) & 0,0 & 0,0 & 0,0 & 0,0 \\
\hline & $\begin{array}{l}\text { Máxima fuerza de difracción } \\
(\mathrm{kN})\end{array}$ & 0,0 & 0,0 & 0,0 & 0,0 \\
\hline & Máxima fuerza total (kN) & 129,3018 & 129,3962 & 216,7337 & 216,8921 \\
\hline
\end{tabular}

Los valores de dichas fuerzas en el fondo marino, en el instante en que producen el máximo momento flector sobre la cimentación, se muestran en la tabla 52:

\begin{tabular}{|c|c|c|c|c|c|}
\hline & & \multicolumn{2}{|c|}{ Método Avanzado } & \multicolumn{2}{|c|}{ Método tradicional } \\
\hline & Parámetro & $\begin{array}{c}\mathrm{T}^{\mathrm{a}}=1 \mathrm{O} \mathrm{C}, \\
\mathrm{S}=0 \%\end{array}$ & $\begin{array}{c}\mathrm{T}^{\mathrm{a}}=22 \mathrm{o} \mathrm{C}, \\
\mathrm{S}=35 \%\end{array}$ & $\begin{array}{c}\mathrm{T}^{\mathrm{a}}=1 \mathrm{O} \mathrm{C} \\
\mathrm{S}=0 \%\end{array}$ & $\begin{array}{c}\mathrm{T}^{\mathrm{a}}=22 \mathrm{o} C \\
\mathrm{~S}=35 \%\end{array}$ \\
\hline \multirow{6}{*}{$\begin{array}{l}0 \\
0 \\
0 \\
0 \\
0\end{array}$} & $\begin{array}{l}\text { Máximo momento flector } \\
(\mathrm{kN} \cdot \mathrm{m})\end{array}$ & 18887,547 & 18901,344 & 34253,375 & 34278,391 \\
\hline & $\begin{array}{l}\text { Máxima fuerza de inercia } \\
(\mathrm{kN})\end{array}$ & 122,1589 & 122,2482 & 216,7333 & 216,8916 \\
\hline & $\begin{array}{l}\text { Máxima fuerza de arrastre } \\
(\mathrm{kN})\end{array}$ & 4,3881 & 4,3913 & 0,0005 & 0,0005 \\
\hline & Máxima fuerza no lineal (kN) & 0,0 & 0,0 & 0,0 & 0,0 \\
\hline & $\begin{array}{l}\text { Máxima fuerza de difracción } \\
(\mathrm{kN})\end{array}$ & 0,0 & 0,0 & 0,0 & 0,0 \\
\hline & Máxima fuerza total (kN) & 126,547 & 126,6394 & 216,7337 & 216,8921 \\
\hline
\end{tabular}

Tabla 52. Fuerzas hidrodinámicas para momento flector máximo, para $\mathrm{H}=3,6 \mathrm{~m}, \mathrm{~h}=22,0 \mathrm{~m}, \mathrm{~T}=18,5 \mathrm{~s}$ y $\mathrm{D}=16,0 \mathrm{~m}$

Por último, se adjuntan en la tabla 53, los valores característicos del número de KeuleganCarpenter $(\mathrm{KC})$, del número de Reynolds $(\mathrm{Re})$ y el parámetro beta de Sarpkaya $(\beta)$ : 
Modelos de predicción de esfuerzos hidrodinámicos y socavación aplicados a ingeniería offshore

\begin{tabular}{|c|c|c|c|c|c|}
\hline & & \multicolumn{2}{|c|}{ Método Avanzado } & \multicolumn{2}{|c|}{ Método tradicional } \\
\hline & Parámetro & $\begin{array}{c}\mathrm{T}^{\mathrm{a}}=10 \mathrm{C} \mathrm{y} \\
\mathrm{S}=0 \%\end{array}$ & $\mathrm{~T}^{\mathrm{a}}=22^{\circ} \mathrm{O} \mathrm{C}$ y $\mathrm{S}=35 \%$ & $\mathrm{~T}^{\mathrm{a}}=1 \stackrel{\circ}{ } \mathrm{C}$ y $\mathrm{S}=0 \%$ & $\mathrm{~T}^{\mathrm{a}}=22{ }^{\circ} \mathrm{C}$ y $\mathrm{S}=35 \%$ \\
\hline \multirow{3}{*}{ 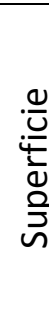 } & $\begin{array}{l}\text { Máximo } \\
\text { KC }\end{array}$ & 2,1195 & 2,1195 & 2,7932 & 2,7932 \\
\hline & $\begin{array}{l}\text { Máximo } \\
\text { Re }\end{array}$ & $\begin{array}{c}16937023,00 \\
96\end{array}$ & 29182905,8915 & 22320692,4239 & 38459100,2841 \\
\hline & Máximo $\beta$ & $\begin{array}{c}7991176,409 \\
4\end{array}$ & 13768992,8735 & 7991176,4094 & 13768992,8735 \\
\hline \multirow{3}{*}{$\begin{array}{l}0 \\
0 \\
0 \\
0 \\
0\end{array}$} & $\begin{array}{l}\text { Máximo } \\
\text { KC }\end{array}$ & 1,6612 & 1,6612 & 1,7796 & 1,7796 \\
\hline & $\begin{array}{l}\text { Máximo } \\
\text { Re }\end{array}$ & $\begin{array}{c}13274631,75 \\
85\end{array}$ & 22872515,9748 & 14220978,618 & 24503094,8153 \\
\hline & Máximo $\beta$ & $\begin{array}{c}7991176,409 \\
4\end{array}$ & 13768992,8735 & 7991176,4094 & 13768992,8735 \\
\hline
\end{tabular}




\subsubsection{Caso 4D}

Los datos de entrada se corresponden con el caso de cálculo para el parque eólico offshore Thornton Bank en Bélgica: altura de ola significativa $6,32 \mathrm{~m}$, profundidad $16,0 \mathrm{~m}$ y periodo de pico 11,06 s. De acuerdo con estos datos, en la figura 48 se muestra la distribución de fuerzas hidrodinámicas, generadas por el oleaje, en el instante en que producen el máximo momento flector, sobre la base del pilote, mostrando el cálculo según el método avanzado a la izquierda y según el método tradicional, a la derecha.
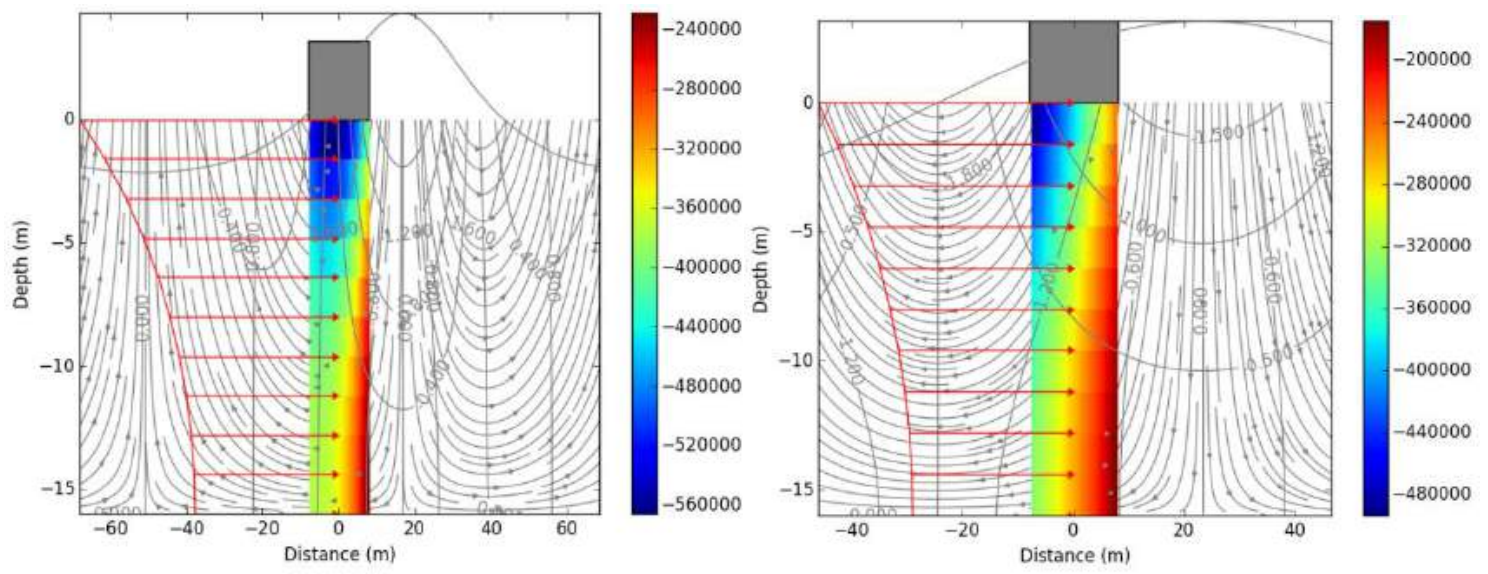

Figura 48. Momento flector máximo para $H=6,32 m, h=16 m, T=11,06$ s y $D=16,0 m$

Los valores más característicos de la simulación, tanto para el caso de temperatura de 1 ํㅡ y salinidad de $0 \%$, como para el caso de temperatura de $22{ }^{\circ} \mathrm{C}$ y salinidad del $35 \%$, se muestran, a continuación, en la tabla 54:

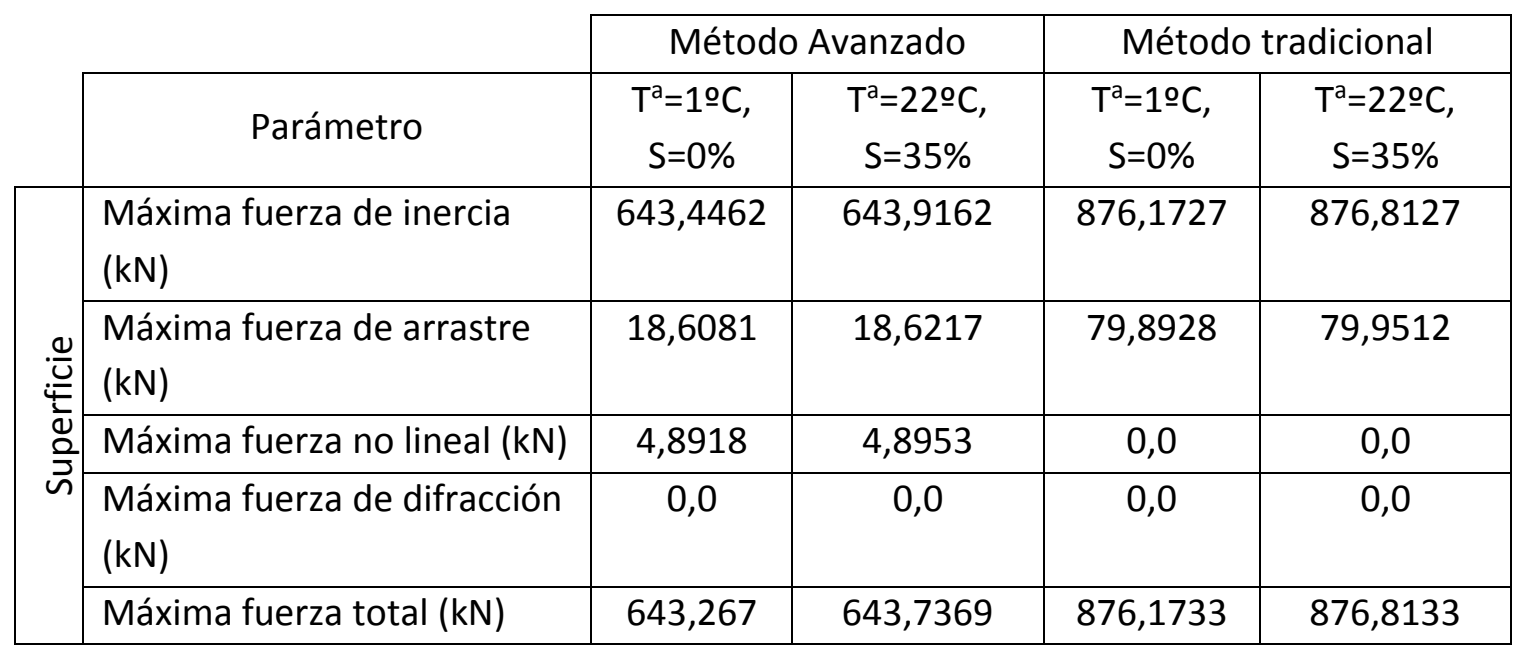




\begin{tabular}{|c|c|c|c|c|c|}
\hline \multirow{5}{*}{$\begin{array}{l}\text { 윰 } \\
\text { 은 } \\
\text { 난 }\end{array}$} & $\begin{array}{l}\text { Máxima fuerza de inercia } \\
(\mathrm{kN})\end{array}$ & 462,2476 & 462,5852 & 529,0371 & 529,4236 \\
\hline & $\begin{array}{l}\text { Máxima fuerza de arrastre } \\
(\mathrm{kN})\end{array}$ & 11,2403 & 11,2485 & 29,1273 & 29,1486 \\
\hline & Máxima fuerza no lineal (kN) & 9,3342 & 9,341 & 0,0 & 0,0 \\
\hline & $\begin{array}{l}\text { Máxima fuerza de difracción } \\
(\mathrm{kN})\end{array}$ & 0,0 & 0,0 & 0,0 & 0,0 \\
\hline & Máxima fuerza total (kN) & 459,5811 & 459,8401 & 529,0373 & 529,4238 \\
\hline
\end{tabular}

Tabla 54. Fuerzas hidrodinámicas para el caso de $H=6,32 m, h=16 m, T=11,06$ s y $D=16,0 m$

Los valores de dichas fuerzas en el fondo marino, en el instante en que producen el máximo momento flector sobre la cimentación, se muestran en la tabla 55:

\begin{tabular}{|c|c|c|c|c|c|}
\hline & & \multicolumn{2}{|c|}{ Método Avanzado } & \multicolumn{2}{|c|}{ Método tradicional } \\
\hline & Parámetro & $\begin{aligned} \mathrm{T}^{\mathrm{a}} & =1 \text { - } \mathrm{C}, \\
\mathrm{S} & =0 \%\end{aligned}$ & $\begin{array}{c}\mathrm{T}^{\mathrm{a}}=22{ }^{\circ} \mathrm{C}, \\
\mathrm{S}=35 \%\end{array}$ & $\begin{array}{c}\mathrm{T}^{\mathrm{a}}=1 \mathrm{O} \mathrm{C}, \\
\mathrm{S}=0 \%\end{array}$ & $\begin{array}{c}\mathrm{T}^{\mathrm{a}}=22{ }^{\circ} \mathrm{C}, \\
\mathrm{S}=35 \%\end{array}$ \\
\hline \multirow{6}{*}{$\begin{array}{l}\frac{0}{0} \\
\frac{1}{0} \\
\text { 닌 }\end{array}$} & $\begin{array}{l}\text { Máximo momento flector } \\
(\mathrm{kN} \cdot \mathrm{m})\end{array}$ & 45012,165 & 45045,046 & 62924,296 & 62970,261 \\
\hline & $\begin{array}{l}\text { Máxima fuerza de inercia } \\
(\mathrm{kN})\end{array}$ & 376,7113 & 376,9864 & 529,0371 & 529,4236 \\
\hline & $\begin{array}{l}\text { Máxima fuerza de arrastre } \\
(\mathrm{kN})\end{array}$ & 4,5844 & 4,5878 & 0,0002 & 0,0002 \\
\hline & Máxima fuerza no lineal (kN) & $-9,1747$ & $-9,1814$ & 0,0 & 0,0 \\
\hline & $\begin{array}{l}\text { Máxima fuerza de difracción } \\
(\mathrm{kN})\end{array}$ & 0,0 & 0,0 & 0,0 & 0,0 \\
\hline & Máxima fuerza total (kN) & 372,121 & 372,3928 & 529,0373 & 529,4238 \\
\hline
\end{tabular}

Tabla 55. Fuerzas hidrodinámicas para momento flector máximo, para $\mathrm{H}=6,32 \mathrm{~m}, h=16 \mathrm{~m}, T=11,06 \mathrm{~s}$ y $D=16,0 \mathrm{~m}$

Por último, se adjuntan en la tabla 56, los valores característicos del número de KeuleganCarpenter $(\mathrm{KC})$, del número de Reynolds $(\mathrm{Re})$ y el parámetro beta de Sarpkaya $(\beta)$ : 

aplicados a ingeniería offshore

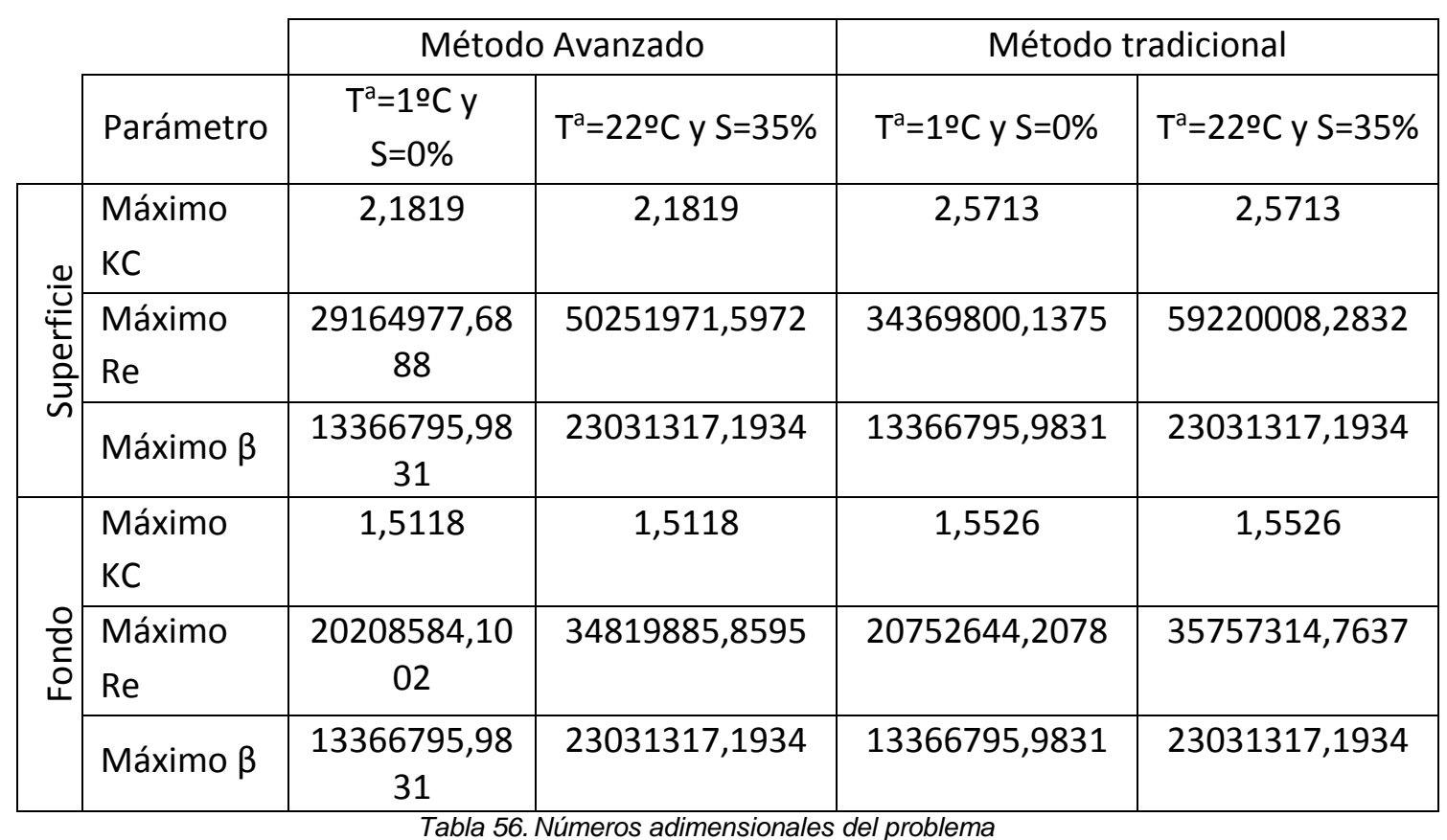




\section{Proceso III: Análisis dinámico}

El tercer proceso se corresponde con el cálculo del comportamiento dinámico de la estructura, a partir de las fuerzas generadas por el oleaje, $F(z, t)$, para cada instante de tiempo, $\mathrm{t}_{\mathrm{i}}$.

\subsection{Caso 1: Diámetros igual a 0,5 m}

\subsubsection{Caso $1 \mathrm{~A}$}

Los datos de entrada se corresponden con el caso de cálculo para el parque eólico offshore Princess Amalia Q7 en Holanda: altura de ola significativa 7,7 m, profundidad 24,0 m y periodo de pico 9,7 s. De acuerdo con estos datos, en la figura 49 se muestra la deformada de la pila, en el instante en que se produce el máximo momento flector, sobre la base del pilote, mostrando el cálculo según el método avanzado a la izquierda y según el método tradicional, a la derecha.
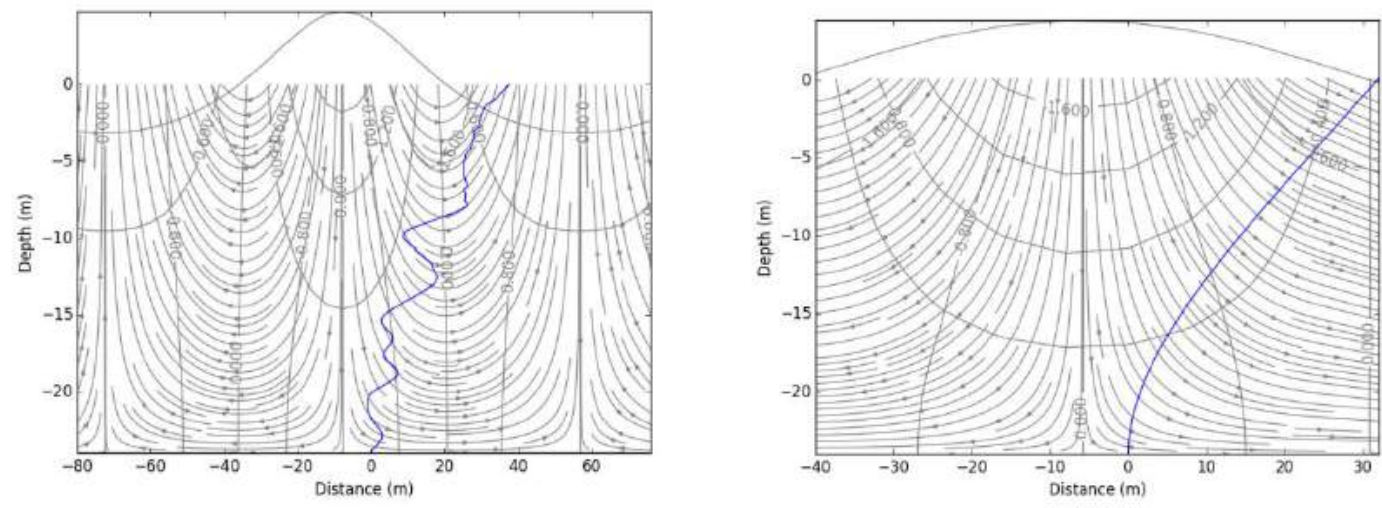

Figura 49. Deformada en el instante de momento flector máximo para $H=7,7 m, h=24,0 m, T=9,7 s$ y $D=0,5 m$

La imagen de las deformadas ha sido escalada en el plano de las abscisas, para poder ser representada junto con el perfil de oleaje.

Los valores más característicos de la simulación, tanto para el caso de temperatura de 1 ํ $\mathrm{C}$ y salinidad de $0 \%$, como para el caso de temperatura de $22^{\circ} \mathrm{C}$ y salinidad del $35 \%$, se muestran, a continuación, en la tabla 57: 
Modelos de predicción de esfuerzos hidrodinámicos y socavación aplicados a ingeniería offshore

\begin{tabular}{|c|c|c|c|c|}
\hline \multirow[b]{2}{*}{ Parámetro } & \multicolumn{2}{|c|}{ Método avanzado } & \multicolumn{2}{|c|}{ Método tradicional } \\
\hline & $\mathrm{T}^{\mathrm{a}}=1 \stackrel{\circ}{ } \mathrm{C}, \mathrm{S}=0 \%$ & $\begin{array}{c}\mathrm{T}^{\mathrm{a}}=22{ }^{\circ} \mathrm{C}, \\
\mathrm{S}=35 \%\end{array}$ & $\mathrm{~T}^{\mathrm{a}}=1 \stackrel{\circ}{ } \mathrm{C}, \mathrm{S}=0 \%$ & $\begin{array}{c}\mathrm{T}^{\mathrm{a}}=22 \mathrm{o} C, \\
\mathrm{~S}=35 \%\end{array}$ \\
\hline $\begin{array}{l}\text { Máximo desplazamiento en } \\
\text { superficie }(\mathrm{m})\end{array}$ & 0,108408 & 0,108488 & 0,067698 & 0,067738 \\
\hline $\begin{array}{l}\text { Máximo esfuerzo cortante en } \\
\text { cimentación }(\mathrm{kN})\end{array}$ & 15,7363 & 15,2471 & 19,6185 & 19,5997 \\
\hline $\begin{array}{l}\text { Máximo momento flector en } \\
\text { cimentación }(\mathrm{kN} \cdot \mathrm{m})\end{array}$ & 205,4122 & 205,3283 & 273,2804 & 273,3697 \\
\hline
\end{tabular}

Tabla 57. Comportamiento de la estructura para el caso de $H=7,7 m, h=24,0 m, T=9,7 s$ y $D=0,5 m$ 


\subsubsection{Caso 1B}

Los datos de entrada se corresponden con el caso de cálculo para el parque eólico offshore North Hoyle en Reino Unido: altura de ola significativa 4,78 m, profundidad $10,0 \mathrm{~m}$ y periodo de pico 6,8 s. De acuerdo con estos datos, en la figura 50 se muestra la deformada de la pila, en el instante en que se produce el máximo momento flector, sobre la base del pilote, mostrando el cálculo según el método avanzado a la izquierda y según el método tradicional, a la derecha.
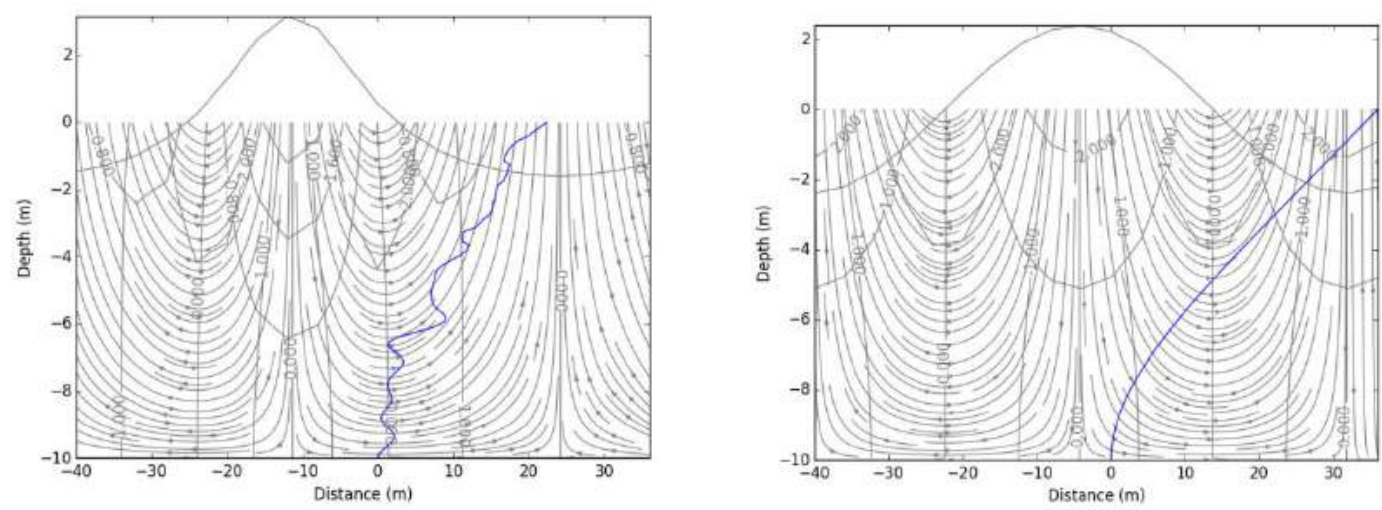

Figura 50. Deformada en el instante de momento flector máximo para $H=4,78 m, h=10,0 m, T=6,8 s$ y $D=0,5 m$

La imagen de las deformadas ha sido escalada en el plano de las abscisas, para poder ser representada junto con el perfil de oleaje.

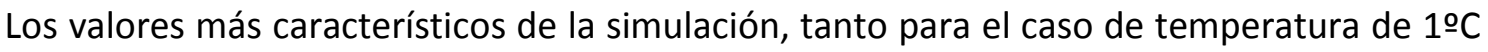
y salinidad de $0 \%$, como para el caso de temperatura de $22{ }^{\circ} \mathrm{C}$ y salinidad del $35 \%$, se muestran, a continuación, en la tabla 58:

\begin{tabular}{|c|c|c|c|c|}
\hline \multirow[b]{2}{*}{ Parámetro } & \multicolumn{2}{|c|}{ Método avanzado } & \multicolumn{2}{|c|}{ Método tradicional } \\
\hline & $\mathrm{T}^{\mathrm{a}}=1 \stackrel{\mathrm{o}}{ } \mathrm{C}, \mathrm{S}=0 \%$ & $\begin{array}{c}\mathrm{T}^{\mathrm{a}}=22{ }^{\circ} \mathrm{C}, \\
\mathrm{S}=35 \%\end{array}$ & $\mathrm{~T}^{\mathrm{a}}=1 \stackrel{\mathrm{o}}{ } \mathrm{C}, \mathrm{S}=0 \%$ & $\begin{aligned} \mathrm{T}^{\mathrm{a}} & =22{ }^{\circ} \mathrm{C}, \\
\mathrm{S} & =35 \%\end{aligned}$ \\
\hline $\begin{array}{l}\text { Máximo desplazamiento en } \\
\text { superficie }(\mathrm{m})\end{array}$ & 0,002749 & 0,002742 & 0,001543 & 0,001544 \\
\hline $\begin{array}{l}\text { Máximo esfuerzo cortante en } \\
\text { cimentación }(\mathrm{kN})\end{array}$ & 8,8607 & 7,9016 & 7,8444 & 7,8213 \\
\hline $\begin{array}{l}\text { Máximo momento flector en } \\
\text { cimentación }(\mathrm{kN} \cdot \mathrm{m})\end{array}$ & 42,4094 & 37,5519 & 39,3826 & 39,3705 \\
\hline
\end{tabular}

Tabla 58. Comportamiento de la estructura para el caso de $H=4,78 m, h=10,0 m, T=6,8$ s y $D=0,5 m$ 


\subsubsection{Caso $1 \mathrm{C}$}

Los datos de entrada se corresponden con el caso de cálculo para el parque eólico offshore Egmond Aan Zee en Holanda: altura de ola significativa 3,6 m, profundidad 22,0 m y periodo de pico 18,5 s. De acuerdo con estos datos, en la figura 51 se muestra la deformada de la pila, en el instante en que se produce el máximo momento flector, sobre la base del pilote, mostrando el cálculo según el método avanzado a la izquierda y según el método tradicional, a la derecha.
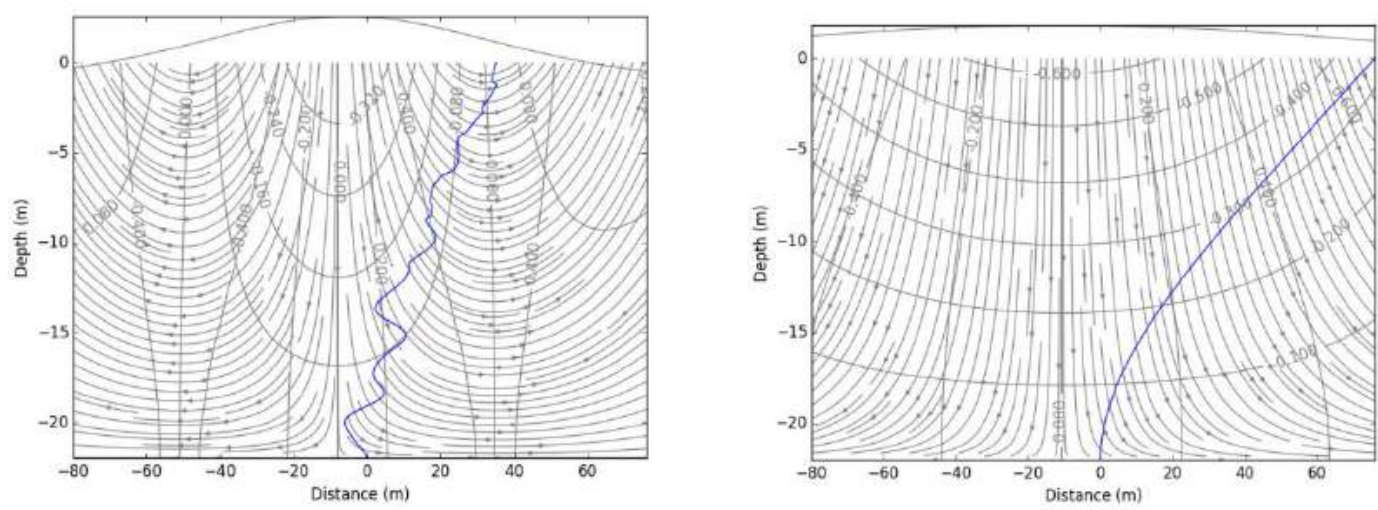

Figura 51. Deformada en el instante de momento flector máximo para $H=3,6 m, h=22,0 m, T=18,5 s$ y $D=0,5 m$

La imagen de las deformadas ha sido escalada en el plano de las abscisas, para poder ser representada junto con el perfil de oleaje.

Los valores más característicos de la simulación, tanto para el caso de temperatura de 1 ํㅡ y salinidad de $0 \%$, como para el caso de temperatura de $22{ }^{\circ} \mathrm{C}$ y salinidad del $35 \%$, se muestran, a continuación, en la tabla 59:

\begin{tabular}{|c|c|c|c|c|}
\hline \multirow[b]{2}{*}{ Parámetro } & \multicolumn{2}{|c|}{ Método avanzado } & \multicolumn{2}{|c|}{ Método tradicional } \\
\hline & $\mathrm{T}^{\mathrm{a}}=1 \stackrel{\mathrm{o}}{ } \mathrm{C}, \mathrm{S}=0 \%$ & $\begin{array}{c}\mathrm{T}^{\mathrm{a}}=22{ }^{\circ} \mathrm{C}, \\
\mathrm{S}=35 \%\end{array}$ & $\mathrm{~T}^{\mathrm{a}}=1 \stackrel{\mathrm{o}}{ } \mathrm{C}, \mathrm{S}=0 \%$ & $\begin{array}{c}\mathrm{T}^{\mathrm{a}}=22{ }^{\circ} \mathrm{C}, \\
\mathrm{S}=35 \%\end{array}$ \\
\hline $\begin{array}{l}\text { Máximo desplazamiento en } \\
\text { superficie }(\mathrm{m})\end{array}$ & 0,044739 & 0,044163 & 0,030625 & 0,030643 \\
\hline $\begin{array}{l}\text { Máximo esfuerzo cortante en } \\
\text { cimentación }(\mathrm{kN})\end{array}$ & 10,0342 & 9,9748 & 12,6867 & 12,6841 \\
\hline $\begin{array}{l}\text { Máximo momento flector en } \\
\text { cimentación }(\mathrm{kN} \cdot \mathrm{m})\end{array}$ & 112,978 & 112,5272 & 151,4389 & 151,4995 \\
\hline
\end{tabular}

Tabla 59. Comportamiento de la estructura para el caso de $H=3,6 m, h=22,0 m, T=18,5$ s y $D=0,5 m$ 


\subsubsection{Caso 1D}

Los datos de entrada se corresponden con el caso de cálculo para el parque eólico offshore Thornton Bank en Bélgica: altura de ola significativa $6,32 \mathrm{~m}$, profundidad $16,0 \mathrm{~m}$ y periodo de pico 11,06 s. De acuerdo con estos datos, en la figura 52 se muestra la deformada de la pila, en el instante en que se produce el máximo momento flector, sobre la base del pilote, mostrando el cálculo según el método avanzado a la izquierda y según el método tradicional, a la derecha.
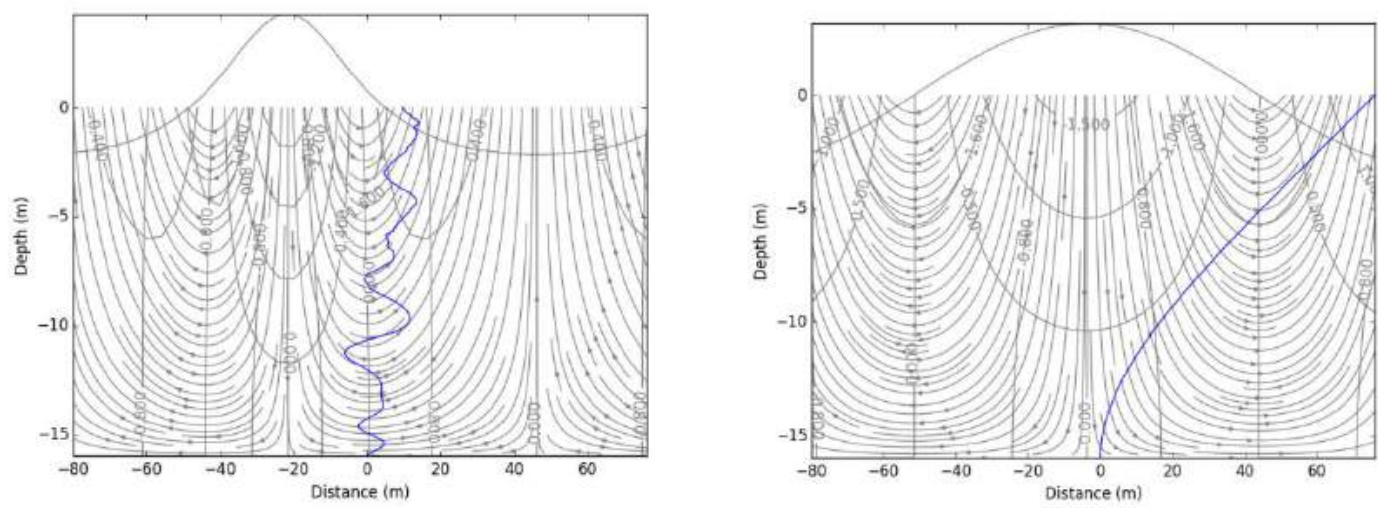

Figura 52. Deformada en el instante de momento flector máximo para $H=6,32 m, h=16,0 m, T=11,06 s$ y $D=0,5 m$

La imagen de las deformadas ha sido escalada en el plano de las abscisas, para poder ser representada junto con el perfil de oleaje.

Los valores más característicos de la simulación, tanto para el caso de temperatura de 1 으 y salinidad de $0 \%$, como para el caso de temperatura de $22^{\circ} \mathrm{C}$ y salinidad del $35 \%$, se muestran, a continuación, en la tabla 60:

\begin{tabular}{|l|c|c|c|c|}
\cline { 2 - 5 } \multicolumn{1}{c|}{} & \multicolumn{2}{c|}{ Método avanzado } & \multicolumn{2}{c|}{ Método tradicional } \\
\hline Parámetro & $\mathrm{T}^{\mathrm{a}}=1 \mathrm{o} \mathrm{C}, \mathrm{S}=0 \%$ & $\begin{array}{c}\mathrm{T}^{\mathrm{a}}=22 \mathrm{o} \mathrm{C}, \\
\mathrm{S}=35 \%\end{array}$ & $\mathrm{~T}^{\mathrm{a}}=1$ o $\mathrm{C}, \mathrm{S}=0 \%$ & $\begin{array}{c}\mathrm{T}^{\mathrm{a}}=22 \text { o } \mathrm{C}, \\
\mathrm{S}=35 \%\end{array}$ \\
\hline $\begin{array}{l}\text { Máximo desplazamiento en } \\
\text { superficie }(\mathrm{m})\end{array}$ & 0,014619 & 0,014625 & 0,018034 & 0,018048 \\
\hline $\begin{array}{l}\text { Máximo esfuerzo cortante en } \\
\text { cimentación }(\mathrm{kN})\end{array}$ & 10,9346 & 9,7122 & 19,9382 & 19,9528 \\
\hline $\begin{array}{l}\text { Máximo momento flector en } \\
\text { cimentación }(\mathrm{kN} \cdot \mathrm{m})\end{array}$ & 87,0082 & 78,6799 & 170,8073 & 170,9321 \\
\hline
\end{tabular}

Tabla 60. Comportamiento de la estructura para el caso de $H=6,32 m, h=16,0 m, T=11,06$ s y $D=0,5 m$ 


\subsection{Caso 2: Diámetros igual a 4,0 m}

\subsubsection{Caso $2 \mathrm{~A}$}

Los datos de entrada se corresponden con el caso de cálculo para el parque eólico offshore Princess Amalia Q7 en Holanda: altura de ola significativa 7,7 m, profundidad 24,0 m y periodo de pico 9,7 s. De acuerdo con estos datos, en la figura 53 se muestra la deformada de la pila, en el instante en que se produce el máximo momento flector, sobre la base del pilote, mostrando el cálculo según el método avanzado a la izquierda y según el método tradicional, a la derecha.
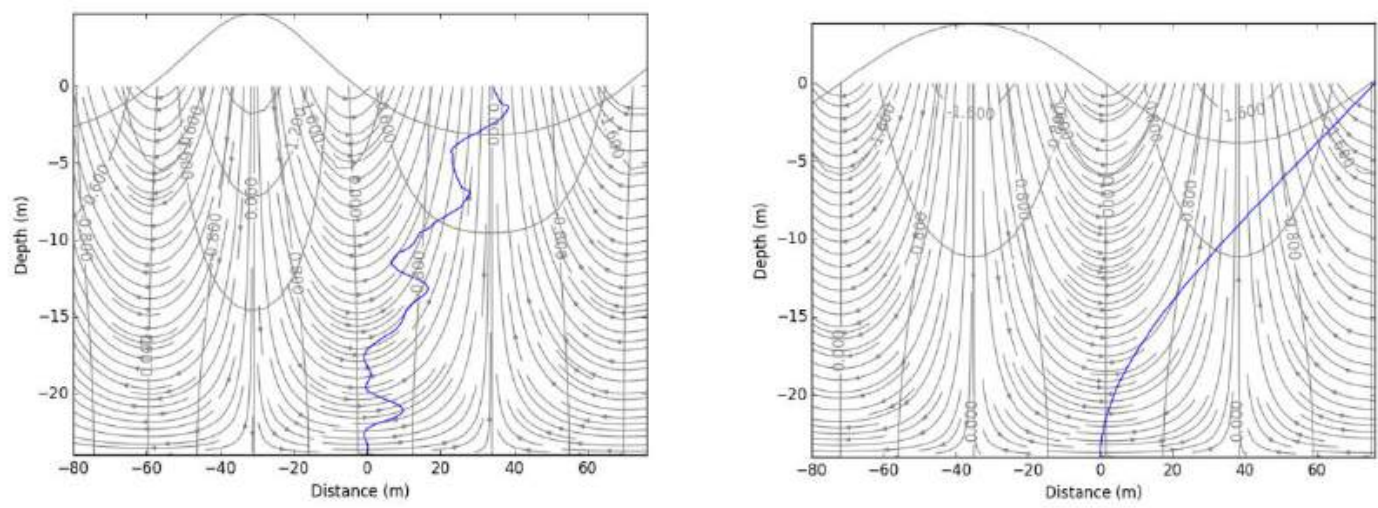

Figura 53. Deformada en el instante de momento flector máximo para $H=7,7 \mathrm{~m}, h=24,0 \mathrm{~m}, T=9,7 \mathrm{~s}$ y $D=4,0 \mathrm{~m}$

La imagen de las deformadas ha sido escalada en el plano de las abscisas, para poder ser representada junto con el perfil de oleaje.

Los valores más característicos de la simulación, tanto para el caso de temperatura de 1 ํㅡ y salinidad de $0 \%$, como para el caso de temperatura de $22^{\circ} \mathrm{C}$ y salinidad del $35 \%$, se muestran, a continuación, en la tabla 61:

\begin{tabular}{|c|c|c|c|c|}
\hline \multirow[b]{2}{*}{ Parámetro } & \multicolumn{2}{|c|}{ Método avanzado } & \multicolumn{2}{|c|}{ Método tradicional } \\
\hline & $\mathrm{T}^{\mathrm{a}}=1 \stackrel{\circ}{ } \mathrm{C}, \mathrm{S}=0 \%$ & $\begin{array}{c}\mathrm{T}^{\mathrm{a}}=22{ }^{\circ} \mathrm{C} \\
\mathrm{S}=35 \%\end{array}$ & $\mathrm{~T}^{\mathrm{a}}=1 \stackrel{\mathrm{o}}{ } \mathrm{C}, \mathrm{S}=0 \%$ & $\begin{array}{c}\mathrm{T}^{\mathrm{a}}=22{ }^{\circ} \mathrm{C}, \\
\mathrm{S}=35 \%\end{array}$ \\
\hline $\begin{array}{l}\text { Máximo desplazamiento en } \\
\text { superficie }(\mathrm{m})\end{array}$ & 0,00226729 & 0,00207231 & 0,00124111 & 0,00124201 \\
\hline $\begin{array}{l}\text { Máximo esfuerzo cortante en } \\
\text { cimentación }(\mathrm{kN})\end{array}$ & 769,7061 & 711,4984 & 796,2792 & 796,8609 \\
\hline $\begin{array}{l}\text { Máximo momento flector en } \\
\text { cimentación }(\mathrm{kN} \cdot \mathrm{m})\end{array}$ & 9761,9266 & 9178,5741 & 10181,8001 & 10189,2379 \\
\hline
\end{tabular}

Tabla 61. Comportamiento de la estructura para el caso de $H=7,7 m, h=24,0 m, T=9,7 s$ y $D=4,0 m$ 


\subsubsection{Caso 2B}

Los datos de entrada se corresponden con el caso de cálculo para el parque eólico offshore North Hoyle en Reino Unido: altura de ola significativa 4,78 m, profundidad $10,0 \mathrm{~m}$ y periodo de pico 6,8 s. De acuerdo con estos datos, en la figura 54 se muestra la deformada de la pila, en el instante en que se produce el máximo momento flector, sobre la base del pilote, mostrando el cálculo según el método avanzado a la izquierda y según el método tradicional, a la derecha.
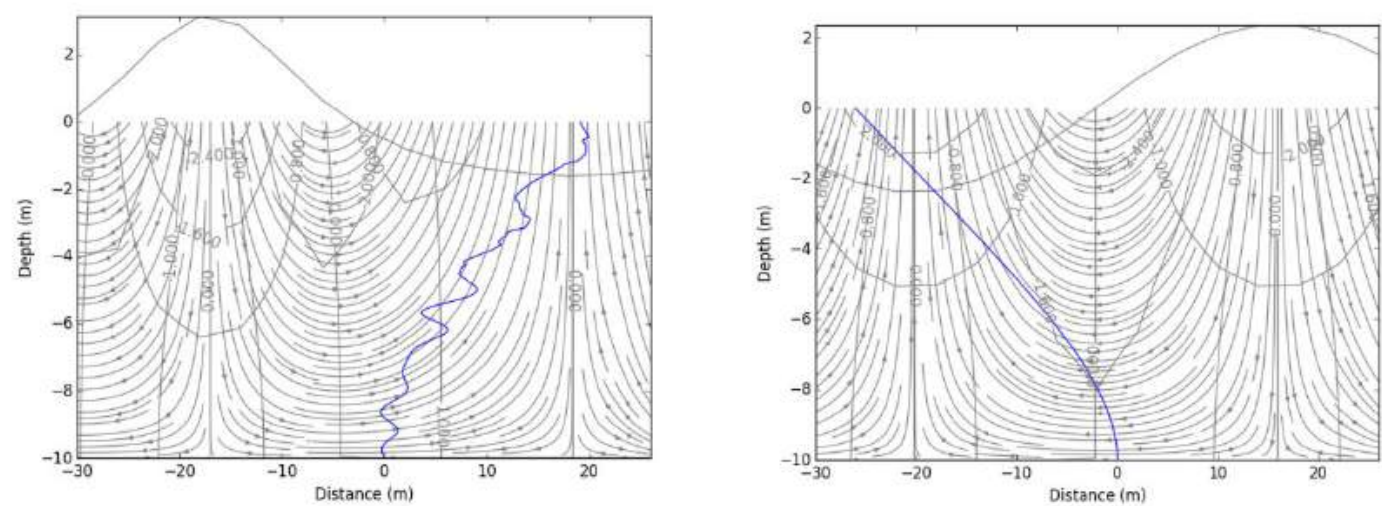

Figura 54. Deformada en el instante de momento flector máximo para $H=4,78 m, h=10,0 m, T=6,8$ s y $D=4,0 \mathrm{~m}$

La imagen de las deformadas ha sido escalada en el plano de las abscisas, para poder ser representada junto con el perfil de oleaje.

Los valores más característicos de la simulación, tanto para el caso de temperatura de 1 ํㅡ y salinidad de $0 \%$, como para el caso de temperatura de $22{ }^{\circ} \mathrm{C}$ y salinidad del $35 \%$, se muestran, a continuación, en la tabla 62:

\begin{tabular}{|c|c|c|c|c|}
\hline \multirow[b]{2}{*}{ Parámetro } & \multicolumn{2}{|c|}{ Método avanzado } & \multicolumn{2}{|c|}{ Método tradicional } \\
\hline & $\mathrm{T}^{\mathrm{a}}=1 \stackrel{\mathrm{o}}{ } \mathrm{C}, \mathrm{S}=0 \%$ & $\begin{array}{c}\mathrm{T}^{\mathrm{a}}=22{ }^{\circ} \mathrm{C}, \\
\mathrm{S}=35 \%\end{array}$ & $\mathrm{~T}^{\mathrm{a}}=1 \stackrel{\mathrm{o}}{ } \mathrm{C}, \mathrm{S}=0 \%$ & $\begin{array}{c}\mathrm{T}^{\mathrm{a}}=22 \mathrm{O} \mathrm{C}, \\
\mathrm{S}=35 \%\end{array}$ \\
\hline $\begin{array}{l}\text { Máximo desplazamiento en } \\
\text { superficie }(\mathrm{m})\end{array}$ & $6,531 \mathrm{e}-05$ & $6,536 e-05$ & $4,130 \mathrm{e}-05$ & $4,133 e-05$ \\
\hline $\begin{array}{l}\text { Máximo esfuerzo cortante en } \\
\text { cimentación }(\mathrm{kN})\end{array}$ & 386,5533 & 348,6344 & 438,3363 & 438,6571 \\
\hline $\begin{array}{l}\text { Máximo momento flector en } \\
\text { cimentación }(\mathrm{kN} \cdot \mathrm{m})\end{array}$ & 1873,845 & 1724,035 & 2100,411 & 2101,9494 \\
\hline
\end{tabular}

Tabla 62. Comportamiento de la estructura para el caso de $H=4,78 m, h=10,0 m, T=6,8$ s y $D=4,0 m$ 


\subsubsection{Caso 2C}

Los datos de entrada se corresponden con el caso de cálculo para el parque eólico offshore Egmond Aan Zee en Holanda: altura de ola significativa 3,6 m, profundidad 22,0 m y periodo de pico $18,5 \mathrm{~s}$. De acuerdo con estos datos, en la figura 55 se muestra la deformada de la pila, en el instante en que se produce el máximo momento flector, sobre la base del pilote, mostrando el cálculo según el método avanzado a la izquierda y según el método tradicional, a la derecha.
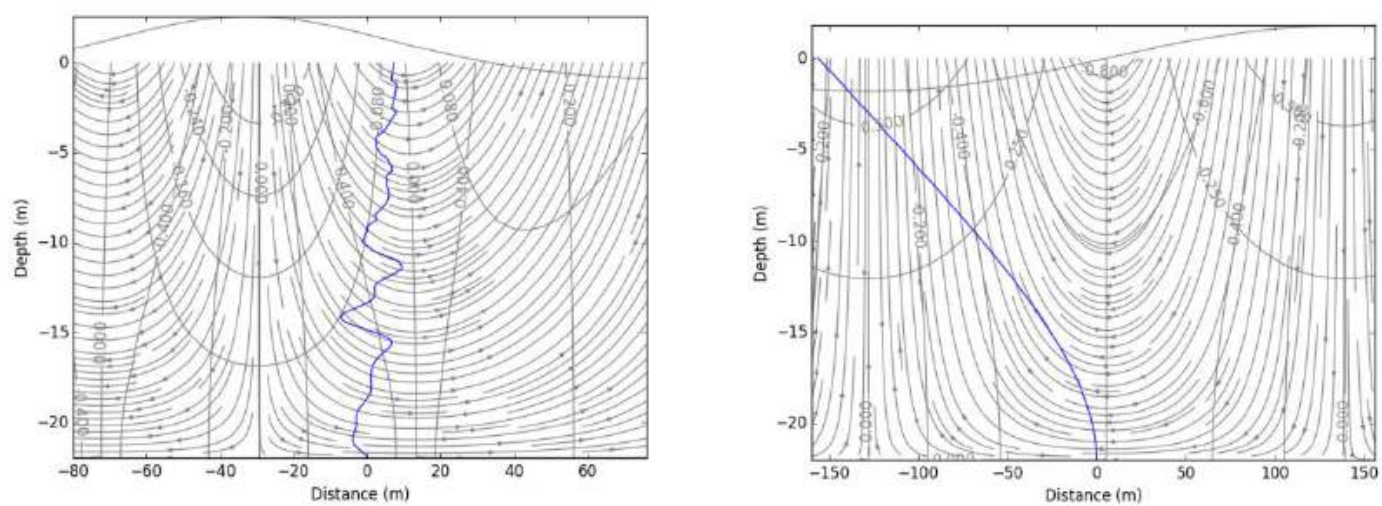

Figura 55. Deformada en el instante de momento flector máximo para $H=3,6 \mathrm{~m}, h=22,0 \mathrm{~m}, T=18,5 \mathrm{~s}$ y $D=4,0 \mathrm{~m}$

La imagen de las deformadas ha sido escalada en el plano de las abscisas, para poder ser representada junto con el perfil de oleaje.

Los valores más característicos de la simulación, tanto para el caso de temperatura de 1 ํㅡ y salinidad de $0 \%$, como para el caso de temperatura de $22{ }^{\circ} \mathrm{C}$ y salinidad del $35 \%$, se muestran, a continuación, en la tabla 63:

\begin{tabular}{|c|c|c|c|c|}
\hline \multirow[b]{2}{*}{ Parámetro } & \multicolumn{2}{|c|}{ Método avanzado } & \multicolumn{2}{|c|}{ Método tradicional } \\
\hline & $\mathrm{T}^{\mathrm{a}}=1 \stackrel{\circ}{ } \mathrm{C}, \mathrm{S}=0 \%$ & $\begin{array}{c}\mathrm{T}^{\mathrm{a}}=22{ }^{\circ} \mathrm{C}, \\
\mathrm{S}=35 \%\end{array}$ & $\mathrm{~T}^{\mathrm{a}}=1 \stackrel{\circ}{ } \mathrm{C}, \mathrm{S}=0 \%$ & $\begin{array}{c}\mathrm{T}^{\mathrm{a}}=22 \mathrm{O} \mathrm{C}, \\
\mathrm{S}=35 \%\end{array}$ \\
\hline $\begin{array}{l}\text { Máximo desplazamiento en } \\
\text { superficie }(\mathrm{m})\end{array}$ & 0,00045336 & 0,0004537 & 0,00037328 & 0,00037355 \\
\hline $\begin{array}{l}\text { Máximo esfuerzo cortante en } \\
\text { cimentación }(\mathrm{kN})\end{array}$ & 204,2277 & 204,3769 & 331,4289 & 331,6711 \\
\hline $\begin{array}{l}\text { Máximo momento flector en } \\
\text { cimentación }(\mathrm{kN} \cdot \mathrm{m})\end{array}$ & 2265,5089 & 2267,1639 & 3717,7389 & 3720,4547 \\
\hline
\end{tabular}

Tabla 63. Comportamiento de la estructura para el caso de $H=3,6 m, h=22,0 m, T=18,5$ s y $D=4,0 m$ 


\subsubsection{Caso 2D}

Los datos de entrada se corresponden con el caso de cálculo para el parque eólico offshore Thornton Bank en Bélgica: altura de ola significativa $6,32 \mathrm{~m}$, profundidad $16,0 \mathrm{~m}$ y periodo de pico $11,06 \mathrm{~s}$. De acuerdo con estos datos, en la figura 56 se muestra la deformada de la pila, en el instante en que se produce el máximo momento flector, sobre la base del pilote, mostrando el cálculo según el método avanzado a la izquierda y según el método tradicional, a la derecha.
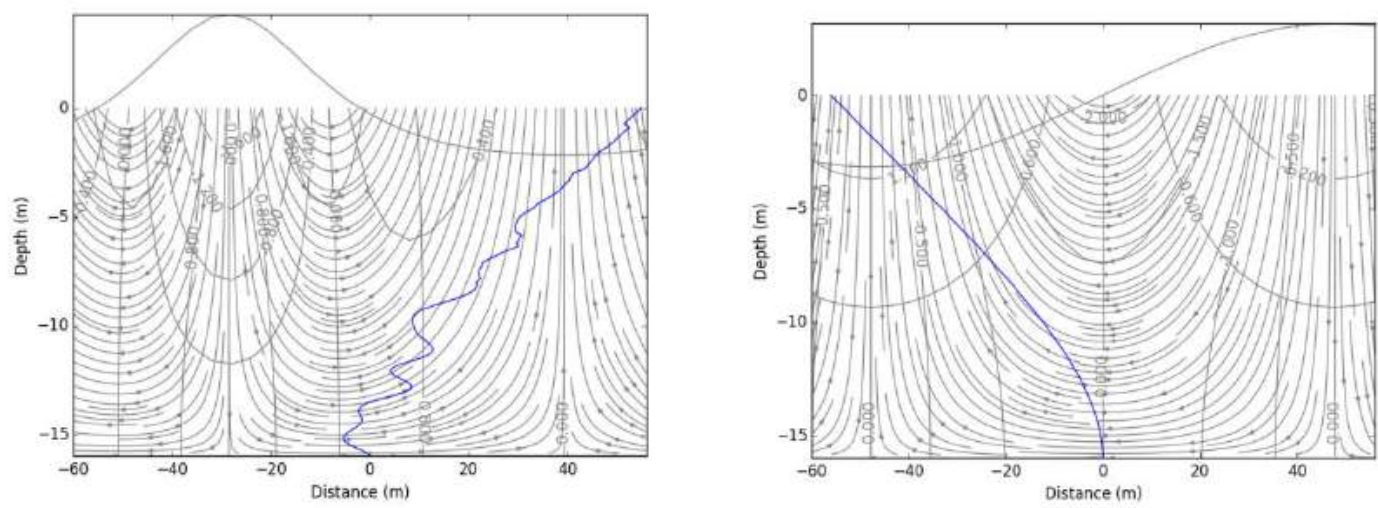

Figura 56. Deformada en el instante de momento flector máximo para $H=6,32 m, h=16,0 m, T=11,06 s$ y $D=4,0 m$

La imagen de las deformadas ha sido escalada en el plano de las abscisas, para poder ser representada junto con el perfil de oleaje.

Los valores más característicos de la simulación, tanto para el caso de temperatura de 1 ํㅡ y salinidad de $0 \%$, como para el caso de temperatura de $22{ }^{\circ} \mathrm{C}$ y salinidad del $35 \%$, se muestran, a continuación, en la tabla 64 :

\begin{tabular}{|l|c|c|c|c|}
\cline { 2 - 5 } \multicolumn{1}{c|}{} & \multicolumn{2}{c|}{ Método avanzado } & \multicolumn{2}{c|}{ Método tradicional } \\
\hline Parámetro & $\mathrm{T}^{\mathrm{a}}=1$ 으, $\mathrm{S}=0 \%$ & $\begin{array}{c}\mathrm{T}^{\mathrm{a}}=22{ }^{\circ} \mathrm{C}, \\
\mathrm{S}=35 \%\end{array}$ & $\mathrm{~T}^{\mathrm{a}}=1$ 으, $\mathrm{S}=0 \%$ & $\begin{array}{c}\mathrm{T}^{\mathrm{a}}=22 \text { 으, } \\
\mathrm{S}=35 \%\end{array}$ \\
\hline $\begin{array}{l}\text { Máximo desplazamiento en } \\
\text { superficie }(\mathrm{m})\end{array}$ & 0,00034047 & 0,00034072 & 0,00024627 & 0,00024645 \\
\hline $\begin{array}{l}\text { Máximo esfuerzo cortante en } \\
\text { cimentación (kN) }\end{array}$ & 466,1613 & 434,8223 & 585,5884 & 586,0162 \\
\hline $\begin{array}{l}\text { Máximo momento flector en } \\
\text { cimentación }(\mathrm{kN} \cdot \mathrm{m})\end{array}$ & 3735,1715 & 3504,0376 & 4700,5443 & 4703,978 \\
\hline
\end{tabular}

Tabla 64. Comportamiento de la estructura para el caso de $H=6,32 m, h=16,0 m, T=11,06$ s y $D=4,0 m$ 


\subsection{Caso 3: Diámetros igual a $8,0 \mathrm{~m}$}

\subsubsection{Caso $3 \mathrm{~A}$}

Los datos de entrada se corresponden con el caso de cálculo para el parque eólico offshore Princess Amalia Q7 en Holanda: altura de ola significativa 7,7 m, profundidad 24,0 m y periodo de pico 9,7 s. De acuerdo con estos datos, en la figura 57 se muestra la deformada de la pila, en el instante en que se produce el máximo momento flector, sobre la base del pilote, mostrando el cálculo según el método avanzado a la izquierda y según el método tradicional, a la derecha.
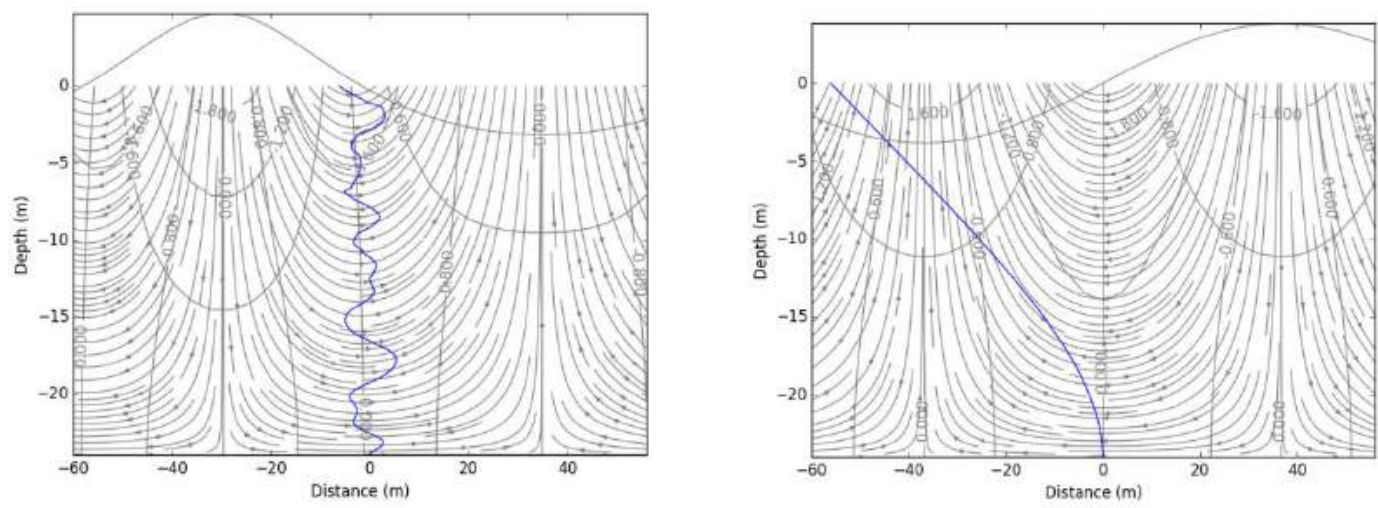

Figura 57. Deformada en el instante de momento flector máximo para $H=7,7 \mathrm{~m}, h=24,0 \mathrm{~m}, T=9,7 \mathrm{~s}$ y $D=8,0 \mathrm{~m}$

La imagen de las deformadas ha sido escalada en el plano de las abscisas, para poder ser representada junto con el perfil de oleaje.

Los valores más característicos de la simulación, tanto para el caso de temperatura de 1 ํㅡ y salinidad de $0 \%$, como para el caso de temperatura de $22^{\circ} \mathrm{C}$ y salinidad del $35 \%$, se muestran, a continuación, en la tabla 65:

\begin{tabular}{|c|c|c|c|c|}
\hline \multirow[b]{2}{*}{ Parámetro } & \multicolumn{2}{|c|}{ Método avanzado } & \multicolumn{2}{|c|}{ Método tradicional } \\
\hline & $\mathrm{T}^{\mathrm{a}}=1 \stackrel{\circ}{ } \mathrm{C}, \mathrm{S}=0 \%$ & $\begin{array}{c}\mathrm{T}^{\mathrm{a}}=22 \stackrel{\circ}{ } \mathrm{C}, \\
\mathrm{S}=35 \%\end{array}$ & $\mathrm{~T}^{\mathrm{a}}=1 \stackrel{\mathrm{o}}{ } \mathrm{C}, \mathrm{S}=0 \%$ & $\begin{array}{c}\mathrm{T}^{\mathrm{a}}=22{ }^{\circ} \mathrm{C} \\
\mathrm{S}=35 \%\end{array}$ \\
\hline $\begin{array}{l}\text { Máximo desplazamiento en } \\
\text { superficie }(\mathrm{m})\end{array}$ & 0,00159107 & 0,0015557 & 0,00105936 & 0,00106013 \\
\hline $\begin{array}{l}\text { Máximo esfuerzo cortante en } \\
\text { cimentación }(\mathrm{kN})\end{array}$ & 2811,7193 & 2721,4694 & 3185,1167 & 3187,4434 \\
\hline $\begin{array}{l}\text { Máximo momento flector en } \\
\text { cimentación }(\mathrm{kN} \cdot \mathrm{m})\end{array}$ & 35618,093 & 33803,9496 & 40727,2003 & 40756,9514 \\
\hline
\end{tabular}

Tabla 65. Comportamiento de la estructura para el caso de $H=7,7 \mathrm{~m}, h=24,0 \mathrm{~m}, T=9,7 \mathrm{~s}$ y $D=8,0 \mathrm{~m}$ 


\subsubsection{Caso 3B}

Los datos de entrada se corresponden con el caso de cálculo para el parque eólico offshore North Hoyle en Reino Unido: altura de ola significativa $4,78 \mathrm{~m}$, profundidad $10,0 \mathrm{~m}$ y periodo de pico 6,8 s. De acuerdo con estos datos, en la figura 58 se muestra la deformada de la pila, en el instante en que se produce el máximo momento flector, sobre la base del pilote, mostrando el cálculo según el método avanzado a la izquierda y según el método tradicional, a la derecha.
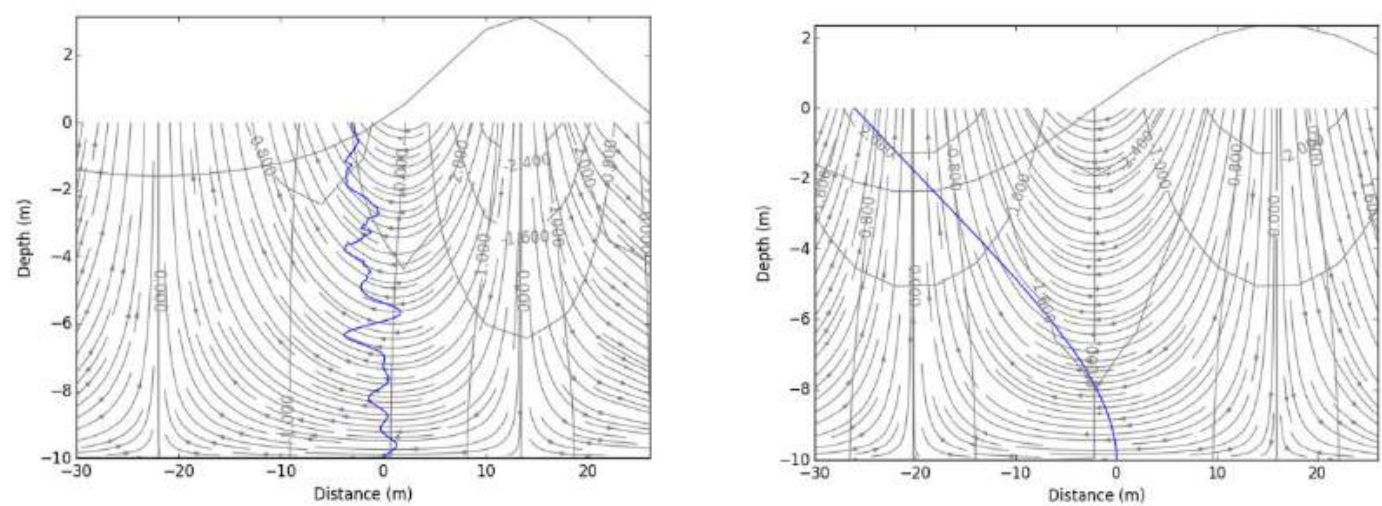

Figura 58. Deformada en el instante de momento flector máximo para $H=4,78 m, h=10,0 m, T=6,8 s$ y $D=8,0 m$

La imagen de las deformadas ha sido escalada en el plano de las abscisas, para poder ser representada junto con el perfil de oleaje.

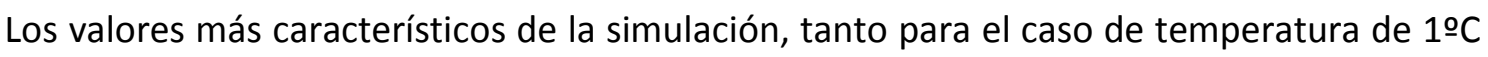
y salinidad de $0 \%$, como para el caso de temperatura de $22{ }^{\circ} \mathrm{C}$ y salinidad del $35 \%$, se muestran, a continuación, en la tabla 66:

\begin{tabular}{|l|c|c|c|c|}
\cline { 2 - 5 } \multicolumn{1}{c|}{} & \multicolumn{2}{c|}{ Método avanzado } & \multicolumn{2}{c|}{ Método tradicional } \\
\hline Parámetro & $\mathrm{T}^{\mathrm{a}}=1 \mathrm{o} \mathrm{C}, \mathrm{S}=0 \%$ & $\begin{array}{c}\mathrm{T}^{\mathrm{a}}=22 \mathrm{o} \mathrm{C}, \\
\mathrm{S}=35 \%\end{array}$ & $\mathrm{~T}^{\mathrm{a}=1 ㅇ} \mathrm{C}, \mathrm{S}=0 \%$ & $\begin{array}{c}\mathrm{T}^{\mathrm{a}}=22 \text { o } \mathrm{C}, \\
\mathrm{S}=35 \%\end{array}$ \\
\hline $\begin{array}{l}\text { Máximo desplazamiento en } \\
\text { superficie }(\mathrm{m})\end{array}$ & $5,775 \mathrm{e}-05$ & $5,502 \mathrm{e}-05$ & $3,525 \mathrm{e}-05$ & $3,525 \mathrm{e}-05$ \\
\hline $\begin{array}{l}\text { Máximo esfuerzo cortante en } \\
\text { cimentación }(\mathrm{kN})\end{array}$ & 1497,5394 & 1341,5715 & 1753,6062 & 1753,3866 \\
\hline $\begin{array}{l}\text { Máximo momento flector en } \\
\text { cimentación }(\mathrm{kN} \cdot \mathrm{m})\end{array}$ & 7014,5558 & 6419,4026 & 8402,9206 & 8401,8584 \\
\hline
\end{tabular}

Tabla 66. Comportamiento de la estructura para el caso de $H=4,78 m, h=10,0 m, T=6,8$ s y $D=8,0 m$ 


\subsubsection{Caso $3 \mathrm{C}$}

Los datos de entrada se corresponden con el caso de cálculo para el parque eólico offshore Egmond Aan Zee en Holanda: altura de ola significativa 3,6 m, profundidad 22,0 m y periodo de pico $18,5 \mathrm{~s}$. De acuerdo con estos datos, en la figura 59 se muestra la deformada de la pila, en el instante en que se produce el máximo momento flector, sobre la base del pilote, mostrando el cálculo según el método avanzado a la izquierda y según el método tradicional, a la derecha.
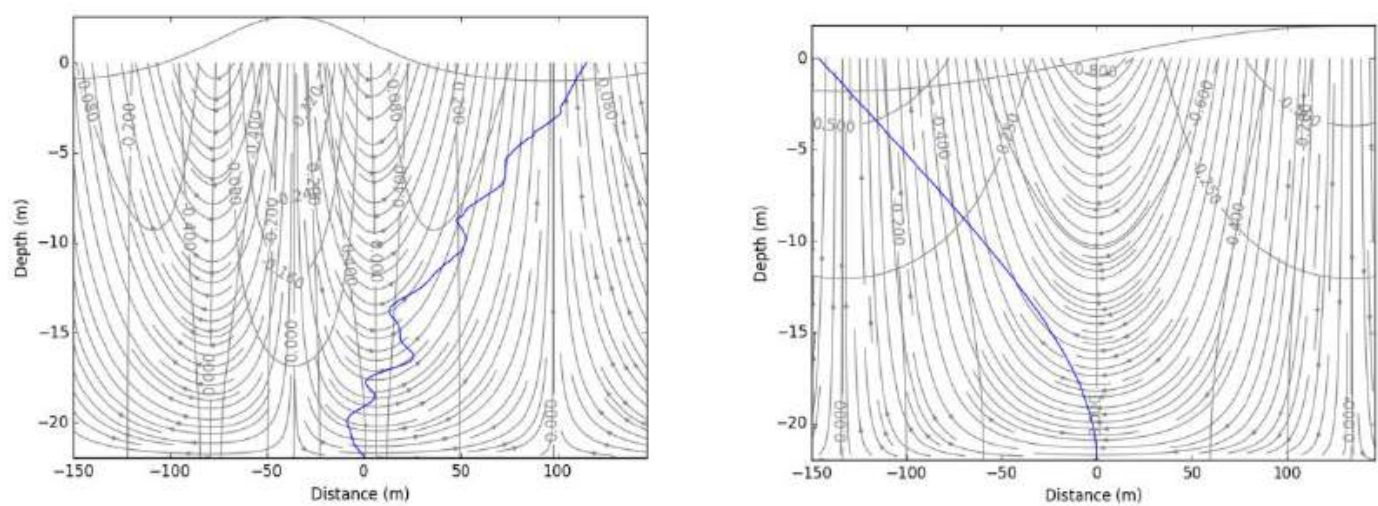

Figura 59. Deformada en el instante de momento flector máximo para $H=3,6 \mathrm{~m}, h=22,0 \mathrm{~m}, T=18,5 \mathrm{~s}$ y $D=8$, $0 \mathrm{~m}$

La imagen de las deformadas ha sido escalada en el plano de las abscisas, para poder ser representada junto con el perfil de oleaje.

Los valores más característicos de la simulación, tanto para el caso de temperatura de 1 ํㅡ y salinidad de $0 \%$, como para el caso de temperatura de $22{ }^{\circ} \mathrm{C}$ y salinidad del $35 \%$, se muestran, a continuación, en la tabla 67:

\begin{tabular}{|l|c|c|c|c|}
\cline { 2 - 5 } \multicolumn{1}{c|}{} & \multicolumn{2}{c|}{ Método avanzado } & \multicolumn{2}{c|}{ Método tradicional } \\
\hline \multicolumn{1}{c|}{ Parámetro } & $\mathrm{T}^{\mathrm{a}}=1 \mathrm{\circ} \mathrm{O}, \mathrm{S}=0 \%$ & $\begin{array}{c}\mathrm{T}^{\mathrm{a}}=22{ }^{\circ} \mathrm{C}, \\
\mathrm{S}=35 \%\end{array}$ & $\mathrm{~T}^{\mathrm{a}=1} \mathrm{O} \mathrm{C}, \mathrm{S}=0 \%$ & $\begin{array}{c}\mathrm{T}^{\mathrm{a}}=22{ }^{\circ} \mathrm{C}, \\
\mathrm{S}=35 \%\end{array}$ \\
\hline $\begin{array}{l}\text { Máximo desplazamiento en } \\
\text { superficie }(\mathrm{m})\end{array}$ & 0,00038994 & 0,00039023 & 0,0003188 & 0,00031883 \\
\hline $\begin{array}{l}\text { Máximo esfuerzo cortante en } \\
\text { cimentación }(\mathrm{kN})\end{array}$ & 769,0136 & 769,5753 & 1325,8628 & 1326,6221 \\
\hline $\begin{array}{l}\text { Máximo momento flector en } \\
\text { cimentación }(\mathrm{kN} \cdot \mathrm{m})\end{array}$ & 8502,4635 & 8508,6745 & 14876,8044 & 14881,1092 \\
\hline
\end{tabular}

Tabla 67. Comportamiento de la estructura para el caso de $H=3,6 m, h=22,0 m, T=18,5$ s y $D=8,0 m$ 


\subsubsection{Caso 3D}

Los datos de entrada se corresponden con el caso de cálculo para el parque eólico offshore Thornton Bank en Bélgica: altura de ola significativa $6,32 \mathrm{~m}$, profundidad $16,0 \mathrm{~m}$ y periodo de pico 11,06 s. De acuerdo con estos datos, en la figura 60 se muestra la deformada de la pila, en el instante en que se produce el máximo momento flector, sobre la base del pilote, mostrando el cálculo según el método avanzado a la izquierda y según el método tradicional, a la derecha.
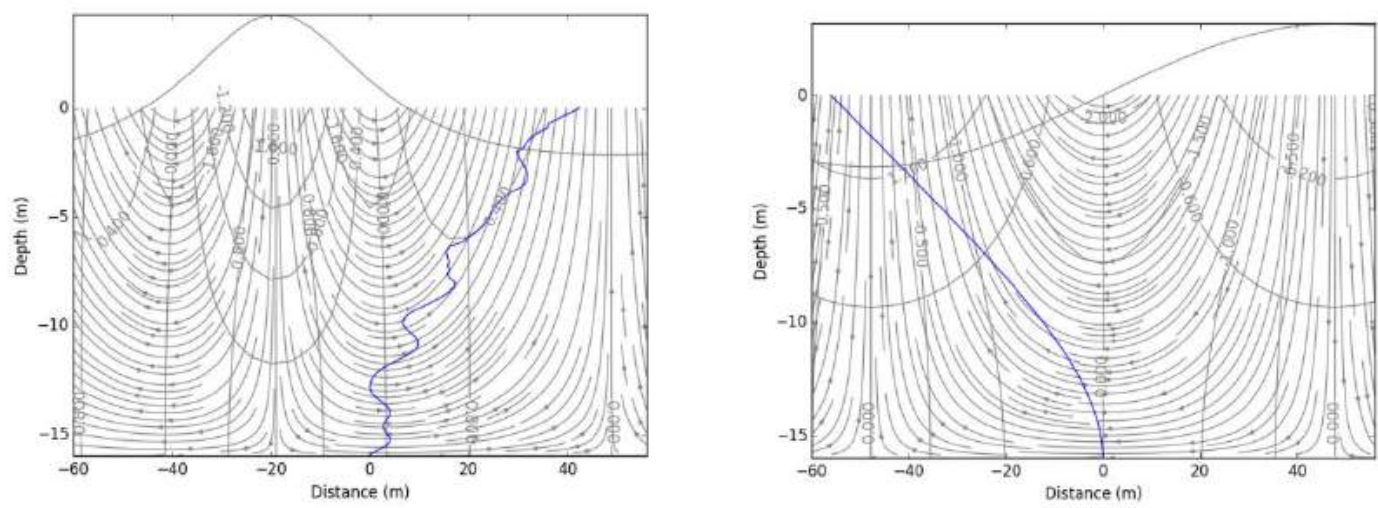

Figura 60. Deformada en el instante de momento flector máximo para $H=6,32 m, h=16,0 m, T=11,06 s$ y $D=8,0 m$

La imagen de las deformadas ha sido escalada en el plano de las abscisas, para poder ser representada junto con el perfil de oleaje.

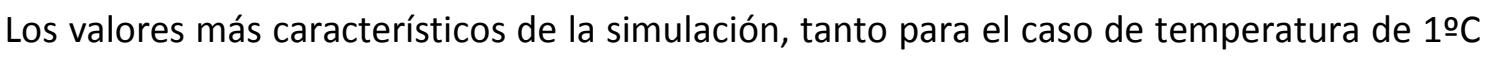
y salinidad de $0 \%$, como para el caso de temperatura de $22{ }^{\circ} \mathrm{C}$ y salinidad del $35 \%$, se muestran, a continuación, en la tabla 68:

\begin{tabular}{|c|c|c|c|c|}
\hline \multirow[b]{2}{*}{ Parámetro } & \multicolumn{2}{|c|}{ Método avanzado } & \multicolumn{2}{|c|}{ Método tradicional } \\
\hline & $\mathrm{T}^{\mathrm{a}}=1 \stackrel{\mathrm{o}}{ } \mathrm{C}, \mathrm{S}=0 \%$ & $\begin{array}{c}\mathrm{T}^{\mathrm{a}}=22{ }^{\circ} \mathrm{C}, \\
\mathrm{S}=35 \%\end{array}$ & $\mathrm{~T}^{\mathrm{a}}=1 \stackrel{\mathrm{o}}{ } \mathrm{C}, \mathrm{S}=0 \%$ & $\begin{array}{c}\mathrm{T}^{\mathrm{a}}=22{ }^{\circ} \mathrm{C} \\
\mathrm{S}=35 \%\end{array}$ \\
\hline $\begin{array}{l}\text { Máximo desplazamiento en } \\
\text { superficie }(m)\end{array}$ & 0,00030661 & 0,00028764 & 0,00021021 & 0,00021036 \\
\hline $\begin{array}{l}\text { Máximo esfuerzo cortante en } \\
\text { cimentación }(\mathrm{kN})\end{array}$ & 1716,623 & 1685,6838 & 2342,3538 & 2344,0649 \\
\hline $\begin{array}{l}\text { Máximo momento flector en } \\
\text { cimentación }(\mathrm{kN} \cdot \mathrm{m})\end{array}$ & 13804,8297 & 13556,2456 & 18802,177 & 18815,912 \\
\hline
\end{tabular}

Tabla 68. Comportamiento de la estructura para el caso de $H=6,32 m, h=16,0 m, T=11,06$ s y $D=8,0 m$ 


\subsection{Caso 4: Diámetros igual a $16,0 \mathrm{~m}$}

\subsubsection{Caso $4 \mathrm{~A}$}

Los datos de entrada se corresponden con el caso de cálculo para el parque eólico offshore Princess Amalia Q7 en Holanda: altura de ola significativa 7,7 m, profundidad 24,0 m y periodo de pico 9,7 s. De acuerdo con estos datos, en la figura 61 se muestra la deformada de la pila, en el instante en que se produce el máximo momento flector, sobre la base del pilote, mostrando el cálculo según el método avanzado a la izquierda y según el método tradicional, a la derecha.
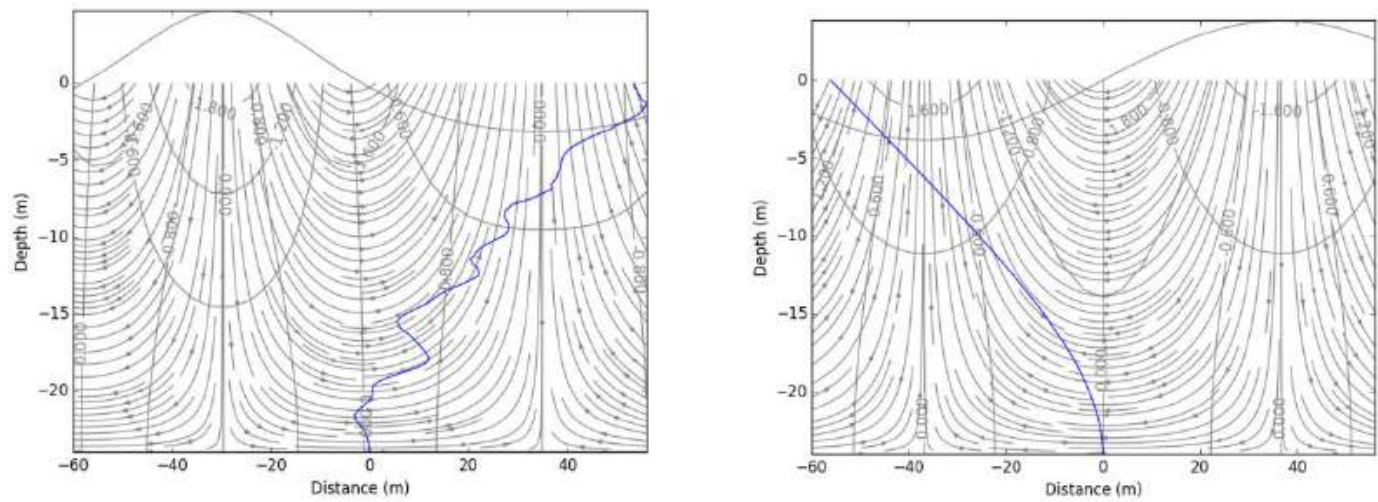

Figura 61. Deformada en el instante de momento flector máximo para $H=7,7 m, h=24,0 m, T=9,7 s$ y $D=16,0 m$

La imagen de las deformadas ha sido escalada en el plano de las abscisas, para poder ser representada junto con el perfil de oleaje.

Los valores más característicos de la simulación, tanto para el caso de temperatura de 1 ํㅡ y salinidad de $0 \%$, como para el caso de temperatura de $22^{\circ} \mathrm{C}$ y salinidad del $35 \%$, se muestran, a continuación, en la tabla 69:

\begin{tabular}{|c|c|c|c|c|}
\hline \multirow[b]{2}{*}{ Parámetro } & \multicolumn{2}{|c|}{ Método avanzado } & \multicolumn{2}{|c|}{ Método tradicional } \\
\hline & $\mathrm{T}^{\mathrm{a}}=1 \stackrel{\circ}{ } \mathrm{C}, \mathrm{S}=0 \%$ & $\begin{array}{c}\mathrm{T}^{\mathrm{a}}=22{ }^{\circ} \mathrm{C}, \\
\mathrm{S}=35 \%\end{array}$ & $\mathrm{~T}^{\mathrm{a}}=1 \stackrel{\mathrm{o}}{ } \mathrm{C}, \mathrm{S}=0 \%$ & $\begin{array}{c}\mathrm{T}^{\mathrm{a}}=22 \mathrm{o} C, \\
\mathrm{~S}=35 \%\end{array}$ \\
\hline $\begin{array}{l}\text { Máximo desplazamiento en } \\
\text { superficie }(\mathrm{m})\end{array}$ & 0,00045362 & 0,00042773 & 0,00026484 & 0,00026503 \\
\hline $\begin{array}{l}\text { Máximo esfuerzo cortante en } \\
\text { cimentación }(\mathrm{kN})\end{array}$ & 10791,5527 & 10448,5318 & 12740,4669 & 12749,7737 \\
\hline $\begin{array}{l}\text { Máximo momento flector en } \\
\text { cimentación }(\mathrm{kN} \cdot \mathrm{m})\end{array}$ & 133698,0572 & 127192,3688 & 162908,8013 & 163027,8057 \\
\hline
\end{tabular}

Tabla 69. Comportamiento de la estructura para el caso de $H=7,7 m, h=24,0 m, T=9,7$ s y $D=16,0 m$ 


\subsubsection{Caso 4B}

Los datos de entrada se corresponden con el caso de cálculo para el parque eólico offshore North Hoyle en Reino Unido: altura de ola significativa 4,78 m, profundidad $10,0 \mathrm{~m}$ y periodo de pico 6,8 s. De acuerdo con estos datos, en la figura 62 se muestra la deformada de la pila, en el instante en que se produce el máximo momento flector, sobre la base del pilote, mostrando el cálculo según el método avanzado a la izquierda y según el método tradicional, a la derecha.
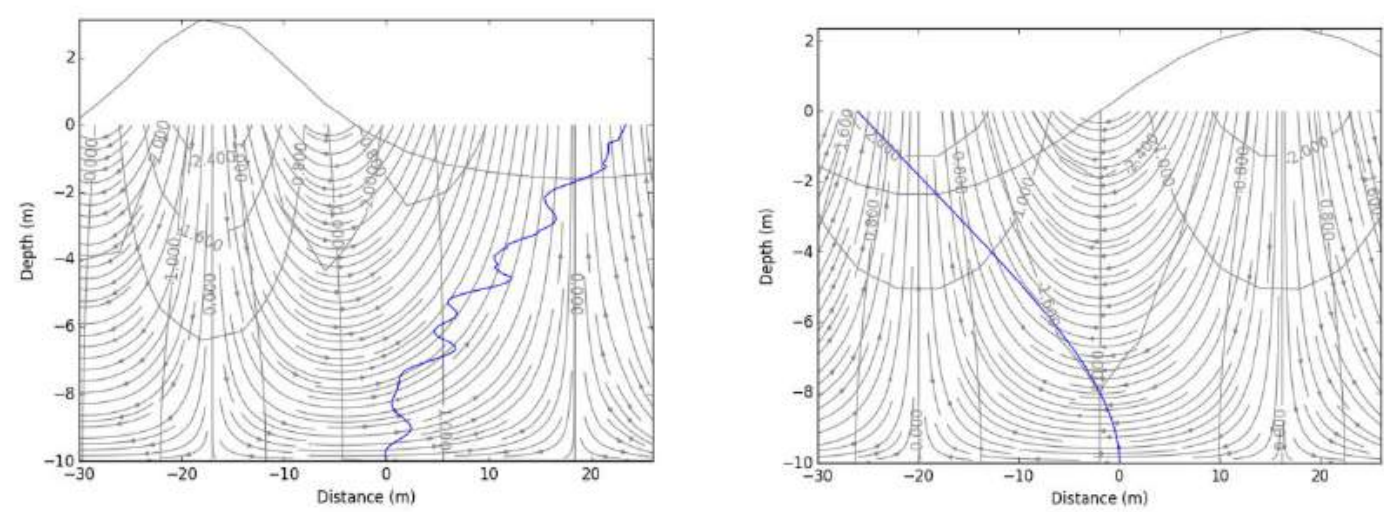

Figura 62. Deformada en el instante de momento flector máximo para $H=4,78 m, h=10,0 m, T=6,8 s$ y $D=16,0 m$

La imagen de las deformadas ha sido escalada en el plano de las abscisas, para poder ser representada junto con el perfil de oleaje.

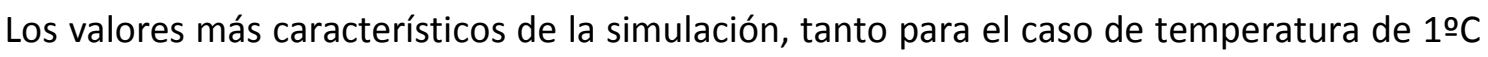
y salinidad de $0 \%$, como para el caso de temperatura de $22{ }^{\circ} \mathrm{C}$ y salinidad del $35 \%$, se muestran, a continuación, en la tabla 70:

\begin{tabular}{|c|c|c|c|c|}
\hline \multirow[b]{2}{*}{ Parámetro } & \multicolumn{2}{|c|}{ Método avanzado } & \multicolumn{2}{|c|}{ Método tradicional } \\
\hline & $\mathrm{T}^{\mathrm{a}}=1 \stackrel{\circ}{ } \mathrm{C}, \mathrm{S}=0 \%$ & $\begin{array}{c}\mathrm{T}^{\mathrm{a}}=22 \stackrel{\circ}{ } \mathrm{C} \\
\mathrm{S}=35 \%\end{array}$ & $\mathrm{~T}^{\mathrm{a}}=1 \stackrel{\circ}{ } \mathrm{C}, \mathrm{S}=0 \%$ & $\begin{array}{c}\mathrm{T}^{\mathrm{a}}=22{ }^{\circ} \mathrm{C} \\
\mathrm{S}=35 \%\end{array}$ \\
\hline $\begin{array}{l}\text { Máximo desplazamiento en } \\
\text { superficie }(\mathrm{m})\end{array}$ & $1,276 \mathrm{e}-05$ & $1,253 e-05$ & $8,81 e-06$ & 8,81e-06 \\
\hline $\begin{array}{l}\text { Máximo esfuerzo cortante en } \\
\text { cimentación }(\mathrm{kN})\end{array}$ & 5296,417 & 5287,2441 & 7014,2914 & 7004,5412 \\
\hline $\begin{array}{l}\text { Máximo momento flector en } \\
\text { cimentación }(\mathrm{kN} \cdot \mathrm{m})\end{array}$ & 26014,96 & 25255,3486 & 33611,0476 & 33576,1034 \\
\hline
\end{tabular}

Tabla 70. Comportamiento de la estructura para el caso de $H=4,78 m, h=10,0 m, T=6,8$ s y $D=16,0 \mathrm{~m}$ 


\subsubsection{Caso 4C}

Los datos de entrada se corresponden con el caso de cálculo para el parque eólico offshore Egmond Aan Zee en Holanda: altura de ola significativa 3,6 m, profundidad 22,0 m y periodo de pico $18,5 \mathrm{~s}$. De acuerdo con estos datos, en la figura 63 se muestra la deformada de la pila, en el instante en que se produce el máximo momento flector, sobre la base del pilote, mostrando el cálculo según el método avanzado a la izquierda y según el método tradicional, a la derecha.
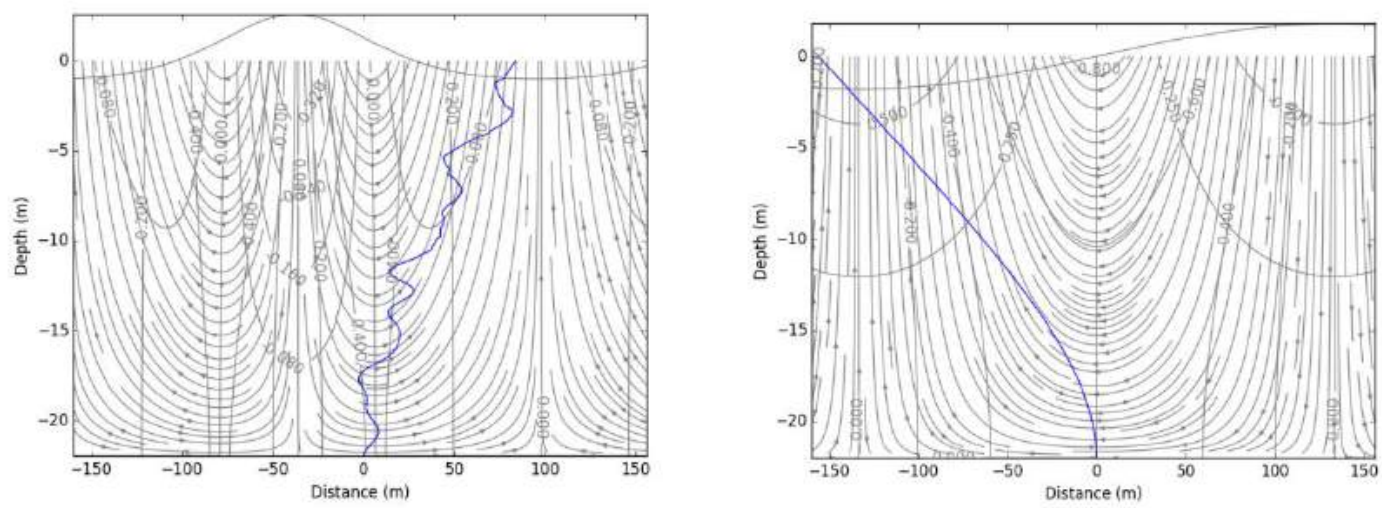

Figura 63. Deformada en el instante de momento flector máximo para $H=3,6 m, h=22,0 m, T=18,5$ s y $D=16,0 m$

La imagen de las deformadas ha sido escalada en el plano de las abscisas, para poder ser representada junto con el perfil de oleaje.

Los valores más característicos de la simulación, tanto para el caso de temperatura de 1 ํㅡ y salinidad de $0 \%$, como para el caso de temperatura de $22{ }^{\circ} \mathrm{C}$ y salinidad del $35 \%$, se muestran, a continuación, en la tabla 71:

\begin{tabular}{|c|c|c|c|c|}
\hline \multirow[b]{2}{*}{ Parámetro } & \multicolumn{2}{|c|}{ Método avanzado } & \multicolumn{2}{|c|}{ Método tradicional } \\
\hline & $\mathrm{T}^{\mathrm{a}}=1 \stackrel{\circ}{ } \mathrm{C}, \mathrm{S}=0 \%$ & $\begin{array}{c}\mathrm{T}^{\mathrm{a}}=22{ }^{\circ} \mathrm{C} \\
\mathrm{S}=35 \%\end{array}$ & $\mathrm{~T}^{\mathrm{a}}=1 \stackrel{\mathrm{o}}{ } \mathrm{C}, \mathrm{S}=0 \%$ & $\begin{array}{c}\mathrm{T}^{\mathrm{a}}=22{ }^{\circ} \mathrm{C} \\
\mathrm{S}=35 \%\end{array}$ \\
\hline $\begin{array}{l}\text { Máximo desplazamiento en } \\
\text { superficie }(m)\end{array}$ & $8,403 e-05$ & $8,409 e-05$ & $7,965 e-05$ & 7,971e-05 \\
\hline $\begin{array}{l}\text { Máximo esfuerzo cortante en } \\
\text { cimentación }(\mathrm{kN})\end{array}$ & 2998,9047 & 3001,0954 & 5302,8631 & 5306,7368 \\
\hline $\begin{array}{l}\text { Máximo momento flector en } \\
\text { cimentación }(\mathrm{kN} \cdot \mathrm{m})\end{array}$ & 33091,2214 & 33115,3944 & 59483,8231 & 59527,2759 \\
\hline
\end{tabular}

Tabla 71. Comportamiento de la estructura para el caso de $H=3,6 m, h=22,0 m, T=18,5$ s y $D=16,0 m$ 


\subsubsection{Caso 4D}

Los datos de entrada se corresponden con el caso de cálculo para el parque eólico offshore Thornton Bank en Bélgica: altura de ola significativa $6,32 \mathrm{~m}$, profundidad $16,0 \mathrm{~m}$ y periodo de pico 11,06 s. De acuerdo con estos datos, en la figura 64 se muestra la deformada de la pila, en el instante en que se produce el máximo momento flector, sobre la base del pilote, mostrando el cálculo según el método avanzado a la izquierda y según el método tradicional, a la derecha.
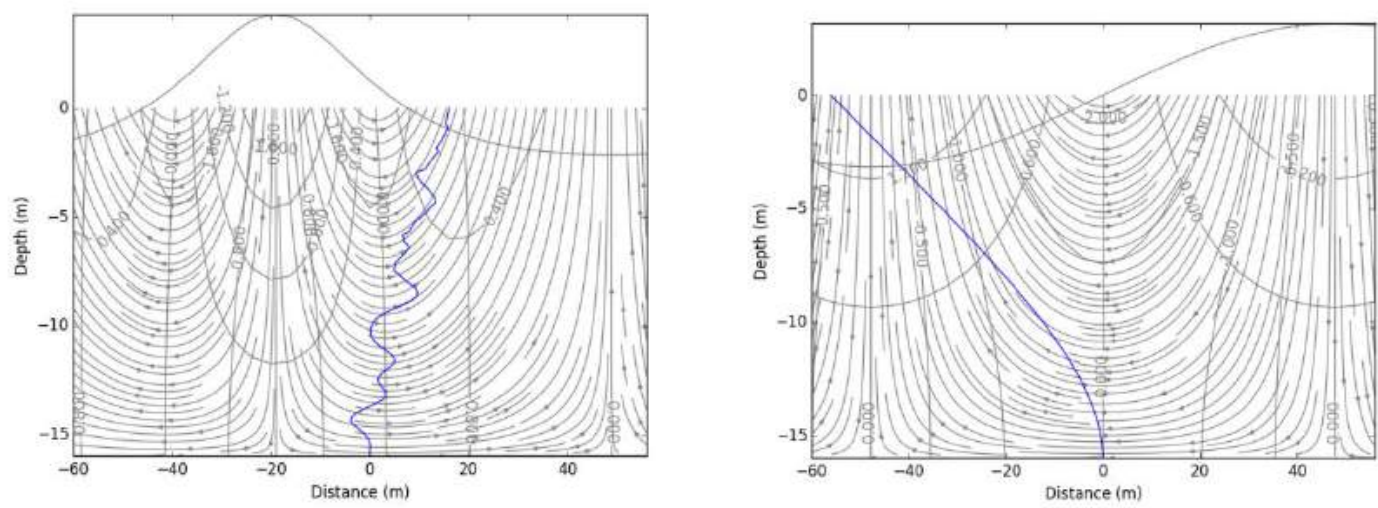

Figura 64. Deformada en el instante de momento flector máximo para $H=6,32 m, h=16,0 m, T=11,06$ s y $D=16,0 m$

La imagen de las deformadas ha sido escalada en el plano de las abscisas, para poder ser representada junto con el perfil de oleaje.

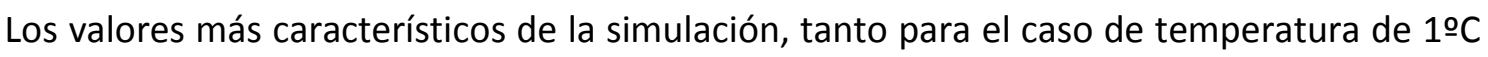
y salinidad de $0 \%$, como para el caso de temperatura de $22{ }^{\circ} \mathrm{C}$ y salinidad del $35 \%$, se muestran, a continuación, en la tabla 72:

\begin{tabular}{|c|c|c|c|c|}
\hline \multirow[b]{2}{*}{ Parámetro } & \multicolumn{2}{|c|}{ Método avanzado } & \multicolumn{2}{|c|}{ Método tradicional } \\
\hline & $\mathrm{T}^{\mathrm{a}}=1 \stackrel{\mathrm{o}}{ } \mathrm{C}, \mathrm{S}=0 \%$ & $\begin{array}{c}\mathrm{T}^{\mathrm{a}}=22{ }^{\circ} \mathrm{C}, \\
\mathrm{S}=35 \%\end{array}$ & $\mathrm{~T}^{\mathrm{a}}=1 \stackrel{\circ}{ } \mathrm{C}, \mathrm{S}=0 \%$ & $\begin{array}{c}\mathrm{T}^{\mathrm{a}}=22 \stackrel{\circ}{ } \mathrm{C}, \\
\mathrm{S}=35 \%\end{array}$ \\
\hline $\begin{array}{l}\text { Máximo desplazamiento en } \\
\text { superficie }(\mathrm{m})\end{array}$ & $7,264 \mathrm{e}-05$ & $7,27 e-05$ & $5,255 e-05$ & $5,259 e-05$ \\
\hline $\begin{array}{l}\text { Máximo esfuerzo cortante en } \\
\text { cimentación }(\mathrm{kN})\end{array}$ & 6664,7017 & 6669,5703 & 9369,4152 & 9376,2595 \\
\hline $\begin{array}{l}\text { Máximo momento flector en } \\
\text { cimentación }(\mathrm{kN} \cdot \mathrm{m})\end{array}$ & 53536,2114 & 53575,3195 & 75208,7082 & 75263,6479 \\
\hline
\end{tabular}

Tabla 72. Comportamiento de la estructura para el caso de $H=6,32 m, h=16,0 m, T=11,06$ s y $D=16,0 m$ 


\section{Proceso IV: Acoplamiento procesos II y III}

El cuarto proceso se corresponde con el cálculo del comportamiento dinámico de la estructura teniendo en cuenta la velocidad y la aceleración relativa que esta tiene en relación con la velocidad y aceleración del fluido que impacta sobre ella.

\subsection{Caso 1: Diámetros igual a $0,5 \mathrm{~m}$}

\subsubsection{Caso $1 \mathrm{~A}$}

Los datos de entrada se corresponden con el caso de cálculo para el parque eólico offshore Princess Amalia Q7 en Holanda: altura de ola significativa 7,7 m, profundidad 24,0 m y periodo de pico 9,7 s. Los valores más característicos de la simulación, tanto para el caso de temperatura de 1 으 $\mathrm{C}$ salinidad de $0 \%$, como para el caso de temperatura de $22{ }^{\circ} \mathrm{C}$ y salinidad del 35\%, se muestran, a continuación, en la tabla 73:

\begin{tabular}{|l|c|c|}
\cline { 2 - 3 } \multicolumn{1}{c|}{} & \multicolumn{2}{c|}{ Método avanzado } \\
\hline \multicolumn{1}{c|}{ Parámetro } & $\mathrm{T}^{\mathrm{a}}=1$ 으, $\mathrm{S}=0 \%$ & $\mathrm{~T}^{\mathrm{a}}=22{ }^{\circ} \mathrm{C}, \mathrm{S}=35 \%$ \\
\hline Máximo desplazamiento en superficie $(\mathrm{m})$ & 0,17926064 & 0,13404809 \\
\hline $\begin{array}{l}\text { Máximo esfuerzo cortante en cimentación } \\
(\mathrm{kN})\end{array}$ & 35,0575 & 31,9846 \\
\hline $\begin{array}{l}\text { Máximo momento flector en cimentación } \\
(\mathrm{kN} \cdot \mathrm{m})\end{array}$ & 519,641 & 474,7644 \\
\hline \multicolumn{2}{|c|}{ Tabla 73. Comportamiento de la estructura para el caso de $H=7,7 \mathrm{~m}, h=24,0 \mathrm{~m}, T=9,7 \mathrm{~s}$ y $\mathrm{D}=0,5 \mathrm{~m}$} \\
\hline
\end{tabular}

\subsubsection{Caso $1 B$}

Los datos de entrada se corresponden con el caso de cálculo para el parque eólico offshore North Hoyle en Reino Unido: altura de ola significativa $4,78 \mathrm{~m}$, profundidad $10,0 \mathrm{~m}$ y periodo de pico 6,8 s. Los valores más característicos de la simulación, tanto para el caso de temperatura de 1 으 $\mathrm{C}$ s salinidad de $0 \%$, como para el caso de temperatura de $22{ }^{\circ} \mathrm{C}$ y salinidad del 35\%, se muestran, a continuación, en la tabla 74:

\begin{tabular}{|c|c|c|}
\hline & \multicolumn{2}{|c|}{ Método avanzado } \\
\hline Parámetro & $\mathrm{T}^{\mathrm{a}}=1 \mathrm{O} \mathrm{C}, \mathrm{S}=0 \%$ & $\mathrm{~T}^{\mathrm{a}}=22^{\circ} \mathrm{C}, \mathrm{S}=35 \%$ \\
\hline Máximo desplazamiento en superficie (m) & 0,00278762 & 0,00278281 \\
\hline
\end{tabular}




\begin{tabular}{|l|c|c|}
\hline $\begin{array}{l}\text { Máximo esfuerzo cortante en cimentación } \\
\text { (kN) }\end{array}$ & 8,9747 & 8,1281 \\
\hline $\begin{array}{l}\text { Máximo momento flector en cimentación } \\
\text { (kN.m) }\end{array}$ & 43,1491 & 39,0315 \\
\hline \multicolumn{2}{|c|}{ Tabla 74. Comportamiento de la estructura para el caso de $H=4,78 m, h=10,0 m, T=6,8$ s y $D=0,5 m$} \\
\hline
\end{tabular}

\subsubsection{Caso 1C}

Los datos de entrada se corresponden con el caso de cálculo para el parque eólico offshore Egmond Aan Zee en Holanda: altura de ola significativa 3,6 m, profundidad 22,0 m y periodo de pico $18,5 \mathrm{~s}$. Los valores más característicos de la simulación, tanto para el caso de temperatura de 1 으 $\mathrm{C}$ salinidad de $0 \%$, como para el caso de temperatura de $22{ }^{\circ} \mathrm{C}$ y salinidad del $35 \%$, se muestran, a continuación, en la tabla 75 :

\begin{tabular}{|c|c|c|}
\hline & \multicolumn{2}{|c|}{ Método avanzado } \\
\hline Parámetro & $\mathrm{T}^{\mathrm{a}}=1 \mathrm{O} \mathrm{C}, \mathrm{S}=0 \%$ & $\mathrm{~T}^{\mathrm{a}}=22^{\circ} \mathrm{C}, \mathrm{S}=35 \%$ \\
\hline Máximo desplazamiento en superficie (m) & 0,03489555 & 0,03387134 \\
\hline $\begin{array}{l}\text { Máximo esfuerzo cortante en cimentación } \\
(\mathrm{kN})\end{array}$ & 12,312 & 11,6557 \\
\hline $\begin{array}{l}\text { Máximo momento flector en cimentación } \\
(\mathrm{kN} \cdot \mathrm{m})\end{array}$ & 149,5193 & 134,1776 \\
\hline
\end{tabular}

\subsubsection{Caso 1D}

Los datos de entrada se corresponden con el caso de cálculo para el parque eólico offshore Thornton Bank en Bélgica: altura de ola significativa $6,32 \mathrm{~m}$, profundidad $16,0 \mathrm{~m}$ y periodo de pico $11,06 \mathrm{~s}$.

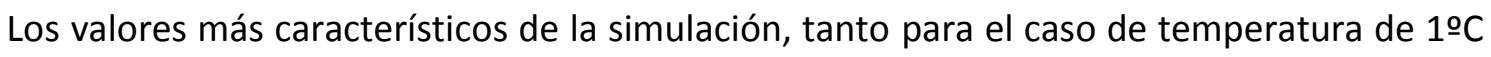
y salinidad de $0 \%$, como para el caso de temperatura de $22{ }^{\circ} \mathrm{C}$ y salinidad del $35 \%$, se muestran, a continuación, en la tabla 76 :

\begin{tabular}{|c|c|c|}
\hline & \multicolumn{2}{|c|}{ Método avanzado } \\
\hline Parámetro & $\mathrm{T}^{\mathrm{a}}=1 \mathrm{o} \mathrm{C}, \mathrm{S}=0 \%$ & $\mathrm{~T}^{\mathrm{a}}=22^{\circ} \mathrm{C}, \mathrm{S}=35 \%$ \\
\hline Máximo desplazamiento en superficie (m) & 0,0115673 & 0,01157783 \\
\hline $\begin{array}{l}\text { Máximo esfuerzo cortante en cimentación } \\
(\mathrm{kN})\end{array}$ & 12,7862 & 11,1992 \\
\hline $\begin{array}{l}\text { Máximo momento flector en cimentación } \\
(\mathrm{kN} \cdot \mathrm{m})\end{array}$ & 106,3268 & 94,3608 \\
\hline
\end{tabular}


Modelos de predicción de esfuerzos hidrodinámicos y socavación aplicados a ingeniería offshore

Tabla 76. Comportamiento de la estructura para el caso de $H=6,32 m, h=16,0 m, T=11,06$ s y $D=0,5 m$ 


\subsection{Caso 2: Diámetros igual a 4,0 m}

\subsubsection{Caso $2 \mathrm{~A}$}

Los datos de entrada se corresponden con el caso de cálculo para el parque eólico offshore Princess Amalia Q7 en Holanda: altura de ola significativa 7,7 m, profundidad 24,0 m y periodo de pico 9,7 s. Los valores más característicos de la simulación, tanto para el caso de temperatura de 1 으 Cy salinidad de $0 \%$, como para el caso de temperatura de $22{ }^{\circ} \mathrm{C}$ y salinidad del 35\%, se muestran, a continuación, en la tabla 77:

\begin{tabular}{|c|c|c|}
\hline & \multicolumn{2}{|c|}{ Método avanzado } \\
\hline Parámetro & $\mathrm{T}^{\mathrm{a}}=1 \mathrm{o} \mathrm{C}, \mathrm{S}=0 \%$ & $\mathrm{~T}^{\mathrm{a}}=22^{\circ} \mathrm{C}, \mathrm{S}=35 \%$ \\
\hline Máximo desplazamiento en superficie (m) & 0,0022614 & 0,00206347 \\
\hline $\begin{array}{l}\text { Máximo esfuerzo cortante en cimentación } \\
(\mathrm{kN})\end{array}$ & 759,4139 & 726,3143 \\
\hline $\begin{array}{l}\text { Máximo momento flector en cimentación } \\
(\mathrm{kN} \cdot \mathrm{m})\end{array}$ & 9439,8115 & 8764,9648 \\
\hline
\end{tabular}

\subsubsection{Caso 2B}

Los datos de entrada se corresponden con el caso de cálculo para el parque eólico offshore North Hoyle en Reino Unido: altura de ola significativa $4,78 \mathrm{~m}$, profundidad $10,0 \mathrm{~m}$ y periodo de pico 6,8 s. Los valores más característicos de la simulación, tanto para el caso de temperatura de 1 으 y salinidad de $0 \%$, como para el caso de temperatura de $22{ }^{\circ} \mathrm{C}$ y salinidad del 35\%, se muestran, a continuación, en la tabla 78:

\begin{tabular}{|c|c|c|}
\hline & \multicolumn{2}{|c|}{ Método avanzado } \\
\hline Parámetro & $\mathrm{T}^{\mathrm{a}}=1 \stackrel{\mathrm{o}}{ } \mathrm{C}, \mathrm{S}=0 \%$ & $\mathrm{~T}^{\mathrm{a}}=22^{\circ} \mathrm{C}, \quad \mathrm{S}=35 \%$ \\
\hline Máximo desplazamiento en superficie (m) & $6,317 \mathrm{e}-05$ & $6,321 \mathrm{e}-05$ \\
\hline $\begin{array}{l}\text { Máximo esfuerzo cortante en cimentación } \\
(\mathrm{kN})\end{array}$ & 407,043 & 383,7881 \\
\hline $\begin{array}{l}\text { Máximo momento flector en cimentación } \\
(\mathrm{kN} \cdot \mathrm{m})\end{array}$ & 1922,7307 & 1784,7974 \\
\hline
\end{tabular}




\subsubsection{Caso 2C}

Los datos de entrada se corresponden con el caso de cálculo para el parque eólico offshore Egmond Aan Zee en Holanda: altura de ola significativa 3,6 m, profundidad 22,0 m y periodo de pico 18,5 s. Los valores más característicos de la simulación, tanto para el caso de temperatura de $1 \stackrel{\circ}{\circ} \mathrm{C}$ y salinidad de $0 \%$, como para el caso de temperatura de $22^{\circ} \mathrm{C}$ y salinidad del $35 \%$, se muestran, a continuación, en la tabla 79:

\begin{tabular}{|l|c|c|}
\cline { 2 - 3 } \multicolumn{1}{c|}{} & \multicolumn{2}{c|}{ Método avanzado } \\
\hline \multicolumn{1}{c|}{ Parámetro } & $\mathrm{T}^{\mathrm{a}}=1$ 으, $\mathrm{S}=0 \%$ & $\mathrm{~T}^{\mathrm{a}}=22$ 으, $\mathrm{S}=35 \%$ \\
\hline Máximo desplazamiento en superficie $(\mathrm{m})$ & 0,00041778 & 0,00041808 \\
\hline $\begin{array}{l}\text { Máximo esfuerzo cortante en cimentación } \\
(\mathrm{kN})\end{array}$ & 202,8313 & 202,9779 \\
\hline $\begin{array}{l}\text { Máximo momento flector en cimentación } \\
\text { (kN.m) }\end{array}$ & 2243,2717 & 2244,8875 \\
\hline \multicolumn{2}{|c|}{ Tabla 79. Comportamiento de la estructura para el caso de $H=3,6 \mathrm{~m}, h=22,0 \mathrm{~m}, \mathrm{~T}=18,5$ s y $\mathrm{D}=4,0 \mathrm{~m}$} \\
\hline
\end{tabular}

\subsubsection{Caso 2D}

Los datos de entrada se corresponden con el caso de cálculo para el parque eólico offshore Thornton Bank en Bélgica: altura de ola significativa $6,32 \mathrm{~m}$, profundidad $16,0 \mathrm{~m}$ y periodo de pico 11,06 s. Los valores más característicos de la simulación, tanto para el caso de temperatura de 1 으 $\mathrm{C}$ salinidad de $0 \%$, como para el caso de temperatura de $22{ }^{\circ} \mathrm{C}$ y salinidad del $35 \%$, se muestran, a continuación, en la tabla 80 :

\begin{tabular}{|l|c|c|}
\cline { 2 - 3 } \multicolumn{1}{c|}{} & \multicolumn{2}{c|}{ Método avanzado } \\
\cline { 2 - 3 } \multicolumn{1}{c|}{ Parámetro } & $\mathrm{T}^{\mathrm{a}}=1$ 으, $\mathrm{S}=0 \%$ & $\mathrm{~T}^{\mathrm{a}}=22{ }^{\circ} \mathrm{C}, \mathrm{S}=35 \%$ \\
\hline Máximo desplazamiento en superficie $(\mathrm{m})$ & 0,00031442 & 0,00031465 \\
\hline $\begin{array}{l}\text { Máximo esfuerzo cortante en cimentación } \\
(\mathrm{kN})\end{array}$ & 453,3752 & 434,9045 \\
\hline $\begin{array}{l}\text { Máximo momento flector en cimentación } \\
(\mathrm{kN} \cdot \mathrm{m})\end{array}$ & 3468,2209 & 3470,7513 \\
\hline \multicolumn{2}{|c|}{ Tabla 80. Comportamiento de la estructura para el caso de $H=6,32 \mathrm{~m}, h=16,0 \mathrm{~m}, T=11,06 \mathrm{~s}$ y $\mathrm{D}=4,0 \mathrm{~m}$} \\
\hline
\end{tabular}




\subsection{Caso 3: Diámetros igual a 8,0 m}

\subsubsection{Caso $3 \mathrm{~A}$}

Los datos de entrada se corresponden con el caso de cálculo para el parque eólico offshore Princess Amalia Q7 en Holanda: altura de ola significativa 7,7 m, profundidad 24,0 m y periodo de pico 9,7 s. Los valores más característicos de la simulación, tanto para el caso de temperatura de $1{ }^{\circ} \mathrm{C}$ y salinidad de $0 \%$, como para el caso de temperatura de $22{ }^{\circ} \mathrm{C}$ y salinidad del $35 \%$, se muestran, a continuación, en la tabla 81 :

\begin{tabular}{|l|c|c|}
\cline { 2 - 3 } \multicolumn{1}{c|}{} & \multicolumn{2}{c|}{ Método avanzado } \\
\hline \multicolumn{1}{|c|}{ Parámetro } & $\mathrm{T}^{\mathrm{a}}=1$ 으, $\mathrm{S}=0 \%$ & $\mathrm{~T}^{\mathrm{a}}=22{ }^{\circ} \mathrm{C}, \mathrm{S}=35 \%$ \\
\hline Máximo desplazamiento en superficie $(\mathrm{m})$ & 0,00154265 & 0,00154359 \\
\hline $\begin{array}{l}\text { Máximo esfuerzo cortante en cimentación } \\
(\mathrm{kN})\end{array}$ & 2864,6665 & 2767,6009 \\
\hline $\begin{array}{l}\text { Máximo momento flector en cimentación } \\
(\mathrm{kN} \cdot \mathrm{m})\end{array}$ & 34349,1768 & 32778,0359 \\
\hline \multicolumn{2}{|c|}{ Tabla 81. Comportamiento de la estructura para el caso de $H=7,7 \mathrm{~m}, h=24,0 \mathrm{~m}, T=9,7 \mathrm{~s}$ y $\mathrm{D=8,0 \textrm {m }}$} \\
\hline
\end{tabular}

\subsubsection{Caso 3B}

Los datos de entrada se corresponden con el caso de cálculo para el parque eólico offshore North Hoyle en Reino Unido: altura de ola significativa $4,78 \mathrm{~m}$, profundidad $10,0 \mathrm{~m}$ y periodo de pico 6,8 s. Los valores más característicos de la simulación, tanto para el caso de temperatura de 1 으 $\mathrm{C}$ salinidad de $0 \%$, como para el caso de temperatura de $22{ }^{\circ} \mathrm{C}$ y salinidad del $35 \%$, se muestran, a continuación, en la tabla 82 :

\begin{tabular}{|c|c|c|}
\hline & \multicolumn{2}{|c|}{ Método avanzado } \\
\hline Parámetro & $\mathrm{T}^{\mathrm{a}}=1 \mathrm{o} \mathrm{C}, \mathrm{S}=0 \%$ & $\mathrm{~T}^{\mathrm{a}}=22^{\circ} \mathrm{C}, \mathrm{S}=35 \%$ \\
\hline Máximo desplazamiento en superficie (m) & $5,521 \mathrm{e}-05$ & $5,407 e-05$ \\
\hline $\begin{array}{l}\text { Máximo esfuerzo cortante en cimentación } \\
(\mathrm{kN})\end{array}$ & 1484,6241 & 1340,9649 \\
\hline $\begin{array}{l}\text { Máximo momento flector en cimentación } \\
(\mathrm{kN} \cdot \mathrm{m})\end{array}$ & 6937,4345 & 6421,2331 \\
\hline
\end{tabular}




\subsubsection{Caso $3 \mathrm{C}$}

Los datos de entrada se corresponden con el caso de cálculo para el parque eólico offshore Egmond Aan Zee en Holanda: altura de ola significativa 3,6 m, profundidad 22,0 m y periodo de pico 18,5 s. Los valores más característicos de la simulación, tanto para el caso de temperatura de $1 \stackrel{\circ}{\circ} \mathrm{C}$ y salinidad de $0 \%$, como para el caso de temperatura de $22^{\circ} \mathrm{C}$ y salinidad del 35\%, se muestran, a continuación, en la tabla 83 :

\begin{tabular}{|l|c|c|}
\cline { 2 - 3 } \multicolumn{1}{c|}{} & \multicolumn{2}{c|}{ Método avanzado } \\
\hline \multicolumn{1}{c|}{ Parámetro } & $\mathrm{T}^{\mathrm{a}}=1$ 으, $\mathrm{S}=0 \%$ & $\mathrm{~T}^{\mathrm{a}}=22$ 으, $\mathrm{S}=35 \%$ \\
\hline Máximo desplazamiento en superficie $(\mathrm{m})$ & 0,0003856 & 0,00038588 \\
\hline $\begin{array}{l}\text { Máximo esfuerzo cortante en cimentación } \\
(\mathrm{kN})\end{array}$ & 764,1877 & 764,7459 \\
\hline $\begin{array}{l}\text { Máximo momento flector en cimentación } \\
\text { (kN.m) }\end{array}$ & 8438,0723 & 8444,2343 \\
\hline \multicolumn{2}{|c|}{ Tabla 83. Comportamiento de la estructura para el caso de $H=3,6 \mathrm{~m}, h=22,0 \mathrm{~m}, \mathrm{~T}=18,5 \mathrm{~s}$ y $\mathrm{D=8,0 \textrm {m }}$} \\
\hline
\end{tabular}

\subsubsection{Caso 3D}

Los datos de entrada se corresponden con el caso de cálculo para el parque eólico offshore Thornton Bank en Bélgica: altura de ola significativa $6,32 \mathrm{~m}$, profundidad $16,0 \mathrm{~m}$ y periodo de pico 11,06 s. Los valores más característicos de la simulación, tanto para el caso de temperatura de 1 으 $\mathrm{C}$ salinidad de $0 \%$, como para el caso de temperatura de $22{ }^{\circ} \mathrm{C}$ y salinidad del $35 \%$, se muestran, a continuación, en la tabla 84 :

\begin{tabular}{|c|c|c|}
\hline & \multicolumn{2}{|c|}{ Método avanzado } \\
\hline Parámetro & $\mathrm{T}^{\mathrm{a}}=1 \mathrm{o}=\mathrm{C}, \mathrm{S}=0 \%$ & $\mathrm{~T}^{\mathrm{a}}=22^{\circ} \mathrm{O}, \mathrm{S}=35 \%$ \\
\hline Máximo desplazamiento en superficie (m) & 0,00027412 & 0,00027432 \\
\hline $\begin{array}{l}\text { Máximo esfuerzo cortante en cimentación } \\
(\mathrm{kN})\end{array}$ & 1720,7101 & 1688,6876 \\
\hline $\begin{array}{l}\text { Máximo momento flector en cimentación } \\
(\mathrm{kN} \cdot \mathrm{m})\end{array}$ & 13612,3551 & 13622,4252 \\
\hline
\end{tabular}




\subsection{Caso 4: Diámetros igual a $16,0 \mathrm{~m}$}

\subsubsection{Caso 4A}

Los datos de entrada se corresponden con el caso de cálculo para el parque eólico offshore Princess Amalia Q7 en Holanda: altura de ola significativa 7,7 m, profundidad 24,0 m y periodo de pico 9,7 s. Los valores más característicos de la simulación, tanto para el caso de temperatura de $1 \stackrel{\circ}{\circ}$ y y salinidad de $0 \%$, como para el caso de temperatura de $22{ }^{\circ} \mathrm{C}$ y salinidad del 35\%, se muestran, a continuación, en la tabla 85 :

\begin{tabular}{|l|c|c|}
\cline { 2 - 3 } \multicolumn{1}{c|}{} & \multicolumn{2}{c|}{ Método avanzado } \\
\hline \multicolumn{1}{|c|}{ Parámetro } & $\mathrm{T}^{\mathrm{a}}=1$ 으, $\mathrm{S}=0 \%$ & $\mathrm{~T}^{\mathrm{a}}=22$ 으, $\mathrm{S}=35 \%$ \\
\hline Máximo desplazamiento en superficie $(\mathrm{m})$ & 0,00036908 & 0,00036921 \\
\hline $\begin{array}{l}\text { Máximo esfuerzo cortante en cimentación } \\
(\mathrm{kN})\end{array}$ & 10787,8815 & 10463,6223 \\
\hline $\begin{array}{l}\text { Máximo momento flector en cimentación } \\
(\mathrm{kN} \cdot \mathrm{m})\end{array}$ & 126709,6659 & 123647,7568 \\
\hline \multicolumn{1}{|c|}{ Tabla 85. Comportamiento de la estructura para el caso de $H=7,7 m, h=24,0 m, T=9,7 \mathrm{~s}$ y $D=16,0 \mathrm{~m}$} \\
\hline
\end{tabular}

\subsubsection{Caso 4B}

Los datos de entrada se corresponden con el caso de cálculo para el parque eólico offshore North Hoyle en Reino Unido: altura de ola significativa $4,78 \mathrm{~m}$, profundidad $10,0 \mathrm{~m}$ y periodo de pico 6,8 s. Los valores más característicos de la simulación, tanto para el caso de temperatura de 1 으 y salinidad de $0 \%$, como para el caso de temperatura de $22{ }^{\circ} \mathrm{C}$ y salinidad del $35 \%$, se muestran, a continuación, en la tabla 86 :

\begin{tabular}{|c|c|c|}
\hline & \multicolumn{2}{|c|}{ Método avanzado } \\
\hline Parámetro & $\mathrm{T}^{\mathrm{a}}=1 \mathrm{O} \mathrm{C}, \mathrm{S}=0 \%$ & $\mathrm{~T}^{\mathrm{a}}=22^{\circ} \mathrm{C}, \mathrm{S}=35 \%$ \\
\hline Máximo desplazamiento en superficie (m) & $1,314 \mathrm{e}-05$ & $1,315 \mathrm{e}-05$ \\
\hline $\begin{array}{l}\text { Máximo esfuerzo cortante en cimentación } \\
(\mathrm{kN})\end{array}$ & 5281,3791 & 5285,236 \\
\hline $\begin{array}{l}\text { Máximo momento flector en cimentación } \\
(\mathrm{kN} \cdot \mathrm{m})\end{array}$ & 25243,4678 & 25261,9016 \\
\hline
\end{tabular}




\subsubsection{Caso 4C}

Los datos de entrada se corresponden con el caso de cálculo para el parque eólico offshore Egmond Aan Zee en Holanda: altura de ola significativa 3,6 m, profundidad 22,0 m y periodo de pico 18,5 s. Los valores más característicos de la simulación, tanto para el caso de temperatura de 1 으 $\mathrm{C}$ s salinidad de $0 \%$, como para el caso de temperatura de $22{ }^{\circ} \mathrm{C}$ y salinidad del $35 \%$, se muestran, a continuación, en la tabla 87 :

\begin{tabular}{|c|c|c|}
\hline & \multicolumn{2}{|c|}{ Método avanzado } \\
\hline Parámetro & $\mathrm{T}^{\mathrm{a}}=1 \mathrm{o} \mathrm{C}, \mathrm{S}=0 \%$ & $\mathrm{~T}^{\mathrm{a}}=22^{\circ} \mathrm{O}, \mathrm{S}=35 \%$ \\
\hline Máximo desplazamiento en superficie (m) & $7,931 \mathrm{e}-05$ & $7,937 \mathrm{e}-05$ \\
\hline $\begin{array}{l}\text { Máximo esfuerzo cortante en cimentación } \\
\text { (kN) }\end{array}$ & 2979,597 & 2981,7741 \\
\hline $\begin{array}{l}\text { Máximo momento flector en cimentación } \\
(\mathrm{kN} \cdot \mathrm{m})\end{array}$ & 32863,5765 & 32887,5268 \\
\hline
\end{tabular}

\subsubsection{Caso 4D}

Los datos de entrada se corresponden con el caso de cálculo para el parque eólico offshore Thornton Bank en Bélgica: altura de ola significativa $6,32 \mathrm{~m}$, profundidad $16,0 \mathrm{~m}$ y periodo de pico 11,06 s. Los valores más característicos de la simulación, tanto para el caso de temperatura de 1 으 $\mathrm{C}$ salinidad de $0 \%$, como para el caso de temperatura de $22{ }^{\circ} \mathrm{C}$ y salinidad del $35 \%$, se muestran, a continuación, en la tabla 88 :

\begin{tabular}{|l|c|c|}
\cline { 2 - 3 } \multicolumn{1}{c|}{} & \multicolumn{2}{c|}{ Método avanzado } \\
\cline { 2 - 3 } \multicolumn{1}{c|}{ Parámetro } & $\mathrm{T}^{\mathrm{a}}=1$ 으, $\mathrm{S}=0 \%$ & $\mathrm{~T}^{\mathrm{a}}=22{ }^{\circ} \mathrm{C}, \mathrm{S}=35 \%$ \\
\hline Máximo desplazamiento en superficie $(\mathrm{m})$ & $6,313 \mathrm{e}-05$ & $6,318 \mathrm{e}-05$ \\
\hline $\begin{array}{l}\text { Máximo esfuerzo cortante en cimentación } \\
(\mathrm{kN})\end{array}$ & 6649,5114 & 6654,3753 \\
\hline $\begin{array}{l}\text { Máximo momento flector en cimentación } \\
(\mathrm{kN} \cdot \mathrm{m})\end{array}$ & 53586,8134 & 53626,0304 \\
\hline \multicolumn{2}{|c|}{ Tabla 88. Comportamiento de la estructura para el caso de $H=6,32 \mathrm{~m}, h=16,0 \mathrm{~m}, T=11,06 \mathrm{~s}$ y $D=16,0 \mathrm{~m}$} \\
\hline
\end{tabular}




\section{Proceso V: Comportamiento de la cimentación.}

El quinto proceso se corresponde con la simulación del fenómeno de socavación en el lecho de la cimentación, teniendo en cuenta las aceleraciones y velocidades halladas en el fondo marino, mediante los procesos de cálculo anteriores.

\subsection{Caso 1: Diámetros igual a $0,5 \mathrm{~m}$}

Se va a evaluar el fenómeno de socavación en pilotes de 0,5 $\mathrm{m}$ de diámetro mediante los cuatro casos de oleaje definidos en el apartado de mecánica de ondas, calculados según la teoría lineal y según las teorías no lineales.

\subsubsection{Caso 1A}

Los datos de entrada se corresponden con el caso de cálculo para el parque eólico offshore Princess Amalia Q7 en Holanda: altura de ola significativa 7,7 m, profundidad 24,0 m, periodo de pico 9,7 s y corriente de 1,30 m/s. Los resultados de la simulación, tanto para el caso analizado mediante el método avanzado, como para el caso del método tradicional, se muestran, a continuación, en la tabla 89:

\begin{tabular}{|l|c|}
\hline \multicolumn{2}{|c|}{ Profundidad máxima de socavación en equilibrio, en metros } \\
\hline Método avanzado & 0,636 \\
\hline $\begin{array}{l}\text { Método avanzado, modelo de socavación } \\
\text { tradicional }\end{array}$ & 0,634 \\
\hline Método tradicional & 0,634 \\
\hline \multicolumn{2}{|c|}{ Tabla 89. Fenómeno de socavación para el caso de $H=7,7 \mathrm{~m}, h=24,0 \mathrm{~m}, T=9,7 \mathrm{~s}, u c=1,3 \mathrm{~m} / \mathrm{s}$ y $\mathrm{D}=0,5 \mathrm{~m}$} \\
\hline
\end{tabular}

\subsubsection{Caso $1 B$}

Los datos de entrada se corresponden con el caso de cálculo para el parque eólico offshore North Hoyle en Reino Unido: altura de ola significativa 4,78 m, profundidad 10,0 m, periodo de pico $6,8 \mathrm{~s}$ y corriente de $0,8 \mathrm{~m} / \mathrm{s}$. Los resultados de la simulación, tanto para el caso analizado mediante el método avanzado, como para el caso del método tradicional, se muestran, a continuación, en la tabla 90: 


\begin{tabular}{|l|c|}
\hline \multicolumn{2}{|c|}{ Profundidad máxima de socavación en equilibrio, en metros } \\
\hline Método avanzado & 0,510 \\
\hline $\begin{array}{l}\text { Método avanzado, modelo de socavación } \\
\text { tradicional }\end{array}$ & 0,505 \\
\hline Método tradicional & 0,505 \\
\hline
\end{tabular}

Tabla 90. Fenómeno de socavación para el caso de $H=4,78 m, h=10,0 m, T=6,8 s, u c=0,8 \mathrm{~m} / \mathrm{s}$ y $D=0,5 \mathrm{~m}$

\subsubsection{Caso $1 \mathrm{C}$}

Los datos de entrada se corresponden con el caso de cálculo para el parque eólico offshore Egmond Aan Zee en Holanda: altura de ola significativa 3,6 m, profundidad 22,0 m, periodo de pico $18,5 \mathrm{~s}$ y corriente de $0,7 \mathrm{~m} / \mathrm{s}$. Los resultados de la simulación, tanto para el caso analizado mediante el método avanzado, como para el caso del método tradicional, se muestran, a continuación, en la tabla 91:

\begin{tabular}{|l|c|}
\hline \multicolumn{2}{|c|}{ Profundidad máxima de socavación en equilibrio, en metros } \\
\hline Método avanzado & 0,642 \\
\hline $\begin{array}{l}\text { Método avanzado, modelo de socavación } \\
\text { tradicional }\end{array}$ & 0,634 \\
\hline Método tradicional & 0,634 \\
\hline \multicolumn{2}{|c|}{ Tabla 91. Fenómeno de socavación para el caso de $H=3,6 m, h=22,0 m, T=18,5 s, u c=0,7 \mathrm{~m} / \mathrm{s}$ y $D=0,5 \mathrm{~m}$} \\
\hline
\end{tabular}

\subsubsection{Caso 1D}

Los datos de entrada se corresponden con el caso de cálculo para el parque eólico offshore Thornton Bank en Bélgica: altura de ola significativa 6,32 m, profundidad 16,0 m, periodo de pico $11,06 \mathrm{~s}$ y corriente de 1,2 m/s. Los resultados de la simulación, tanto para el caso analizado mediante el método avanzado, como para el caso del método tradicional, se muestran, a continuación, en la tabla 92: 
Modelos de predicción de esfuerzos hidrodinámicos y socavación aplicados a ingeniería offshore

\begin{tabular}{|l|c|}
\hline \multicolumn{2}{|c|}{ Profundidad máxima de socavación en equilibrio, en metros } \\
\hline Método avanzado & 0,636 \\
\hline $\begin{array}{l}\text { Método avanzado, modelo de socavación } \\
\text { tradicional }\end{array}$ & 0,636 \\
\hline Método tradicional & 0,635 \\
\hline
\end{tabular}

Tabla 92. Fenómeno de socavación para el caso de $H=6,32 \mathrm{~m}, h=16,0 \mathrm{~m}, \mathrm{~T}=11,06 \mathrm{~s}, \mathrm{c}=1,2 \mathrm{~m} / \mathrm{s}$ y $\mathrm{D}=0,5 \mathrm{~m}$ 


\subsection{Caso 2: Diámetros igual a 4,0 m}

Se va a evaluar el fenómeno de socavación en pilotes de 4,0 m de diámetro mediante los cuatro casos de oleaje definidos en el apartado de mecánica de ondas, calculados según la teoría lineal y según las teorías no lineales.

\subsubsection{Caso $2 \mathrm{~A}$}

Los datos de entrada se corresponden con el caso de cálculo para el parque eólico offshore Princess Amalia Q7 en Holanda: altura de ola significativa 7,7 m, profundidad 24,0 m, periodo de pico $9,7 \mathrm{~s}$ y corriente de 1,30 m/s. Los resultados de la simulación, tanto para el caso analizado mediante el método avanzado, como para el caso del método tradicional, se muestran, a continuación, en la tabla 93:

\begin{tabular}{|l|c|}
\hline \multicolumn{2}{|c|}{ Profundidad máxima de socavación en equilibrio, en metros } \\
\hline Método avanzado & 1,716 \\
\hline $\begin{array}{l}\text { Método avanzado, modelo de socavación } \\
\text { tradicional }\end{array}$ & 1,664 \\
\hline Método tradicional & 1,672 \\
\hline \multicolumn{1}{|c|}{ Tabla 93. Fenómeno de socavación para el caso de $H=7,7 m, h=24,0 \mathrm{~m}, T=9,7 \mathrm{~s}, u c=1,3 \mathrm{~m} / \mathrm{s}$ y $\mathrm{D=}=4,0 \mathrm{~m}$} \\
\hline
\end{tabular}

\subsubsection{Caso 2B}

Los datos de entrada se corresponden con el caso de cálculo para el parque eólico offshore North Hoyle en Reino Unido: altura de ola significativa 4,78 m, profundidad 10,0 m, periodo de pico $6,8 \mathrm{~s}$ y corriente de $0,8 \mathrm{~m} / \mathrm{s}$. Los resultados de la simulación, tanto para el caso analizado mediante el método avanzado, como para el caso del método tradicional, se muestran, a continuación, en la tabla 94:

\begin{tabular}{|l|c|}
\hline \multicolumn{2}{|c|}{ Profundidad máxima de socavación en equilibrio, en metros } \\
\hline Método avanzado & 0,548 \\
\hline $\begin{array}{l}\text { Método avanzado, modelo de socavación } \\
\text { tradicional }\end{array}$ & 0,528 \\
\hline Método tradicional & 0,528 \\
\hline
\end{tabular}

Tabla 94. Fenómeno de socavación para el caso de $H=4,78 m, h=10,0 m, T=6,8 s, u c=0,8 \mathrm{~m} / \mathrm{s}$ y $D=4,0 \mathrm{~m}$ 


\subsubsection{Caso $2 \mathrm{C}$}

Los datos de entrada se corresponden con el caso de cálculo para el parque eólico offshore Egmond Aan Zee en Holanda: altura de ola significativa 3,6 m, profundidad 22,0 m, periodo de pico $18,5 \mathrm{~s}$ y corriente de $0,7 \mathrm{~m} / \mathrm{s}$. Los resultados de la simulación, tanto para el caso analizado mediante el método avanzado, como para el caso del método tradicional, se muestran, a continuación, en la tabla 95:

\begin{tabular}{|l|c|}
\hline \multicolumn{2}{|c|}{ Profundidad máxima de socavación en equilibrio, en metros } \\
\hline Método avanzado & 1,967 \\
\hline $\begin{array}{l}\text { Método avanzado, modelo de socavación } \\
\text { tradicional }\end{array}$ & 1,649 \\
\hline Método tradicional & 1,649 \\
\hline
\end{tabular}

Tabla 95. Fenómeno de socavación para el caso de $H=3,6 m, h=22,0 m, T=18,5 s, u c=0,7 \mathrm{~m} / \mathrm{s}$ y $D=4,0 \mathrm{~m}$

\subsubsection{Caso 2D}

Los datos de entrada se corresponden con el caso de cálculo para el parque eólico offshore Thornton Bank en Bélgica: altura de ola significativa 6,32 m, profundidad 16,0 m, periodo de pico $11,06 \mathrm{~s}$ y corriente de $1,2 \mathrm{~m} / \mathrm{s}$. Los resultados de la simulación, tanto para el caso analizado mediante el método avanzado, como para el caso del método tradicional, se muestran, a continuación, en la tabla 96:

\begin{tabular}{|l|c|}
\hline \multicolumn{2}{|c|}{ Profundidad máxima de socavación en equilibrio, en metros } \\
\hline Método avanzado & 1,704 \\
\hline $\begin{array}{l}\text { Método avanzado, modelo de socavación } \\
\text { tradicional }\end{array}$ & 1,699 \\
\hline Método tradicional & 1,696 \\
\hline \multicolumn{2}{|c|}{ Tabla 96. Fenómeno de socavación para el caso de $H=6,32 m, h=16,0 m, T=11,06 s, u c=1,2 \mathrm{~m} / \mathrm{s}$ y $D=4,0 \mathrm{~m}$} \\
\hline
\end{tabular}




\subsection{Caso 3: Diámetros igual a 8,0 m}

Se va a evaluar el fenómeno de socavación en pilotes de 8,0 m de diámetro mediante los cuatro casos de oleaje definidos en el apartado de mecánica de ondas, calculados según la teoría lineal y según las teorías no lineales.

\subsubsection{Caso $3 A$}

Los datos de entrada se corresponden con el caso de cálculo para el parque eólico offshore Princess Amalia Q7 en Holanda: altura de ola significativa 7,7 m, profundidad 24,0 m, periodo de pico 9,7 s y corriente de 1,30 m/s. Los resultados de la simulación, tanto para el caso analizado mediante el método avanzado, como para el caso del método tradicional, se muestran, a continuación, en la tabla 97:

\begin{tabular}{|l|c|}
\hline \multicolumn{2}{|c|}{ Profundidad máxima de socavación en equilibrio, en metros } \\
\hline Método avanzado & 1,495 \\
\hline $\begin{array}{l}\text { Método avanzado, modelo de socavación } \\
\text { tradicional }\end{array}$ & 1,428 \\
\hline Método tradicional & 1,440 \\
\hline \multicolumn{1}{|c|}{ Tabla 97. Fenómeno de socavación para el caso de $H=7,7 m, h=24,0 \mathrm{~m}, T=9,7 \mathrm{~s}, u c=1,3 \mathrm{~m} / \mathrm{s}$ y $\mathrm{D=}=8,0 \mathrm{~m}$} \\
\hline
\end{tabular}

\subsubsection{Caso 3B}

Los datos de entrada se corresponden con el caso de cálculo para el parque eólico offshore North Hoyle en Reino Unido: altura de ola significativa 4,78 m, profundidad 10,0 m, periodo de pico $6,8 \mathrm{~s}$ y corriente de $0,8 \mathrm{~m} / \mathrm{s}$. Los resultados de la simulación, tanto para el caso analizado mediante el método avanzado, como para el caso del método tradicional, se muestran, a continuación, en la tabla 98:

\begin{tabular}{|l|c|}
\hline \multicolumn{2}{|c|}{ Profundidad máxima de socavación en equilibrio, en metros } \\
\hline Método avanzado & 0,101 \\
\hline $\begin{array}{l}\text { Método avanzado, modelo de socavación } \\
\text { tradicional }\end{array}$ & 0,079 \\
\hline Método tradicional & 0,080 \\
\hline
\end{tabular}

Tabla 98. Fenómeno de socavación para el caso de $H=4,78 m, h=10,0 m, T=6,8 s, u c=0,8 \mathrm{~m} / \mathrm{s}$ y $D=8,0 \mathrm{~m}$ 


\subsubsection{Caso $3 \mathrm{C}$}

Los datos de entrada se corresponden con el caso de cálculo para el parque eólico offshore Egmond Aan Zee en Holanda: altura de ola significativa 3,6 m, profundidad 22,0 m, periodo de pico $18,5 \mathrm{~s}$ y corriente de $0,7 \mathrm{~m} / \mathrm{s}$. Los resultados de la simulación, tanto para el caso analizado mediante el método avanzado, como para el caso del método tradicional, se muestran, a continuación, en la tabla 99:

\begin{tabular}{|l|c|}
\hline \multicolumn{2}{|c|}{ Profundidad máxima de socavación en equilibrio, en metros } \\
\hline Método avanzado & 1,815 \\
\hline $\begin{array}{l}\text { Método avanzado, modelo de socavación } \\
\text { tradicional }\end{array}$ & 1,403 \\
\hline Método tradicional & 1,402 \\
\hline
\end{tabular}

Tabla 99. Fenómeno de socavación para el caso de $H=3,6 m, h=22,0 m, T=18,5 s, u c=0,7 m / s$ y $D=8,0 m$

\subsubsection{Caso 3D}

Los datos de entrada se corresponden con el caso de cálculo para el parque eólico offshore Thornton Bank en Bélgica: altura de ola significativa $6,32 \mathrm{~m}$, profundidad $16,0 \mathrm{~m}$, periodo de pico $11,06 \mathrm{~s}$ y corriente de $1,2 \mathrm{~m} / \mathrm{s}$. Los resultados de la simulación, tanto para el caso analizado mediante el método avanzado, como para el caso del método tradicional, se muestran, a continuación, en la tabla 100:

\begin{tabular}{|l|c|}
\hline \multicolumn{2}{|c|}{ Profundidad máxima de socavación en equilibrio, en metros } \\
\hline Método avanzado & 1,474 \\
\hline $\begin{array}{l}\text { Método avanzado, modelo de socavación } \\
\text { tradicional }\end{array}$ & 1,467 \\
\hline Método tradicional & 1,464 \\
\hline
\end{tabular}




\subsection{Caso 4: Diámetros igual a 16,0 m}

Se va a evaluar el fenómeno de socavación en pilotes de 16,0 m de diámetro mediante los cuatro casos de oleaje definidos en el apartado de mecánica de ondas, calculados según la teoría lineal y según las teorías no lineales.

\subsubsection{Caso $4 \mathrm{~A}$}

Los datos de entrada se corresponden con el caso de cálculo para el parque eólico offshore Princess Amalia Q7 en Holanda: altura de ola significativa 7,7 m, profundidad 24,0 m, periodo de pico 9,7 s y corriente de 1,30 m/s. Los resultados de la simulación, tanto para el caso analizado mediante el método avanzado, como para el caso del método tradicional, se muestran, a continuación, en la tabla 101:

\begin{tabular}{|l|c|}
\hline \multicolumn{2}{|c|}{ Profundidad máxima de socavación en equilibrio, en metros } \\
\hline Método avanzado & 0,666 \\
\hline $\begin{array}{l}\text { Método avanzado, modelo de socavación } \\
\text { tradicional }\end{array}$ & 0,590 \\
\hline Método tradicional & 0,617 \\
\hline \multicolumn{2}{|c|}{ Tabla 101. Fenómeno de socavación para el caso de $H=7,7 \mathrm{~m}, h=24,0 \mathrm{~m}, T=9,7 \mathrm{~s}, u c=1,3 \mathrm{~m} / \mathrm{s}$ y $D=16,0 \mathrm{~m}$} \\
\hline
\end{tabular}

\subsubsection{Caso 4B}

Los datos de entrada se corresponden con el caso de cálculo para el parque eólico offshore North Hoyle en Reino Unido: altura de ola significativa 4,78 m, profundidad 10,0 m, periodo de pico $6,8 \mathrm{~s}$ y corriente de $0,8 \mathrm{~m} / \mathrm{s}$. Los resultados de la simulación, tanto para el caso analizado mediante el método avanzado, como para el caso del método tradicional, se muestran, a continuación, en la tabla 102:

\begin{tabular}{|l|c|}
\hline \multicolumn{2}{|c|}{ Profundidad máxima de socavación en equilibrio, en metros } \\
\hline Método avanzado & 0,0 \\
\hline $\begin{array}{l}\text { Método avanzado, modelo de socavación } \\
\text { tradicional }\end{array}$ & 0,0 \\
\hline Método tradicional & 0,0 \\
\hline \multicolumn{2}{|c|}{ Tabla 102. Fenómeno de socavación para el caso de $H=4,78 m, h=10,0 \mathrm{~m}, T=6,8 \mathrm{~s}, u c=0,8 \mathrm{~m} / \mathrm{s}$ y $\mathrm{D}=16,0 \mathrm{~m}$}
\end{tabular}




\subsubsection{Caso $4 \mathrm{C}$}

Los datos de entrada se corresponden con el caso de cálculo para el parque eólico offshore Egmond Aan Zee en Holanda: altura de ola significativa 3,6 m, profundidad 22,0 m, periodo de pico $18,5 \mathrm{~s}$ y corriente de $0,7 \mathrm{~m} / \mathrm{s}$. Los resultados de la simulación, tanto para el caso analizado mediante el método avanzado, como para el caso del método tradicional, se muestran, a continuación, en la tabla 103:

\begin{tabular}{|l|c|}
\hline \multicolumn{2}{|c|}{ Profundidad máxima de socavación en equilibrio, en metros } \\
\hline Método avanzado & 1,016 \\
\hline $\begin{array}{l}\text { Método avanzado, modelo de socavación } \\
\text { tradicional }\end{array}$ & 0,547 \\
\hline Método tradicional & 0,545 \\
\hline Tabla 103. Fenómeno de socavación para el caso de $H=3,6 m, h=22,0 m, T=18,5 \mathrm{~s}, u c=0,7 \mathrm{~m} / \mathrm{s}$ y $D=16,0 \mathrm{~m}$
\end{tabular}

\subsubsection{Caso 4D}

Los datos de entrada se corresponden con el caso de cálculo para el parque eólico offshore Thornton Bank en Bélgica: altura de ola significativa 6,32 m, profundidad 16,0 m, periodo de pico $11,06 \mathrm{~s}$ y corriente de $1,2 \mathrm{~m} / \mathrm{s}$. Los resultados de la simulación, tanto para el caso analizado mediante el método avanzado, como para el caso del método tradicional, se muestran, a continuación, en la tabla 104:

\begin{tabular}{|l|c|}
\hline \multicolumn{2}{|c|}{ Profundidad máxima de socavación en equilibrio, en metros } \\
\hline Método avanzado & 0,629 \\
\hline $\begin{array}{l}\text { Método avanzado, modelo de socavación } \\
\text { tradicional }\end{array}$ & 0,622 \\
\hline Método tradicional & 0,617 \\
\hline Tabla 104. Fenómeno de socavación para el caso de $H=6,32 \mathrm{~m}, h=16,0 \mathrm{~m}, T=11,06 \mathrm{~s}, u c=1,2 \mathrm{~m} / \mathrm{s}$ y $D=16,0 \mathrm{~m}$ \\
\hline
\end{tabular}


Apéndice 2

INDICIOS DE CALIDAD 



\section{Apéndice 2}

\section{INDICIOS DE CALIDAD}

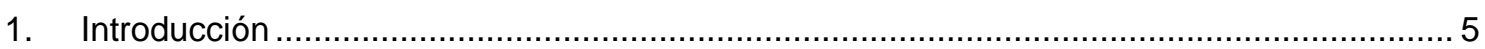

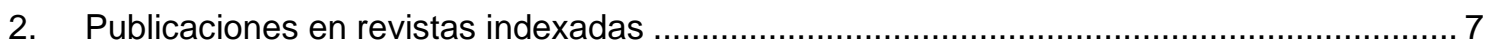

2.1. Journal of Coastal Research (Coastal Education \& Research Foundation) .................. 7

3. Publicaciones en revistas internacionales con revisión por pares...................................... 9

3.1. Journal of Ocean Engineering and Science (Revista de Elsevier) ............................ 9

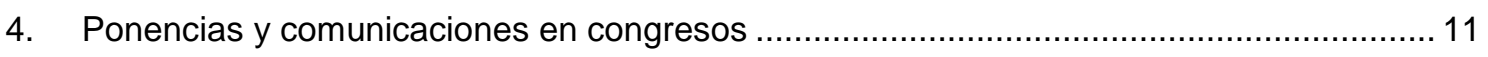

4.1. The 14th International Coastal Symposium (ICS), 2016 (Sidney, Australia) .............. 11

4.2. XIV Jornadas Españolas de Ingeniería de Costas y Puertos 2017 (Alicante, España) 11 



\section{Apéndice 2}

\section{INDICIOS DE CALIDAD}

\section{Introducción}

Durante el desarrollo de la investigación se ha realizado la publicación de dos artículos en revistas internacionales, donde se han recogido los resultados y las principales conclusiones de la comparación de los métodos tradicionales de cálculo, con los resultados aportados por el método avanzado de cálculo, desarrollado mediante la presente Tesis Doctoral.

Además, se han realizado dos ponencias, una de carácter internacional y otra nacional.

A continuación, se muestran los índicos de calidad de la presente Tesis Doctoral. 



\section{Publicaciones en revistas indexadas}

2.1. Journal of Coastal Research (Coastal Education \& Research Foundation) 

Software for Predicting Hydrodynamic Pressures on Offshore Pile Foundations: The Next Step in Ocean Energy Development

\author{
Adrian Escobar ${ }^{\dagger^{*}}$, Vicente Negro ${ }^{\dagger}$, Jose Santos Lopez-Gutierrez ${ }^{\dagger}$, and M. Dolores Esteban ${ }^{\dagger}$ \\ ${ }^{\dagger}$ Research Group on Marine, Coastal and Port Environment and other Sensitive Areas \\ Technical University of Madrid \\ Madrid, Spain
}

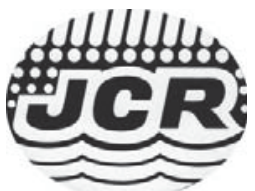

www.JCRonline.org

\begin{abstract}
Escobar, A.; Negro, V.; Lopez-Gutierrez, J.S., and Esteban, M.D., 2016. Software for predicting hydrodynamic pressures on offshore pile foundations. The next step in ocean energy development. In: Vila-Concejo, A.; Bruce, E.; Kennedy, D.M., and McCarroll, R.J. (eds.), Proceedings of the 14th International Coastal Symposium (Sydney, Australia). Journal of Coastal Research, Special Issue, No. 75, pp. 841-845. Coconut Creek (Florida), ISSN 07490208.

The purpose of this study is to carry out a thorough, critical analysis of the Morison equation as far as calculating wave action on large diameter piles like those used nowadays in offshore wind farms is concerned. The aim is to observe whether models currently used to estimate wave forces on piles are valid for large diameter piles apart from observing what the main forces in play in scouring are. This equation enables wave produced forces on a cylinder supported on the sea bed to be calculated. The study includes observations on the calculation model's sensitivity as to a variation in the cylinder's diameter, on the one hand and, on the other, as to temperature and salinity variation. With this in mind, specific software has been developed to simulate equations in fluid mechanics applied to solve the wave-structure interaction problem in the separation, inertial and diffraction range. This software will enable an iterative calculation to be made for finding out the shape of the pressure wave caused when a wave passes over and will show the results for different pile diameters and water temperature.
\end{abstract}

\title{
ADDITIONAL INDEX WORDS: Morison equation, hydrodynamic wave actions, offshore foundations.
}

\section{INTRODUCTION}

Nowadays, most standards applied in the world in the field of offshore wind farm design have the Morison formula as a main pylon of the calculation method for predicting hydrodynamic forces on a vertical circular cylinder. The following points will provide a brief description of the work performed by the main authors, who have developed the different theories solving the problem for all hydrodynamic ranges, and in addition, the main differences between the results obtained referring to those provided by the Morison equation will be shown. The whole analysis will focus on the estimation of stresses suffered by the pile foundation. Furthermore, as innovative approaches, two key points will be developed: temperature and salinity will be included as parameters of the calculation to observe their influence on the dynamic amplification factor and, an iterative method will be introduced for taking the interaction between structure and waves into account.

As the development of renewable energies increased, the main concern of researchers has been to find new sources for obtaining energy and new processes enabling cleaner, less expensive and, therefore, more efficient energy to be created. With this in mind, the use of offshore wind farms has been one of the major achievements of modern science for achieving this

DOI: $10.2112 / S 175-169.1$ received 15 October 2015 ; accepted in revision 15 January 2016.

*Corresponding author: adrian.escobar.pastor@alumnos.upm.es

${ }^{\circ}$ Coastal Education and Research Foundation, Inc. 2016 aim, as suggested by Esteban et al. (2011). The main difficulty these structures display from the construction standpoint lies in the correct estimation of hydrodynamic loads the structure will have to withstand. It could be said that calculating hydrodynamic forces in offshore structures is one of the major, key issues for design engineers participating in maritime engineering, as suggested by Negro et al. (2014). In addition, they will be very important for the study of the influence of each of the forces on possible scouring at the pile's base, as suggested by Matutano et al. (2013). Even though wave action is stochastic in nature, studying wave action as if it behaved in a regular fashion and estimating the hydrodynamic forces it will generate on the structure and its resulting movements are of the utmost interest to the engineer. This type of analysis is completely determinist and rests on two fundamental variables; the wave height and its period. On the other hand, there is another approach which, by using the probabilistic theory, works with the wave energy's spectra results.

\section{Background}

Morison et al. (1950) suggested that the two flow regimes generated by inertia forces and drag forces may be superimposed to obtain the total load varying in time per unit of length of an immobile cylinder in a plane flow field with an arbitrary free flow speed. The wave force on circular piles, within the diffraction range, was first calculated by MacCamy et al. (1954), who proposed that there was an exact mathematical solution presented by wave action according to the linear wave theory. Chakrabarti (1972) obtained the hydrodynamic forces 
caused by nonlinear waves on a vertical cylinder, including the use of the diffraction theory by means of a compact equation and then compared the equation proposed with experimental results. Lighthill (1979) made a methodological analysis describing the basic physical phenomena together with the applicable methods used for estimating hydrodynamic forces. His main contribution to the calculation of wave force by using the Morison equation lies in the proposal of a second order correction thereto. The 17th ITFC Ocean Engineering Committee undertook to compare the methods in order to calculate the movement of a semi-submergible body. They concluded with a summary of the final project results in which 34 computer programs from 28 different organizations were later described by Takagi et al. (1985).

Existing methods for finding hydrodynamic forces in offshore structures are mainly based on the following techniques: the use of the Morison equation or techniques using the potential of speeds for sources and sumps, in two-dimensional or threedimensional flow. Faltinsen (1990) made a detailed analysis of wave induced linear movements and second order loads as well as nonlinear problems and viscous loads and concluded that the Morison equation and methods based on the theory of potential are favorably similar between them and with the field or laboratory made experimental measurements.

As computing power increased by means of the development of hardware and software engineering, new and more sophisticated methods have been introduced in recent years to calculate the wave-structure interaction problem. Koutandos (2009) presented a numerical study on wave interaction with rigid vertical barriers and computed the velocities and turbulence kinetic energy in the vicinity of the structure. Isaacson et al. (1998) and Zhu (2013) investigated wave interaction with a row of piles using the full wave theory. Zheng et al. (2015) presents an analytical model to predict the threedimensional wave diffraction of a floating cylinder located in front of a vertical wall at a finite water depth. Feng et al. (2015), provide a solution for a two-dimensional non linear wavestructure interaction problem by a desingularized integral method in combination with a mixed Euler-Lagrange method. Dongfeng et al. (2015) develop a 1/10 scale model to study the influence of foundation degradation on the dynamic response of an offshore jacket platform, including the design of finite element models to analyze the dynamic behavior of the jacket platform.

\section{METHODS}

The methodology developed to suitably solve wave-structure interaction must be divided into three main sections or, in other words, into three separate problems. The first uses environmental conditions: wave period $(T)$ and height $(H)$ and sea depth $(d)$ as first order variables which define the wave mechanics to develop in the calculation as a base for the whole assessment. Moreover, this section takes into account the influence of temperature $(\mathrm{Ta})$ and salinity $(\mathrm{S})$ over the water density $(\rho)$ as a second order effect. In second place, section two is earmarked to assessing hydrodynamic loads on the basis of flow speeds $(v)$ and accelerations $(a)$ obtained in section one. This problem has the diameter of the pile $(D)$ as a first order variable. Together with wave mechanics, the pile diameter defines the range (separate flow, inertial or diffraction) in which the problem must be solved. The sea temperature and salinity are included again because they play a key role in this part, due to their influence on water viscosity and, hence, on drag forces. Finally, the third section takes the sea wave loads $(F)$ obtained from section two and assesses their effect on the foundation and on movements of the structure along the vertical axis. Finally, the structure movements and loads generated by them $(R)$ feed the second section again and allow the calculation to be iteratively re-done until the final solution is achieved.

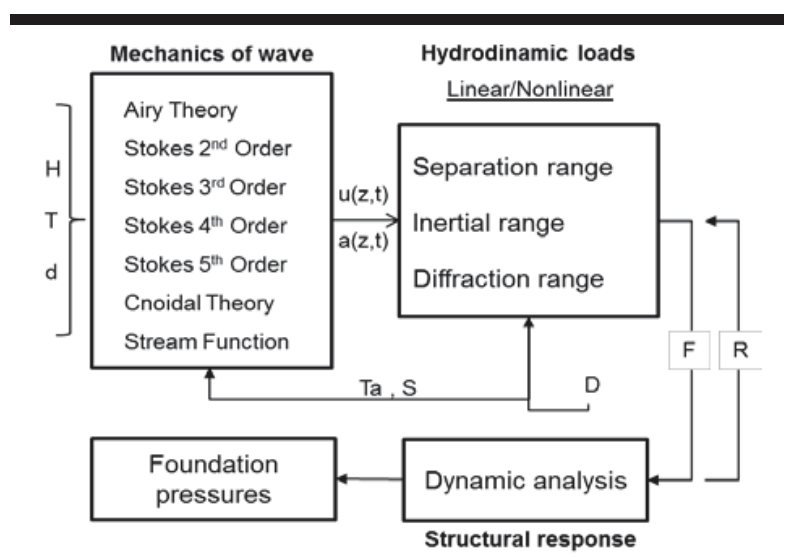

Figure 1. Calculation scheme as followed by the software

The wave mechanics model is designed to identify the range of the problem (deep water, intermediate depth and shallow water) and to solve the equations which define water wave motion (Euler equations of motion and continuity equation). To solve the system, the software will use the pertinent wave theory (such as Airy, Stokes, Cnoidal or Stream Function).

Hydrodynamic loads will be assessed by using six different calculation modules which provide the solution for one of the ranges: separate flow, inertial and diffraction; taking into account nonlinear effects when $H / L>0.5 \cdot H / L_{\max }$ (Table 1 ), where $L$ is the wavelength.

Table 1. Characterization of the hydrodynamic range performed by means of two parameters: Keulegan-Carpenter number (KC) and ratio $D / L$

\begin{tabular}{lcc}
\hline \hline Direction & $\mathrm{KC}<4$ & $\mathrm{KC}>4$ \\
\hline $\mathrm{D} / \mathrm{L}<0.2$ & Inertial Range & Separate Flow Range \\
$\mathrm{D} / \mathrm{L}>0.2$ & Diffraction Range & - \\
\hline
\end{tabular}

The separate flow range is solved by means of the Morison equation. The Reynolds number, $\mathrm{Re}$, is used to obtain the two parameters: coefficient of inertia due to the hydrodynamic mass $\mathrm{C}_{\mathrm{M}}$ and drag coefficient $\mathrm{C}_{\mathrm{D}}$. Furthermore, nonlinear effects of wave motion will be introduced analytically for the cases in which the problem is located in the nonlinear separate flow range:

$$
F(z, t)=F_{1}+F_{2}+F_{3}+F_{4}
$$




$$
\begin{aligned}
& F_{1}=-\rho \int_{\partial \Omega}\left[\frac{\partial \varphi_{1}}{\partial t} n\right] d s \\
& F_{2}=\frac{1}{2} C_{D} \rho D \cdot u(z, t) \cdot|u(z, t)| \\
& F_{3}=\frac{1}{2} \rho \int_{S}\left(\nabla \varphi_{1}\right)^{2} \cos \theta d s \\
& F_{4}=\frac{\rho}{2 g} \oint_{C}\left(\frac{\partial \varphi_{l}}{\partial t}\right)^{2} \cos \theta d l
\end{aligned}
$$

The inertial range uses the same equation, shown above for the separate flow range, but removes the term F2 corresponding to the drag force. On the other hand, when the assumption that the kinematics of the undisturbed flow in the region near the structure does not change in the incident wave direction is not complied with, the diffraction theory has to be used. The theory provided by MacCamy et al. (1954) is used as a basis (F1) but adding additional terms to include the nonlinear effects in the calculation (F2).

$$
\begin{gathered}
F_{1}=-D \rho \int_{0}^{2 \pi}\left(\frac{\partial \varphi^{(1)}}{\partial t}\right)_{r=D} \cos (\pi-\theta) d \theta \\
F_{2}=-D \rho \int_{0}^{2 \pi}\left[\int_{0}^{\eta^{(1)}}\left(\frac{\partial \varphi^{(1)}}{\partial t}+g z\right) d z+\frac{\partial \varphi^{(2)}}{\partial t}+\right. \\
\left.\frac{1}{2}\left(\frac{\partial \varphi^{(1)}}{\partial z}\right)^{2}+\frac{1}{2 D^{2}}\left(\frac{\partial \varphi^{(1)}}{\partial \theta}\right)^{2}\right]_{r=D} \cos (\pi-\theta) d \theta
\end{gathered}
$$

The structural response is analyzed in the field of elastic deformation. The vertical circular cylinder is assumed to be a cantilever beam fixed in the sea bed. Calculations of structural movements and bending moments at a given time can be obtained by means of the superposition method as shown below:

$$
\begin{gathered}
x_{\text {static }}(z)=\int_{h=z}^{h=d}\left[\frac{z^{2}}{6 E I}(3 h-z)\right] d F-\frac{F z^{3}}{3 E I}+ \\
\int_{h=0}^{h=z}\left[\frac{h^{2}}{6 E I}(3 z-h)\right] d F \\
M_{\text {static }}(z)=\int_{h=z}^{h=d}(h-z) d F
\end{gathered}
$$

The main problem shown in the above equation is that both expressions solve the static case without taking into account the inertial effects of the mass of the cylinder, so it is not suitable for applying in a dynamic analysis with dynamic loads. The calculation must apply a dynamic assessment in the frequency domain, which takes into account the inertial effects. The problem of that kind of analysis is the difficulty of finding a solution and has a substantial computing cost. However, this study has developed an efficient algorithm to solve these issues without increasing the calculation time or the computing cost. The differential equation which defines the behavior of the cantilever beam is:

$$
E I \frac{\partial^{4} x(z, t)}{\partial z^{4}}+m \frac{\partial^{2} x(z, t)}{\partial t^{2}}=0
$$

The above equation is best solved by separating variables. If it is assumed that the displacement can be separated into two parts, one depends on position and the other on time $x(z, t)=Z \cdot T$, which are both independent of each other, so that the equation can now be written as two differential equations:

$$
\begin{aligned}
& \frac{\partial^{4} Z}{\partial z^{4}}-k_{n}^{4} Z=0 \\
& \frac{\partial^{2} T}{\partial t^{2}}+\omega_{n}^{2} T=0 \\
& k_{n}^{4}=\frac{w_{n}^{2} m}{E I}
\end{aligned}
$$

The solution to the system is given by:

$$
x=\sum_{m=1}^{\infty} Z_{n}(z)\left[A_{n} \cos \left(\omega_{n} t\right)+B_{n} \sin \left(\omega_{n} t\right)\right]
$$

$A_{n}$ depends on the initial position at time $t=0, B_{n}$ depends on the initial velocity, and both of them must be determined with the boundary conditions.

This kind of analysis leads to obtaining the speed and acceleration of each point of the structure as a response of hydrodynamic loads for a given time. These speeds and accelerations can be subtracted from the initial $\mathrm{v}(\mathrm{z}, \mathrm{t})$ and $\mathrm{a}(\mathrm{z}, \mathrm{t})$, and the process is repeated until its convergence for each fraction of time. The final result is a whole record of the movements of the structure along the whole length, as originated by sea waves. Finally, the bending moment on the foundation can easily be obtained from these structural movements.

\section{RESULTS}

The sensitivity of the model to temperature and salinity for the two extreme cases is shown below (Table 2). The percentage of error has been calculated as follows:

$$
\text { \%error }=\left(M_{2}-M_{1}\right) / M_{1}
$$

Table 2. Differences in the model results with regard to the maximum bending moment on a pile foundation between using fresh water $M_{2}$ with low temperature $S=0 \%$ and $T a=1{ }^{\circ} \mathrm{C}$ and saturated warm water $M_{1}$ with $S=3.5 \%$ and $T a=22^{\circ} \mathrm{C}$, for the case of $H=7.0 \mathrm{~m}, d=30.0 \mathrm{~m}$ and values of $T$ between 16 seconds and 6 seconds.

\begin{tabular}{lll}
\hline \hline Diameter & Max \% error & Min \% error \\
\hline$D=1 \mathrm{~m}$ & $1.77 \%$ & $-0.07 \%$ \\
$\mathrm{D}=2 \mathrm{~m}$ & $0.76 \%$ & $-0.03 \%$ \\
$\mathrm{D}=4 \mathrm{~m}$ & $0.45 \%$ & $-0.01 \%$ \\
$\mathrm{D}=8 \mathrm{~m}$ & $2.02 \%$ & $-0.04 \%$ \\
$\mathrm{D}=16 \mathrm{~m}$ & $10.73 \%$ & $-0.07 \%$ \\
$\mathrm{D}=24 \mathrm{~m}$ & $10.52 \%$ & $-0.07 \%$ \\
\hline
\end{tabular}

In addition, based on the above equation, results are shown (Figure 3) regarding the maximum bending moment on the pile foundation in terms of the ratio $\mathrm{H} / \mathrm{gT}^{2}$, and the flow of accelerations for the case of $\mathrm{T}=10 \mathrm{~s}$. Table 3 shows the error in using the simplified model (Airy \& Morison) with regard to the results provided by the software which uses the correct formulation to match each calculation range. 

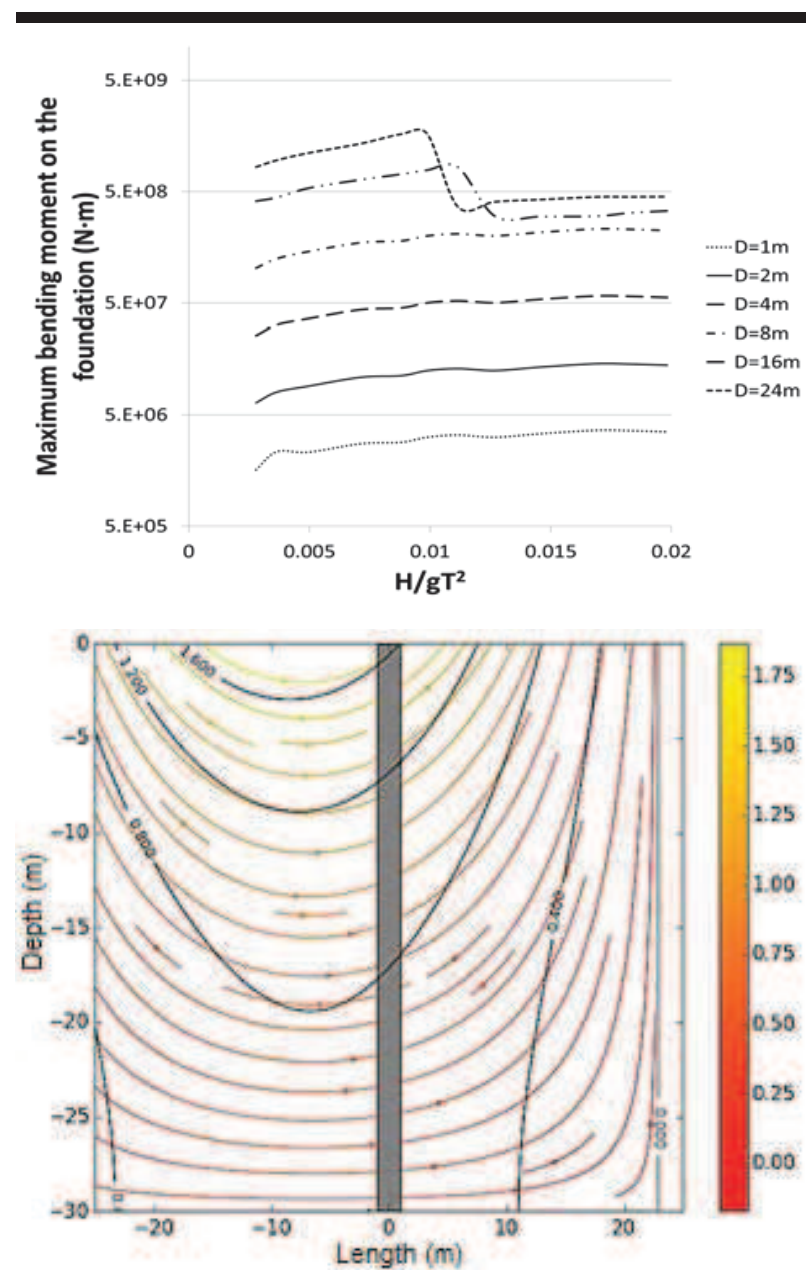

Figure 3. Maximum bending moment on the foundation for the case: $\mathrm{H}=7.0 \mathrm{~m}, \mathrm{~d}=30.0 \mathrm{~m}, \mathrm{~S}=3.5 \%$ and $\mathrm{Ta}=22.0{ }^{\circ} \mathrm{C}$ (top figure); flow of accelerations $\left(\mathrm{m} / \mathrm{s}^{2}\right)$ for the case of $\mathrm{H}=7.0 \mathrm{~m}, \mathrm{~d}=30.0 \mathrm{~m}, \mathrm{~S}=3.5 \%, \mathrm{Ta}=22.0$ ${ }^{\circ} \mathrm{C} \mathrm{T}=10 \mathrm{~s}$ and $\mathrm{D}=2 \mathrm{~m}$, with depth as vertical axis (bottom figure).

Table 3. Differences in the model results with regard to the maximum bending moment on a pile foundation between using Morison \& Linear theory $\left(M_{2}\right)$ or corresponding wave and hydrodynamic theory $\left(M_{1}\right)$, for the case of $S=3.5 \%, T a=22^{\circ} \mathrm{C}, H=7.0 \mathrm{~m}, d=30.0 \mathrm{~m}$ and values of $\mathrm{T}$ between 16 seconds and 6 seconds.

\begin{tabular}{lll}
\hline \hline Diameter & Max \% error & Min \% error \\
\hline$D=1 \mathrm{~m}$ & $122.05 \%$ & $9.71 \%$ \\
$D=2 \mathrm{~m}$ & $86.64 \%$ & $8.74 \%$ \\
$D=4 \mathrm{~m}$ & $68.76 \%$ & $6.60 \%$ \\
$D=8 \mathrm{~m}$ & $59.84 \%$ & $5.54 \%$ \\
$D=16 \mathrm{~m}$ & $232.33 \%$ & $4.46 \%$ \\
$D=24 \mathrm{~m}$ & $434.00 \%$ & $9.07 \%$ \\
\hline
\end{tabular}

The loads over the cylinder are shown, for the case of $\mathrm{T}=10 \mathrm{~s}$, in Figure 4. The type of force acting on a foundation is then shown for several periods in Table 4 .

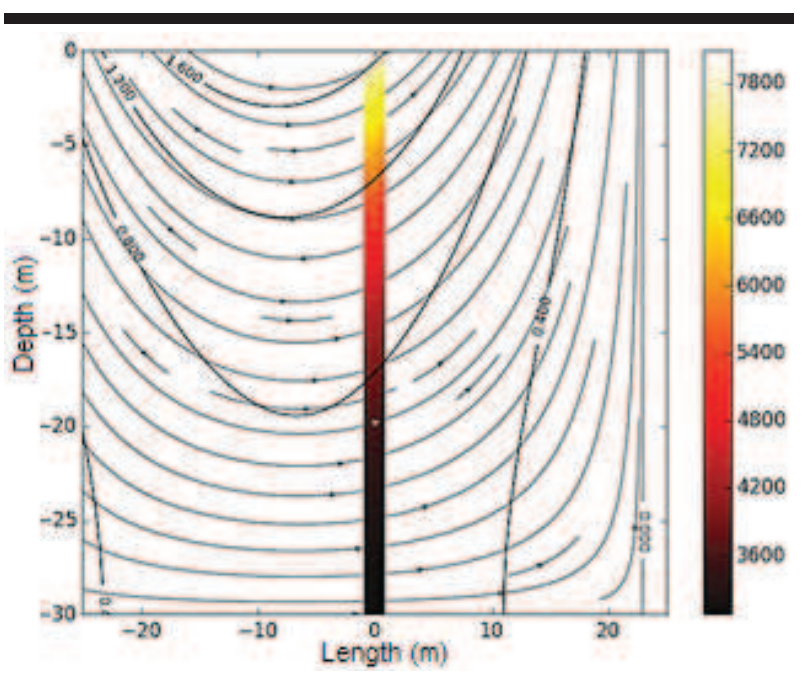

Figure 4. Wave generated loads $(\mathrm{N})$ on the cylinder for the case of $\mathrm{H}=7.0 \mathrm{~m}, \mathrm{~d}=30.0 \mathrm{~m}, \mathrm{~S}=3.5 \%$ and $\mathrm{Ta}=22.0^{\circ} \mathrm{C}$ and $\mathrm{D}=2 \mathrm{~m}$.

Table 4. Type of forces acting on the foundation for the case of $H=7 \mathrm{~m}$, $T=10 \mathrm{~s}, d=30 \mathrm{~m}, \mathrm{~S}=3.5 \%$ and $\mathrm{Ta}=22^{\circ} \mathrm{C}$

\begin{tabular}{lll}
\hline \hline Diameter & Inertial F. & Drag F. \\
\hline $\mathrm{D}=1 \mathrm{~m}$ & $205.42 \mathrm{~N}$ & $0.031 \mathrm{~N}$ \\
$\mathrm{D}=2 \mathrm{~m}$ & $273.90 \mathrm{~N}$ & $0.055 \mathrm{~N}$ \\
$\mathrm{D}=4 \mathrm{~m}$ & $365.20 \mathrm{~N}$ & $0.098 \mathrm{~N}$ \\
$\mathrm{D}=12 \mathrm{~m}$ & $469.55 \mathrm{~N}$ & $0.162 \mathrm{~N}$ \\
$\mathrm{D}=16 \mathrm{~m}$ & $547.80 \mathrm{~N}$ & $0.220 \mathrm{~N}$ \\
\hline
\end{tabular}

Finally, results from the Morison equation are compared against those provided by the diffraction theory for different diameters. It can be observed that for higher values of diameter, the effect of diffraction significantly increases the load dispersed.

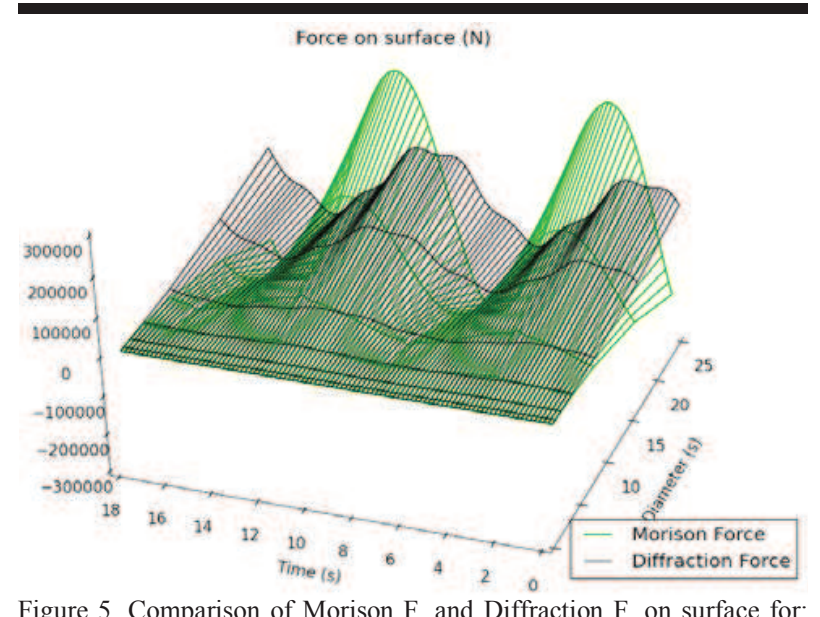

H $=7$. Morison $F$. and Diffract 


\section{DISCUSSION}

The large dispersion in the results which are shown in Table 3 has been a consequence of the choice of the range of pile diameters and the range of wave mechanic which is between $3^{\text {rd }}$ Stokes and $5^{\text {th }}$ Stokes ranges. The simulations which take into account large diameters have to be solved by means of diffraction models which consider the effects of the scatter flow in the calculation; this is the main cause that Morison formulation gives results four times higher, as shown Figure 5 for the biggest diameters. Furthermore, the nonlinear effects, which appear for some of the periods considered, have to be taken into account in the model in order to consider the real load profiles. This point is of key importance for assessing the foundation bending moment correctly, because nonlinear effects deform the distribution of loads along the whole pile, or in other words, they usually generate worse load cases than if only linear effects are considered. In addition, this dispersion is a consequence of vibration modes. For linear cases, vibration is harmonic and accumulates more energy in the pile. These three effects generate a difference in the results which shows that linear results can be four times higher than results taking all effects into account.

\section{CONCLUSIONS}

This study has shown the sensitivity of calculations with regard to the right choice of equations to characterize wave mechanics and hydrodynamic pressures. The results show a very important difference in the simplified model which is able to provide results up to five times higher than the results provided by using the suitable theory. In addition, the most important differences are caused in the diffraction range, where the simplified model considers that the scatter flow is negligible. In ranges with the smallest diameters, these differences are associated to the nonlinear effects, and mainly with drag forces, which are able to produce results up to two times higher. The smallest differences are produced for the cases of diameters of intermediate size because these calculations are mainly in the inertial range where diffraction effects and drag forces are neglected with regard to inertial forces. Hence, it has been demonstrated that the use of the Morison Equation and the Airy Theory is not valid to design large diameter offshore wind farms as used nowadays.

Furthermore, the effect of changing salinity and temperature has been presented and the conclusion drawn is that both of them have an insignificant effect in the total bending moment on the foundation. The maximum effect is found for the largest diameters and is $10.73 \%$. This result is caused by the threshold between the diffraction theory and the inertial theory. Changes in salinity and temperature influence the wave length and this produces a light movement of the threshold to the left side generating a bigger difference. As a second conclusion, the differences in salinity and temperature cannot be neglected for the case of large diameter piles.

Finally, the predominance of inertial loads on foundation scouring is observed and this is due to of the high attenuation of drag forces with depth. Drag forces have attenuation with depth of a higher order than the attenuation of inertial forces. In the following years, more research and monitoring activities are needed in this regard.

\section{LITERATURE CITED}

Chakrabarti, S.K., 1972. Nonlinear wave forces on vertical Cylinder. Journal Hydraulics Division, ASCE, 98, No. HY11, 1895-1909.

Esteban, M.D.; Diez, J.J.; López-Gutiérrez, J.S., and Negro, V., 2011. Why Offshore Wind Energy?. Renewable Energy Journal, 36, 444-450.

Faltinsen, O.M., 1990. Wave loads on offshore structures. Annual Review of Fluid Mechanics, 22, 35-56.

Feng, A.; Chen, Z., and Price, W., 2015. A desingularized Rankine source method for nonlinear wave-body interaction problems, Ocean Engineering, 101, 131-141.

Isaacson, M.; Premasirl, S., and Yang, G., 1998. Wave interaction with vertical slotted barrier. Journal of Waterway Port Coastal and Ocean Engineering, 124 (3), 118-126.

Koutandos, E.V., 2009. Hydrodynamics of vertical semiimmersed slotted barrier. WSEAS Transactions on Fluid Mechanics, 4 (3), 85-96.

Lighthill, J., 1979. Waves and hydrodynamic loading. Proceedings of the 2nd International Conference on the Behaviour of Offshore Structures, (London, United Kingdom), pp. 1-40.

MacCamy, R.C. and Fuchs, R.A., 1954. Wave forces on piles: a diffraction theory. Washington: U.S. Corps of Engineers, Beach Erosion Board, Technical Memorandum No. 69.

Mao, D.; Zhong, C.; Zhang, L., and Chu, G., 2015. Dynamic response of offshore jacket platform including foundation degradation under cyclic loadings. Ocean Engineering, 100, $35-45$.

Matutano, C.; Negro, V.; López-Gutiérrez, J.S., and Esteban, M.D., 2013. Scour Prediction and Scour Protection in Offshore Wind Farms. Renewable Energy Journal, 57, 358365.

Morison, J.R.; Johnson, J.W., and Schaaf, S.A., 1950. The force exerted by surface waves on piles. Journal of Petroleum Technology, 2(5), 149-154.

Negro, V.; López-Gutiérrez, J.S.; Esteban, M.D., and Matutano, C., 2014, Uncertainties in the design of support structures and foundation for offshore wind turbines. Renewable Energy Journal, 63, 125-132.

Takagi, M.; Arai, S.I.; Takezawa, S.; Tanaka, K., and Takarada, N., 1985. A comparison of methods for calculating the motion of a semi-submersible. Ocean Engineering, 12, 4597.

Zheng, S. and Zhang, Y., 2015. Wave diffraction from a truncated cylinder in front of a vertical wall. Ocean Engineering, 104, 329-343.

Zhu, D., 2013. Full wave solution for hydrodynamic behaviors of pile breakwater. China Ocean Engineering, 27(3), 323334. 

3. Publicaciones en revistas internacionales con revisión por pares.

3.1. Journal of Ocean Engineering and Science (Revista de Elsevier) 



\title{
Influence of temperature and salinity on hydrodynamic forces
}

\author{
A. Escobar*, V. Negro, J.S. López-Gutiérrez, M.D. Esteban \\ Technical University of Madrid, C/Profesor Aranguren S/N, 28040 Madrid, Spain
}

Received 26 June 2016; received in revised form 31 August 2016; accepted 21 September 2016

Available online 9 November 2016

\begin{abstract}
The purpose of this study is to introduce an innovative approach to offshore engineering so as to take variations in sea temperature and salinity into account in the calculation of hydrodynamic forces. With this in mind, a thorough critical analysis of the influence of sea temperature and salinity on hydrodynamic forces on piles like those used nowadays in offshore wind farms will be carried out. This influence on hydrodynamic forces occurs through a change in water density and viscosity due to temperature and salinity variation. Therefore, the aim here is to observe whether models currently used to estimate wave forces on piles are valid for different ranges of sea temperature and salinity apart from observing the limit when diffraction or nonlinear effects arise combining both effects with the magnitude of the pile diameter. Hence, specific software has been developed to simulate equations in fluid mechanics taking into account nonlinear and diffraction effects. This software enables wave produced forces on a cylinder supported on the sea bed to be calculated. The study includes observations on the calculation model's sensitivity as to a variation in the cylinder's diameter, on the one hand and, on the other, as to temperature and salinity variation. This software will enable an iterative calculation to be made for finding out the shape the pressure wave caused when a wave passes over will have for different pile diameters and water with different temperature and salinity.
\end{abstract}

(C) 2016 Shanghai Jiaotong University. Published by Elsevier B.V.

This is an open access article under the CC BY-NC-ND license (http://creativecommons.org/licenses/by-nc-nd/4.0/).

Keywords: Sea temperature; Salinity; Hydrodynamic forces; Wave action; Offshore wind farms.

\section{Introduction}

Nowadays, most standards applied in the world in the field of offshore wind farm design, have the Morison formula as a central pillar of the calculation method for predicting hydrodynamic forces on a vertical circular cylinder, using Airy's theory to assess the field of speeds and accelerations of sea waves. On the other hand, for large diameter piles, the equation proposed by MacCamy \& Fuchs is the basis for the calculation to take diffraction effects into account. This second equation also uses the linear theory to obtain the flow of speed and acceleration. In this research, special software has been developed to take all the different wave theories into account: Stokes, Cnoidal and Stream Function; to suitably characterize the field of fluid speed and accelerations whatever may be the height, period and depth of sea waves. In addition, this

\footnotetext{
* Corresponding author.

E-mail address: adrian.escobar.pastor@alumnos.upm.es (A. Escobar).
}

software allows the nonlinear and diffraction effects to be calculated for all cases: separate flow, inertial and diffraction range; and takes into account the wave-structure interaction.

The limit between the uses of each of the theories is an important issue which must be defined taking into account the temperature and salinity effect, not only the ratio between wave-length, pile diameter and the Keulegan-Carpenter number. According to available measures, extreme data regarding sea temperatures may be found in the Arctic and Southern Ocean, with sea temperatures of $1{ }^{\circ} \mathrm{C}$; and in the Persian Gulf or Gulf of Mexico, with temperatures reaching $35^{\circ} \mathrm{C}$. Regarding sea salinity, the variation is less profound except near the coastal region. River run-off introduces enough fresh water in circulation near the coast producing variable horizontal as well as vertical salinity. In the open sea, values range from 3.4 to $3.7 \%$ and reach values of $35 \%$ in some bodies of water, such as the Red Sea.

In this research, the scope of works encompasses, in first place, the assessment of the range of use of each of the theories taking into account variations in salinity and sea 
temperature. Secondly, a sensibility analysis is undertaken in order to assess whether the influence of temperature and salinity is greater for large or small pile diameters. Finally, an estimation of the percentages of diffraction and nonlinear effects caused by changes in salinity and temperature is included.

\section{State of the art}

Wind energy has begun to play an increasingly relevant role in society over the last few decades. As the development of renewable energies increased, the main concern of researchers has been to find new sources for obtaining energy and new processes enabling cleaner, less expensive and, therefore, more efficient energy to be created. With this in mind, the use of offshore wind farms has been one of the major achievements of modern science for achieving this aim as suggested by Esteban et al. [1]. The main difficulty these structures display from the construction standpoint lies in the correct estimation of hydrodynamic loads the structure will have to withstand. It could be said that calculating hydrodynamic forces in offshore structures is one of the major, key issues for design engineers participating in maritime engineering as suggested by Negro et al. [2]. In addition, they will be very important for the study of the influence of each of the forces on a possible scouring at the pile's base as suggested by Matutano et al. [3].

Calculating hydrodynamic forces is a very difficult task since environmental conditioning factors are highly complex and there are interaction phenomena between structure and incident waves. Nevertheless, even though wave action is stochastic in nature, studying wave action as if it behaved in a regular fashion and estimating the hydrodynamic forces it will generate on the structure and its resulting movements are of the utmost interest to the engineer. This method is known as the approach from the design wave. This type of analysis is completely determinist and rests on three fundamental variables, the wave height, its period and the depth. There is another approach which, by using the probabilistic theory, works with the wave energy's spectrum. Statistic design parameters, extreme forces and major dynamic responses may be predicted by using this approach.

Different calculation methods are used in predicting hydrodynamic forces and resulting responses, depending on the type and size of a structure's members in the high seas in comparison with the wave length [4-7].

Existing methods for finding hydrodynamic forces in offshore structures are mainly based on the following techniques: the use of the Morison equation or techniques using the potential of speeds for sources and sumps in two-dimensional or three-dimensional flow. The three-dimensional method is generally used from amongst these methods for structures comprising large structural elements. If a structure is made up of small elements, the Morison equation or the two-dimensional method of source-sump distribution will be required.

Morison et al. [8] suggested that the two flow regimes generated by inertia forces and drag forces may be superimposed to obtain the total load varying in time per unit of length of a cylinder immobile in a plane flow field with an arbitrary free flow speed. The wave force on circular piles, within the diffraction range, was first calculated by MacCamy and Fuchs [9], who proposed that there was an exact mathematical solution presented by wave action according to the linear wave theory. Chakrabarti [10] obtained the hydrodynamic forces caused by nonlinear waves on a vertical cylinder, including the use of the diffraction theory by means of a compact equation and then compared the equation proposed with experimental results. Chakrabarti et al. [11] proposed a general equation for the wave force equation applicable to a randomly oriented submerged or semi-submerged cylinder. Then expressions were also proposed for wave pressures and forces on several different sized vertical cylinders with different distances between them. Hydrodynamic forces due to the interaction of the different linear waves propagating with arbitrary directions were found. Lighthill [12] made a methodological analysis describing the basic physical phenomena together with the applicable methods used for estimating hydrodynamic forces. His main contribution to the calculation of wave force by using the Morison equation lies in the proposal of a second order correction thereto. Turbulent forces induced (separate flow) in wave action subjected cylinders and some methods for predicting such forces are discussed by Graham [13].

Wave forces acting on a cylinder emerging in a regular wave field were measured and compared with those of the calculations based on the Morison equation [14]. Cook and Simiu [15] also worked on hydrodynamic forces on vertical cylinders applying Lighthill's correction with the aim of including nonlinear effects in the calculation. The 17th ITFC Ocean Engineering Committee undertook to compare the methods in order to calculate the movement of a semisubmergible body. They concluded with a summary of the final project results in which 34 computer programs from 28 different organizations were later described by Takagi et al. [16].

Faltinsen [17] made a detailed analysis of wave induced linear movements and second order loads as well as nonlinear problems and viscous loads. This study concluded that the Morison equation and methods based on the theory of potential are favorably similar to each other and to field or laboratory made experimental measurements.

As computing power increased by the development of hardware and software engineering, new, more sophisticated methods have been introduced in recent years to calculate the wave-structure interaction problem. Koutandos [18] presented a numerical study on wave interaction with rigid vertical barriers and computed the velocities and turbulence kinetic energy in the vicinity of the structure. Isaacson et al. [19] and Zhu [20] investigated wave interaction with a row of piles using the full wave theory. Zheng and Zhang [21] present an analytical model to predict the three-dimensional wave diffraction of a floating cylinder located in front of a vertical wall at a finite water depth. Feng et al. [22], provide a solution for a two-dimensional nonlinear wave-structure interaction problem by a desingularized integral method in combination with 
a mixed Euler-Lagrange method. Cao and Beck [23], present the derivations of different forms of integral desingularized boundary equations and discuss their fundamental aspects and advantages for studying water wave dynamics and body motion dynamics. Meng and Zhang [24] develop a third-order $\mathrm{KdV}$ solution for internal solitary waves by means of a new method based on weakly nonlinear assumptions in a rigidlid two-layer system. Das [25] provides the solution to wave scattering by a horizontal circular cylinder in a three-layer fluid.

According to the current state of the art in this field, this research can be seen to represents the first investigation focused on the influence of temperature and salinity on hydrodynamic forces.

\section{Method}

The different forces acting must be broken down in order to study the wave regime affecting a fixed cylindrical body in a water domain. To do this, the forces waves exert on such a body may be classed into:

a. Drag forces (also called the viscous type).

a.1 "Form drag" $\bar{F}_{p}$ associated with normal tensions.

a.2 "Friction drag" $\bar{F}_{f}$ associated with surface friction.

b. Forces of inertia.

According to the undulatory theory, these forces originate from the speed potential, $\phi$ :

$\varphi=\varphi_{I}+\varphi_{D}$

$\phi_{I}$ Incident waves.

$\phi_{D}$ Wave diffraction.

When the structure's diameter is much smaller than the wave length, $D<<L$, incident waves are not significantly modified by the presence of the body (where $D$ is the cylinder's diameter and $L$ the wave length); the diffraction component, $\varphi_{D}$ can therefore be ignored. The force due to continuous current flow acting on a cylinder is:

$F_{D}=\frac{1}{2} \cdot \rho \cdot C_{D} \cdot D \cdot u \cdot|u|$

where, $F_{D}$ is the force per unit of length of the cylinder, $\rho$ the fluid's density and $C_{D}$ the coefficient of drag. The speed squared is written in the form $u \cdot|u|$ to ensure that the direction of the drag force is the same as the speed's flow. However, wave action is an oscillatory current and, in this case, there will be two additional terms contributing to the whole of the force.

$F=\frac{1}{2} \cdot \rho \cdot C_{D} \cdot D \cdot u \cdot|u|+m^{\prime} \cdot a+\rho \cdot V \cdot a$

where $m$ ' $a$, is called the "force of the additional mass mobilized" (hydrodynamic-mass force), $m$ ' is the additional mass mobilized or hydrodynamic mass, $\rho \cdot V \cdot a$, is called the "Froude-Krylov force", and $V$ is the cylinder's volume which, if we take a unit of length of the cylinder, is reduced to $A$, the cross section area. The additional mass mobilized is defined as the mass of fluid around the body which is accelerated with the body's movement due to the action of pressure.

There are two effects when the body remains immobile and the water moves with an acceleration $a$. First, the water surrounding the body will accelerate as described. The force of the hydrodynamic mass will therefore be present. The second effect will be the fluid's accelerated movement in the external region of the flow generating a pressure gradient according to:

$\frac{\partial p}{\partial x}=-\rho \cdot \frac{d u}{d t}$

where $u$ is the speed of the fluid far from the cylinder. This pressure gradient will produce an additional force on the cylinder, which we call the Froude-Krylov force. This force may be calculated by the following integration:

$F_{p}=-\int_{S} p \cdot d S$

where $S$, is the body's surface area. From the OstrogadskiGauss theorem, we have:

$F_{p}=-\int_{V} \frac{\partial p}{\partial x} \cdot d V=\int_{V} \rho \cdot \frac{d u}{d t} \cdot d V$

If we bear in mind that the pressure gradient is constant, the force per unit of length, $F_{p}$, for a cylinder with a cross section area, $A$, is:

$F_{p}=\rho \cdot A \cdot a$

The total force, $F$, is given by the foregoing expressions bearing in mind the force of the hydrodynamic mass and the Froude-Krylov force:

$F=\frac{1}{2} \cdot \rho \cdot C_{D} \cdot D \cdot u \cdot|u|+\rho \cdot C_{m} \cdot A \cdot a+\rho \cdot A \cdot a$

Knowing that $C_{M}=C_{m}+1$, the classic expression of Morison, Johnson, Schaaf \& O'Brien is given by:

$$
\begin{aligned}
F= & \frac{1}{2} \cdot \rho \cdot C_{D} \cdot u \cdot|u|+\rho \cdot C_{M} \cdot A \cdot a=\rho \cdot \frac{D}{2} \cdot C_{D} \cdot u \cdot|u| \\
& +\rho \cdot \frac{\pi \cdot D^{2}}{4} \cdot C_{M} \cdot a
\end{aligned}
$$

where:

- $D$ : pile diameter

- A: cross section area of the vertical cylinder

- $C_{M}$ : coefficient of inertia due to the hydrodynamic mass

- $C_{D}$ : drag coefficient.

Morison proposed that inertial forces should be superimposed on drag forces in order to calculate the hydrodynamic forces on the cylinder.

The coefficients $C_{M}$ and $C_{D}$ are found empirically and basically depend on the Reynolds number, $R_{E}$, the KeuleganCarpenter number and the cylinder's surface roughness. A multitude of studies has been carried out by many authors in 
order to establish $C_{M}$. The criterion proposed by the Shore Protection Manual for establishing the coefficient $C_{M}$ will be used as from the different studies shown in the foregoing table. In addition, the criterion used by the Shore Protection Manual is also taken into account to find the coefficient $C_{D}$, as from the studies of Achenbach (1968).

$C_{M}=2.0 \quad$ when $R_{E}<2.5 \cdot 10^{5}$

$C_{M}=2.5-R_{E} / 5 \cdot 10^{5} \quad$ when $2.5 \cdot 10^{5}<R_{E}<5 \cdot 10^{5}$

$C_{M}=1.5 \quad$ when $R_{E}>5 \cdot 10^{5}$

$C_{D}=1.2 \quad$ when $R_{E}<1 \cdot 10^{5}$

$C_{D}=1.2-\left(1.2-0.7 / 3 \cdot 10^{5}\right) \cdot\left(R_{E}-10^{5}\right)$ when $1 \cdot 10^{5}<R_{E}<4 \cdot 10^{5}$

$C_{D}=0.7 \quad$ when $R_{E}>4 \cdot 10^{5}$.

The Reynolds number is calculated as:

$R_{E}=\frac{u \cdot D}{v}$

Variations in the sea temperature and salinity will change viscosity, $v$, and water density, $\rho$. Hence, they will influence the total force generated because of the variation in density and, therefore will also influence the percentage of each type of force because they influence the Reynolds number, so affecting the $C_{M}$ and $C_{D}$ coefficients. Water viscosity and density are obtained in the calculation by relations established by Chakrabarti [26] from values of water temperature and salinity.

On the other hand, the diffraction equations are included in the problem statement, by taking into account the cylindrical coordinates:

$\Delta \varphi_{I}=\frac{\partial^{2} \varphi_{I}}{\partial r^{2}}+\frac{1}{r} \frac{\partial \varphi_{I}}{\partial r}+\frac{1}{r^{2}} \frac{\partial^{2} \varphi_{I}}{\partial \theta^{2}}+\frac{\partial^{2} \varphi_{I}}{\partial z^{2}}=0, \quad$ in $\Omega$

$\frac{\partial \varphi_{D}}{\partial r}=-\frac{\partial \varphi_{I}}{\partial r}, \quad$ when $r=b$

$\frac{\partial \varphi_{I}}{\partial z}=0, \quad$ when $z=0$

$\frac{\partial^{2} \varphi_{I}}{\partial t^{2}}+g \frac{\partial \varphi_{I}}{\partial z}=0, \quad$ when $z=d$.

If the Sommerfeld condition is shown as a complex expression:

$\lim _{r \rightarrow \infty} \sqrt{r}\left(\frac{\partial \varphi_{D}}{\partial r}-i k \varphi_{D}\right)=0$
With $D / 2=b$, the velocity potential can be obtained in terms of the first type of the Bessel function $J_{n}$ and Hankel functions of the first type $H_{n}$ :

$\varphi_{I}=\operatorname{Re}\left[-\frac{g H}{2 \omega} \frac{\cosh k z}{\cosh k d} \sum_{n=0}^{\infty} \varepsilon_{n} i^{n} J_{n}(k r) \cos n \theta e^{-i \omega t}\right]$

$\varphi_{D}=\operatorname{Re}\left[+\frac{g H}{2 \omega} \frac{\cosh k z}{\cosh k d} \sum_{n=0}^{\infty} \varepsilon_{n} i^{n} \frac{J_{n}^{\prime}(k b)}{H_{n}^{\prime}(k b)} H_{n}(k r) \cos n \theta e^{-i \omega t}\right]$

The velocity potential can be expressed as:

$\varphi=\varphi_{I}+\varphi_{D}=\operatorname{Re}\left[-\frac{g H}{2 \omega} \frac{\cosh k z}{\cosh k d}\right.$
$\left.\sum_{n=0}^{\infty} \varepsilon_{n} i^{n}\left[J_{n}(k r)-\frac{J_{n}^{\prime}(k b)}{H_{n}^{\prime}(k b)} H_{n}(k r)\right] \cos n \theta e^{-i \omega t}\right]$

From the above expression, the Froude-Krylov force exerted by the waves on the cylinder is obtained by the following expression:

$$
\begin{aligned}
& F=\frac{2 \pi}{k} \rho g \frac{H D}{4} \tanh k d R e \\
& {\left[-\left(J_{1}(k b)-\frac{J_{1}^{\prime}(k b)}{H_{1}^{\prime}(k b)} H_{1}(k b)\right) e^{-i \omega t}\right]}
\end{aligned}
$$

\section{Calculation model}

The methodology developed to suitably solve the problem must follow the following scheme (Figs. 1 and 2).

In the first place, the software takes the environmental conditions: wave period $(T)$ and height $(H)$, and sea depth $(d)$; as first order variables which define the wave mechanics to handle in the calculation as the basis of the whole assessment. Moreover, this section takes the influence of temperature $(T a)$ and salinity $(S)$ on the water density $(\rho)$ into account as a second order effect. In this step of the calculation, the range of the problem (deep water, intermediate depth and shallow water) has to be identified and the equations which define the water wave motion solved (Euler equations of motion and continuity equation).

$$
\begin{aligned}
& \frac{\partial u_{i}}{\partial t}+u_{j} \frac{\partial u_{i}}{\partial x_{j}}+w \frac{\partial u_{i}}{\partial z}=-\frac{1}{\rho} \frac{\partial p}{\partial x_{i}} \\
& \frac{\partial w}{\partial t}+u_{j} \frac{\partial w}{\partial x_{j}}+w \frac{\partial w}{\partial z}=-g-\frac{1}{\rho} \frac{\partial p}{\partial z} \\
& \frac{\partial u_{j}}{\partial x_{j}}+\frac{\partial w}{\partial z}=0
\end{aligned}
$$

To solve the system, the software will use the pertinent wave theory (such as Airy, Stokes, Cnoidal or Stream Function) according to the classification of Le Méhauté shown in Fig. 3.

In second place, the software assesses the hydrodynamic loads on the basis of flow speeds $(v)$ and accelerations $(a)$ 


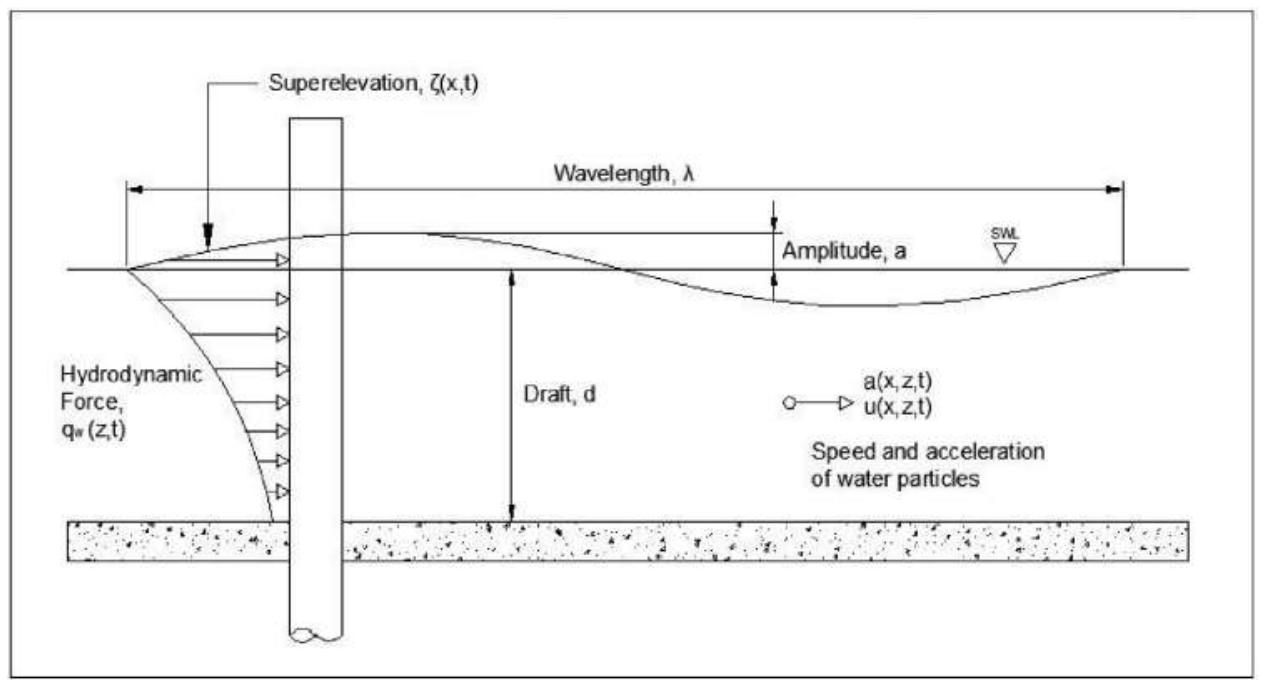

Fig. 1. Diagram of wave action on a pile.

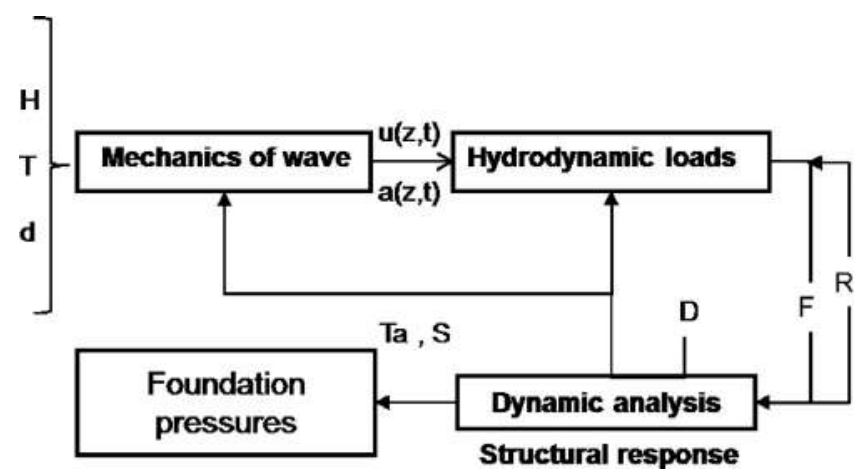

Fig. 2. Calculation scheme.

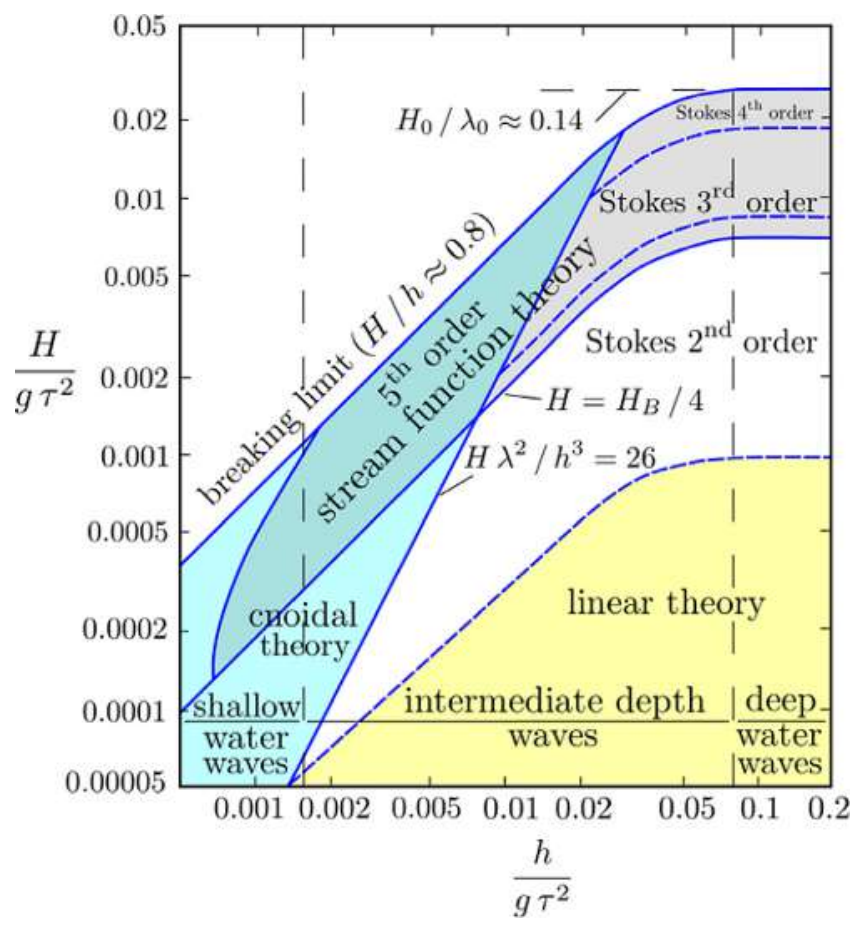

Fig. 3. Wave theories classification by Le Méhauté. obtained in the last step. This problem has the diameter of the pile $(D)$ as a first order variable, In conjunction with wave mechanics the pile diameter defines the range (separate flow, inertial or diffraction) in which the problem must be solved. Sea temperature and salinity are included again because they play a key role in this part because of their influence on water viscosity and hence on drag forces. The assessment of hydrodynamic loads will provide the solution for one of the ranges: separate flow, inertial and diffraction; taking into account nonlinear effects, as appropriate. The range is identified by three parameters: Keulegan-Carpenter number, ratio $H / L$ and ratio $D / L$ :

The separate flow range is solved by means of Morison's equation. Furthermore, nonlinear effects of the second order terms of the wave motion will be introduced analytically in accordance with the corrections provided by Lighthill (as $F_{3}$, $F_{4}$ and $F_{5}$ ) for the cases in which the problem is located in the separate nonlinear flow range:

$F(z, t)=F_{1}+F_{2}+F_{3}+F_{4}+F_{5}$

$F_{1}=-\rho \int_{\partial \mathbf{\Omega}} \frac{\partial \varphi_{1}}{\partial t} n d s=\frac{1}{4} C_{M} \rho \pi D^{2} \cdot a(z, t) ;$

$F_{2}=\frac{1}{2} C_{D} \rho D \cdot u(z, t) \cdot|u(z, t)|$

$F_{3}=-\rho \int_{z=d} w\left(\nabla \varphi_{1}\right)^{2} d s ; \quad F_{4}=\frac{1}{2} \rho \int_{S}\left(\nabla \varphi_{1}\right)^{2} \cos \theta d s ;$

$F_{5}=\frac{\rho}{2 g} \oint_{C}\left(\frac{\partial \varphi_{l}}{\partial t}\right)^{2} \cos \theta d l$

The inertial range uses the same equation, shown above for the separate flow range, but removes the term $F_{2}$ corresponding to drag force (Table.1). On the other hand, when 
Table 1

Range classification.

\begin{tabular}{|c|c|c|c|c|}
\hline & $\mathrm{KC}<4 H / L<0.5 H / L_{\max }$ & $\mathrm{KC}<4 H / L>0.5 H / L_{\max }$ & $\mathrm{KC}>4 H / L<0.5 H / L_{\max }$ & $\mathrm{KC}>4 H / L>0.5 H / L_{\max }$ \\
\hline$D / L<0,2$ & Linear inertial range & Nonlinear inertial range & Linear separate flow range & Nonlinear separate flow range \\
\hline$D / L>0,2$ & Linear diffraction range & Nonlinear diffraction range & - & - \\
\hline
\end{tabular}

the assumption that the kinematics of the undisturbed flow in the region near the structure do not change in the incident wave direction is not fulfilled, the diffraction theory has to be used. The theory provided by MacCamy and Fuchs is used as a basis $\left(F_{1}\right)$ but adding additional terms to include nonlinear effects in the calculation $\left(F_{2}\right)$, using the perturbation parameter $(\varepsilon)$.

$F(z, t)=\varepsilon F_{1}+\varepsilon^{2} F_{2}$

$F_{1}(z, t)=-D \rho \int_{0}^{2 \pi}\left(\frac{\partial \varphi^{(1)}}{\partial t}\right)_{r=D} \cos (\pi-\theta) d \theta$

$F_{2}(z, t)=-D \rho \int_{0}^{2 \pi}\left[\int_{0}^{\eta^{(1)}}\left(\frac{\partial \varphi^{(1)}}{\partial t}+g z\right) d z+\frac{\partial \varphi^{(2)}}{\partial t}\right.$

$\left.+\frac{1}{2}\left(\frac{\partial \varphi^{(1)}}{\partial z}\right)^{2}+\frac{1}{2 D^{2}}\left(\frac{\partial \varphi^{(1)}}{\partial \theta}\right)^{2}\right]_{r=D} \cos (\pi-\theta) d \theta$

Finally, the third step takes the sea wave loads obtained and assesses their effect on the foundation and on the movements of the structure along the vertical axis. Finally, the structure movements and the loads generated by them feed the second section again and allow the calculation to be iteratively made again until a final solution is achieved. The structural response analysis is performed in the field of elastic deformation. The vertical circular cylinder is assumed to be a cantilever beam fixed in the sea bed. The calculations must apply a dynamic assessment in the frequency domain, which takes the inertial effects into account. The differential equation which defines the behavior of the cantilever beam is:

$E I \frac{\partial^{4} x(z, t)}{\partial z^{4}}+m \frac{\partial^{2} x(z, t)}{\partial t^{2}}=0$

where, $E I$ is the beam stiffness and $m$ the beam mass. The above equation is best solved by separating variables. If it is assumed that the displacement can be separated into two parts, one depends on position and the other on time, which are both independent of each other, so that the equation can now be written as two differential equations:

$x(z, t)=Z \cdot T ; \quad \frac{\partial^{4} Z}{\partial z^{4}}-k_{n}^{4} Z=0 ; \quad \frac{\partial^{2} T}{\partial t^{2}}+\omega_{n}^{2} T=0$

where $\omega_{n}$ represents the natural frequencies of the beam, and $k_{n}$ is as follows:

$k_{n}^{4}=\frac{w_{n}^{2} m}{E I}$
The solution of the system is given by:

$x(z, t)=\sum_{m=1}^{\infty} Z_{n}(z)\left[A_{n} \cos \left(\omega_{n} t\right)+B_{n} \sin \left(\omega_{n} t\right)\right]$.

$A_{n}$ depends on the initial position at time $t=0, B_{n}$ depends on the initial velocity, and both of them must be determined with the boundary conditions.

$A_{n}=\frac{\int_{0}^{d} x_{0}(z) Z_{n}(z) d z}{\int_{0}^{d} Z_{n}^{2}(z) d z} ; \quad B_{n}=\frac{1}{\omega_{n}} \frac{\int_{0}^{d} v_{0}(z) Z_{n}(z) d z}{\int_{0}^{d} Z_{n}^{2}(z) d z}$

Then, the load $F(z, t)$ is introduced into the differential equations as a boundary condition to obtain $A_{n, \mathrm{i}+1}$ and $B_{n, \mathrm{i}+1}$, for the next time step. For each time step, the speed and acceleration of the following time step can be obtained by the following equation

$\begin{aligned} A_{n, i+1} & =\frac{1}{\omega_{n}^{2} \rho_{e s t} A} \frac{\int_{0}^{d} \Delta F(z) Z_{n}(z) d z}{\int_{0}^{d} Z_{n}^{2}(z) d z} ; \\ B_{n, i+1} & =\frac{1}{\omega_{n}} \frac{\int_{0}^{d} v_{i}(z) Z_{n}(z) d z}{\int_{0}^{d} Z_{n}^{2}(z) d z}\end{aligned}$

$\Delta F(z)=F\left(z, t=t_{i+1}\right)-R\left(z, t=t_{i}\right)$

where:

- $F\left(z, t=t_{i+1}\right)$ : hydrodynamic forces in $t i+1$.

- $R\left(z, t=t_{i}\right)$ : structural response in $t i$.

- $\rho_{\text {est }}$ : density of structural element.

- $A$ : area of structural element.

The function $Z_{n}$ and the modes of vibration $k_{n}$ are determined by the following equations:

$$
\begin{aligned}
& Z_{n}(z)=\frac{1}{2}\left\{\left[\cos \left(k_{n} z\right)-\cosh \left(k_{n} z\right)\right]\right. \\
& \left.\quad+\left[\frac{-\cos \left(k_{n} d\right)-\cosh \left(k_{n} d\right)}{\sin \left(k_{n} d\right)-\sinh \left(k_{n} d\right)}\right]\left[\sin \left(k_{n} z\right)-\sinh \left(k_{n} z\right)\right]\right\}
\end{aligned}
$$

$\cos \left(k_{n} d\right) \cosh \left(k_{n} d\right)=-1$

Using this kind of analysis, the speed and acceleration of each point of the structure can be obtained as a response of the hydrodynamic loads to each time step. These speeds and accelerations can be subtracted from the initial $v(z, t)$ and $a(z, t)$, and the process repeated until its convergence for each fraction of time. The final result is a full record of the movements of the structure along the whole length originated by sea waves. Finally, the bending moment on the foundation can be easily obtained from these structural movements. 


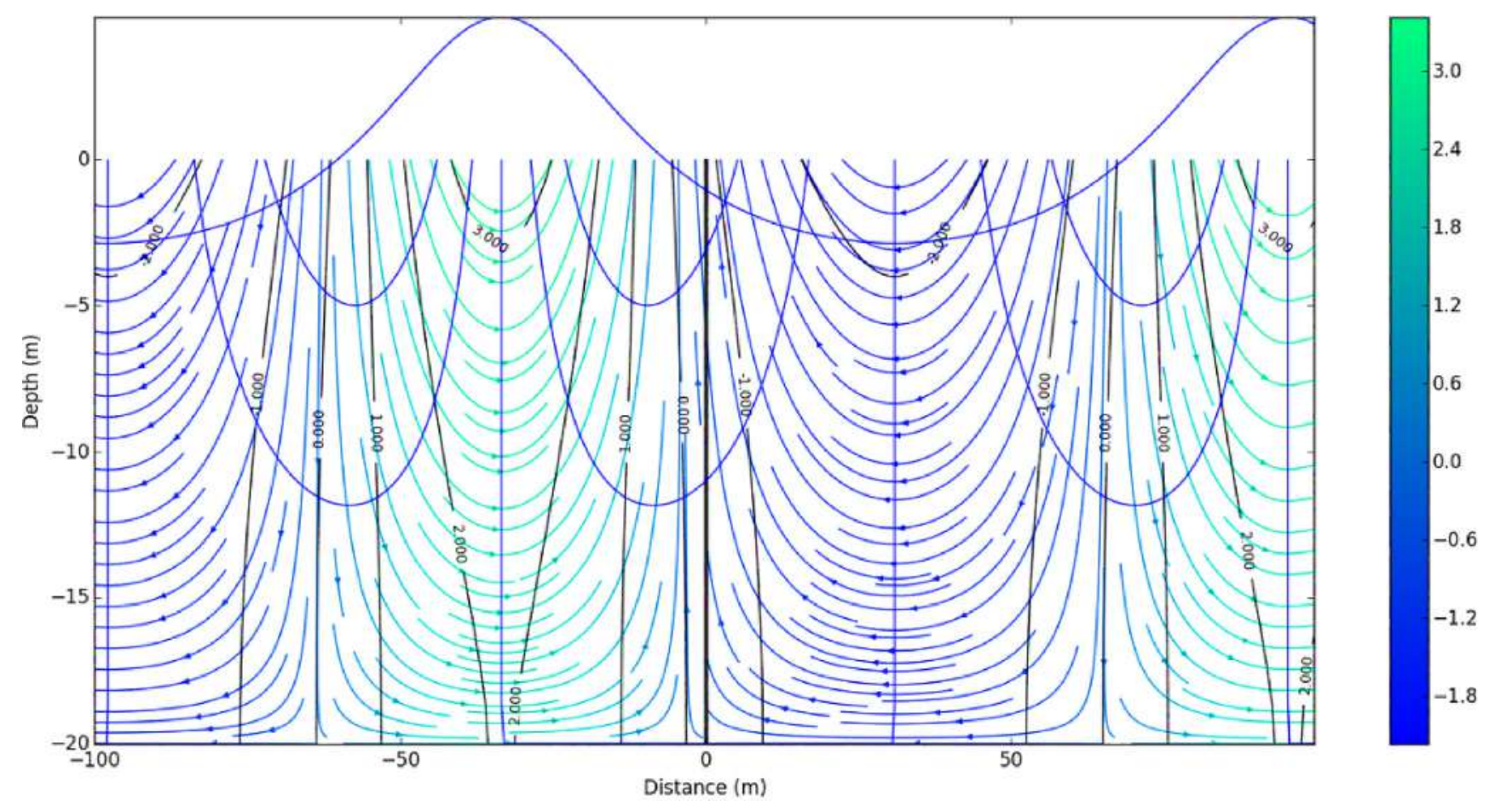

Fig. 4. Field of sea wave speeds.

\section{Results}

Seven cases were defined for undertaking the study and only the cylinder's diameter, the sea's temperature and salinity varies in them whilst waves are taken to be regular. Case 1 will address a diameter of $0.5 \mathrm{~m}$, case 2 , a diameter of $2 \mathrm{~m}$, case 3 , a diameter of $4 \mathrm{~m}$, case 4 , a diameter of $8 \mathrm{~m}$, case 5 , a diameter of $16 \mathrm{~m}$, case 6 , a diameter of $20 \mathrm{~m}$, and case 7 , a diameter of $26 \mathrm{~m}$. All these diameters have been chosen according to the standard types of offshore wind farms, from the first models with $0.5 \mathrm{~m}$ to those used nowadays as in: Scarweather Sands (United Kingdom) with $2.2 \mathrm{~m}$, Princess Amalia (Netherlands) with $4.0 \mathrm{~m}$ and, Nysted (Denmark) or Thornton Bank (Belgium) with diameters from $6.5 \mathrm{~m}$ up to $17 \mathrm{~m}$; the values of 20 and $26 \mathrm{~m}$ have been included to take into account the future piles due to the fact that, maybe in the next few years, pile diameters will keep increasing to support a heavier and more powerful wind turbine in keeping with the requirements of the offshore industry (Figs. 4-Fig. 12).

The flow of speeds and accelerations has been calculated for them all, taking into account the possible diffraction and nonlinear effects for two sub-cases. Case A will have a sea temperature of $1{ }^{\circ} \mathrm{C}$ and $0 \%$ salinity, and case B will be performed with a sea temperature of $22^{\circ} \mathrm{C}$ and salinity of $35 \%$. The common details for regular sea waves to be entered in all the study cases have been obtained from those used in the design of Princess Amalia (Netherlands):

- Wave height: $7.5 \mathrm{~m}$.

- Depth: $20.0 \mathrm{~m}$.

- Period: $10 \mathrm{~s}$.
According to this data, the wave theory will be Stokes' 3rd order in transition depth.

$$
\begin{aligned}
& \delta_{1}=\frac{H}{g T^{2}}=0.00764 \quad \delta_{2}=\frac{h}{g T^{2}}=0.02038 \\
& \frac{d}{L}=\frac{20}{128.7} \rightarrow 0.4<0.155<0.5 \rightarrow \text { Transition depth }
\end{aligned}
$$

The field of accelerations and speeds for the case of height $7.5 \mathrm{~m}$, depth $20.0 \mathrm{~m}$ and period $10 \mathrm{~s}$, calculated by the software developed is shown below:

According to those values, the force for the case of a sea temperature of $1{ }^{\circ} \mathrm{C}$ and $0 \%$ salinity and for the case of a sea temperature of $22{ }^{\circ} \mathrm{C}$ and salinity of $35 \%$ will calculated, as, respectively, $F_{1}$ and $F_{2}$. The following figures show the percentages of $\left|F_{i 1}-F_{i 2}\right| / F_{t 2}$, where $F_{i 1}$ and $F_{i 2}$ are the different components of the total force: inertial forces, drag forces, diffraction forces and nonlinear forces; and $F_{t 2}$, the total forces obtained in the second case. All this is for the different time steps throughout the whole time period of $10 \mathrm{~s}$, for the sea surface and for the bottom.

According to the values shown above, the maximum variations for all cases are summarized below:

The variation suffered by the drag forces can initially be seen to drastically reduce on the pile diameter increasing whilst the variation of inertial force is increasing enormously and becomes the predominant variation. The influence of temperature and salinity is shown to be clearly higher on the bottom than on the free surface for the cases with diameters: 0.5 , 8.0, 16.0, 20.0 and 26.0. For those cases with diameter 2.0 and 4.0, there is almost the same influences on the bottom and the surface. 


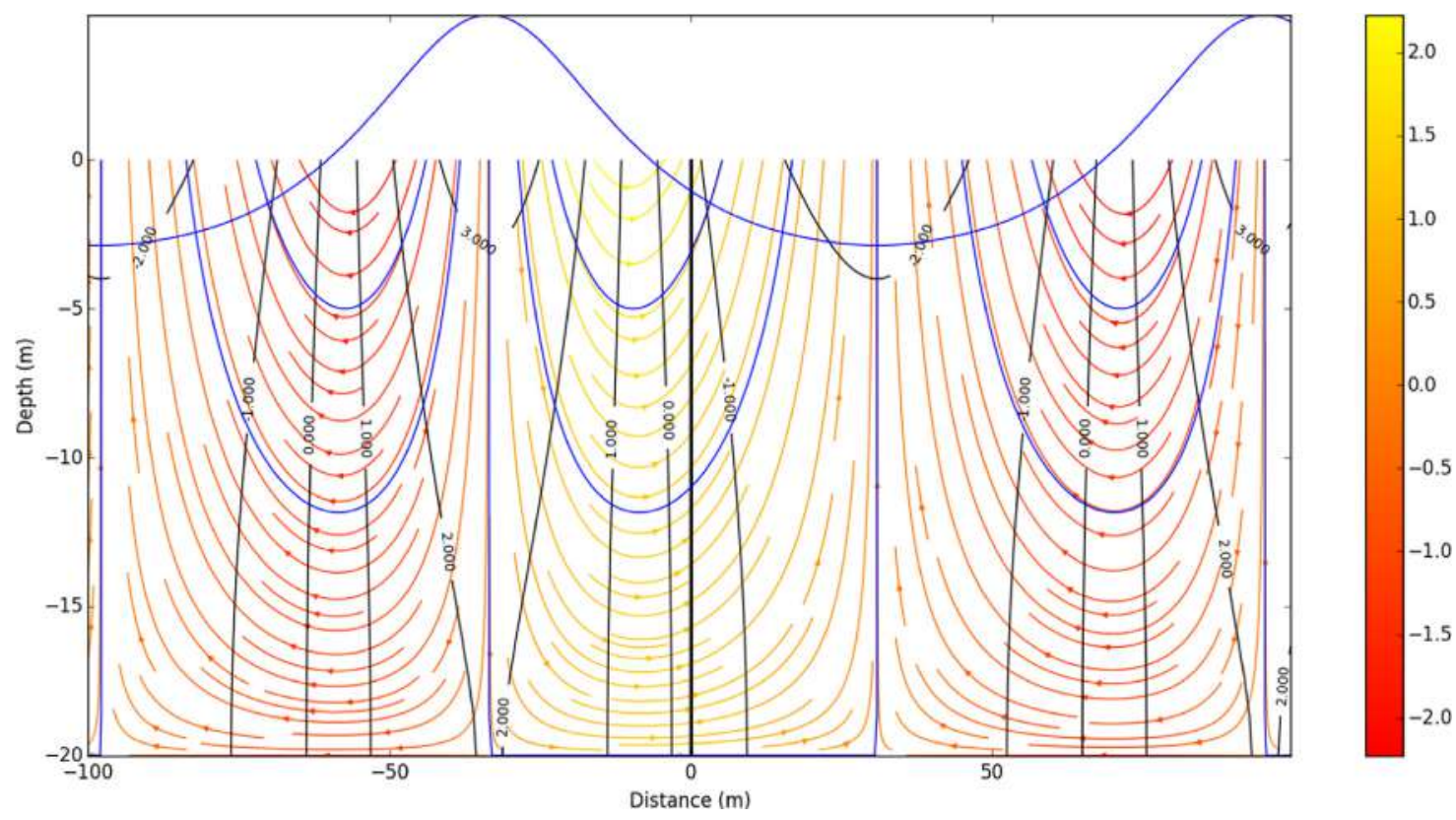

Fig. 5. Field of sea wave accelerations.

\section{Case 1: Diameter $=0.5 \mathrm{~m}$}

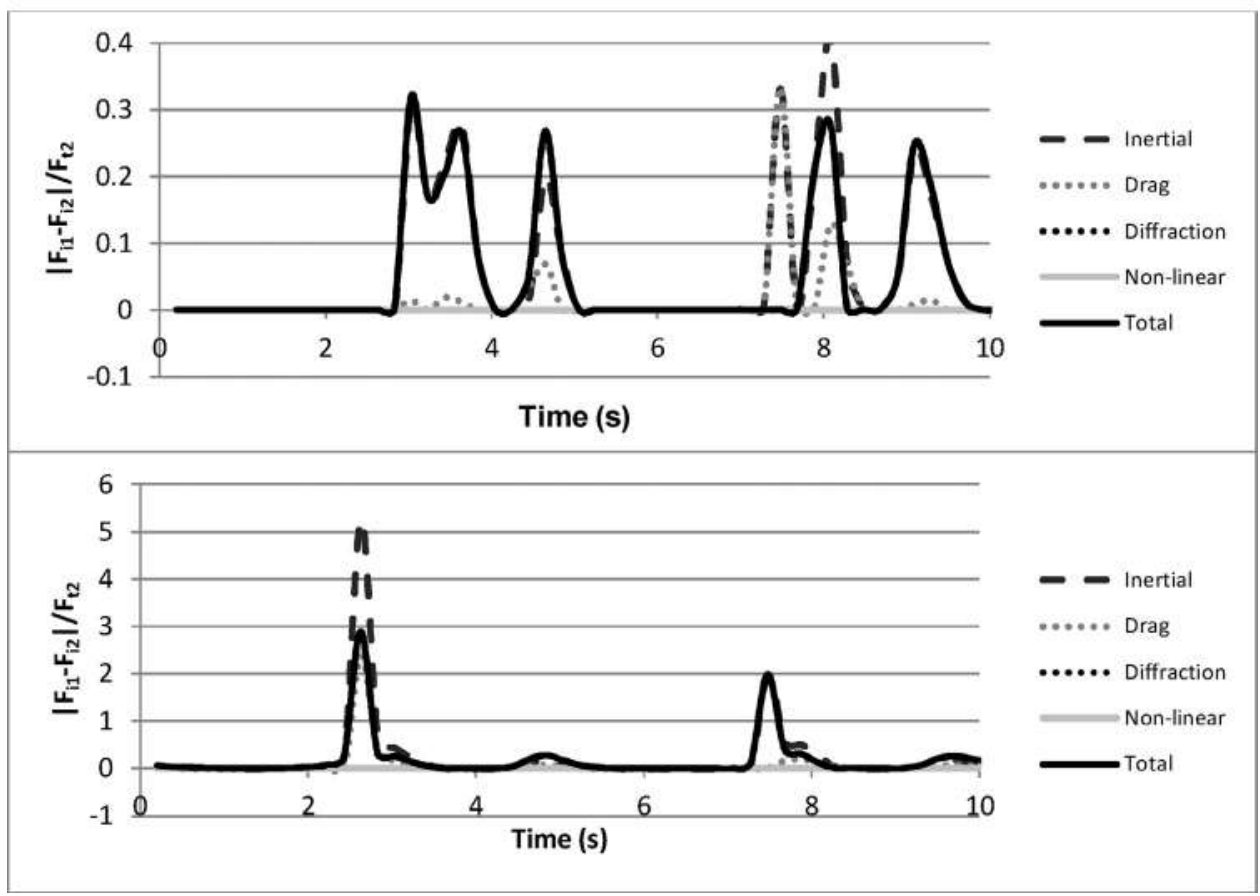

Fig. 6. Simulation with $D=0.5 \mathrm{~m}$, on a free surface in the figure above, and at the sea bed in the figure below

The reason for this is that the inertial force is discontinuous for the largest diameters. This manifests as a leap or step in the wave crest. This leap gradually disappears as the diameter increases and is caused by a change in the coefficients $C_{D}$ and $C_{M}$. As explained earlier, these coefficients vary with the Reynolds number and as the dynamic viscosity and diameter remain constant during the calculation of one case, the leap is indicating that at that point, speed is varying enough for the Reynolds number to become less than $2.5 \times 10^{5}$, making $C_{M}$ 2.0.

There is a different reason for the $0.5 \mathrm{~m}$ diameter as drag forces which generate a large variation if the load profile for changes in temperature and salinity are heavily influential.

The diameters of $2.0 \mathrm{~m}$ and $4.0 \mathrm{~m}$ are cases in the transition between the end of the range of separate flow and the beginning of the inertial range, so the effect generated by 
Case 2: Diameter $=\mathbf{2} .0 \mathrm{~m}$

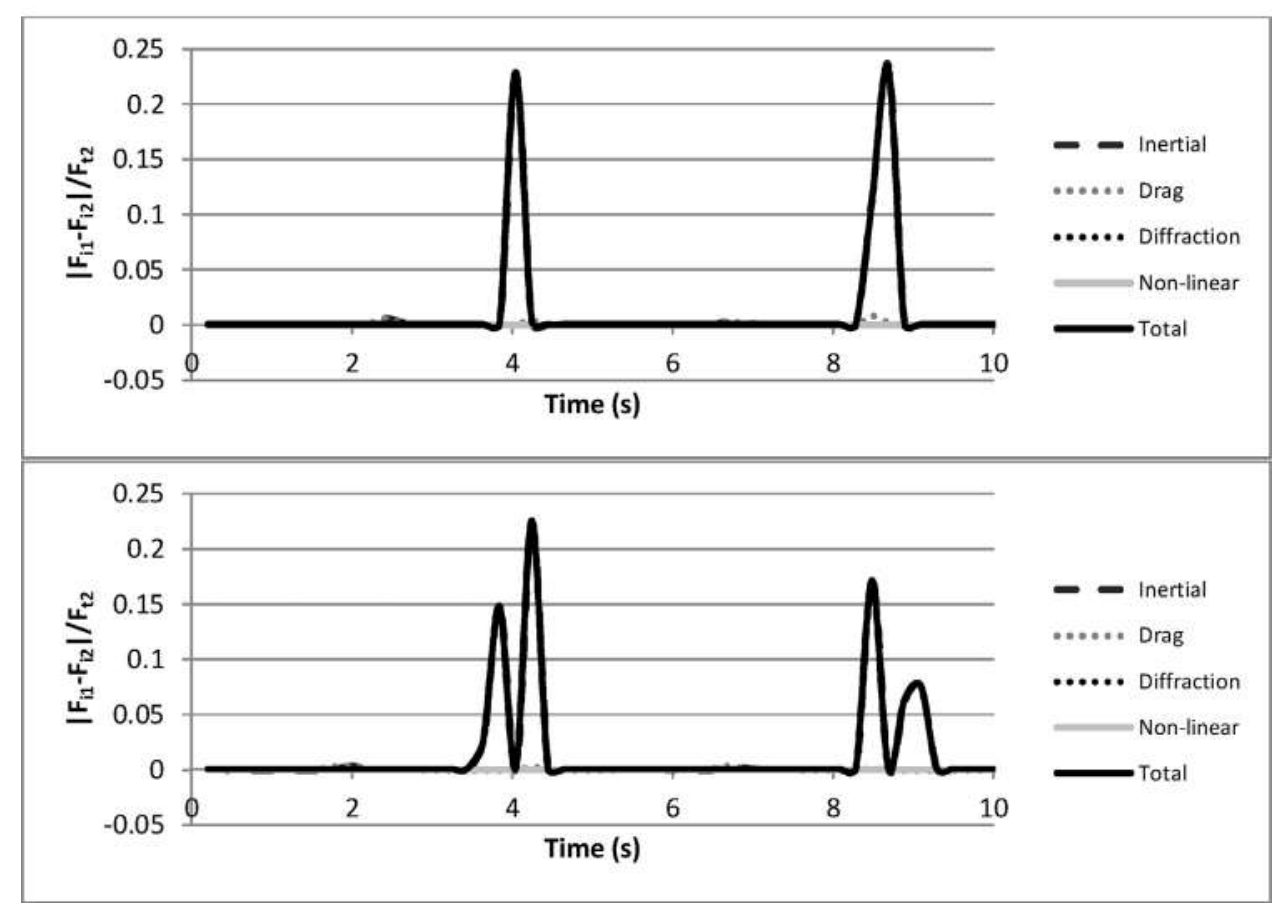

Fig. 7. Simulation with $D=2.0 \mathrm{~m}$, on a free surface in the figure above, and at the sea bed in the figure below.

\section{Case 3: Diameter $=4.0 \mathrm{~m}$}

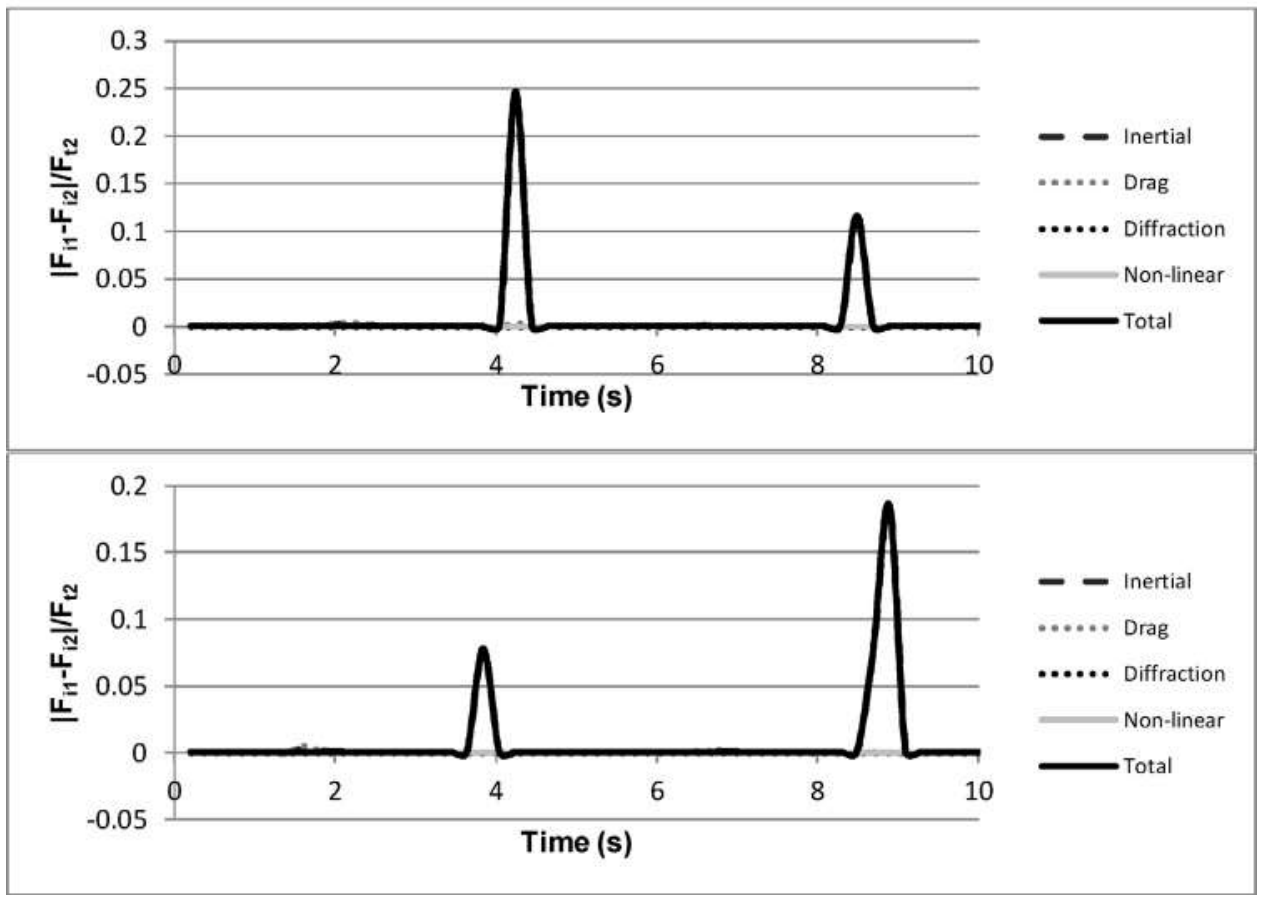

Fig. 8. Simulation with $D=4.0 \mathrm{~m}$, on a free surface in the figure above, and at the sea bed in the figure below.

drag forces is disappearing but the effect of discontinuity in inertial forces influences the whole depth.

The higher the temperature values, the greater is the wave deformation observed. Discontinuities can also be observed in the case with a sea temperature of $22^{\circ} \mathrm{C}$ and salinity of
$35 \%$ showing a narrower width than those appearing in the case of a sea temperature of $1{ }^{\circ} \mathrm{C}$ and salinity of $0.0 \%$, so the temperature increase could be assimilated to a virtual pile diameter increase in terms of the shape of the load profile of the wave. In addition, the width this discontinuity shows can 
Case 4: Diameter $=8.0 \mathrm{~m}$

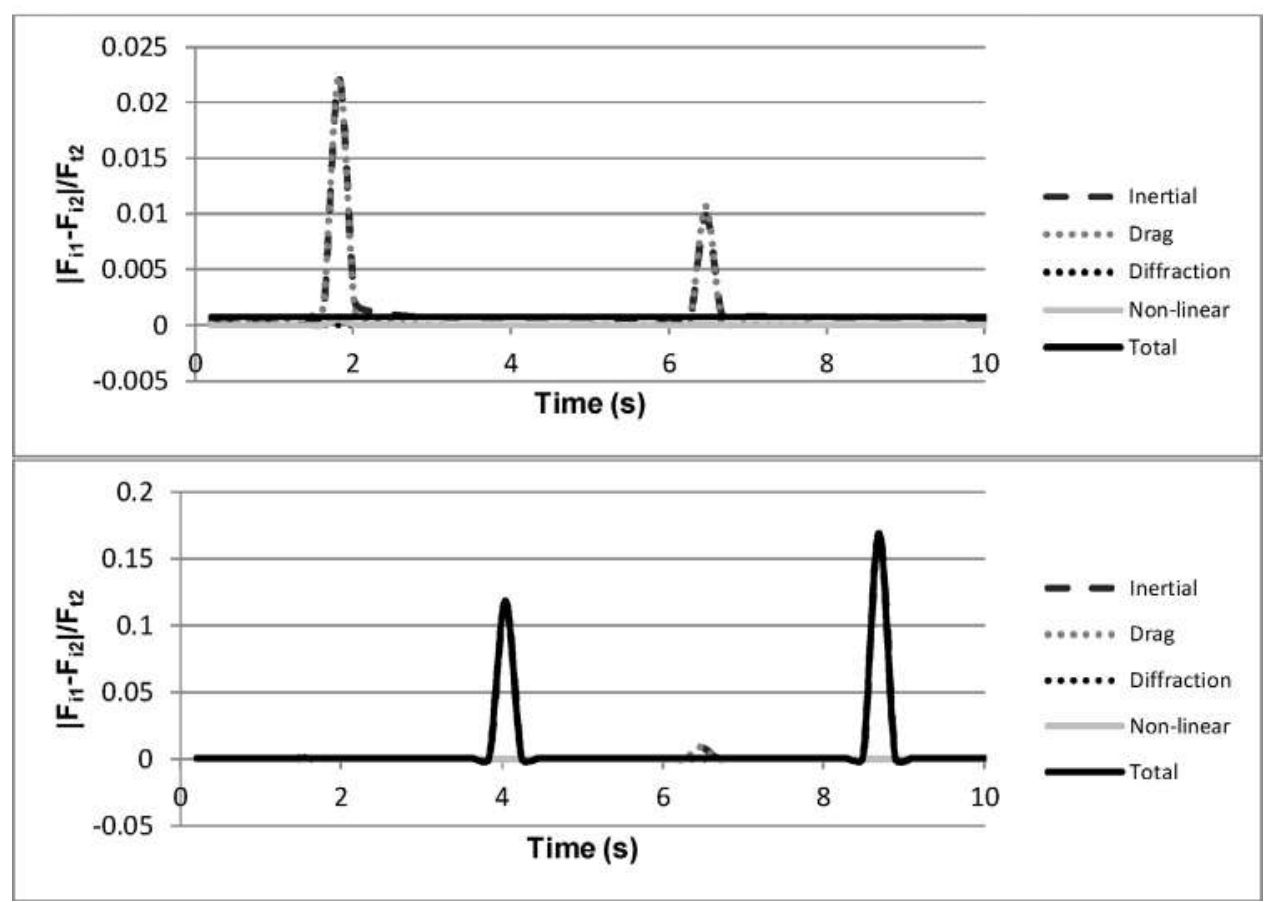

Fig. 9. Simulation with $D=8.0 \mathrm{~m}$, on a free surface in the figure above, and at the sea bed in the figure below.

\section{Case 5: Diameter $=16.0 \mathrm{~m}$}

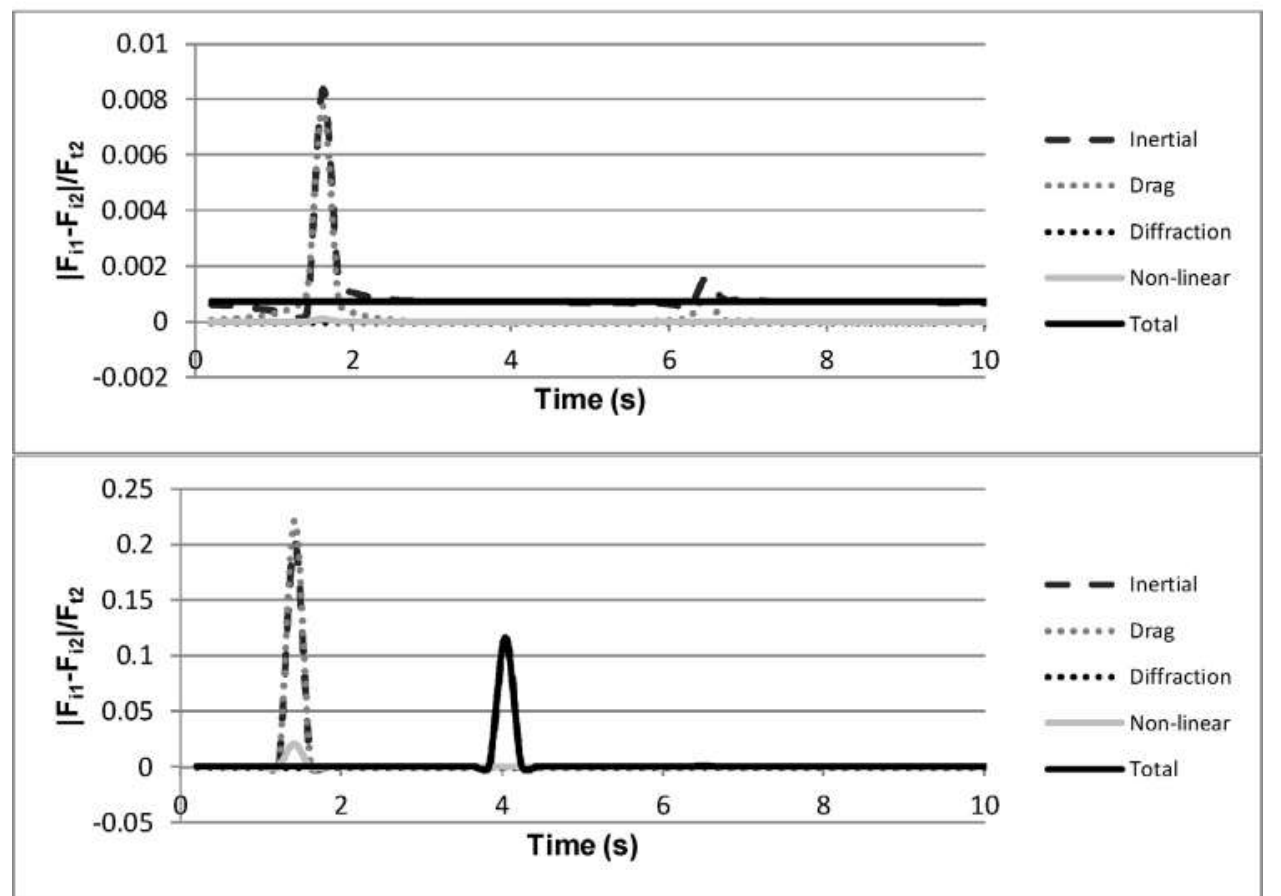

Fig. 10. Simulation with $D=16.0 \mathrm{~m}$, on a free surface in the figure above, and at the sea bed in the figure below.

also be observed to be always narrower at the surface than at the sea bed.

All of the results shown above demonstrate the major differences generated in the load profile of sea waves after the temperature and salinity change. However, the maximum influence has to be demonstrated by using the ratio between the maximum total forces generated in both temperature cases for each diameter, in order to obtain the actual influence in terms 


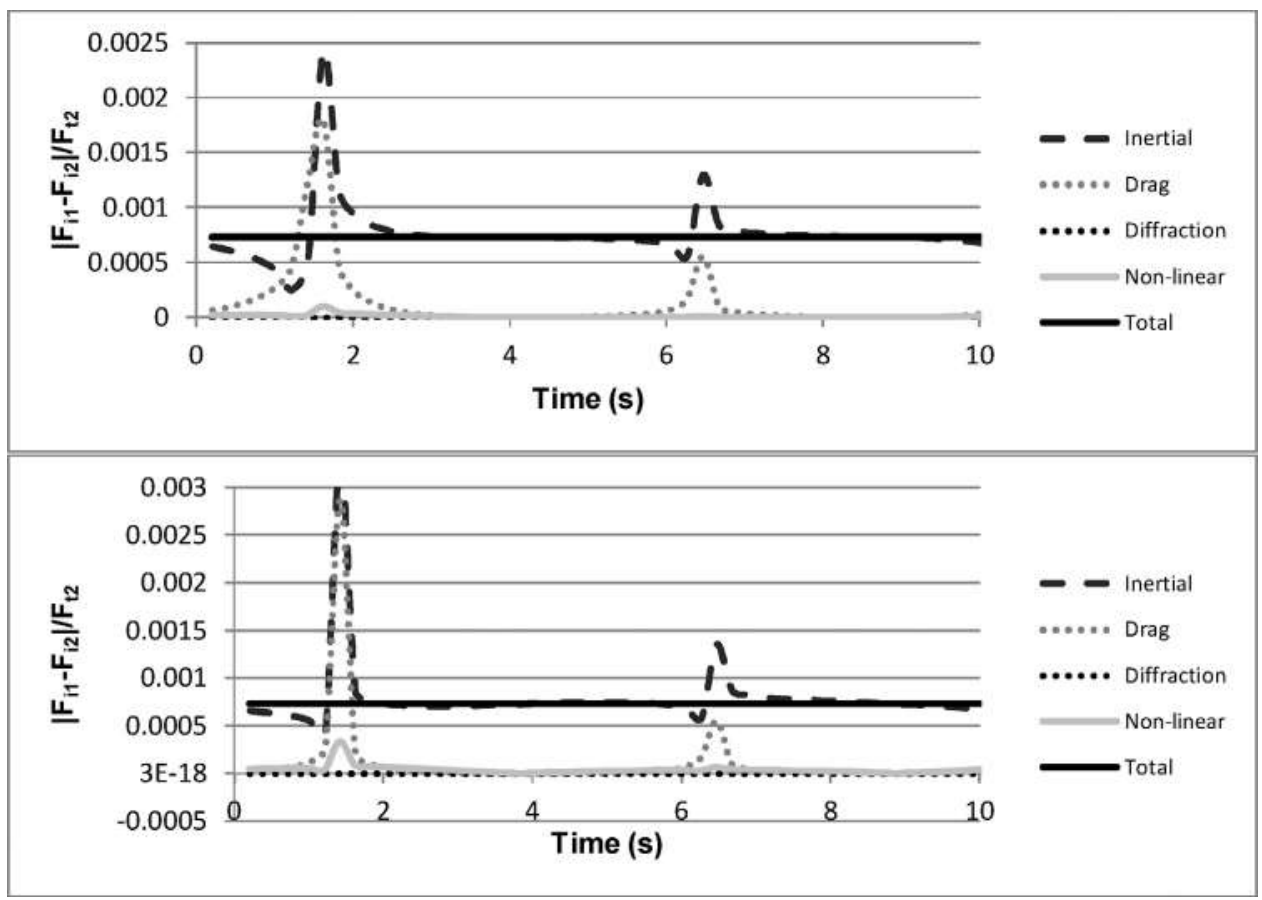

Fig. 11. Simulation with $D=20.0 \mathrm{~m}$, on a free surface in the figure above, and at the sea bed in the figure below.

Case 7: Diameter $=\mathbf{2 6 . 0} \mathrm{m}$

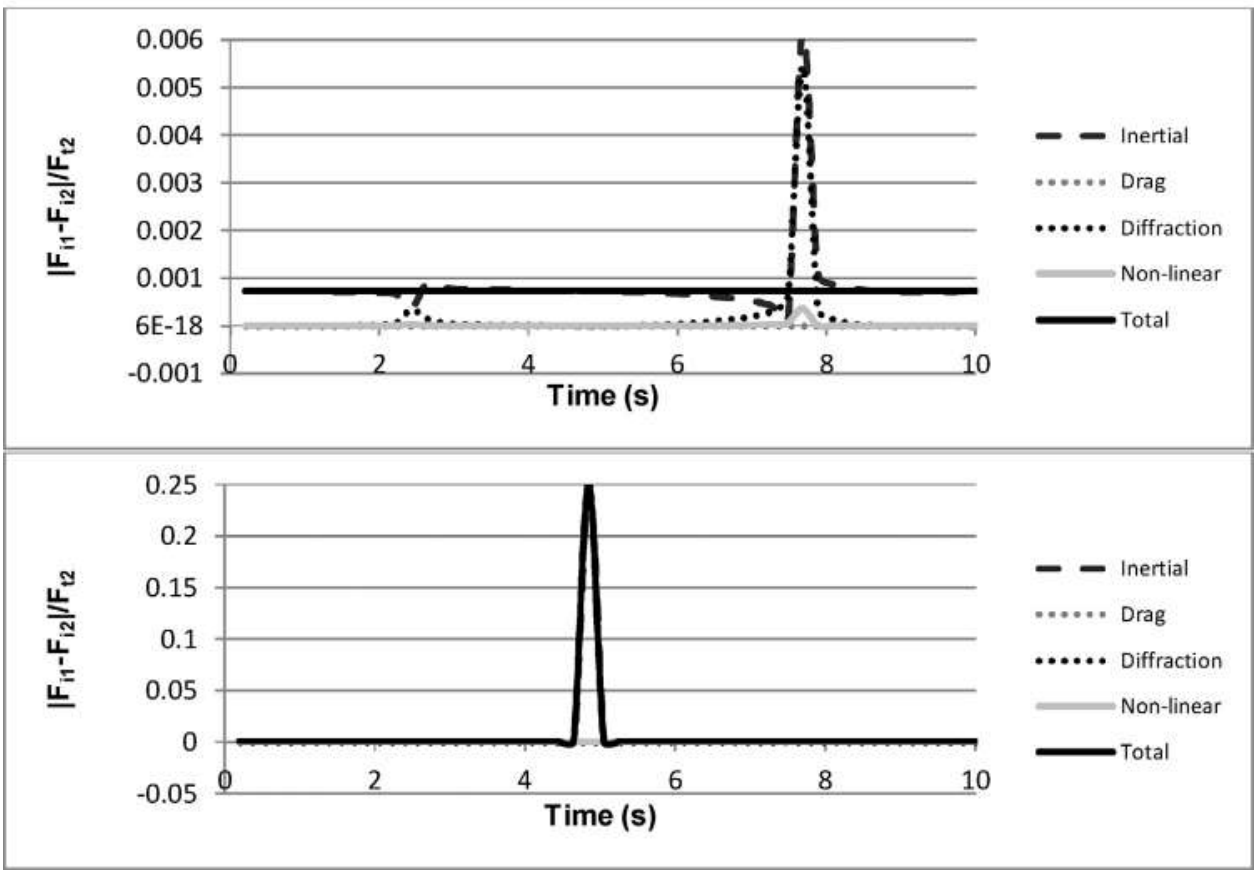

Fig. 12. Simulation with $D=26.0 \mathrm{~m}$, on a free surface in the figure above, and at the sea bed in the figure below.

of maximum values.

$\%=\frac{F_{22^{\circ} \mathrm{C}, 35 \% S}-F_{1{ }^{\circ} \mathrm{C}, 0 \% S}}{F_{1{ }^{\circ} \mathrm{C}, 0 \% S}}$

Table 3 shows that the maximum value of the total force generated is higher for the case of $1^{\circ} \mathrm{C}$ and salinity of
$0.0 \%$ than the force generated in the case of $22^{\circ} \mathrm{C}$ and salinity of $35 \%$. But the higher the diameter values, the smaller are the influences of temperature and salinity. However, the variations observed in Table 3 for the total load are quite smaller than those shown in Table 2, so maximum variations are not necessarily related to maximum loads. 
Table 2

Maximum variations shown for each type of force.

\begin{tabular}{|c|c|c|c|c|c|c|c|c|c|c|}
\hline \multirow[b]{2}{*}{ Diameter } & \multicolumn{2}{|c|}{ Inertial $\mathrm{F}$. } & \multicolumn{2}{|l|}{ Drag F. } & \multicolumn{2}{|c|}{ Diffraction F. } & \multicolumn{2}{|c|}{ Nonlinear F. } & \multicolumn{2}{|l|}{ Total } \\
\hline & Surface & Bottom & Surface & Bottom & Surface & Bottom & Surface & Bottom & Surface & Bottom \\
\hline$D=0.5$ & $40.80 \%$ & $527.27 \%$ & $33.44 \%$ & $238.02 \%$ & $0.00 \%$ & $0.00 \%$ & $0.19 \%$ & $0.14 \%$ & $31.95 \%$ & $289.11 \%$ \\
\hline$D=2$ & $23.47 \%$ & $22.27 \%$ & $0.83 \%$ & $0.35 \%$ & $0.00 \%$ & $0.00 \%$ & $0.02 \%$ & $0.03 \%$ & $23.22 \%$ & $22.54 \%$ \\
\hline$D=4$ & $24.30 \%$ & $18.54 \%$ & $0.44 \%$ & $0.54 \%$ & $0.00 \%$ & $0.00 \%$ & $0.01 \%$ & $0.04 \%$ & $24.65 \%$ & $18.54 \%$ \\
\hline$D=8$ & $2.22 \%$ & $16.94 \%$ & $2.24 \%$ & $0.95 \%$ & $0.00 \%$ & $0.00 \%$ & $0.05 \%$ & $0.04 \%$ & $0.07 \%$ & $16.93 \%$ \\
\hline$D=16$ & $0.84 \%$ & $20.17 \%$ & $0.78 \%$ & $22.17 \%$ & $0.00 \%$ & $0.00 \%$ & $0.01 \%$ & $2.08 \%$ & $0.07 \%$ & $11.61 \%$ \\
\hline$D=20$ & $0.24 \%$ & $0.33 \%$ & $0.18 \%$ & $0.29 \%$ & $0.00 \%$ & $0.00 \%$ & $0.01 \%$ & $0.03 \%$ & $0.07 \%$ & $0.07 \%$ \\
\hline$D=26$ & $0.62 \%$ & $25.08 \%$ & $0.00 \%$ & $0.00 \%$ & $0.55 \%$ & $0.05 \%$ & $0.04 \%$ & $0.02 \%$ & $0.07 \%$ & $25.08 \%$ \\
\hline
\end{tabular}

Table 3

Influence on the maximum force generated.

\begin{tabular}{llc}
\hline & \multicolumn{2}{l}{ Total } \\
\cline { 2 - 3 } Diameter & Surface & Bottom \\
\hline$D=0.5$ & $0.07 \%$ & $-10.73 \%$ \\
$D=2$ & $-2.12 \%$ & $-1.06 \%$ \\
$D=4$ & $-0.96 \%$ & $-0.47 \%$ \\
$D=8$ & $-0.46 \%$ & $-0.19 \%$ \\
$D=16$ & $-0.12 \%$ & $-0.07 \%$ \\
$D=20$ & $-0.10 \%$ & $-0.04 \%$ \\
$=26$ & $-0.09 \%$ & $-0.01 \%$ \\
\hline
\end{tabular}

\section{Conclusions and discussion}

The intention throughout this study was to observe the influence of temperature and salinity on hydrodynamic forces. As observed, temperature and salinity act as a diameter amplifying component allowing the discontinuity or leap very clearly observed in inertia forces, to reduce in width. This width is the amount of time during which that discontinuity manifests. This effect is also observed when increasing the pile's diameter and therefore the increase in temperature and salinity is partly assimilated to the effect that an increase in diameter would have. However, viewed from the perspective of maximum loads generated, an increase in temperature has the opposite effect, giving maximum forces for the lower values of temperature and salinity.

It may be noted also that the maximum influence is exerted on elements with the smallest diameters and at the sea bed, so for large diameters, the influence has almost disappeared on the free surface.

Finally, it may be concluded that the influence of temperature and salinity acts over the whole load profile of the wave, but has minimum influence on maximum load values. Hence, neither parameter will have any influence on simplified models of calculation which do not take the dynamic behavior of the structure and the coupling between the sea waves and the structural elements into account. However, correct values of temperature and salinity will have to be observed in complex calculation models as developed for the current research which takes into account the whole load profile of the sea wave due to the variations of the load profile generating a variation in the induced vibrations over the structure. Therefore, these temperature and salinity variations will be able to generate load increments in the range of $15-30 \%$ in some cases, and need to be considered in modern calculation models.

\section{References}

[1] M.D. Esteban, J.J. Diez, J.S. López-Gutiérrez, V. Negro, Renew. Energy J. 36 (2011) 444-450, doi:10.1016/j.renene.2010.07.009.

[2] V. Negro, J.S. López-Gutiérrez, M.D. Esteban, C. Matutano, Renew. Energy J. 63 (2014) 125-132, doi:10.1016/j.rene.2013.08.041.

[3] C. Matutano, V. Negro, J.S. López-Gutiérrez, M.D. Esteban, Renew. Energy J. 57 (2013) 358-365 Beach Erosion Board, doi:10.1016/j.renene. 2013.01.048

[4] S.K. Chakrabarti, Nonlinear Methods in Offshore Engineering, Elsevier Science, New York, 1990

[5] O.M. Faltinsen, Sea Loads on Ships and Offshore Structures, Cambridge University Press, Cambridge, 1990

[6] J.P. Hooft, Advanced Dynamics of Marine Structures, John Wiley, New York, 1982.

[7] T. Sarpkaya, M. Isaacson, Mechanics of Wave Forces on Offshore Structures, Van Nostrand-Reinhold, New York, 1981.

[8] J.R. Morison, M.P. O’Brien, J.W. Johnson, S.A. Schaaf, Petrol. Trans. AIME 189 (1950) 149-154; A.M. Muir Wood, Coastal Hydraulics, Macmillan, New York, 1969.

[9] MacCamy, R.C. and Fuchs, R.A. 1954. Technical Memorandum No. 69 , Beach Erosion Board, Corps of Engineers.

[10] S.K. Chakrabarti, J. Hydraul. Div., ASCE 98 (HY11) (1972) 1895-1909.

[11] S.K. Chakrabarti, W.A. Tam, A.L. Wolbert, in: Proceedings of the 7th Offshore Technology Conference, OTC 2190, Houston, Texas, 1975, pp. 433-447.

[12] J. Lighthill, in: Proceedings of the 2nd International Conference on the Behaviour of Offshore Structures, BOSS'79, London, 1979, pp. 1-40.

[13] J.M.R. Graham, Proceedings in Mechanics of Wave-induced Forces on Cylinders, Pitman, London, 1979, pp. 133-151.

[14] W. Koterayama, M. Nakamura, ASME Trans. J. Offshore Mech. Arctic Eng. 110 (1988) 315-319.

[15] G.R. Cook, E. Simiu, Ocean Eng. 16 (1989) 355-372.

[16] M. Takagi, S-I. Arai, S. Takezawa, K. Tanaka, N. Takarada, Ocean Eng. 12 (1985) 45-97.

[17] O.M. Faltinsen, Ann. Rev. Fluid Mech. 22 (1990) 35-56.

[18] E.V. Koutandos, WSEAS Trans. Fluid Mech. 4 (3) (2009) 85-96.

[19] M. Isaacson, S. Premasirl, G. Yang, J. Waterw. Port Coast. Ocean Eng. 124 (3) (1998) 118-126.

[20] D. Zhu, China Ocean Eng. 27 (3) (2013) 323-334.

[21] S. Zheng, Y. Zhang, Ocean Eng. 104 (2015) 329-343.

[22] A. Feng, Z. Chen, W. Price, Ocean Eng. 101 (2015) 131-141.

[23] Y. Cao, R.F. Beck, J. Ocean Eng. Sci. 1 (2016) 11-29.

[24] Q. Meng, C. Zhang, J. Ocean Eng. Sci. 1 (2016) 93-108.

[25] D. Das, J. Ocean Eng. Sci. 1 (2016) 135-148.

[26] S.K. Chakrabarti, Handbook of Offshore Engineering, Elsevier Science, New York, 2005 
4. Ponencias y comunicaciones en congresos

4.1. The 14th International Coastal Symposium (ICS), 2016 (Sidney, Australia)

4.2. XIV Jornadas Españolas de Ingeniería de Costas y Puertos 2017 (Alicante, España) 

SOFTWARE TO PREDICT HYDRODYNAMIC PRESSURES ON OFFSHORE PILE FOUNDATIONS. THE NEXT STEP IN OCEAN ENERGY DEVELOPMENT.

Escobar A. ${ }^{1}$, Negro V. ${ }^{2}$, López-Gutiérrez J.S. ${ }^{3}$, Esteban M.D. ${ }^{4}$

${ }^{1}$ Technical University of Madrid, C/Profesor Aranguren S/N, 28040 Madrid, adrian.escobar.pastor@alumnos.upm.es

${ }^{2}$ Technical University of Madrid, C/Profesor Aranguren S/N, 28040 Madrid, vicente.negro@upm.es

${ }^{3}$ Technical University of Madrid, C/Profesor Aranguren S/N, 28040 Madrid, josesantos.lopez@upm.es

${ }^{4}$ Technical University of Madrid, C/Profesor Aranguren S/N, 28040 Madrid, mariadolores.esteban@upm.es

The purpose of this study is to carry out a thorough, critical analysis of the Morison equation as far as calculating wave action on large diameter piles like those used nowadays in offshore wind farms is concerned. The aim is to observe whether models currently used to estimate wave forces on piles are valid for large diameter piles apart from observing which the main forces in play are in scouring. This equation enables wave produced forces on a cylinder supported on the sea bed to be calculated. The study includes observations on the calculation model's sensitivity as to a variation in the cylinder's diameter, on the one hand and, on the other, as to temperature variation. With this in mind, specific software has been developed which develops and simulates equations in fluid mechanics applied to the Morison equation, as shown in the figure 1 . This software will enable an iterative calculation to be made for finding out the shape the pressure wave caused when a wave passes over will have for different pile diameters and water temperature.

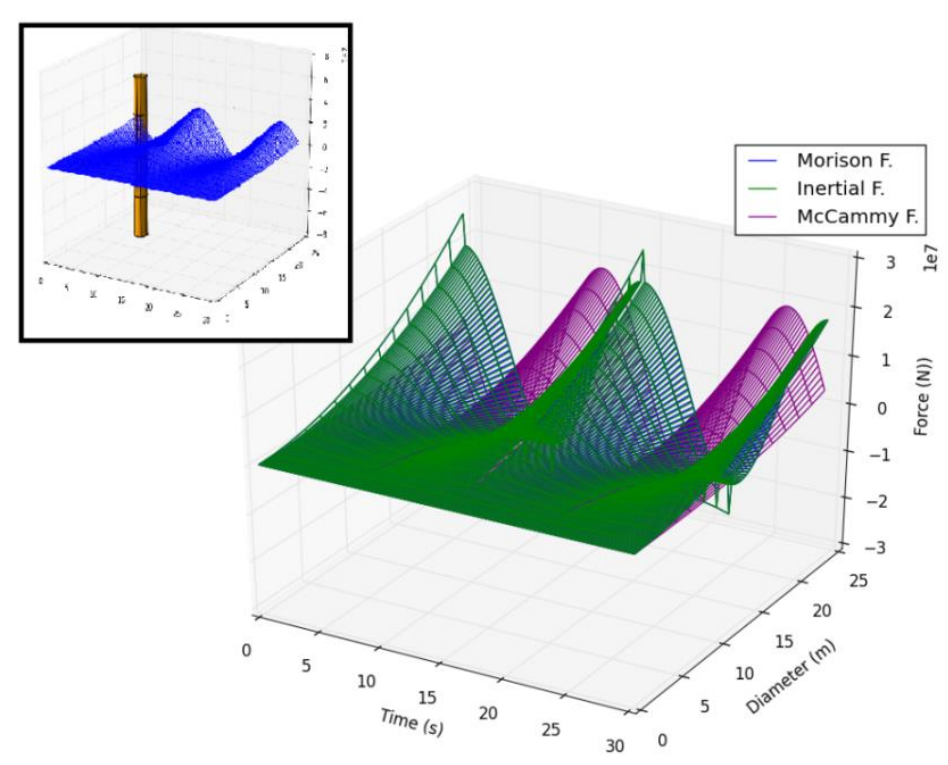

Figure 1. Development of software to calculate the pressures in offshore pile foundations. 



\title{
COMPORTAMIENTO HIDRODINÁMICO Y SENSIBILIDAD DE LOS ESQUEMAS DE DISEÑO EN ESTRUCTURAS DE GRAVEDAD APLICADAS A ENERGÍAS EÓLICAS MARINAS
}

\author{
A. Escobar, J.S. López, M.D. Esteban, V. Negro
}

Universidad Politécnica de Madrid, Grupo de Medio Marino, Costero y Portuario y otras Áreas Sensibles, C/Profesor Aranguren S/N, 28040 Madrid, España. adrian.escobar.pastor@alumnos.upm.es, josesantos.lopez@upm.es, mariadolores.esteban@upm.es, vicente.negro@upm.es

\section{INTRODUCCIÓN}

Las distintas tipologías estructurales empleadas en energías eólicas marinas dependen básicamente de la capacidad portante del terreno, las profundidades del emplazamiento, las características del parque y las afecciones a la costa, tanto a nivel línea de orilla, como de percepción visual y paisajística. El empleo de estructuras, tanto de gravedad (GBS), como pilotadas, trípodes, flotantes o de tecnología offshore, queda condicionada por los datos de partida descritos anteriormente. Además, podría añadirse que el cálculo de las fuerzas hidrodinámicas en las estructuras offshore, es uno de los problemas clave, para los ingenieros de diseño de hoy en día, que participan en la ingeniería marítima, como sugiere Negro et al. (2014).

\section{METODOLOGÍA}

El objetivo de esta reflexión es facilitar la toma de decisiones en estructuras de gravedad empleando esquemas de cálculo en dominios hidrodinámicos diferenciados, $D / L<$ 0.20 (Morison), D/L > 0.20 (difracción), observando cómo se pueden complementar los modelos basados en el cálculo de las fuerzas de inercia y arrastre, con los esquemas en presiones dinámicas derivados de la mecánica de ondas, en el diseño previo.

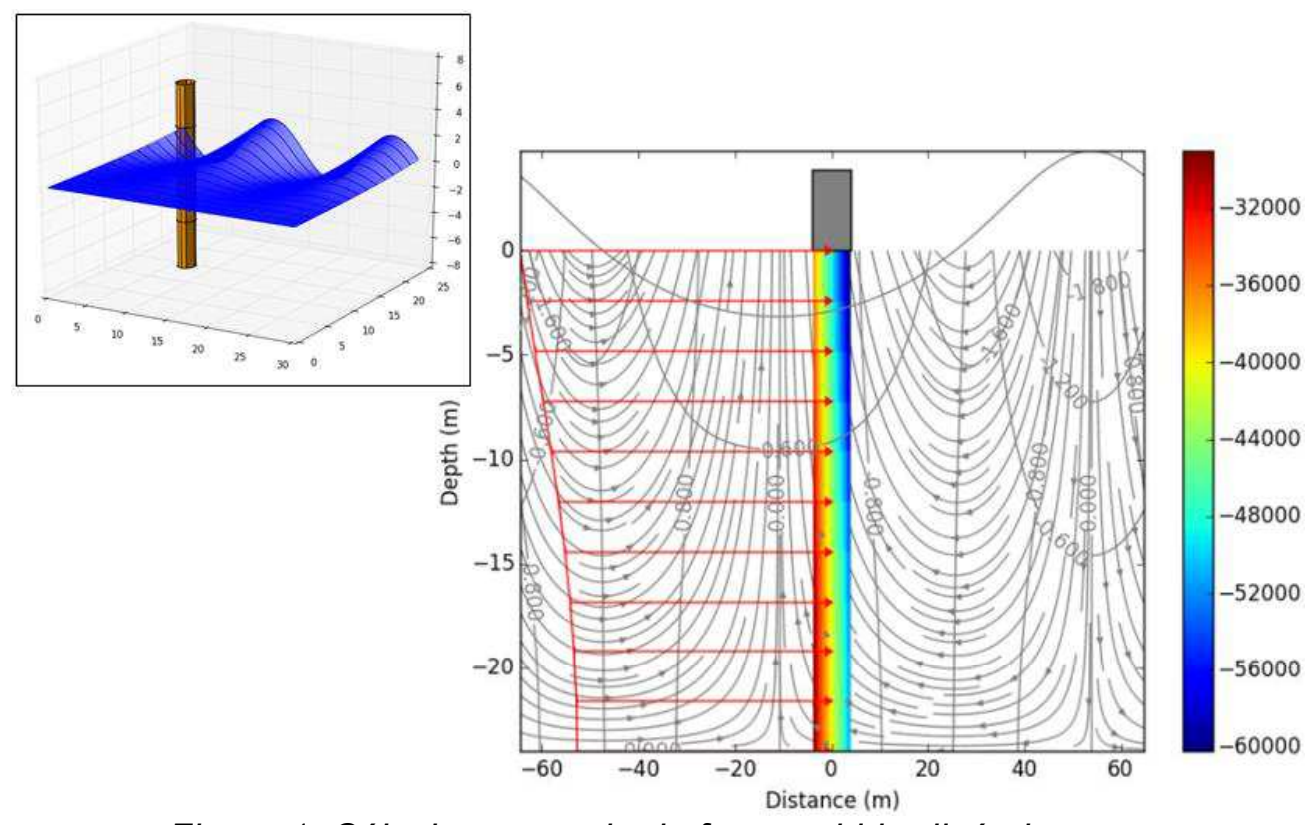

Figura 1. Cálculo avanzado de fuerzas hidrodinámicas

Para ello se va a utilizar el modelo de cálculo avanzado, desarrollado por Escobar et al. (2016a), que tendrá en cuenta: la caracterización completa de la mecánica de ondas, proporcionando los valores del campo de velocidades, aceleraciones y presiones, en cada 
punto del fluido; la caracterización de las fuerzas hidrodinámicas sobre la estructura, teniendo en cuenta la aparición de efectos de no lineales y de difracción; y por último, incorporando en el análisis, el comportamiento dinámico de la estructura y el efecto de acoplamiento que sufre la misma, con el oleaje incidente.

Además, el modelo de cálculo avanzado incorporará por primera vez en la historia, la influencia de la temperatura del agua y la salinidad, sobre las fuerzas hidrodinámicas generadas, tal y como describe Escobar et al. (2016b).

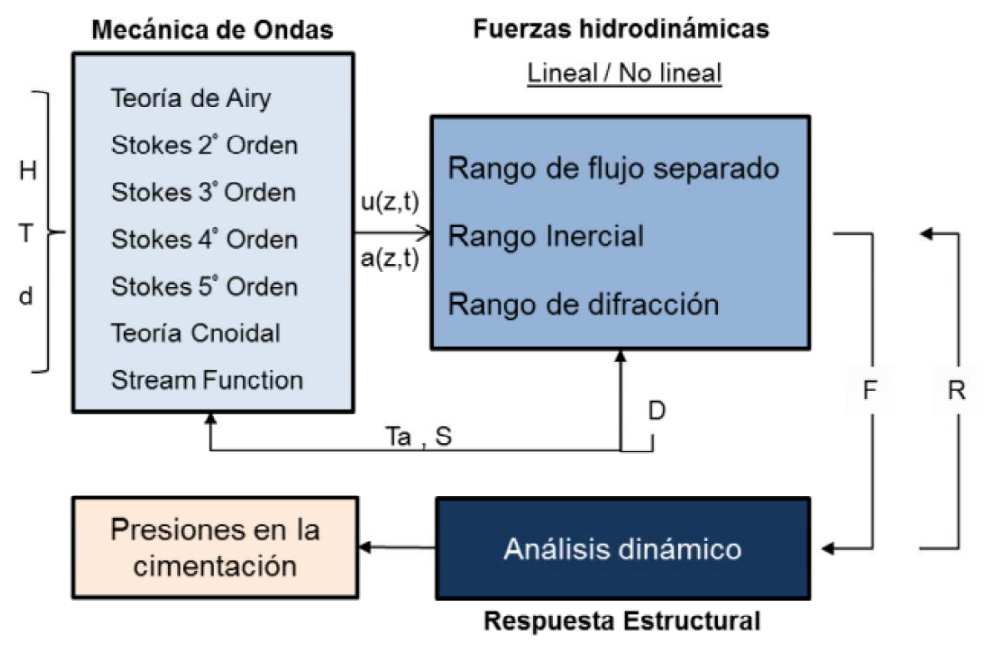

Figura 2. Esquema de cálculo avanzado de fuerzas hidrodinámicas

Donde las variables mostradas son: la altura de ola $(\mathrm{H})$, el periodo $(\mathrm{T})$, la profundidad $(\mathrm{d})$, la temperatura del agua (Ta), la salinidad (S), el campo de velocidades (u) y el de aceleraciones (a), el diámetro de la estructura (D), la fuerza hidrodinámica generada (F) y la respuesta estructural $(R)$.

\section{RESULTADOS}

Con este objetivo en mente, se plantean tres casos de estudio; el primero, tratando la estructura de la figura 1 , como si fuese directamente un dique vertical con diagrama cuasiestacionario y modelo de cálculo tipo Goda; el segundo, como si la estructura fuese un pilote de gran diámetro sometido a las fuerzas de inercia y arrastre de Morison y, el tercero, dividiendo la estructura en la zona gravitatoria con esquema Goda y en la zona de torre con esquema Morison, observando el nivel de confianza y la bondad del ajuste para esta tipología de estructuras.

\section{CONCLUSIÓN}

El concepto evolutivo es, en estos momentos, la gran profundidad, para la tecnología oil and gas $u$ offshore, en instalaciones flotantes o de tipo jacket. Esta primera recomendación puede servir para emplazamientos entre 20 y 40 metros de lámina de agua, y terrenos muy competentes, donde las estructuras de gravedad pueden emplearse.

\section{REFERENCIAS}

Escobar, A., Negro, V., López-Gutiérrez, J.S., Esteban, M.D, 2016a. Software for predicting hydrodynamic pressures on offshore pile foundations. The next step in ocean energy development. Journal of Coastal Research, Special Issue, 75, 841-845.

Escobar, A., Negro, V., López-Gutiérrez, J.S., Esteban, M.D, 2016b. Influence of temperature and salinity on hydrodynamic forces. Journal of Ocean Engineering and Science 1, 325-336.

Negro, V., López-Gutiérrez, J.S., Esteban, M.D., Matutano, C., 2014, Uncertainties in the design of support structures and foundation for offshore wind turbines, Renewable Energy Journal, 63, 125-132. 